Universidad Politécnica de Madrid

Escuela Técnica Superior de Edificación

\title{
ESTUDIO EXPERIMENTAL Y MODELIZACIÓN DE LA ILUMINACIÓN NATURAL EN LA EDIFICACIÓN MEDIANTE MODELOS A ESCALA
}

TESIS DOCTORAL
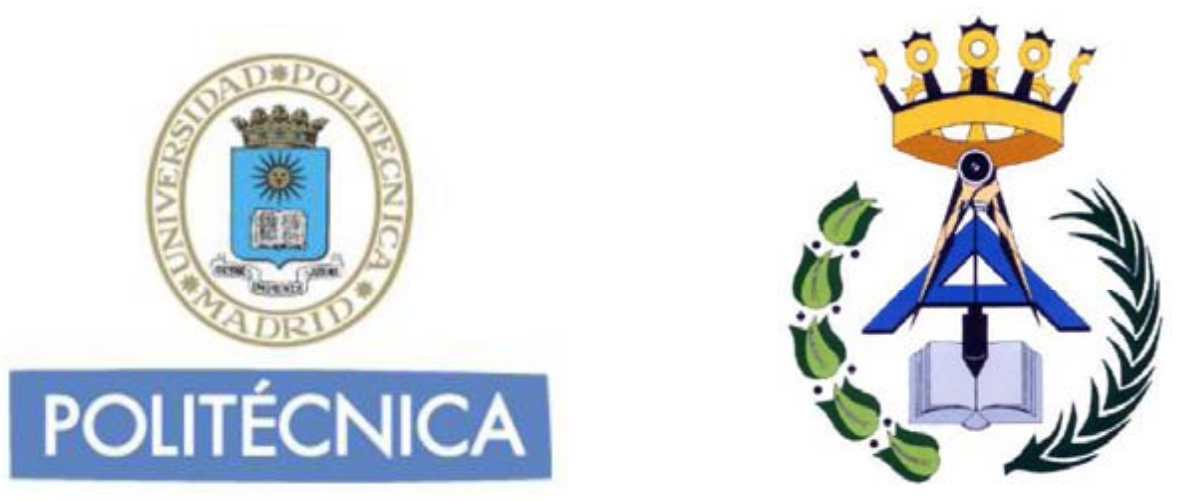

CAROLINA CABRERA MERTENS

(Ingeniero de Edificación) 

Departamento de Tecnología de la Edificación

Escuela Técnica Superior de Edificación

\section{ESTUDIO EXPERIMENTAL Y MODELIZACIÓN DE LA ILUMINACIÓN NATURAL EN LA EDIFICACIÓN MEDIANTE MODELOS A ESCALA}

Autor

CAROLINA CABRERA MERTENS

Director/a o Directores/as

CARLOS MORÓN FERNÁNDEZ

MERCEDES GONZÁLEZ REDONDO 

Tribunal nombrado por el Mgco. y Excmo. Sr. Rector de la Universidad

EL TRIBUNAL CALIFICADOR

Presidente:

Vocal:

Vocal:

Vocal:

Secretario:

Realizado el acto de defensa y lectura de la Tesis: 

AGRADECIMIENTOS

Quisiera dar las gracias a mis directores de tesis, por darme la oportunidad de trabajar en este proyecto así como por su ayuda y disponibilidad.

Agradecer especialmente a mi familia y amigos por su paciencia, apoyo y comprensión. 


\section{RESUMEN}

La presente tesis tiene como principal objetivo estudiar el comportamiento de la luz natural en distintos puntos de una estancia para, de este modo, no solo optimizar el número de iluminarias necesarias manteniendo una adecuada iluminación (según la normativa), sino que también conocer los puntos adecuados donde colocar dichas luminarias para optimizar al máximo la iluminación en una estancia.

Para lograr dicho objetivo la presente tesis propone un método con el cual predecir la iluminación natural que va a tener una estancia (en fase de diseño) a través de medidas de iluminancias tomadas en un modelo a escala de dicha estancia y de las medidas de iluminancia tomadas en el exterior donde vaya a estar situada dicha estancia. El método consiste en aplicar a una ecuación los valores de iluminancias tomadas en un modelo a escala y las medidas de iluminancia tomadas en el exterior a una distancia concreta, y de este modo hallar las iluminancias de la estancia que simulaba el modelo a escala. La presente tesis se centra en hallar dicha ecuación.

Inicialmente se ha realizado un estudio teórico sobre la luz natural a lo largo de la historia, así como un análisis de los fundamentos teóricos sobre las magnitudes luminosas con sus unidades de medida, la propagación de la luz, sistemas de iluminación natural y de la normativa vigente respecto a la iluminación en edificación.

Posteriormente se ha realizado un estudio experimental, en el que se ha analizado el comportamiento de la luz natural que recibe tanto el aula 2S2 de la Escuela Técnica Superior de Arquitectura de la Universidad Politécnica de Madrid (E.T.S.A.M), que tienen la fachada orientada al Norte, así como su modelo a escala $1 / 15$,

Este estudio experimental comenzó distribuyendo tanto el aula como el modelo a escala en cuatro columnas, de tal manera que la primera denominada Zona 1 
se sitúa a $0.28 \mathrm{~m}$ de la pared Este, la segunda (Zona 2) a $1.56 \mathrm{~m}$ de dicha pared (alineada con el centro de la ventana situada al Este del Aula), la tercera (Zona 3) a $3.17 \mathrm{~m}$ de la pared y la última columna (Zona 4) a $4.78 \mathrm{~m}$ de la pared Este (alineada con el centro de la otra ventana). En cada columna se colocaron sensores a distintas distancias de la cara interior de la fachada tanto en el aula objeto de estudio como a distancias proporcionales en su modelo a escala. Tras obtener y analizar las medidas registradas por los sensores se han hallado distintas ecuaciones para distintas zonas del aula, así como una ecuación general para las cuatro zonas de forma conjunta. Finalmente se han comprado los resultados obtenidos tanto en el aula objeto de estudio como en el modelo a escala del mismo, así como con los resultado obtenidos tras aplicar las ecuaciones halladas en distintas zonas del aula y para las cuatro zonas conjuntamente, con las simulaciones obtenidas a través del programa informático de iluminación Dialux 4.11. 


\section{ABSTRACT}

The present thesis has as main purpose to study the behaviour of daylight at different points inside a room, not only to optimize the number of luminaries needed for a proper illumination (according to the regulation), but also, to know the proper points where to set those luminaries in order to maximize the optimization of the illumination inside a room

In order to reach this goal the present thesis proposes a method with which to predict daylight inside a room (at a design stage) through illuminance measures taken inside a scale model of that room and illuminance measures taken outside the room and the scale model. This method consist of applying an equation to the illuminance values taken inside a scale model at a certain distance from the facade and on the outside, and through this way to find out the illuminances of the room the scale model was simulating. The present thesis has been focused on finding this equation.

Firstly a theoretical study about daylight throughout history has been done, as well as an analysis of the theoretical foundations about light magnitudes with their measurement units, light spread, daylight systems and current regulation on construction field.

Subsequently an experimental study has been carried out, in which the behaviour of daylight inside the 2S2 classroom of the Escuela Técnica Superior de Arquitectura de la Universidad Politécnica de Madrid (E.T.S.A.M) has been analysed, which façade faces the north, as well as its $1 / 15$ scale model, this last one located

This experimental study started dividing the classroom as well as the scale model into four rows, the first one named zone 1, located at $0.28 \mathrm{~m}$ from the eastern wall, the second one (zone 2) at $1.56 \mathrm{~m}$ (align with the centre of the eastern window), the third one (zone 3) at $3.17 \mathrm{~m}$ and the last one (zone 4) at $4.78 \mathrm{~m}$ of the eastern wall (align with the centre of the other window). On each row sensores where located at different distances from the inner side of the façade inside the classroom as well as inside the scale model. After analysing 
the measures recorded by the sensors, different equations for each classroom zone have been obtained, as well as a general equation for the four zones jointly. Finally the results obtained inside the classroom and the scale model, as well as the results obtain after applying the equations obtained for each zone inside the classroom and for the equation obtained from the four zones jointly inside the scale model has been verified, have been verified with simulations obtained with the lighting software Dialux 4.11. 


\section{INDICE}

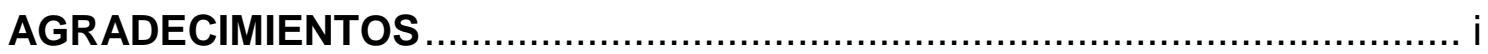

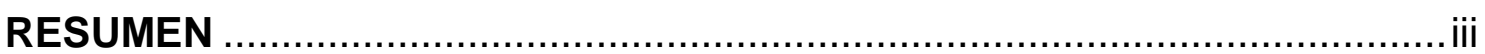

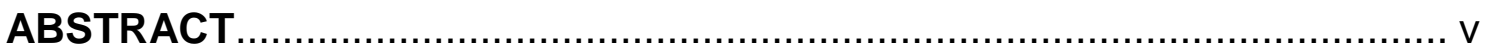

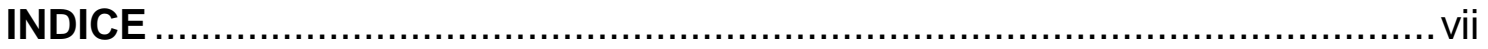

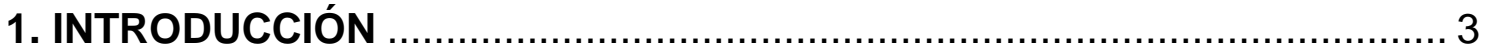

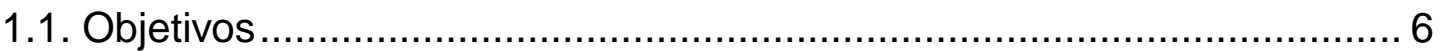

1.2. Antecedentes históricos de la iluminación en la Arquitectura ................. 7

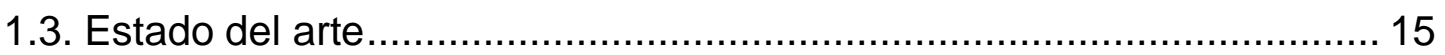

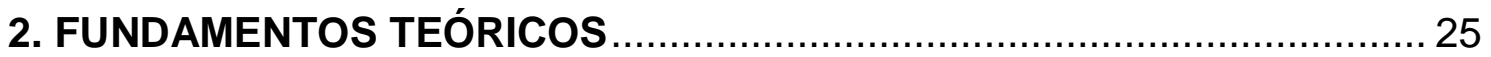

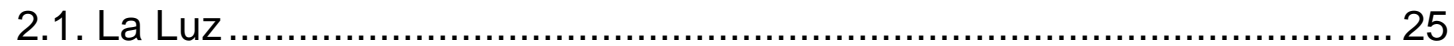

2.2. Magnitudes y unidades luminosas .............................................. 25

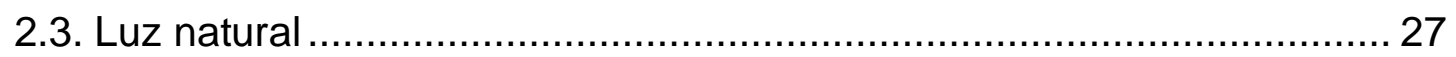

2.4. Relación de la luz con la materia ............................................... 30

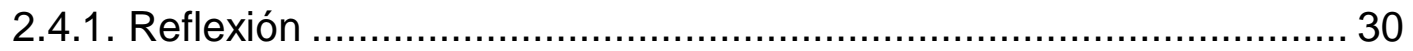

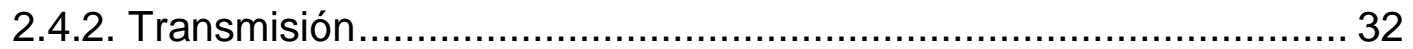

2.4.3. Absorción .............................................................................. 34

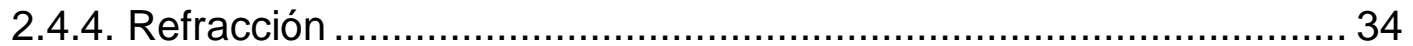

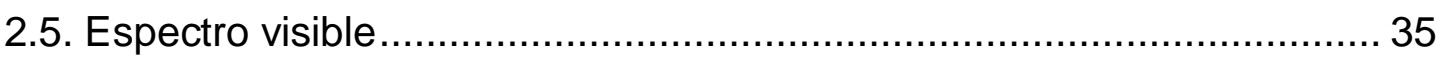

2.6. Sistemas de iluminación natural ....................................................... 35

2.6.1. Estrategias de iluminación natural......................................... 36

2.6.1.1. Orientación ........................................................................ 36

2.6.1.2. Forma, posición y tamaño .................................................. 37

2.6.1.3. Materiales empleados.................................................. 37

2.6.2. Sistemas de aprovechamiento o captación de iluminación natural. 38

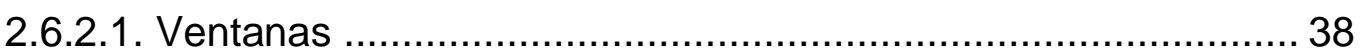

2.6.2.2. Claraboyas.................................................................... 42

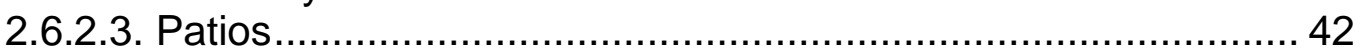




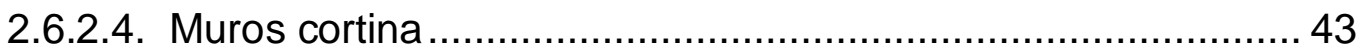

2.6.2.5. Paredes translucidas ...................................................... 43

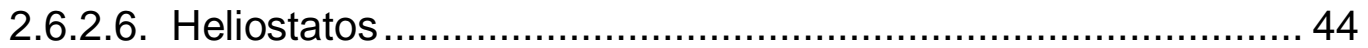

2.6.2.7. Conductos o pozos de luz ................................................. 44

2.6.3. Sistemas de control de iluminación natural ................................... 45

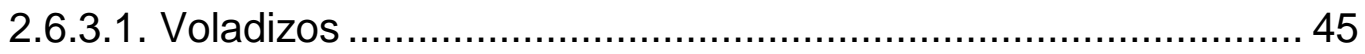

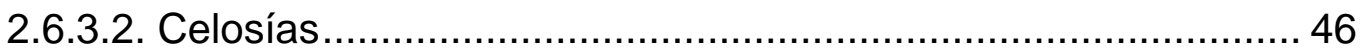

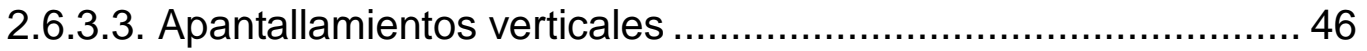

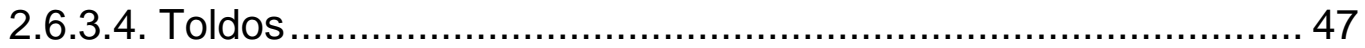

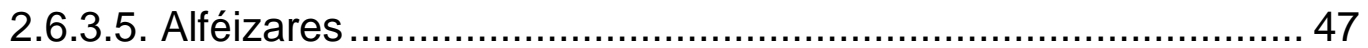

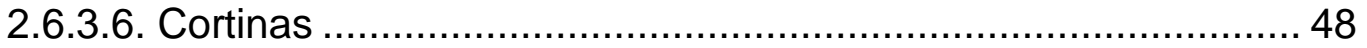

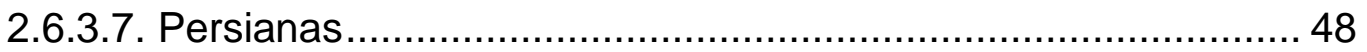

3. NORMATIV A

3.1. Código Técnico de la Edificación (CTE). Ministerio de Vivienda, Gobierno

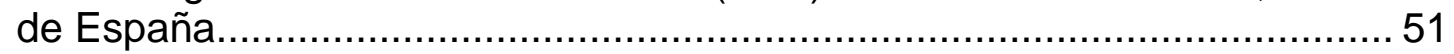

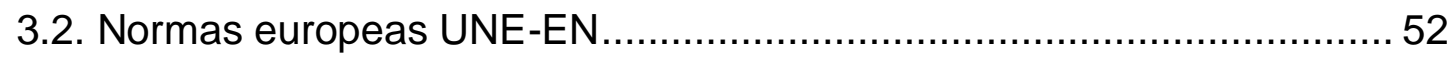

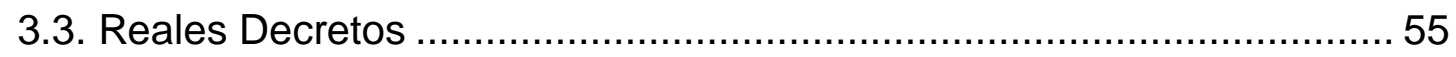

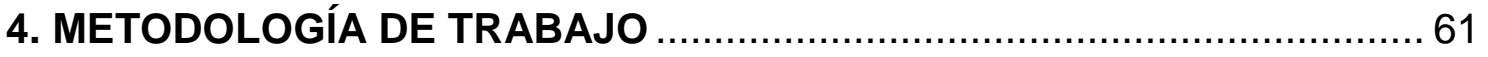

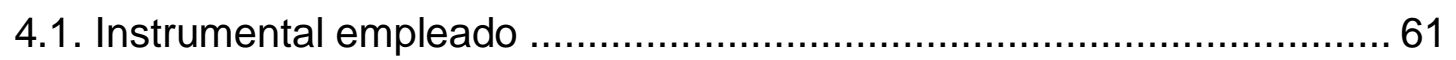

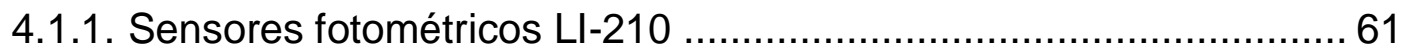

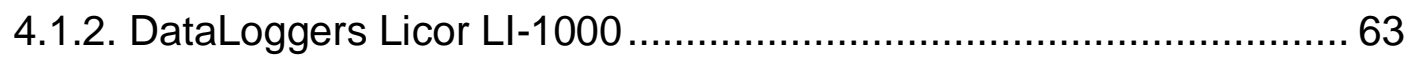

4.2. Aula

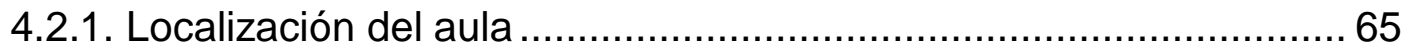

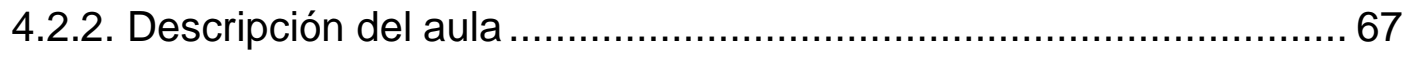

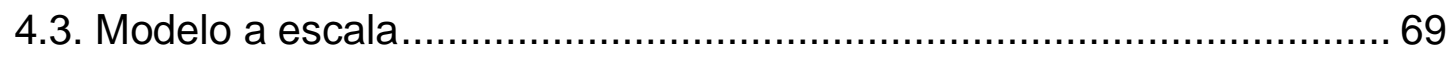

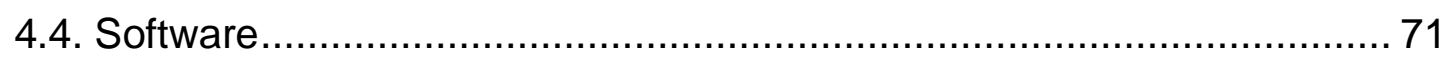

4.4.1. Almacenamiento y tratamiento de datos. ..................................... 71

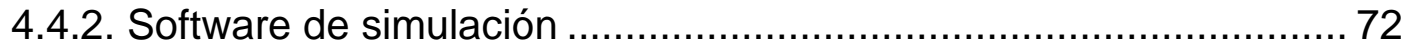

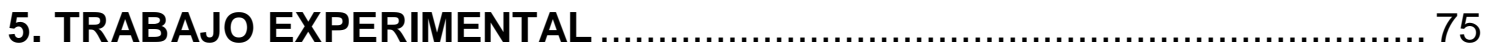

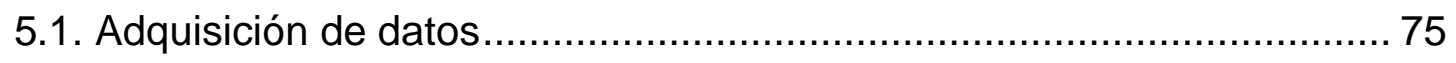

5.1.1. Colocación de los sensores en el aula ........................................ 75

5.1.1.1 Obtención de los datos por zona de manera individual......... 76 
5.1.1.2.Obtención de datosen las cuatro zonas simultáneamente .... 77

5.1.2. Colocación de sensores en el modelo a escala 78

5.1.2.1 Obtención de los datos por zona de manera individual......... 80

5.1 .2 .2$. Obtención de datos en las cuatro zonas simultáneamente ... 81

5.1.3. Colocación del sensor exterior ................................................... 81

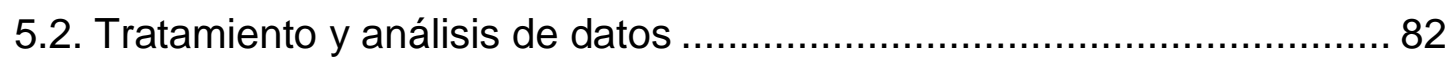

6. RESULTADOS

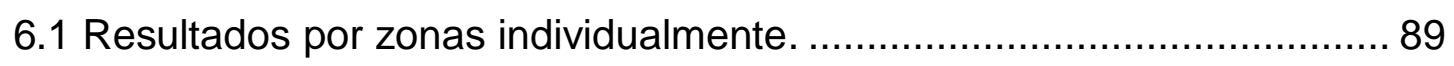

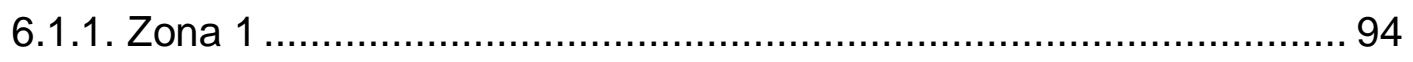

6.1.1.1. Evolución diaria de las iluminancias ......................................99 94

6.1.1.2. Relación entre las iluminancias del aula y del modelo a escala en función de las horas del día........................................................ 100 6.1.1.3. Relación entre las iluminancias del aula y del modelo a escala en función de la iluminancia exterior................................................. 103

6.1.1.4. Modelo matemático para determinar la iluminancia del aula.. 106

6.1.1.5. Análisis estadístico .......................................................... 108

6.1.1.6. Simulación con software de iluminación y comprobación de

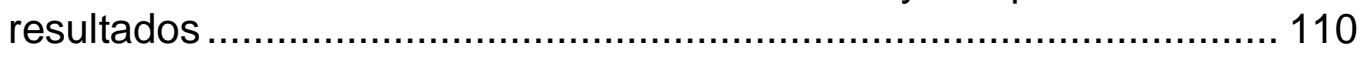

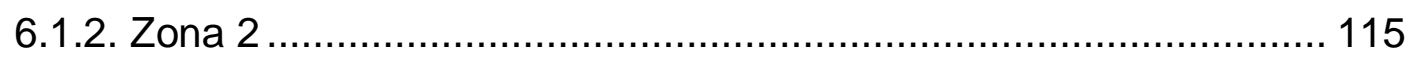

6.1.2.1. Evolución diaria de las iluminancias ..................................... 115

6.1.2.2. Relación entre las iluminancias del aula y del modelo a escala en función de las horas del día....................................................... 120

6.1.2.3. Relación entre las iluminancias del aula y del modelo a escala en función de la iluminancia exterior................................................ 123

6.1.2.4. Modelo matemático para determinar la iluminancia del aula.. 126

6.1.2.5. Análisis estadístico ........................................................... 128

6.1.2.6. Simulación con software de iluminación y comprobación de

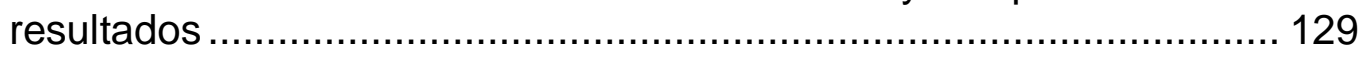

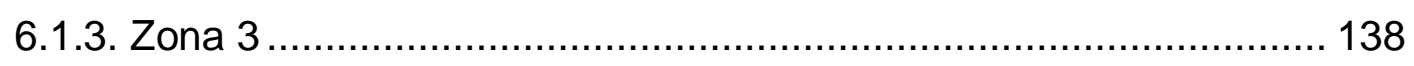

6.1.3.1. Evolución diaria de las iluminancias .................................... 138

6.1.3.2. Relación entre las iluminancias del aula y del modelo a escala en función de las horas del día...................................................... 144

6.1.3.3. Relación entre las iluminancias del aula y del modelo a escala en función de la iluminancia exterior................................................ 147

6.1.3.4. Modelo matemático para determinar la iluminancia del aula. . 150

6.1.3.5. Análisis estadístico ......................................................... 151

6.1.3.6. Simulación con software de iluminación y comprobación de

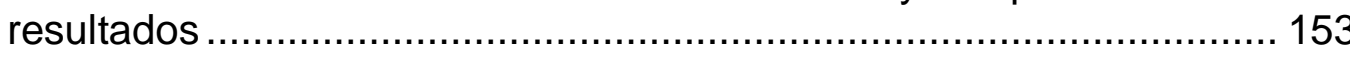


6.1.4.1. Evolución diaria de las iluminancias 160

6.1.4.2. Relación entre las iluminancias del aula y del modelo a escala en función de las horas del día

6.1.4.3. Relación entre las iluminancias del aula y del modelo a escala en función de la iluminancia exterior.

6.1.4.4. Modelo matemático para determinar la iluminancia del aula. . 171

6.1.4.5. Análisis estadístico 173

6.1.4.6. Simulación con software de iluminación y comprobación de resultados

6.2. Resultado de las cuatro zonas conjuntamente. ................................ 180

6.2.1. Evolución diaria de las iluminancias............................................ 180 6.2.2. Relación entre las iluminancias del aula y del modelo en función de la iluminancia exterior....................................................................... 182 6.2.3. Modelo matemático para determinar la iluminancia del aula......... 184 6.2.4. Simulación con software de iluminación y comprobación de resultados.

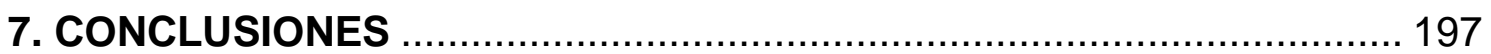

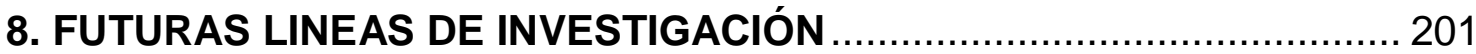

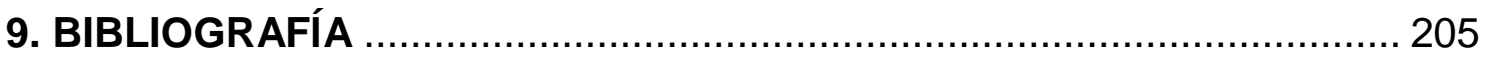

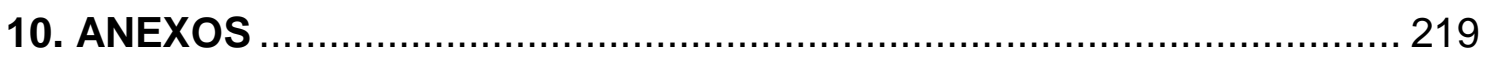

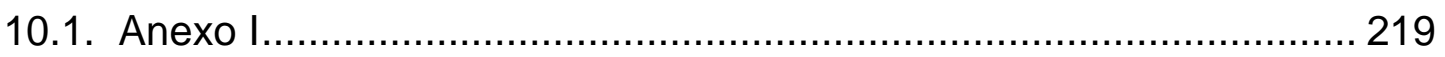

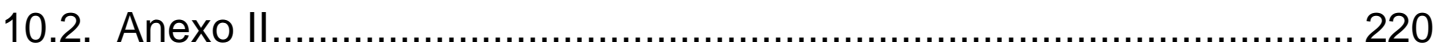

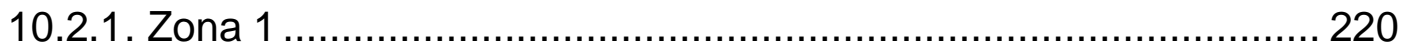

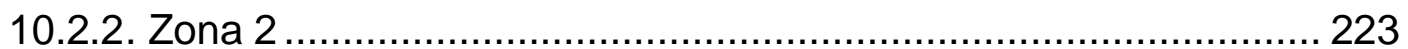

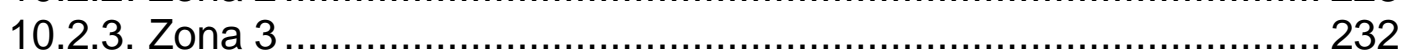

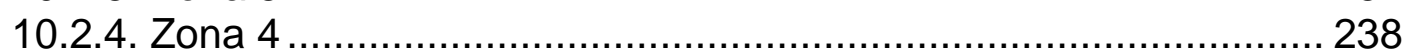


1.INTRODUCCIÓN 



\section{INTRODUCCIÓN}

El cambio climático es uno de los mayores retos a los que nos enfrentamos en la actualidad, generado en parte por un excesivo consumo energético, que aumenta de forma consecutiva el avance de las nuevas tecnologías. Por ello, el ahorro energético es un objetivo clave hoy en día. A raíz de esto se está promoviendo el desarrollo de nuevas estrategias [European Union Directive 2010/31/EU,2010], técnicas y dispositivos tecnológicos destinados a reducir el consumo energético, optimizando el diseño y desarrollando sistemas pasivos de acondicionamiento [Casablanca et al., 2003].

Dado que en los países industrializados el consumo energético debido a la luz artificial supone entre un 5-15\% del consumo energético total en los edificios de viviendas [Ryckaert et al., 2010] y sobre un $25-35 \%$ en edificios de oficinas [Pandharipande et al., 2011], un correcto diseño de los niveles de iluminación en una estancia (tanto en cantidad como calidad), para asegurar el normal desarrollo de las actividades en ellos previstas, buscan minimizar el uso de la iluminación artificial y por lo tanto resulta muy eficaz para reducir el consumo energético [Gonzalo et al., 2001].

Si además de procurar un idóneo diseño del aprovechamiento de la iluminación natural se diseña un adecuado control del intercambio térmico en las superficies transparentes, unido al confort visual de los ocupantes, esto puede suponer un ahorro del $70 \%$ de la energía eléctrica. Si se combina lo anterior con un sistema automático de encendido y apagado de luminarias, este ahorro puede incrementarse hasta un nivel próximo al 90\% [Pattini, 2005].

Algunos de estos sistemas para el aprovechamiento de la iluminación natural y la reducción del consumo energético debido a la luz artificial, se han centrado en diversos elementos para controlar la penetración de luz natural en el interior de las estancias de los edificios [De Carli et al., 2009] [Stanislav et al, 2010] o dispositivos como sensores para controlar el consumo de luz artificial [Van de Meugheuvel et al., 2014]. 
El mal aprovechamiento de la luz natural en edificios no sólo provoca un mayor consumo de energía, sino que también puede favorecer el desarrollo de enfermedades fisiológicas y psicológicas [Boyce, 1998], especialmente en los meses de invierno cuando hay menos horas de sol [Küller, 2002]. Un alto número de estudios [Klein et al., 1991] [Wehr, 1991] [Thorington, 1971] [De Carli et al., 2008] demuestran cómo la luz natural reduce la posibilidad de padecer problemas de salud causados por las rápidas fluctuaciones de la potencia luminosa usual en el alumbrado artificial que afectan tanto al sistema visual humano como a su ritmo biológico.

En la actualidad algunos estudios se están centrando en el análisis de la influencia de la luz natural en el ser humano en estancias de trabajo tales como aulas escolares y despachos, puesto que son los lugares donde las personas desarrollan gran parte de su actividad [Joseph et al., 1999] [Pattini et al., 1998] [Ledesma et al., 2005].

Se ha comprobado que en las aulas escolares con mayor luz natural sus estudiantes progresan un 20-26\% más rápido que aquellos alumnos situados en aulas con menos luz natural en su interior. Además, en el caso de aulas con ventanas de mayores dimensiones los alumnos aumentan su rendimiento escolar en un 15-23\% y en las aulas en las que se puede graduar la entrada de luz diurna, en un 19-20\% [Loisos, 1999].

Un estudio realizado en Canadá [Hathaway et al., 1992] demostró que los alumnos situados en aulas con mejor iluminación, no sólo tenían un bajo índice de absentismo escolar con respecto a aquellos alumnos situados en aulas con peor iluminación, sino que también incrementaban su desarrollo físico.

Otro estudio en Suecia [Küller et al., 1992] afirmó que alumnos situados en aulas sin luz natural tenían un desarrollo físico menor y eran menos sociables que aquéllos situados en aulas con luz natural. Estos estudios permiten afirmar que la luz natural afecta a la capacidad de socialización de los niños así como a su crecimiento físico, estado de ánimo y salud mental. 
Otros estudios realizados en edificios de oficinas [Çakir et al., 1990] [ Küller, 1987] revelan cómo los trabajadores situados más próximos a las ventanas sufren menos problemas de salud que aquéllos situados en lugares donde no alcanza por igual la luz natural. Los empleados situados en oficinas sin ventanas buscan un sistema sustitutorio de las vistas a través de pósters

[De Carli et al., 2008], ya que los seres humanos necesitan la luz natural puesto que los mantiene en conexión con su entorno físico. Los empleados prefieren trabajar en estancias con ventanas, dado que prefieren la luz natural a la artificial, en especial las ubicadas con vistas a la naturaleza, ya que a nivel psicológico estas vistas ayudan a tener una actitud positiva y reducir el estrés [Royce, 2003].

En edificios destinados a personas de la tercera edad, es importante prestar especial atención a un correcto diseño del sistema de iluminación [Royce, 2003] [Edwards et al., 2002], puesto que a medida que el ser humano va envejeciendo su capacidad visual se va deteriorando, de tal manera que conforme el cristalino del ojo humano va perdiendo transmitancia, aumenta la necesidad de luz para poder ver.

Lograr un alto nivel de confort en el interior de los edificios resulta de vital importancia, puesto que el ser humano pasa el $80 \%$ de su vida en el interior de ellos [Alexandridis et al., 2007].

Cuando se alcanza el confort en una estancia o habitación mediante un adecuado uso de la iluminación natural, no sólo aumenta la sensación de bienestar y se reduce el deterioro visual prematuro, sino que también se favorece el aumento de la productividad en dicho habitáculo, e incluso disminuye el absentismo laboral. 


\subsection{Objetivos}

El objetivo principal de este trabajo de investigación es conocer la iluminación natural (en la fase de diseño) en el interior de una estancia para optimizar el número de luminarias necesarias manteniendo una adecuada iluminación (conforme a la normativa vigente). Además, conocer la iluminación en función de la distancia a la fachada para colocar en los puntos adecuados las distintas luminarias necesarias para una iluminación óptima.

Para ello se pretende obtener una ecuación mediante la cual se puedan conocer las iluminancias de un aula (sin tener que tomar medidas in situ) a partir de las iluminancias en su modelo a escala y en el exterior, así como de la distancia a la cara interior de la fachada.

Los objetivos específicos han sido:

-Tener un modelo a escala lo más semejante posible al aula de estudio para realizar comparaciones lo más fidedignas entre éste y el aula.

-Determinar la relación físico-matemática entre la iluminancia del aula y del modelo a escala a lo largo del día, dependiendo de la iluminancia exterior.

-Comparar los resultados de la iluminación del aula obtenidos experimentalmente con los obtenidos por el software de iluminación Dialux 4.11. en el momento actual (2016).

-Analizar cómo varia la iluminancia en los distintos puntos de la estancia dependiendo de la distancia que haya desde éstos a la cara interior de la fachada. 


\subsection{Antecedentes históricos de la iluminación en la Arquitectura}

\section{Prehistoria}

Desde la prehistoria el ser humano ha mostrado gran interés por la luz, asociando la arquitectura y la luz natural a través de monumentos de carácter religioso. Un ejemplo de ello, que todavía se sigue estudiando, es el monumento prehistórico Stonehenge (figura 1.1), emplazamiento neolítico que fue un centro de ritual religioso orientado a la luz recibida y a la energía de los cuerpos celestes, dedicada al movimiento natural del sol, la luna y las estrellas.

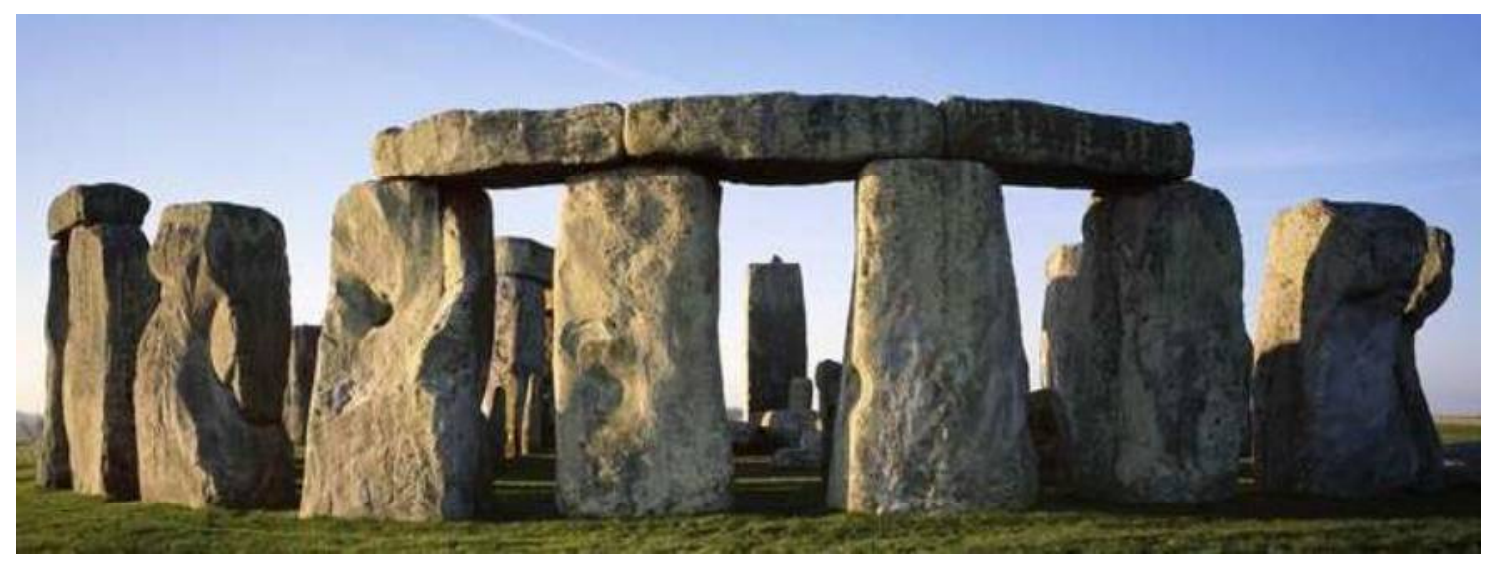

Figura 1.1. Stonehenge (Reino Unido $2500 \mathrm{AC})^{1}$.

\section{Antiguo Egipto}

En el antiguo Egipto los edificios de gran importancia, como los edificios religiosos, estaban concebidos en torno a una planta con un eje longitudinal, que simbolizaba el río Nilo, y construidos de tal forma que la techumbre y el suelo de las salas se iban aproximando a medida que se penetraba en el interior de los mismos, favoreciendo de este modo una disminución gradual de la luz solar en función de la importancia de la sala hasta quedar prácticamente todo el espacio interior sumido en la oscuridad, como se puede apreciar en la figura 1.2 .

\footnotetext{
${ }^{1}$ http://www.english-heritage.org.uk/visit/places/stonehenge/history/. (Última visita 17/12/2016).
} 


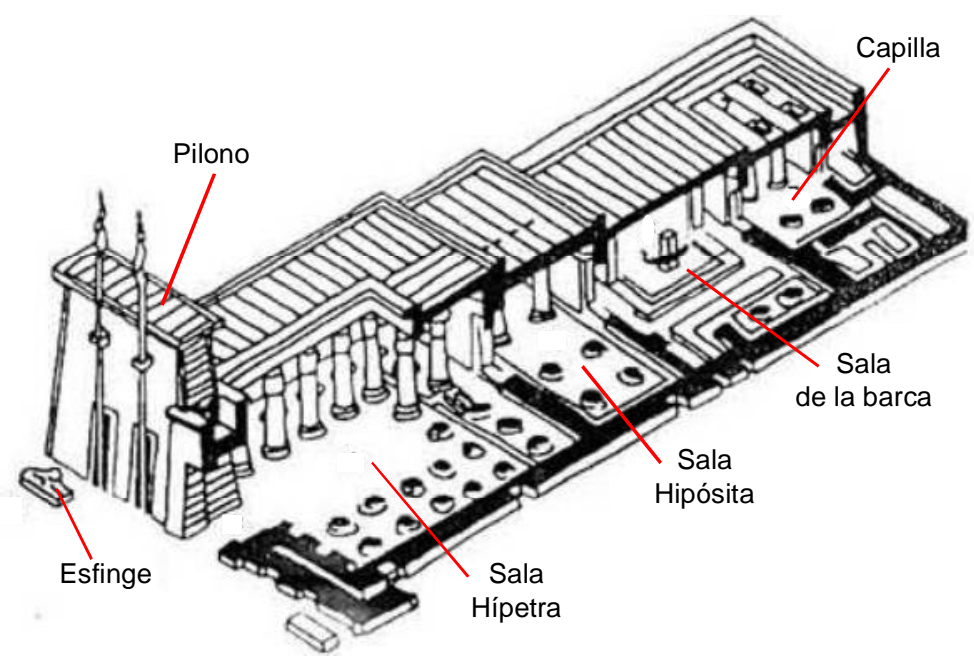

Figura 1.2. Reconstrucción de un templo clásico egipcio.

Algunos arqueólogos aseguran que los antiguos egipcios usaban un sistema complejo de espejos (en aquella época discos de cobre pulimentado) para captar la luz exterior; sin embargo, en este tipo de objetos la absorción de la luz era de algo más que el $30 \%$, por consiguiente al poner más de tres espejos la claridad reflejada era poco apreciable. Este método pues, en la práctica, sólo permitía iluminar lugares poco profundos [Vallejo, 2002].

\section{Antigua Grecia}

Una de las principales edificaciones en la antigua Grecia era el templo, diseñado de forma cuidadosa de tal manera que todos sus componentes guardaran una determinada relación matemática. La planta de los templos griegos podía variar (díptero, tholos, hípetro, períptero, etc...); sin embargo en todas ellas las columnas se disponían alrededor de la cella, estancia dedicada al Dios del templo al que estaba dedicado esta construcción. Los detalles de los templos tales como los triglifos en el friso, las molduras de la cornisa, las estrías de las columnas, etc..., estaban diseñados de tal forma que incidiera directamente sobre ellos la luz del sol y que con los movimientos de la traslación de éste a lo largo del día, se creara un juego de luces que lograra que la apariencia del tiempo cambiara continuamente. Un ejemplo de esto puede apreciarse en el Partenón, construido en el año 440 A.C., en la ciudad de Grecia, que se muestra en la figura 1.3. 


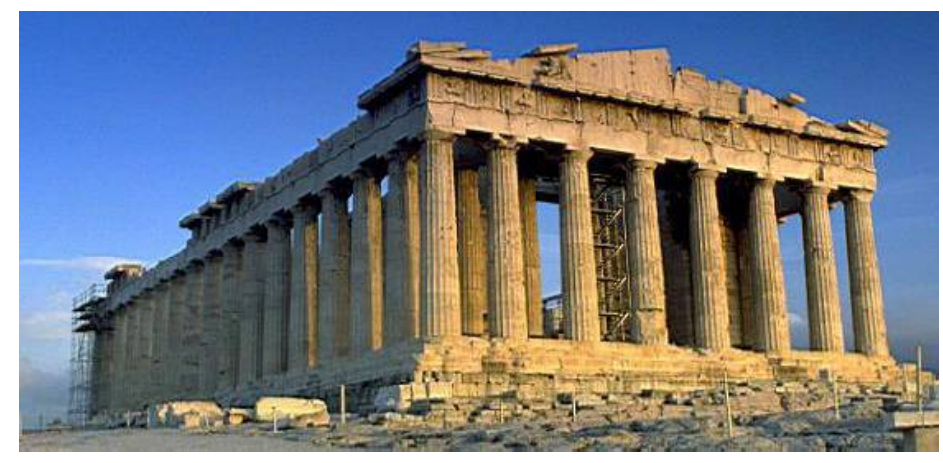

Figura 1.3. Partenón de Atenas. $440 \mathrm{AC}^{2}$.

\section{Antigua Roma}

Los antiguos romanos fueron conocidos, entre otras realizaciones, por sus grandiosas y prácticas construcciones. Su arquitectura se caracterizaba principalmente por su solidez y grandes dimensiones, el empleo de arcos y bóvedas como elementos estructurales de su sistema de construcción, uso de mortero y ladrillo y abundancia de ventanas. Estos arcos, cúpulas, bóvedas, etc..., no sólo permitían construir estancias más grandes sino también más altas.

En ciertos casos el uso de un óculo realzaba el efecto de la iluminación natural en el interior de la construcción. El ejemplo más destacado de ello es el Panteón de Agripa (figura 1.4), con un ojo central (óculo) de $8.92 \mathrm{~m}$ de diámetro en el centro de la cúpula.

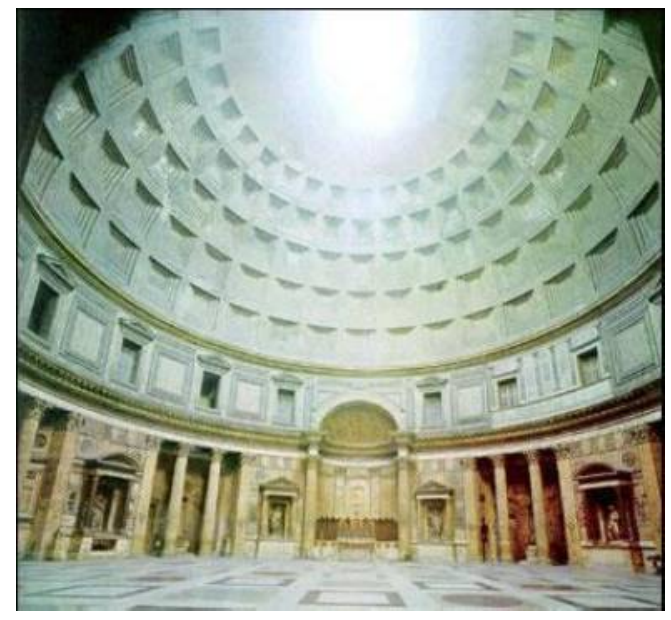

Figura 1.4. Panteón de Agripa ${ }^{3}$.

\footnotetext{
${ }^{2} \mathrm{http}: / /$ www.artehistoria.com/v2/obras/7927.htm. (Última visita 17/12/2016).

${ }^{3} \mathrm{http}: / /$ www.exploraroma.com/el-panteon-de-agripa.htm. (Última visita 17/12/2016).
} 


\section{Arquitectura Bizantina}

La arquitectura bizantina se caracteriza por el uso de materiales pobres como la piedra y el ladrillo en el exterior; en cambio el interior se recubría de materiales lujosos como mosaicos con mármoles, cerámica vidriada y láminas de oro y plata.

La bóveda de Santa Sofía (figura 1.5), construida en Constantinopla entre los años 532-537 sobre pechinas ejecutadas con materiales cerámicos muy ligeros (ánforas), fue diseñada de tal forma que en su base se encuentra una línea de 40 ventanas (noventa originalmente) por las que se infiltra una gran cantidad de luz natural en el templo creando así la sensación óptica de que la cúpula flota sobre el edificio. Esta luz natural se ve intensificada en el interior del edificio gracias a la reflexión aportada por sus mosaicos, sus paredes revestidas de mármol y sus colores dorados y plateados.

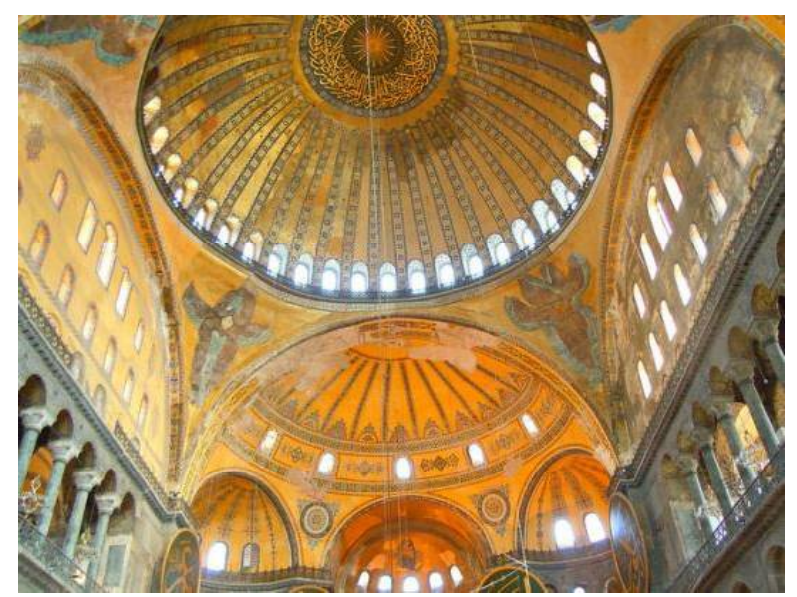

Figura 1.5. Santa Sofía de Constantinopla ${ }^{4}$.

\section{Arquitectura Románica}

La arquitectura románica se caracteriza por la grandeza y robustez de sus templos construidos con objeto de que perduren en el tiempo. Fue buscando la perfección a través de un continuo proceso evolutivo aumentando cada vez más la altura. Debido al gran peso que suponían las bóvedas, los elementos de soporte como los muros eran muy gruesos. Para no reducir la resistencia de los

\footnotetext{
${ }^{4}$ http://seordelbiombo.blogspot.com.es/2012/09/analisis-y-comentario-de-santa-sofia-de.html. (Última visita 17/12/2016).
} 
muros, se abrían pocos y estrechos vanos que tenían una forma abocinada con derrame hacia el interior para extender mejor la luz en el interior del templo.

La tenue luz en el interior de estos templos, que estaba en consonancia con el propósito religioso de aquel tiempo, se centraba en la parte más importante de éste, la cabecera, donde se ubicaba el altar. Esta zona noble del templo se encontraba situada en el extremo oriental del mismo, de tal manera que los primeros rayos de luz bañaran el altar. Un ejemplo de esto es la Iglesia de San Juan de Alba de Tormes en Salamanca (figura 1.6).

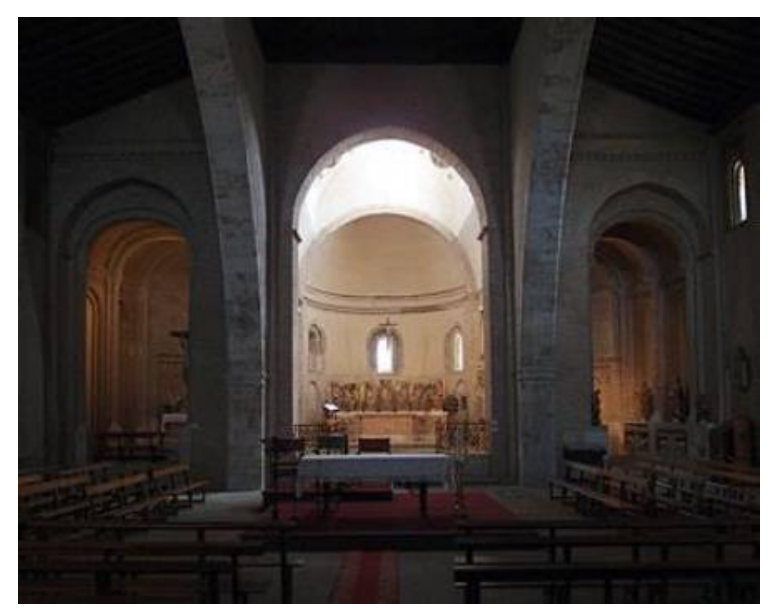

Figura 1.6. Iglesia de San Juan de Alba de Tormes (Salamanca) ${ }^{5}$.

\section{Edad Media (gótico)}

El arte gótico destaca por su búsqueda de la altura y de la luz natural. El edificio en el que el gótico alcanza su mayor esplendor es la catedral, dado que en ella se refleja la vitalidad de las ciudades, la riqueza y prestigio de sus obispos.

Los arquitectos góticos lograron reducir la estructura sustentante al mínimo imprescindible, lo que permitió abrir grandes huecos en los muros de las fachadas. Estos grandes huecos fueron cubiertos por coloridas vidrieras, inundando así el interior de la catedral de luz natural y a la vez mística, porque ésta quedaba transfigurada y coloreada por vitrales (figura 1.7),

\footnotetext{
${ }^{5}$ http://www. arteguias.com/iglesia/sanjuanalbadetormes.htm. (Última visita 17/12/2016).
} 
los cuales cumplían además una función didáctica, semejante a biblias visuales que llevaban el mensaje de Dios a todas las personas.

Entre los elementos decorativos del gótico, destaca el rosetón, que se colocaba en los vanos circulares ubicados en los altos de las fachadas principales.

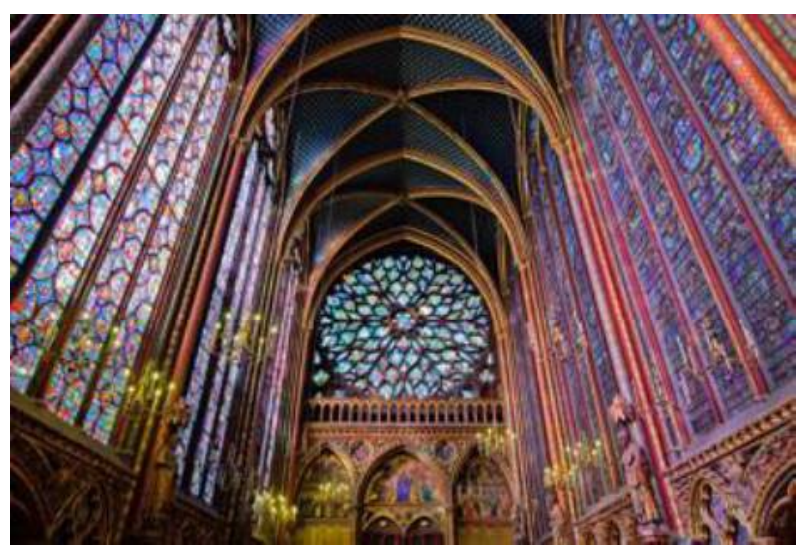

Figura 1.7. Catedral de Santa María de Sydney (Australia) ${ }^{6}$.

\section{Renacimiento}

El renacimiento destaca por su preocupación por las proporciones, la perspectiva y la luz. Admiraba la naturaleza y el modo en que funcionaba la luz en ésta, su armonía y homogeneidad; la naturaleza se veía como lo más próximo a la perfección. Este funcionamiento de la luz sobre la naturaleza, fue llevado al mundo de la nobleza y la burguesía, lo que consiguió que las iglesias compartieran protagonismo con palacios y grandes villas, es decir, que los edificios emblemáticos ya no fueran sólo las iglesias.

Las ventanas eran consideradas un portal hacia la naturaleza, hacia lo que era perfecto. Esto hizo que las fachadas estuvieran dominadas por grandes ventanales, miradores y un mayor número de ventanas con mayores dimensiones.

El arquitecto renacentista buscaba siempre una luz homogénea, con lo que conseguía que ésta reinara el espacio hasta el último rincón de la misma, así que en el interior de los recintos reinaba una luz difusa y uniforme. Puede verse

\footnotetext{
${ }^{6}$ https://lifeaperture.files.wordpress.com/2012/01/dsc0051.jpg. (Última visita 17/12/2016).
} 
un ejemplo de esto en la Iglesia de San Lorenzo en Italia (figura 1.8), donde se une gradualmente la luz natural con la luz interior sin que éstas sean tamizadas.

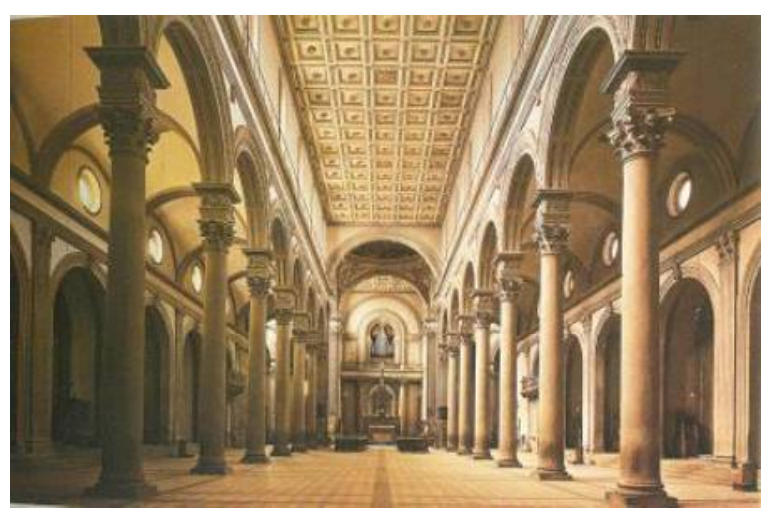

Figura 1.8. Iglesia de San Lorenzo ${ }^{7}$.

\section{Barroco}

La arquitectura barroca se caracteriza por la predilección de lo curvilíneo, la sensualidad de las formas, la abundancia de ornamentación y los reflejos (figura 1.9). Los arquitectos barrocos estudiaban cuidadosamente los efectos de la luz natural en fachadas e interiores, buscaban crear efectos ópticos ilusionistas a base de luces indirectas que a través de claraboyas y ventanas ocultas se proyectaban en el interior. La luz natural se convierte así en un elemento esencial de la arquitectura barroca, pues se usaba para realzar la movilidad de los edificios, multiplicar los ángulos de perspectiva y el dinamismo de las formas.

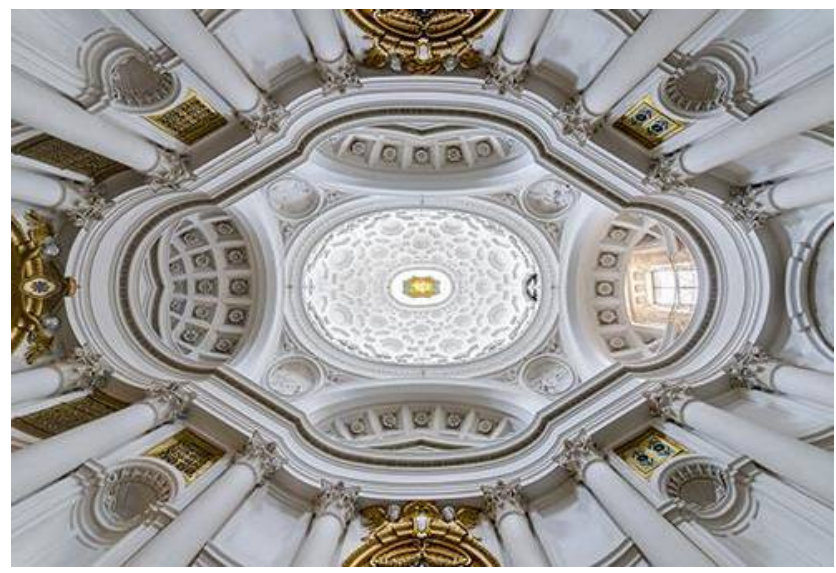

Figura 1.9. Iglesia de San Carlo alle Quattro Fontane (Roma) ${ }^{8}$.

\footnotetext{
7 http://ekokultur.blogspot.com.es/2014/03/brunelleschi-arquitecto-quattrocento.html. (Última visita 17/12/2016).

8 http://www.jmhdezhdez.com/2014/07/frases-bernini-gian-lorenzo-escultor.html. (Última visita 17/12/2016).
} 


\section{Revolución industrial}

El crecimiento industrial del siglo XIX generó nuevas necesidades en los edificios y, para satisfacerlas, se utilizaron nuevos materiales. Debido a la importancia que adquirió el lugar de trabajo y las nuevas formas de producción, como el trabajo en cadena, se necesitó de edificios con altos niveles de iluminación (aparición del efecto estroboscópico). Ello conllevó a la construcción de largas ventanas horizontales.

La revolución industrial potenció el uso de materiales tales como el hierro y el cristal, cuyas cualidades permitían construcciones más esbeltas, rápidas y baratas. Se buscaba una arquitectura más diáfana y con espacios más amplios y prácticos para el trabajo. El acero, debido a su resistencia, permitía minimizar los soportes internos lo que lo hizo ideal para este tipo de arquitectura. Un ejemplo, que permanece en la actualidad, es el mercado de San Miguel en Madrid (figura 1.10).

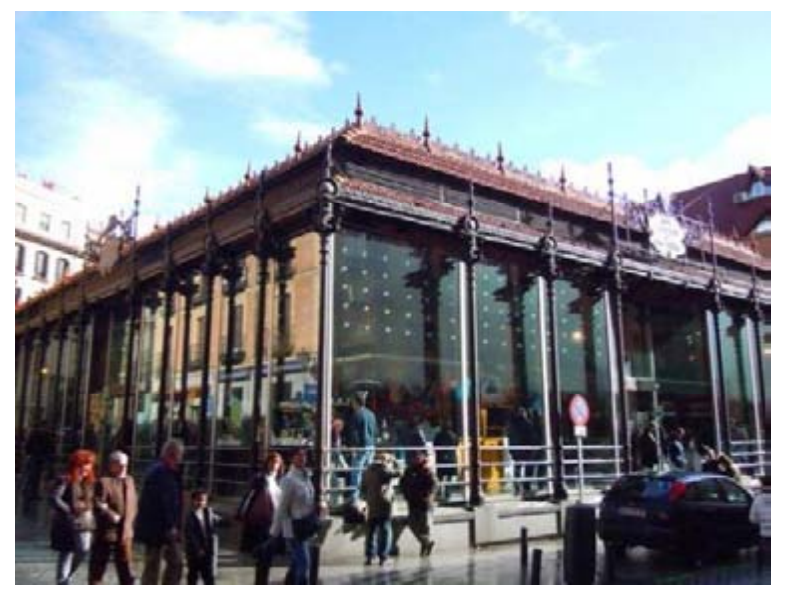

Figura 1.10. Mercado de San Miguel ${ }^{9}$.

\section{Arquitectura Moderna}

La arquitectura moderna se desarrolló a lo largo del Siglo XX. Se caracterizó por el uso de líneas limpias de las fachadas tanto en horizontal como en vertical, el uso de nuevos materiales, como el acero y el hormigón armado, y el centrarse en construir espacios para la vida y la actividad humana a gran escala.

\footnotetext{
${ }^{9}$ http://seordelbiombo.blogspot.com.es/2012/03/el-mercado-de-san-miguel-del-abandono.html. (Última visita 17/12/2016).
} 
Un gran notable representante de este tipo de arquitectura fue Le Corbusier, el cual atendía en sus diseños a cinco puntos: planta libre (nivel que reservaba para los automóviles), terraza-jardín como quinta fachada, los pilotes (estructura que permitía elevar las casas del nivel basal), fachada libre (se retrasaban los pilares para liberar a la fachada de su función estructural) y las importantes ventanas longitudinales que se extendían a lo largo de la construcción para captar la luz natural, mejorando así la relación con el exterior (figura 1.11).

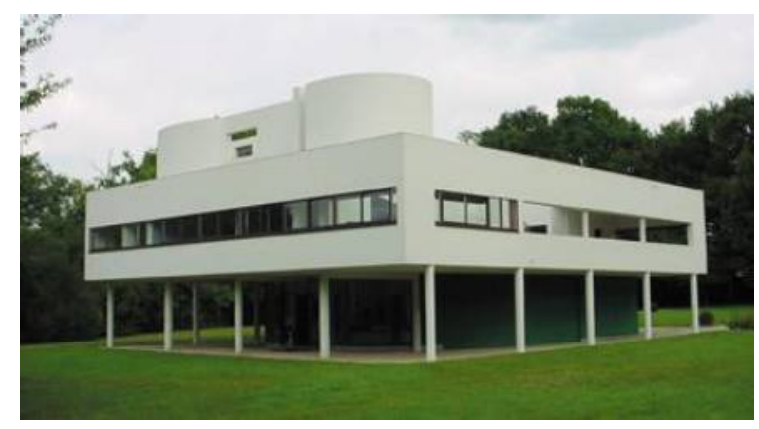

Figura 1.11. La casa Savoya en Poissy ${ }^{10}$.

\subsection{Estado del arte}

El consumo de energía debido a la luz artificial necesaria para iluminar el interior de los edificios depende, entre otros factores, de las actividades que desarrollen los ocupantes, las horas de permanencia dentro de las distintas estancias del edificio, la

realizan diversas investigaciones sobre el comportamiento de la luz natural en el interior de estancias y así poder calcular el aporte de luz artificial mínimo (para que el consumo energético sea mínimo) que requerirán dichas estancias.

Estas investigaciones se han llevado a cabo de diversas maneras, centrándose en distintos factores (distintas orientaciones, tipos de cielos, etc...) y mediante distintos tipos de medidas (medidas en superficies horizontales o verticales, en el exterior del edificio así como en las cubiertas e incluso en el interior de variedad de herramientas (programas de simulación, distintos dispositivos de medición, etc...).

\footnotetext{
${ }^{10}$ http://www.galenusrevista.com/Los-cinco-puntos-de-la.html. (Última visita 17/12/2016).
} 
Parte de estos estudios se han centrado en el análisis del comportamiento de la luz. Como por ejemplo, en Bangkok, Surapong Chirarattananon et al. [2002], llevaron a cabo un estudio sobre iluminancia y radiación solar en regiones tropicales, midiendo iluminancias de luz natural e irradiación solar por el "Asian Institute of Technology" (AIT). Esta investigación demostró que el cielo tropical es un cielo de tipo luminoso, puesto que los rayos tienen un recorrido menor por la atmósfera que en otras regiones no tropicales debido a la posición del sol. También afirma que parte de esta luminosidad del cielo trópico se debe a la presencia de nubes a lo largo de todo el año.

En Hong Kong, Li et al. [2004] desarrollaron un estudio sobre distintos parámetros, entre otros, horas de sol, irradiancia solar e iluminancia de la luz natural, con el fin de clasificar los distintos tipos de cielo en despejado, cubierto y parcialmente cubierto.

En Grecia, Markou et al. [2009] realizaron un estudio sobre la variación de los niveles de iluminancia diurna durante el invierno y el verano en Grecia. Llegaron a la conclusión de que en invierno las iluminancias globales seguían seis tipos de recorridos semi-diurnos característicos (desde el amanecer hasta el mediodía y del medio día hasta el atardecer), y en verano había seis tipos de recorridos característicos desde el amanecer hasta el mediodía y cinco desde el mediodía hasta el atardecer. En algunos de estos recorridos las iluminancias presentaban variaciones leves; sin embargo, cuando se daba la presencia de nubes había variaciones bastante más acusadas.

En Nigeria, Okobue et al. [2008], estudiaron la relación entre la estación del año, la iluminancia global a lo largo del día y el rendimiento lumínico en lle-lfe (7.5ํN, $4.57^{\circ} \mathrm{E}$, Nigeria). Afirmaron que la probabilidad de tener luz natural con horas de rendimiento lumínico con más de $100 \mathrm{~lm} / \mathrm{W}$ es mayor durante las estaciones de lluvia que durante las estaciones secas (conocidas como Harmattan). 
En Italia, Bella et al. [febrero 2015], analizaron el impacto, mediante simulaciones con el programa DIVA, de usar un archivo de datos meteorológicos $u$ otro para predecir la luz natural en el interior de una oficina orientada al Norte. Compararon cuatro archivos de datos meteorológicos IWEC (International Weather for International Calculation), Meteonorm, Satel-Light y TRY (Test Reference Year); en cinco ciudades europeas (Copenhague, Londres, Milán, Nancy y Roma). Demostraron que los archivos IWEC, Meteonorm y Satel-Light weather, presentaban resultados similares, sin embargo el archivo TRY siempre daba resultados considerablemente más bajos.

Más tarde realizaron un estudio similar [mayo 2015], en este caso realizando las simulaciones sólo con los archivos meteorológicos de IWEC, Meteonorm and Satel-Light, para dos ciudades europeas (Copenhagen y Roma), y orientado la oficina simulada en las cuatro orientaciones (Norte, Sur, Este y Oeste). Demostraron que la diferencia entre usar estos tres archivos meteorológicos seleccionados pueden generar diferencias del $1 \%$ hasta del $20 \%$, siendo las diferencias mayores en verano que en inviernos si las ventanas están orientadas al Norte, Este u Oeste, y de forma viceversa si éstas están orientada al Sur.

En España, Claros et al. [2002] realizaron un estudio sobre cómo afectan distintos tipos de voladizos a la iluminancia en el interior de los edificios, en Madrid.

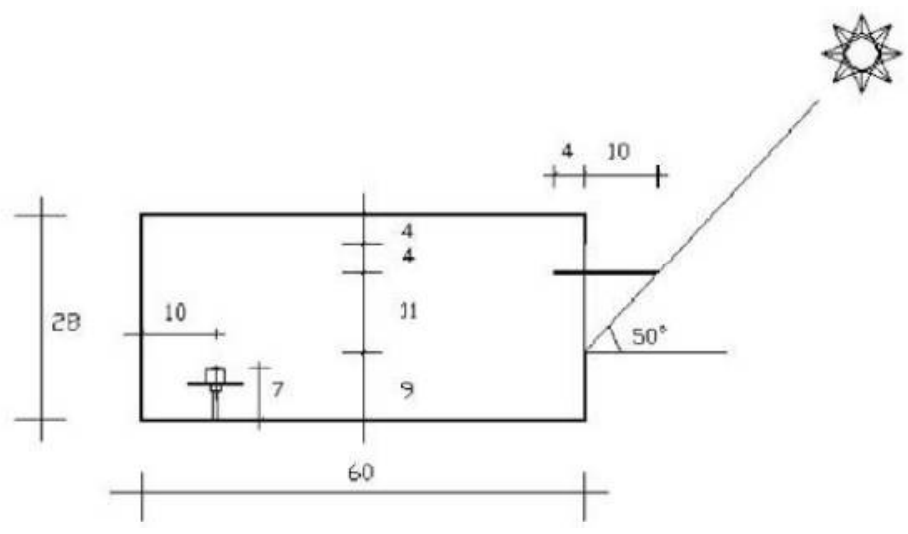

Figura 1.12. Modelo a escala [Claros et al., 2002] 
Por otro lado hay estudios que se han centrado en el análisis de elementos de control pasivo de entrada de luz natural al interior de edificios. Como por ejemplo, en Malasia, Zain-Ahmed et al. [2002], realizaron un estudio en el que afirman que un correcto diseño de un sistema pasivo de luz natural, como por ejemplo, sería establecer el correcto tamaño y tipo de ventana, podría ahorrar al menos un $10 \%$ de la energía destinada a luz artificial.

Hee et al. [2015], recalcaron la importancia que tiene una correcta elección del acristalamiento de las ventanas en el ahorro energético. Lim et al. [2016] recalcaron la importancia de emplear sistemas pasivos de control de luz natural en los rascacielos en Malasia, para evitar el disconfort visual de sus ocupantes debido al deslumbramiento provocado por las grandes superficies acristaladas de estos edificios.

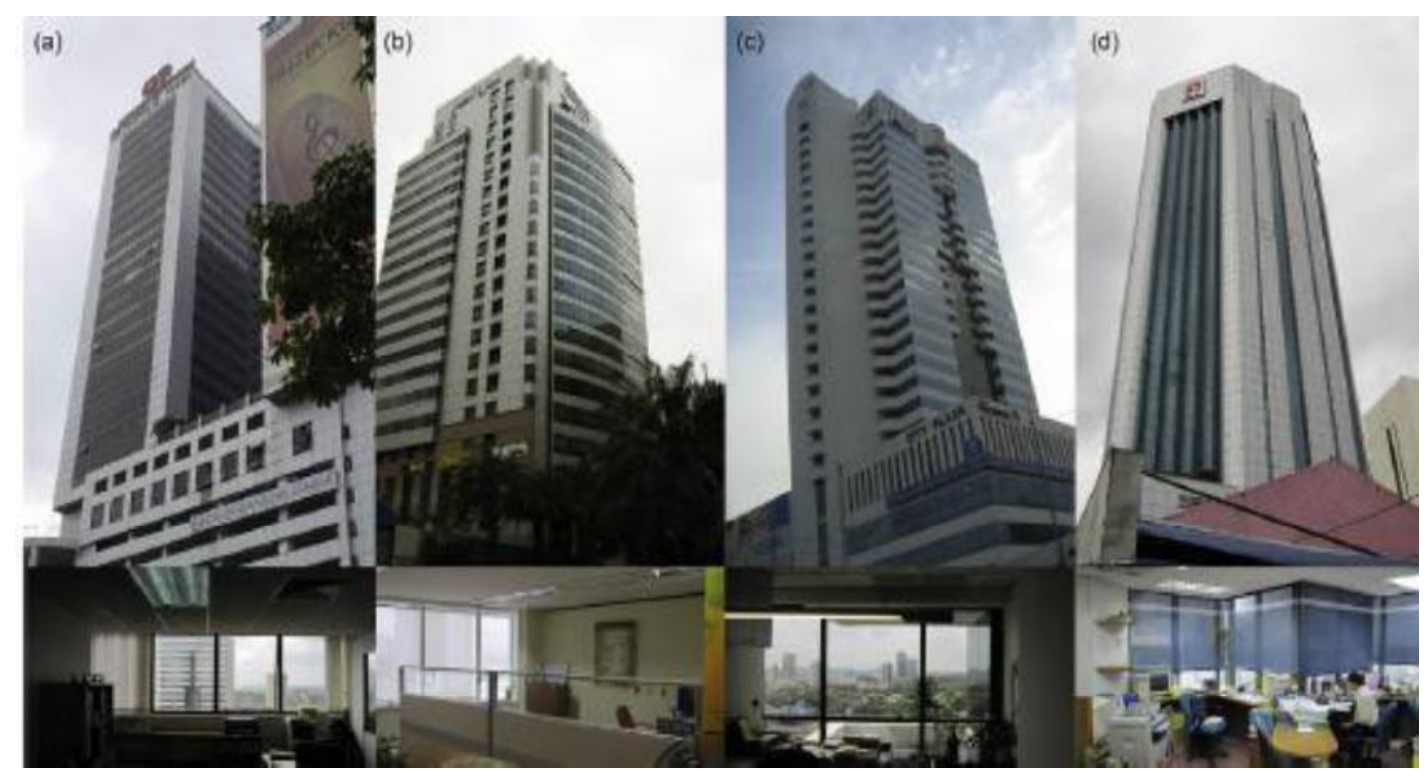

Figura 1.13. Ejemplos de rascacielos acristalados de oficinas Johor Bahru, Malaysia: (a) Metrópolis Tower; (b) Ansar Tower; (c) City Plaza; (d) Public Tower [Hee et al., 2015].

En china, Huang et al. [2014], tras analizar el comportamiento térmico y el de la luz natural de ventanas en oficinas con distintas orientaciones en zonas de clima predominantemente frío, llegaron a las siguientes conclusiones: el diseño energéticamente eficiente de ventanas orientadas al Este y al Oeste son los más rentables, el diseño más costoso se da en las ventanas orientadas al norte, y según aumenta la latitud la diferencia entre las cuatro orientaciones disminuye. 
Por otro lado, Konstantzos et al. [2015], se centraron en analizar las persianas en ventanas de edificios de oficinas, llegaron a la conclusión de que estas persianas debían tener un porcentaje de ranuras inferior al $2 \%$ o bien ser de materiales mates para evitar por completo los deslumbramientos.

En India, Singh et al. [2010] emplearon el programa de simulación ADELINE 3.0. En esta investigación se afirmó que el ahorro de consumo de energía de luz artificial está muy relacionado con el tipo de ventana que se emplee, siendo este consumo superior, por ejemplo, en ventanas de doble acristalamiento transparente que en ventanas con revestimientos reflectantes.

Hay estudios que se centran en encontrar los factores que provocan un alto consumo de energía debido a la luz artificial, o en combinar luminarias tipo LED (Light Emitting Diode) y sensores para el control del consumo de la luz artificial. En china, Xin Zhou et al. [2015], realizaron un estudio sobre los principales factores que influyen en el uso de energía destinado a luz artificial en oficinas. El resultado de este estudio fue que el principal factor del que depende el consumo de energía debido a luz artificial en edificios de oficina en China es el horario de los trabajadores.

Otros estudios similares especifican que instalando un sistema de control de luz artificial conforme al horario de ocupación de las oficinas se puede reducir el consumo de luz artificial en este tipo de oficinas hasta en un $43 \%$ [Yun et al., 2012] [Xu et al., 2017].

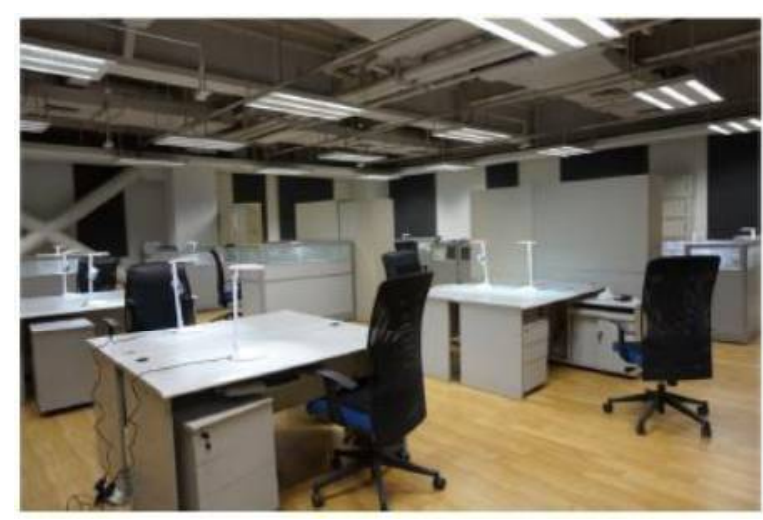

Figura 1.14 lluminación en edificio de oficinas [Xu et al., 2017]. 
Por otro lado, hay otro estudio que afirma que al instalar un correcto sistema de control de luz artificial teniendo en cuenta el horario de ocupación de las oficinas, no sólo proporciona un ahorro energético destinado a luz artificial, sino también respecto del aire acondicionado [Li et al., 2001].

Varios estudios [Bangali et al., 2012] [Hertoget al., 20015] [Caicedoet al., 2014] proponen un sistema de control de iluminación basado en emplear e incluso combinar sensores, microcontroladores y/o luminarias LED. De este modo consiguen demostrar que la tecnología LED puede llegar a ahorrar un $70 \%$ más de energía que un fluorescente tradicional de $40 \mathrm{~W}$ [Bangali et al., 2012].

Hay otros estudios que se centran en encontrar un modelo matemático con el cual poder predecir la iluminación de luz natural en el interior de edificios, con el objetivo de establecer sistemas de control para aportar la luz artificial mínima necesaria para alcanzar el grado de confort proyectado.

En España, Robledo, Soler y Ruiz [Robledo et al., 2000] [Ruiz et al., 2002] [Soler et al., 2004] desarrollaron varios modelos para predecir la iluminancia de una estancia. Por un lado se obtuvieron dos modelos empíricos, uno para estimar la iluminancia global para cielo despejado con una desviación media del $5 \%$ y otro con desviación de la media cuadrática del $7 \%$ respecto a las iluminancias medidas [Robledo et al., 2000]. Ruiz et al. [2002], también comprobaron la eficacia del modelo Olmo [Olmo et al., 1999], modelo por el cual se puede estimar la irradiancia global en planos inclinados a partir de la irradiancia global horizontal, así como de la elevación del sol y su azimut.

En otro estudio de Santiago-Tomás et al. [2002], obtuvieron unos perfiles para la media de la iluminancia cenital, a partir de los cuales se puede calcular la iluminancia cenital media para distintos tipos de cielo a distintas horas y épocas del año.

En Valladolid (España), Pérez-Burgos et al. [2010] [2007], tomaron medidas de iluminancias tanto horizontales como verticales durante varios días a lo largo 
de un año (2007), llegando a la conclusión de que las superficies predominantemente orientadas al Norte están la mayor parte del día en sombra y que sólo están iluminadas por iluminación reflejada y difusa, mientras que las superficies orientadas al Oeste y Este están la mitad del día en sombra. Desarrollaron una ecuación paramétrica para obtener iluminancias verticales a partir de medidas de iluminancias horizontales y la altitud solar local.

En Grecia, Tsangrassoulis et al [1996], proponen un método basado en el cálculo de coeficientes, mediante el método de Monte Carlo [Tregenza, 1983], para la evolución de la luz natural en el interior de una estancia según distintos tipos de sistemas de control de penetración de la luz natural en el interior de un edificio.

En Italia, De la Rosa et al. [2008], obtuvieron un método sencillo de cálculo para evaluar la iluminancia difusa natural en superficies verticales, que consiste en multiplicar una constante obtenida a través de valores de eficacia luminosa en cada superficie por la irradiancia difusa de la misma superficie. Por otro lado Cucumo et al. [2008], realizaron un estudio comparativo entre la iluminancia natural difusa calculada por el método anteriormente citado y la obtenida experimentalmente en diversas superficies con distintas orientaciones sin distinguir entre distintos tipos de cielo, obteniendo como resultado que tanto los resultados experimentales obtenidos como los calculados eran similares.

En China, Li et al. [2009] [2010], propusieron calcular un factor de luz natural para determinar la iluminancia de la luz natural en superficies verticales bajo cielos cubiertos.

Otro grupo de investigaciones están centradas en la toma de datos de iluminancias en modelos a escala. Como por ejemplo la de Cheng et al. [2007], que proponen estudiar y predecir el comportamiento de la luz reflejada sobre elementos de sombra verticales a través de una tabla obtenida a partir de un modelo a escala $(1 / 20)$. 
En Italia, De Rosa et al. [2009], tomaron medidas de iluminancias en un modelo a escala $(1 / 5)$ de una estancia real, durante días de cielo cubierto, para validar el programa de simulación de iluminación llamado INLUX. De este programa se creó una versión modificada denominada INLUX-DBR, que se validó, mediante medidas de iluminancia tomadas en un modelo a escala (1/5) pero en este caso bajo distintos tipos de cielo [Ferraro et al., 2010].

Por otro lado, Boccia et al [2015], realizaron un estudio con el fin de averiguar cómo afecta el factor de escala en las medidas de iluminación tomadas en una estancia y en su modelo a escala 1/5. Llegaron a conclusiones como por ejemplo que a la hora de realizar trabajos en los que se tomen datos de iluminancia en una estancia y en su modelo a escala, es preferible desarrollar estos trabajos en ausencia de luz solar directa puesto que el comportamiento de esta presenta anomalías en el modelo que en muchas ocasiones son inevitables y podrían llevar a datos no fiables. 
2. FUNDAMENTOS TEÓRICOS 



\section{FUNDAMENTOS TEÓRICOS}

\subsection{La Luz}

Luz es un término que proviene del latín: lux, lucis y que según el diccionario de La Real Academia Española es el agente físico que hace visibles los objetos.

La Física clásica la define como una radiación electromagnética, una onda. Su amplitud proporciona la luminosidad y las diferentes longitudes de onda conforman los diversos colores. La teoría cuántica nos indica que las partículas de energía llamadas fotones producen la luz. El número de fotones proporciona la luminosidad, y la energía de los mismos crea su color. Sin embargo ambas teorías son correctas, ya que la Teoría corpuscular unifica ambas teorías anteriores al definir la luz como partícula y como onda.

\subsection{Magnitudes y unidades luminosas}

\section{Flujo luminoso}

Se entiende por flujo luminoso, la potencia $(W$ emitida en forma de radiación luminosa a la que el ojo humano es sensible. Se designa con la letra griega $\Phi$ y tiene como unidad en el Sistema Internacional (S.I.) el lúmen (Im).

\section{Intensidad luminosa}

La intensidad luminosa se define como la cantidad de flujo luminoso $(\Phi)$ que atraviesa o incide sobre una superficie por unidad de ángulo sólido $(\omega)$ en una dirección concreta. Se designa con la letra $l$, y tiene como unidad en el S.I la candela $(\mathrm{cd}=\mathrm{Im} /$ estr) (estereorradián: unidad de ángulo sólido en el que la superficie abarcada en una esfera es igual al cuadrado del radio).

$$
I=\frac{\Phi}{\omega}
$$

\section{Luminancia}

Se conoce como luminancia a la relación entre la intensidad luminosa y la superficie aparente vista por el ojo humano en una dirección determinada. Se simboliza con la letra $L$, y se mide en candelas por metro cuadrado $\left(\mathrm{cd} / \mathrm{m}^{2}\right)$. 


$$
L=\frac{I}{S_{\text {aparente }}}=\frac{I}{S \cdot \cos \alpha}
$$

\section{Iluminancia}

La iluminancia es el flujo luminoso recibido por una superficie. Se simboliza con la letra $E$, y su unidad en el S.I. es el lux (Ix).

$$
E=\frac{\Phi}{S} ; \quad \text { lux }=\frac{\text { lumen }}{m^{2}}
$$

Estas cuatro magnitudes pueden apreciarse en la figura 2.1.

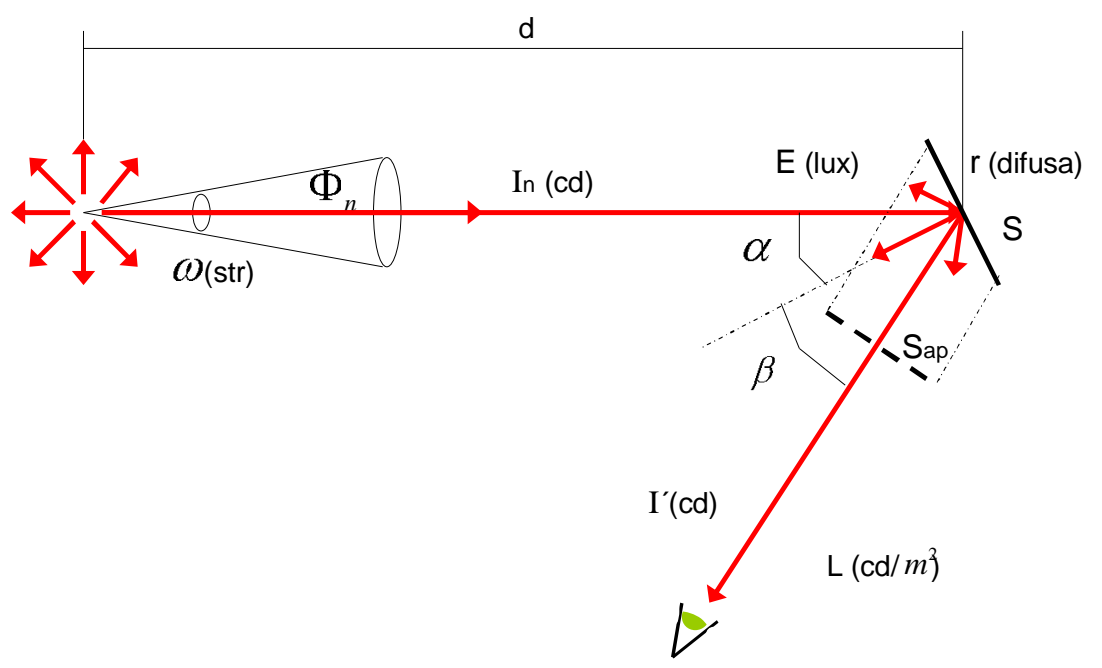

Siendo:

Ф: Flujo, I: intensidad, E: lluminancia, L: luminancia, r: coeficiente de reflexión, S: superficie iluminada.

$$
\begin{aligned}
& I_{n}=\frac{\Phi_{n}}{\omega} \\
& E=\frac{I_{n}}{d^{2}} \cdot \cos \alpha \\
& L=\frac{I^{\prime}}{S_{a p}},\left(S_{a p}=S \cdot \cos \beta\right)
\end{aligned}
$$

Figura 2.1. Esquema de las magnitudes luminosas. 


\subsection{Luz natural}

La luz natural es un tipo de energía emitida por fuentes que provienen de la naturaleza. Su principal fuente es el Sol, aunque físicamente existen otras como los relámpagos, las estrellas e incluso la emitida por algunos seres vivos.

La luz natural puede llegar al interior de un local de manera directa, indirecta o de manera difusa dispersada por la atmósfera y reflejada por las superficies cercanas a dicho local. Puede afirmarse que el Sol, el cielo, los obstáculos artificiales (construcciones) y las obstrucciones naturales (montañas, vegetación, terreno) influyen en el grado de variación de la iluminación natural de los interiores. Esta variación se ve afectada por los movimientos del Sol, los cambios en la nubosidad y, en parte, por la vegetación y la reflexión del suelo que cambian a lo largo del año con las estaciones.

La denominada luz solar directa es aquella que incide en un lugar específico directamente desde el Sol. Sus principales características son su continuo cambio de dirección, probabilidad de ocurrencia, temperatura del color así como la iluminancia que produce en una superficie horizontal no obstruida. A este tipo de luz natural debe prestársele especial atención a la hora de realizar el diseño de un sistema de iluminación natural, puesto que genera excesivos contrastes y es causa de deslumbramientos, lo que debe evitarse.

La luz solar indirecta es aquella que llega a un espacio determinado por reflexión. En zonas de climas soleados, la luz natural indirecta puede suponer un gran aporte a los sistemas de iluminación natural, puesto que mediante el uso de superficies reflectoras se puede redirigir la luz solar directa, aumentando de esta forma la luz natural disponible y mejorando su distribución.

Se considera luz natural difusa aquella que tiene aproximadamente la misma intensidad en diferentes direcciones. 
Como se ha mencionado en párrafos anteriores, el cambio en la nubosidad afecta a la variación de la iluminación natural. Estos cambios de nubes dan lugar a tres principales tipos de cielo: cielo cubierto, cielo semicubierto y cielo despejado.

Se considera cielo cubierto cuando éste lo está en un 70-100\% por nubes con un Sol no visible. La CIBSE (Chartered Institution of building Services Engineers), fija este porcentaje en un 90\%. La distribución de las luminancias para cielo cubierto de define por la siguiente ecuación:

$L_{z}$ : luminancia en el cenit

$\theta$ : el ángulo de altitud del punto

$$
L_{\theta}=L_{Z} \cdot \frac{1+\operatorname{sen} \theta}{3}
$$

considerado

Un cielo cubierto es unas 3 veces más luminoso en el cenit que en el horizonte (figura 2.2), lo que beneficia a la iluminación cenital (ventanas altas o en el techo), aprovechando de este modo la parte más luminosa de la bóveda celeste, sin la contingencia de excesivos contrastes provocados por la luz solar directa. Sus valores y distribuciones varían dependiendo de parámetros como la localización, densidad y uniformidad de las nubes, las características climáticas y condiciones atmosféricas. El valor medio anual de iluminancia exterior sobre una superficie horizontal considerada normalmente para los cálculos es de 5000 lux.

Existe una simplificación de este tipo de cielo, denominada cielo de luminancias uniformemente distribuidas o cielo uniforme (figura 2.3), implica un espesor constante tanto de la turbidez de la atmósfera como una capa de nubes blancas. Consecuentemente su distribución de luminancias es de la forma: 


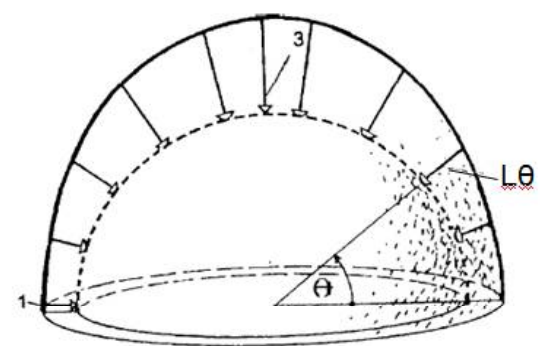

Figura 2.2. lluminancias con cielo nublado [Pattini].

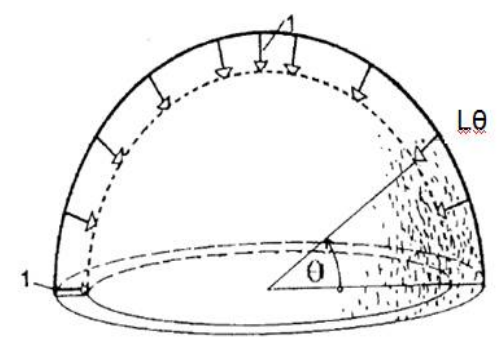

Figura 2.3. Iluminancias con cielo uniforme [Pattini].

Se define como cielo semicubierto o parcialmente despejado, aquel en el que se alterna la presencia del Sol con períodos de nubosidad variable (climas templados húmedos y cálidos húmedos).

La iluminancia en una superficie horizontal exterior bajo este tipo de cielos (no obstruida), oscila entre los 100.000 lux (sin nubes) y 10.000 lux (con nubes interceptando el sol). Este tipo de cielo es el más complicado de predecir por la gran variabilidad que presenta y por lo tanto, no se dispone de un modelo particular y simple para ello.

Cielo claro o despejado se define como aquél en el que el Sol no está obstruido por nubes. La CIBSE lo define como cielo no obstruido por nubes y la IESNA (Illuminating Enbineering Society of North America), como un cielo obstruido por nubes en un porcentaje menor al $30 \%$. Un cielo despejado es la mitad de luminoso en el cenit que en el horizonte (figura 2.4).

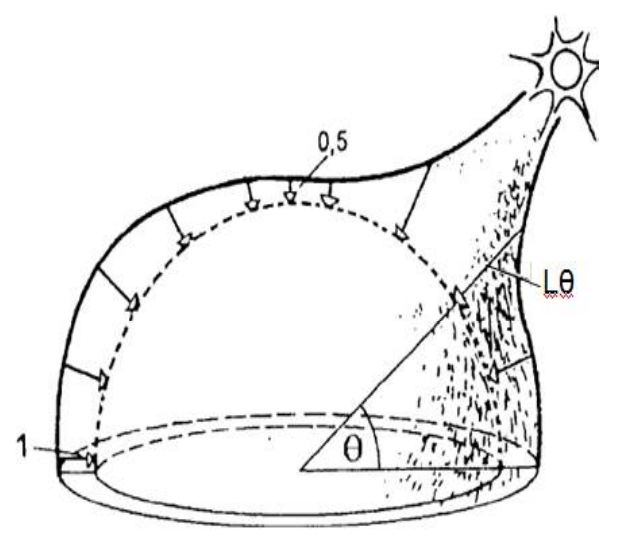

Figura 2.4. Iluminancias con cielo despejado [Pattini]. 


\subsection{Relación de la luz con la materia}

La luz se propaga por un medio $y$ al alcanzar otro medio $u$ objeto pueden suceder varios fenómenos: que se refleje, se absorba o se transmita (figura 2.5). Los coeficientes de reflexión ( $r$ ), de absorción (a) y de transmisión $(t)$ nos informan, del porcentaje de luz incidente que es reflejado, absorbido y transmitido por una superficie. La suma de los tres coeficientes, expresados en tantos por uno, siempre valdrá la unidad: $r+a+t=1$.

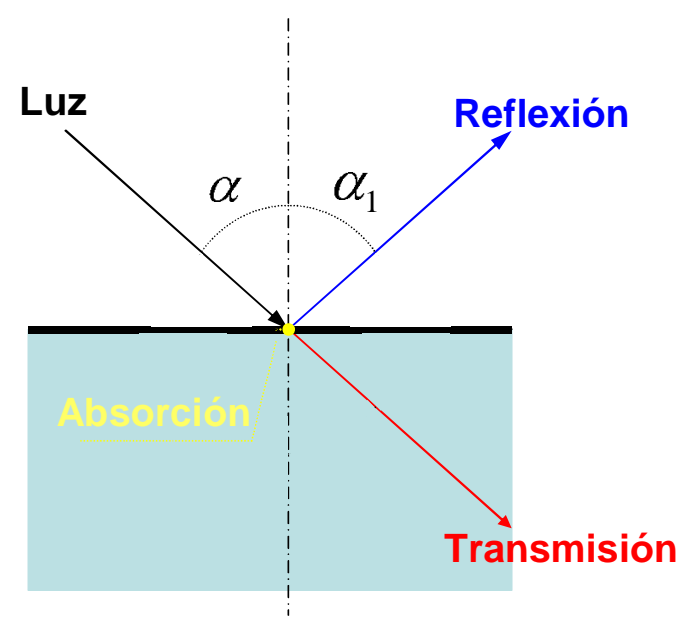

Figura 2.5. Reflexión, transmisión y absorción de la luz.

A continuación se van a explicar estos fenómenos por separado y además, la refracción, otro fenómeno que se produce cuando la luz pasa de un medio a otro.

\subsubsection{Reflexión}

La reflexión se produce cuando la luz incide en una superficie y es reflejada por ésta. La reflectancia de la superficie (relación entre la luz reflejada y la luz incidente) depende de la longitud de onda del rayo incidente y del tipo de superficie. Existen cinco tipos de reflexión:

Reflexión especular: Tiene lugar en superficies lisas y pulidas. Los haces de luz inciden de forma paralela entre sí sobre la superficie, y al entrar en contacto con esta última se reflejan en la misma dirección (figura 2.6). 


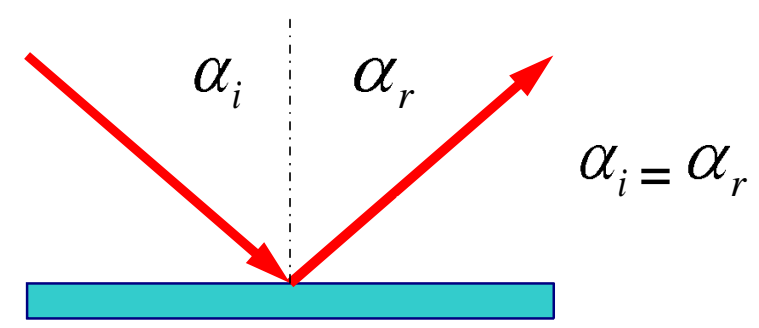

Figura 2.6. Reflexión especular.

Reflexión difusa: Es aquélla que se da en superficies rugosas. Los haces de luz inciden de forma paralela entre sí sobre la superficie, sin embargo se reflejan en distintas direcciones (figura 2.7).

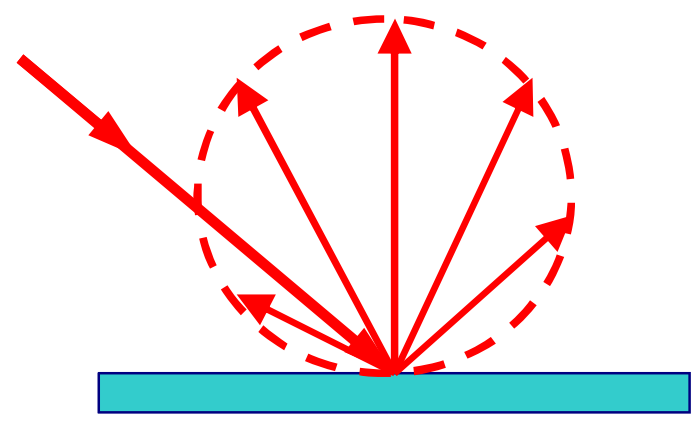

Figura 2.7. Reflexión difusa.

Reflexión extendida: Es una combinación entre la reflexión especular y la difusa. Se diferencia de las anteriores en que tiene un componente direccional dominante en la reflexión (figura 2.8).

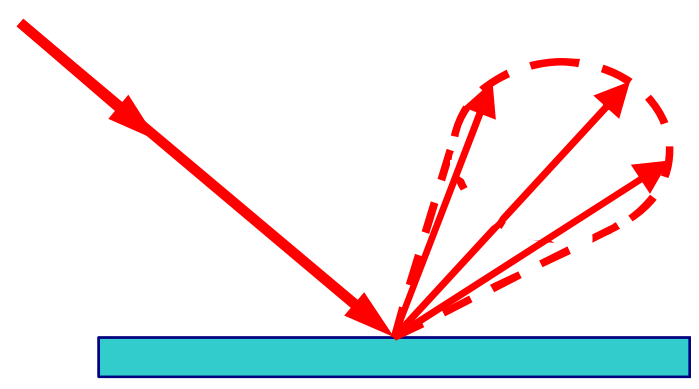

Figura 2.8. Reflexión extendida.

Reflexión mixta: Es una combinación entre las reflexiones especular, difusa y extendida. Es la que se da en la mayoría de los materiales que nos rodean (figura 2.9). 


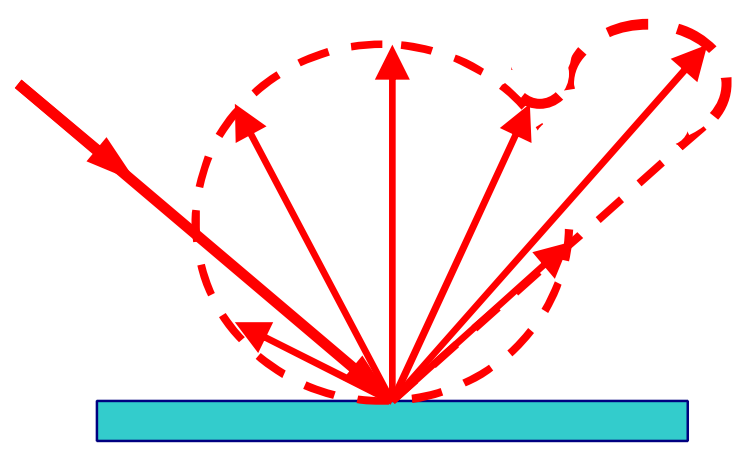

Figura 2.9. Reflexión mixta.

Reflexión expandida: Es la más irregular de la reflexiones, al no seguir ninguna ley (figura 2.10), ni la ley de reflexión ni la de Lambert. La ley de reflexión establece que el ángulo de incidencia $(\theta)$ es igual al ángulo de reflexión $\left(\theta_{1}\right)$ (figura 2.5).

La Ley de Lambert establece que la cantidad de luz que se refleja se ve mermada debido a tres fenómenos físicos: la concentración, la distancia que la luz debe atravesar a través del objeto $y$ absorbencia (probabilidad de que el rayo sea absorbido por el material). Se calcula coeficiente molar de extinción, $d$ es la distancia y $c$ la concentración molar.

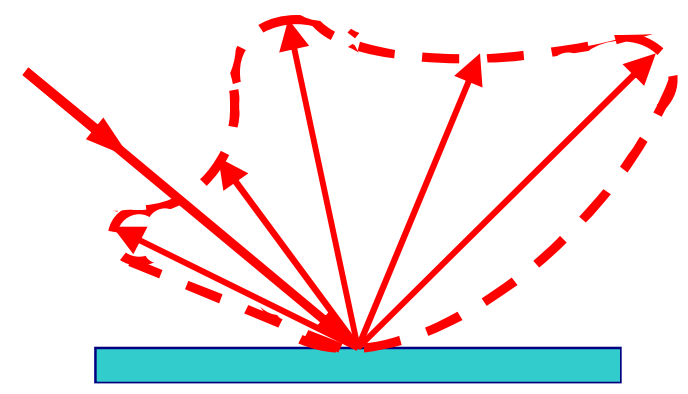

Figura 2.10. Reflexión expandida.

\subsubsection{Transmisión}

La transmisión se produce cuando la luz pasa a través de un medio sin que cambie la frecuencia de las radiaciones monocromáticas que lo componen. Hay varios tipos de transmisión: 
Transmisión regular: En este caso el haz de luz incide sobre un medio y al atravesarlo no sufre ningún cambio en su dirección (figura 2.11). Este tipo de transmisión es propia de materiales transparentes.

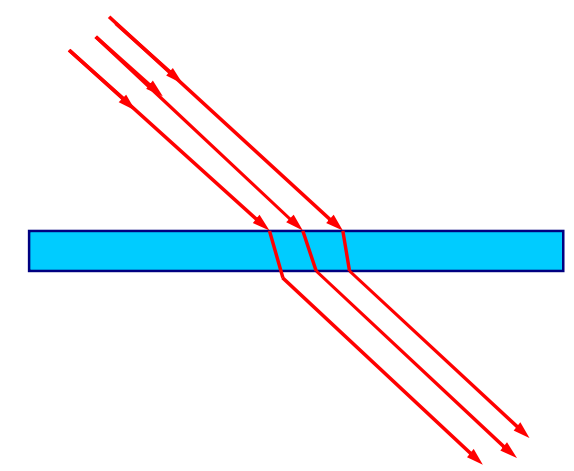

Figura 2.11. Transmisión regular.

Transmisión difusa: En este caso el haz de luz incide sobre un medio y al atravesarlo sufre cambios en su dirección (figura 2.12). Este tipo de transmisión es propia de materiales translúcidos.

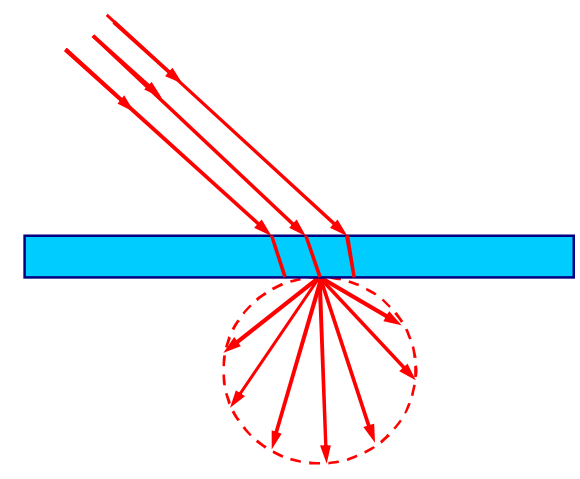

Figura 2.12. Transmisión difusa.

Transmisión mixta: Es una combinación entre la transmisión regular y la difusa (figura 2.13). Se da en materiales como los vidrios orgánicos o los cristales de superficie labrada.

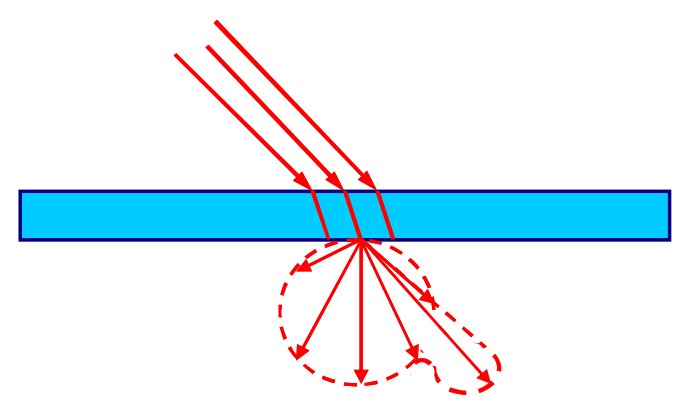

Figura 2.13. Transmisión mixta. 


\subsubsection{Absorción}

La absorción se produce cuando la luz (energía radiante) alcanza un medio y éste absorbe toda o parte de esta luz, es decir, esta energía radiante se transforma en otra forma de energía (generalmente calor).

Cuando un objeto no absorbe nada de luz (refleja toda la luz), el color del objeto será teóricamente de color blanco. Si por el contrario el objeto absorbiera toda la luz, el objeto sería de color negro.

\subsubsection{Refracción}

La refracción (figura 2.14) se produce cuando un rayo de luz pasa de un medio a otro cambiando su dirección, debido a la alteración de la velocidad de la luz. Esta velocidad resulta ser menor cuanto más denso sea el medio al que pase la luz.

La refracción sigue dos leyes principales. La primera ley establece que cuando la onda pasa de un medio a otro, el rayo incidente, el rayo refractado y la normal a la superficie de separación de los medios en el punto de incidencia, están en el mismo plano. Y la segunda ley, es la Ley de Snell, que establece que la razón del seno del ángulo de incidencia y el seno del ángulo de refracción es una constante para los medios comprendidos.

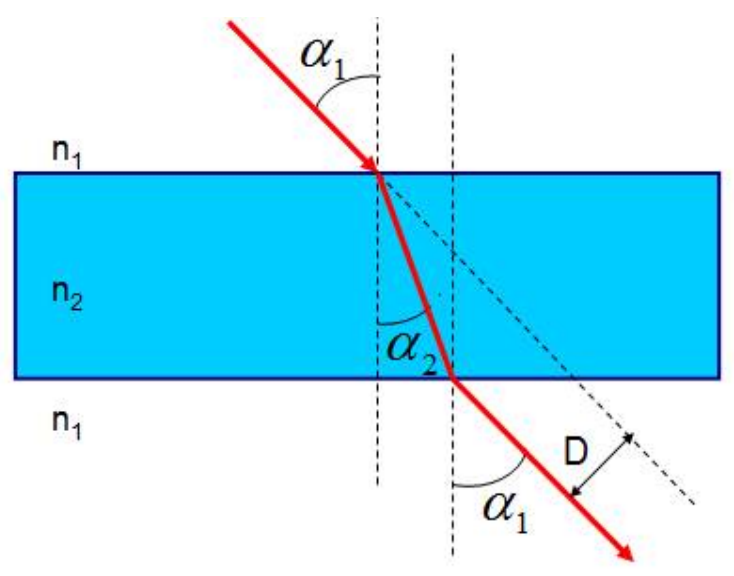

$\mathrm{n}_{1}=$ índice de refracción medio 1 .

$\mathrm{n}_{2}=$ índice de refracción medio 2.

$\mathrm{a}_{1}=$ Ángulo de incidencia

$\mathrm{a}_{2}=$ Ángulo de refracción

$\mathrm{D}=$ Desplazamiento

$n_{1} \cdot \operatorname{sen} \alpha_{1}>\quad-\begin{array}{lll}\operatorname{sen} \alpha_{1} & n_{1} & \\ \operatorname{sen} \alpha_{2} & n_{2} & n\end{array}$

${ }^{*} n_{i}$ se calcula mediante el cociente entre la velocidad de la luz en el aire y el de la luz en el medio i. Cuando el primer medio es

Figura 2.14. Refracción. el aire $n_{1}=1$. 


\subsection{Espectro visible}

El espectro visible es el espectro de radiación electromagnética que es visible para el ojo humano y abarca desde una longitud de onda de $400 \mathrm{~nm}$ hasta los $700 \mathrm{~nm}$. La energía se reparte en las diferentes longitudes de onda del espectro visible y cada longitud de onda se corresponde con un color específico (Tabla 2.1). Se denomina luz blanca a la luz procedente del sol dado que cubre todas las zonas del espectro visible.

\begin{tabular}{|c|c|c|}
\hline Color & Frecuencia & $\begin{array}{c}\text { Longitud de } \\
\text { onda }\end{array}$ \\
\hline Violeta & $668-789 \mathrm{~T} \mathrm{~Hz}$ & $380-450 \mathrm{~nm}$ \\
\hline Azul & $631-668 \mathrm{~T} \mathrm{~Hz}$ & $450-475 \mathrm{~nm}$ \\
\hline Cian & $606-630 \mathrm{~T} \mathrm{~Hz}$ & $476-495 \mathrm{~nm}$ \\
\hline Verde & $526-606 \mathrm{~T} \mathrm{~Hz}$ & $495-570 \mathrm{~nm}$ \\
\hline Amarillo & $508-526 \mathrm{~T} \mathrm{~Hz}$ & $570-590 \mathrm{~nm}$ \\
\hline Naranja & $484-508 \mathrm{~T} \mathrm{~Hz}$ & $590-620 \mathrm{~nm}$ \\
\hline Rojo & $400-484 \mathrm{~T} \mathrm{~Hz}$ & $620-750 \mathrm{~nm}$ \\
\hline
\end{tabular}

Tabla 2.1. Espectro de luz visible.

\subsection{Sistemas de iluminación natural}

Los sistemas de iluminación natural son aquéllos utilizados para controlar la cantidad, calidad y distribución de la luz natural en una estancia. Al diseñar un sistema de iluminación natural en interiores no sólo hay que tener en cuenta el nivel de iluminación, sino también los deslumbramientos (por ser perturbadores y molestos) y los contrastes, tanto elevados como demasiado bajos (fatigan los ojos).

Hay tres sistemas principales de iluminación natural: iluminación lateral, cenital y combinada.

La iluminación lateral es aquélla que llega desde aberturas laterales de los muros, de ahí que cuanto más cercana sea la posición a la apertura más altos serán los valores de iluminación directa y cuanto más lejana sea esta posición, la iluminancia directa decrece y aumenta la proporción relativa de la luz difusa y reflejada (indirecta). 
La iluminación cenital es aquélla que procede de las aperturas en las cubiertas. Se usa en zonas donde predomina el cielo cubierto, siendo la proporción de iluminación indirecta usual del $25 \%$ o menor.

El sistema de iluminación combinada, como su propio nombre indica, es una combinación de los dos sistemas anteriores, en la que encontramos aperturas tanto en las cubiertas como en los muros.

\subsubsection{Estrategias de iluminación natural}

\subsubsection{Orientación}

La cantidad de luz que penetra en una estancia a través de una apertura depende, en gran parte, de la orientación en la que se sitúe. Las ventanas situadas en fachadas orientadas al Sur (figura 2.15) pueden recibir iluminación directa del Sol a lo largo de todo el día.

En el caso de que las ventanas estén situadas en una fachada orientada hacia el Norte (figura 2.16), la iluminación que recibirá el interior de la estancia será difusa o reflejada. En el caso de ser verano, el sol puede apantallarse de forma simple al encontrarse este en posiciones altas

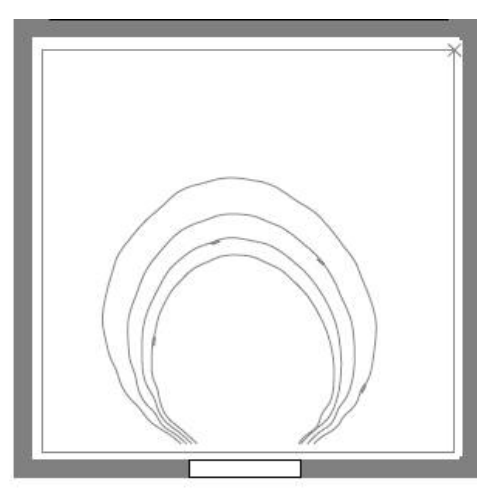

Fig.2.15. Curvas isolux, ventana situada en fachada con orientación Sur [Pattini].

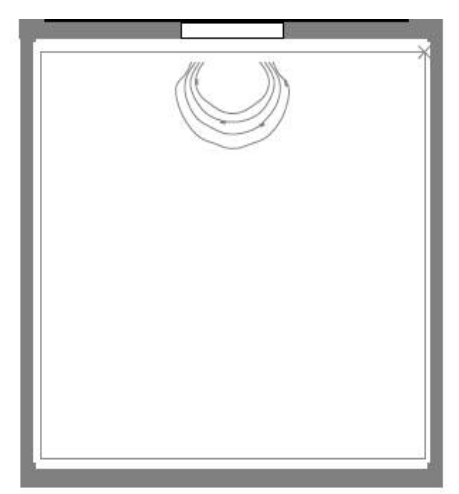

Figura 2.16. Curvas isolux, ventana situada en fachada con orientación Norte [Pattini].

Las ventanas situadas en fachadas orientadas hacia el Este (figura 2.18) sólo reciben radiación directa desde el amanecer hasta el mediodía, mientras que aquellas situadas en fachadas con orientación Oeste (2.17) sólo desde el 
mediodía hasta el atardecer. Ambas orientaciones reciben niveles muy variables de luz durante el resto de las horas de sol.

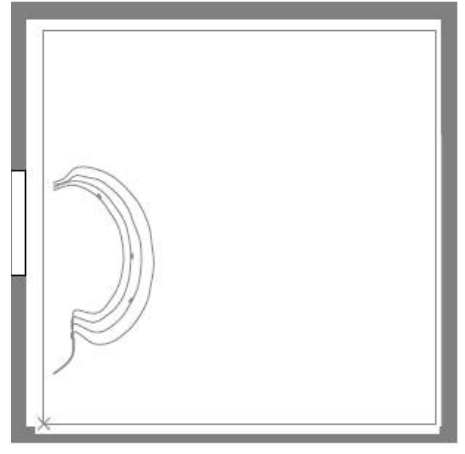

Figura 2.17. Curvas isolux ventana situada en fachada con orientación Oeste [Pattini].

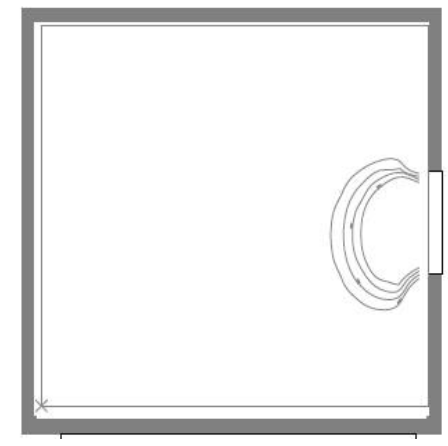

Figura 2.18. Curvas isolux ventana situada en fachada con orientación Este [Pattini].

\subsubsection{Forma, posición y tamaño}

La forma, tamaño y posición de las aperturas influyen considerablemente en la penetración de la luz natural al interior de un edificio. En cuanto a la forma, una apertura horizontal proporcionará una banda de iluminación natural paralela a la pared en la que se encuentra, experimentando a lo largo del día poca diferencia de la distribución de la luz, reduciendo en consecuencia, el efecto deslumbramiento. En el caso de las ventanas verticales, la iluminación en el interior de la estancia se distribuye de forma perpendicular a la ventana y varía a lo largo del día, y aunque la luz penetra más en el interior de las estancias, sin embargo la probabilidad de deslumbramiento es mayor.

Las ventanas de mayor tamaño permitirán una mayor penetración de luz natural que aquellas ventanas de menor tamaño en una estancia. Por otro lado, ventanas posicionadas muy cerca del techo sólo iluminaran el fondo de ésta, y si se sitúan muy cerca del suelo sólo iluminarán el suelo de dicha estancia, por tanto, es importante un correcto posicionamiento de las aperturas.

\subsubsection{Materiales empleados}

Los materiales empleados en los sistemas de iluminación natural deben filtrar correctamente la radiación ultravioleta (U.V.) para evitar el deterioro del material en el interior del edificio, así como por motivos basados en la salud de los ocupantes de éste (figura 2.19). También deben ser los suficientemente 
transparentes para dejar pasar la luz natural, así como cumplir, como mínimo, los requisitos del coeficiente de transmitancia térmica y factor solar modificado exigidos en CTE HE1.

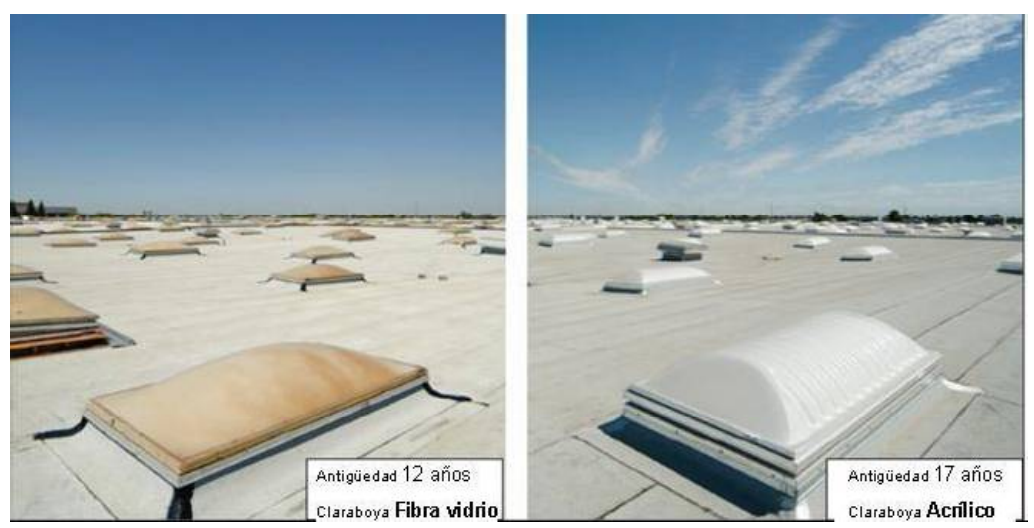

Figura 2.19. Degradación material ${ }^{1}$.

\subsubsection{Sistemas de aprovechamiento o captación de iluminación natural}

Entre los distintos sistemas de iluminación natural destinados a la captación de luz natural se deben destacar los que se exponen a continuación:

\subsubsection{Ventanas}

Las ventanas son aperturas en las paredes o muros de un edificio, cuyo principal objetivo es permitir la entrada de luz natural y ventilación a una estancia. Existen diversos tipos de ventanas, pero todas ellas están

Hay múltiples tipos de ventana, así como diversas formas de distinguirlas (por por lo que, se suelen clasificar según su tipo de apertura. Entre los muchos tipos de ventana practicables, caben destacar los siguientes:

\section{De apertura abatible}

La ventana abatible dispone de un eje horizontal sobre el cual bascula la hoja de la ventana. Este eje puede situarse en la parte superior de la ventana (la ventana abrirá hacia fuera normalmente) o en la parte inferior como en la figura 2.20 (la ventana abre hacia dentro en la mayor parte de los casos).

\footnotetext{
${ }^{1}$ http://www.Iluminación natural y eficiencia energética LLEDO.pdf. (Última visita 28/12/2016)
} 


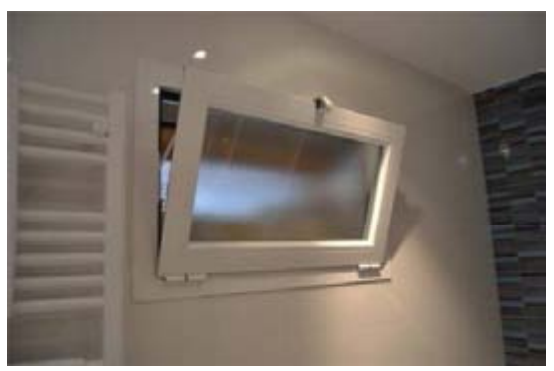

Figura 2.20. Ventana de apertura abatible ${ }^{2}$.

\section{De apertura practicable}

Este tipo de ventana gira para abrirse sobre un eje vertical situado en el lateral del bastidor de la ventana; esta apertura puede ser hacia el interior o hacia el exterior (figura 2.21).

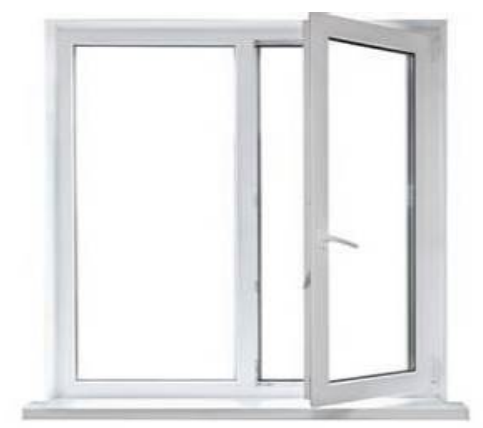

Figura 2.21. Ventana Practicable ${ }^{3}$.

\section{Oscilo- batiente}

La ventana Oscilo- batiente combina tanto la apertura practicable como la horizontal (figura 2.22). Actualmente es muy utilizada en viviendas de alto standing. Para evitar que la ventana se desplome, la hoja de la ventana debe tener una medida mayor en la altura que en la anchura.

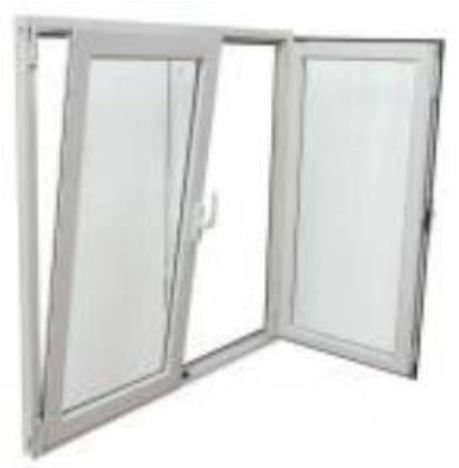

Figura 2.22. Ventana oscilo-batiente ${ }^{4}$.

\footnotetext{
${ }^{2}$ http://www.windart.com.mx/abatibles. (Última visita 31/07/2016).

${ }^{3} \mathrm{http} / / /$ www.hogar.mapfre.es/hogar/ahorro-en-el-hogar/1754/tipos-de-ventanas(Última visita $31 / 07 / 2016)$.

${ }^{4} \mathrm{http}: / /$ www.ventanaspvc.com/interesante/tipos-de-ventana/(Última visita 31/07/2016).
} 


\section{Pivotante}

Las ventanas pivotantes son aquellas cuyas hojas giran alrededor de un eje, horizontal (figura 2.23) o vertical (figura 2.24) situado centralmente. El giro de apertura es de $180^{\circ}$ lo que permite limpiarlas con facilidad.

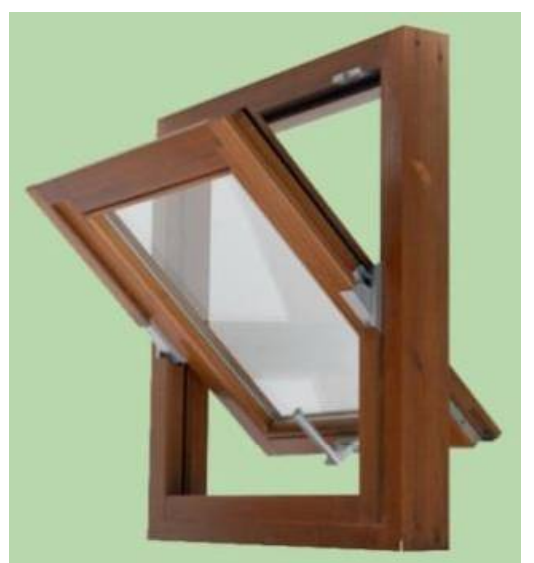

Figura 2.23. Ventana pivotante de eje horizontal ${ }^{5}$.

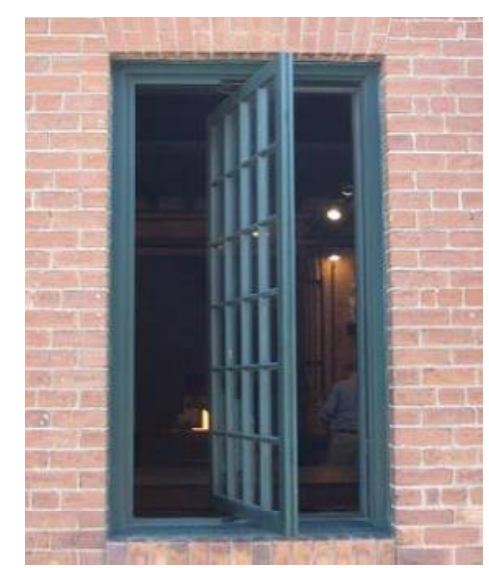

Figura 2.24. Ventana pivotante de eje vertical ${ }^{6}$.

\section{Corredera}

La ventana corredera es aquella cuyas hojas se mueven desplazándose en el mismo plano de la ventana de forma horizontal, de tal manera que ninguna de las piezas de la misma sobresale ni hacia el interior ni hacia el exterior de la estancia. Este tipo de ventana requiere como mínimo de dos hojas, y es fácilmente combinable con ventanas fijas (figura 2.25). Presenta el inconveniente en que sólo es practicable la mitad de su superficie.

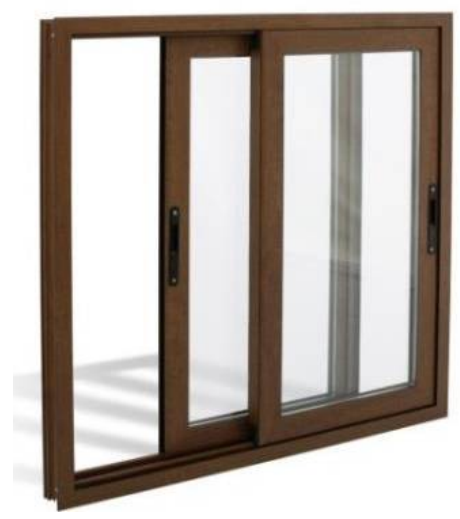

Figura 2.25. Ventana corredera ${ }^{7}$.

\footnotetext{
${ }^{15} \mathrm{http}: / /$ www.persianesmiquel.com/fusteria-especials-pivotants.php?lang=es(Última visita $31 / 07 / 2016)$.

${ }^{6} \mathrm{http}: / /$ www.ventanasbarcelona.com/ventanas-pivotante-vertical.html(Última visita 31/07/2016).

${ }^{7} \mathrm{http} / / /$ zutrok.com/sin-persiana/11-ventana-corredera-de-2-hojas-sin-persiana-sin-rpt-it205.html(Última visita 31/07/2016).
} 


\section{Guillotina}

La ventana de guillotina tiene una apertura corredera vertical (Figura 2.26). Generalmente tiene una hoja móvil y otra fija, aunque también pueden ser las dos móviles. En el caso de que ambas hojas sean móviles se deslizan verticalmente por guías separadas.

Cabe la posibilidad de incorporar un cajón cabecero a estas ventanas, donde introducir una o ambas hojas móviles, lo que permite una ampliación de la apertura y en consecuencia del campo de visión.

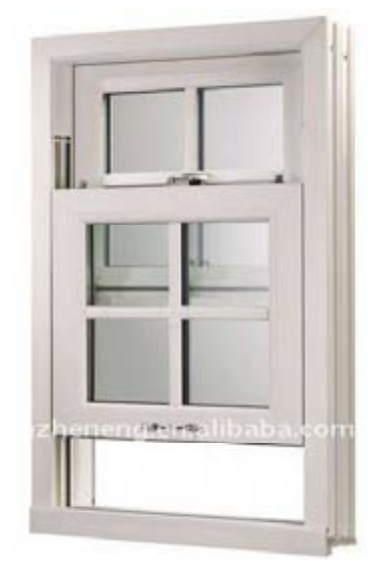

Figura 2.26. Ventana de guillotina ${ }^{8}$.

\section{Plegable}

La ventana plegable también es conocida con el nombre de ventana corredera de acordeón (figura 2.27). Está compuesta usualmente por un sistema de entre 3 y 7 hojas que se pliegan tanto hacia el interior como hacia el exterior gracias a los rieles que tiene en la parte superior y en la inferior, proporcionando una apertura casi total del hueco donde están instaladas.

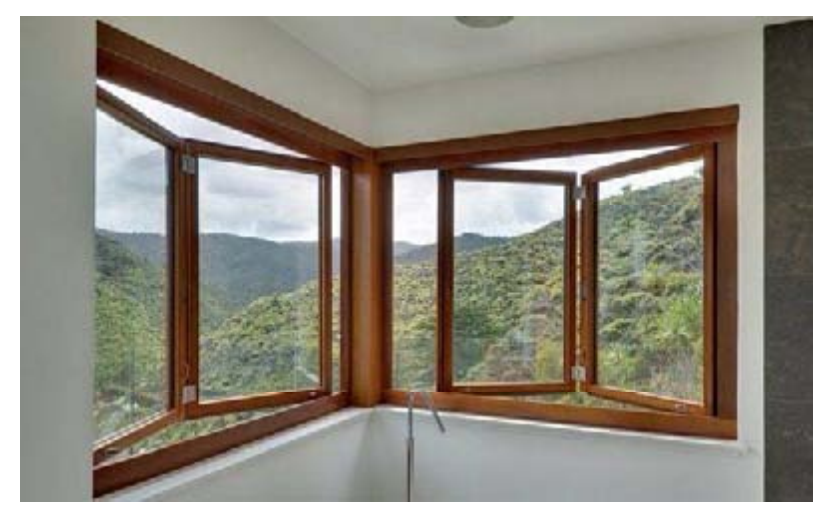

Figura 2.27. Ventana plegable ${ }^{9}$.

\footnotetext{
${ }^{18} \mathrm{http}: / /$ french.alibaba.com/product-gs/pvc-vertical-sliding-sash-window-pvc-window481205700.html(Última visita 31/07/2016).

9 http://www.archiexpo.es/prod/panda-windows-doors/product-65376-1087043.html. (Última visita 31/07/2016).
} 


\subsubsection{Claraboyas}

Es una abertura cubierta generalmente con vidrio o plástico tipo metacrilato 0 similar, situada en la cubierta o en la parte alta de una pared que permite la entrada de luz al interior de un edificio (figura 2.28.). Pueden ser fijas 0 practicables (ya sea de forma manual o automatizada). Algunas de las pocas desventajas de este sistema, son su limpieza y los problemas de condensación que pueden originar.

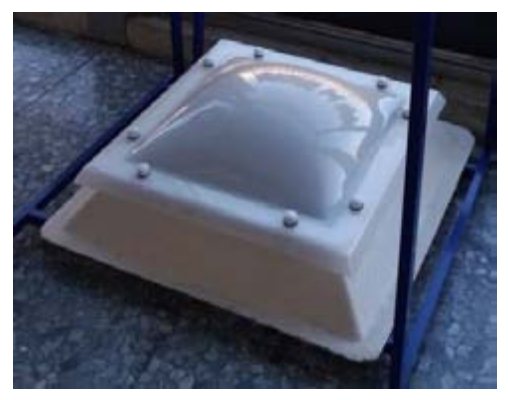

Figura 2.28. Claraboya practicable.

\subsubsection{Patios}

Los patios son espacios abiertos en el interior de edificios. Ya en la época romana se usaban para iluminar las estancias más interiores de las casas (que en aquel entonces tenían, por lo general, poca altura). Actualmente con la altura de nuestros edificios esta técnica no es suficiente para los pisos más bajos; sin embargo, se está imponiendo un sistema de reflectores de luz para resolver este problema (figura 2.29).

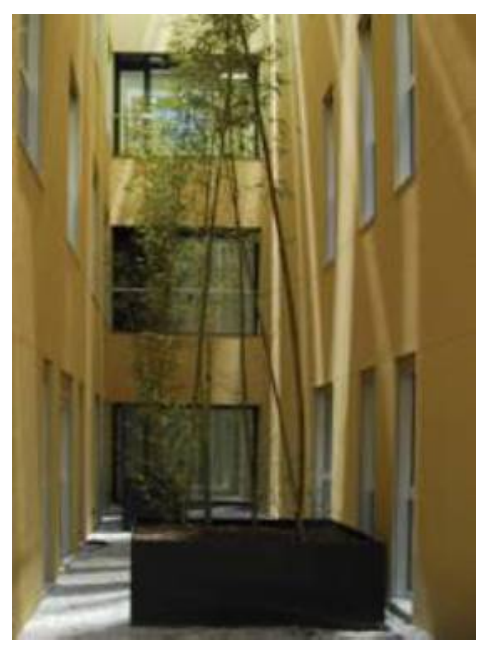

Figura 2.29. Patio iluminado con espejos ${ }^{10}$.

${ }^{10} \mathrm{http}: / /$ espaciosolar.com/patios-de-luz.htm. (Última visita 31/07/2016). 
Estos reflectores se colocan en la zona superior del patio, captando y reflejando de este modo la luz natural al interior, llegando a aumentar el nivel de iluminación natural hasta en 7 veces con respecto al nivel original.

\subsubsection{Muros cortina}

Un muro cortina es una estructura auxiliar, que actúa como fachada y que cubre la estructura principal del edificio. Está formada principalmente por elementos resistentes de tipo metálico y vidrio. Al igual que cualquier cerramiento exterior, debe cumplir la normativa vigente correspondiente, de ahí que deba garantizar la estanqueidad y el aislamiento tanto térmico como acústico. En la estructura auxiliar se debe establecer tanto un sistema de evacuación de agua procedente de la condensación, como un sistema de rotura de puente térmico. También debe diseñarse de tal manera que sus componentes individuales sean fácilmente reemplazables o reparables.

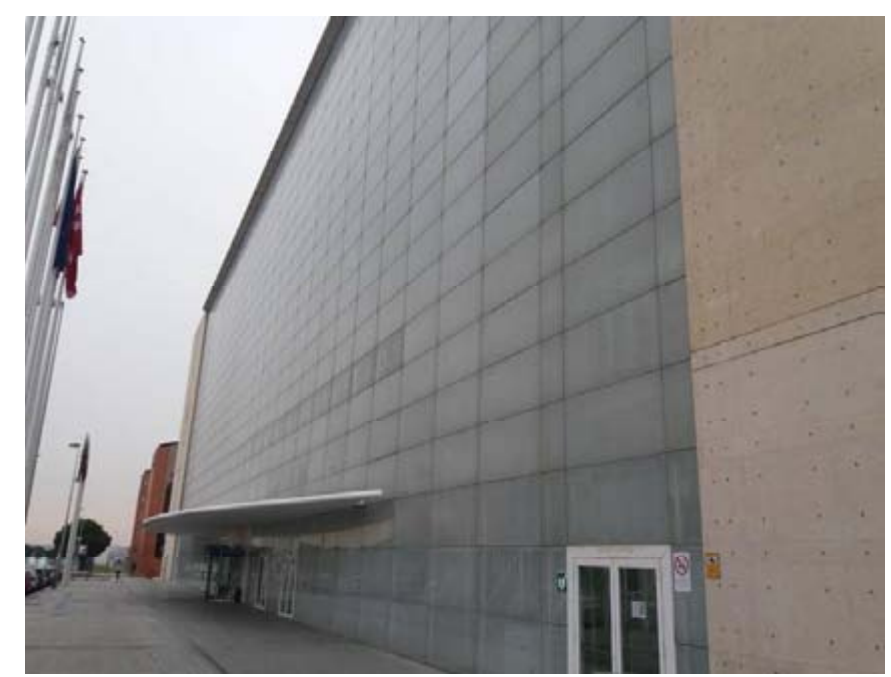

Figura 2.30. Muro cortina (Palacio de Congresos Madrid).

\subsubsection{Paredes translúcidas}

Como su propio nombre indica son paredes formadas por elementos translúcidos, es decir, con materiales con cierta transparencia como vidrios, telas $u$ hormigón translúcido. Este último material está formado por fibras ópticas y hormigón con áridos finos, y se puede emplear tanto en paredes como en pilares; además de aportar luminosidad también permite la conducción de electricidad sin necesidad de cables. 


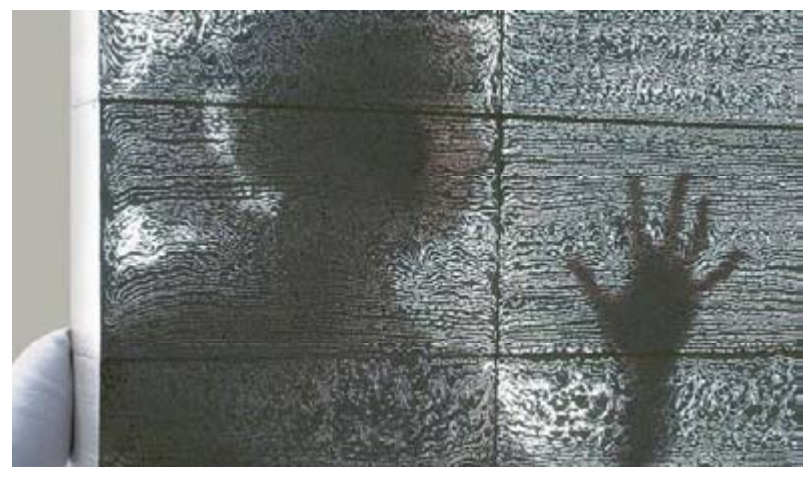

Figura 2.31. Pared translúcida ${ }^{11}$.

\subsubsection{Heliostatos}

El heliostato es un dispositivo que consiste en uno o varios espejos que se mueven sobre dos ejes, con el objetivo de seguir los movimientos del sol, de tal manera que es capaz de mantener el reflejo de los rayos solares sobre un punto o superficie pequeña a lo largo de todo el día.

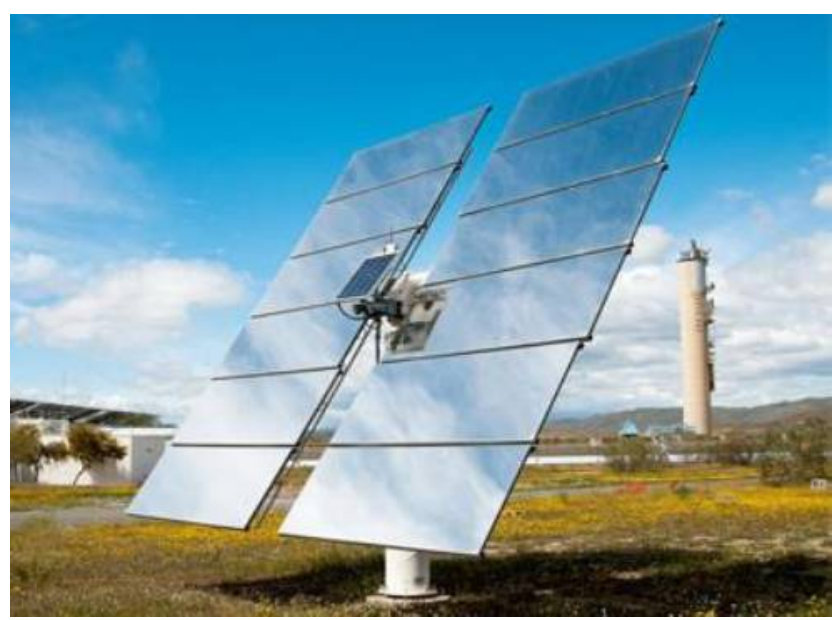

Figura 2.32. Heliostato ${ }^{12}$.

\subsubsection{Conductos o pozos de luz}

Los conductos son captadores de luz (figura 2.33) que actúan en forma de embudo con longitudes, hasta ahora, no superiores a los $10 \mathrm{~m}$. Los rayos solares atraviesan una claraboya transparente, penetrando así en el conducto el cual está forrado de material reflectante, de tal manera que la luz va recorriendo el conducto rebotando hasta llegar al terminal difusor.

\footnotetext{
11 https://cdn3.decoracion2.com/imagenes/2015/02/aprovechar-la-luz1-1.jpg. (Última visita 13/07/2016)

${ }^{12}$ http://www.dforcesolar.com/energia-solar/que-son-los-heliostatos/(Última visita 31/07/2016).
} 


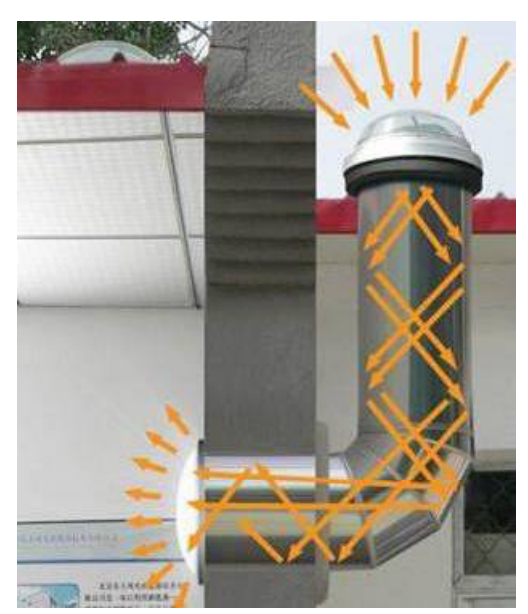

Figura 2.33. Sistema de captación de luz por conducto de luz tubular ${ }^{13}$.

\subsubsection{Sistemas de control de iluminación natural}

Por otro lado, también existen sistemas de iluminación destinados a controlar la entrada de luz natural al interior de las estancias situadas en un edificio. Entre sus objetivos está evitar los posibles deslumbramientos. Algunos de estos sistemas se exponen a continuación.

\subsubsection{Voladizos}

Los voladizos (figura 2.34) son elementos con resistencia estructural que sobresale más allá de la fachada. Son protecciones fijas situadas generalmente en la parte superior de la ventana, que impiden la entrada de radiación solar directa a las horas centrales del día principalmente.

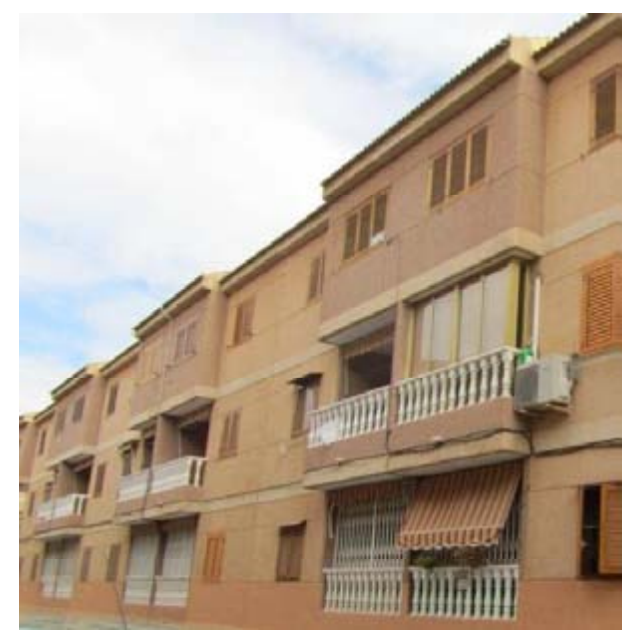

Figura 2.34. Voladizo.

\footnotetext{
${ }^{23}$ http://es.made-in-china.com/co_teksupply/product_Solar-Tubular-Skylight-System_hrhounsng .html. (Última visita 31/07/2016).
} 


\subsubsection{Celosías}

Las celosías son enrejados tupidos que actúan como elementos de control de la entrada de luz natural al interior de una estancia (figura 2.35). Hay de diversos tipos, pueden ser simples láminas ajustables según el ángulo de incidencia del sol, o bien complejos enrejados ornamentales que permiten ver a través de la ventana en el que están colocados sin ser visto.

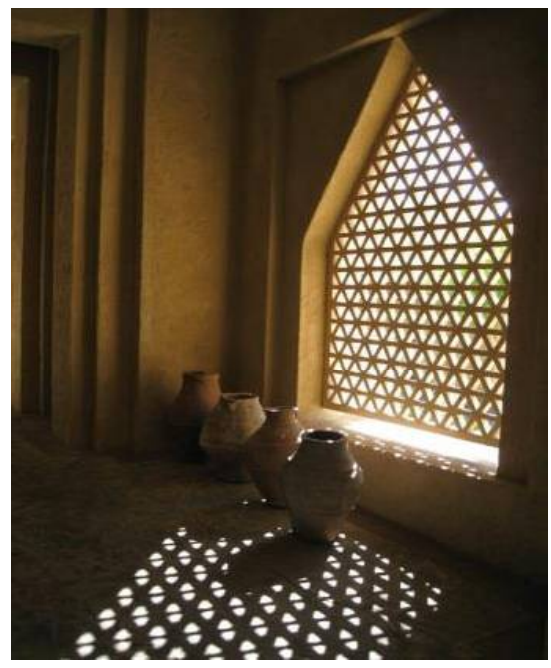

Figura 2.35. Celosía ${ }^{14}$.

\subsubsection{Apantallamientos verticales}

Los apantallamientos verticales son elementos de control fijos que se colocan a los lados de una ventana en dirección perpendicular a la fachada, para impedir el paso de radiación solar directa al interior de la estancia (figura 2.36). No es de los sistemas más comunes pues no sólo dificulta la entrada de aire por la ventana para ventilar la estancia, sino que limita las vistas desde el interior

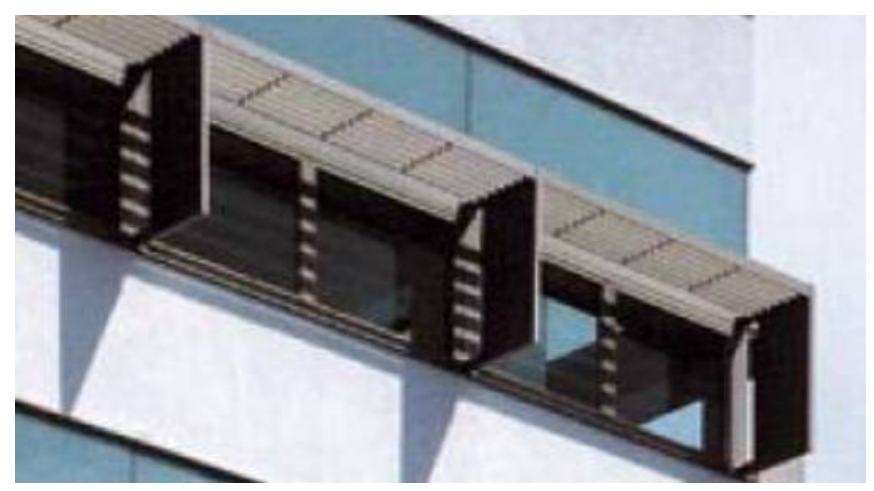

Figura 2.36. Sistema de Apantallamiento vertical ${ }^{15}$.

\footnotetext{
${ }^{14} \mathrm{http}: / / \mathrm{mm}$-actualidad.blogspot.com.es/2013/10/celosia.html. (Última visita 31/07/2016).

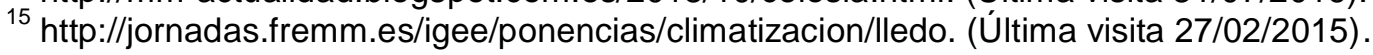




\subsubsection{Toldos}

Los toldos son elementos formados por una sencilla estructura, generalmente metálica o de PVC, y una lona o tejido resistente, que se puede extender 0 recoger según el ángulo de incidencia de los rayos solares (figura 2.37). Es uno de los elementos de protección solar más comunes y eficaces para impedir la penetración de radiación solar directa por las ventanas al interior de una estancia. Puede llegar a usarse como sistema de protección contra la lluvia. Hay de diversos tipos, puede ser fijo o móvil, vertical u horizontal, extensible de forma manu

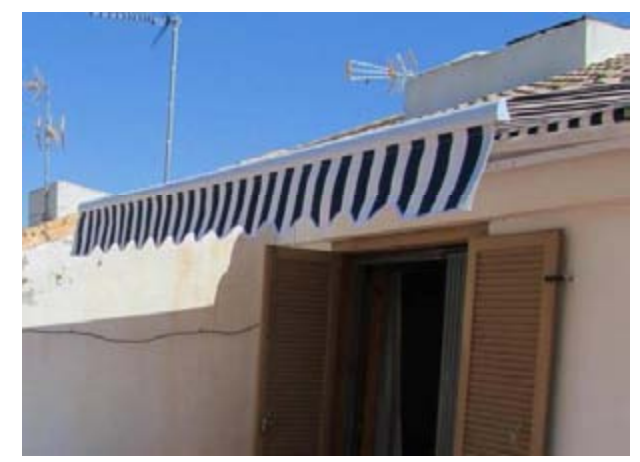

Figura 2.37. Toldo.

\subsubsection{Alféizares}

El alféizar es una pieza horizontal que se coloca en la base del hueco de una ventana (figura 2.37) generalmente hacia el exterior para que también cumpla la función de goterón. De esta manera obliga a la ventana a colocarse a haces interiores en lugar de a intermedios o exteriores, es decir, que se retranquea la ventana hacia el interior, y se crea un barrera protectora contra la radiación solar directa parecida a la de los voladizos. Este elemento, si además es de un color claro, puede reflejar y dirigir la luz hacia el interior del edificio.

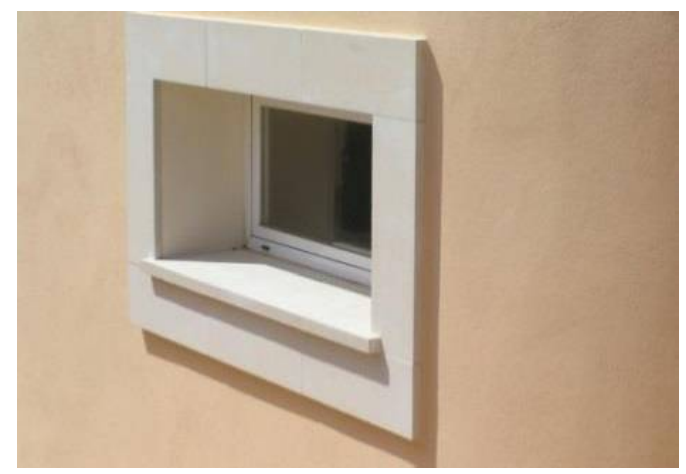

Figura 2.37. Alféizar en una ventana retranqueada ${ }^{16}$.

${ }^{16}$ http://premares.com/ampits.php?lang=ES. (Última visita 27/12/2016) 


\subsubsection{Cortinas}

Las cortinas son piezas de material flexible (generalmente tela) que cuelga de la parte superior de aperturas con el fin de cubrirlo o adornarlo. Son los dispositivos menos eficaces en verano, puesto que al ser colocados detrás del vidrio, en la cara que da al interior de la estancia, controlan la incidencia directa del sol pero no evitan la penetración del calor de la radiación solar. En invierno, sin embargo, actúan como un buen aislante dado que disminuye las pérdidas de energía calorífica.

\subsubsection{Persianas}

Las persianas son estructuras sencillas, colocadas encima de aperturas, formada por finas y estrechas láminas enganchadas entre si, de tal manera, que se puedan enrollar y extender. Pueden colocarse, en ventanas con doble acristalamiento, entremedias de los dos vidrios. Esto supone un menor mantenimiento, pero también un menor aislamiento que si se colocan por el exterior. Las persianas de colores claros serán más eficaces que las de color oscuro, puesto que reflejaran más la luz y absorberán menos calor. 
3. NORMATIVA 



\section{NORMATIVA}

La normativa vigente actualmente con respecto al tema de la iluminación no es muy abundante y está dirigida, mayormente, a la iluminación artificial. Se centra en establecer niveles mínimos de iluminación en estancias según el uso prioritario al que estén destinadas. Algunas de estas normas se mencionan a continuación:

\subsection{Código Técnico de la Edificación (CTE). Ministerio de Vivienda, Gobierno de España.}

\section{DB HE-3. 2013. Eficiencia energética de las instalaciones de iluminación.}

Este código exige que los edificios (tanto nuevos como reformados), dispongan de instalaciones de iluminación adecuadas que cubran las necesidades de sus usuarios y al mismo tiempo, sean energéticamente eficientes. Para alcanzar esta eficiencia energética, el sistema de iluminación artificial no deberá superar unos valores establecidos y así mismo debe contar con un sistema de control que permita ajustar el encendido a la ocupación real de la zona, y también servir como un sistema de regulación que optimice el aprovechamiento de la luz natural.

EI CTE establece niveles mínimos de luz artificial en el interior de edificios, sin embargo no establece ningún criterio sobre los niveles mínimos de iluminación natural en los edificios. Curiosamente ningún país del mundo en la actualidad, tiene una normativa que establezca niveles mínimos de iluminación natural en espacios interiores. Esto probablemente se deba a la dificultad de poder calcular adecuadamente la luz natural en el interior de un edificio (en la fase de diseño), ya que depende de múltiples factores (localización, clima, horas de luz, etc.). Aunque, en un futuro, esto podría cambiar debido a la creciente búsqueda de herramientas para predecir la iluminación con luz natural en el interior de edificios. 


\subsection{Normas europeas UNE-EN}

UNE-EN 12464-1: 2012. Iluminación de los lugares de trabajo. Parte 1: lugares de trabajo interiores.

En esta norma se establecen los requisitos de iluminación que deben tener los lugares de trabajo en el interior de edificios, de tal manera que queden satisfechas las necesidades tanto de confort como del rendimiento visual (para personas con una capacidad visual normal) de los ocupantes de cada zona laboral.

UNE-EN 12464-2: 2008. Iluminación de los lugares de trabajo. Parte 2: lugares de trabajo exteriores.

En esta norma se establecen los requisitos de iluminación que deben tener los lugares de trabajo en el exterior, de tal manera que queden satisfechas las necesidades tanto de confort como del rendimiento visual (para personas con una capacidad visual normal) de los ocupantes de cada zona laboral.

UNE-EN 12665: 2012. Términos básicos y criterios para la especificación de los requisitos de alumbrado.

Esta norma establece los términos básicos, así como las definiciones, a emplear en todas las aplicaciones de alumbrado. También establece que el alumbrado de un espacio debe cumplir con los siguientes requisitos: proporcionar la iluminación adecuada para garantizar la seguridad y el movimiento; facilidad para la prestación y percepción visual de los colores; y un confort visual adecuado para los ocupantes del espacio alumbrado.

UNE-EN 12193: 2009. lluminación. lluminación de instalaciones deportivas. Esta norma se centra en establecer las recomendaciones y requisitos necesarios para un alumbrado en instalaciones deportivas (dedicada a los deportes más practicados en la Unión Europea), tanto exteriores como interiores, que asegure unas buenas condiciones visuales para optimizar la percepción de la información visual, un nivel de confort visual aceptable, el 
mantenimiento del nivel de adecuadas prestaciones visuales y la limitación de luz perturbadora o deslumbramientos.

UNE-EN 15193: 2008. Eficiencia energética de los edificios. Requisitos energéticos para la iluminación.

En esta norma se recoge una metodología para calcular la cantidad de energía que se utiliza en un edificio para iluminar su interior, así como para calcular el uso de energía debido a iluminación en un edificio y así poder estimar el rendimiento energético total del edificio. Por otro lado aporta un procedimiento para hallar un indicador numérico de rendimiento energético de edificios, para así proporcionar valores para un certificado energético.

UNE-EN 15193:2008/AC: 2010. Eficiencia energética de los edificios. Requisitos energéticos para la iluminación.

Se modifica el anexo F de la Norma europea UNE-EN 15193: 2008.

\section{UNE-EN 50172: 2005. Sistemas de alumbrado de seguridad.}

El objetivo de esta norma es establecer los requisitos que deben cumplir las señales y el alumbrado de las vías de evacuación en el interior de edificios. Esta normativa está dirigida a espacios de trabajo y locales de ámbito público. Aunque no está dirigida al interior de viviendas particulares, sí es aplicable a las zonas comunes de edificios de viviendas de uso colectivo.

UNE-EN 72153: 1985. Niveles de iluminación. Asignación a tareas visuales. En esta norma se establecen los niveles de iluminancia media requeridos para cada zona dependiendo de la exigencia del campo visual que corresponde a la tarea que se vaya a llevar a cabo en dicha zona.

\section{UNE-EN 72161: 1985. Niveles de iluminación. Especificación.}

Esta disposición legal establece las magnitudes y los términos que deben emplearse al especificar el nivel de iluminación en un punto, superficie o volumen para los que no hay una normativa específica. 


\section{UNE-EN 72162: 1985. Clasificación y designación.}

Esta norma tiene como objetivo clasificar los niveles de iluminación tanto de un punto superficie o volumen específico, por categorías (establece 15 categorías de niveles de iluminación), clases (establece 4 clases de niveles de iluminación), tipos (establece 30 tipos de niveles de iluminación) y bandas (establece 5 bandas de niveles de iluminación).

\section{UNE-EN 72160: 1984. Niveles de iluminación. Definiciones.}

En esta norma se definen tanto los conceptos como las magnitudes necesarias para especificar el nivel de iluminación en una superficie o espacio determinado.

UNE-EN 72163: 1984. Niveles de iluminación. Asignación a tareas visuales. La finalidad de esta norma es establecer el método adecuado para determinar la iluminancia media, es decir el nivel de iluminación, que debe tener un punto, superficie o volumen específico, teniendo en cuenta la tarea visual que se vaya a desarrollar en tal punto, superficie o volumen específico.

\section{UNE-EN 72502: 1984. Sistemas de iluminación. Clasificación general.}

Se realiza una clasificación de los distintos sistemas de iluminación teniendo en cuenta para ello las fuentes de luz empleadas, la distribución de la luz así como los objetivos particulares de la iluminación.

UNE-EN ISO 28803: 2012. Ergonomía del ambiente físico. Aplicación de las normas internacionales a las personas con necesidades especiales.

Se establecen unos estándares internacionales para la ergonomía de ambientes físicos, estudia algunos temas enfocados hacia el confort térmico, acústico, lumínico, calidad del aire, etc...

Aconseja prestar especial atención al diseño de los sistemas de iluminación en aquellas estancias donde vayan a estar ancianos o personas con algún tipo de trastorno visual. Clasifica los trastornos visuales en 4 grupos: 
-Visión parcial: Visión afectada por traumas, síndromes degenerativos o enfermedades.

-Baja visión: Se aplica a aquellas personas que no pueden leer el periódico a una distancia de lectura normal aun llevando gafas o lentillas.

-Legalmente ciego: Se aplica a aquellas personas con una visión inferior a 6/60 dioptrías en su mejor ojo o tienen un campo de visión limitado.

-Totalmente ciego: Se aplica en caso de que haya incapacidad para distinguir entre luz y oscuridad.

\subsection{Reales decretos}

Real Decreto 486/1997, por el que se establecen las disposiciones mínimas de seguridad y salud en los lugares de trabajo. BOE no 97 de 2304-1997.

Este Real decreto establece las disposiciones mínimas de seguridad y salud que debe reunir un lugar de trabajo. En el campo de la iluminación especifica los valores mínimos que deben tener los lugares de trabajo según las exigencias visuales (bajas, moderadas, altas o muy altas) y uso (ocasional o habitual) de la zona de trabajo o parte de ésta, de tal manera que los trabajadores puedan desarrollar sus actividades bajo condiciones de visibilidad adecuadas sin riesgos para su seguridad y salud.

Real Decreto 1890/2008, por el que se aprueba el Reglamento de eficiencia energética en instalaciones de alumbrado exterior. BOE no 279 de 19-112008.

Este Real Decreto tiene como finalidad establecer las características que deben reunir las instalaciones de alumbrado exterior para su mayor eficiencia energética y que contaminen lumínicamente lo menos posible. De acuerdo con esta normativa, todos los proyectos de iluminación en el interior de edificios deben incluir en su memoria los siguientes parámetros:

-Cálculo del valor de eficiencia energética de la instalación (VEEI): 
VEEI $=\frac{\text { Potencia instalada } \cdot 100}{\text { Superficieiluminada }\left(\mathrm{m}^{2}\right) \cdot \| l \text { minanciamediamantenida }}$

Las unidades en el Sistema Internacional: W/m² por cada 100 Lux.

-Comprobación de la existencia del sistema de control y regulación que optimice el aprovechamiento de la luz natural.

-Verificación de la existencia de un plan de mantenimiento.

-Índice del local (K) utilizado en el cálculo:

$$
K=\frac{L \cdot A}{H \cdot(L+A)}
$$

Siendo: $L$ la longitud del local, $A$ la anchura del local, $H$ la distancia del plano de trabajo a las luminarias.

-Número de puntos considerados.

-Factor de mantenimiento previsto ( $F m$ ): Este índice depende del grado de mantenimiento del local objeto de estudio, el cual está comprendido entre el 50 y el $80 \%$.

-lluminancia media mantenida (Em): Es el límite inferior admitido de iluminancia media en un área concreta. Debe especificarse en la unidad en el Sistema Internacional (Lux).

-Índice de deslumbramiento unificado (UGR):

$$
U G R=8 \log \left(\frac{0,25}{L b} \cdot \frac{S L 2 w}{P 2}\right)
$$




\section{Dónde:}

Lb: Luminancia de fondo $(\mathrm{cd} / \mathrm{m})$

L: Iluminancia de las partes luminosas de cada luminaria en dirección del observador $(\mathrm{cd} / \mathrm{m})$

w: Ángulo sólido obtenido por las partes luminosas de cada luminaria a la vista del observador (estereorradianes)

P2: Índice de posición para cada luminaria individual, relacionado con su desviación de la línea visual.

-Índice de rendimiento del color (Ra): Es la medida de la calidad de reproducción de los colores. Se obtiene como una nota de examen in situ; esta nota es el resultado sobre la comparación de 8 ó 14 colores de muestra. Un nivel 100 significa que todos los colores se reproducen perfectamente, y conforme nos vamos alejando de ese nivel 100, podemos esperar una menor definición sobre los colores.

$$
\begin{array}{ll}
\mathrm{Ra}<60 & \text { Pobre } \\
60<\mathrm{Ra}<80 & \text { Bueno } \\
80<\mathrm{Ra}<90 & \text { Muy Bueno } \\
\mathrm{Ra}>90 & \text { Excelente }
\end{array}
$$

-Las potencias de los conjuntos lámpara más equipo auxiliar.

-Tipo de sistema de control y regulación de la iluminación que corresponda. 



$$
\begin{aligned}
& \text { 4. METODOLOGÍA } \\
& \text { DE TRABAJO }
\end{aligned}
$$





\section{METODOLOGIA DE TRABAJO}

\subsection{Instrumental empleado}

Para llevar a cabo el estudio de los niveles de iluminación (iluminancias) en el interior del aula objeto de estudio, así como en su modelo a escala, se emplearon diversos instrumentos de medida y registro de datos de iluminancias. Los instrumentos y equipos empleados cuyas características se exponen a continuación, fueron facilitados por el Departamento de Estructuras y Física de Edificación de la Escuela Técnica Superior de Arquitectura (E.T.S.A.M) de la universidad Politécnica de Madrid (U.P.M)

\subsubsection{Sensores fotométricos $\mathrm{LI}-210$}

Este tipo de sensores permiten captar la radiación electromagnética que emite una fuente de luz y la transforman en intensidad eléctrica, la cual es transmitida a un equipo o instrumento de registro (Data Logger) mediante cableado, el cual es capaz de interpretar ese impulso eléctrico como una medida de iluminancia.

Están formados por una cabeza donde se encuentra una superficie de captación de la radiación electromagnética (fotodiodo de silicio con filtro de precisión que es sensible a la luz en el espectro visible), una base y un cable de transmisión del impulso eléctrico (figura 4.1.). En el Anexo I se especifican las características técnicas completas de este sensor.
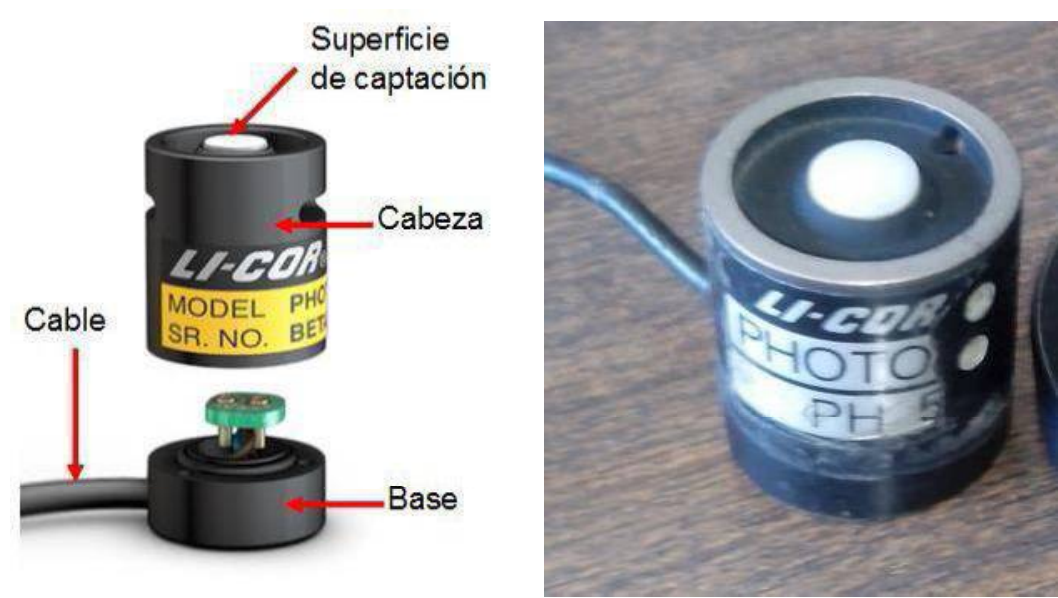

Figura 4.1. Sensor fotométrico LI-210. 
Para que las medidas tomadas sean válidas, es necesario aplicar una constante de calibración. Esta constante de calibración viene determinada por el fabricante del producto; sin embargo, es recomendable calibrarlo todos los años. Las constantes de calibración de los sensores utilizados se recogen en la tabla 4.1, que se muestra a continuación.

\begin{tabular}{|c|c|c|c|c|}
\hline & Datalogger & Sensor & $\begin{array}{l}\text { Número de } \\
\text { referencia }\end{array}$ & $\begin{array}{c}\text { Constante } \\
\text { de } \\
\text { calibración }\end{array}$ \\
\hline \multirow{12}{*}{ Aula } & \multirow{6}{*}{ D3 } & AA & 5024 & 2,8814 \\
\hline & & $A B$ & 5818 & 3,8064 \\
\hline & & $A C$ & 5783 & 3,0321 \\
\hline & & $A D$ & 5781 & 2,9872 \\
\hline & & $\mathrm{AE}$ & 5000 & 3,3734 \\
\hline & & $\mathrm{AF}$ & 5020 & 3,1984 \\
\hline & \multirow{6}{*}{ D4 } & $A G$ & 4731 & 3,1161 \\
\hline & & $\mathrm{AH}$ & 5018 & 3,4846 \\
\hline & & $\overline{A l}$ & 5046 & 3,1015 \\
\hline & & AJ & 5045 & 3,608 \\
\hline & & $\mathrm{AK}$ & 5026 & 3,4622 \\
\hline & & $\mathrm{AL}$ & 5022 & 3,3487 \\
\hline \multirow{12}{*}{ Modelo } & \multirow{6}{*}{ D1 } & $\mathrm{MA}$ & 5021 & 3,7004 \\
\hline & & $\mathrm{MB}$ & 4730 & 3,5098 \\
\hline & & $\mathrm{MC}$ & 5148 & 3,4324 \\
\hline & & $\mathrm{MD}$ & 5785 & 3,6966 \\
\hline & & $\mathrm{ME}$ & 5779 & 2,9379 \\
\hline & & MF & 5780 & 3,1951 \\
\hline & \multirow{6}{*}{ D2 } & $M G$ & 4993 & 3,718 \\
\hline & & $\mathrm{MH}$ & 5817 & 3,0897 \\
\hline & & $\mathrm{Ml}$ & 5019 & 3,1841 \\
\hline & & $\mathrm{MJ}$ & 5784 & 4,1225 \\
\hline & & $\mathrm{MK}$ & 4707 & 3,4175 \\
\hline & & $\mathrm{ML}$ & 5786 & 3,4615 \\
\hline Exterior & D5 & $\mathrm{C} 8$ & 5044 & 3,783 \\
\hline
\end{tabular}

Tabla 4.1. Constantes de calibración. 
Para aportar a los sensores más estabilidad y sujeción, éstos han sido colocados en unos soportes (figura 4.2. y figura 4.3.) creados a partir de una impresora 3D. Estos soportes además elevan los sensores 0.80 centímetros, dando al conjunto soporte más sensor una altura total de 3.30 centímetros.

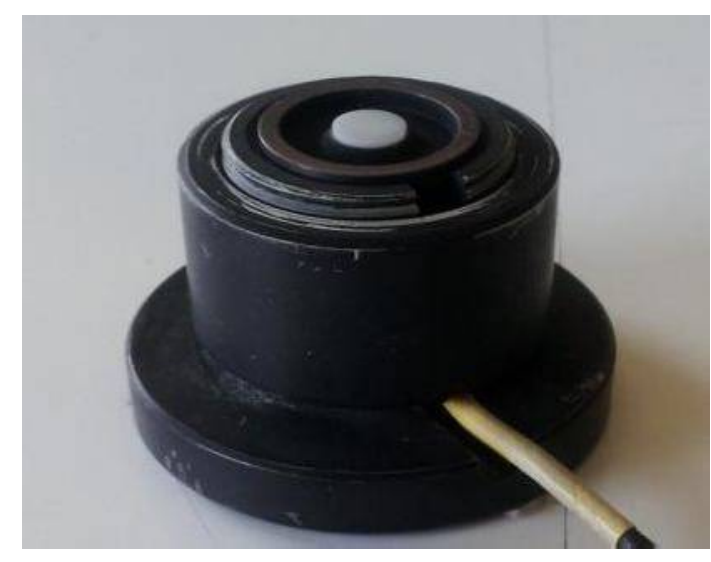

Figura 4.2. Sensor fotométrico LI-210 con soporte horizontal en aula.

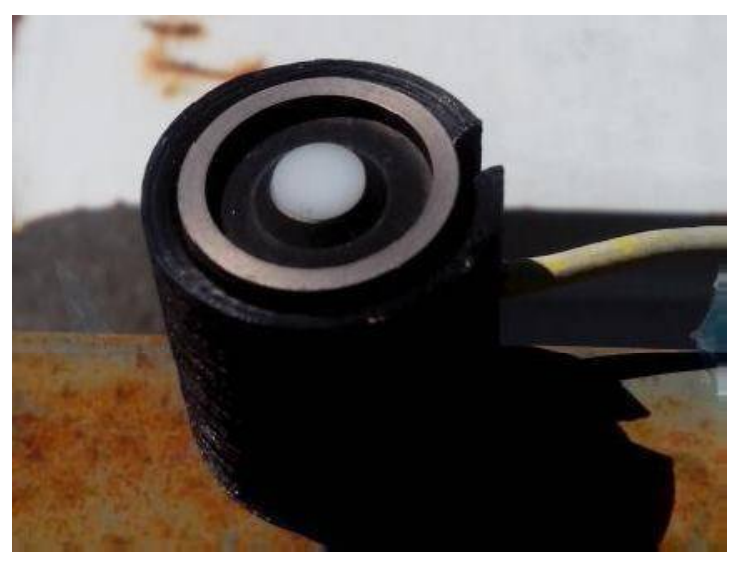

Figura 4.3. Sensor fotométrico LI-210 con soporte horizontal en modelo a escala.

En total se han utilizado 25 de estos sensores, 12 de ellos colocados en el aula objeto de estudio, otros 12 en el modelo a escala de dicho aula y uno en una torreta exterior situada al lado del modelo en la cubierta norte de la E.T.S.A.M.

\subsubsection{DataLoggers Licor LI-1000}

Los DataLoggers son instrumentos de registro y almacenamiento de datos. Este modelo, dependiendo del tipo de sensor que se le conecte puede tomar medidas de diferentes magnitudes físicas: de radiación solar (piranómetros), de iluminancias (fotómetros), de temperatura (termopares), etc. En este caso al haber sido conectados a sensores fotométricos se han registrado medidas de iluminancias.

Estos instrumentos ofrecen la opción de fijar la frecuencia con la que se registran los datos, la hora de comienzo del registro, así como los niveles mínimos de la magnitud de medida a partir de los que se comienzan a registrar datos. En este estudio se fijó un mínimo para los valores de iluminancias de $1 \times 10^{-5}$ klux. 
A cada DataLogger utilizado se pueden conectar hasta 8 sensores, dos a través de las conexiones BCN y otros seis a través de un puerto de conexión de una placa Terminal Block (figura 4.4). En esta investigación se han empleado 5 DataLoggers, dos de ellos en el aula conectados con 6 sensores cada uno, otros dos en el modelo a escala igualmente conectados con 6 sensores cada uno y uno conectado únicamente al sensor exterior. Los datos registrados se descargaron al ordenador a través del puerto RS-232 del mismo al ordenador.

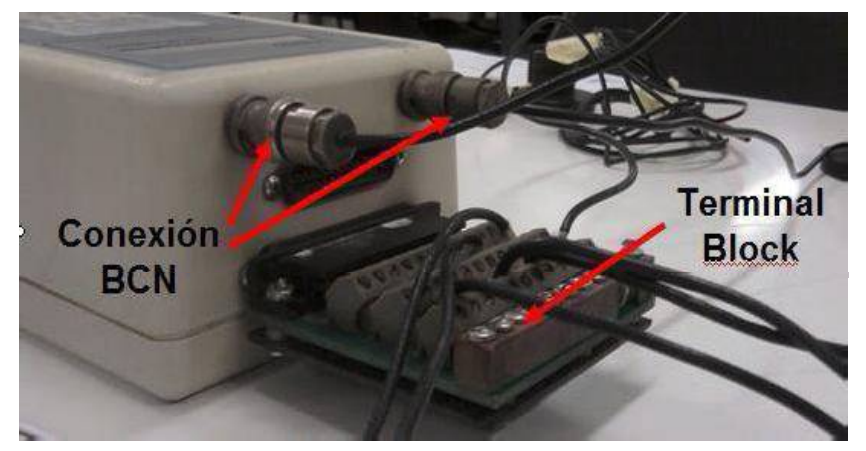

Figura 4.4. Conexiones BCN y Terminal Block.

Estos dispositivos también constan de un display y un teclado para la configuración de sus funciones (figura 4.5). Su misión es poder establecer que las medidas se visualicen de forma instantánea en la pantalla (modo INST) o que almacene las medidas de forma automática (Modo LOG).

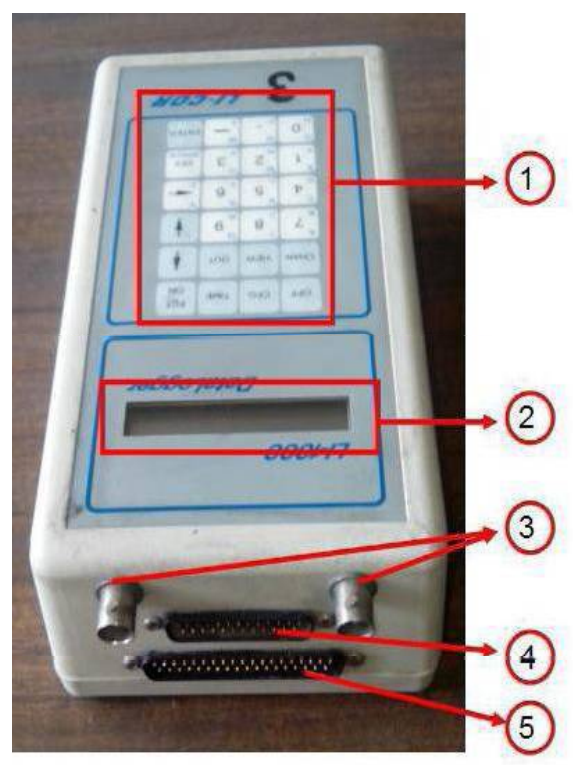

1. Teclado de configuración.

2. Display de 16 dígitos.

3. Canales de conexión BNC para sensores.

4. Puerto de conexión de 25 pines para cable PC.

5. Puerto de conexión de 37 pines para el Terminal Block.

Figura 4.5. Datalogger LI-1000. 
La memoria de estos aparatos de almacenamiento de datos, de $32 \mathrm{~Kb}$, para nuestro trabajo es considerablemente limitada, lo que implica la descarga de datos al ordenador cada poco tiempo, a fin de evitar la superposición de unos datos con otros.

Conviene destacar que su fuente de alimentación son seis pilas de 1.5 V LR20 cada una y dispuestas en serie (figura 4.6), lo que implica cierta planificación y control en el tiempo óptimo de uso de éstas. Se puede encontrar más información sobre estos aparatos en su manual de utilización [LI-1000 Datalogger Instruction Manual, 1987].

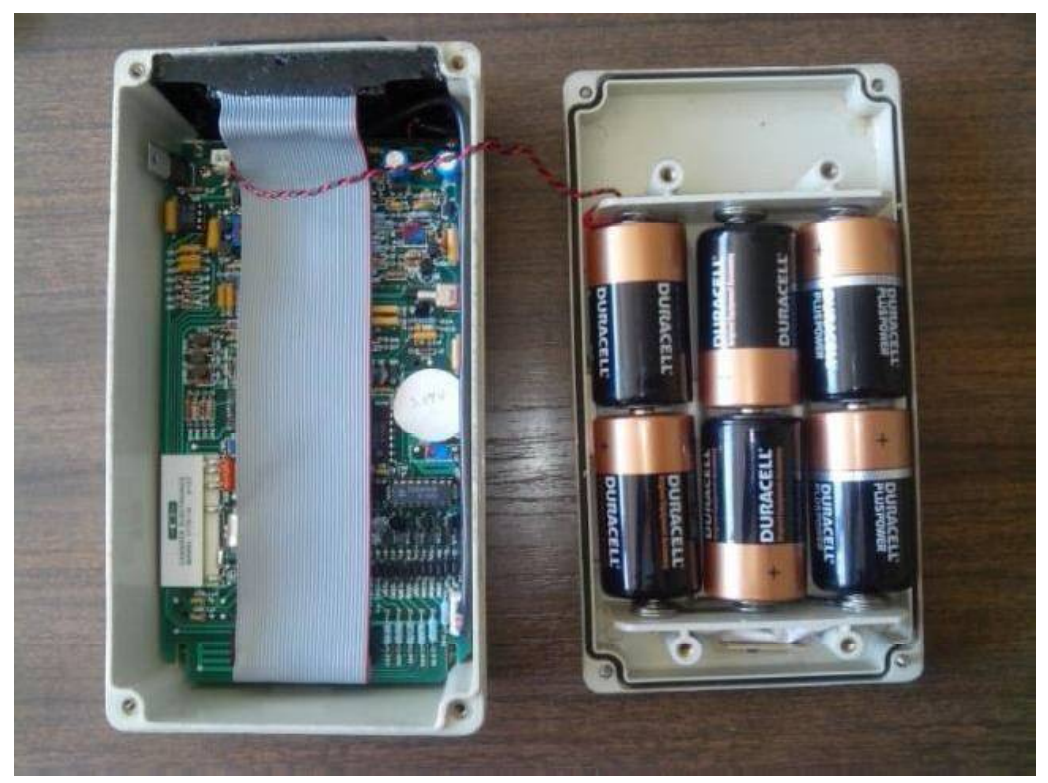

Figura 4.6. Interior de Datalogger LI-1000.

\subsection{Aula de estudio}

\subsubsection{Localización del aula}

El recinto utilizado en este trabajo de investigación es el aula 2S2 de la Escuela Técnica Superior de Arquitectura de Madrid (E.T.S.A.M), situada en la ciudad de Madrid, en el campus de la U.P.M de Ciudad Universitaria perteneciente al distrito de Moncloa-Aravaca. Este edificio se encuentra delimitado por la Casa de Velázquez (Norte), el Museo del Traje (Este), la Escuela Técnica Superior de Edificación (Sur) y la facultad de Bellas Artes (Oeste) (figura 4.7). 


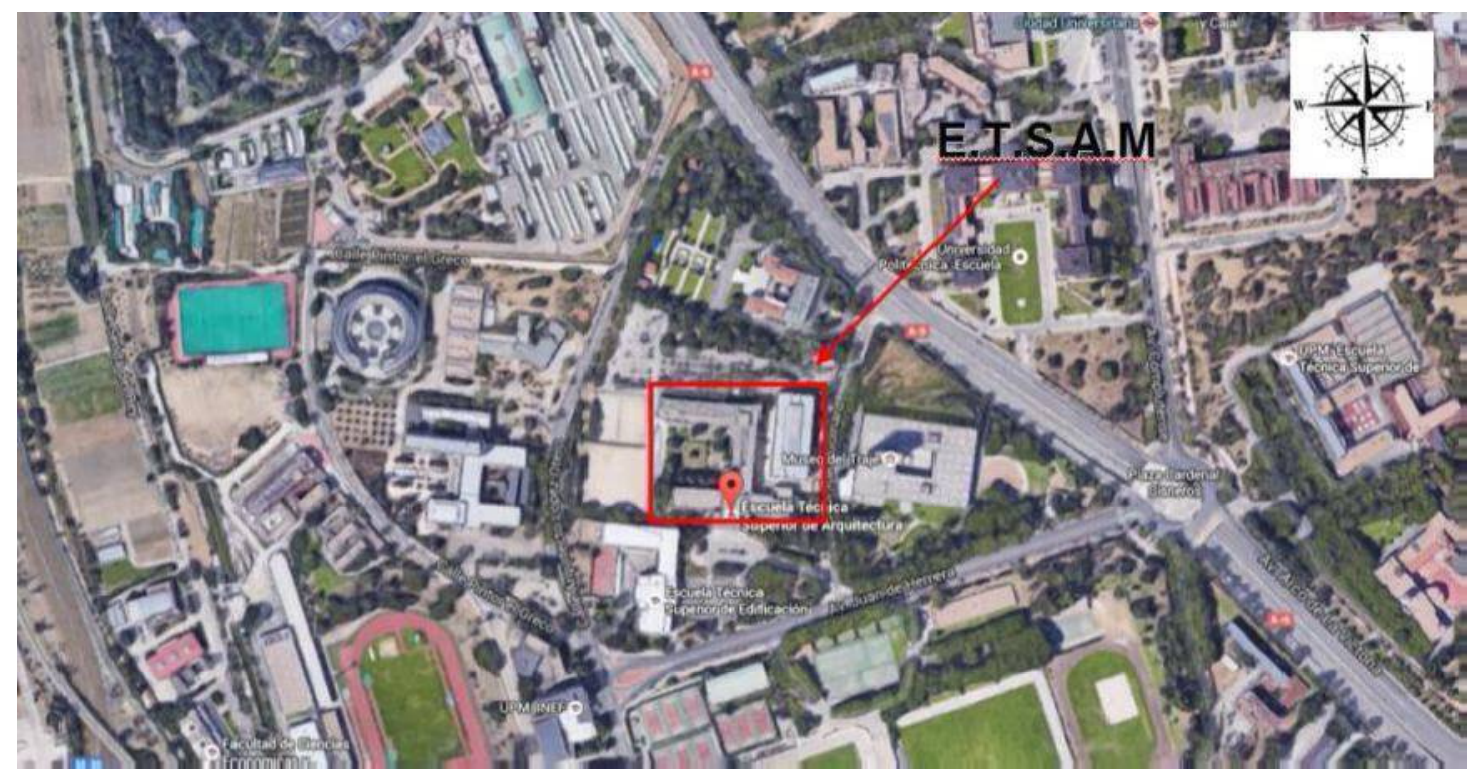

Figura 4.7. Localización de la E.T.S.A.M mediante Google maps.

El aula para medición in situ (figura 4.9) está situada en el segundo piso del Ala Norte de esta Escuela Técnica Superior (figura 4.8). Dicha ala Norte conforma una construcción con tres plantas sobre rasante y dos sótanos.

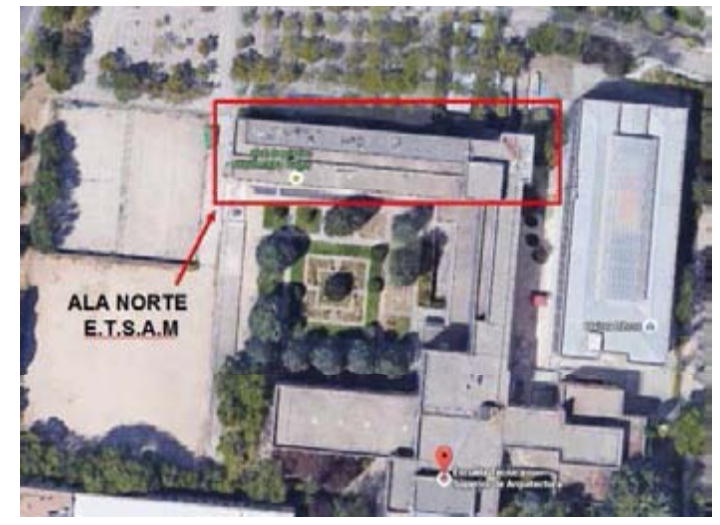

Figura 4.8. Ala norte de la E.T.S.A.M vista mediante Google maps.

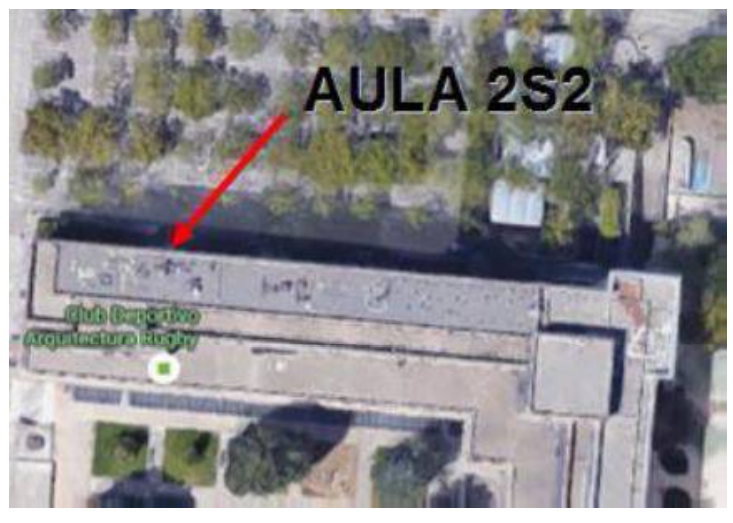

Figura 4.9. Situación aula2S2 en el ala norte de la E.T.S.A.M mediante Google maps.

El aula objeto de estudio está orientada al Norte y no presenta colindancia con fachadas ni vegetación arbórea cercana que, aparentemente, pudieran llegar a obstruir o interferir en la penetración de la luz natural a través de sus ventanas. Lo más cercano que pudiera interferir está a más de 40 m (figura 4.10). 


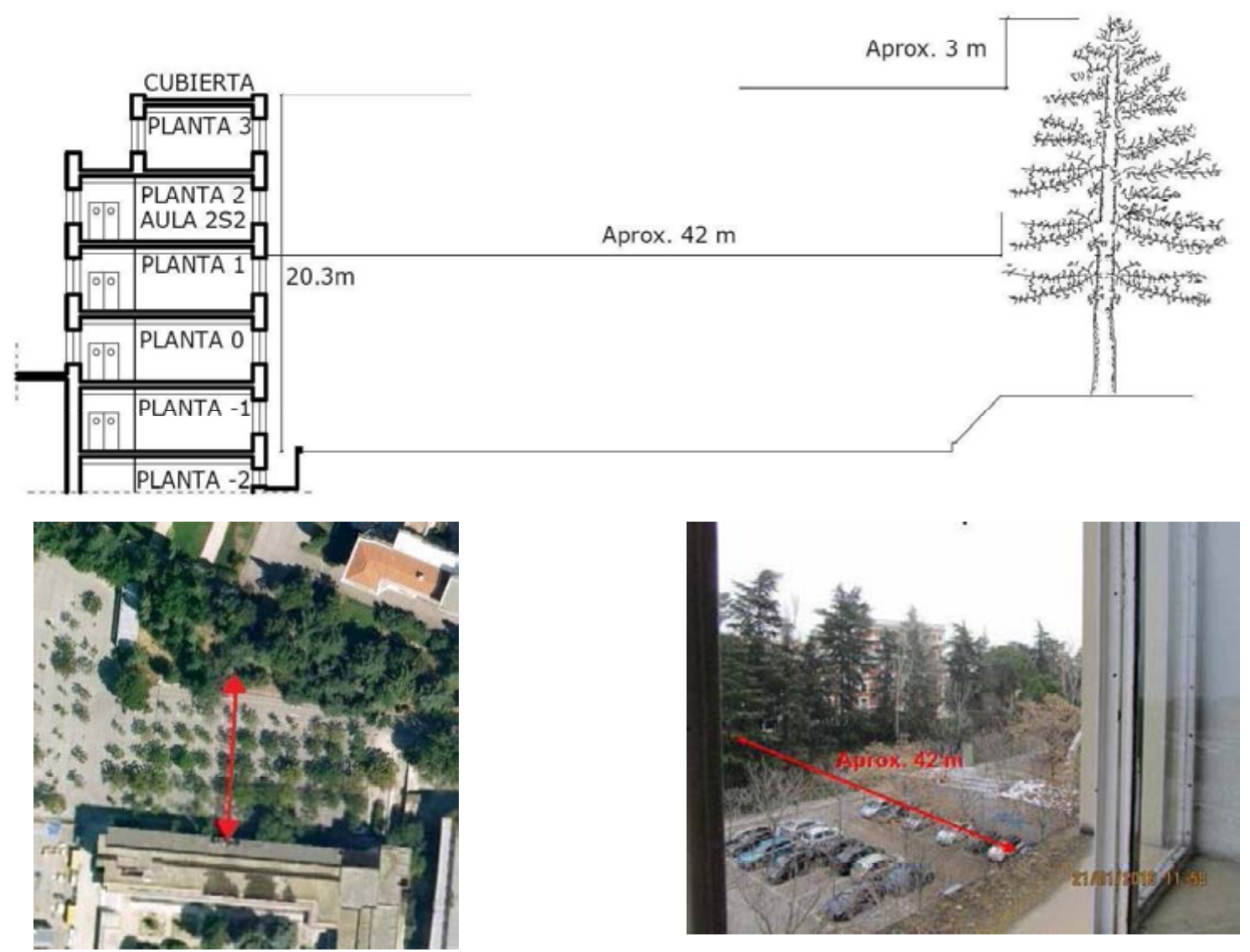

Figura 4.10. Distancia desde la ventana del aula 2S2 a la vegetación colindante.

\subsubsection{Descripción del aula}

El aula objeto de estudio tiene una planta rectangular, con las siguientes dimensiones: longitud de $6.30 \mathrm{~m}$, anchura de $6.65 \mathrm{~m}$ y $3.28 \mathrm{~m}$ de altura (figura 4.11).

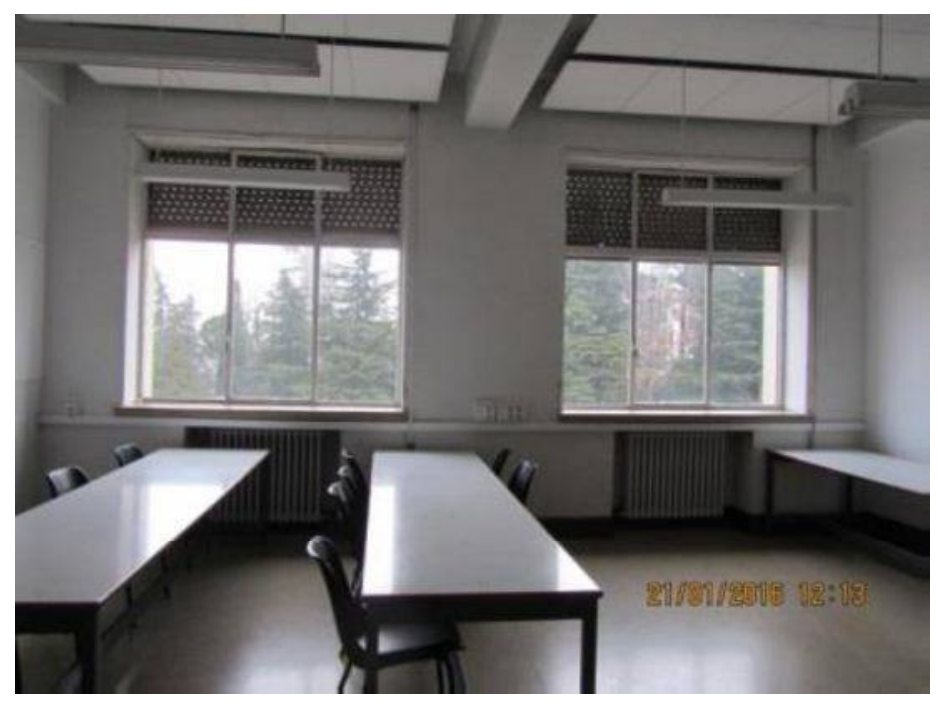

Figura 4.11. Aula $2 S 2$ de la E.T.S.A.M. 
El mobiliario del aula está compuesto por 5 mesas de tablero chapado en blanco y 4 sillas por mesa (20 sillas en total). Las paredes interiores del aula están acabadas en yeso pintado al temple de color blanco, el suelo con baldosas de terrazo pulimentado en color beige grisáceo, y se dispone de un falso techo de color blanco. En la pared Este se encuentran la pizarra de color verde oliva y una puerta corredera de color blanco que da paso al aula contigua. En la pared Oeste se dispone de un corcho blanco y otra puerta corredera que da a otra aula contigua. La puerta de acceso al aula es de doble hoja con abertura hacia el pasillo exterior, de color madera está situada frente a la fachada Norte.

En la fachada norte se encuentran situadas dos ventanas cuyas medidas son de $2.00 \mathrm{~m}$ de ancho por $2.00 \mathrm{~m}$ de altura $\left(4 \mathrm{~m}^{2}\right)$. Debido a que las persianas de las ventanas no pueden subirse completamente para el presente estudio sólo se ha considerado un hueco de $2 \mathrm{~m}$ de ancho por $1.20 \mathrm{~m}$ de altura $\left(2.40 \mathrm{~m}^{2}\right)$, fijando las persianas a esa altura, como se muestra en las figuras 4.11 y 4.12 .
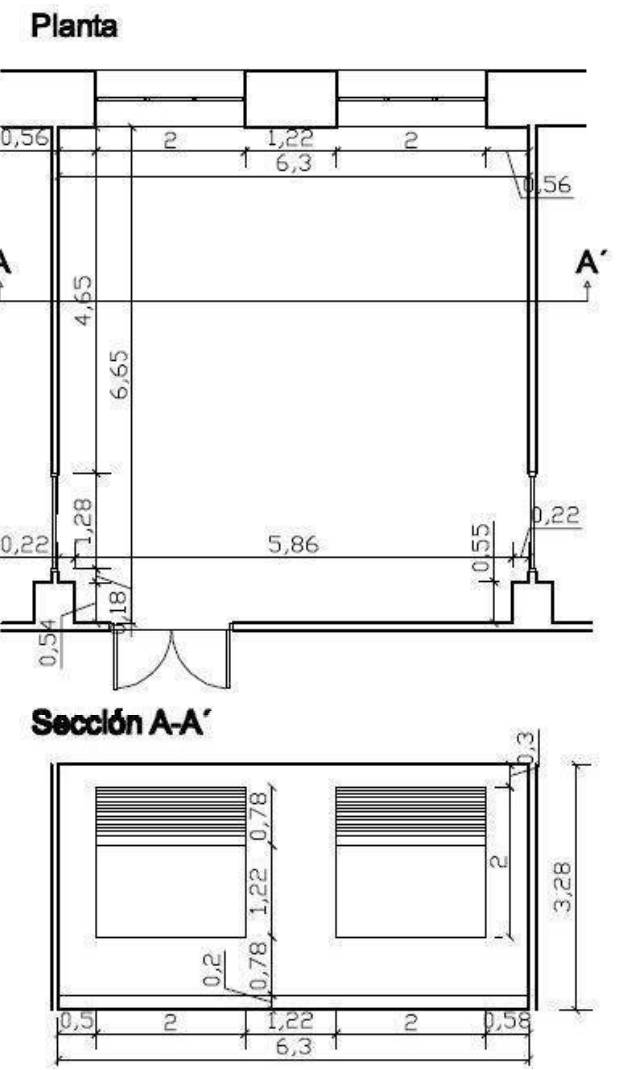

Figura 4.12. Planta y sección Aula $2 S 2$ de la E.T.S.A.M. (cotas en m) [García, 2013]. 


\subsection{Modelo a escala}

Para llevar a cabo el desarrollo de esta tesis se ha utilizado un modelo a escala 1/15 (figura 4.13), escala que ha considerado la más adecuada ya que no sólo facilita el transporte y la construcción del mismo, sino que además proporciona el suficiente espacio en el interior de éste para colocar los sensores necesarios para llevar a cabo la toma de datos. Este modelo ha sido construido por Paula García Palencia [García, 2013], en el laboratorio de maquetas de la E.T.S.A.M.

El material utilizado en la construcción del modelo ha sido Poliestireno Expandido (EPS) en planchas de $5 \times 1 \mathrm{~m}^{2}$ y $5 \mathrm{~cm}$ de espesor. Se decidió emplear este material por diversas razones, entre ellas, por su ligereza (lo que facilita su manipulación y transporte), por su permeabilidad y resistencia a la intemperie, cualidad imprescindible ya que va a estar sometida a las inclemencias del tiempo en la cubierta de la E.T.S.A.M.; y por su espesor, puesto que los muros de la E.T.S.A.M. son de 0.75 m que a escala $1 / 15$ serian $0.05 \mathrm{~m}$, coincidiendo de esta manera con el espesor de las placas de EPS.

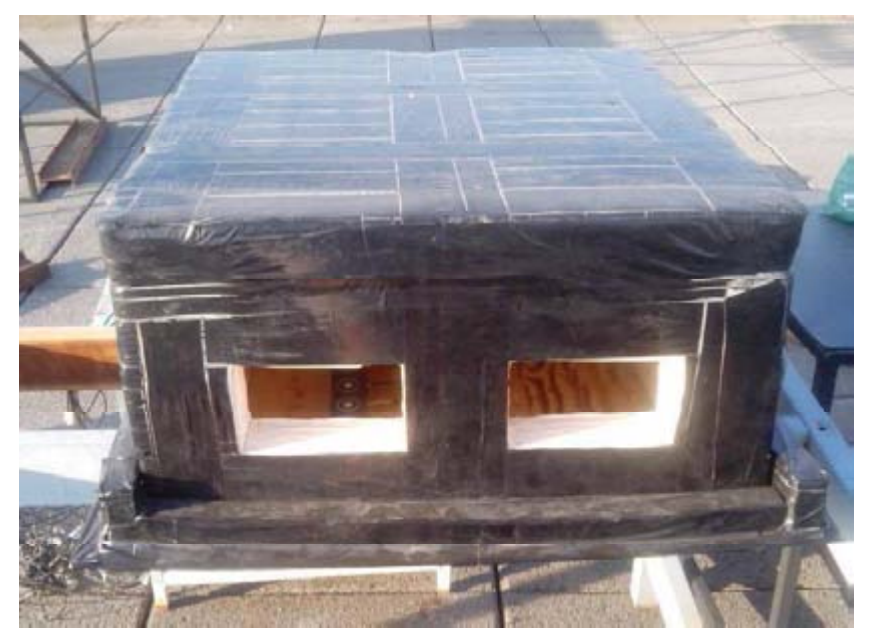

Figura 4.13. Modelo a escala 1/15.

El Poliestireno Expandido es un material opaco, sin embargo, no lo suficiente. Para asegurar que no hubiera filtraciones de luz al interior del modelo que desvirtuasen las iluminancias reales, las planchas EPS fueron recubiertas de cinta adhesiva opaca de color negro. 
Para la base del modelo se ha empleado un tablero de fibras de madera contrachapada con una superficie de $0.55 \mathrm{~m} \times 0.55 \mathrm{~m}$, dado que se considera lo suficientemente ligera para transportar cómodamente el modelo a escala, y lo suficientemente rígida para mantenerse horizontal.

Los huecos que simulan las ventanas en el modelo, no han sido cubiertos con ningún tipo de vidrio ni material que simule ese último, para evitar que en el interior del modelo se alcanzasen temperaturas excesivamente altas por efecto invernadero y para eliminar el efecto lupa que podría originar el uso de un vidrio sobre los materiales empleados en la maqueta.

Para obtener la máxima fiabilidad posible en las medidas, el modelo a escala debe ser lo más fiel al espacio real (el aula). Por ello no sólo se debe tener en cuenta los acabados exteriores sino también los interiores. Para esto último se recubrió el interior del modelo con papel canson $160 \mathrm{~g} / \mathrm{m}^{2}$ de colores con reflectancias similares a las interiores del aula (tabla 4.1). En relación a las reflectancias del mobiliario en el interior del aula se consideraron elementos con reflectancias similares a la del suelo, techo y paredes del mismo, y así mismo se hizo en el modelo, a excepción de la pizarra que al ser un elemento especialmente oscuro puede diferir en gran medida con la superficie donde está colocado.

\begin{tabular}{|c|c|c|}
\hline SUPERFICIE & REFLECTANCIA & $\begin{array}{c}\text { PAPEL } \\
\text { CANSON 160 } \\
\mathbf{g} / \mathbf{m}^{2}\end{array}$ \\
\hline PAREDES & $68 \%$ & 407 lichen \\
\hline TECHO & $68 \%$ & 407 lichen \\
\hline SUELO & $51 \%$ & 470 maïs \\
\hline PIZARRA & $3 \%$ & 425 noir \\
\hline
\end{tabular}

Tabla 4.1. Valores reflectancias. 
Este modelo a escala 1/15 del aula objeto se ha colocado en la azotea Norte de la Escuela Técnica Superior de Arquitectura de la Universidad Politécnica de Madrid (ETSAM), cuyas coordenadas GPS son: 40 $25^{\prime} \mathrm{N}, 3^{\circ} 41^{\prime} \mathrm{W}$. El modelo se encuentra orientado exactamente igual que el aula al que simula, ubicado justo con las mismas coordenadas pero una planta en altura por encima de dicho aula objeto de estudio.

\subsection{Software}

Una vez se han recogido los datos de iluminancia necesarios tanto en el aula como en el modelo a escala, con los instrumentos de medida mencionados en el apartado anterior, estos datos deben ser guardados y gestionados de forma adecuada para poder ser analizados e interpretados posteriormente; así como para compararlos con otros datos obtenidos por simulación y obtener, de ese modo, resultados gráficos que faciliten su interpretación. Para ello se han empleado diversos programas con distintas funciones que facilitaran estas tareas.

\subsubsection{Almacenamiento y tratamiento de datos}

Para procesar los datos de medidas de iluminancias se ha utilizado un Software en entorno MS-DOS, compatible con los Dataloggers LI-1000 empleados para registrar los datos de iluminancias. Este software es el LI-1000 2.0, de la misma marca que los Dataloggers (LICOR), que permite descargar los datos registrados y almacenados en los mismos cuando éstos se conectan al PC y guardar los datos descargados. El programa ofrece la opción de descargar los datos por orden de sucesión horaria y sensor específico, pudiendo clasificarlos tanto en filas como en columnas, según el tratamiento posterior que se quiera realizar. Una vez descargados los datos éstos fueron tratados y analizados mediante el programa Excell. Programa que también se ha empleado para realizar algunas gráficas.

Para elaborar la mayor parte de las gráficas se ha empleado el programa Grapher 8.0, con el que es posible crear gráficos tanto en 2D como en 3D a partir de ecuaciones simples y complejas. Ofrece la posibilidad de modificar el 
aspecto de los gráficos, cambiar el color y grosor de línea, cambiar fuentes y estilos utilizados de letra, etc.

El programa escogido para el análisis estadístico de los datos, fue el Statgraphics Centurion. Se trata de un programa para gestionar y analizar valores estadísticos con opciones para representar gráficas tanto estadísticas como de desarrollo de experimentos, previsiones y simulaciones en función del comportamiento de los valores. Consta de cuatro módulos principales: StatReport (prepara informes con datos variables), StatWizard (asistente estadístico que sugiere los métodos más adecuados para recopilar y analizar datos), StatLink (enlaza el libro de análisis con la fuente de datos) y el Statfolio (es la fuente de datos).

\subsubsection{Software de simulación}

Con el fin de verificar si los resultados obtenidos del análisis de los datos son representativos, éstos han sido comparados con valores obtenidos por simulación, a través del programa de simulación de iluminación DiaLux 4.11. Este programa fue el escogido para realizar las simulaciones principalmente debido a que es de acceso gratuito, puede emplearse tanto en la simulación de luz natural como artificial y debido a que su manejo no resulta excesivamente complejo. El programa permite establecer parámetros como la orientación del espacio modelado, franja horaria, día del mes y año, tipo de cielo que hay el día que se quiere realizar la simulación, etc... Por otro lado si se desea trabajar con luz artificial, este programa permite elaborar proyectos de luz artificial con las luminarias de todos los fabricantes. 


$$
\begin{aligned}
& \text { 5. TRABAJO } \\
& \text { EXPERIMENTAL }
\end{aligned}
$$





\section{TRABAJO EXPERIMENTAL}

\subsection{Adquisición de datos}

En este apartado se especifica cómo se ha llevado a cabo la adquisición de datos, tanto en el aula como en el modelo. Además se expone cómo han sido tratados, analizados y ordenados dichos datos.

\subsubsection{Colocación de los sensores en el aula}

Para medir las iluminancias en el aula, se ha dividido ésta en cuatro zonas (figura 5.1). La primera zona (Z1) está a $0.28 \mathrm{~m}$ de la pared Este, alineada con la parte central del machón de fábrica de la fachada. La zona 2 (Z2) está situada a $1.56 \mathrm{~m}$ de la pared Este, alineada a la mitad de la ventana situada más al Este del aula objeto de estudio. La zona 3 (Z3) está situada a $3.17 \mathrm{~m}$ de la pared Este, centrado con el machón central de la fachada del aula. La zona 4 (Z4) está a $4.78 \mathrm{~m}$ de la pared Este, alineada a la mitad de la ventana situada más al Oeste del aula objeto de estudio.

La toma de datos, tanto en el aula como en la maqueta, se ha realizado de dos formas diferentes. En la primera los 12 sensores se han colocado en una única fila, a distintas distancias de la fachada $(0.5,1,1.5,2,2.5,3,3.5,4,4.5,5,5.5$ y $6 \mathrm{~m}$ ), de tal manera que los datos se registran de forma individual por zona; como se muestra en la figura 5.1.
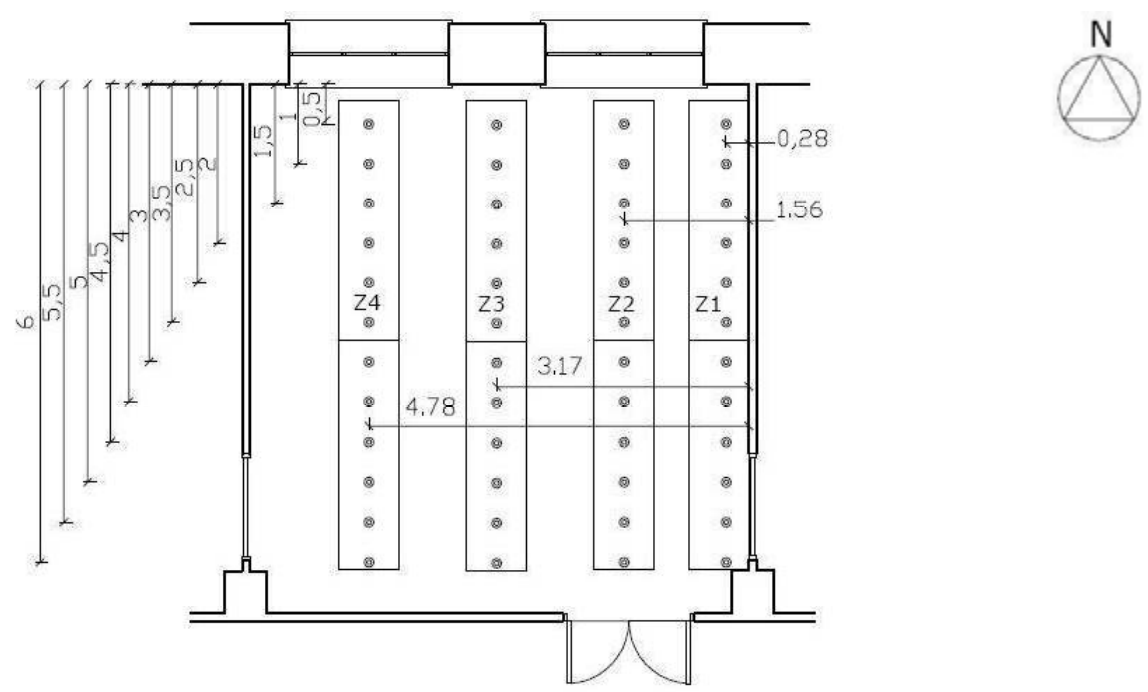

Figura 5.1. Esquema de las cuatro zonas del aula 2S2 (Cotas en $\mathrm{m}$ ). 
En el segundo caso se han colocado 3 sensores (alineados a las distancias de 1,2 y 3 m de la fachada), simultáneamente en las cuatro zonas, con lo que se tienen tres sensores en la zona 1 , tres en la zona 2 , tres en la zona 3 y tres en la zona 4 ( 12 sensores en total), tanto en el aula como en el modelo a escala.

\subsubsection{Obtención de los datos por zona de manera individual}

De forma individual en cada zona, se han colocado los sensores en una única fila separados entre sí $0.5 \mathrm{~m}$ encima de las mesas disponibles en el aula (a una altura de $0.7 \mathrm{~m}$ ). El primer sensor se ha colocado a $0.5 \mathrm{~m}$ de la fachada de tal manera que el segundo sensor queda a $1 \mathrm{~m}$ de ésta, el tercer a $1.5 \mathrm{~m}$, el cuarto a $2 \mathrm{~m}$, el quinto a $2.5 \mathrm{~m}$, el sexto a $3 \mathrm{~m}$, el séptimo a $3.5 \mathrm{~m}$, el octavo a $4 \mathrm{~m}$, el noveno a $4.5 \mathrm{~m}$, el décimo a $5 \mathrm{~m}$, el undécimo a $5.5 \mathrm{~m}$ y el último queda a $6 \mathrm{~m}$ de la fachada (figura 5.2 y 5.3 ).

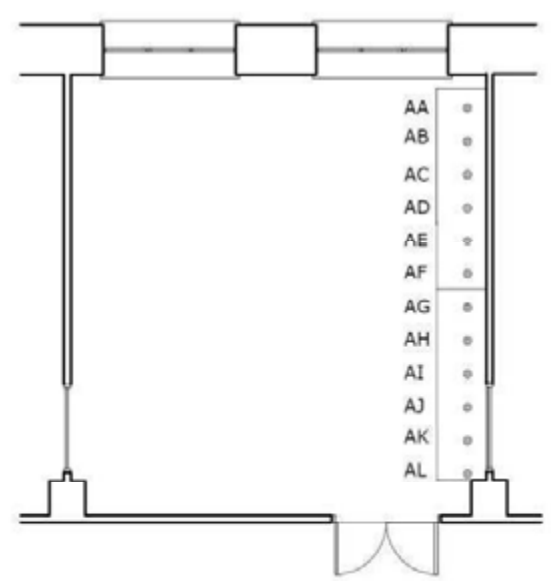

(a) Zona 1.

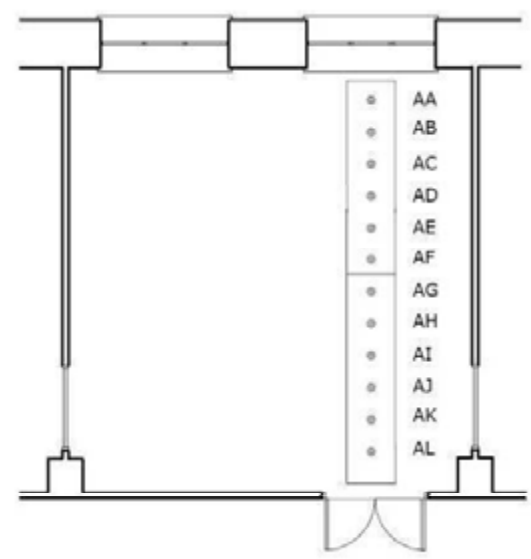

(b) Zona 2.

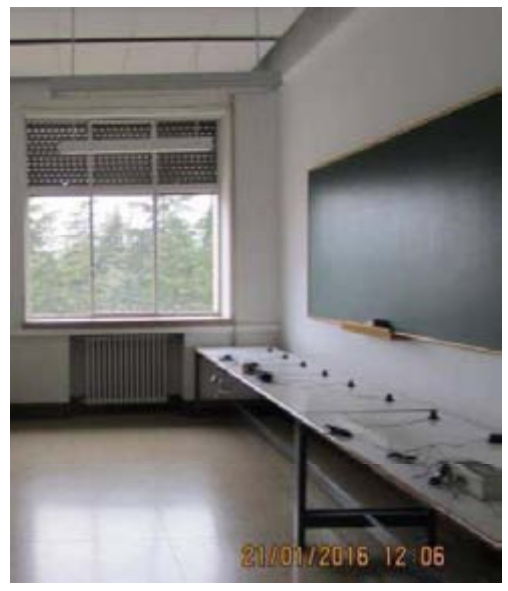

(a) Zona 1.

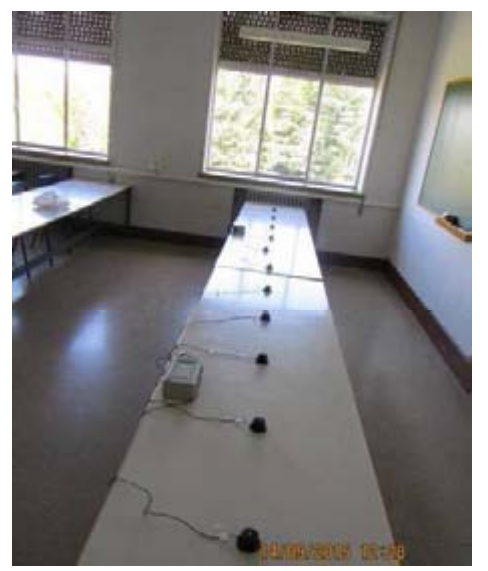

(b) Zona 2. 


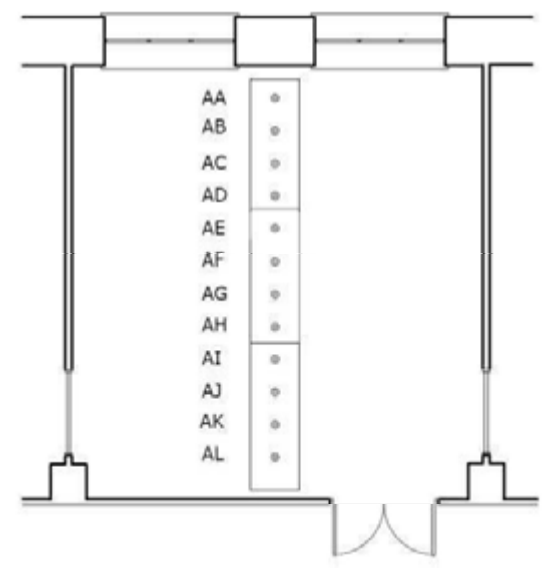

(c) Zona 3.

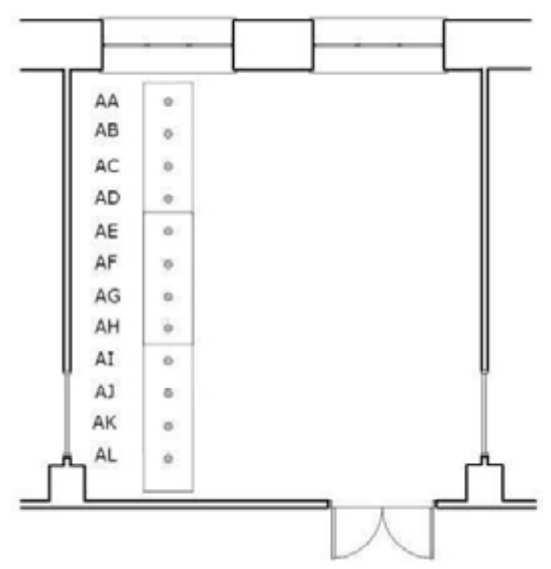

(d) Zona 4.

Figura 5.2. Esquema sensores por zona (cotas en $\mathrm{m}$ ).

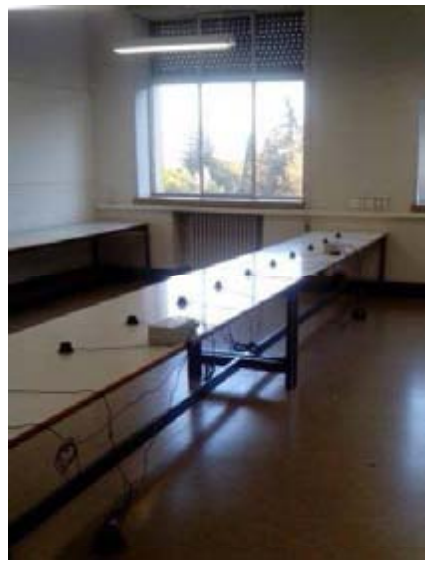

(c) Zona 3.

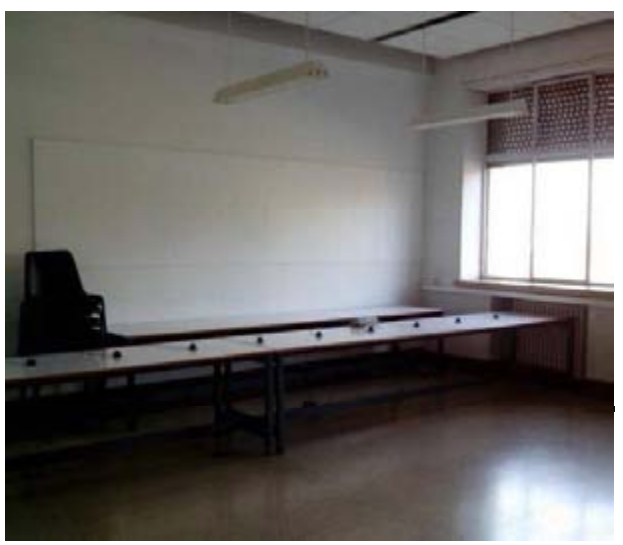

(d) Zona 4.

Figura 5.3. Colocación sensores por zona.

\subsubsection{Obtención de los datos en las cuatro zonas simultáneamente}

Para medir en las cuatro zonas de forma simultánea se han colocado 3 sensores en cada zona, a la distancias respectivas de 1, 2 y $3 \mathrm{~m}$ de la fachada (figuras 5.4 y 5.5 ).

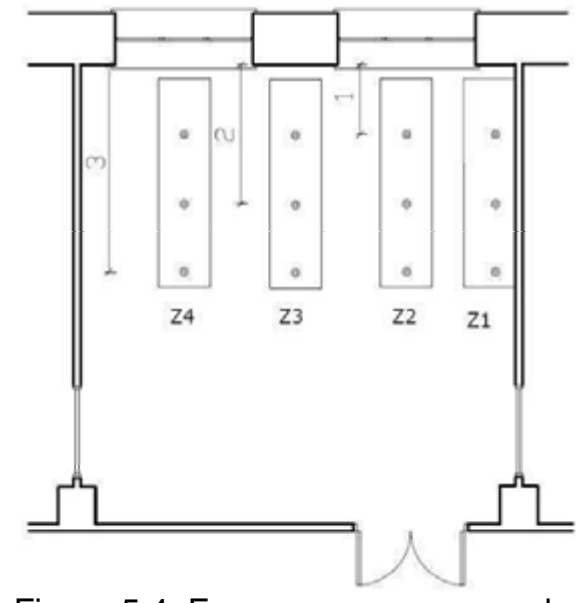

Figura 5.4. Esquema sensores en las cuatro zonas (cotas en $\mathrm{m}$ ).

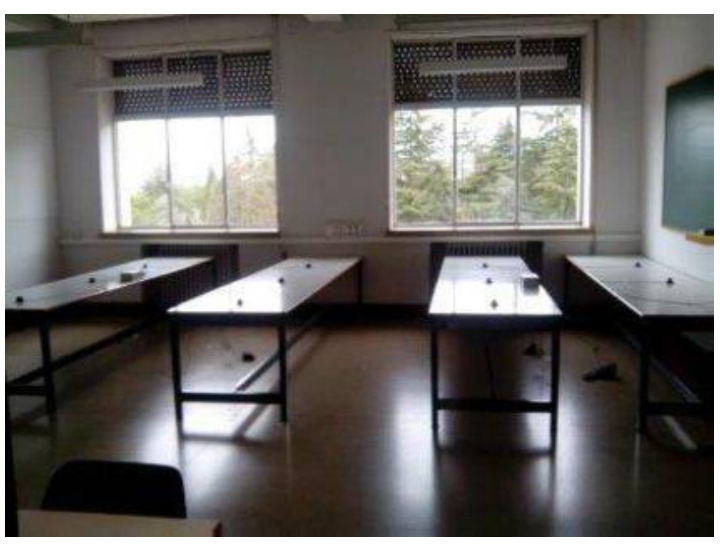

Figura 5.5. Colocación sensores en las cuatro zonas. 


\subsubsection{Colocación de sensores en el modelo a escala}

Los sensores en el modelo a escala $1 / 15$ se han colocado de forma proporcional a los colocados en el aula, para poder posteriormente comparar las iluminancias obtenidas en el modelo y en el aula.

El modelo ha sido dividido también en cuatro zonas al igual que el aula (figura 5.6). La primera zona (Z1) está situada a $1.85 \mathrm{~cm}$ de la pared Este, alineada con la parte central del machón de la fachada. La denominada zona 2 (Z2) está situada a $10.30 \mathrm{~cm}$ de la pared Este, alineada a la mitad del hueco de la ventana situada más al Este. La zona 3 (Z3) está ubicada a $20.92 \mathrm{~cm}$ de la pared Este, centrado con el machón central de la fachada del modelo. La zona 4 (Z4) está situada a $31.54 \mathrm{~m}$ de la pared Este, alineada a la mitad del hueco de la ventana situada más al Oeste del modelo a escala.
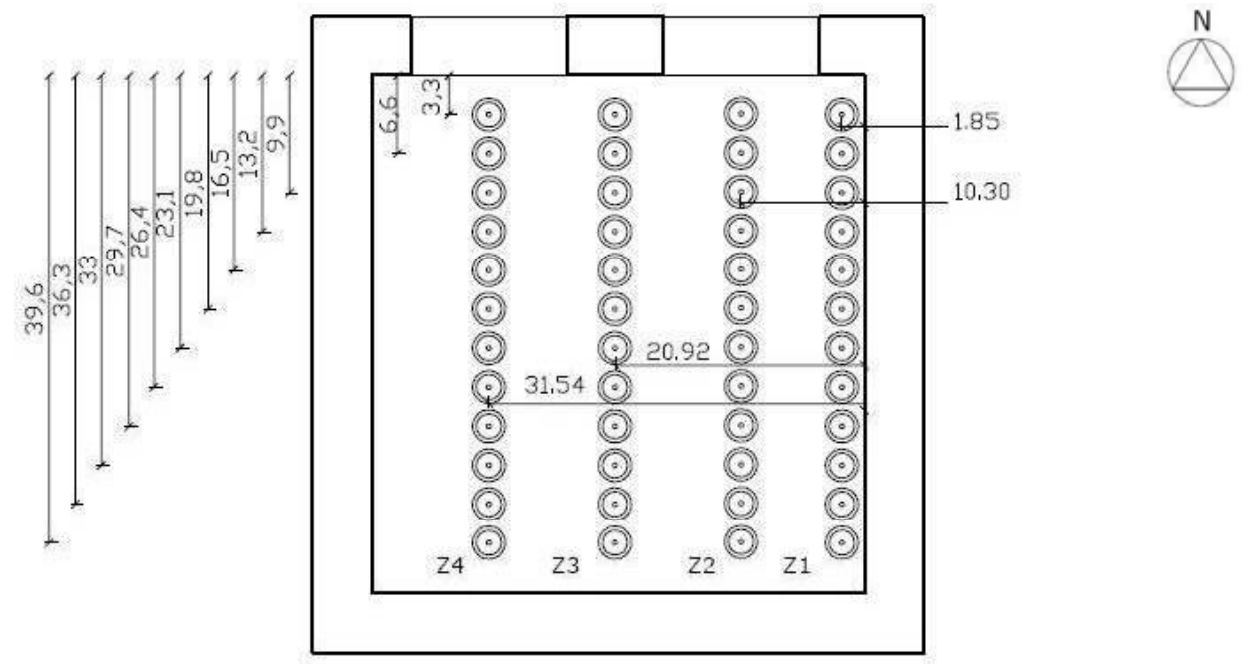

Figura 5.6. Esquema de las cuatro zonas del modelo a escala $2 \mathrm{~S} 2$ (Cotas en $\mathrm{cm}$ ).

Tras comprobar que el modelo a escala estaba totalmente horizontal, se dispusieron en su interior los sensores de forma análoga a como habían sido colocados en el aula objeto de estudio. Colocados los sensores y comprobado que el interior de la maqueta no presenta filtraciones de luz, se colocó un tablero y dos perfiles IPE encima de la maqueta, con la precaución de que no proyectasen sombras en la fachada del modelo, para que la acción del viento que pudiera actuar en la cubierta no moviese ni deteriorase la maqueta. 
Para asegurar que los sensores en el interior del modelo se sitúen de forma proporcional a la misma altura correspondiente a los del aula, y al mismo tiempo ofrecer suficiente estabilidad a los mismos, se colocaron sobre un soporte. Debido a que la toma de datos se realizó de dos maneras distintas se fabricaron dos soportes distintos. Para la toma de datos por zona de forma individual se empleó una tabla de madera (figura 5.7) de $1.50 \mathrm{~cm}$ de espesor, $10.40 \mathrm{~cm}$ de ancho y $40.00 \mathrm{~cm}$ de largo. En esta tabla se colocaron pequeños clavos justo en la posición donde debía ir el sensor. En la cabeza de cada clavo se colocaba el sensor con el soporte horizontal fabricado con la impresora 3D previamente mencionada (apartado 5.1).

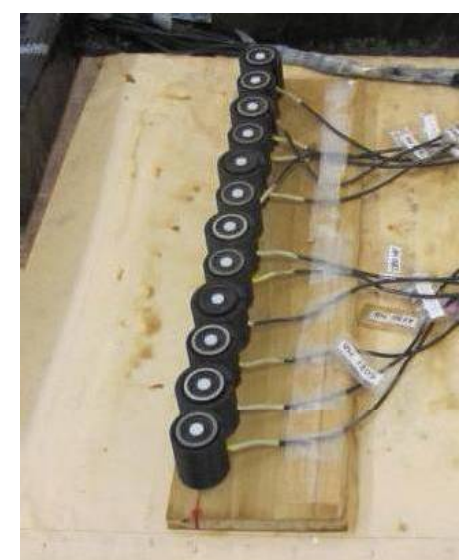

Figura 5.7. Disposición de los sensores sobre la tabla de madera.

Para el la adquisición de datos de iluminancias en las cuatro zonas de modo simultáneo, se fabricó un soporte de "goma eva" (figura 5.8) de $1.50 \mathrm{~cm}$ de espesor, $41.60 \mathrm{~cm}$ de ancho y $26.00 \mathrm{~cm}$ de largo, cuya superficie se pintó con pintura acrílica con el mismo tono que el suelo del aula para que la reflexión fuera la misma. En este caso no se empleó una tabla de madera, dado que una tabla de madera de estas dimensiones aumentaba considerablemente el peso de la maqueta.
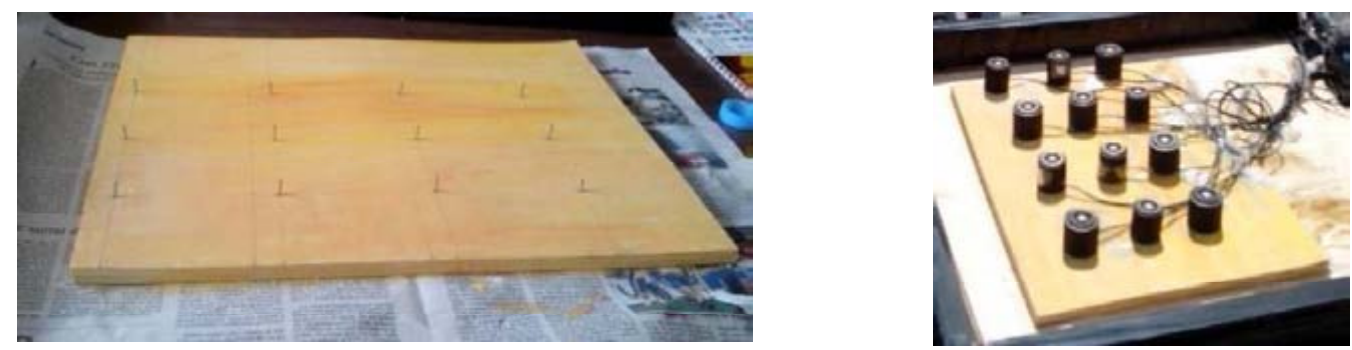

Figura 5.8. Disposición de los sensores sobre soporte de goma eva. 


\subsubsection{Obtención de los datos por zona de manera individual}

De forma individual en cada zona, se han colocado los sensores en una única fila separados entre sí $3.30 \mathrm{~cm}$. El primer sensor se ha colocado a $3.30 \mathrm{~m}$ de la fachada de tal manera que el segundo sensor queda a $6.60 \mathrm{~cm}$ de esta, el tercer sensor a $9.90 \mathrm{~cm}$, el cuarto a $13.20 \mathrm{~cm}$, el quinto a $16.50 \mathrm{~cm}$, el sexto a $19.80 \mathrm{~cm}$, el séptimo a $23.10 \mathrm{~cm}$, el octavo a $26.40 \mathrm{~cm}$, el noveno a $29.70 \mathrm{~m}$, el décimo a $33.00 \mathrm{~cm}$, el undécimo a $36.30 \mathrm{~cm}$ y el último queda a $39.60 \mathrm{~cm}$ de la fachada (figuras 5.9 y 5.10 ).

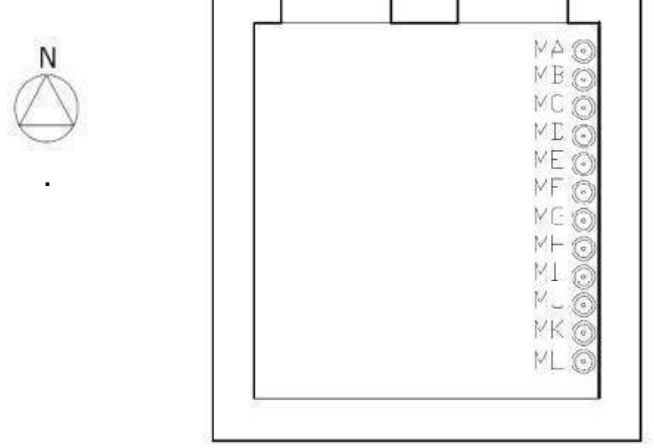

(a) Zona 1.

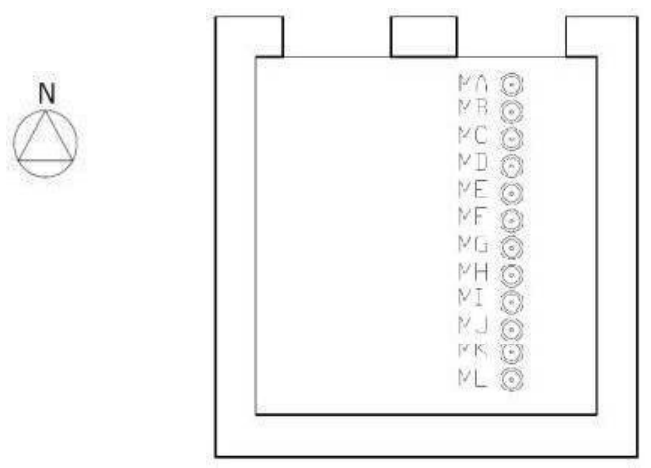

(b) Zona 2.

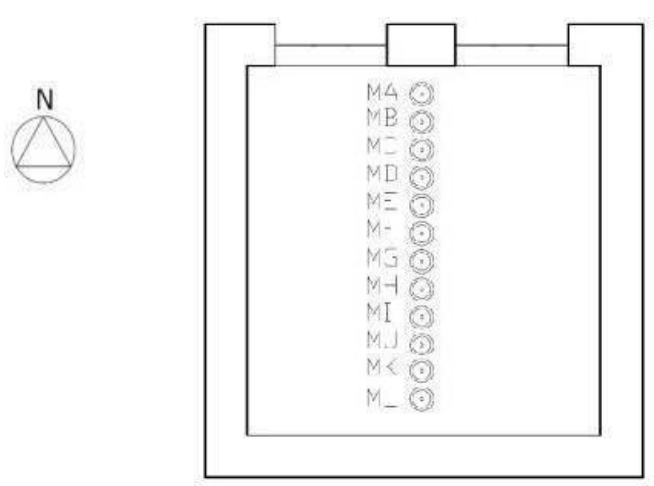

(c) Zona 3.

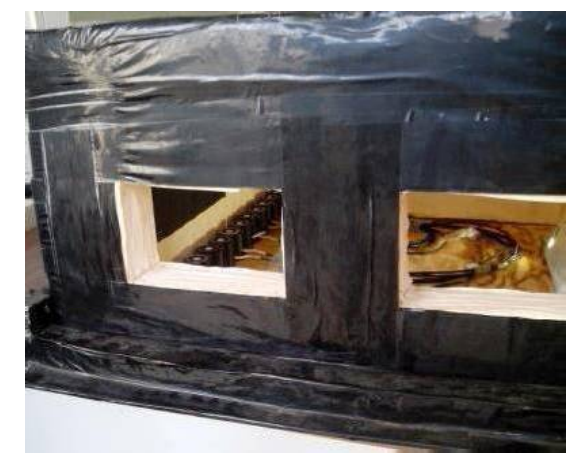

(a) Zona 1.

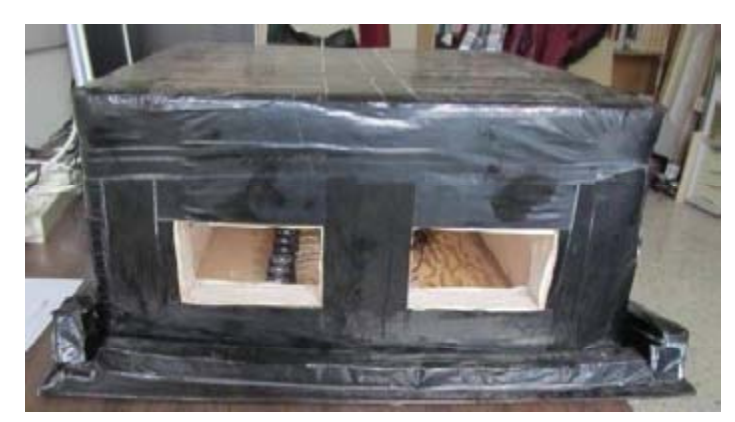

(b) Zona 2.

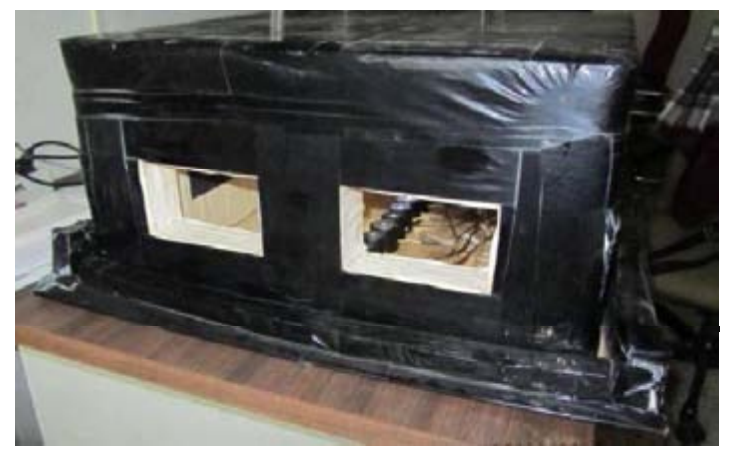

(c) Zona 3. 


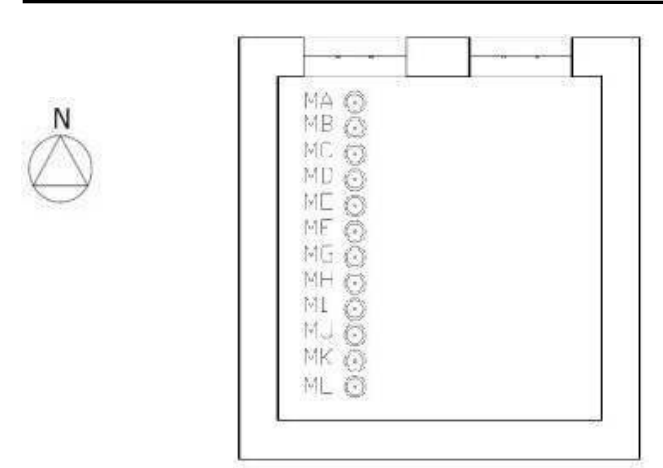

(d) Zona 4.

Figura 5.9. Esquema sensores por zona $(\operatorname{cotas} \mathrm{cm})$.

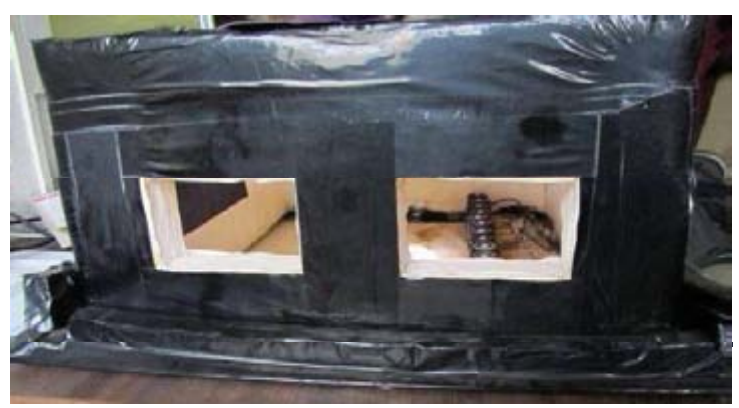

(d) Zona 4 .

Figura 5.10. Colocación sensores por zona.

\subsubsection{Obtención de los datos en las cuatro zonas simultáneamente}

Para medir en las cuatro zonas de forma simultánea se han colocado 3 sensores en cada zona a las distancias de $6.60,13.20$ y $19.80 \mathrm{~cm}$ de la fachada (figuras 5.11 y 5.12 ).

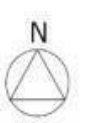

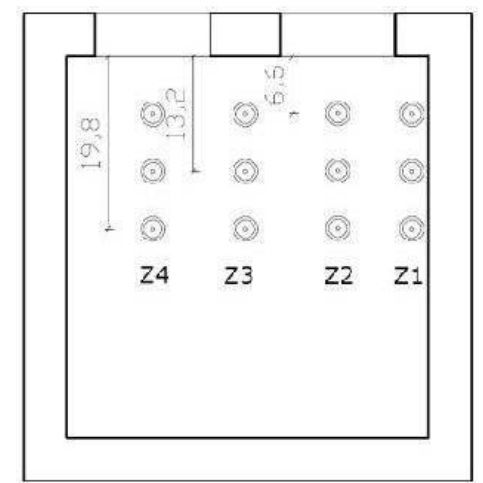

Figura 5.11. Esquema de los sensores en las cuatro zonas (cotas en $\mathrm{m}$ ).

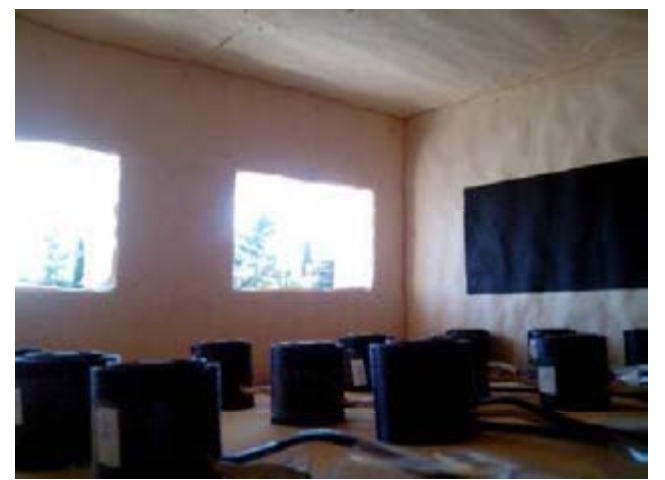

Figura 5.12. Colocación de los sensores en las cuatro zonas.

\subsubsection{Colocación del sensor exterior}

El sensor exterior se colocó en una torreta metálica habilitada para ello (figuras 5.13 y 5.14), en la cubierta norte de la E.T.S.A.M, justo al lado del modelo a escala $\left(40^{\circ} 25^{\prime} \mathrm{N}, 3^{\circ} 41^{\prime} \mathrm{W}\right)$.

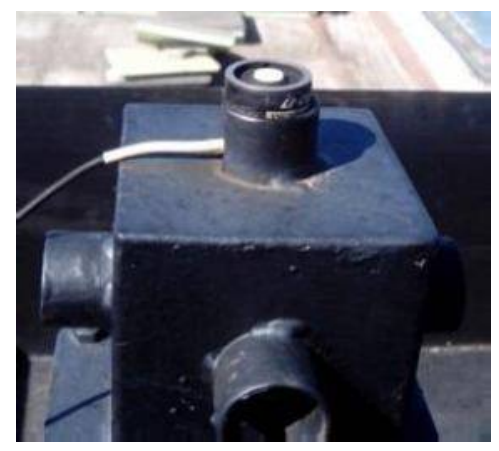

Figura 5.13. Sensor exterior.

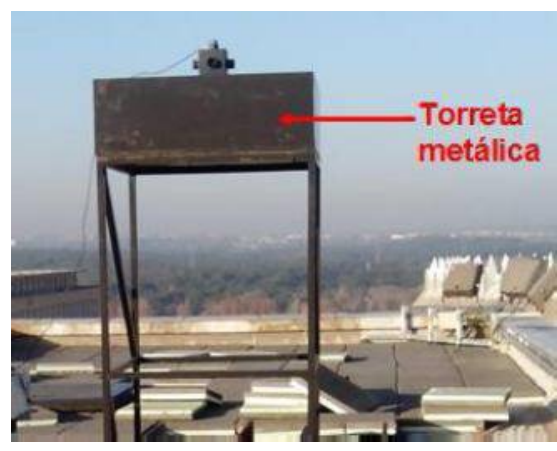

Figura 5.14. Torreta metálica. 


\subsection{TRATAMIENTO Y ANÁLISIS DE DATOS}

Al trabajar con tantos sensores es necesario usar una nomenclatura sencilla para distinguirlos, dependiendo de si está en el aula o en el modelo a escala, y a la distancia de la fachada a la que se encuentran. En el datalogger hay que distinguirlos con 2 dígitos (letras o números). En nuestro caso se designó a cada sensor con dos letras según se muestra en la tabla 5.1.

\begin{tabular}{|c|c|c|}
\hline DISTANCIA (m) & AULA & MODELO \\
\hline 0.5 & $\mathrm{AA}$ & $\mathrm{MA}$ \\
\hline 1 & $\mathrm{AB}$ & $\mathrm{MB}$ \\
\hline 1.5 & $\mathrm{AC}$ & $\mathrm{MC}$ \\
\hline 2 & $\mathrm{AD}$ & $\mathrm{MD}$ \\
\hline 2.5 & $\mathrm{AE}$ & $\mathrm{ME}$ \\
\hline 3 & $\mathrm{AF}$ & $\mathrm{MF}$ \\
\hline 3.5 & $\mathrm{AG}$ & $\mathrm{MG}$ \\
\hline 4 & $\mathrm{AH}$ & $\mathrm{MH}$ \\
\hline 4.5 & $\mathrm{Al}$ & $\mathrm{Ml}$ \\
\hline 5 & $\mathrm{AJ}$ & $\mathrm{MJ}$ \\
\hline 5,5 & $\mathrm{AK}$ & $\mathrm{MK}$ \\
\hline 6 & $\mathrm{AL}$ & $\mathrm{ML}$ \\
\hline
\end{tabular}

Tabla 5.1. Nomenclatura sensores.

La planificación para la adquisición de datos estuvo muy condicionada a la disponibilidad del aula 2S2 objeto de nuestro estudio. Debido a que en ella se impartían clases de lunes a viernes, la toma de datos estuvo restringida de modo temporal a fines de semana y días festivos, además de periodos de vacaciones.

El registro de medidas se realizó al mismo tiempo en el aula, en el modelo a escala y en el sensor exterior. Todos los Datalogger fueron programados para registrar medidas de iluminancias cada 5 minutos, siempre y cuando los valores de iluminancia captados por los sensores fueran superiores a $1 \times 10^{-5}$ Klux.

A continuación, en la tabla 5.2, se exponen los días en los que se realizaron medidas, así como el tipo de cielo que hubo cada día y la zona donde se realizaron las mediciones. 


\begin{tabular}{|c|c|c|}
\hline FECHA & ZONA & TIPO DE CIELO \\
\hline $31 / 03 / 2015$ & 2 & Parcialmente cubierto \\
\hline $01 / 04 / 2015$ & 2 & Despejado \\
\hline $02 / 04 / 2015$ & 2 & Parcialmente cubierto \\
\hline $03 / 04 / 2015$ & 2 & Parcialmente cubierto \\
\hline $04 / 04 / 2015$ & 2 & Despejado \\
\hline $05 / 04 / 2015$ & 2 & Despejado \\
\hline $18 / 04 / 2015$ & 2 & Parcialmente cubierto \\
\hline $19 / 04 / 2015$ & 2 & Parcialmente cubierto \\
\hline $20 / 04 / 2015$ & 2 & Parcialmente cubierto \\
\hline $21 / 04 / 2015$ & 2 & Parcialmente cubierto \\
\hline $22 / 04 / 2015$ & 2 & Parcialmente cubierto \\
\hline $25 / 04 / 2015$ & 2 & Parcialmente cubierto \\
\hline $26 / 04 / 2015$ & 2 & Parcialmente cubierto \\
\hline $27 / 04 / 2015$ & 2 & Parcialmente cubierto \\
\hline $28 / 04 / 2015$ & 2 & Parcialmente cubierto \\
\hline $29 / 04 / 2015$ & 2 & Parcialmente cubierto \\
\hline $01 / 05 / 2015$ & 3 & Cubierto \\
\hline $02 / 05 / 2015$ & 3 & Cubierto \\
\hline $03 / 05 / 2015$ & 3 & Cubierto \\
\hline $09 / 05 / 2015$ & 3 & Parcialmente cubierto \\
\hline $10 / 05 / 2015$ & 3 & Parcialmente cubierto \\
\hline $16 / 05 / 2015$ & 3 & Cubierto \\
\hline $17 / 05 / 2015$ & 3 & Cubierto \\
\hline $06 / 06 / 2015$ & 4 & Parcialmente cubierto \\
\hline $06 / 06 / 2015$ & 4 & Parcialmente cubierto \\
\hline $01 / 07 / 2015$ & 2 & Parcialmente cubierto \\
\hline $16 / 07 / 2015$ & 3 & Parcialmente cubierto \\
\hline $17 / 07 / 2015$ & 3 & Parcialmente cubierto \\
\hline $19 / 07 / 2015$ & 4 & Cubierto \\
\hline $20 / 07 / 2015$ & 4 & Parcialmente cubierto \\
\hline $21 / 07 / 2015$ & 4 & Despejado \\
\hline $23 / 07 / 2015$ & 1 & Despejado \\
\hline $24 / 07 / 2015$ & 1 & Parcialmente cubierto \\
\hline $26 / 09 / 2015$ & 1 & Cubierto \\
\hline $27 / 06 / 2015$ & 1 & Cubierto \\
\hline $17 / 10 / 2015$ & 1 & Parcialmente cubierto \\
\hline $18 / 10 / 2015$ & 1 & Cubierto \\
\hline $07 / 11 / 2015$ & 1 & Parcialmente cubierto \\
\hline $08 / 11 / 2015$ & 1 & Parcialmente cubierto \\
\hline $21 / 11 / 2015$ & 1 & \\
\hline & & \\
\hline
\end{tabular}




\begin{tabular}{|c|c|c|}
\hline FECHA & ZONA & TIPO DE CIELO \\
\hline $22 / 11 / 2015$ & 1 & Parcialmente cubierto \\
\hline $05 / 12 / 2015$ & 3 & Despejado \\
\hline $06 / 12 / 2015$ & 3 & Despejado \\
\hline $07 / 12 / 2015$ & 3 & Parcialmente cubierto \\
\hline $08 / 12 / 2015$ & 3 & Cubierto \\
\hline $12 / 12 / 2015$ & 3 & Cubierto \\
\hline $13 / 12 / 2015$ & 3 & Cubierto \\
\hline $19 / 12 / 2015$ & 3 & Cubierto \\
\hline $20 / 12 / 2015$ & 3 & Cubierto \\
\hline $09 / 01 / 2016$ & 1 & Parcialmente cubierto \\
\hline $10 / 01 / 2016$ & 1 & Cubierto \\
\hline $16 / 01 / 2016$ & 4 & Parcialmente cubierto \\
\hline $17 / 01 / 2016$ & 4 & Parcialmente cubierto \\
\hline $13 / 02 / 2016$ & 4 & Cubierto \\
\hline $14 / 02 / 2016$ & 4 & Cubierto \\
\hline $20 / 02 / 2016$ & 4 & Parcialmente cubierto \\
\hline $21 / 02 / 2016$ & 4 & Parcialmente cubierto \\
\hline $05 / 03 / 2016$ & 4 & Parcialmente cubierto \\
\hline $06 / 03 / 2016$ & 4 & Cubierto \\
\hline $12 / 03 / 2016$ & 4 & Parcialmente cubierto \\
\hline $13 / 03 / 2016$ & 4 & Cubierto \\
\hline $19 / 04 / 2016$ & $1-2-3-4$ & Cubierto \\
\hline $20 / 04 / 2016$ & $1-2-3-4$ & Cubierto \\
\hline $21 / 04 / 2016$ & $1-2-3-4$ & Cubierto \\
\hline $22 / 04 / 2016$ & $1-2-3-4$ & Cubierto \\
\hline $23 / 04 / 2016$ & $1-2-3-4$ & Cubierto \\
\hline $24 / 04 / 2016$ & $1-2-3-4$ & Parcialmente cubierto \\
\hline $25 / 04 / 2016$ & $1-2-3-4$ & Parcialmente cubierto \\
\hline $28 / 04 / 2016$ & $1-2-3-4$ & Parcialmente cubierto \\
\hline $29 / 04 / 2015$ & $1-2-3-4$ & Parcialmente cubierto \\
\hline $30 / 04 / 2016$ & $1-2-3-4$ & Parcialmente cubierto \\
\hline $01 / 05 / 2016$ & $1-2-3-4$ & Despejado \\
\hline $02 / 05 / 2016$ & $1-2-3-4$ & Despejado \\
\hline $12 / 05 / 2016$ & $1-2-3-4$ & Cubierto \\
\hline $13 / 05 / 2016$ & $1-2-3-4$ & Cubierto \\
\hline $14 / 05 / 2016$ & $1-2-3-4$ & Cubierto \\
\hline $15 / 05 / 2016$ & $1-2-3-4$ & Parcialmente cubierto \\
\hline $16 / 05 / 2016$ & $1-2-3-4$ & Parcialmente cubierto \\
\hline $16 / 07 / 2016$ & 2 & Parcialmente cubierto \\
\hline
\end{tabular}

Tabla 5.2. Días de mediciones. 
Una vez tomadas las medidas se procedió al volcado de datos a un ordenador para su posterior análisis y tratamiento, y además de borrar los datos de la memoria de los Dataloggers para dejarla totalmente vacía y preparada para la siguiente toma de datos.

El fichero que genera el software de almacenamiento de datos de los Datalogger está en formato txt (figura 5.15). Por ello primero hay que ordenarlo para llevarlo a formato estándar de Excel (figura 5.16).

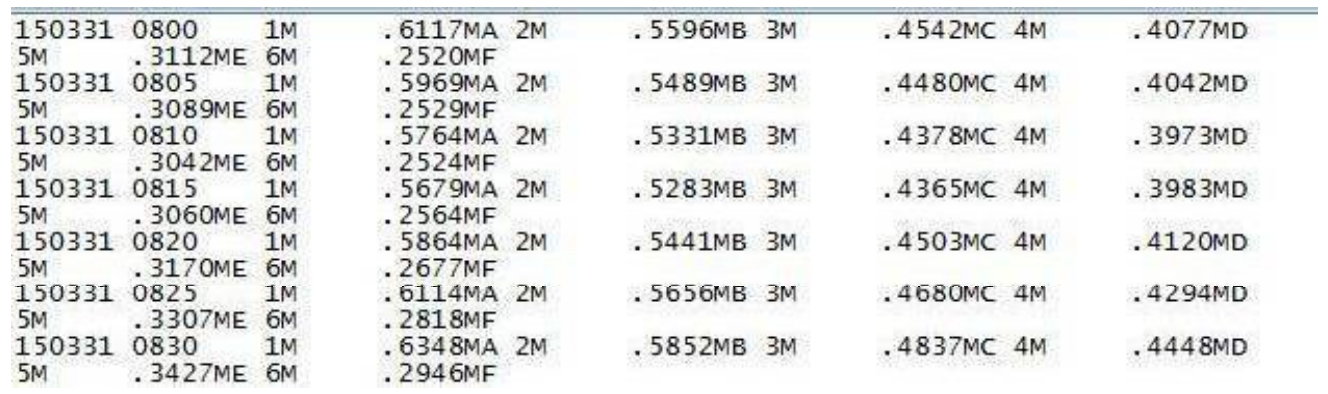

Figura 5.15. Formato .txt al descargar los datos.

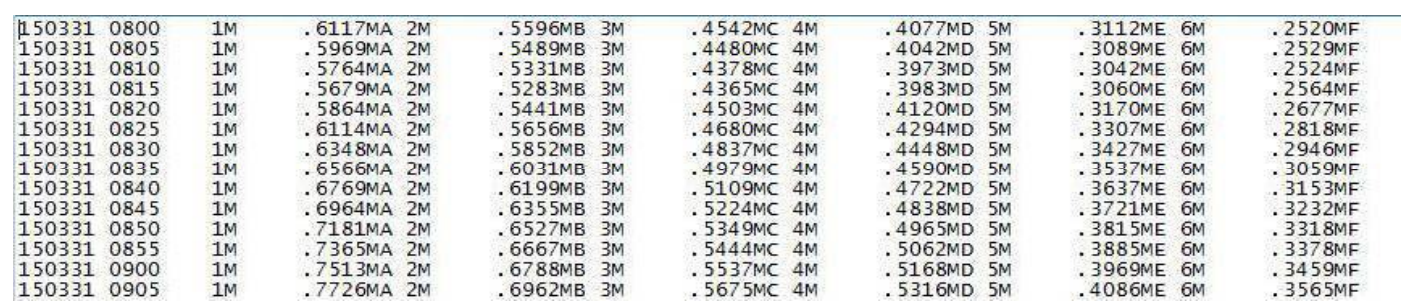

Figura 5.16. Datos .txt ordenados.

Una vez realizada esta última operación se puede abrir el fichero en Excel donde tendremos todos los datos perfectamente colocados y podrán manejarse con comodidad con los software estándar de análisis de datos y simulación. 

6. RESULTADOS 



\section{RESULTADOS}

A continuación se exponen los resultados obtenidos del presente estudio en forma de gráficos para su mejor interpretación. Los resultados se han dividido en dos bloques, en el primero se presentan los resultados obtenidos a distintas distancias, para cada zona, de forma individual. En el segundo bloque se han presentado los resultados obtenidos a las distancias 1,2 y $3 \mathrm{~m}$ en las cuatro zonas simultáneamente.

\subsection{Resultados por zonas individualmente}

Se han medido iluminancias horizontales simultáneamente en el aula 2S2 de la E.T.S.A.M y en el modelo a escala 1:15 de dicho aula cada 5 minutos, durante varios días a las distintas distancias de las ventanas $(0.5 \mathrm{~m}, 1 \mathrm{~m}, 1.5 \mathrm{~m}, 2 \mathrm{~m}$, $2.5 \mathrm{~m}, 3 \mathrm{~m}, 3.5 \mathrm{~m}, 4 \mathrm{~m}, 4.5 \mathrm{~m}, 5 \mathrm{~m}, 5.5 \mathrm{~m}, 6 \mathrm{~m}$ ) y en distintas zonas.

A continuación se muestran los resultados obtenidos tanto en el aula (figura 6.1) como en la maqueta (figura 6.2) para todo tipo de cielo (cubierto, parcialmente cubierto o despejado), épocas del año (verano, otoño, invierno o primavera) y zonas (zona 1, zona 2, zona 3 o zona 4), a lo largo del día (desde las 10:00 de la mañana hasta las 20:00 de la tarde hora local).

MODELO

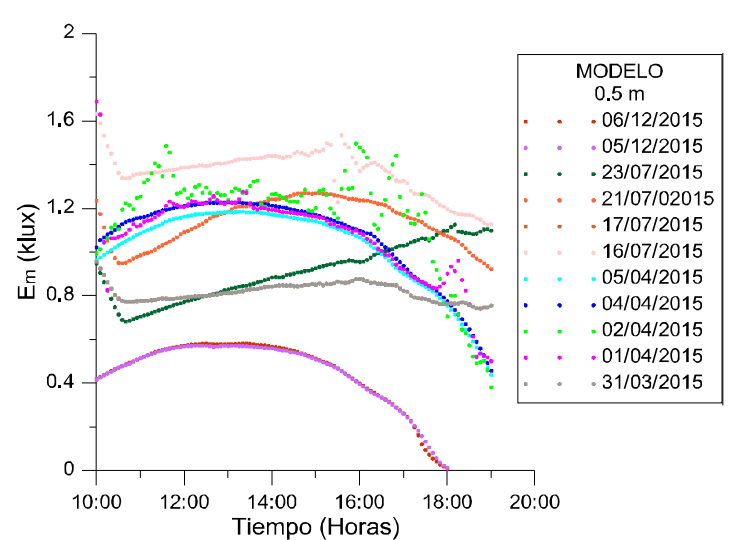

(a) $0.5 \mathrm{~m}$.
AULA

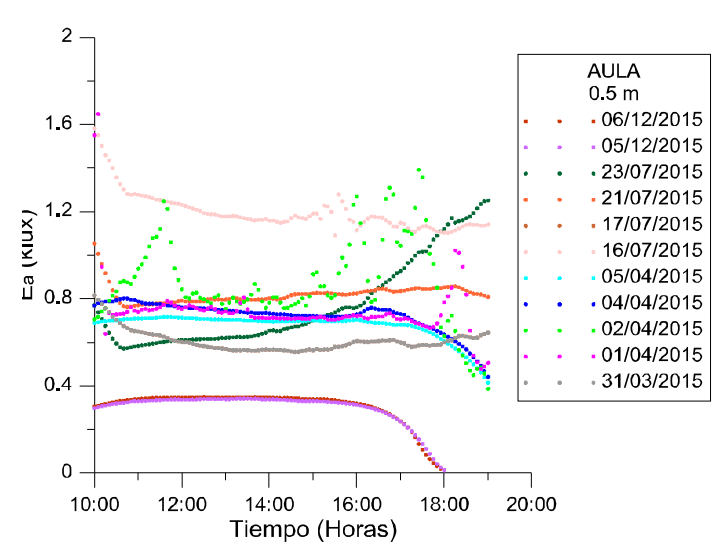

(a) $0.5 \mathrm{~m}$. 


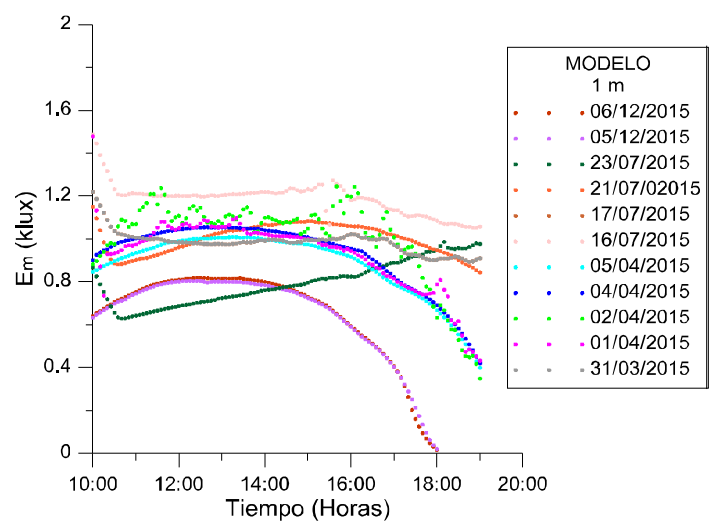

(b) $1 \mathrm{~m}$.

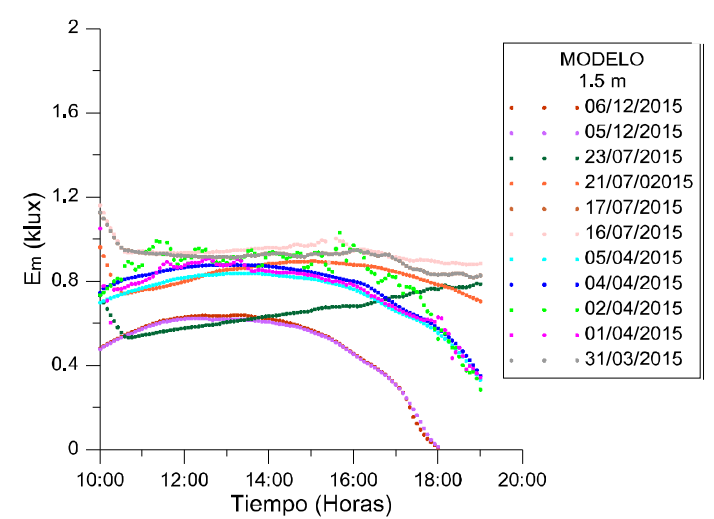

(c) $1.5 \mathrm{~m}$.

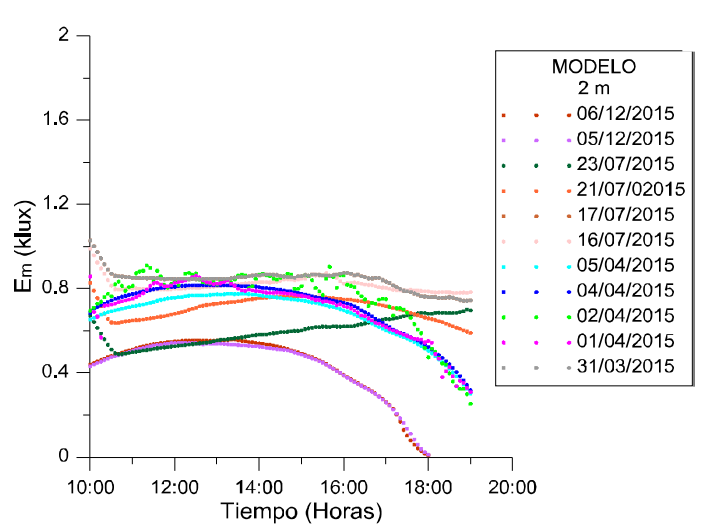

(d) $2 \mathrm{~m}$.

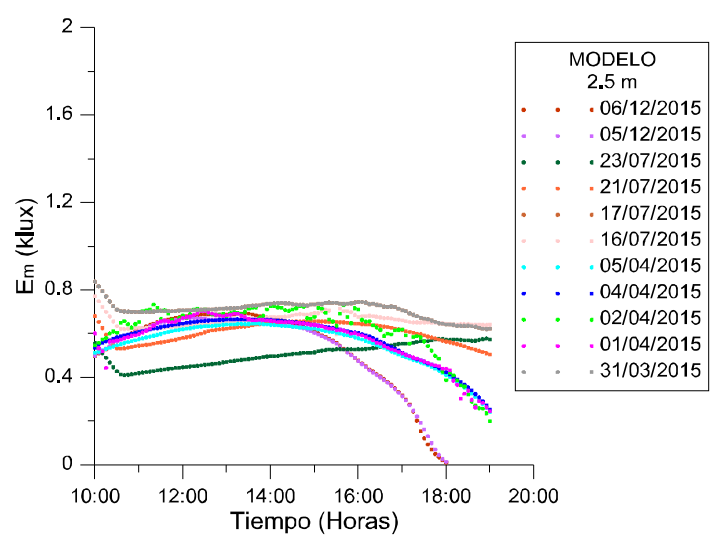

(e) $2.5 \mathrm{~m}$.

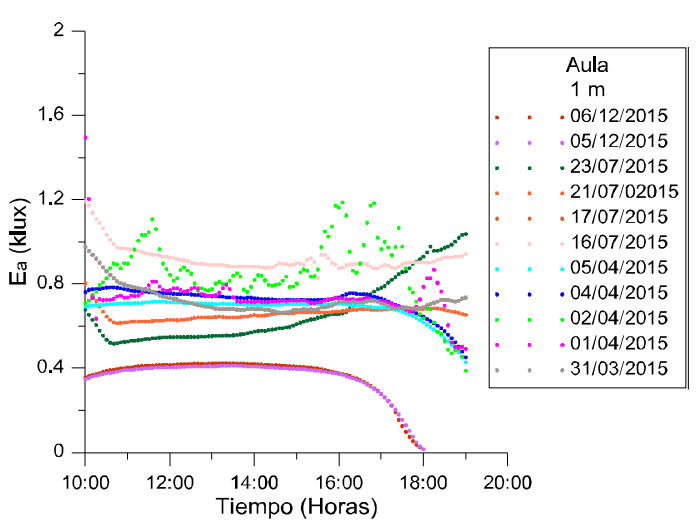

(b) $1 \mathrm{~m}$.

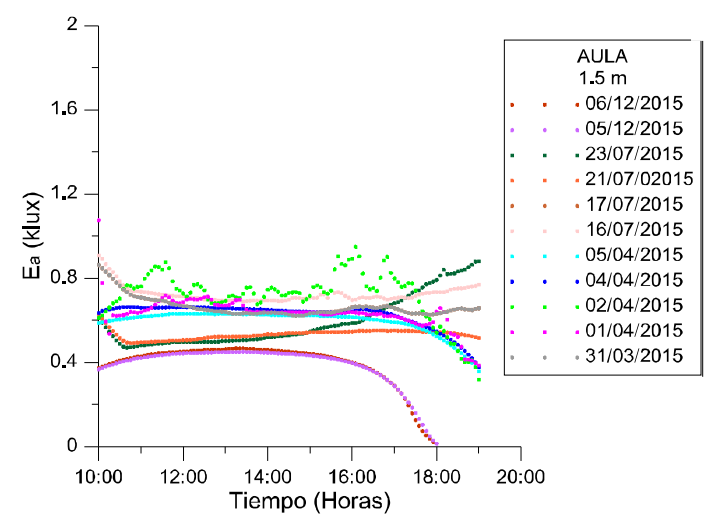

(c) $1.5 \mathrm{~m}$.

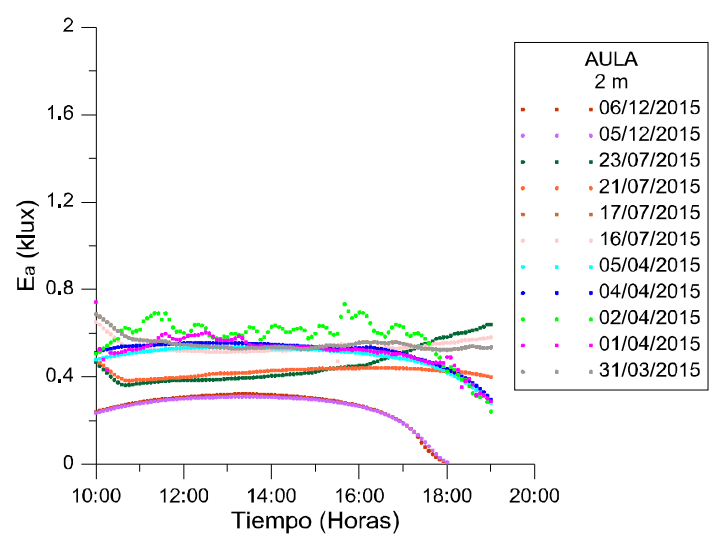

(d) $2 \mathrm{~m}$.

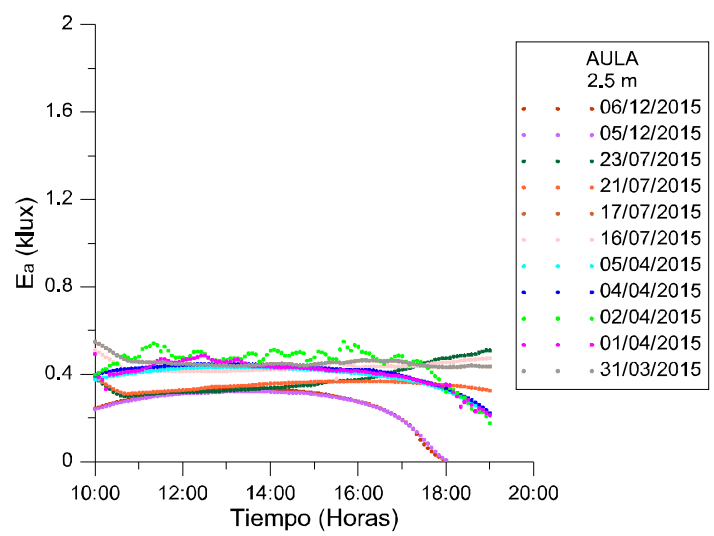

(e) $2.5 \mathrm{~m}$. 
Estudio experimental y modelización de la iluminación natural en la edificación mediante modelos a escala

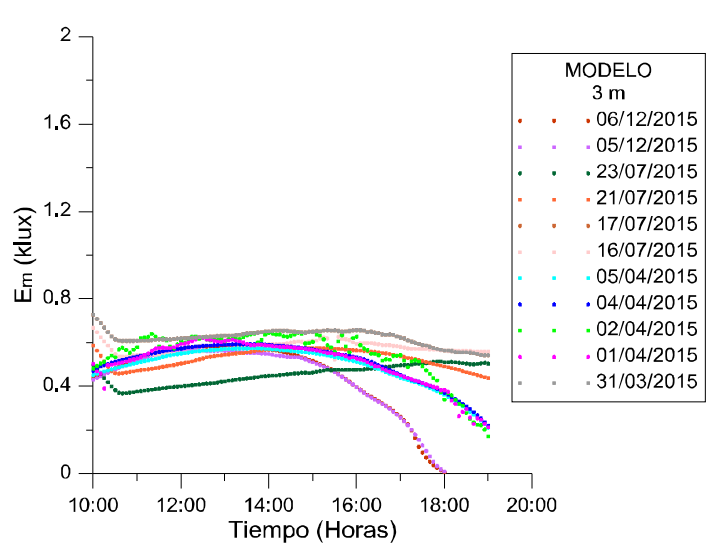

(f) $3 \mathrm{~m}$.

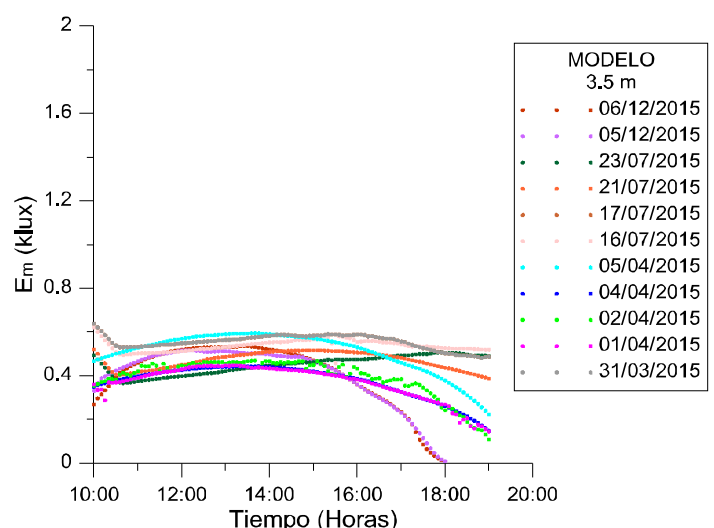

(g) $3.5 \mathrm{~m}$.

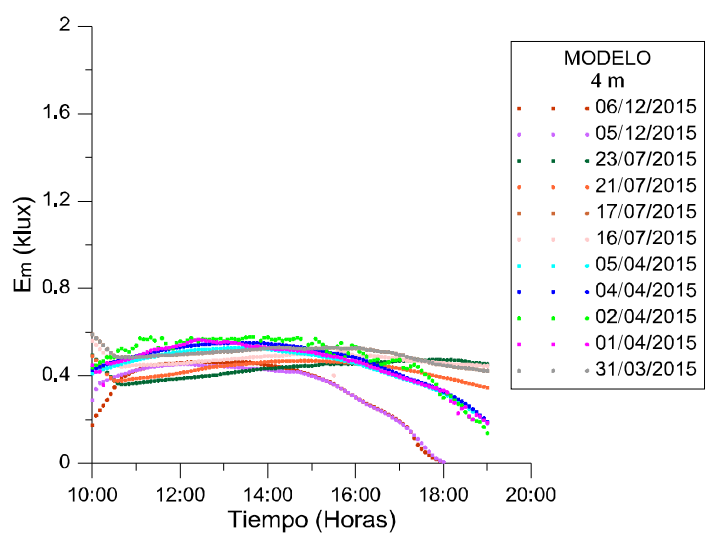

(h) $4 \mathrm{~m}$.

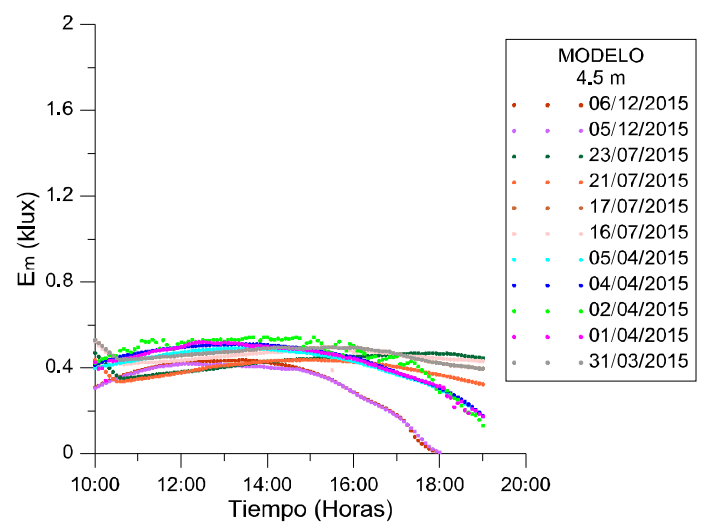

(i) $4.5 \mathrm{~m}$

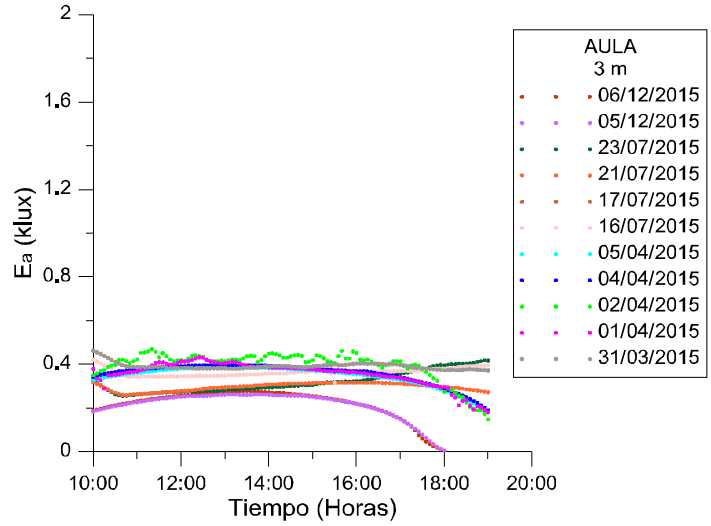

(f) $3 \mathrm{~m}$.

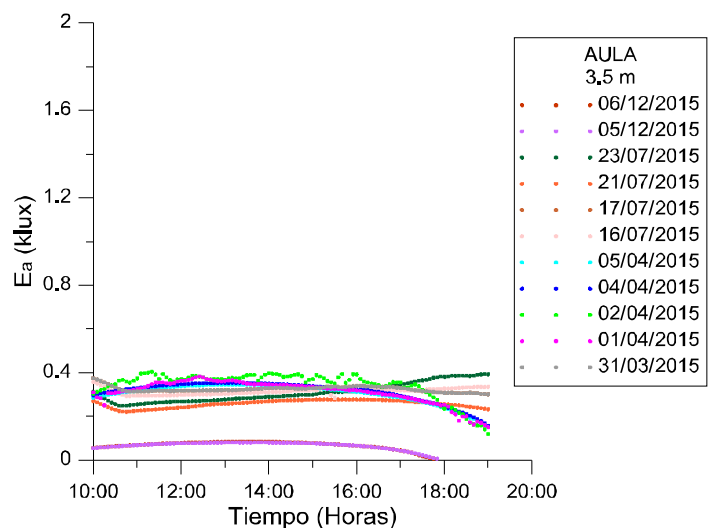

(g) $3.5 \mathrm{~m}$.

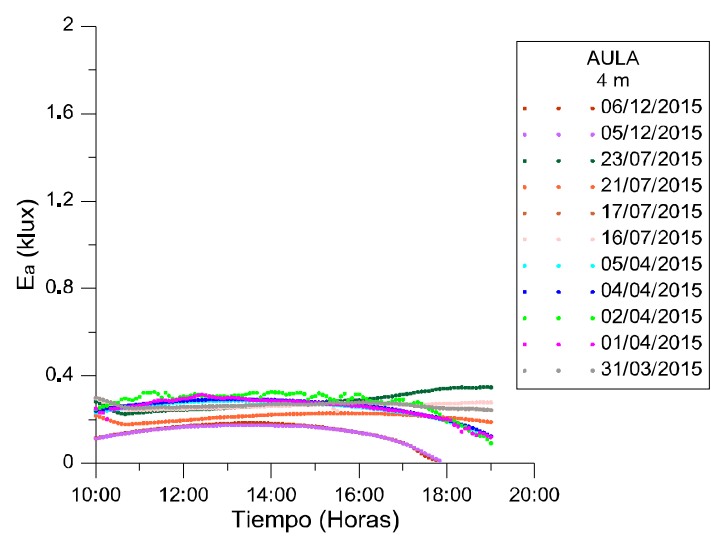

(h) $4 \mathrm{~m}$.

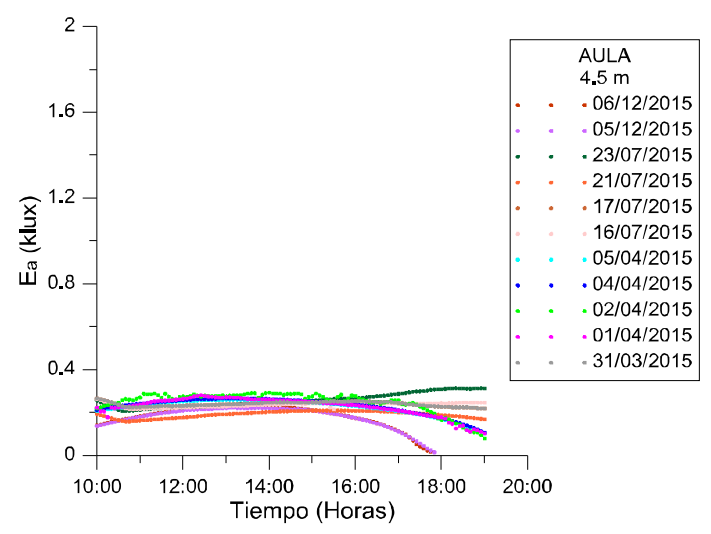

(i) $4.5 \mathrm{~m}$. 


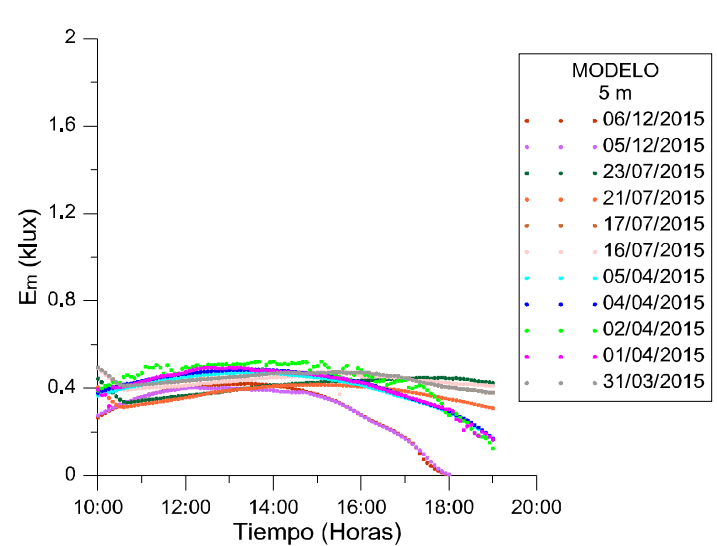

(j) $5 \mathrm{~m}$.

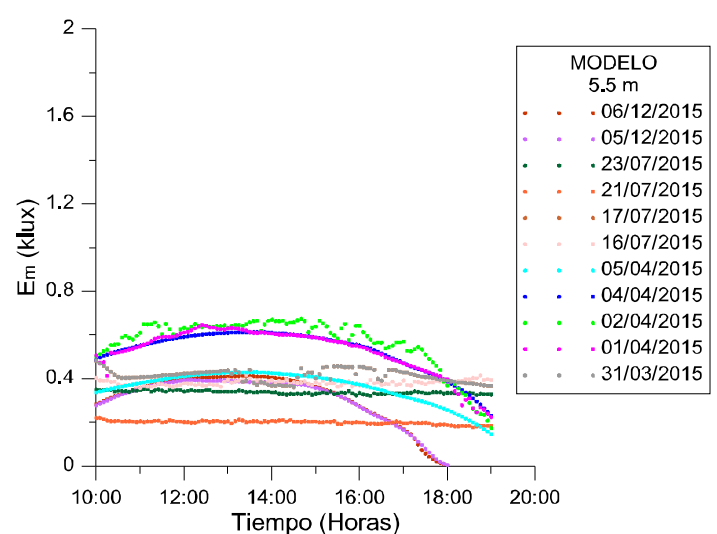

(k) $5.5 \mathrm{~m}$.

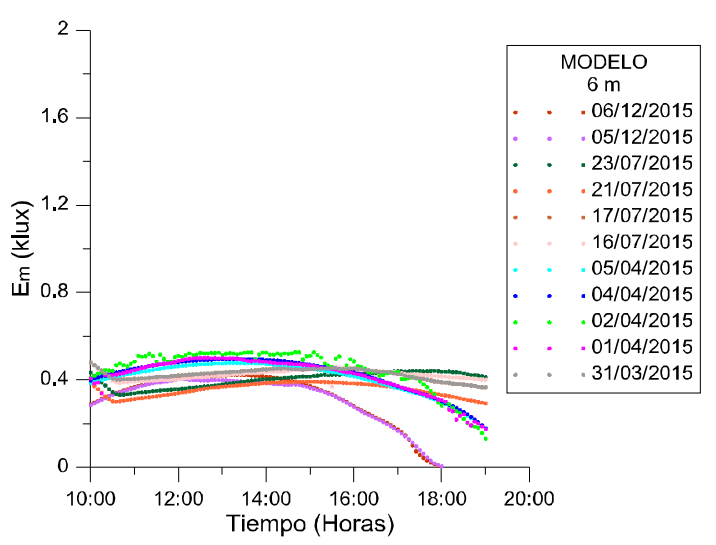

(I) $6 \mathrm{~m}$.

Figura 6.1. Iluminancia diaria en el modelo todos los días considerados a las distancias de la fachada indicadas.

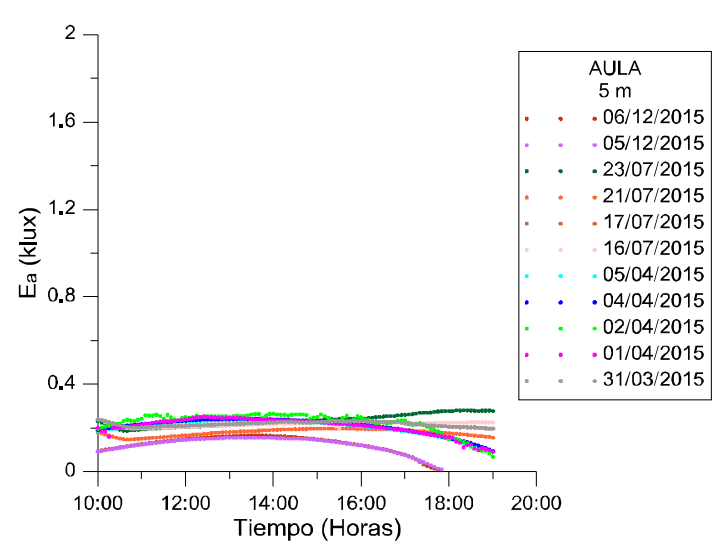

(j) $5 \mathrm{~m}$.

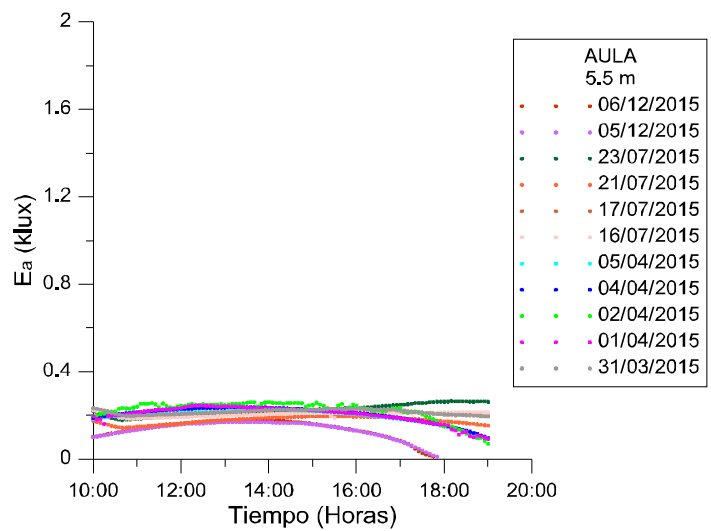

(k) $5.5 \mathrm{~m}$.

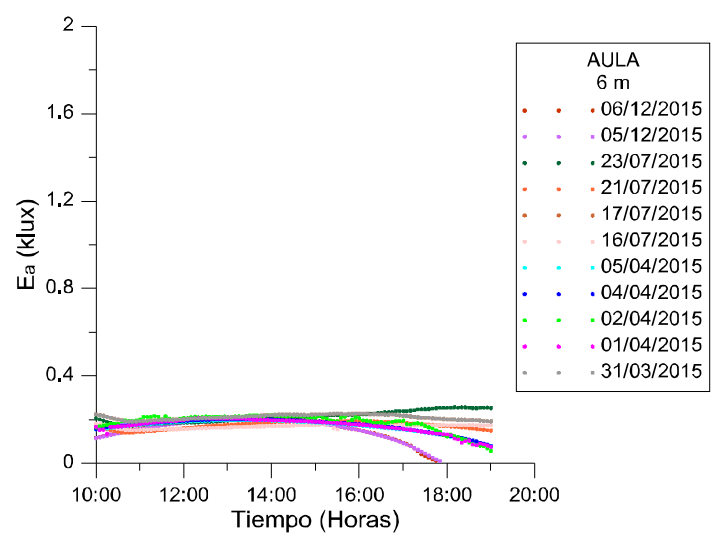

(l) $6 \mathrm{~m}$.

Figura 6.2. lluminancia diaria en el aula todos los días considerados a las distancias de la fachada indicadas.

Al haber tanta disparidad entre los resultados obtenidos dependiendo del tipo de cielo y de la zona a considerar, la presente tesis doctoral se ha centrado únicamente en el estudio de días con cielo despejado, por lo que se reduce, separado por zonas (zona 1, zona 2, zona 3 y zona 4), al estudio de los días recogidos en la tabla 6.1 . 


\begin{tabular}{|c|c|c|}
\hline FECHA & ZONA & TIPO DE CIELO \\
\hline $23 / 07 / 2015$ & 1 & Despejado \\
\hline $01 / 04 / 2015$ & 2 & Despejado \\
\hline $04 / 04 / 2015$ & 2 & Despejado \\
\hline $05 / 04 / 2015$ & 2 & Despejado \\
\hline $05 / 12 / 2015$ & 3 & Despejado \\
\hline $06 / 12 / 2015$ & 3 & Despejado \\
\hline $21 / 07 / 2015$ & 4 & Despejado \\
\hline $01 / 05 / 2016$ & $1-2-3-4$ & Despejado \\
\hline $02 / 05 / 2016$ & $1-2-3-4$ & Despejado \\
\hline
\end{tabular}

Tabla 6.1. Días de mediciones de cielo despejado

Para cada zona, el estudio realizado se estructura de la forma siguiente:

Evolución diaria de las iluminancias en el aula y el modelo para cada distancia y en el exterior.

Relación entre iluminancias del aula y del modelo en función del tiempo para cada distancia a la cara interna de la fachada.

Relación entre iluminancias del aula y del modelo en función de la iluminancia exterior para cada distancia a la cara interna de la fachada.

Modelo matemático que relaciona las iluminancias del aula, del modelo, del exterior y la distancia a la cara interna de la fachada. 


\subsubsection{Zona 1}

Se ha comenzado el análisis por la zona 1 que se encuentra a $0.28 \mathrm{~m}$ de distancia de la pared Este del aula. La distancia de los sensores a la 'parte interior de la fachada se muestra en las figuras 6.3 y 6.4 .

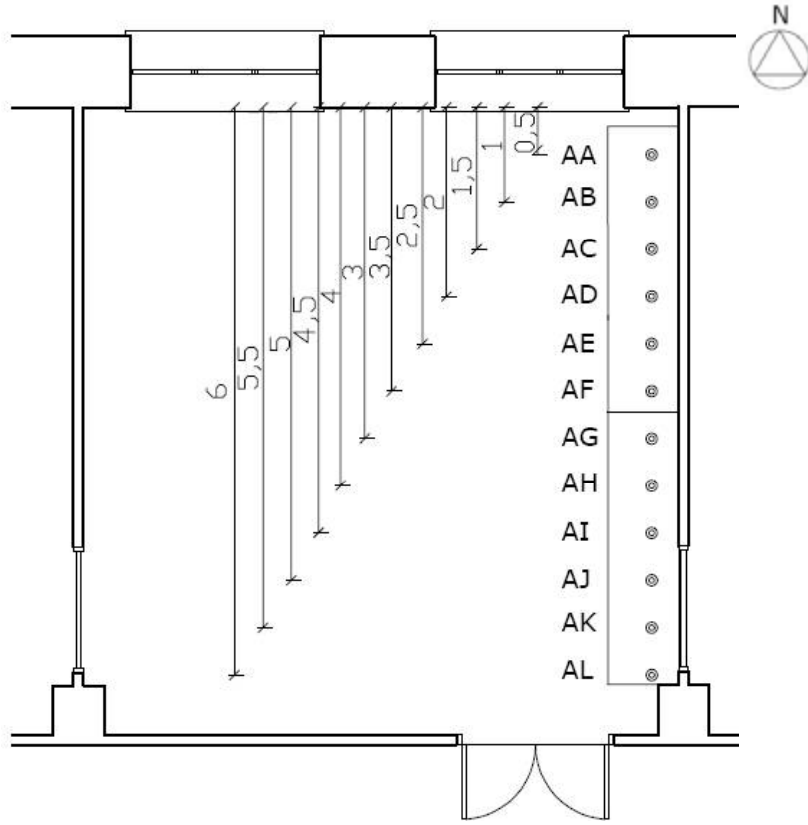

Figura 6.3.Esquema sensores zona 1.

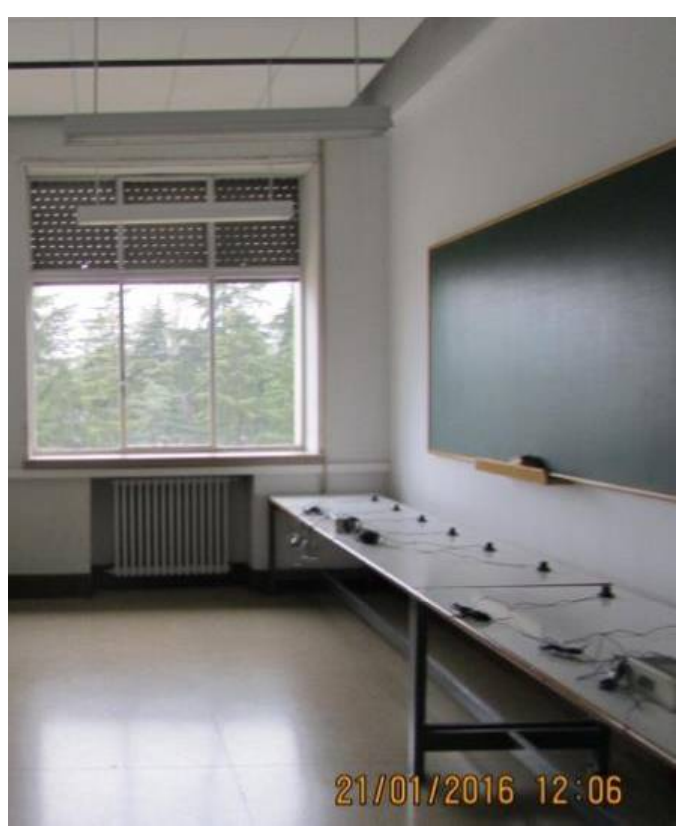

Figura 6.4.Colocación sensores zona 1.

Se han registrado medidas de iluminancias horizontales en el exterior y a todas las distancias consideradas $(0.5 \mathrm{~m}, 1 \mathrm{~m}, 1.5 \mathrm{~m}, 2 \mathrm{~m}, 2.5 \mathrm{~m}, 3 \mathrm{~m}, 3.5 \mathrm{~m}, 4 \mathrm{~m}, 4.5$ $\mathrm{m}, 5 \mathrm{~m}, 5.5 \mathrm{~m}, 6 \mathrm{~m})$, tanto en el aula objeto de estudio como en el modelo a escala 1/15 del mismo el día 23/07/2014 con cielo totalmente despejado desde las 11:00 de la mañana hasta las 19:00 de la tarde (hora local).

\subsubsection{Evolución diaria de las iluminancias}

La evolución diaria de la iluminancia exterior $\left(\mathrm{E}_{\mathrm{ext}}\right)$ del día considerado se muestra en la figura 6.5 . 


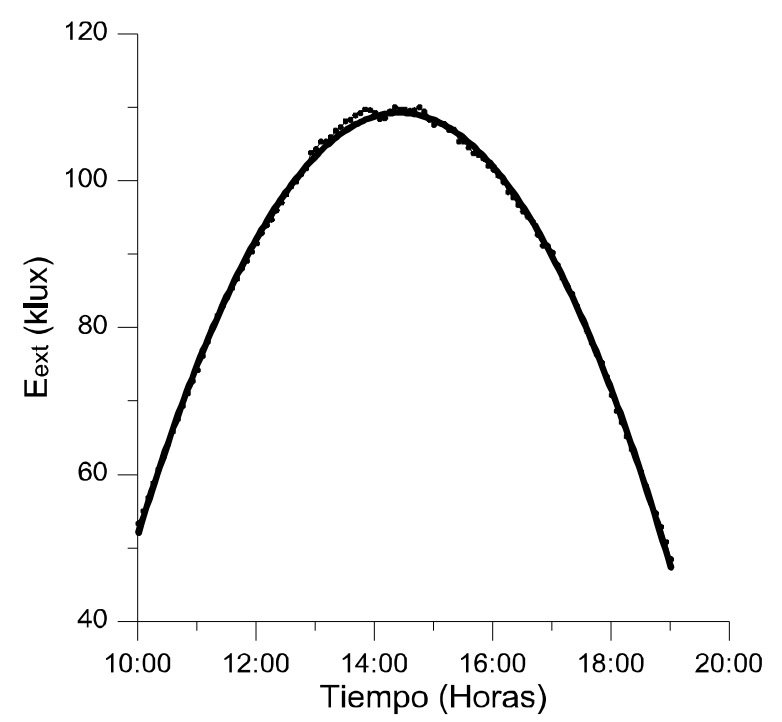

Figura 6.5. Evolución diaria de la iluminancia exterior 23/07/2015.

A continuación se muestran en las figuras 6.6 y 6.7, las gráficas que representan la evolución de las iluminancias horizontales del modelo $\left(E_{m}\right)$ y del aula $\left(E_{a}\right)$ a las distintas distancias de la cara interior de la fachada a lo largo del día. En el eje vertical se representan las iluminancias en klux y en el eje horizontal el tiempo expresado en horas. También se muestra la ecuación de segundo grado a la que se ajusta la nube de puntos, así como su coeficiente de ajuste $\left(r^{2}\right)$. En la zona de la izquierda en verde están representados los datos correspondientes al modelo y a la derecha en rojo los del aula. Aunque las medidas exteriores están consideradas desde que amanece, el análisis del aula y del modelo a escala se ha realizado, para todas las zonas desde las 11:00, porque las iluminancias a horas más tempranas eran muy bajas y producían muchas fluctuaciones.

\section{ZONA 1}

MODELO

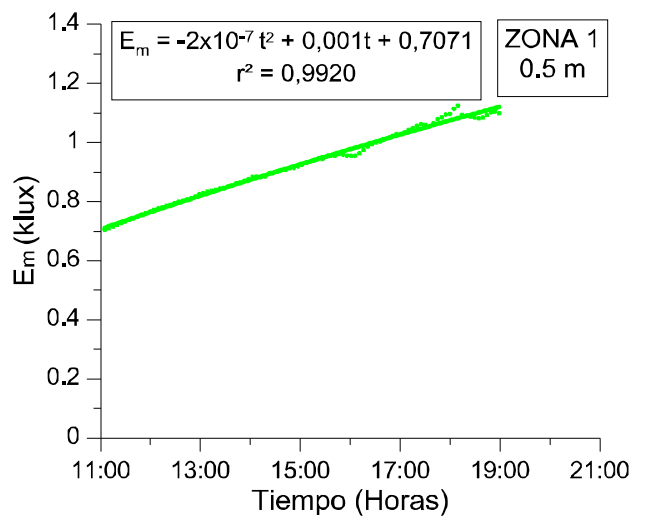

(a) $0.5 \mathrm{~m}$.
AULA

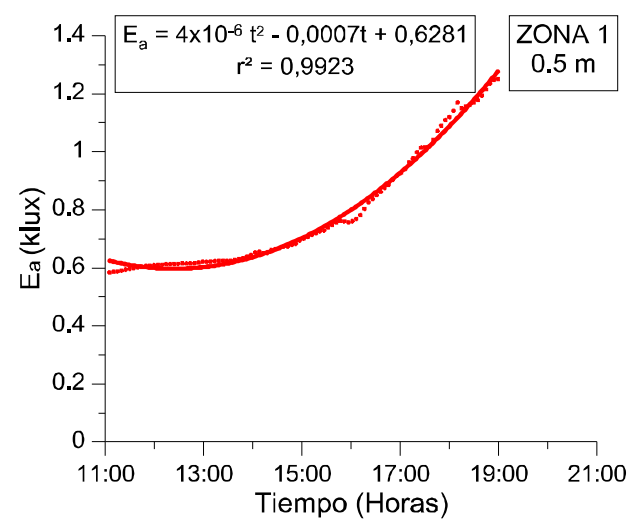

(a) $0.5 \mathrm{~m}$. 
Estudio experimental y modelización de la iluminación natural en la edificación mediante modelos a escala

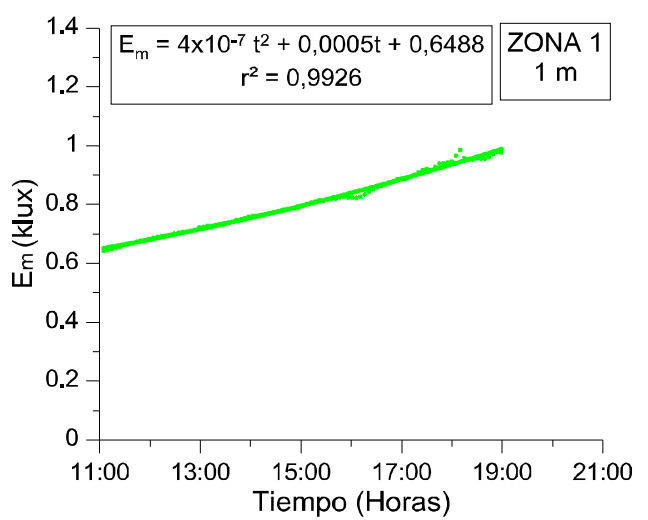

(b) $1 \mathrm{~m}$.

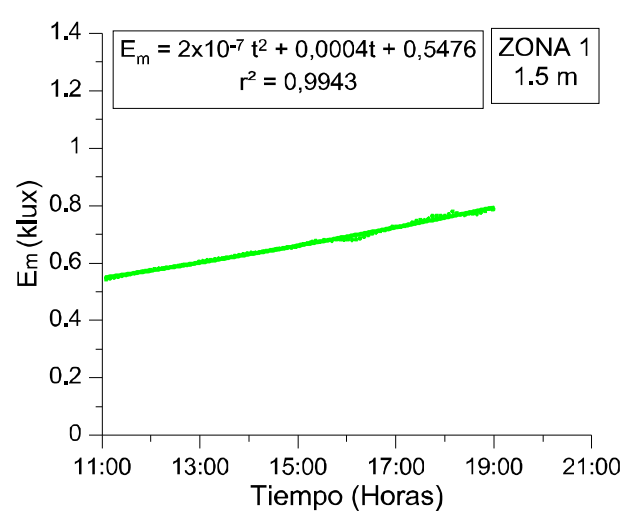

(c) $1.5 \mathrm{~m}$.

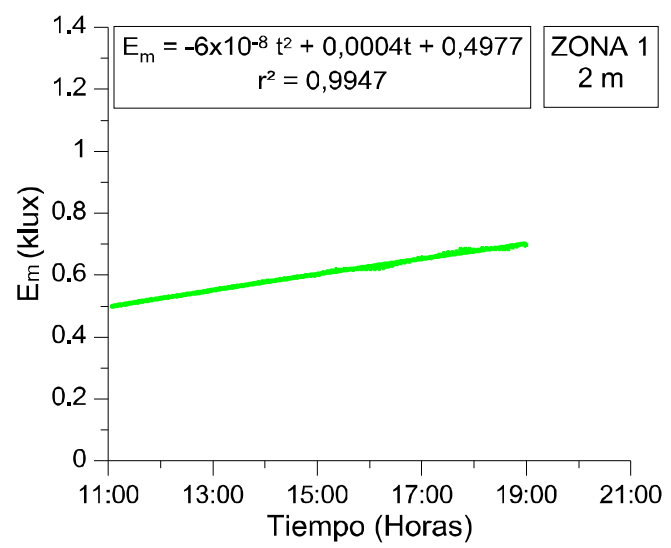

(d) $2 \mathrm{~m}$.

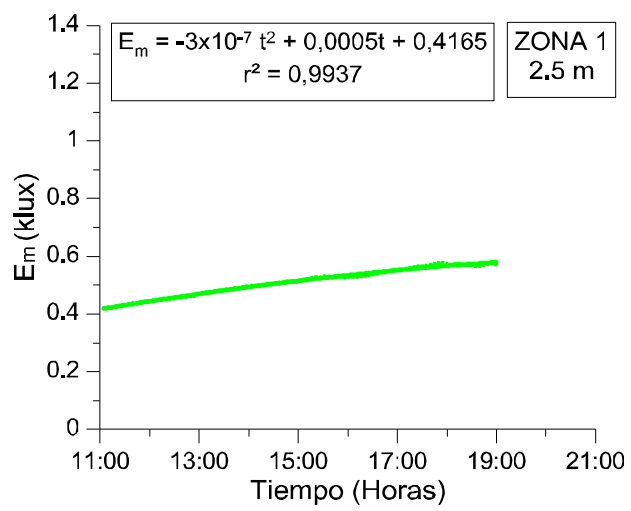

(e) $2.5 \mathrm{~m}$.

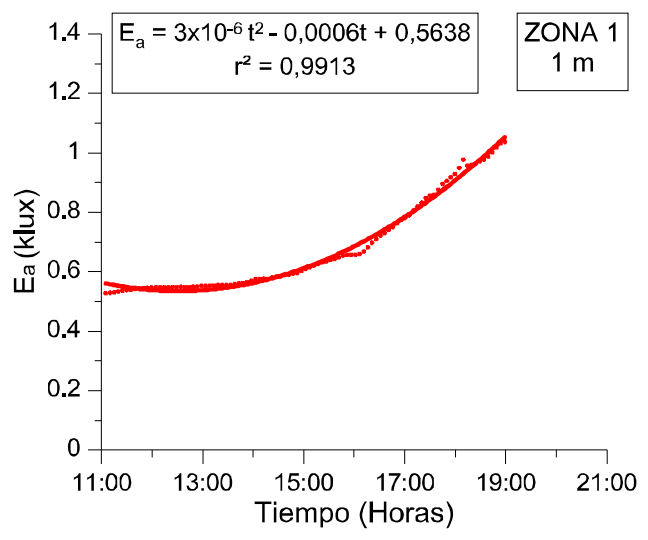

(b) $1 \mathrm{~m}$.

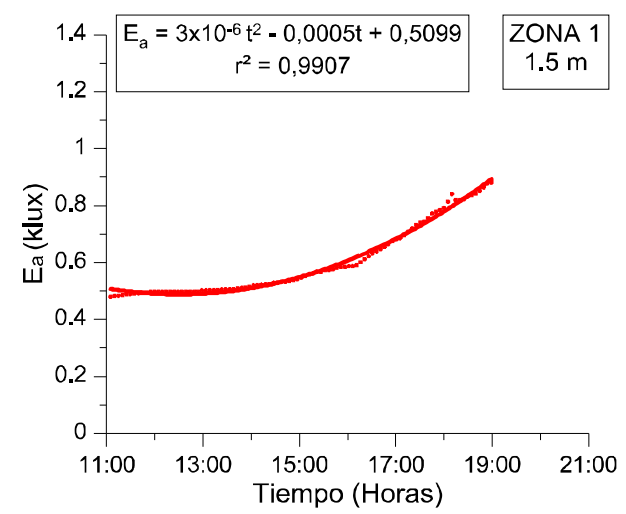

(c) $1.5 \mathrm{~m}$.

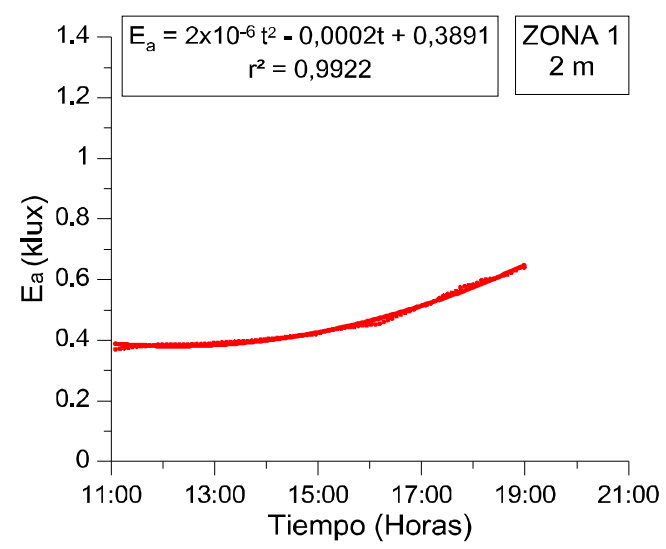

(d) $2 \mathrm{~m}$.

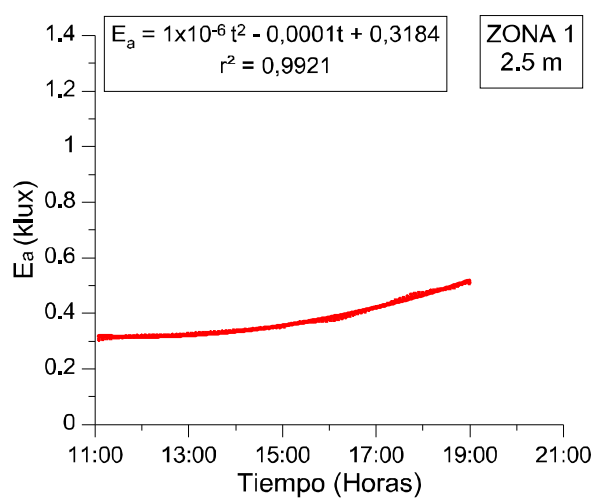

(e) $2.5 \mathrm{~m}$. 
Estudio experimental y modelización de la iluminación natural en la edificación mediante modelos a escala

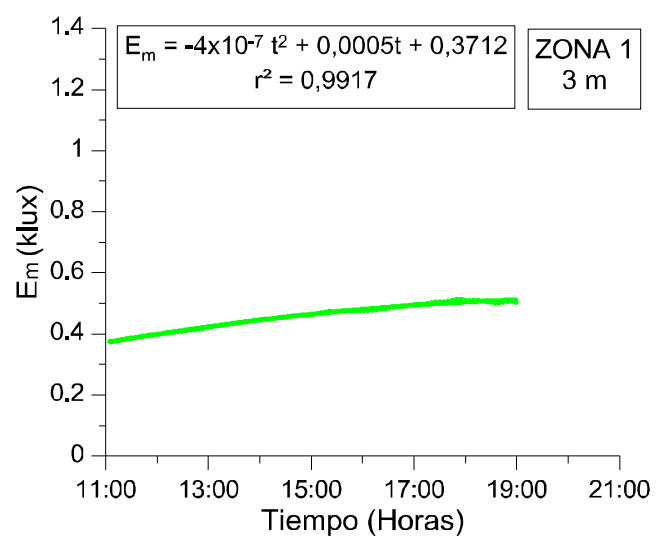

(f) $3 \mathrm{~m}$.

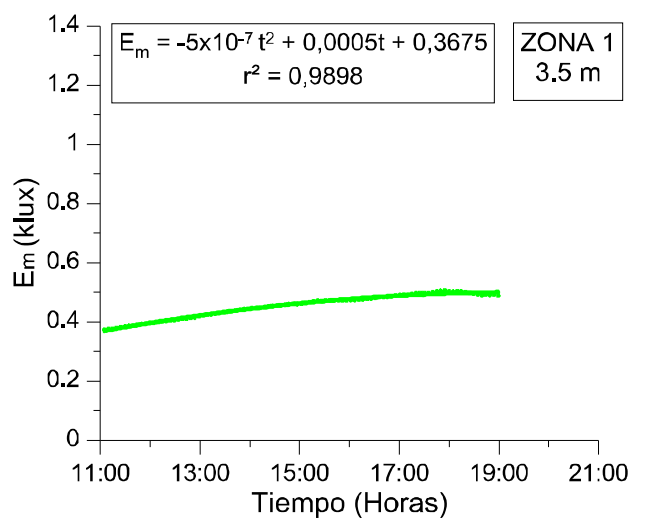

(g) $3.5 \mathrm{~m}$.

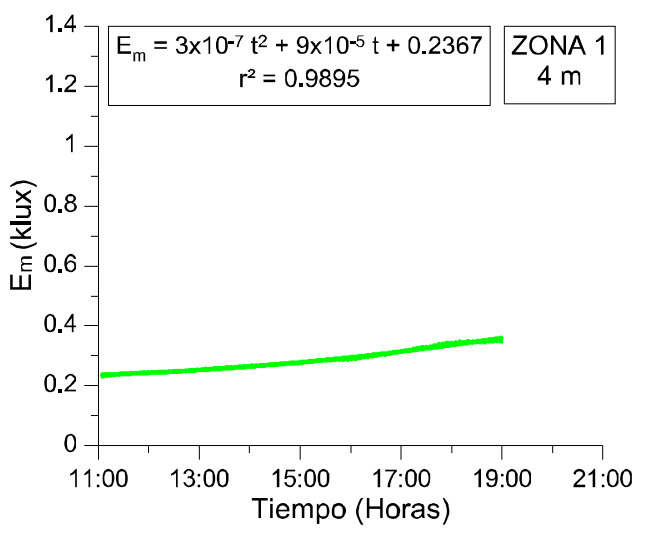

(h) $4 \mathrm{~m}$.

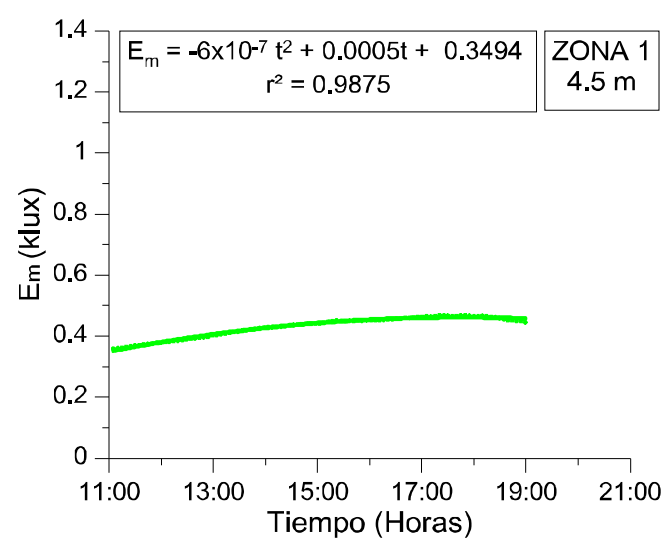

(i) $4.5 \mathrm{~m}$

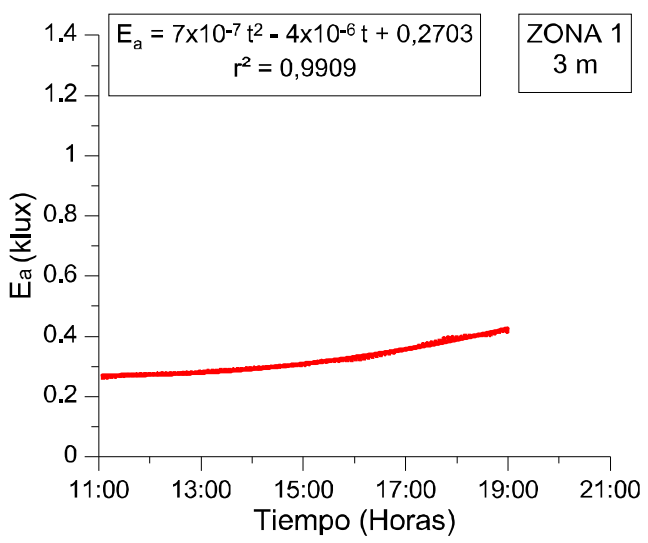

(f) $3 \mathrm{~m}$.

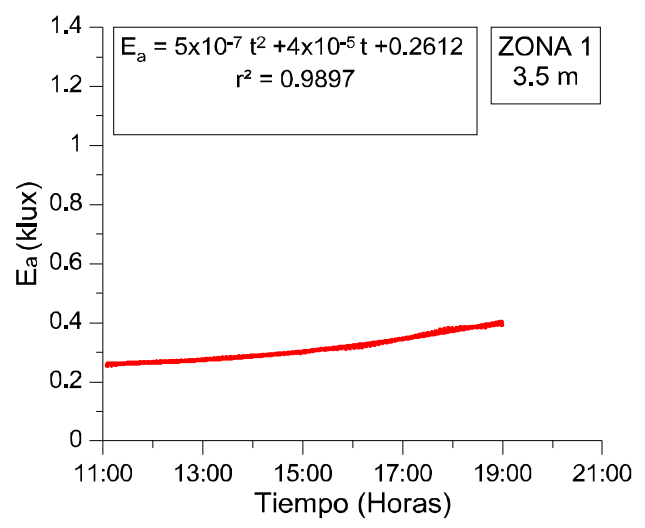

(g) $3.5 \mathrm{~m}$.

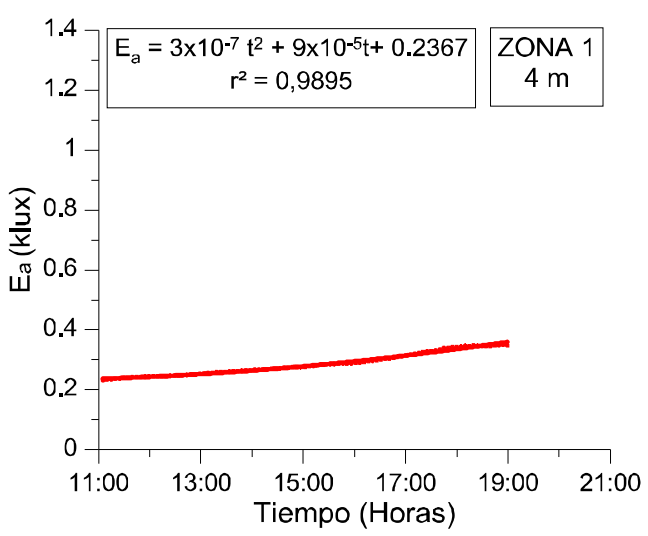

(h) $4 \mathrm{~m}$.

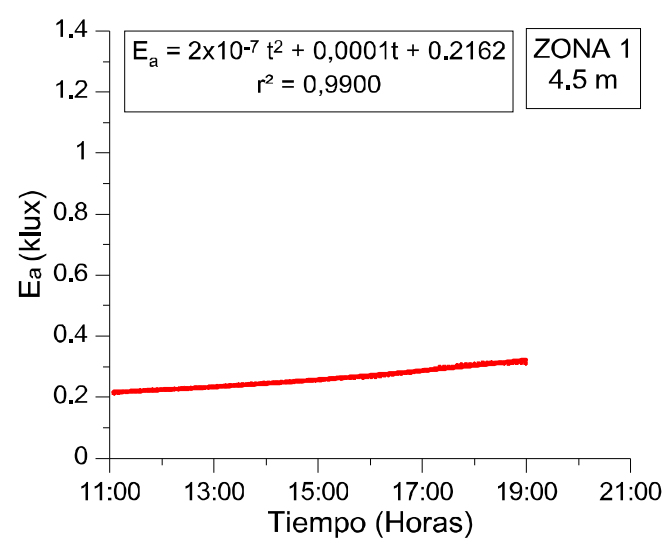

(i) $4.5 \mathrm{~m}$ 


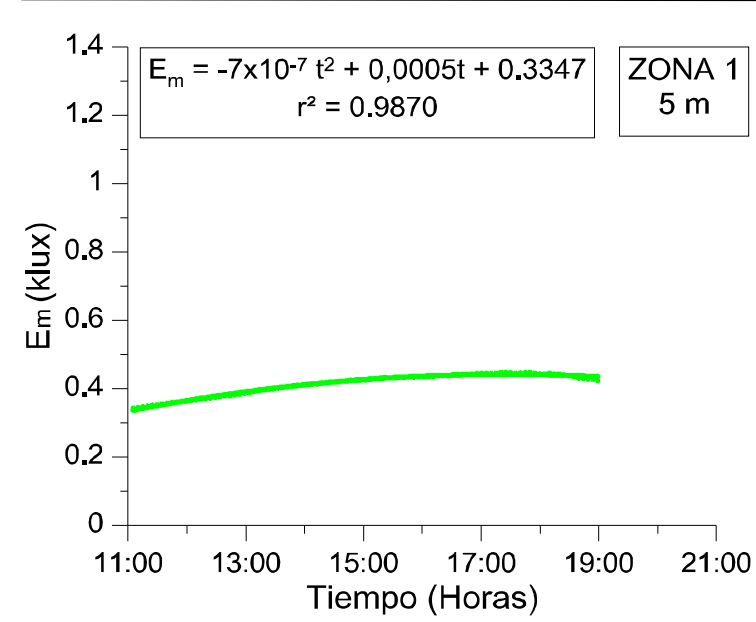

(j) $5 \mathrm{~m}$.

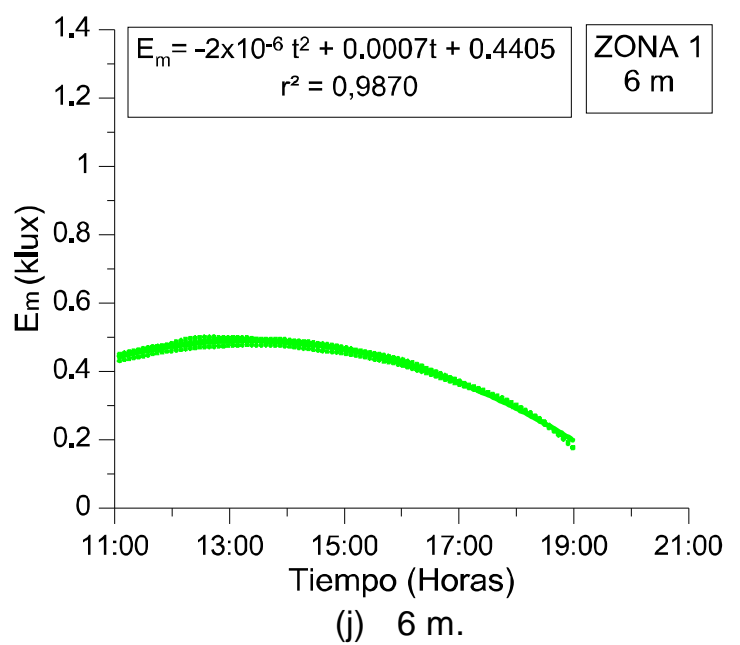

Figura 6.6. Evolución diaria de la iluminancia en el modelo a las distancias indicadas.

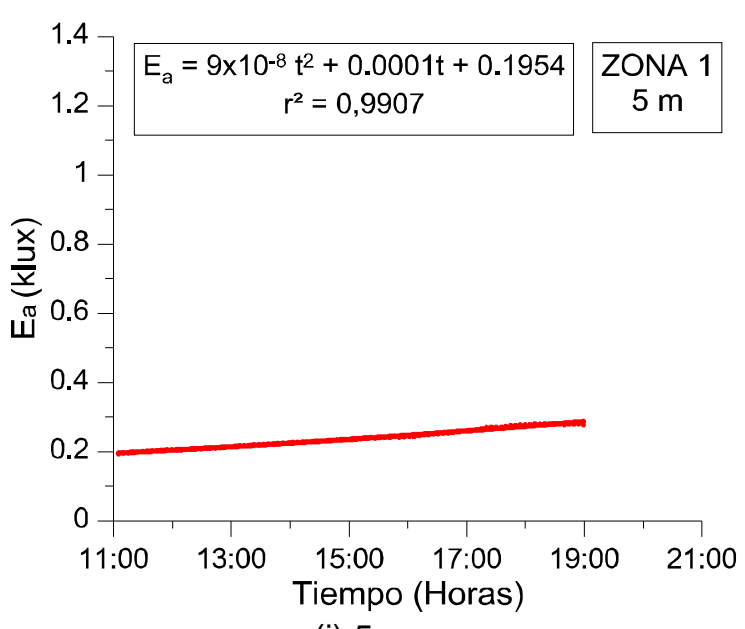

(j) $5 \mathrm{~m}$.

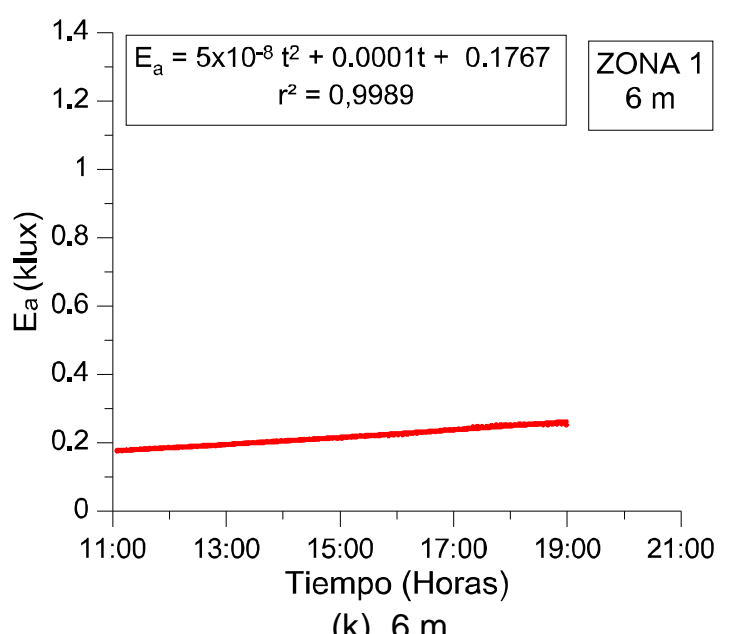

(k) $6 \mathrm{~m}$.

Figura 6.7. Evolución diaria de la iluminancia en el aula a las distancias indicadas.

A continuación, en las figuras 6.8 y 6.9, se muestran todas las curvas de ajuste, obtenidas anteriormente, de forma conjunta en una única gráfica, tanto del modelo como del aula. Se ha descartado la correspondiente a la distancia 5.5 $\mathrm{m}$ por haber medido mal el sensor del modelo a esta distancia.

En ellas se observa cómo a medida que aumenta la distancia a la fachada disminuye el valor de las iluminancias, salvo en el caso de los primeros sensores que al estar tan cerca de la fachada les llega menos luz de la ventana más próxima. 
MODELO

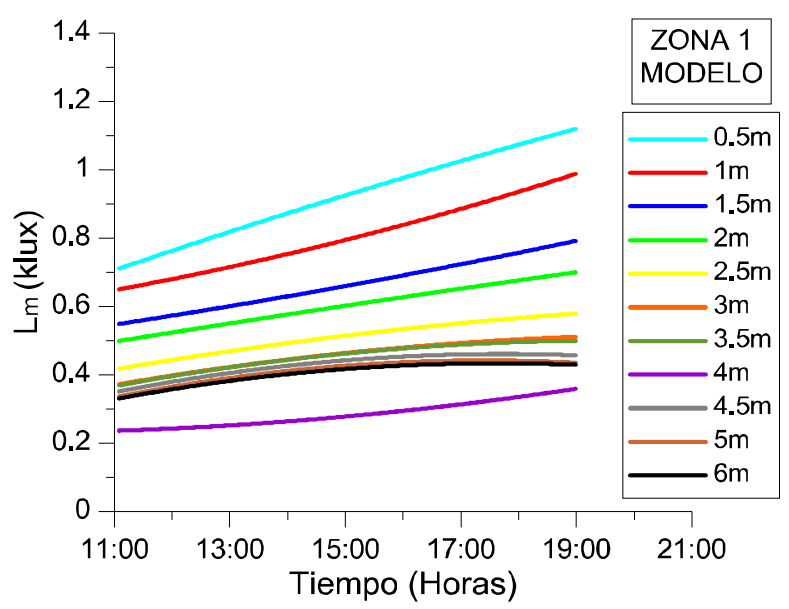

Figura 6.8. Curvas de ajuste de las iluminancias en el modelo a escala a las distintas distancias.

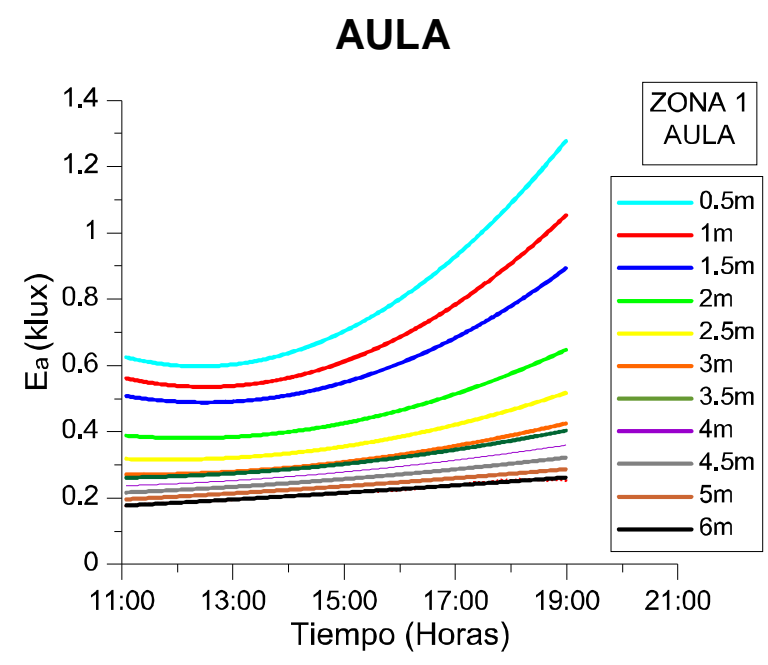

Figura 6.9. Curvas de ajuste de las iluminancias en el aula a las distintas distancias.

Las ecuaciones correspondientes a las curvas representadas en las figuras 6.8 y 6.9, así como su correspondiente coeficiente de ajuste $\left(r^{2}\right)$, se recogen en la tabla 6.2 .

\begin{tabular}{|c|c|c|c|c|}
\hline ZONA 1 & \multicolumn{2}{|l|}{ MODELO } & \multicolumn{2}{|l|}{ AULA } \\
\hline $\begin{array}{l}\text { DISTANCIA } \\
\text { (m) }\end{array}$ & ECUACIÓN & $r^{2}$ & ECUACIÓN & $r^{2}$ \\
\hline 0,5 & $E_{m}=-2 \times 10^{-7} t^{2}+0.001 t+0.7071$ & 0.992 & $E_{s}=4 \times 10^{-6} t^{2}-0.0007 t+0.6281$ & 0.9923 \\
\hline 1 & $E_{m}=4 \times 10^{-7} t^{2}+0.0005 t+0.6488$ & 0.9926 & $E_{s}=3 \times 10^{-6} t^{2}-0.0006 t+0.5638$ & 0.9913 \\
\hline 1,5 & $E_{m}=2 \times 10^{-7} t^{2}+0.0004 t+0.5476$ & 0.9943 & $E_{s}=3 \times 10^{-6} t^{2}-0.0005 t+0.5099$ & 0.9907 \\
\hline 2 & $E_{m}=-6 \times 10^{-8} t^{2}+0,0004 t+0.4977$ & 0.9947 & $E_{a}=2 \times 10^{-6} t^{2}-0.0002 t+0.3891$ & 0.9922 \\
\hline 2,5 & $E_{m}=-3 \times 10^{-7} t^{2}+0.0005 t+0.4165$ & 0.9937 & $E_{g}=1 \times 10^{-6} x^{2}-0.0001 t+0.3184$ & 0.9921 \\
\hline 3 & $E_{m}=-4 \times 10^{-7} t^{2}+0,0005 t+0.3712$ & 0.9917 & $E_{s}=7 \times 10^{-7} t^{2}-4 \times 10^{-6} t+0.2703$ & 0.9909 \\
\hline 3,5 & $E_{m}=-5 \times 10^{-7} t^{2}+0.0005 t+0.3675$ & 0.9898 & $E_{g}=5 \times 10^{-7} t^{2}+4 \times 10^{-5} t+0.2612$ & 0.9897 \\
\hline 4 & $E_{m}=3 \times 10^{-7} t^{2}+9 \times 10^{-5} t+0.2367$ & 0.9895 & $E_{a}=3 \times 10^{-7} t^{2}+9 \times 10^{-5} t+0.2367$ & 0.9895 \\
\hline 4,5 & $E_{m}=-6 \times 10^{-7} t^{2}+0.0005 t+0.3494$ & 0.9875 & $E_{a}=2 \times 10^{-7} t^{2}+0.0001 t+0.2162$ & 0.9900 \\
\hline 5 & $E_{m}=-7 \times 10^{-7} t^{2}+0,0005 t+0.3347$ & 0.9870 & $E_{s}=9 \times 10^{-8} t^{2}+0.0001 t+0.1954$ & 0.9907 \\
\hline 6 & $E_{m}=-2 \times 10^{-6} t^{2}+0.0007 t+0.4405$ & 0.9870 & $E_{g}=5 \times 10^{-8} t^{2}+0.0001 t+0.1767$ & 0.9989 \\
\hline
\end{tabular}

Tabla 6.2. Ecuaciones de ajuste de las iluminancias diarias a distintas distancias tanto del modelo como del aula.

En dicha tabla se puede observar cómo todos los coeficientes de ajuste del aula son superiores a 0.9 , y los del modelo a escala prácticamente también, lo que indica un alto grado de fiabilidad. 


\subsubsection{Relación entre las iluminancias del aula y del modelo a escala en función de las horas del día}

A continuación se ha representado, en la figura 6.10, el cociente obtenido entre las iluminancias del aula y del modelo durante el día, frente al tiempo (expresado en horas), según las distintas a la fachada. La ecuación de la curva obtenida para cada distancia así como su coeficiente de ajuste se muestran en cada gráfica.

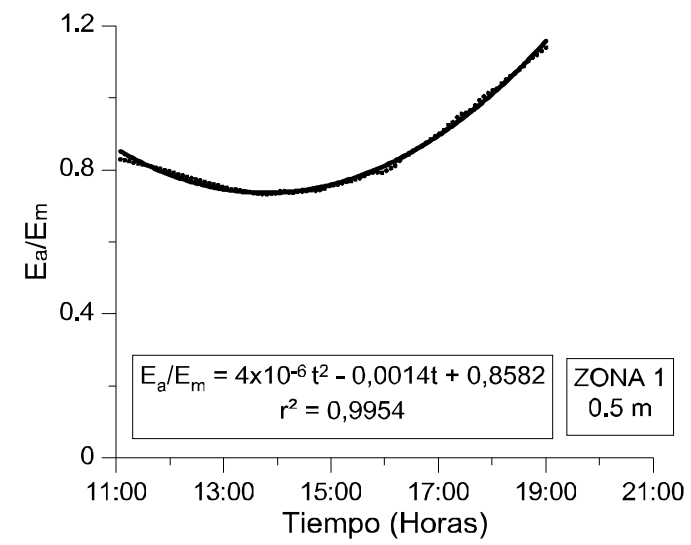

(a) $0.5 \mathrm{~m}$.

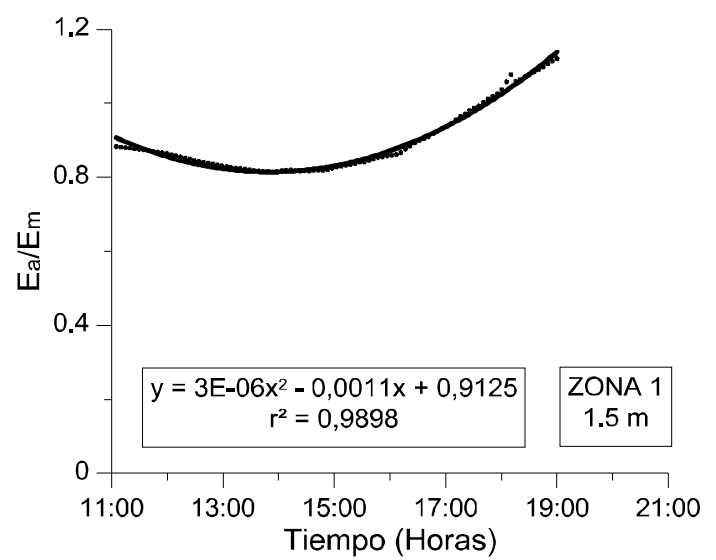

(c) $1.5 \mathrm{~m}$.

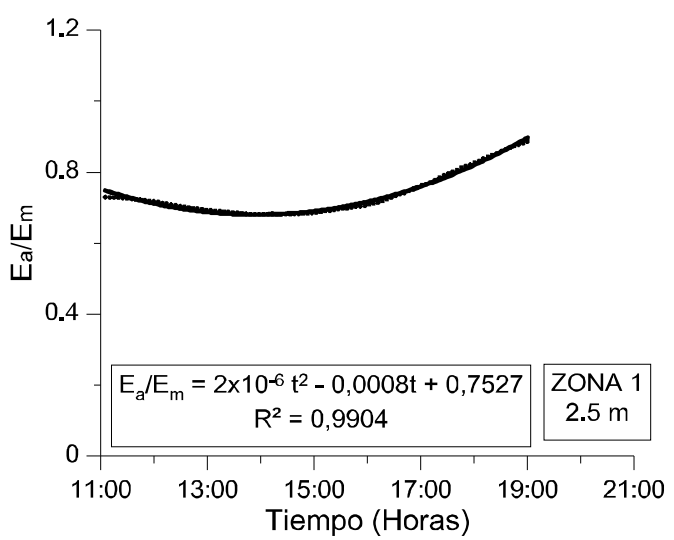

(e) $2.5 \mathrm{~m}$

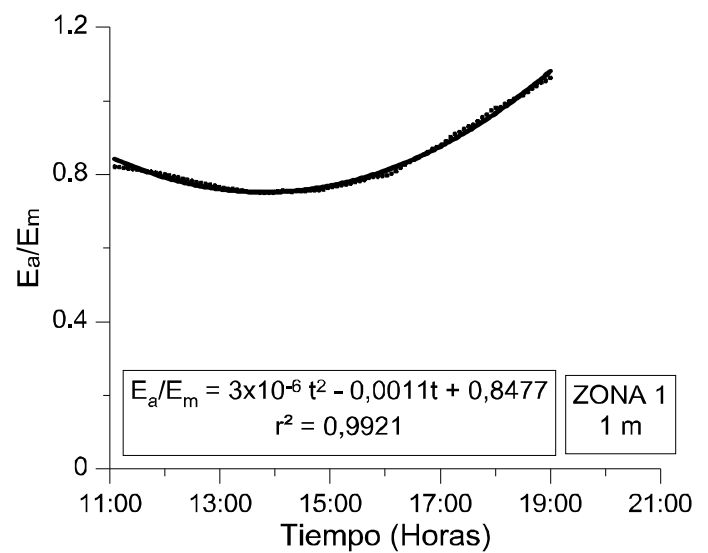

(b) $1 \mathrm{~m}$.

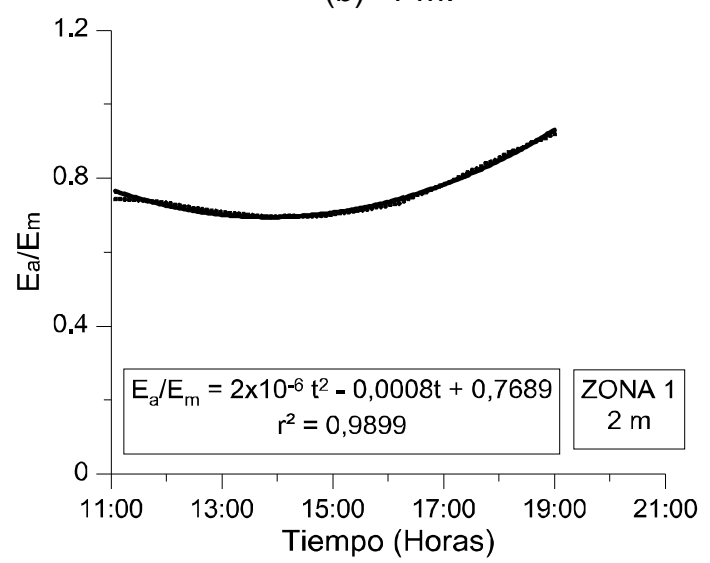

(d) $2 \mathrm{~m}$.

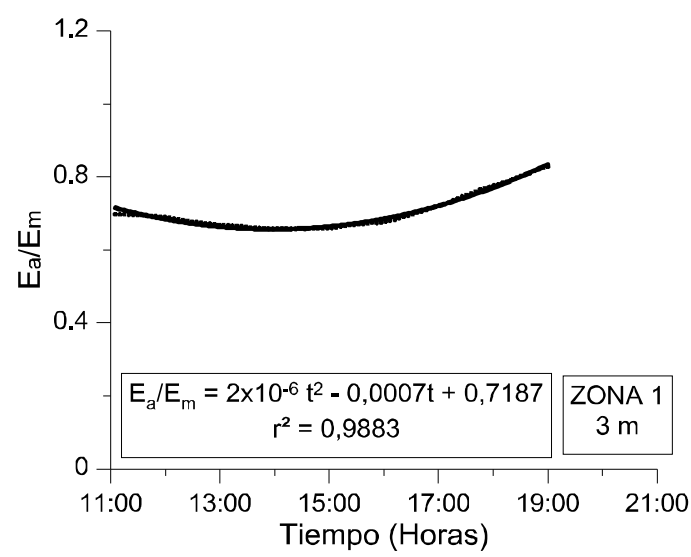

(f) $3 \mathrm{~m}$. 


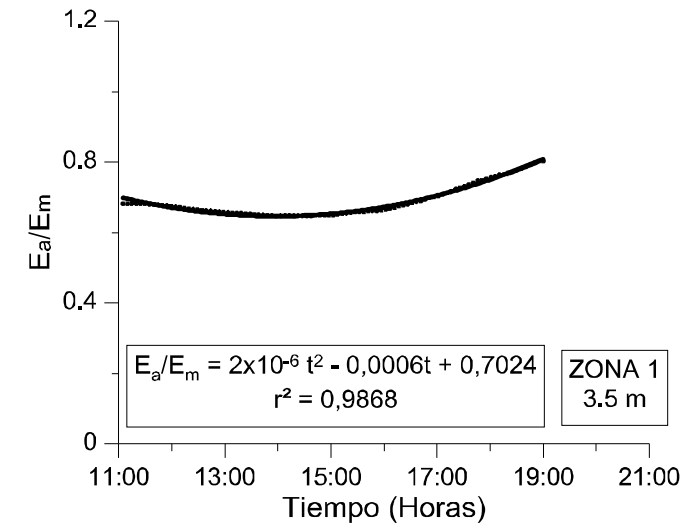

(g) $3.5 \mathrm{~m}$.

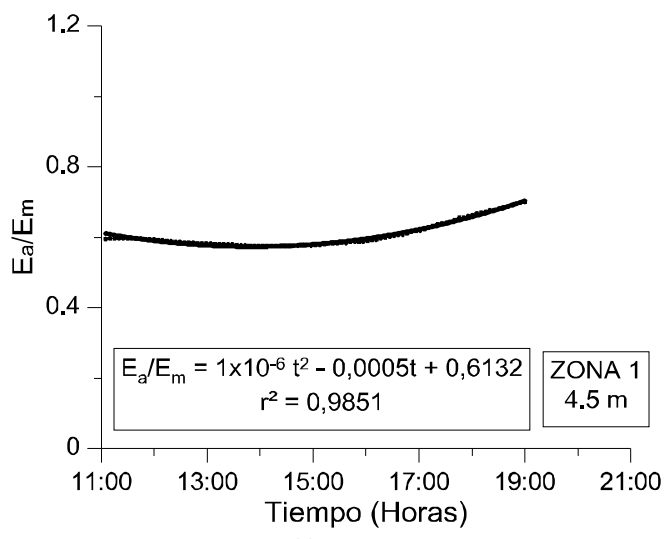

(i) $4.5 \mathrm{~m}$.

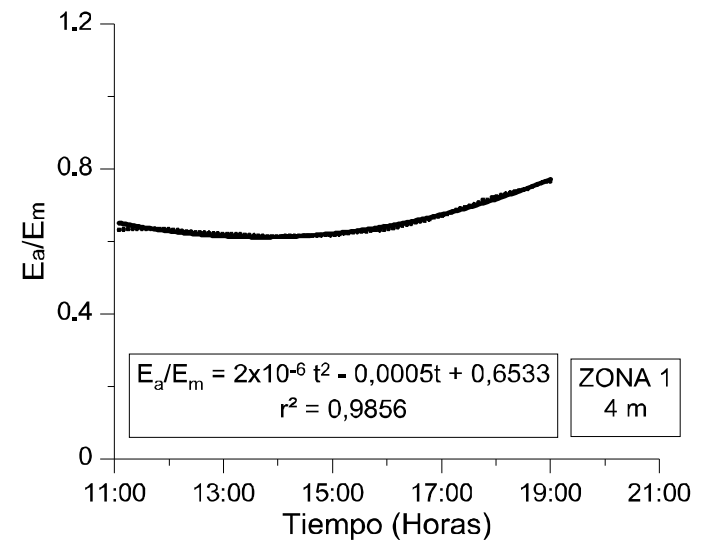

(h) $4 \mathrm{~m}$.

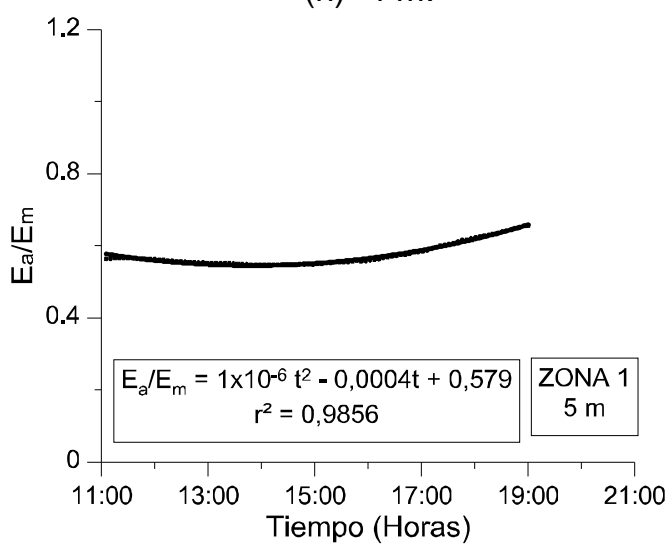

(j) $5 \mathrm{~m}$.

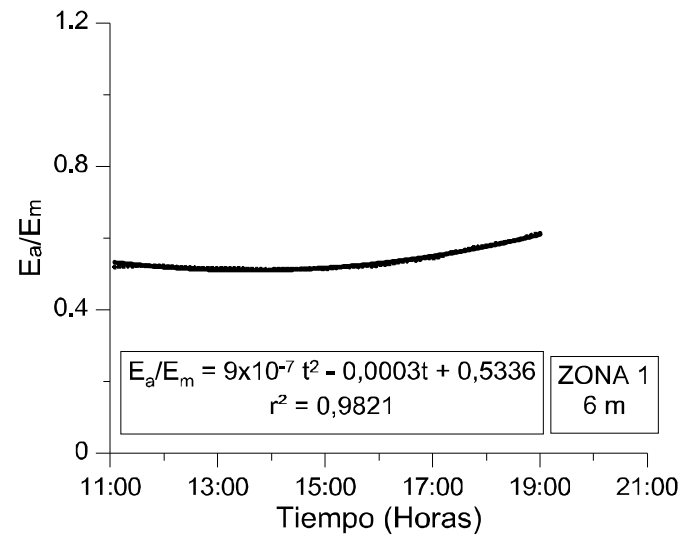

(k) $6 \mathrm{~m}$.

Figura 6.10. Cociente entre las iluminancias del aula y del modelo frente al tiempo a las distancias consideradas.

En este apartado se pretendía encontrar una relación lineal entre las iluminancias del aula y del modelo por medio de la cual hallar un factor de escala que se pudiera aplicar a las iluminancias del modelo para obtener las del aula sin necesidad de medir en el aula (como se establece en los objetivos). Sin embargo, como se puede observar en las gráficas, esta relación no es lineal sino que, para todas las distancias, se ajusta a una ecuación de segundo grado. 
A continuación se muestran de forma conjunta las curvas de los cocientes de las iluminancias aula/ modelo frente al tiempo para las distintas distancias (figura 6.11). Se puede apreciar cómo según aumenta la distancia a la ventana la curvatura va disminuyendo.

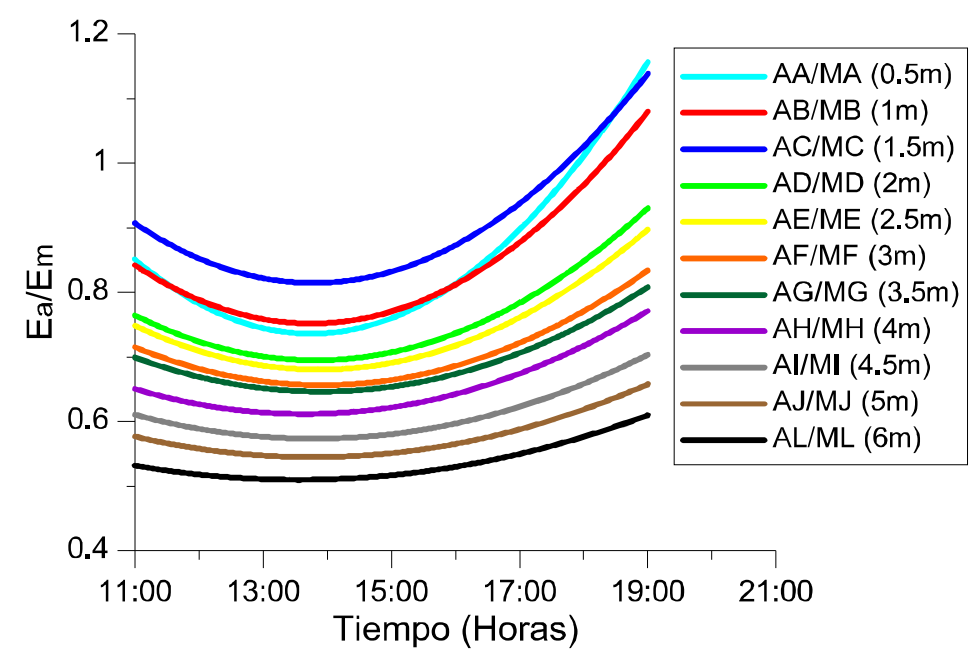

Figura 6.11. Curvas de ajuste de los cocientes de las iluminancias aula/ modelo frente al tiempo a las distintas distancias.

En la tabla 6.3 se recogen las ecuaciones de ajuste obtenidas de la relación entre las iluminancias aula/modelo y el tiempo, así como su correspondiente coeficiente de ajuste, a las distintas distancias a la fachada.

\begin{tabular}{|c|c|c|}
\hline DISTANCIA $(\boldsymbol{m})$ & ECUACIÓN & $\mathbf{r}^{2}$ \\
\hline $\mathbf{0 . 5}$ & $\mathrm{E}_{\mathrm{a}} / \mathrm{E}_{\mathrm{m}}=4 \times 10^{-6} \mathrm{t}^{2}-0.0014 \mathrm{t}+0.8582$ & 0.9954 \\
\hline $\mathbf{1}$ & $\mathrm{E}_{\mathrm{a}} / \mathrm{E}_{\mathrm{m}}=3 \times 10^{-6} \mathrm{t}^{2}-0.0011 \mathrm{t}+0.8477$ & 0.9921 \\
\hline $\mathbf{1 . 5}$ & $\mathrm{E}_{\mathrm{a}} / \mathrm{E}_{\mathrm{m}}=3 \times 10^{-6} \mathrm{t}^{2}-0.0011 \mathrm{t}+0.9125$ & 0.9898 \\
\hline $\mathbf{2}$ & $\mathrm{E}_{\mathrm{a}} / \mathrm{E}_{\mathrm{m}}=2 \times 10^{-6} \mathrm{t}^{2}-0.0008 \mathrm{t}+0.7689$ & 0.9899 \\
\hline $\mathbf{2 . 5}$ & $\mathrm{E}_{\mathrm{a}} / \mathrm{E}_{\mathrm{m}}=2 \times 10^{-6} \mathrm{t}^{2}-0.0008 \mathrm{t}+0.7527$ & 0.9904 \\
\hline $\mathbf{3}$ & $\mathrm{E}_{\mathrm{a}} / \mathrm{E}_{\mathrm{m}}=2 \times 10^{-6} \mathrm{t}^{2}-0.0007 \mathrm{t}+0.7187$ & 0.9883 \\
\hline $\mathbf{3 . 5}$ & $\mathrm{E}_{\mathrm{a}} / \mathrm{E}_{\mathrm{m}}=2 \times 10^{-6} \mathrm{t}^{2}-0.0006 \mathrm{t}+0.7024$ & 0.9868 \\
\hline $\mathbf{4}$ & $\mathrm{E}_{\mathrm{a}} / \mathrm{E}_{\mathrm{m}}=2 \times 10^{-6} \mathrm{t}^{2}-0.0005 \mathrm{t}+0.6533$ & 0.9856 \\
\hline $\mathbf{4 . 5}$ & $\mathrm{E}_{\mathrm{a}} / \mathrm{E}_{\mathrm{m}}=1 \times 10^{-6} \mathrm{t}^{2}-0.0005 \mathrm{t}+0.6132$ & 0.9851 \\
\hline $\mathbf{5}$ & $\mathrm{E}_{\mathrm{a}} / \mathrm{E}_{\mathrm{m}}=1 \times 10^{-6} \mathrm{t}^{2}-0.0004 \mathrm{t}+0.579$ & 0.9856 \\
\hline $\mathbf{6}$ & $\mathrm{E}_{\mathrm{a}} / \mathrm{E}_{\mathrm{m}}=9 \times 10^{-7} \mathrm{t}^{2}-0.0003 \mathrm{t}+0.5336$ & 0.9821 \\
\hline
\end{tabular}

Tabla 6.3. Ecuaciones a las distintas distancias, de los cocientes de las iluminancias aula/ modelo frente al tiempo. 
En la tabla se puede apreciar cómo todas las ecuaciones tienen un coeficiente de ajuste superior a 0.98 , lo que indica que tienen una alta fiabilidad.

\subsubsection{Relación entre iluminancias del aula y del modelo a escala en función de la iluminancia exterior}

Ahora se va a analizar la relación entre las iluminancias del aula $\left(E_{a}\right)$ y del modelo $\left(E_{m}\right)$ en función de la iluminancia exterior $\left(E_{\text {ext }}\right)$ expresada en klux, teniendo en cuenta los datos utilizados anteriormente, para las distintas distancias a la fachada. En las gráficas de la figura 6.12 se ha representado en el eje vertical el cociente entre las iluminancias del aula y el modelo, y en el eje horizontal las iluminancias obtenidas con el sensor exterior.

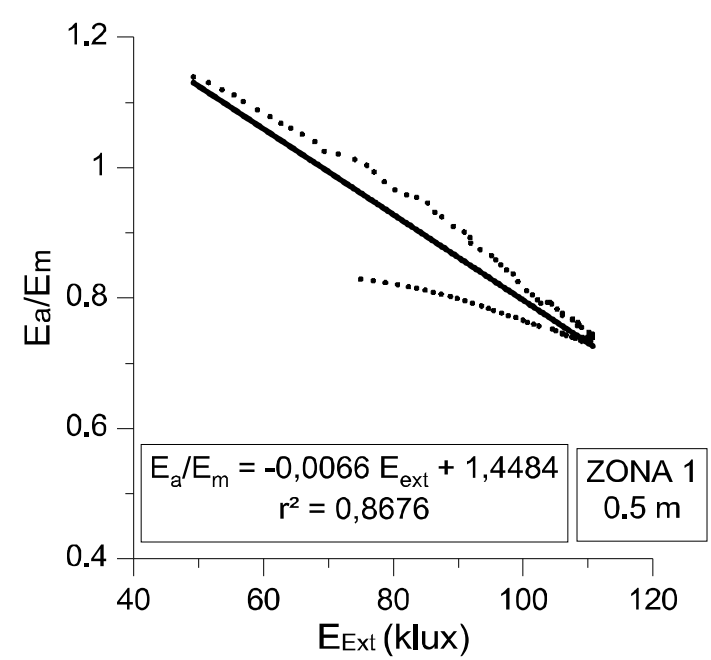

(a) $0.5 \mathrm{~m}$.

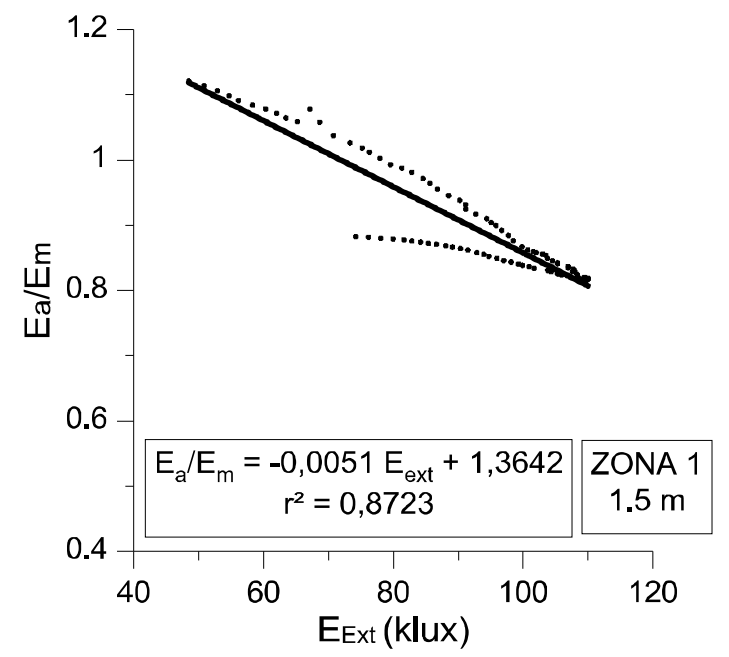

(c) $1.5 \mathrm{~m}$.

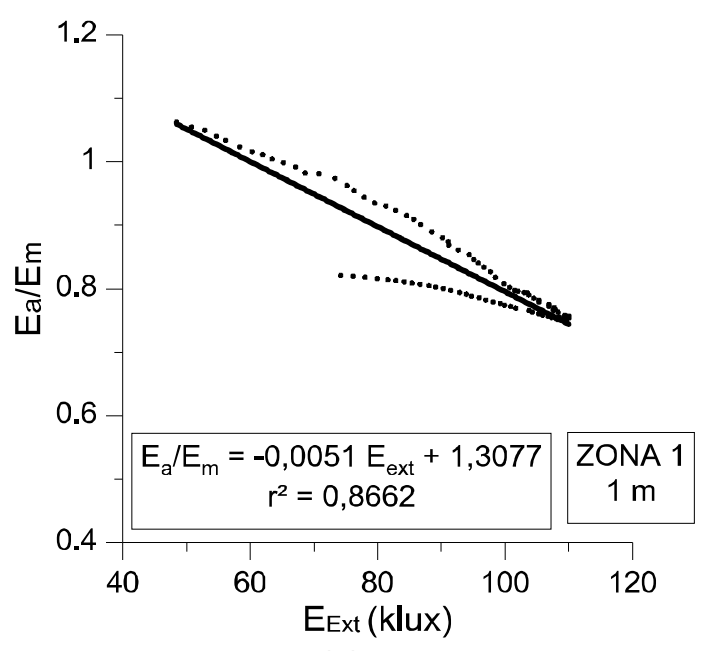

(b) $1 \mathrm{~m}$.

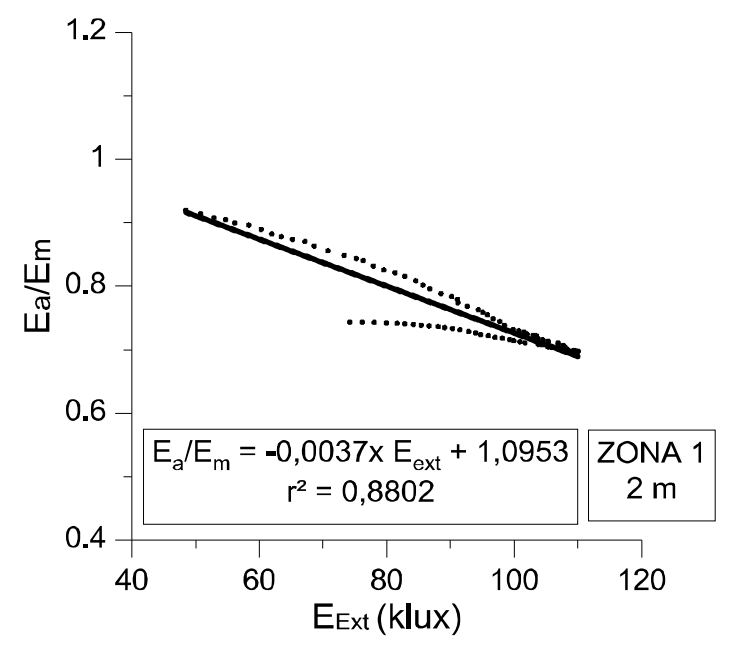

(d) $2 \mathrm{~m}$. 


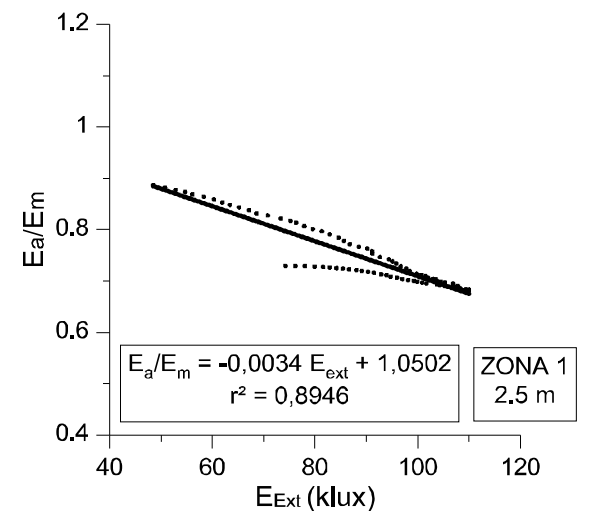

(e) $2.5 \mathrm{~m}$.

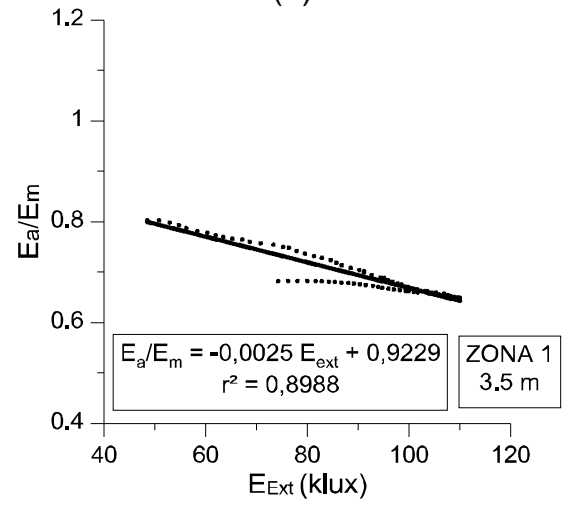

(g) $3.5 \mathrm{~m}$.

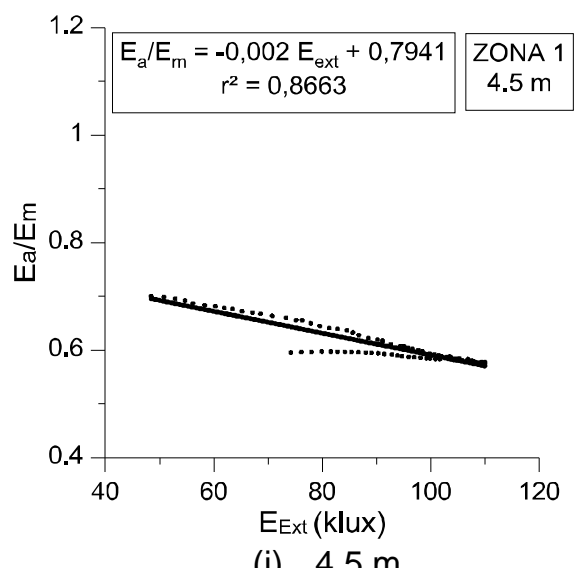

(i) $4.5 \mathrm{~m}$.

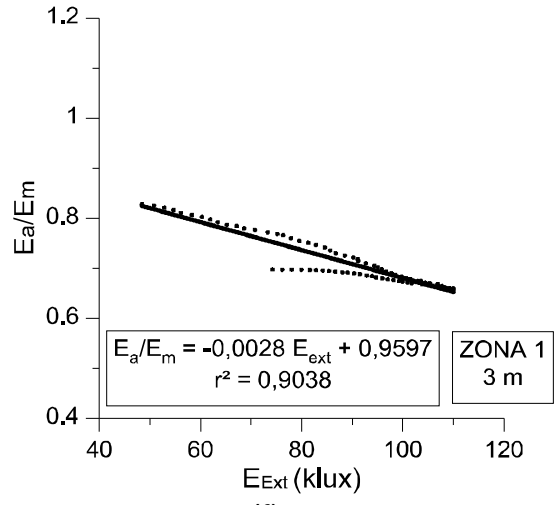

(f) $3 \mathrm{~m}$.

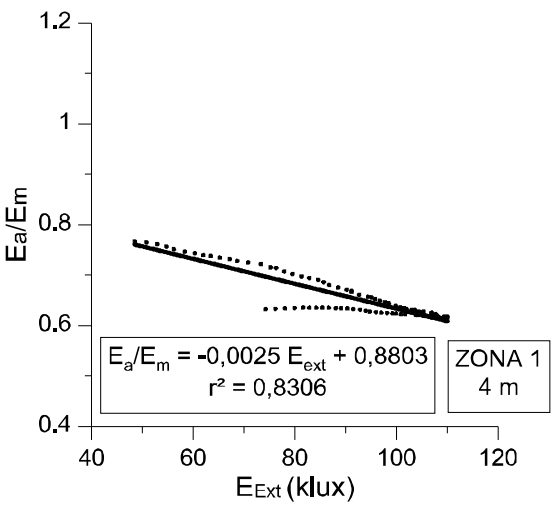

(h) $4 \mathrm{~m}$.

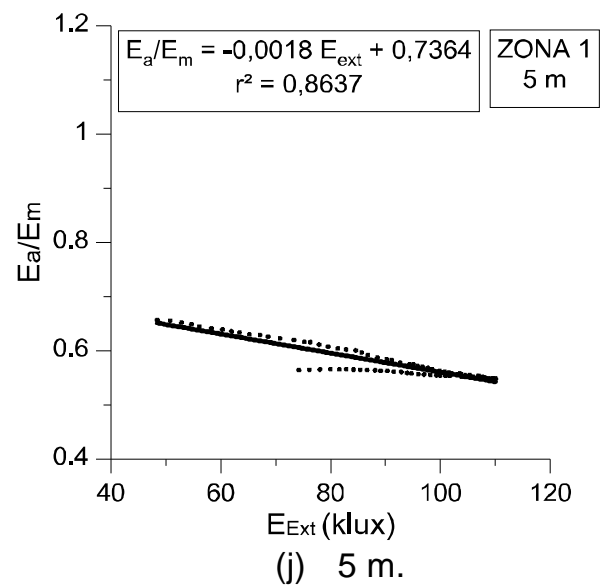

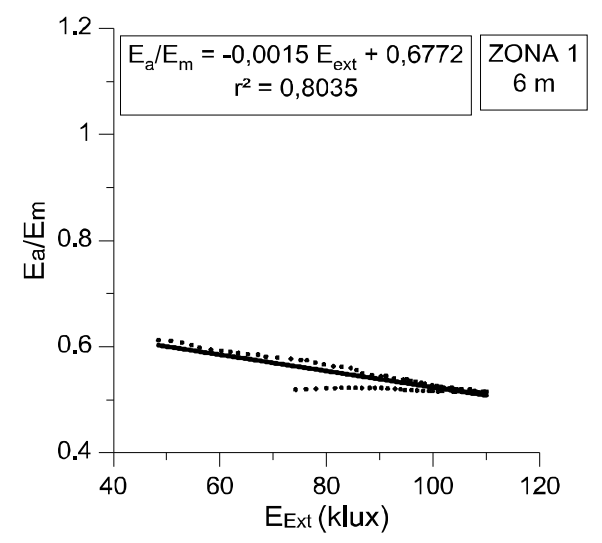

(k) $6 \mathrm{~m}$.

Figura 6.12. Cociente de las iluminancias del aula y del modelo frente a la iluminancia exterior a las distancias indicadas. 
Como se puede observar la relación iluminancias aula/modelo frente a la iluminancia exterior se ajusta a rectas, es decir, en este caso sí se ha obtenido una relación lineal.

A medida que aumenta la distancia a la ventana la relación iluminancia aula/modelo con la iluminancia exterior va disminuyendo. Si se representan las distintas rectas de ajuste, obtenidas anteriormente, de forma conjunta, se obtiene la gráfica de la figura 6.13 .

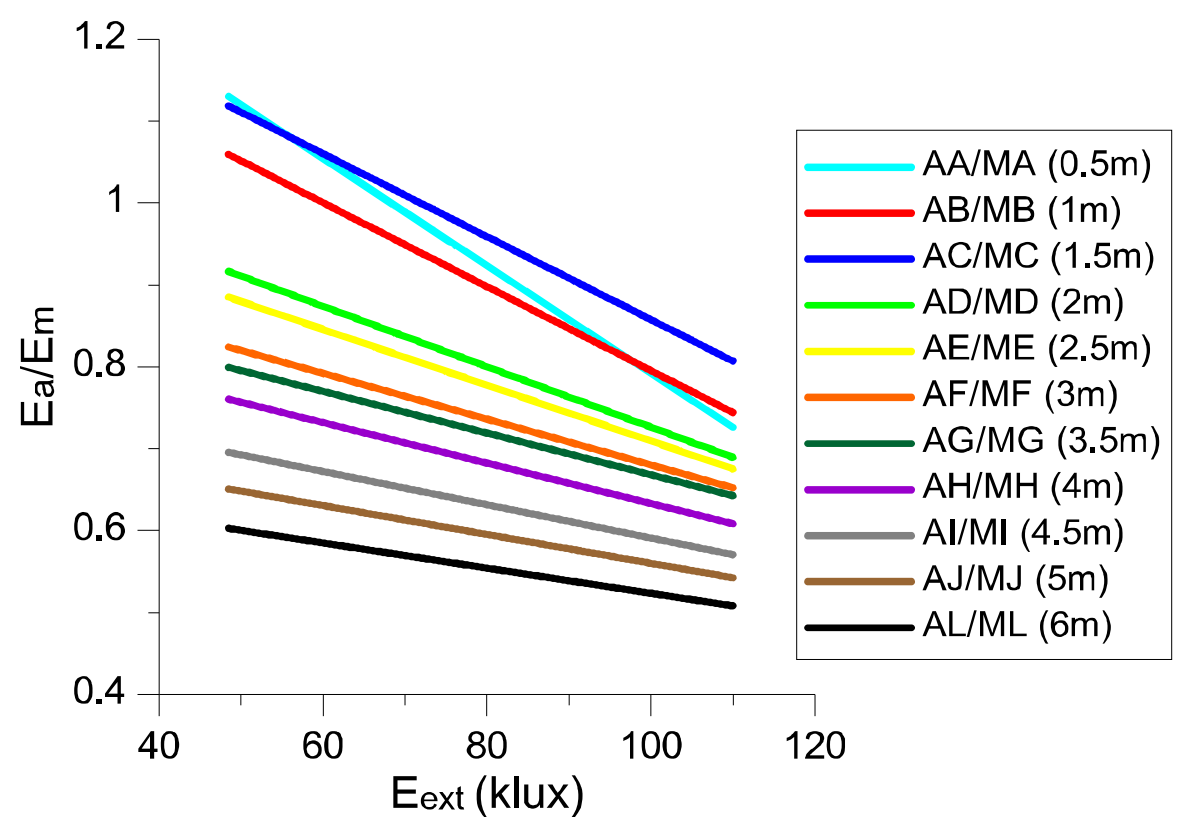

Figura 6.13. Rectas ajustadas de la relaciones entre el cociente de las iluminancias aula/ modelo frente a la iluminancia exterior a las distintas distancias.

Se observa que al aumentar la distancia a la fachada, la pendiente de estas rectas va disminuyendo.

Las ecuaciones correspondientes a las rectas anteriores, así como su coeficiente de ajuste $\left(r^{2}\right)$ se recogen en la tabla 6.4. En ésta se observa que el coeficiente de ajuste de todas las rectas es superior a 0.8 . 


\begin{tabular}{|c|c|c|}
\hline $\begin{array}{c}\text { DISTANCIA } \\
(\mathbf{m})\end{array}$ & ECUACIÓN & $\mathbf{r}^{2}$ \\
\hline $\mathbf{0 . 5}$ & $\mathrm{E}_{\mathrm{a}} / \mathrm{E}_{\mathrm{m}}=-0.0066 \mathrm{E}_{\mathrm{ext}}+1.4484$ & 0.8676 \\
\hline $\mathbf{1}$ & $\mathrm{E}_{\mathrm{a}} / \mathrm{E}_{\mathrm{m}}=-0.0051 \mathrm{E}_{\mathrm{ext}}+1.3077$ & 0.8662 \\
\hline $\mathbf{1 . 5}$ & $\mathrm{E}_{\mathrm{a}} / \mathrm{E}_{\mathrm{m}}=-0.0051 \mathrm{E}_{\mathrm{ext}}+1.3642$ & 0.8723 \\
\hline $\mathbf{2}$ & $\mathrm{E}_{\mathrm{a}} / \mathrm{E}_{\mathrm{m}}=-0.0037 \mathrm{E}_{\mathrm{ext}}+1.0953$ & 0.8802 \\
\hline $\mathbf{2 . 5}$ & $\mathrm{E}_{\mathrm{a}} / \mathrm{E}_{\mathrm{m}}=-0.0034 \mathrm{E}_{\mathrm{ext}}+1.0502$ & 0.8946 \\
\hline $\mathbf{3}$ & $\mathrm{E}_{\mathrm{a}} / \mathrm{E}_{\mathrm{m}}=-0.0028 \mathrm{E}_{\mathrm{ext}}+0.9597$ & 0.9038 \\
\hline $\mathbf{3 . 5}$ & $\mathrm{E}_{\mathrm{a}} / \mathrm{E}_{\mathrm{m}}=-0.0025 \mathrm{E}_{\mathrm{ext}}+0.9229$ & 0.8988 \\
\hline $\mathbf{4}$ & $\mathrm{E}_{\mathrm{a}} / \mathrm{E}_{\mathrm{m}}=-0.0025 \mathrm{E}_{\mathrm{ext}}+0.8803$ & 0.8306 \\
\hline $\mathbf{4 . 5}$ & $\mathrm{E}_{\mathrm{a}} / \mathrm{E}_{\mathrm{m}}=-0.002 \mathrm{E}_{\mathrm{ext}}+0.7941$ & 0.8663 \\
\hline $\mathbf{5}$ & $\mathrm{E}_{\mathrm{a}} / \mathrm{E}_{\mathrm{m}}=-0.0018 \mathrm{E}_{\mathrm{ext}}+0.7364$ & 0.8637 \\
\hline $\mathbf{6}$ & $\mathrm{E}_{\mathrm{a}} / \mathrm{E}_{\mathrm{m}}=-0.0015 \mathrm{E}_{\mathrm{ext}}+0.6772$ & 0.8035 \\
\hline
\end{tabular}

Tabla 6.4. Ecuaciones que relacionan las iluminancias del aula y del modelo con la del exterior a las distintas distancias.

\subsubsection{Modelo matemático para determinar la iluminancia en el aula}

En el apartado anterior se han obtenido unas ecuaciones que relacionan las iluminancias entre el aula $\left(E_{a}\right)$ y el modelo $\left(E_{m}\right)$ en función de la iluminancia exterior $\left(\mathrm{E}_{\mathrm{ext}}\right)$, para las distintas distancias desde la fachada a puntos del interior, de la forma:

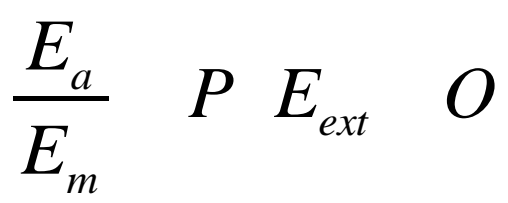

Siendo $P$ la Pendiente y $O$ la Ordenada en el origen.

Como el objetivo es obtener una expresión que permita relacionar las variables anteriores también con la distancia interior a la fachada, se van a analizar, por separado, las pendientes (figura 6.14) y las ordenadas en el origen (figura 6.15) de las ecuaciones de las rectas recogidas en la tabla 6.4 en función de la distancia. Estos datos se muestran en la tabla 6.5. 


\begin{tabular}{|c|c|c|}
\hline Distancia $(\mathbf{m})$ & Pendiente & Ordenada \\
\hline 0.5 & -0.0066 & 1.4484 \\
\hline 1 & -0.0051 & 1.3077 \\
\hline 1.5 & -0.0051 & 1.3642 \\
\hline 2 & -0.0037 & 1.0953 \\
\hline 2.5 & -0.0034 & 1.0502 \\
\hline 3 & -0.0028 & 0.9597 \\
\hline 3.5 & -0.0025 & 0.9229 \\
\hline 4 & -0.0025 & 0.8803 \\
\hline 4.5 & -0.002 & 0.7941 \\
\hline 5 & -0.0018 & 0.7364 \\
\hline 6 & -0.0015 & 0.6772 \\
\hline
\end{tabular}

Tabla 6.5. Pendientes y ordenadas en el origen a las distintas distancias de las rectas procedentes del cociente de las iluminancias aula/modelo frente a la iluminancia exterior.

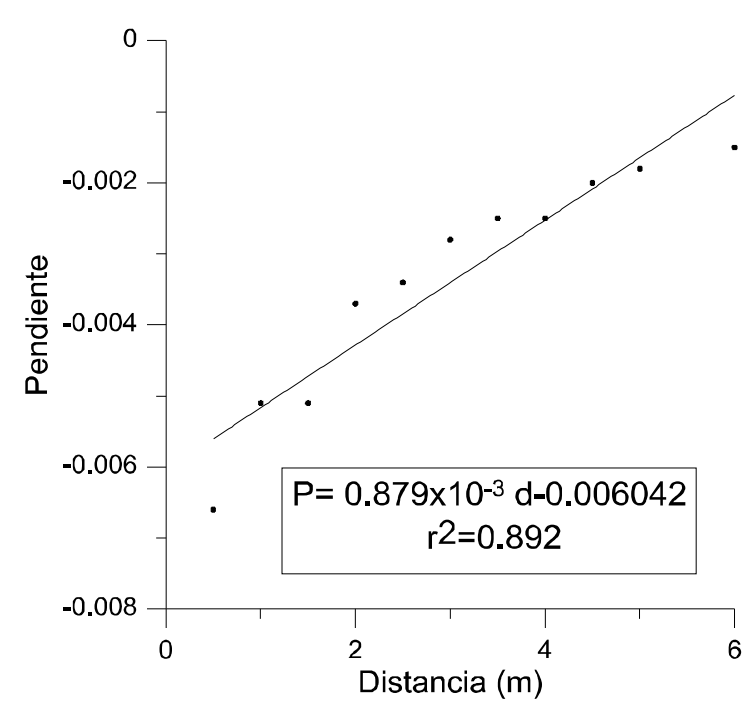

Figura 6.14. Pendiente en función de la distancia a la fachada.

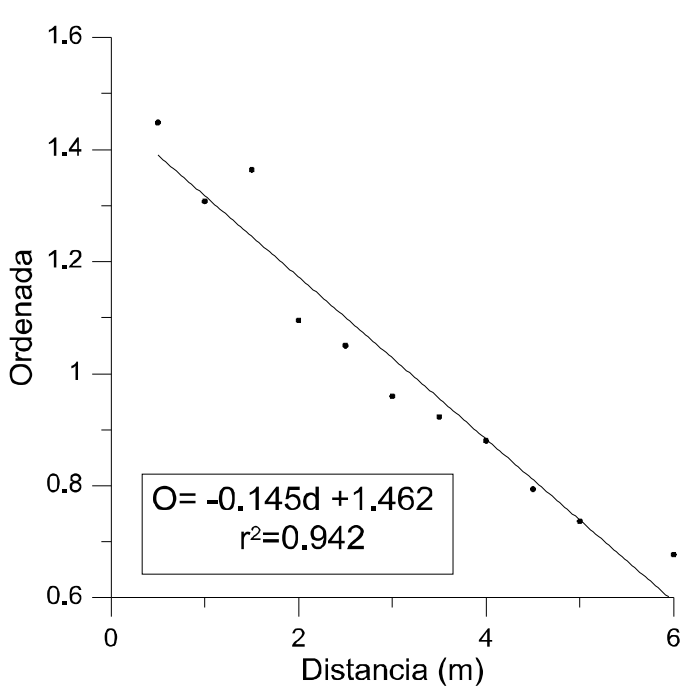

Figura 6.15. Ordenada en el origen en función de la distancia a la fachada.

En la figura 6.14 se representan los distintos valores de las pendientes $(P)$ para cada distancia $(d)$, observándose que existe una relación lineal definida por la recta: $P=0.879 \times 10^{-3}$ d $\quad 0.006042$, con $r^{2}=0.975$.

Analógamente, en la figura 6.15 se representan los valores de las ordenadas en el origen $(O)$ frente a la distancia $(d)$. Su respuesta también ha sido lineal, cuya recta de ajuste es: $O=-0.145 d+1.462$, con $r^{2}=0.969$. 
En ambos casos el coeficiente de ajuste es superior a 0.95 lo que muestra su alta fiabilidad.

Por lo tanto, el modelo matemático que nos permite determinar la iluminancia del aula a partir de la del modelo a escala, la exterior y la distancia en la zona 1 es:

$$
E_{a}=\left[(0.879 d-6.042) \times 10^{-3} E_{e x t}-0.145 d+1.462\right] E_{m} \quad[Z 1]
$$

Siendo:

$$
\begin{aligned}
& E_{a}: \text { Iluminancia del aula (klux) } \\
& E_{m}: \text { lluminancia del modelo (klux) } \\
& E_{\text {ext: }} \text { Iluminancia exterior (klux) } \\
& d: \text { Distancia a la fachada (m) }
\end{aligned}
$$

Esta ecuación permite calcular las iluminancias en cualquier punto de la zona 1, determinado por su distancia hasta la fachada, del aula a partir las iluminancias en la maqueta $\left(E_{m}\right)$ y en el exterior $\left(E_{\text {ext }}\right)$. De esta manera se alcanza el objetivo de poder conocer las iluminancias de una estancia real (el aula) sin necesidad de medir en dicha estancia; sólo sería necesario conocer las iluminancias en el exterior y en el interior de la maqueta de la estancia estudiada así como la distancia a la que hayamos colocado los sensores.

\subsubsection{Análisis estadístico}

A continuación se va a realizar un análisis estadístico de los valores obtenidos para las pendientes y las ordenadas en el origen a las distintas distancias como comprobación de la fiabilidad del modelo obtenido.

Por un lado se ha analizado la pendiente (eje vertical) frente a la distancia a la fachada (eje horizontal) y por otro lado la ordenada en el origen (eje vertical) frente a la distancia a la fachada (eje horizontal). 
En la gráfica en la que se han representado las pendientes de las rectas obtenidas $(P)$ en función de la distancia a la fachada ( $($ ) (figura 6.16) se puede observar cómo esta relación es lineal, que se ajusta a la ecuación de primer grado con pendiente positiva: $P=(0.879 \mathrm{~d}-6.042) \times 10^{-3}$ (como se había obtenido en el apartado anterior). La mayor parte de los datos se encuentran dentro de los límites de confianza al 95\% (verde) y todos los datos se encuentran entre los límites de predicción (rojo) por lo que no se aprecian valores atípicos.

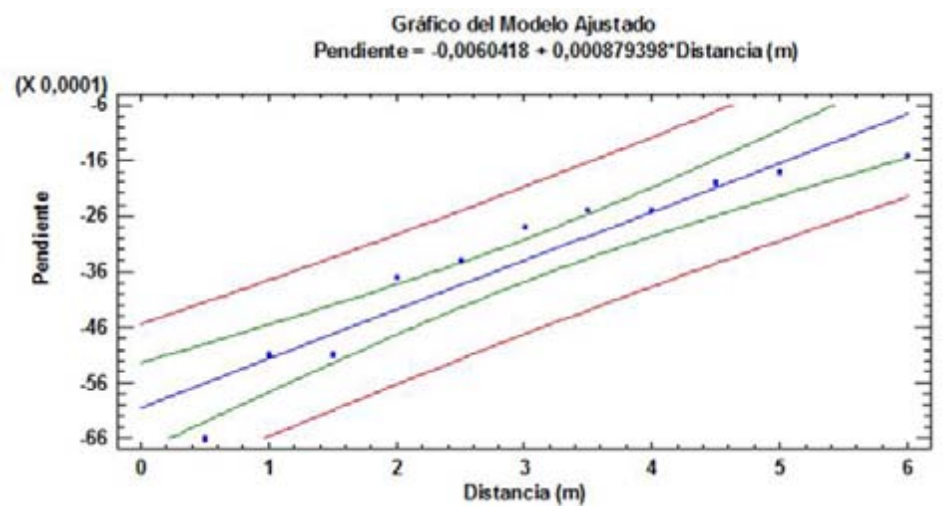

Figura 6.16. Gráfico del modelo ajustado de la pendiente en función de la distancia a la fachada.

De la representación de las ordenadas en el origen de las rectas obtenidas $(O)$ en función de la distancia a la fachada (d) (figura 6.17) también se deduce que esta relación es lineal, que se ajusta a una ecuación de primer grado con pendiente negativa: $O=0.145 d+1.462$, como ya se había obtenido en el apartado anterior. La mayor parte de los datos se encuentran dentro de los límites de confianza al 95\% (verde) y todos los datos se encuentran entre los límites de predicción (rojo), por lo que no se aprecian valores atípicos.

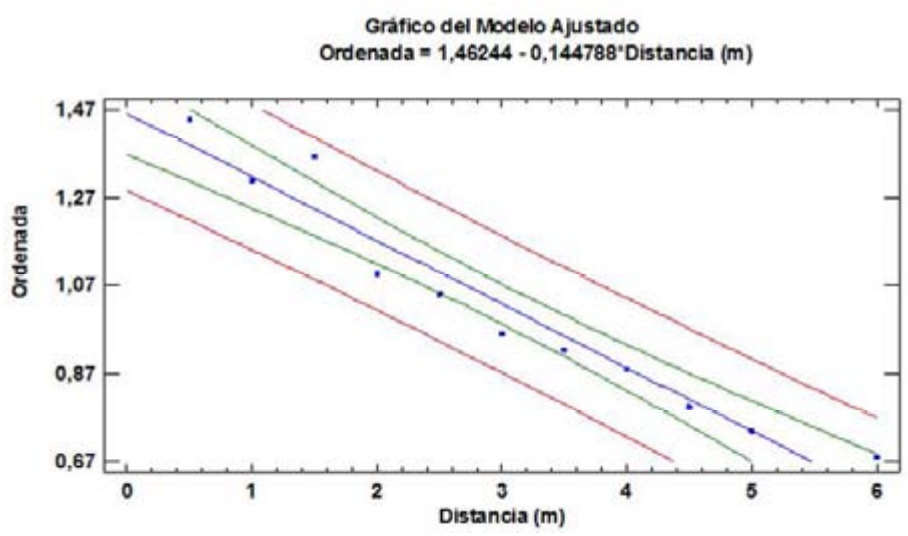

Figura 6.17. Gráfico del modelo ajustado de la ordenada en el origen en función de la distancia a la fachada. 
En ambos casos se obtiene un p-valor de $0.00<0.5$, lo que indica que hay una relación estadística significante entre las variables consideradas con un nivel de confianza de por lo menos el $95 \%$, es decir que la fiabilidad de los datos es $\geq 95 \%$. El p-valor del análisis de los residuos es superior a 0.05 lo que indica que la probabilidad de que existan errores en este análisis es menor al $5 \%$.

\subsubsection{Simulación con software de iluminación y comprobación de resultados}

En este apartado se van a comparar las iluminancias del aula entre las 11:00 y las 19:00 horas, obtenidas de tres formas diferentes:

a) Midiendo directamente en el aula.

b) Calculando mediante la ecuación [Z1] a partir de las iluminancias medidas en el modelo a escala y en el exterior.

c) Utilizando un software de iluminación (Dialux 4.11)

En la figura 6.18 se muestran gráficamente los resultados obtenidos en los tres casos para las distintas distancias consideradas en el estudio. Se han representado en rojo las iluminancias medidas directamente, en azul claro las obtenidas de la ecuación Z1 y en azul oscuro las del programa de simulación.

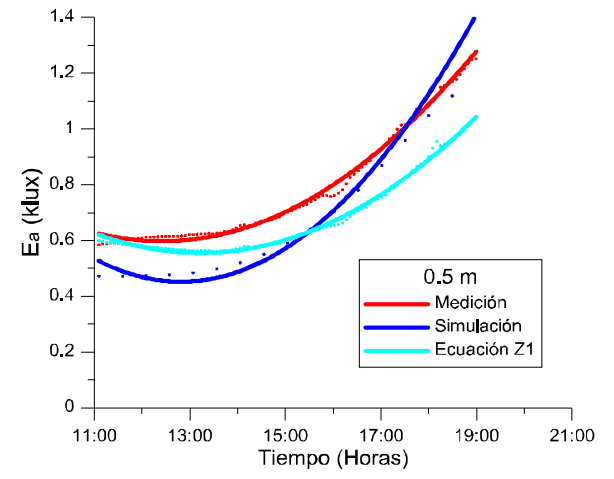

(a) $0.5 \mathrm{~m}$.

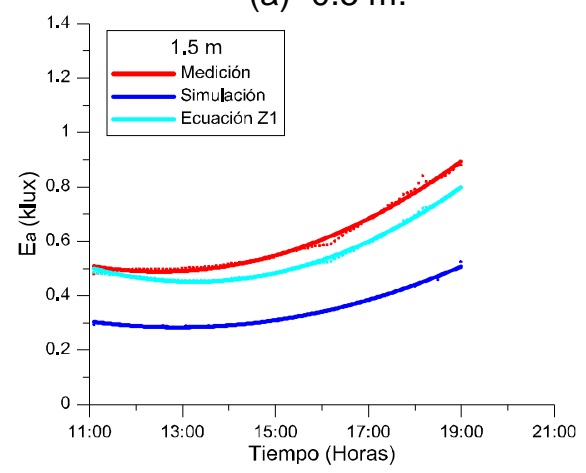

(c) $1.5 \mathrm{~m}$.

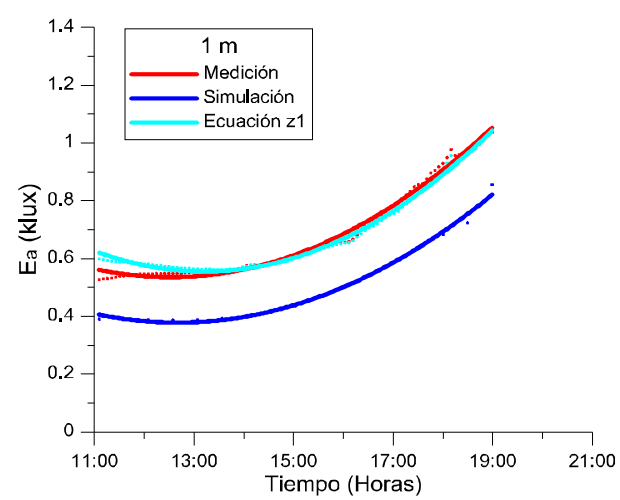

(b) $1 \mathrm{~m}$.

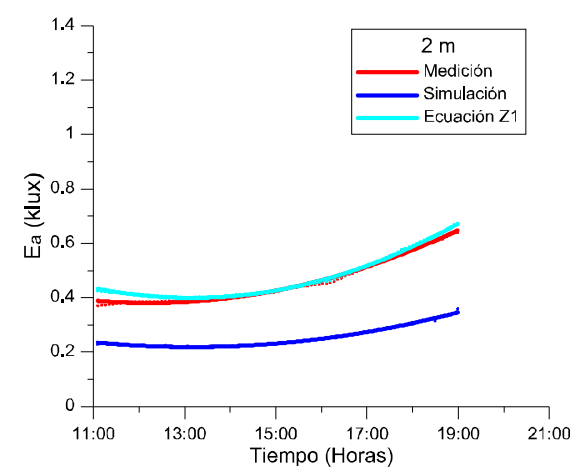

(d) $2 \mathrm{~m}$. 
Estudio experimental y modelización de la iluminación natural en la edificación mediante modelos a escala

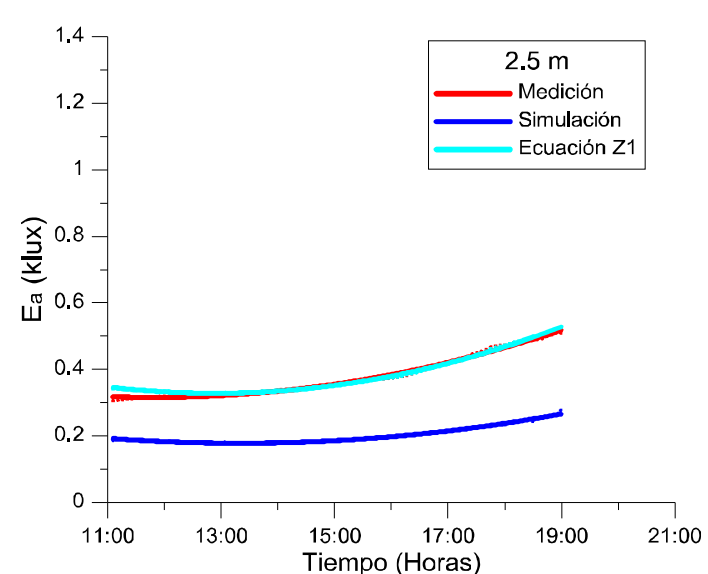

(e) $2.5 \mathrm{~m}$.

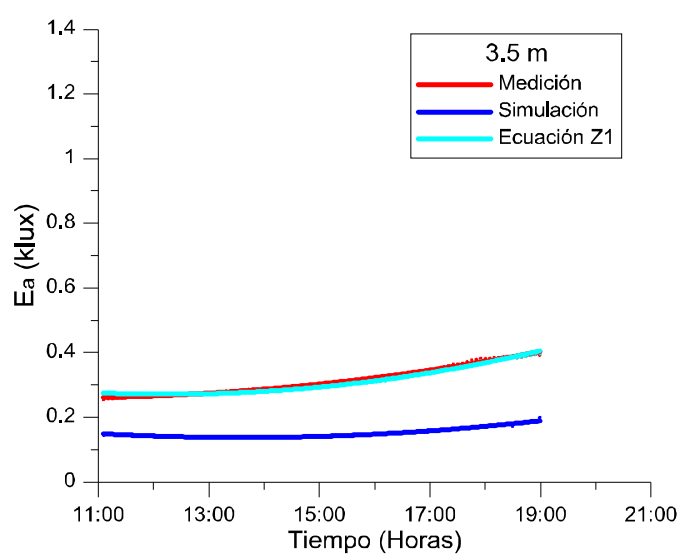

(g) $3.5 \mathrm{~m}$.

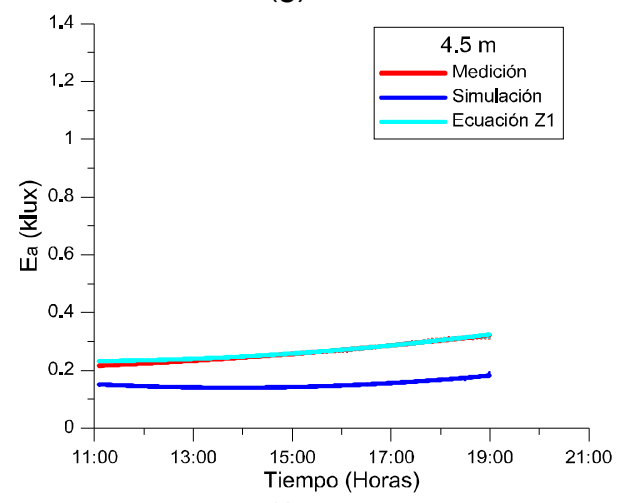

(i) $4.5 \mathrm{~m}$.

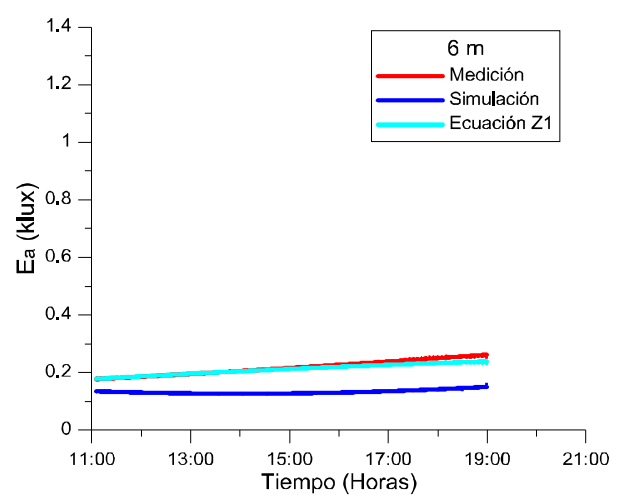

(k) $6 \mathrm{~m}$.

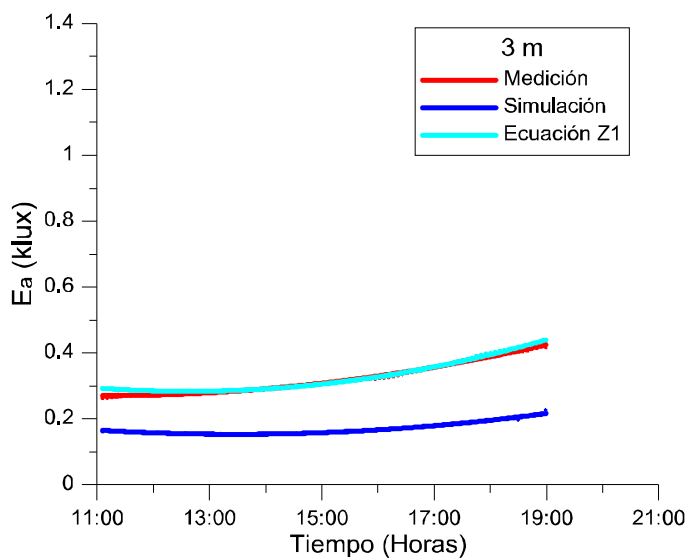

(f) $3 \mathrm{~m}$.

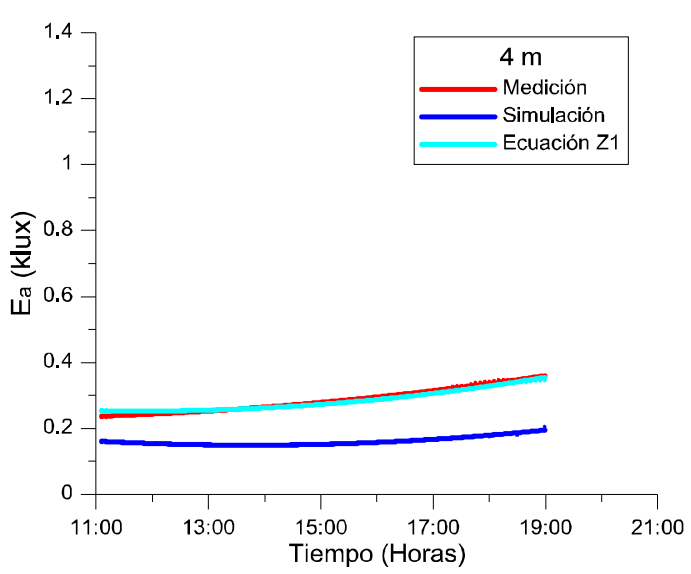

(h) $4 \mathrm{~m}$.

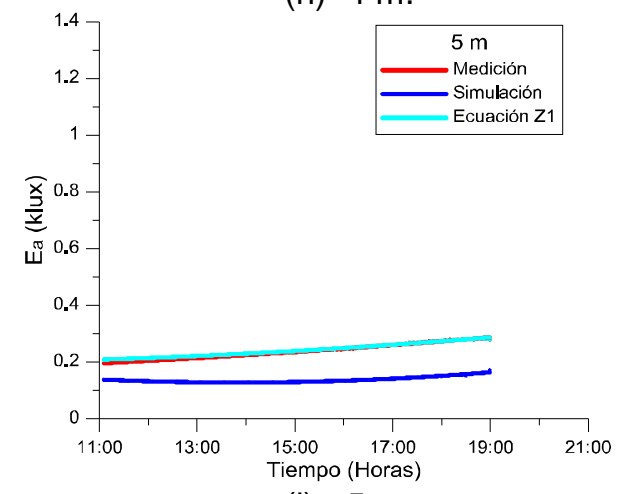

(j) $5 \mathrm{~m}$.

Figura 6.18. Iluminancias en el aula obtenidas de aplicar la ecuación Z1, por simulación y de forma experimental a las distancias de la fachada indicadas. 
En las gráficas de la figura 6.18 se puede observar cómo para todas las distancias $(0.5 \mathrm{~m}, 1 \mathrm{~m}, 1.5 \mathrm{~m}, 2 \mathrm{~m}, 2.5 \mathrm{~m}, 3 \mathrm{~m}, 3.5 \mathrm{~m}, 4 \mathrm{~m}, 4.5 \mathrm{~m}, 5 \mathrm{~m}$ y $6 \mathrm{~m})$ las iluminancias obtenidas de aplicar la ecuación Z1 y las medidas directamente en el aula son similares mientras que las obtenidas de la simulación son relativamente menores a lo largo de todo el día. Esto supone que los valores obtenidos mediante esta ecuación son ligeramente más fiables que aquellos obtenidos mediante simulación.

Continuando con la comprobación de las iluminancias se va a realizar ahora un análisis para todas las distancias a distintas horas del día (11:00, 12:00, 13:00, 14:00, 15:00, 16:00 y 17:00). Estos resultados se muestran en las gráficas de la figura 6.19. En dichas gráficas no está representada la iluminancia obtenida de aplicar la ecuación Z1 a la distancia de $5.5 \mathrm{~m}$ porque, como se había dicho anteriormente, el sensor de la maqueta a esta distancia no había medido correctamente.

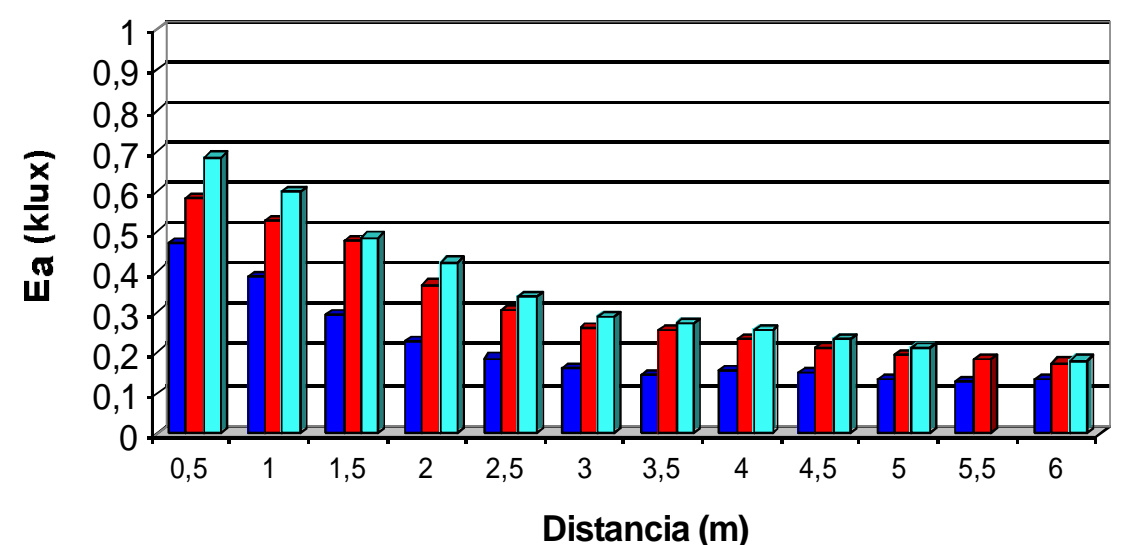

(a) 11:00.

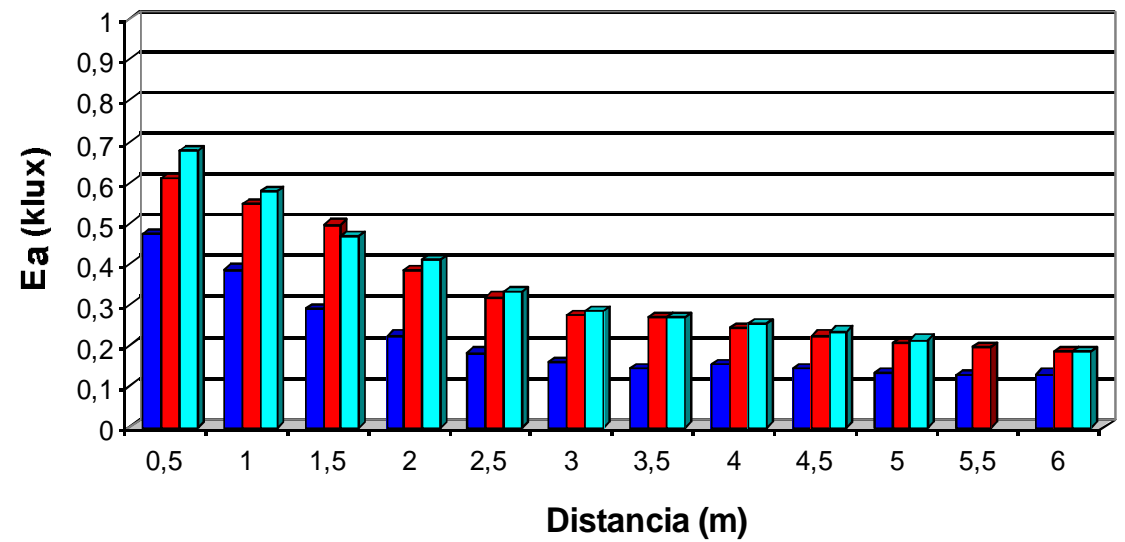

(b) $12: 00$
- SIMULACIÓN

$\square$ MEDICIÓN

$\square$ ECUACIÓN Z1

口MEDICIÓN 


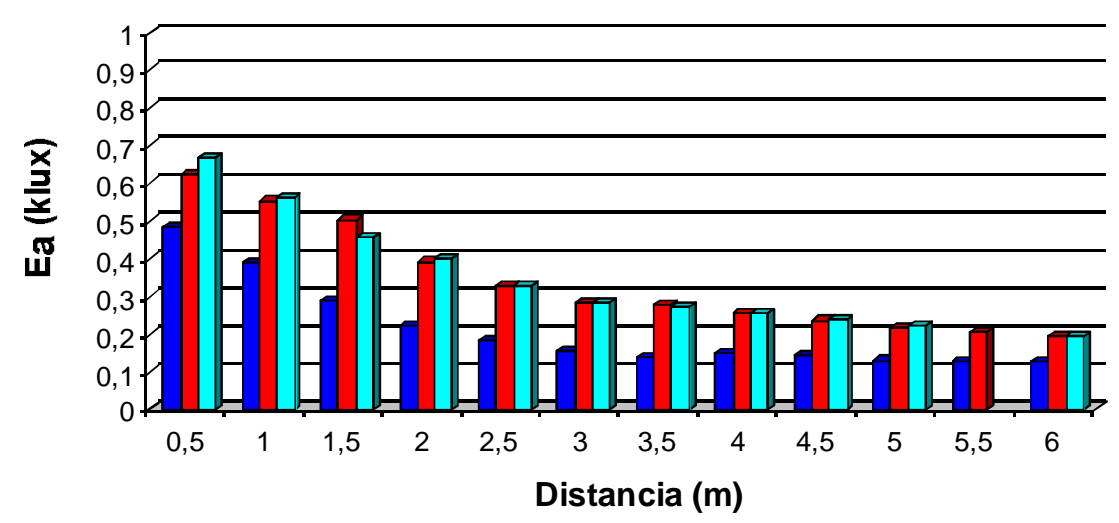

— SIMULACIÓN

口MEDICIÓN

口ECUACIÓN Z1

(c) $13: 00$.

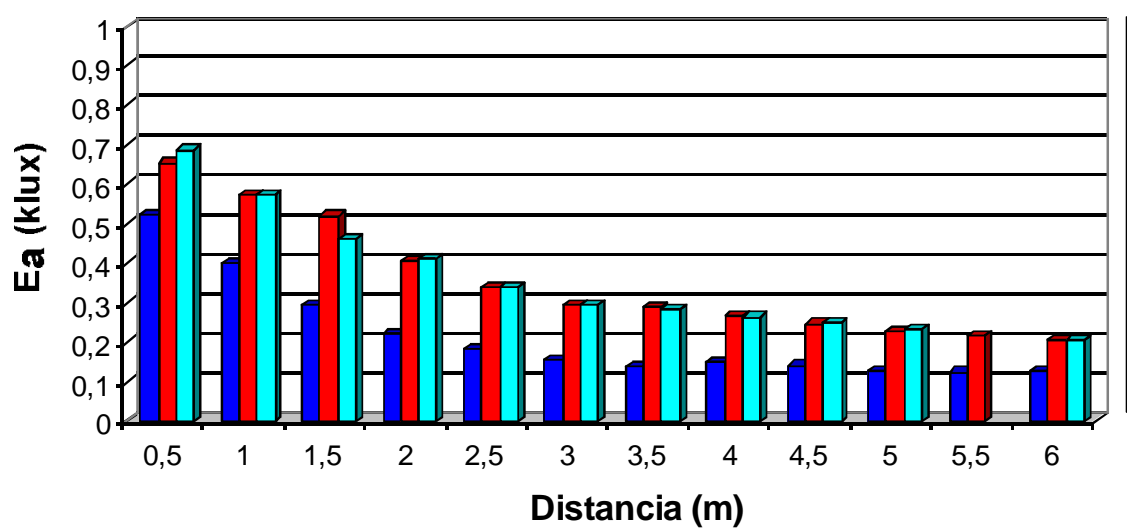

- SIMULACIÓN

口 MEDICIÓN

口 ECUACIÓN Z1

(d) $14: 00$.

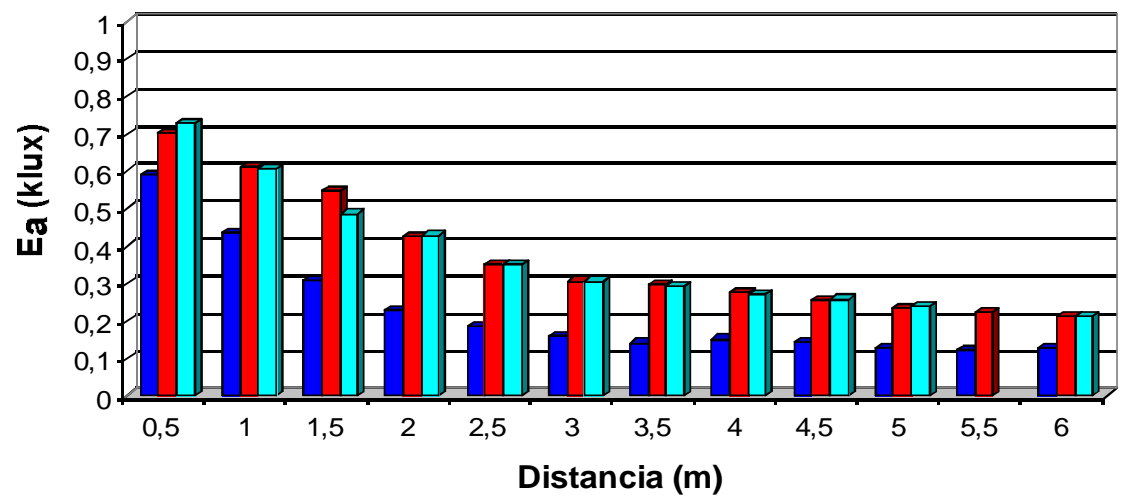

- SIMULACIÓN

口MEDICIÓN

口ECUACIÓN Z1

(e) 15:00.

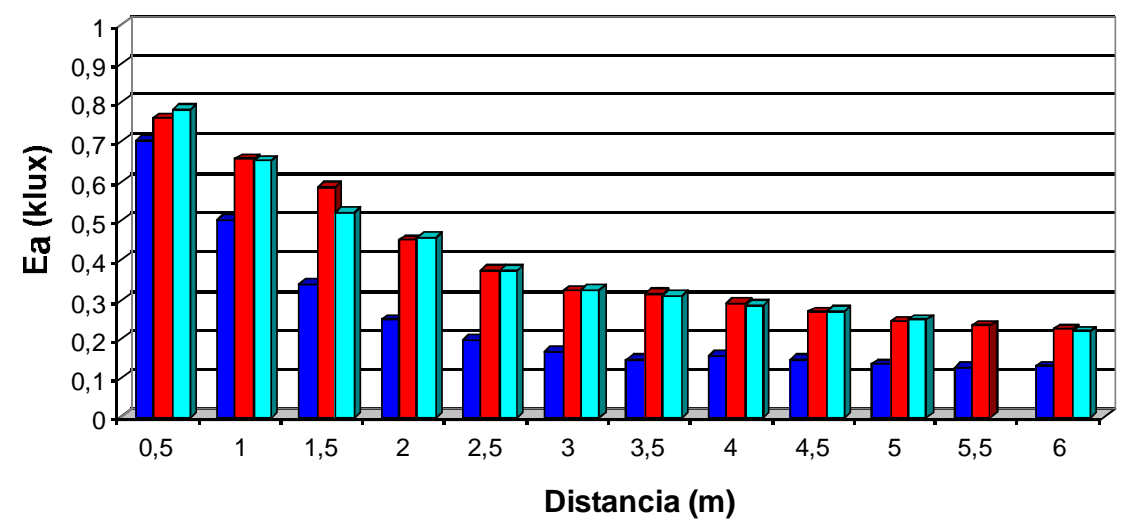

- SIMULACIÓN

๑ MEDICIÓN

口ECUACIÓN Z1

(f) $16: 00$. 


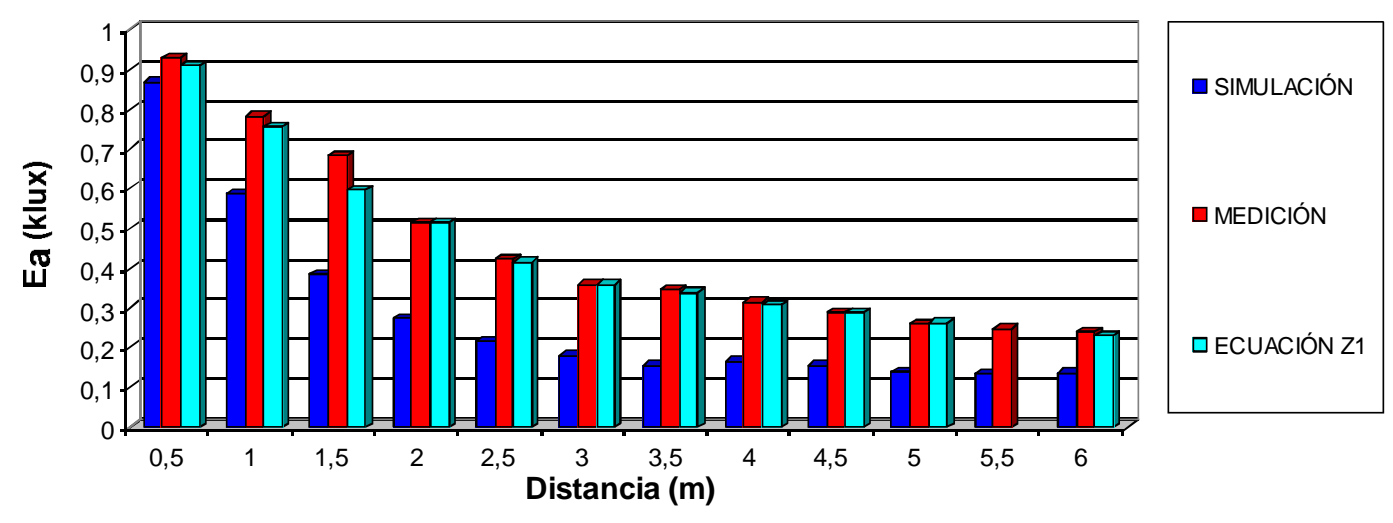

(g) 17:00.

Figura 6.19. Iluminancias del aula obtenidas midiendo en el aula, por simulación y aplicando la ecuación Z1 a distintas distancias de la fachada a las horas indicadas.

En estas gráficas se observa mucho mejor la similitud entre las iluminancias obtenidas por la ecuación Z1 y las medidas directamente, y que ambas son ligeramente superiores a las obtenidas por simulación.

Las curvas ajustadas de las iluminancias del aula obtenidas por los tres procedimientos a distintas horas del día representadas en la figura 6.19 están recogidas en el Anexo II (figura 10.2.1), así como sus correspondientes ecuaciones. Estas ecuaciones son todas de segundo grado y tienen un coeficiente de ajuste superior a 0.9 en todos los casos. 


\subsubsection{Zona 2}

A continuación se va a analizar la zona 2, que es está situada a $1.56 \mathrm{~m}$ de la pared Este, alineada a la mitad de la ventana situada más al Este del aula objeto de estudio. La distribución de los sensores en esta zona se muestra en las figuras 6.20 y 6.21 .

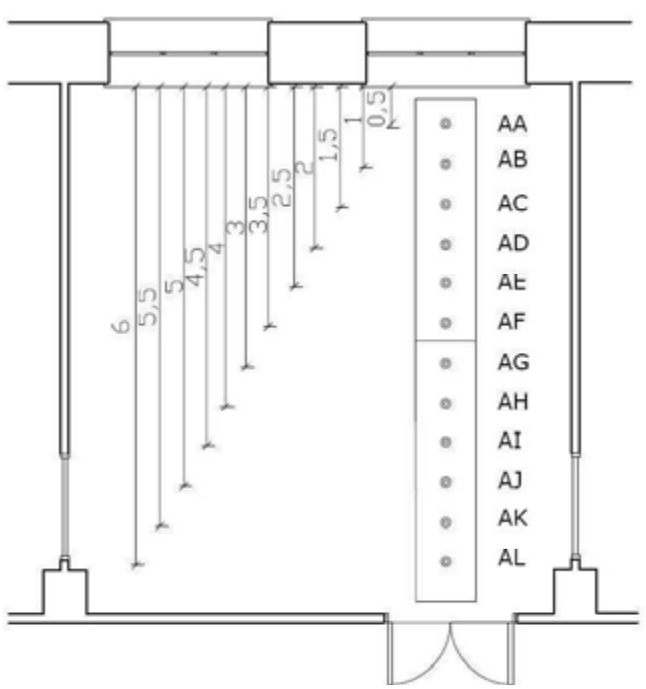

Figura 6.20. Esquema sensores zona 2.

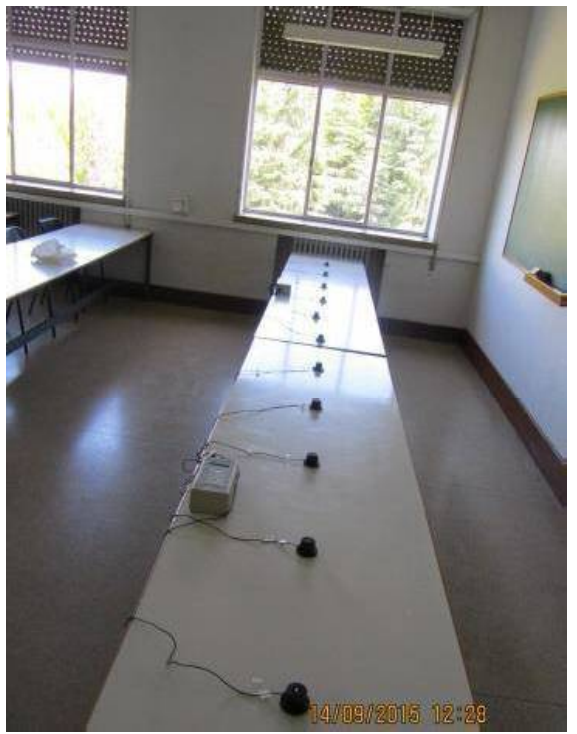

Figura 6.21. Colocación sensores zona 2.

El estudio de esta zona se ha realizado con las medidas de iluminancias realizadas los días 01/04/2015,04/04/2015 y 05/04/2015 con cielo totalmente despejado desde las 11:00 de la mañana hasta las 19:00 de la tarde (hora local).

Se han registrado datos en el exterior y a todas las distancias consideradas $(0.5 \mathrm{~m}, 1 \mathrm{~m}, 1.5 \mathrm{~m}, 2 \mathrm{~m}, 2.5 \mathrm{~m}, 3 \mathrm{~m}, 3.5 \mathrm{~m}, 4 \mathrm{~m}, 4.5 \mathrm{~m}, 5 \mathrm{~m}, 5.5 \mathrm{~m}, 6 \mathrm{~m})$ en el aula y en el modelo a escala. Sin embargo se han suprimido los datos correspondientes a las distancias de $3.5 \mathrm{~m}$ y $5.5 \mathrm{~m}$, porque los sensores del modelo a estas distancias proporcionales no funcionaron de forma correcta.

\subsubsection{Evolución diaria de las iluminancias}

La evolución diaria de la iluminancia exterior $\left(\mathrm{E}_{\text {ext }}\right)$ de los días considerados en esta zona se muestra en la figura 6.22. 


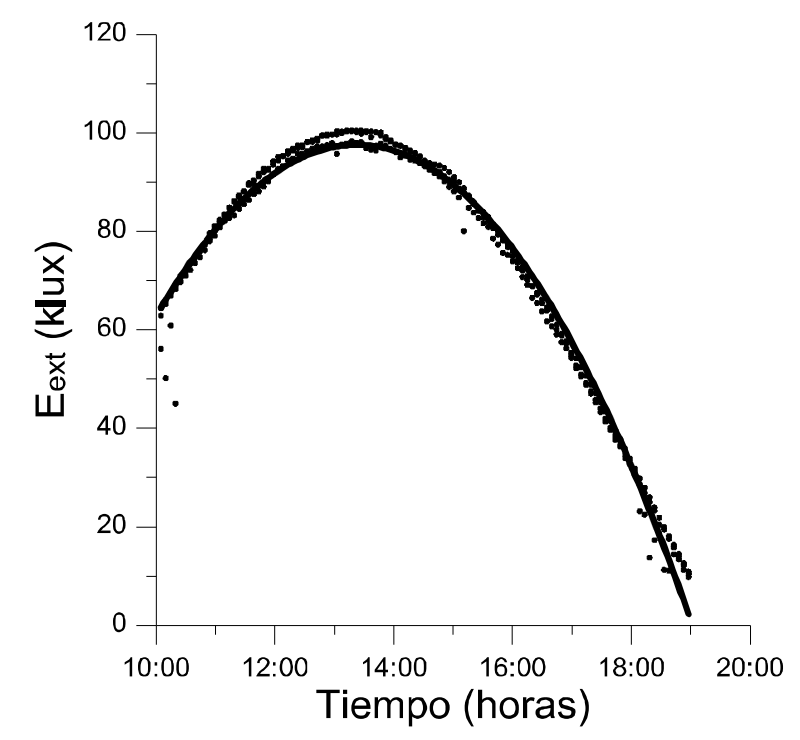

Figura 6.22. Evolución diaria de la iluminancia exterior (Abril 2015).

A continuación, en las figuras 6.23 y 6.24 , se muestran las gráficas que representan la evolución de las iluminancias horizontales del modelo $\left(E_{m}\right)$ y del aula $\left(E_{a}\right)$ a las distintas distancias de la ventana durante los tres días considerados en esta zona.

En el eje vertical aparecen representadas las iluminancias en klux y en el eje horizontal el tiempo expresado en horas. También se muestra la ecuación de segundo grado a la que se ajusta la nube de puntos, así como su coeficiente de ajuste $\left(r^{2}\right)$. En la columna de la izquierda, en color verde, están representados los datos correspondientes al modelo y a la derecha en color rojo los del aula.

\section{ZONA 2}

MODELO

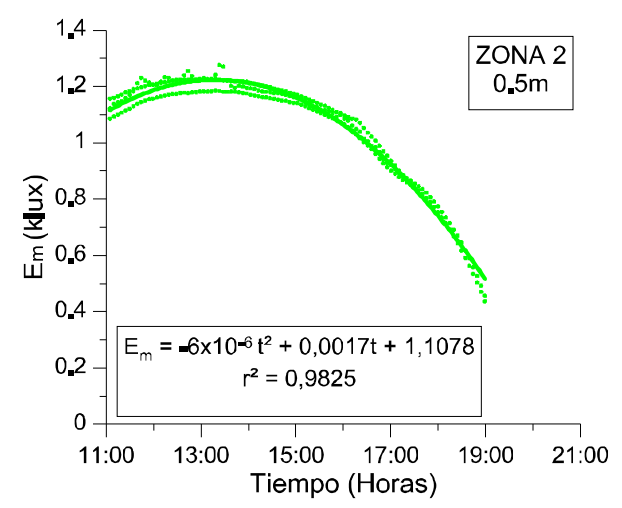

(a) $0.5 \mathrm{~m}$.
AULA

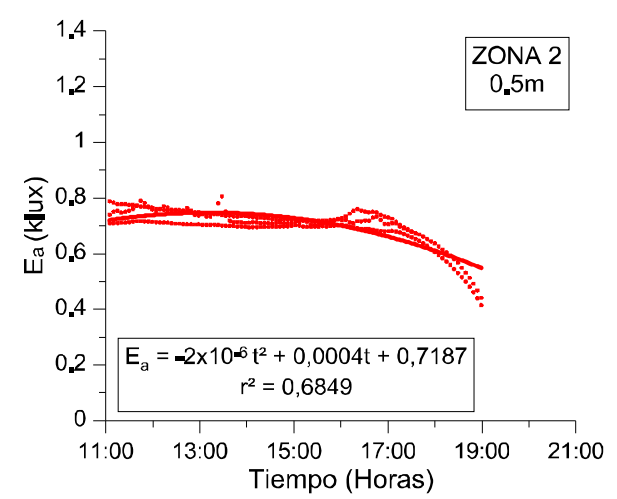

(b) $0.5 \mathrm{~m}$. 


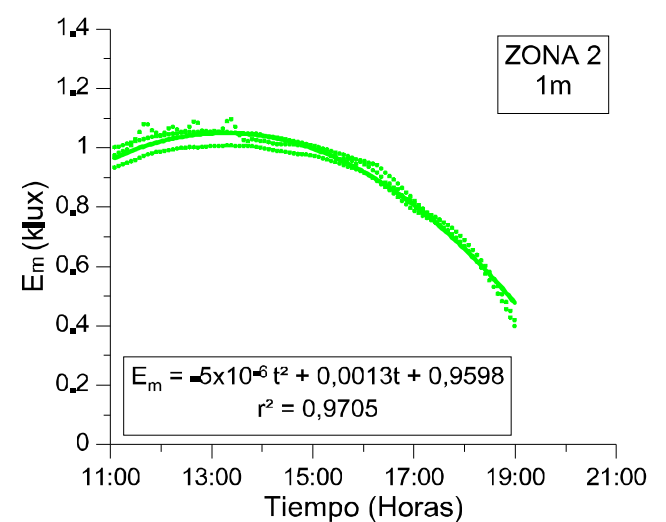

(b) $1 \mathrm{~m}$.

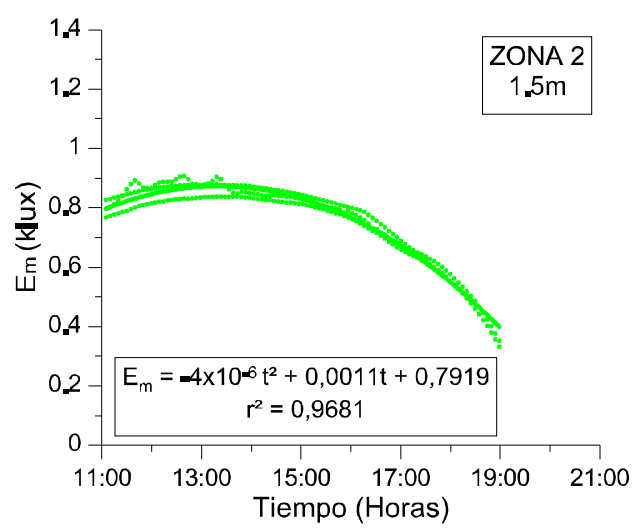

(c) $1.5 \mathrm{~m}$.

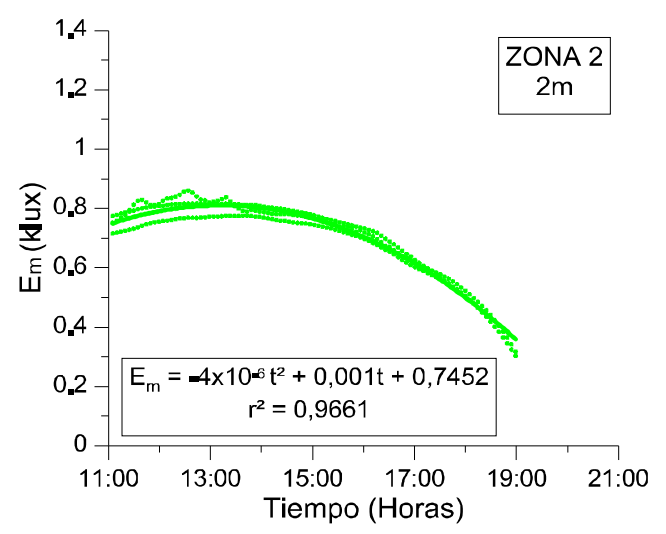

(d) $2 \mathrm{~m}$.

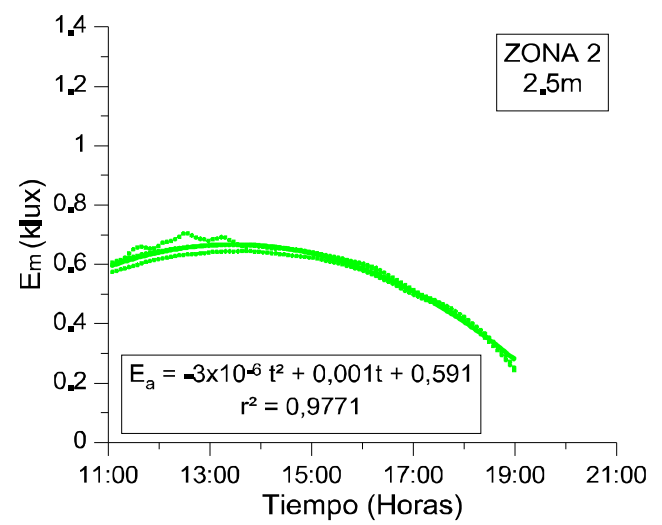

(e) $2.5 \mathrm{~m}$.

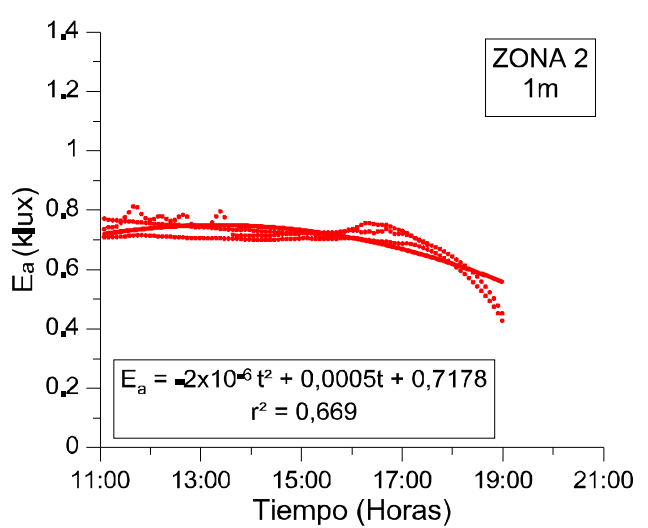

(b) $1 \mathrm{~m}$.

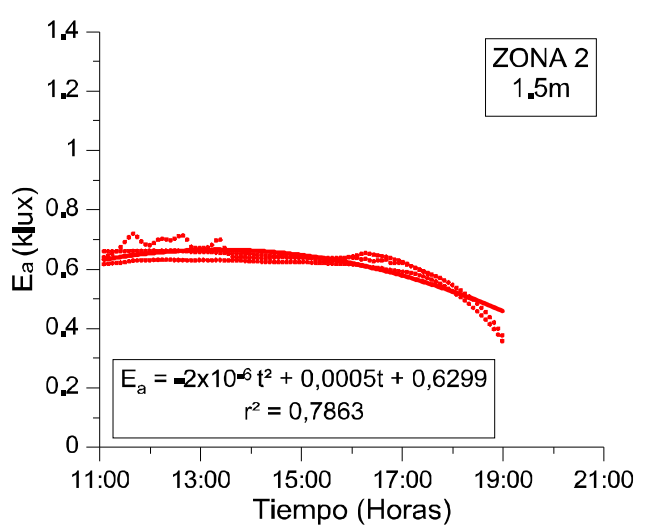

(c) $1.5 \mathrm{~m}$.

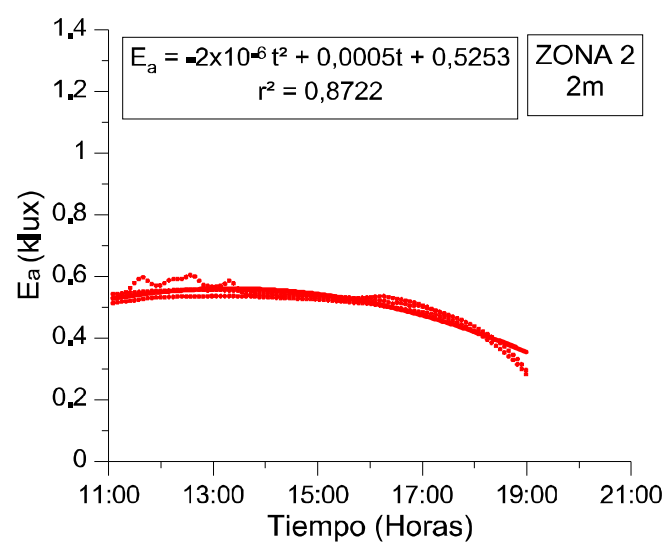

(d) $2 \mathrm{~m}$.

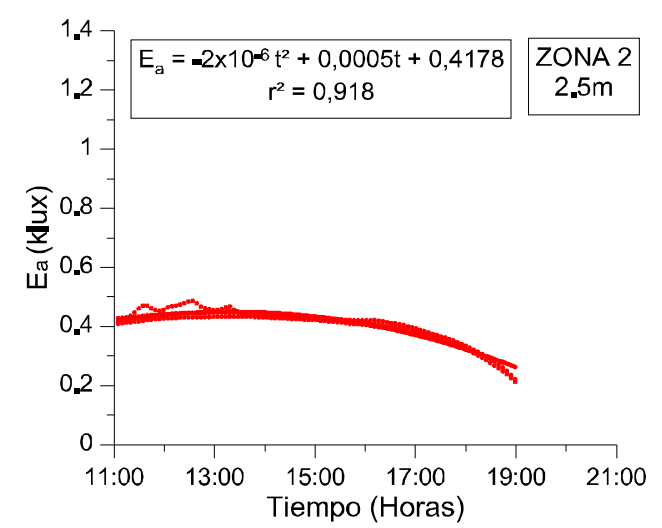

(e) $2.5 \mathrm{~m}$. 
Estudio experimental y modelización de la iluminación natural en la edificación mediante modelos a escala

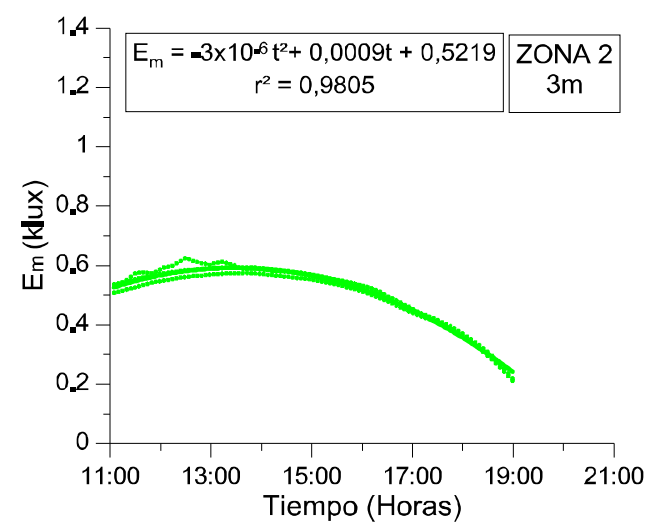

(f) $3 \mathrm{~m}$.

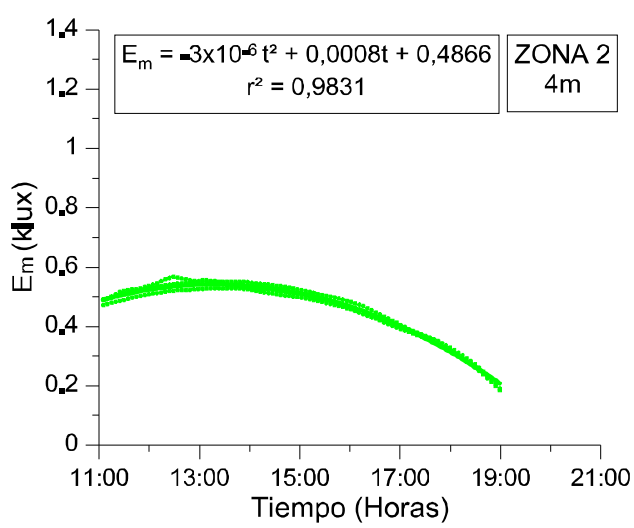

(g) $4 \mathrm{~m}$.

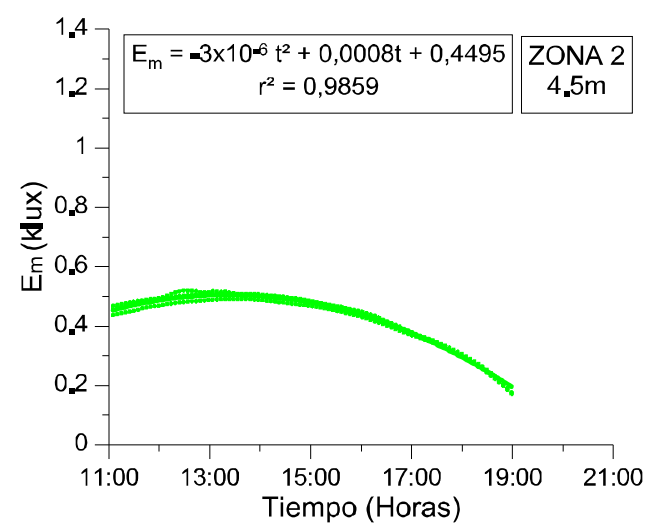

(h) $4.5 \mathrm{~m}$

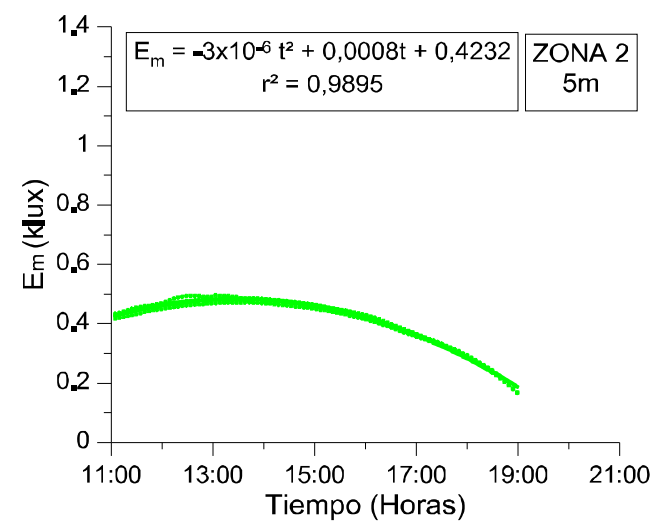

(i) $5 \mathrm{~m}$.

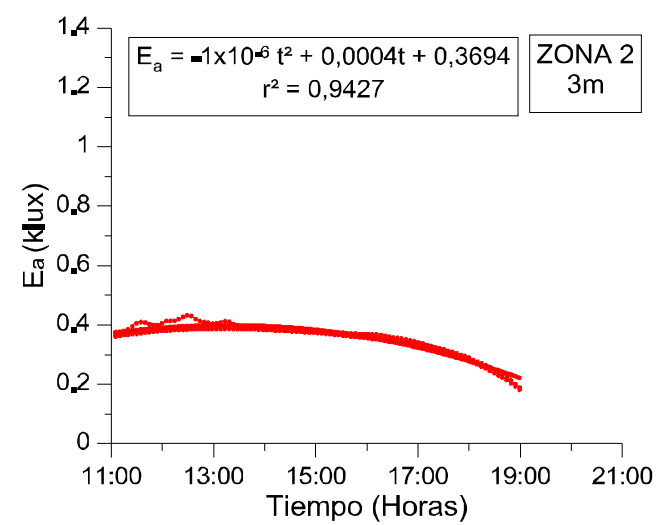

(f) $3 \mathrm{~m}$.

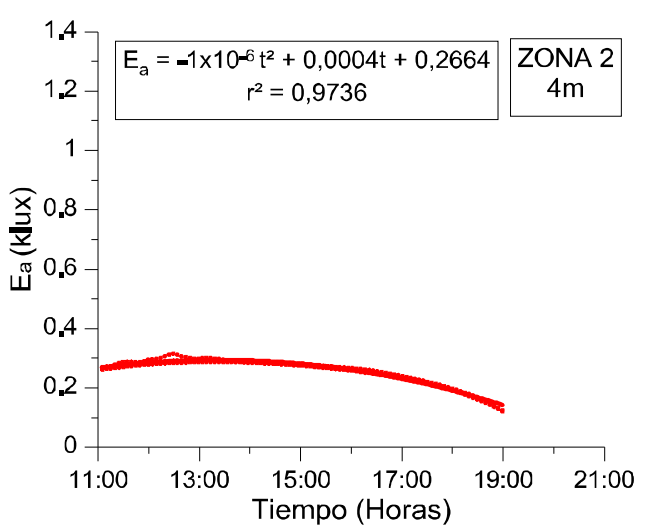

(g) $4 \mathrm{~m}$.

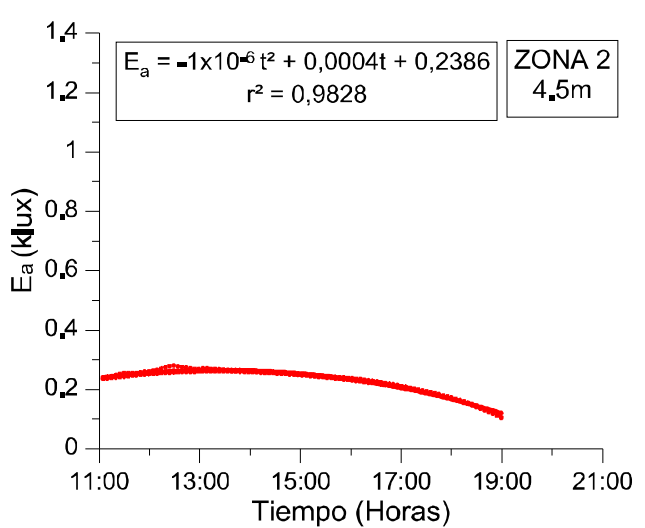

(h) $4.5 \mathrm{~m}$.

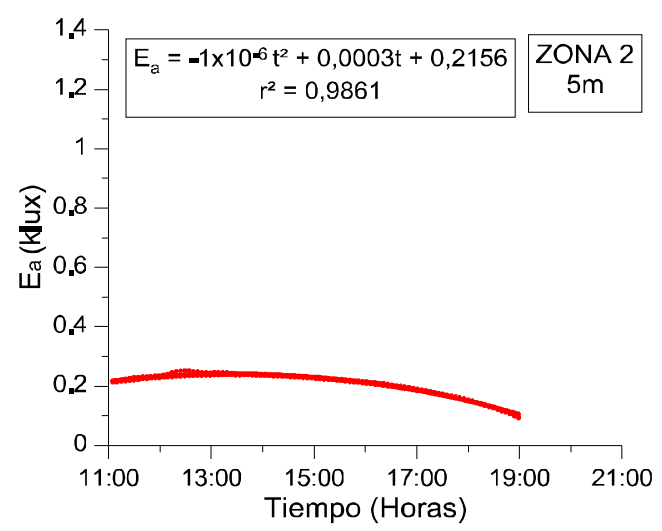

(i) $5 \mathrm{~m}$. 


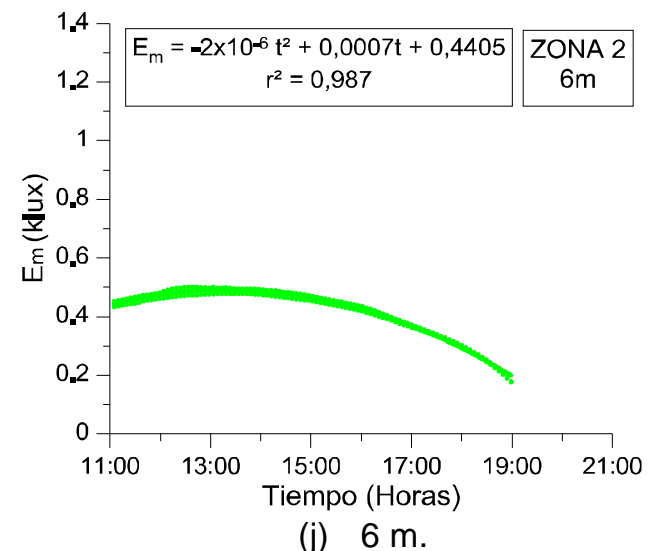

Figura 6.23. Evolución diaria de la iluminancia en el modelo a las distancias indicadas.

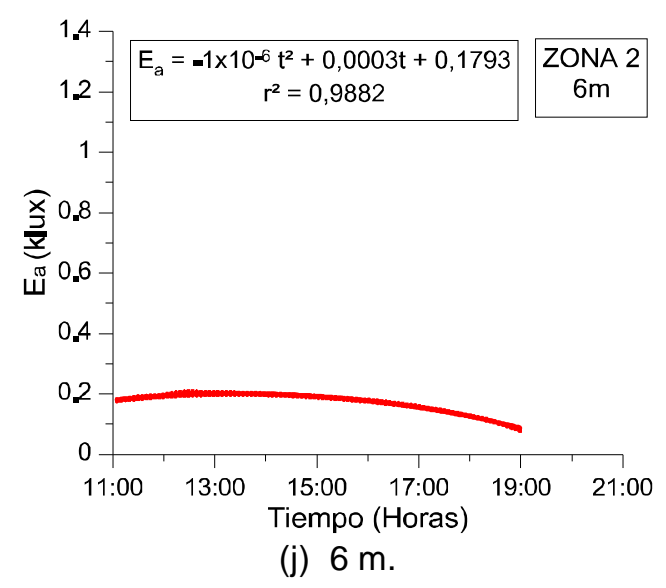

Figura 6.24. Evolución diaria de la iluminancia en el aula a las distancias indicadas.

A continuación, en las figuras 6.25 y 6.26 , se muestran de forma conjunta todas las curvas ajustadas de las figuras 6.23 y 6.24. En ellas se observa, cómo a medida que aumenta la distancia a la ventana disminuye el valor de las iluminancias. También se puede observar que las curvas siguen un comportamiento lógico pues los valores crecen con el amanecer y decrecen con el atardecer.

MODELO

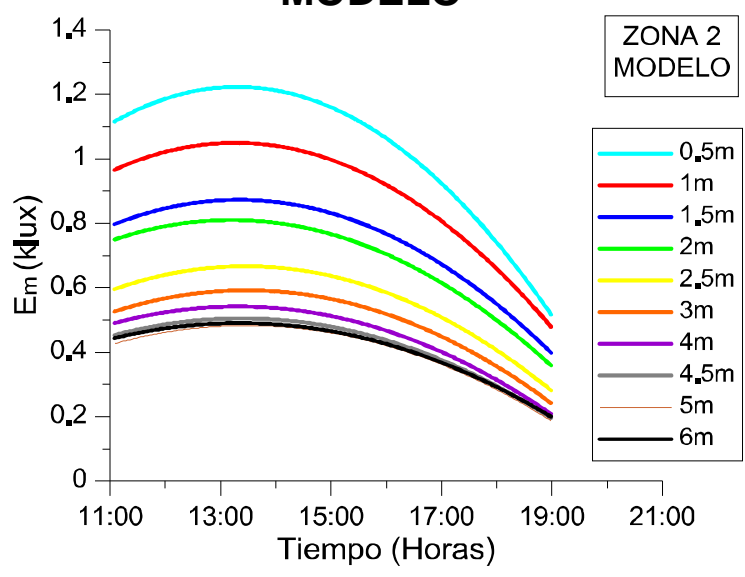

Figura 6.25. Curvas de ajuste de las Figura 6.26. Curvas de ajuste de las iluminancias en el modelo a distintas distancias. iluminancias en el aula a distintas distancias.

Las ecuaciones correspondientes a las curvas de las figuras 6.25 y 6.26 y sus correspondientes coeficientes de ajuste $\left(r^{2}\right)$ se recogen en la tabla 6.6 . 


\begin{tabular}{|c|c|c|c|c|}
\hline \begin{tabular}{c} 
ZONA 2 \\
\hline $\begin{array}{c}\text { DISTANCIA } \\
(\mathbf{m})\end{array}$
\end{tabular} & ECUACIÓN & $r^{2}$ & ECUACIÓN & $\mathbf{r}^{2}$ \\
\hline $\mathbf{0 . 5}$ & $\mathrm{E}_{\mathrm{m}}=-6 \times 10^{-6} \mathrm{t}^{2}+0.0017 \mathrm{t}+1.1078$ & 0.9825 & $\mathrm{E}_{\mathrm{a}}=-2 \times 10^{-6} \mathrm{t}^{2}+0.0004 \mathrm{t}+0.7187$ & 0.6849 \\
\hline $\mathbf{1}$ & $\mathrm{E}_{\mathrm{m}}=5 \times 10^{-6} \mathrm{t}^{2}+0.0013 \mathrm{t}+0.9598$ & 0.9705 & $\mathrm{E}_{\mathrm{a}}=-2 \times 10^{-6} \mathrm{t}^{2}+0.0005 \mathrm{t}+0.7178$ & 0.669 \\
\hline $\mathbf{1 . 5}$ & $\mathrm{E}_{\mathrm{m}}=-4 \times 10^{-6} \mathrm{t}^{2}+0.0011 \mathrm{t}+0.7919$ & 0.9681 & $\mathrm{E}_{\mathrm{a}}=-2 \times 10^{-6} \mathrm{t}^{2}-0.0005 \mathrm{t}+0.6299$ & 0.7863 \\
\hline $\mathbf{2}$ & $\mathrm{E}_{\mathrm{m}}=-4 \times 10^{-6} \mathrm{t}^{2}+0.001 \mathrm{t}+0.7452$ & 0.9661 & $\mathrm{E}_{\mathrm{a}}=-2 \times 10^{-6} \mathrm{t}^{2}+0.0005 \mathrm{t}+0.5253$ & 0.8722 \\
\hline $\mathbf{2 . 5}$ & $\mathrm{E}_{\mathrm{m}}=-3 \times 10^{-6} \mathrm{t}^{2}+0.001 \mathrm{t}+0.5910$ & 0.9771 & $\mathrm{E}_{\mathrm{a}}=-2 \times 10^{-6} \mathrm{x}^{2}+0.0005 \mathrm{t}+0.4178$ & 0.918 \\
\hline $\mathbf{3}$ & $\mathrm{E}_{\mathrm{m}}=-3 \times 10^{-6} \mathrm{t}^{2}+0.0009 \mathrm{t}+0.5219$ & 0.9805 & $\mathrm{E}_{\mathrm{a}}=-1 \times 10^{-6} \mathrm{t}^{2}+0.0004 \mathrm{t}+0.3694$ & 0.9427 \\
\hline $\mathbf{4}$ & $\mathrm{E}_{\mathrm{m}}=-3 \times 10^{-6} \mathrm{t}^{2}+0.0008 \mathrm{t}+0.4866$ & 0.9831 & $\mathrm{E}_{\mathrm{a}}=-1 \times 10^{-6} \mathrm{t}^{2}+0.0004 \mathrm{t}+0.26664$ & 0.9736 \\
\hline $\mathbf{4 . 5}$ & $\mathrm{E}_{\mathrm{m}}=-3 \times 10^{-6} \mathrm{t}^{2}+0.0008 \mathrm{t}+0.4495$ & 0.9859 & $\mathrm{E}_{\mathrm{a}}=-1 \times 10^{-6} \mathrm{t}^{2}+0.0004 \mathrm{t}+0.2386$ & 0.9828 \\
\hline $\mathbf{5}$ & $\mathrm{E}_{\mathrm{m}}=-3 \times 10^{-6} \mathrm{t}^{2}+0.0008 \mathrm{t}+0.4232$ & 0.9895 & $\mathrm{E}_{\mathrm{a}}=-1 \times 10^{-6} \mathrm{t}^{2}+0.0003 t+0.2156$ & 0.9861 \\
\hline $\mathbf{6}$ & $\mathrm{E}_{\mathrm{m}}=-2 \times 10^{-6} \mathrm{t}^{2}+0.0007 \mathrm{t}+0.4405$ & 0.987 & $\mathrm{E}_{\mathrm{a}}=-1 \times 10^{-6} \mathrm{t}^{2}+0.0003 t+0.1793$ & 0.9882 \\
\hline
\end{tabular}

Tabla 6.6. Ecuaciones de las iluminancias diarias a distintas distancias tanto del modelo como del aula.

En la tabla se puede observar cómo todos los coeficientes de ajuste del modelo y la mayoría de los del aula, son superiores a 0,9 lo que indica un alto grado de fiabilidad.

\subsubsection{Relación entre las iluminancias del aula y del modelo a escala en función de las horas del día}

Siguiendo el mismo desarrollo realizado en la zona 1, a continuación se han representado, en la figura 6.27, las gráficas del cociente de las iluminancias del aula y del modelo frente al tiempo (expresado en horas), a las distintas distancias consideradas. También se muestra la ecuación de la curva de ajuste para cada distancia y su coeficiente de ajuste. En el eje vertical se muestra el cociente de las iluminancias aula/ modelo y en el eje horizontal el tiempo.

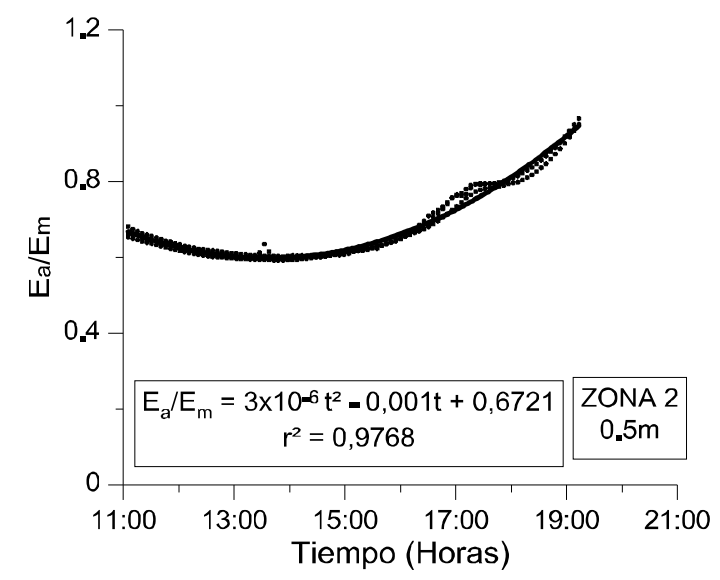

(a) $0.5 \mathrm{~m}$.

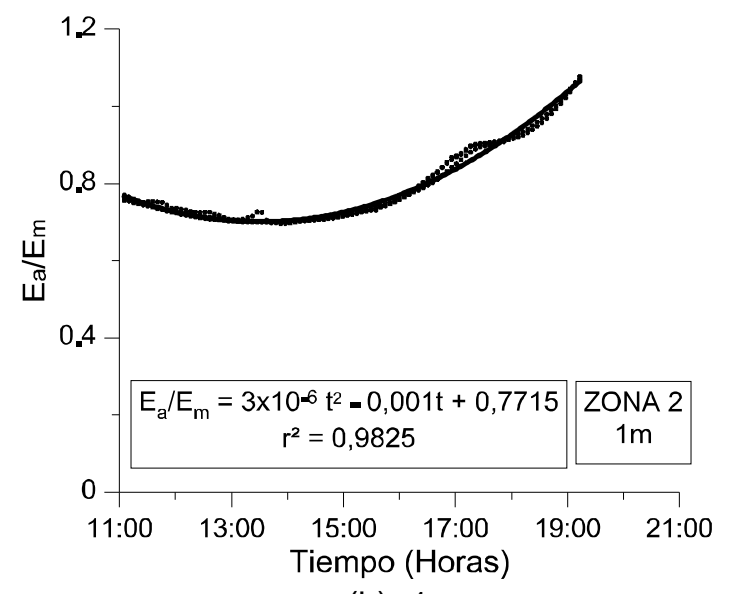

(b) $1 \mathrm{~m}$. 
Estudio experimental y modelización de la iluminación natural en la edificación mediante modelos a escala

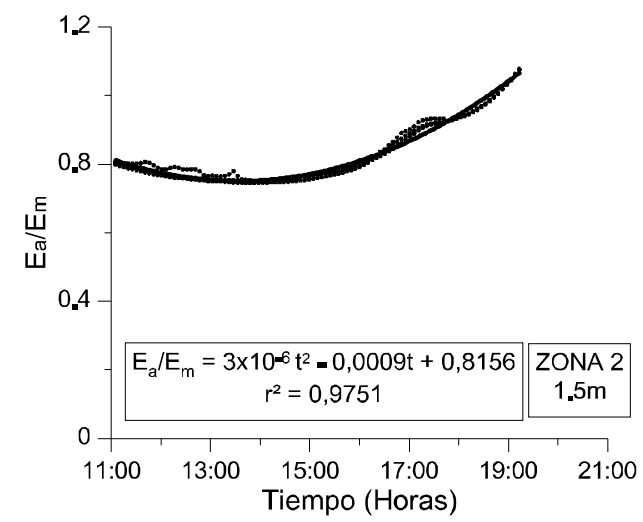

(c) $1.5 \mathrm{~m}$.

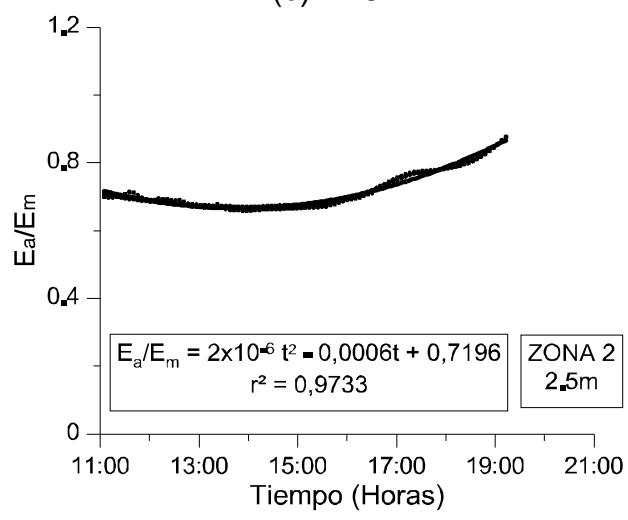

(e) $2.5 \mathrm{~m}$.

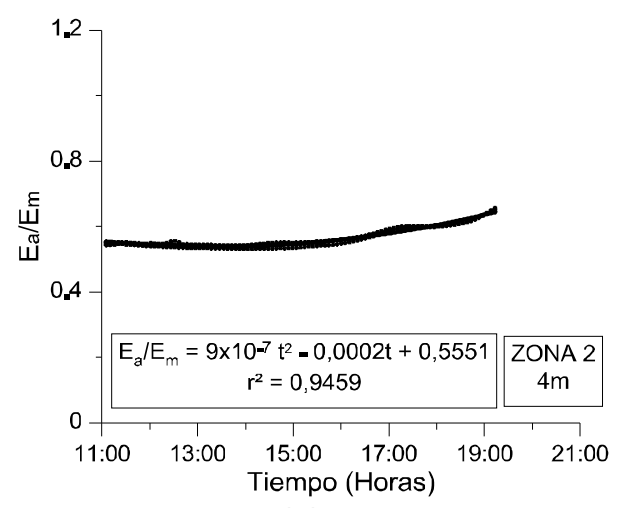

(g) $4 \mathrm{~m}$.

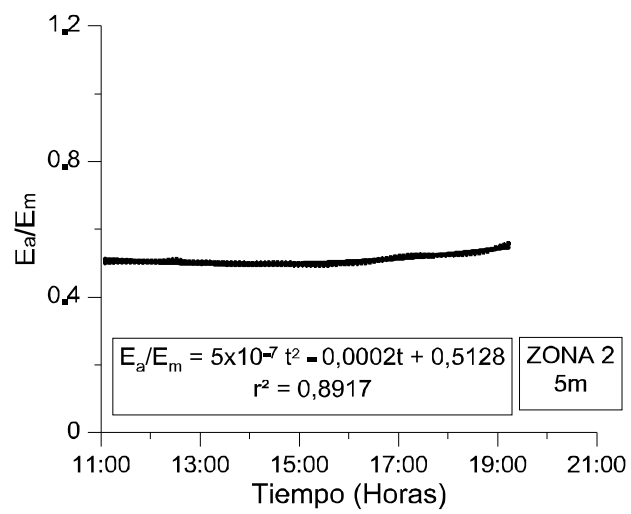

(i) $5 \mathrm{~m}$.

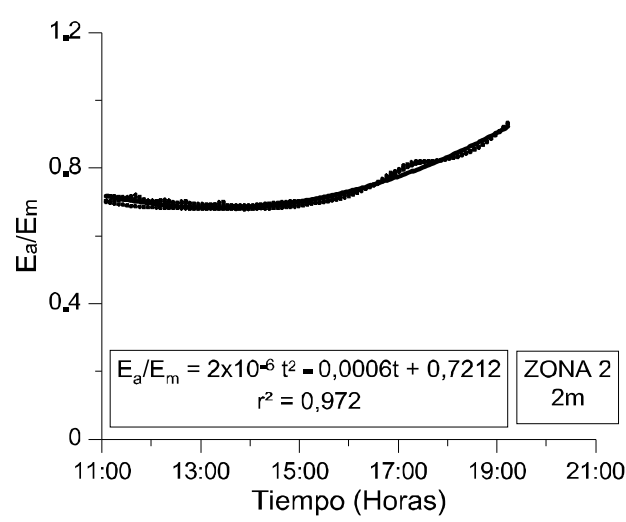

(d) $2 \mathrm{~m}$.

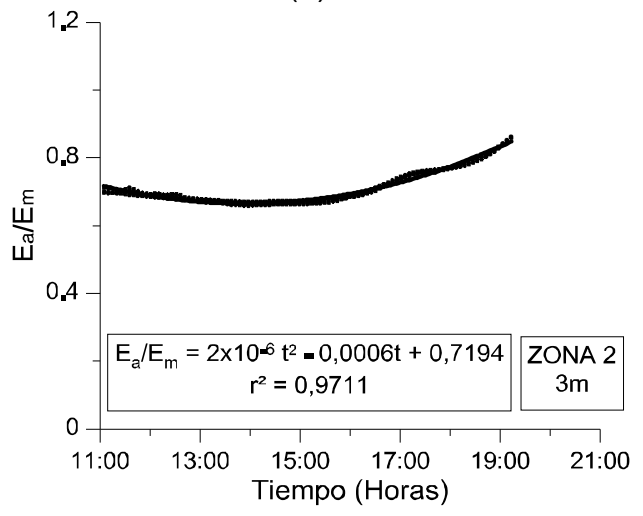

(f) $3 \mathrm{~m}$.

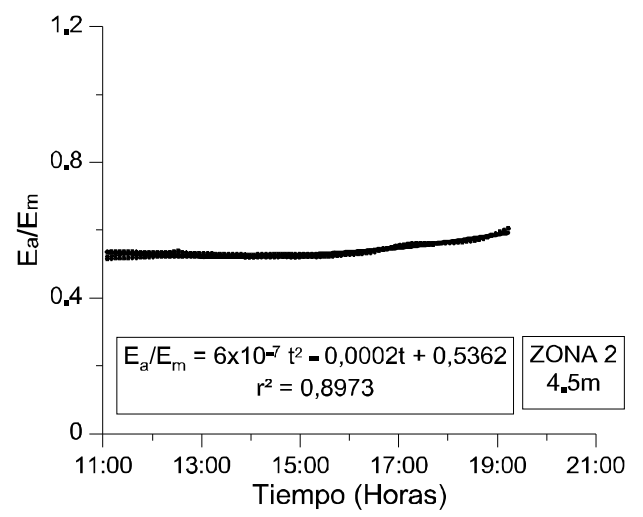

(h) $4.5 \mathrm{~m}$.

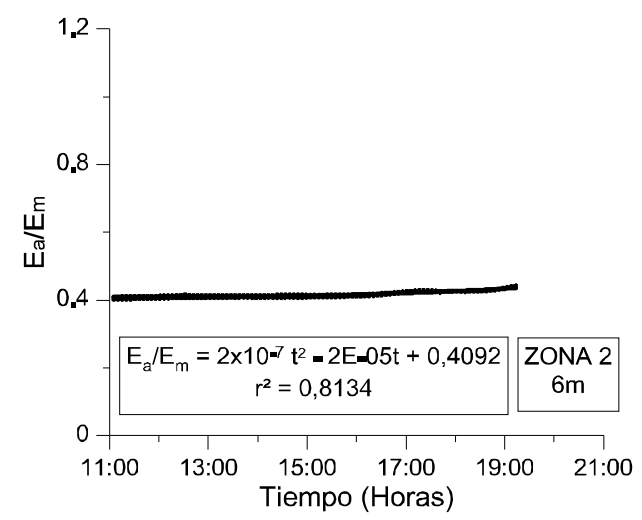

(j) $6 \mathrm{~m}$.

Figura 6.27. Cociente entre las iluminancias del aula y del modelo a escala frente al tiempo a las distancias indicadas. 
Se comprueba que tampoco en esta zona hay una relación lineal entre las iluminancias del aula y del modelo.

A continuación se muestran de forma conjunta las curvas de los cocientes de las iluminancias aula/modelo frente al tiempo para las distintas distancias (figura 6.28). Se puede apreciar cómo según aumenta la distancia a la ventana la curvatura de estas ecuaciones va disminuyendo.

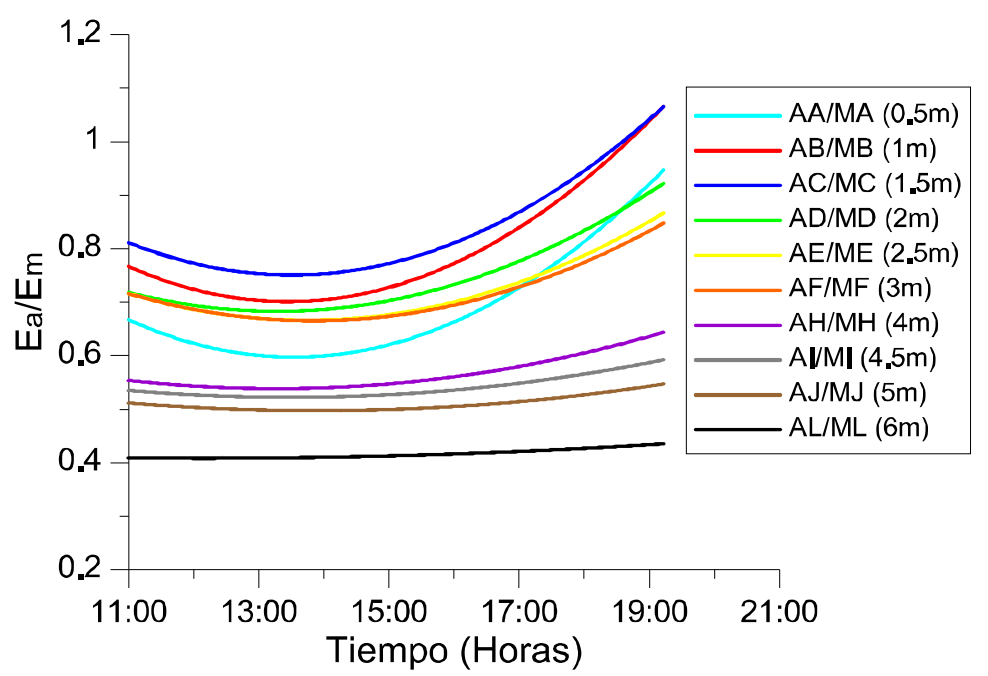

Figura 6.28. Curvas de ajuste a las distintas distancias de la relaciones entre el cociente de las iluminancias aula/ modelo frente al tiempo.

En la tabla 6.7, se muestran las ecuaciones de ajuste obtenidas de la relación entre las iluminancias aula/modelo y el tiempo, así como su correspondiente coeficiente de ajuste, para las distancias consideradas.

\begin{tabular}{|c|l|c|}
\hline DISTANCIA $(\mathrm{m})$ & ECUACIÓN & $\mathbf{r}^{2}$ \\
\hline $\mathbf{0 . 5}$ & $\mathrm{E}_{\mathrm{a}} / \mathrm{E}_{\mathrm{m}}=3 \times 10^{-6} \mathrm{t}^{2}-0.001 \mathrm{t}+0.6721$ & 0.9768 \\
\hline $\mathbf{1}$ & $\mathrm{E}_{\mathrm{a}} / \mathrm{E}_{\mathrm{m}}=3 \times 10^{-6} \mathrm{t}^{2}-0.001 \mathrm{t}+0.7715$ & 0.9825 \\
\hline $\mathbf{1 . 5}$ & $\mathrm{E}_{\mathrm{a}} / \mathrm{E}_{\mathrm{m}}=3 \times 10^{-6} \mathrm{t}^{2}-0.0009 \mathrm{t}+0.8156$ & 0.9751 \\
\hline $\mathbf{2}$ & $\mathrm{E}_{\mathrm{a}} / \mathrm{E}_{\mathrm{m}}=2 \times 10^{-6} \mathrm{t}^{2}-0.0006 \mathrm{t}+0.7212$ & 0.972 \\
\hline $\mathbf{2 . 5}$ & $\mathrm{E}_{\mathrm{a}} / \mathrm{E}_{\mathrm{m}}=2 \times 10^{-6} \mathrm{t}^{2}-0.0006 \mathrm{t}+0.7196$ & 0.9733 \\
\hline $\mathbf{3}$ & $\mathrm{E}_{\mathrm{a}} / \mathrm{E}_{\mathrm{m}}=2 \times 10^{-6} \mathrm{t}^{2}-0.0006 \mathrm{t}+0.7194$ & 0.9711 \\
\hline $\mathbf{4}$ & $\mathrm{E}_{\mathrm{a}} / \mathrm{E}_{\mathrm{m}}=9 \times 10^{-7} \mathrm{t}^{2}-0.0002 \mathrm{t}+0.5551$ & 0.9459 \\
\hline $\mathbf{4 . 5}$ & $\mathrm{E}_{\mathrm{a}} / \mathrm{E}_{\mathrm{m}}=6 \times 10^{-7} \mathrm{t}^{2}-0.0002 \mathrm{t}+0.5362$ & 0.8973 \\
\hline $\mathbf{5}$ & $\mathrm{E}_{\mathrm{a}} / \mathrm{E}_{\mathrm{m}}=5 \times 10^{-7} \mathrm{t}^{2}-0.0002 \mathrm{t}+0.5128$ & 0.8917 \\
\hline $\mathbf{6}$ & $\mathrm{E}_{\mathrm{a}} / \mathrm{E}_{\mathrm{m}}=2 \times 10^{-7} \mathrm{t} 2-2 \times 10^{-5} \mathrm{t}+0.4092$ & 0.8134 \\
\hline
\end{tabular}

Tabla 6.7. Ecuaciones, a las distintas distancias, de los cocientes de las iluminancias aula/ modelo frente al tiempo. 
En la tabla se puede apreciar cómo según aumenta la distancia a la ventana el coeficiente de ajuste va disminuyendo. Todos los coeficientes de ajuste son superiores a 0.8 .

\subsubsection{Relación entre iluminancias del aula y del modelo a escala en función de la iluminancia exterior}

En este apartado se va a analizar la relación entre las iluminancias del aula $\left(E_{a}\right)$ y del modelo $\left(E_{m}\right)$ a escala en función de la iluminancia exterior $\left(E_{\text {ext }}\right)$ expresada en klux, teniendo en cuenta los datos empleados anteriormente, para las distintas distancias a la ventana.

En las gráficas de la figura 6.29 se ha representado en el eje vertical el cociente entre las iluminancias del aula y el modelo, y en el eje horizontal las iluminancias obtenidas con el sensor exterior.

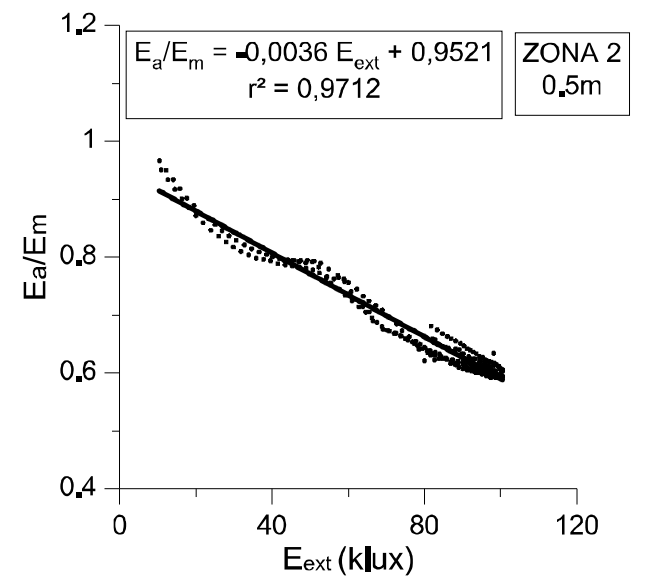

(a) $0.5 \mathrm{~m}$.

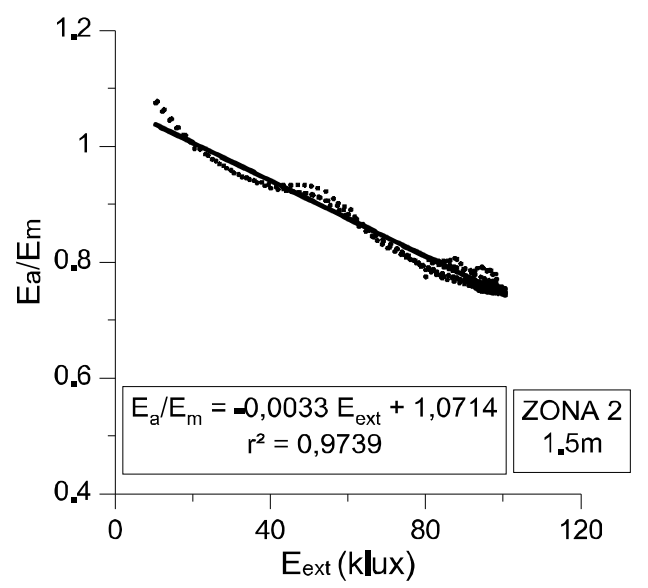

(c) $1.5 \mathrm{~m}$.

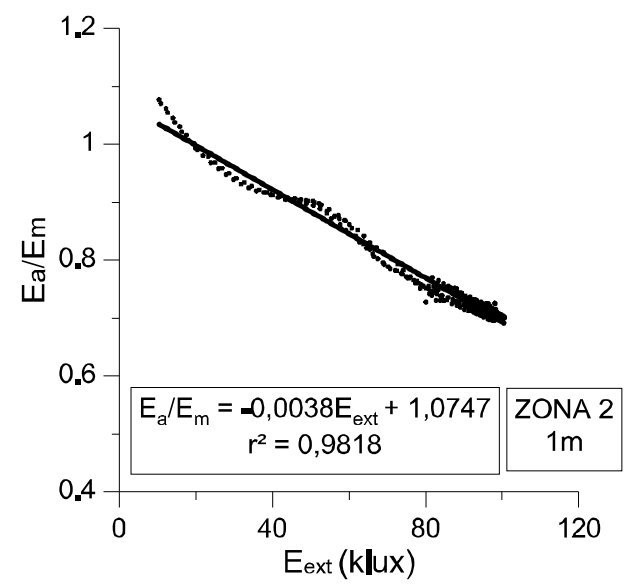

(b) $1 \mathrm{~m}$.

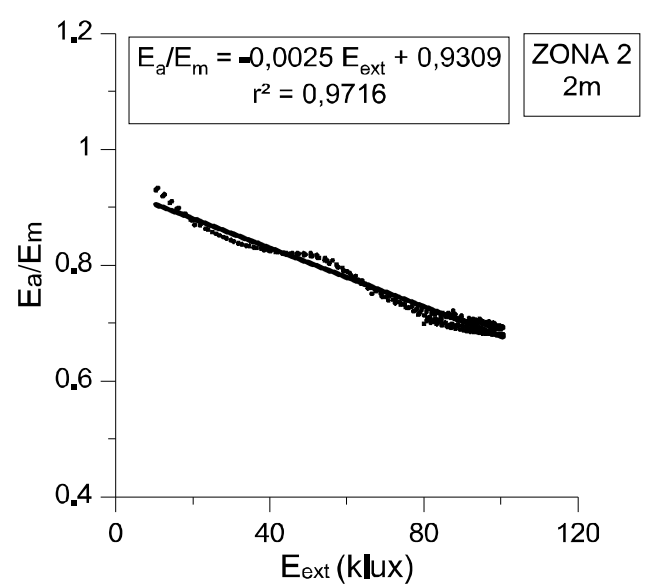

(d) $2 \mathrm{~m}$. 


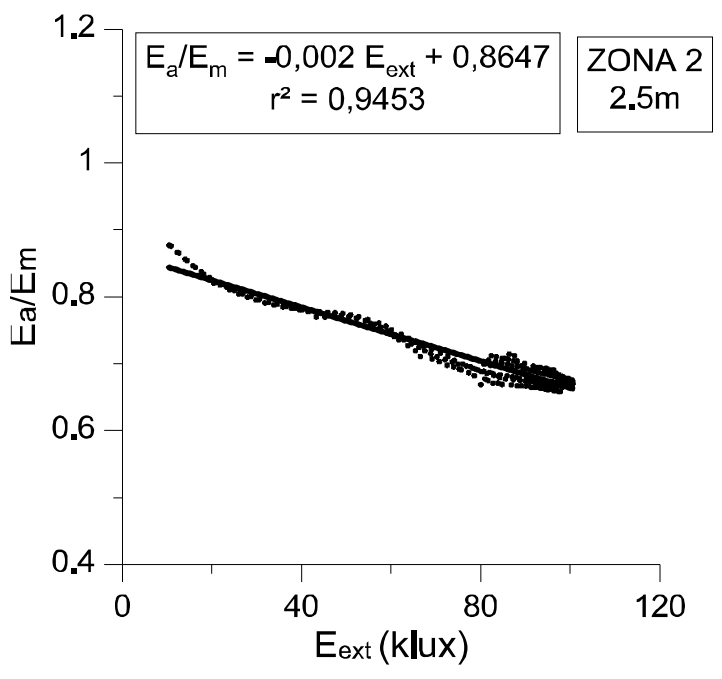

(e) $2.5 \mathrm{~m}$.

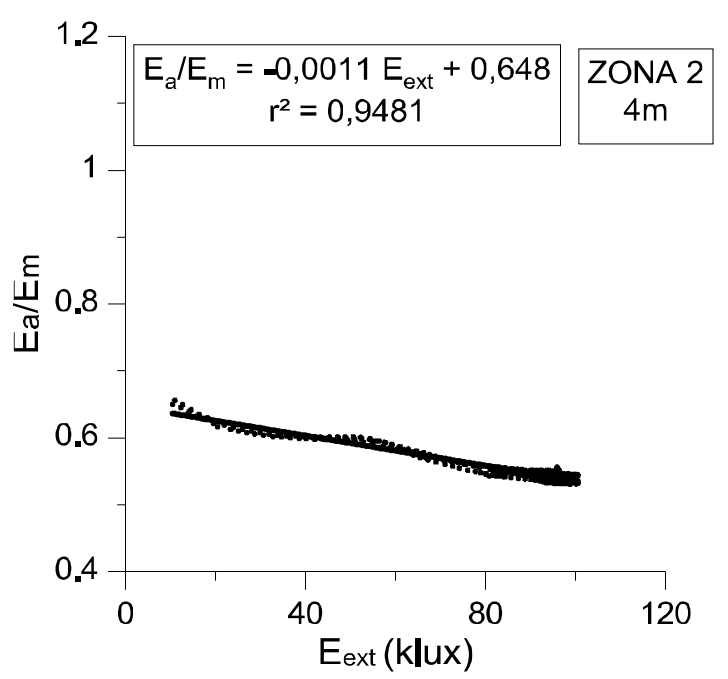

(g) $4 \mathrm{~m}$.

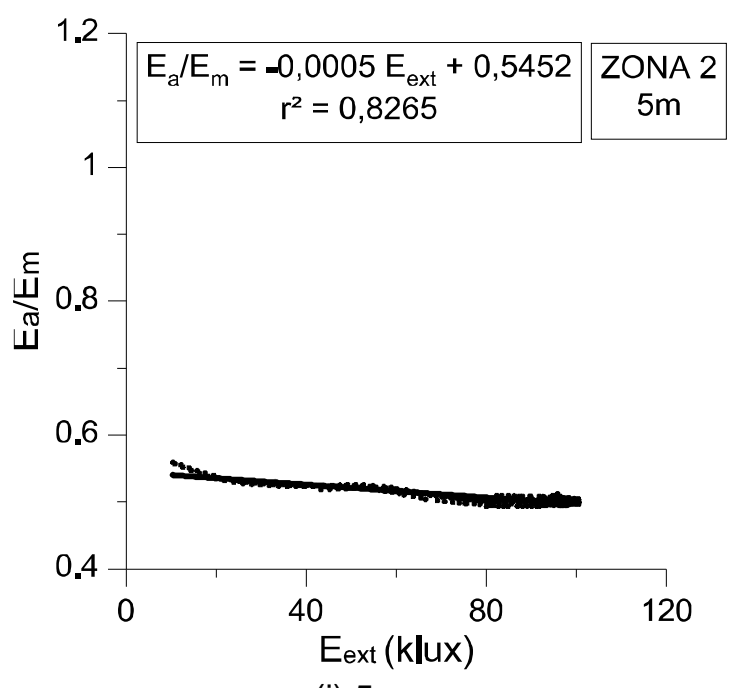

(i) $5 \mathrm{~m}$.

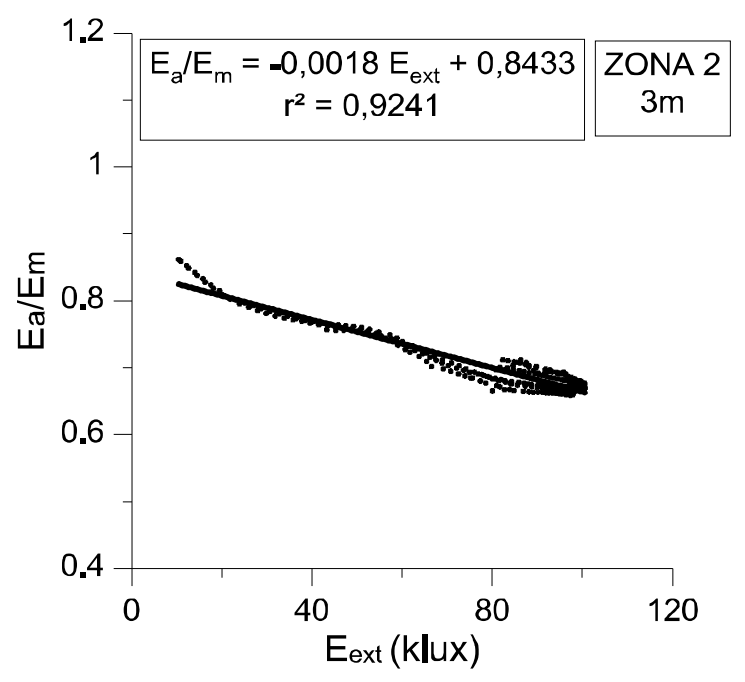

(f) $3 \mathrm{~m}$.

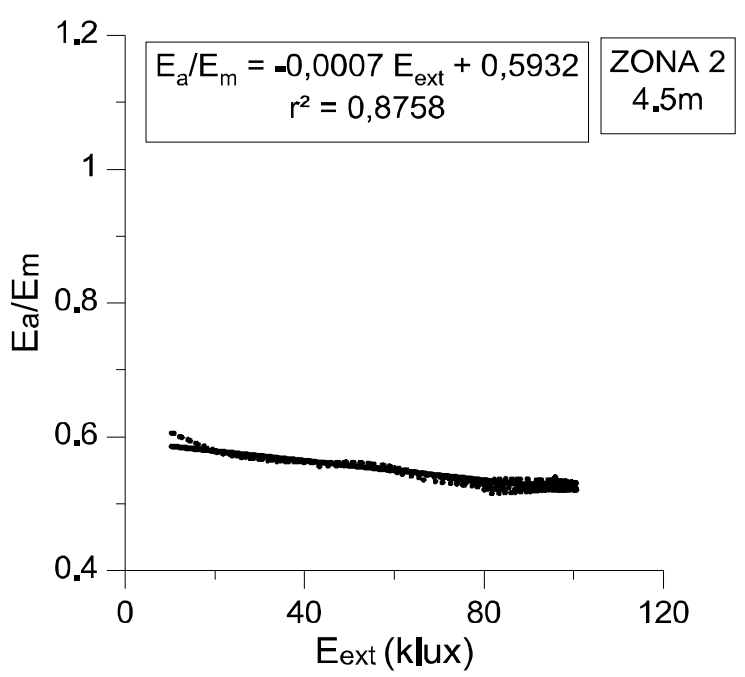

(h) $4.5 \mathrm{~m}$.

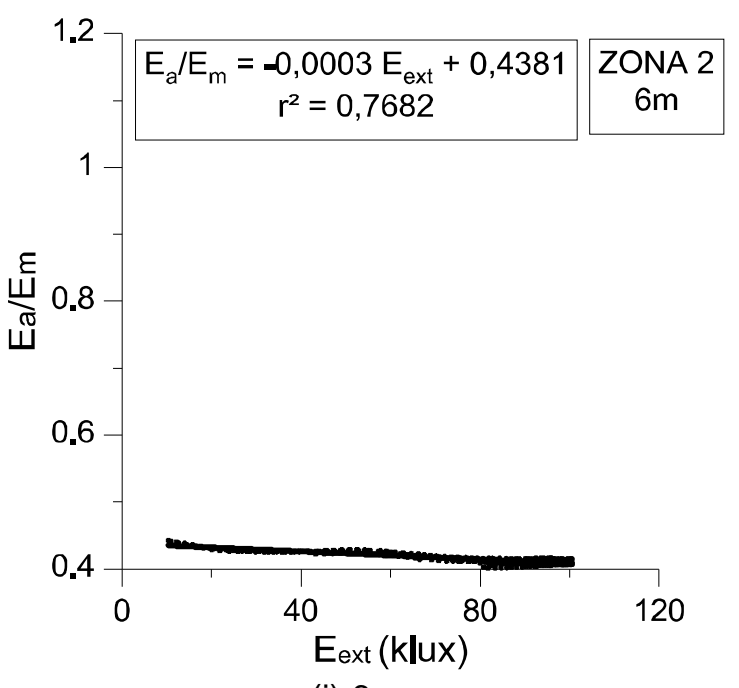

(j) $6 \mathrm{~m}$.

Figura 6.29. Cociente de las iluminancias del aula y del modelo frente a la iluminancia exterior a las distancias de la ventana indicadas. 
Como se puede observar la relación de las iluminancias aula/modelo frente a la iluminancia exterior es una relación lineal. A medida que aumenta la distancia a la ventana el cociente entre iluminancia aula/modelo con la iluminancia exterior va disminuyendo.

Si se representan las distintas rectas ajustadas, obtenidas anteriormente, de forma conjunta, se obtiene gráfica de la figura 6.30.

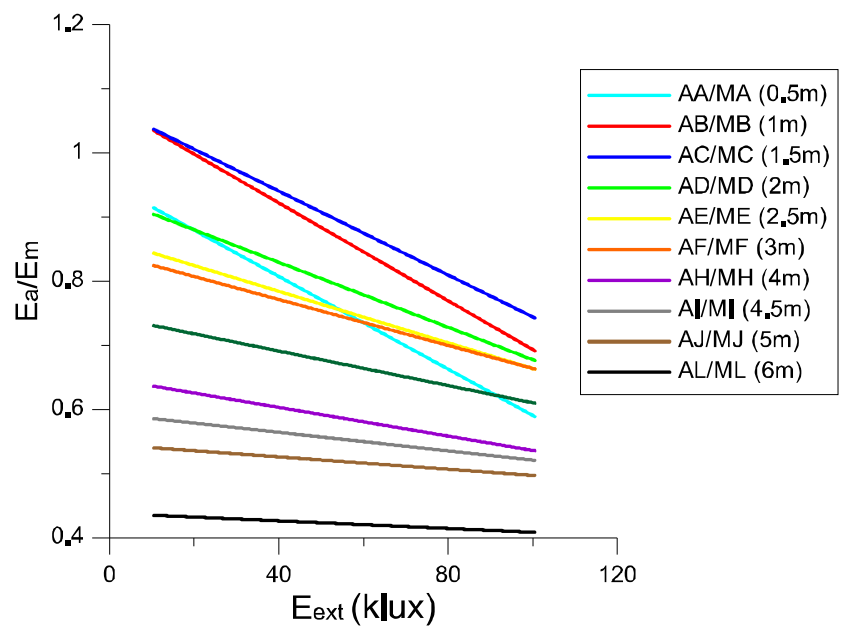

Figura 6.30 Rectas ajustadas de la relaciones entre el cociente de las iluminancias aula/ modelo frente a la iluminancia exterior a las distintas distancias.

Se observa que según aumenta la distancia a la ventana, la pendiente de estas rectas va disminuyendo. Las ecuaciones correspondientes a las rectas anteriores, así como su coeficiente de ajuste $\left(r^{2}\right)$ se recogen en la tabla 6.8. En ésta se puede apreciar cómo el coeficiente de ajuste de todas las rectas es superior a 0.8 , salvo a la distancia de $6 \mathrm{~m}$.

\begin{tabular}{|c|c|c|}
\hline DISTANCIA (m) & ECUACIÓN & $\mathbf{r}^{\mathbf{2}}$ \\
\hline $\mathbf{0 , 5}$ & $\mathrm{E}_{a} / \mathrm{E}_{\mathrm{m}}=-0,0036 \mathrm{E}_{\mathrm{ext}}+0,9521$ & 0,9712 \\
\hline $\mathbf{1}$ & $\mathrm{E}_{\mathrm{a}} / \mathrm{E}_{\mathrm{m}}=-0,0038 \mathrm{E}_{\mathrm{ext}}+1,0747$ & 0,9818 \\
\hline $\mathbf{1 , 5}$ & $\mathrm{E}_{a} / \mathrm{E}_{\mathrm{m}}=-0,0033 \mathrm{E}_{\mathrm{ext}}+1,0714$ & 0,9739 \\
\hline $\mathbf{2}$ & $\mathrm{E}_{a} / \mathrm{E}_{\mathrm{m}}=-0,0025 \mathrm{E}_{\mathrm{ext}}+0,9309$ & 0,9716 \\
\hline $\mathbf{2 , 5}$ & $\mathrm{E}_{a} / \mathrm{E}_{\mathrm{m}}=-0,002 \mathrm{E}_{\mathrm{ext}}+0,8647$ & 0,9453 \\
\hline $\mathbf{3}$ & $\mathrm{E}_{a} / \mathrm{E}_{\mathrm{m}}=-0,0018 \mathrm{E}_{\mathrm{ext}}+0,8433$ & 0,9241 \\
\hline $\mathbf{4}$ & $\mathrm{E}_{\mathrm{a}} / \mathrm{E}_{\mathrm{m}}=-0,0011 \mathrm{E}_{\mathrm{ext}}+0,648$ & 0,9481 \\
\hline $\mathbf{4 , 5}$ & $\mathrm{E}_{a} / \mathrm{E}_{\mathrm{m}}=-0,0007 \mathrm{E}_{\mathrm{ext}}+0,5932$ & 0,8758 \\
\hline $\mathbf{5}$ & $\mathrm{E}_{a} / \mathrm{E}_{\mathrm{m}}=-0,0005 \mathrm{E}_{\mathrm{ext}}+0,5452$ & 0,8265 \\
\hline $\mathbf{6}$ & $\mathrm{E}_{a} / \mathrm{E}_{\mathrm{m}}=-0,0003 \mathrm{E}_{\mathrm{ext}}+0,4381$ & 0,7682 \\
\hline
\end{tabular}

Tabla 6.8. Ecuaciones que relacionan las iluminancias del aula y del modelo con la iluminancia exterior a distintas distancias. 


\subsubsection{Modelo matemático para determinar la iluminancia en el aula}

Hasta el momento se han obtenido unas ecuaciones, recogidas en la tabla 6.8, que relacionan las iluminancias entre el aula $\left(E_{a}\right)$ y el modelo $\left(E_{m}\right)$ en función de la iluminancia exterior $\left(E_{\text {ext }}\right)$, para las distintas distancias desde la ventana a puntos del interior, de la forma:

$$
\frac{E_{a}}{E_{m}}=P \cdot E_{\text {ext }}+O
$$

Siendo $P$ la Pendiente y $O$ la Ordenada en el origen.

Como el objetivo es obtener una expresión que permita relacionar las variables anteriores también con la distancia interior a la fachada, se van a analizar, por separado, las pendientes (figura 6.31) y las ordenadas en el origen (figura 6.32) de las rectas recogidas en la tabla 6.8 en función de la distancia. Estos datos se muestran en la tabla 6.9.

\begin{tabular}{|c|c|c|}
\hline $\begin{array}{c}\text { Distancia } \\
(\mathbf{m})\end{array}$ & Pendiente & Ordenada \\
\hline 0,5 & $-0,0036$ & 0,9521 \\
\hline 1 & $-0,0038$ & 1,0747 \\
\hline 1,5 & $-0,0033$ & 1,0714 \\
\hline 2 & $-0,0025$ & 0,9309 \\
\hline 2,5 & $-0,002$ & 0,8647 \\
\hline 3 & $-0,0018$ & 0,8433 \\
\hline 4 & $-0,0011$ & 0,648 \\
\hline 4,5 & $-0,0007$ & 0,5932 \\
\hline 5 & $-0,0005$ & 0,5452 \\
\hline 6 & $-0,0003$ & 0,4381 \\
\hline
\end{tabular}

Tabla 6.9. Pendientes y ordenadas en el origen a las distintas distancias de las rectas de ajuste procedentes del cociente de las iluminancias aula/ modelo frente a la iluminancia exterior. 


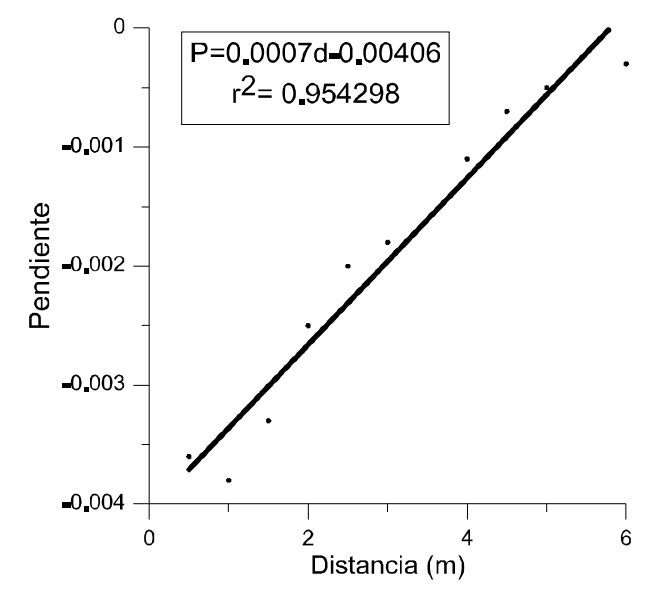

Figura 6.31. Pendiente en función de la distancia a la fachada.

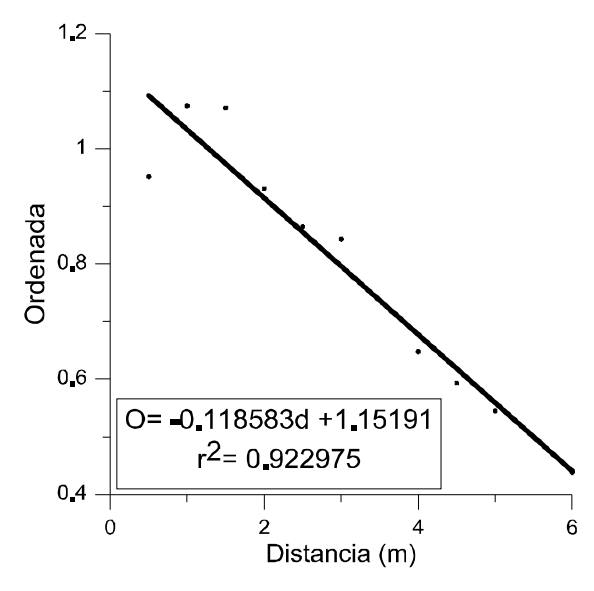

Figura 6.32. Ordenada en el origen en función de la distancia a la fachada.

En la figura 6.31 se representan los valores de las pendientes $(P)$ para cada distancia $(d)$, que se ajustan a una recta cuya ecuación es: $P=(0.700 d-4.060)$ $\times 10^{-3}$ con $r^{2}=0.954$. En la figura 6.32 se representan las ordenadas en el origen $(O)$ frente a la distancia $(d)$, con una respuesta lineal cuya recta es: $O=-0.119 \mathrm{~d}$ $+1.152 ; r^{2}=0.923$. Por lo tanto, el modelo matemático para determinar la iluminancia del aula a partir de la del modelo a escala, la exterior y la distancia, en la zona 2, es:

$$
E_{a}=\left[(0.700 d-4.060) \times 10^{-3} E_{\text {ext }}-0.119 d+1.152\right] E_{m} \quad[Z 2]
$$

Siendo:

$$
\begin{aligned}
& E_{a}: \text { lluminancia del aula (klux) } \\
& E_{m} \text { : lluminancia del modelo (klux) } \\
& E_{\text {ext }} \text { Iluminancia exterior (klux) } \\
& d \text { : Distancia a la fachada (m) }
\end{aligned}
$$

Esta ecuación permite calcular las iluminancias del aula en cualquier punto de la zona 2, determinado por su distancia a la ventana (d), a partir de las iluminancias medidas en la maqueta $\left(E_{m}\right)$ y en el exterior $\left(E_{e x t}\right)$. De esta manera se alcanza el objetivo de poder determinar las iluminancias de una estancia real (el aula) sin necesidad de medir en dicha estancia; sólo sería necesario conocer las iluminancias en el exterior y en el interior de la maqueta de la estancia estudiada así como la distancia a la que hayamos colocado los sensores. 


\subsubsection{Análisis estadístico}

Como comprobación de la fiabilidad del modelo matemático obtenido en el apartado anterior, se va a realizar un análisis estadístico de los valores obtenidos para las pendientes y las ordenadas en el origen a las distintas distancias como comprobación. Por un lado se ha analizado la pendiente (eje vertical) frente a la distancia a la fachada (eje horizontal) y por otro lado la ordenada en el origen (eje vertical) frente a la distancia a la fachada (eje horizontal).

En la gráfica en la que se han representado las pendientes de las rectas obtenidas $(P)$ en función de la distancia a la fachada ( $d$ (figura 6.33) se puede comprobar cómo esta relación es lineal, que se ajusta a la recta con pendiente positiva: $P=0.007 d-0.00406$. Se observa que la mayor parte de los datos se encuentran dentro de los límites de confianza al 95\% (verde) y todos los datos se encuentran entre los límites de predicción (rojo) por lo que no se aprecian valores atípicos.

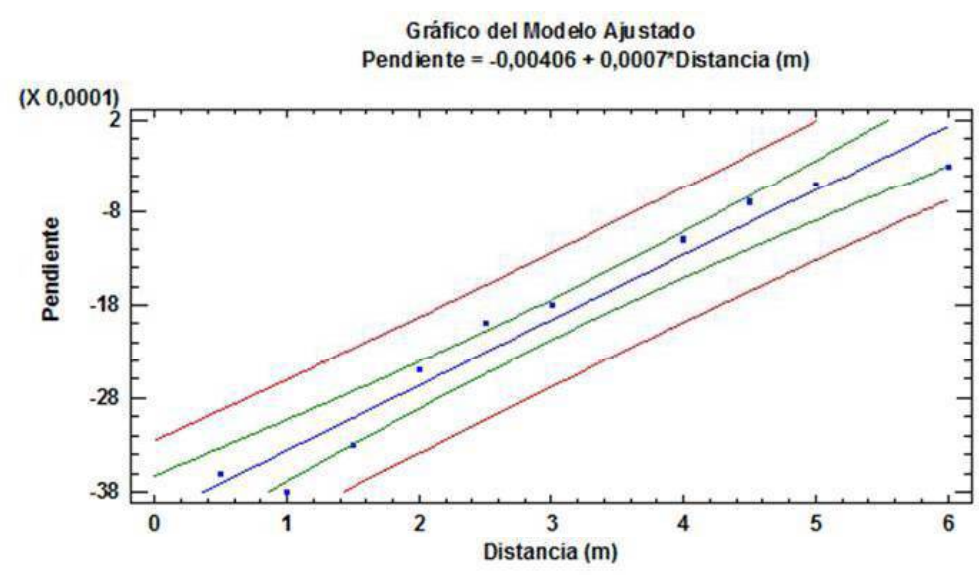

Figura 6.33. Gráfico del modelo ajustado de la pendiente en función de la distancia a la ventana.

Al representar las ordenadas en el origen de las rectas $(O)$ en función de la distancia a la fachada (d) (figura 6.34) se obtiene una recta con pendiente negativa cuya ecuación es: $O=-0.119 d+1.152$ (como se había obtenido anteriormente). La mayor parte de los datos se encuentran dentro de los límites de confianza al 95\% (verde) y todos los datos se encuentran entre los límites de predicción (rojo), por lo que no se aprecian valores atípicos. 


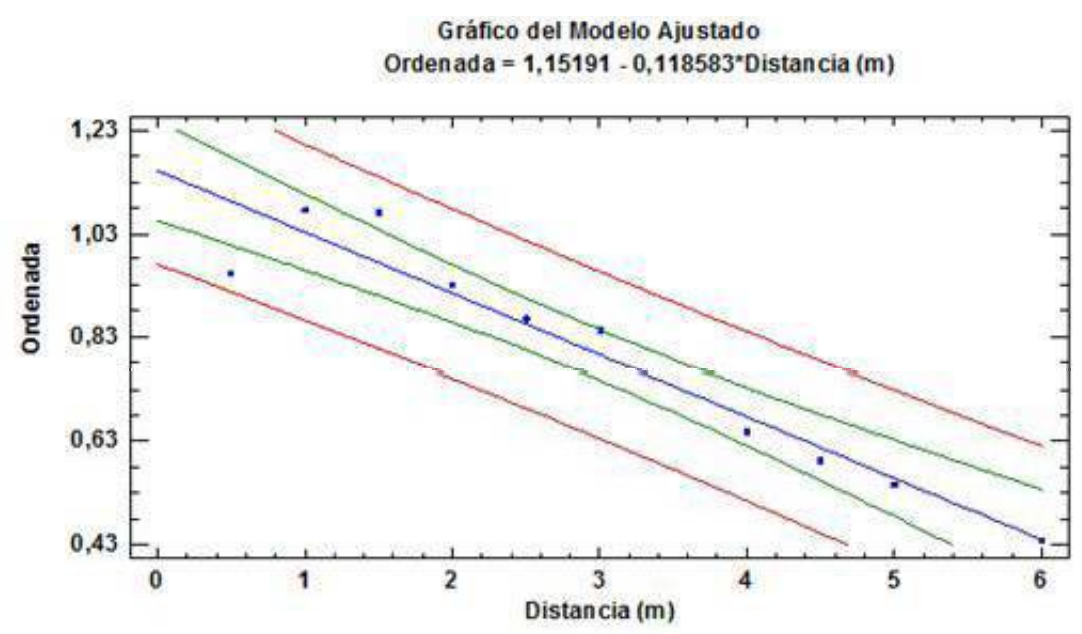

Figura 6.34. Gráfico del modelo ajustado de la ordenada en el origen en función de la distancia a la ventana.

En ambos casos se obtiene un p-valor de $0.00<0.5$, lo que indica que hay una relación estadística significante entre las variables consideradas con un nivel de confianza de por lo menos el $95 \%$, es decir que la fiabilidad de los datos es $\geq 95 \%$. En el análisis de los residuos el p-valor es superior a 0.05 lo que indica que la probabilidad de que haya errores en este análisis es menor al $5 \%$.

\subsubsection{Simulación con software de iluminación y comprobación de resultados}

En este apartado se van a comparar las iluminancias del aula entre las 11:00 y las 19:00 horas, obtenidas de tres formas diferentes:

a) Midiendo directamente en el aula.

b) Calculando mediante la ecuación [Z2] a partir de las iluminancias medidas en el modelo a escala y en el exterior.

c) Utilizando un software de iluminación (Dialux 4.11)

En la figura 6.35 se muestran gráficamente los resultados obtenidos en los tres casos para las distintas distancias consideradas en el estudio. Se han representado en rojo las iluminancias medidas directamente, en azul claro las obtenidas de la ecuación Z2 y en azul oscuro las del programa de simulación. 


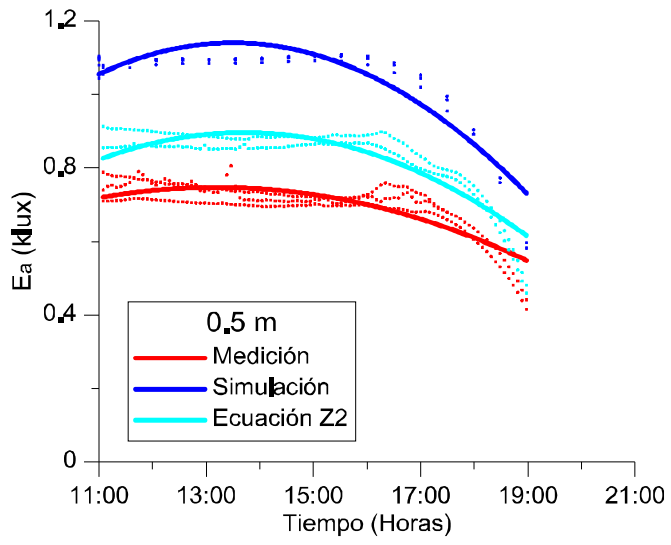

(a) $0.5 \mathrm{~m}$.

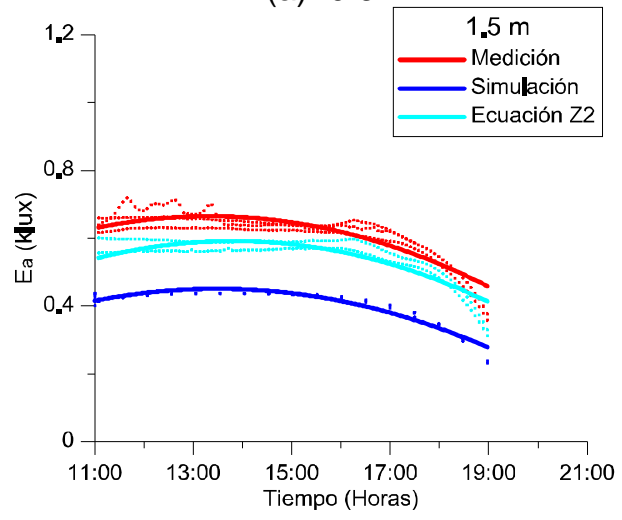

(c) $1.5 \mathrm{~m}$.

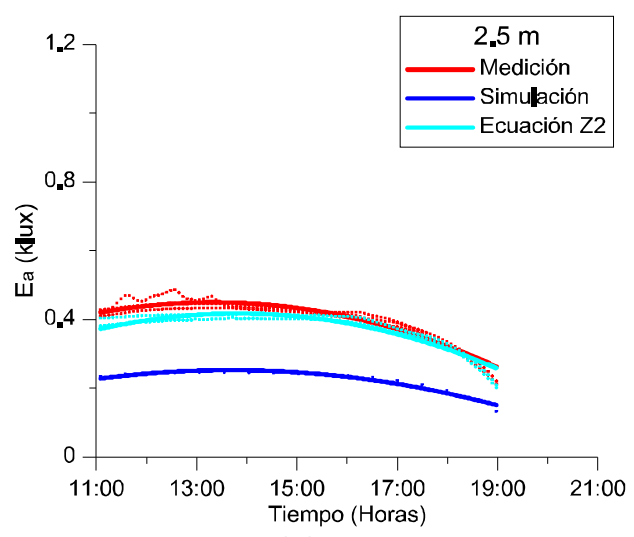

(e) $2.5 \mathrm{~m}$

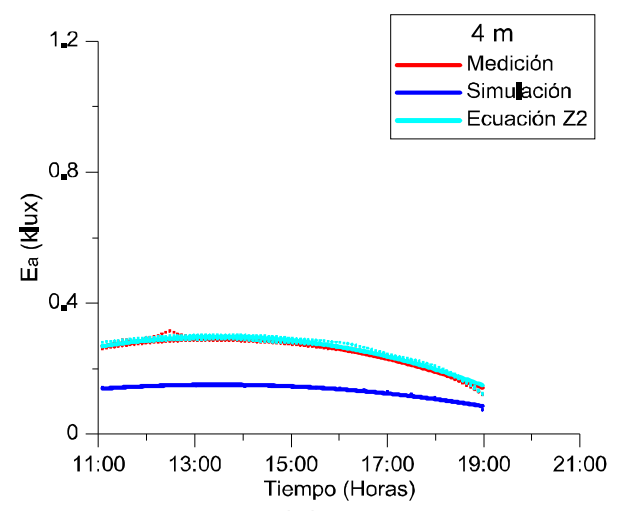

(g) $4 \mathrm{~m}$.

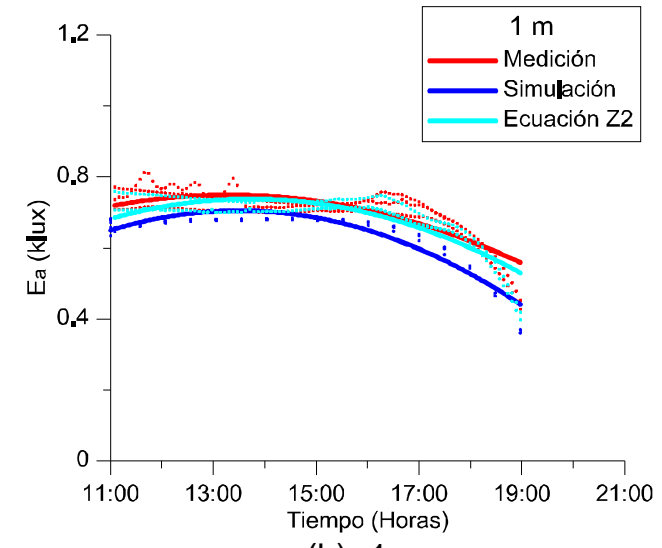

(b) $1 \mathrm{~m}$.

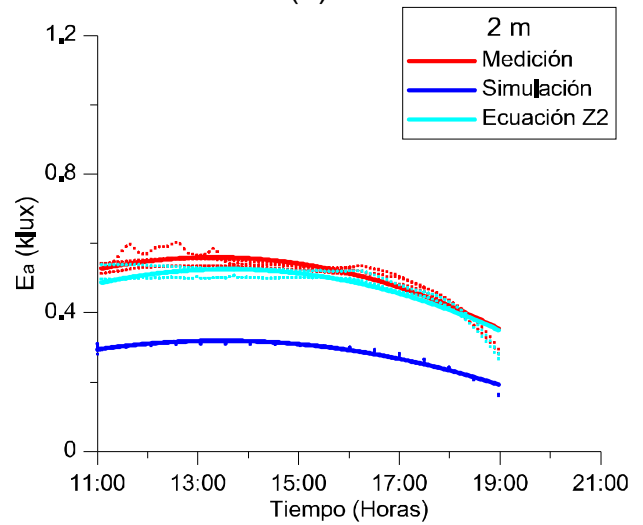

(d) $2 \mathrm{~m}$.

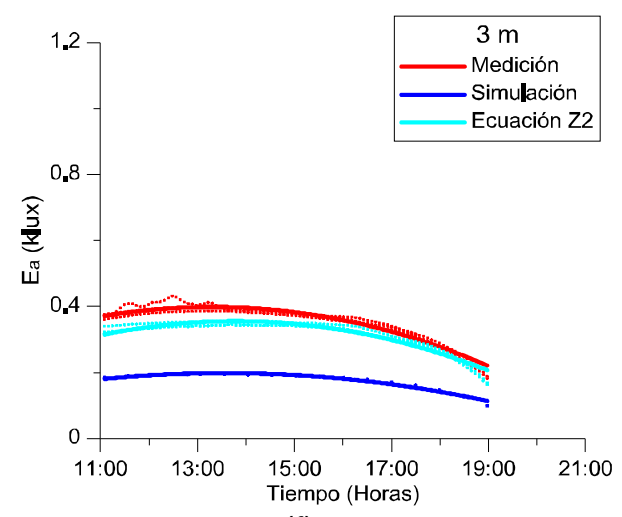

(f) $3 \mathrm{~m}$.

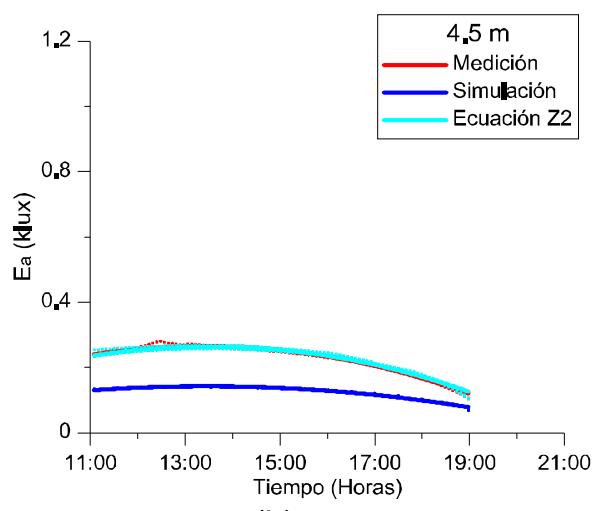

(h) $4.5 \mathrm{~m}$. 


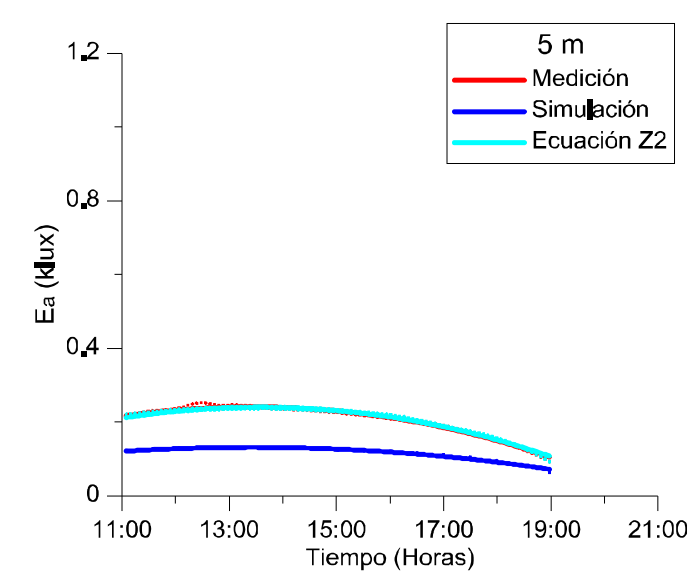

(i) $5 \mathrm{~m}$.

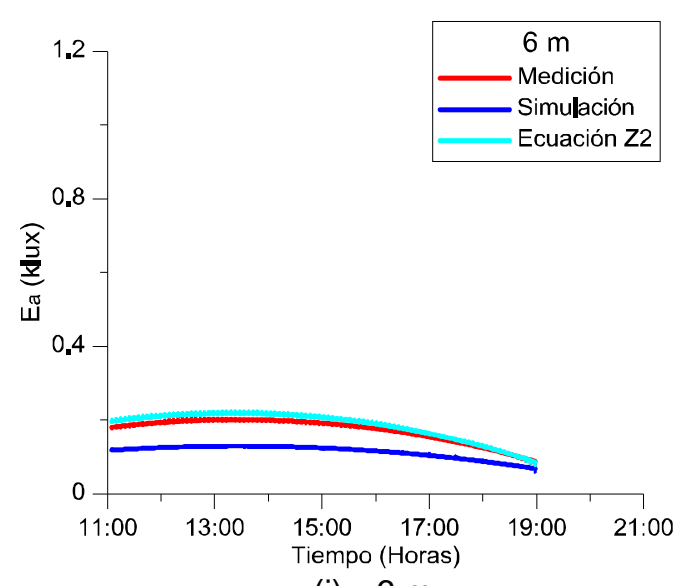

(j) $6 \mathrm{~m}$.

Figura 6.35. Iluminancias en el aula obtenidas de aplicar la ecuación Z2, por simulación y de forma experimental a las distancias de la fachada indicadas.

En las gráficas de la figura 6.35 se puede observar cómo para todas las distancias consideradas los valores de iluminación más bajos se han obtenido en la simulación con la única excepción del punto más cercano a la ventana situado a $0.5 \mathrm{~m}$ de ésta. También se puede observar que las iluminancias obtenidas aplicando la ecuación Z2 son más próximas a las del aula que aquéllas obtenidas por simulación. Esto supone que los valores obtenidos aplicando la ecuación son ligeramente más fiables que los obtenidos mediante simulación.

Para completar esta comparación de las iluminancias, se va a realizar un análisis para todas las distancias simultáneamente a distintas horas del día $(11: 00,12: 00,13: 00,14: 00,15: 00,16: 00$ y 17:00) de forma separada para los distintos días en los que se han realizado medidas en el aula 05/04/2015, 04/04/2015 y 01/04/2015. Los resultados obtenidos para el día 05/04/2015 se muestran en los diagramas de barras de la figura 6.36.

En estos diagramas de barras no se han representado las iluminancias obtenidas de la ecuación Z2 a las distancias de $3.5 \mathrm{~m}$ y $5.5 \mathrm{~m}$ porque, como ya se ha indicado anteriormente, los sensores del modelo a escala a esas distancias no midieron correctamente. 


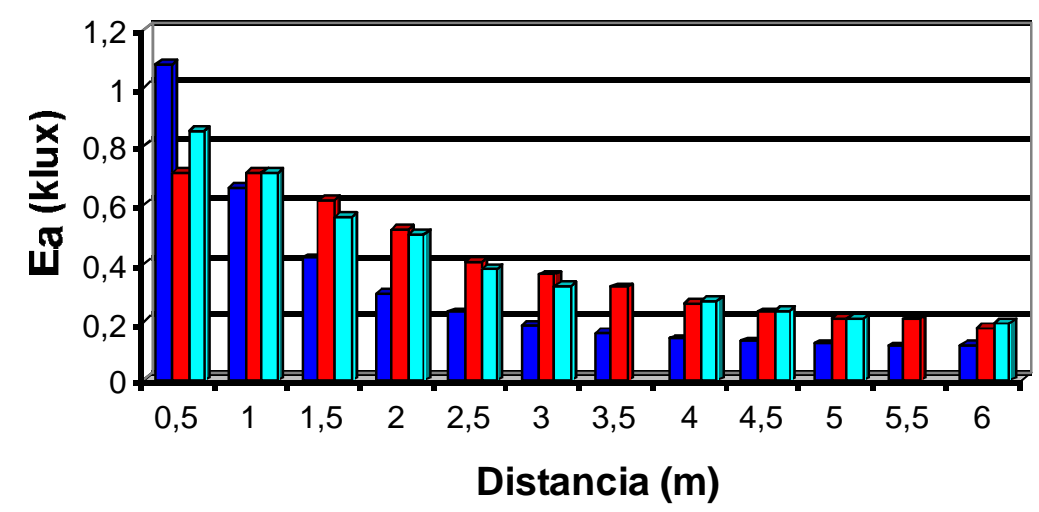

- SIMULACIÓN

$\square$ AULA

口 ECUACIÓNZ2

(a) 11:00.

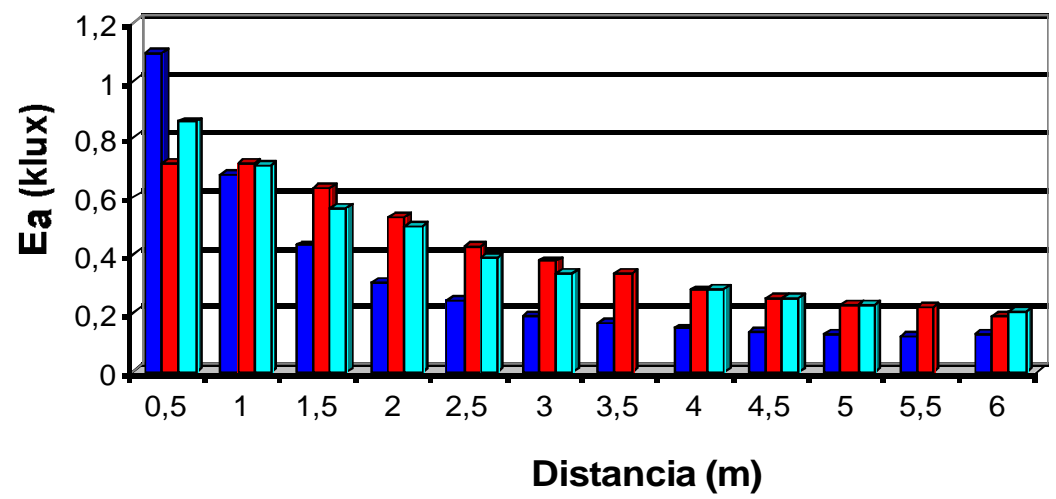

- SIMULACIÓN

口AULA

口 ECUACIÓNZ2

(b) $12: 00$.

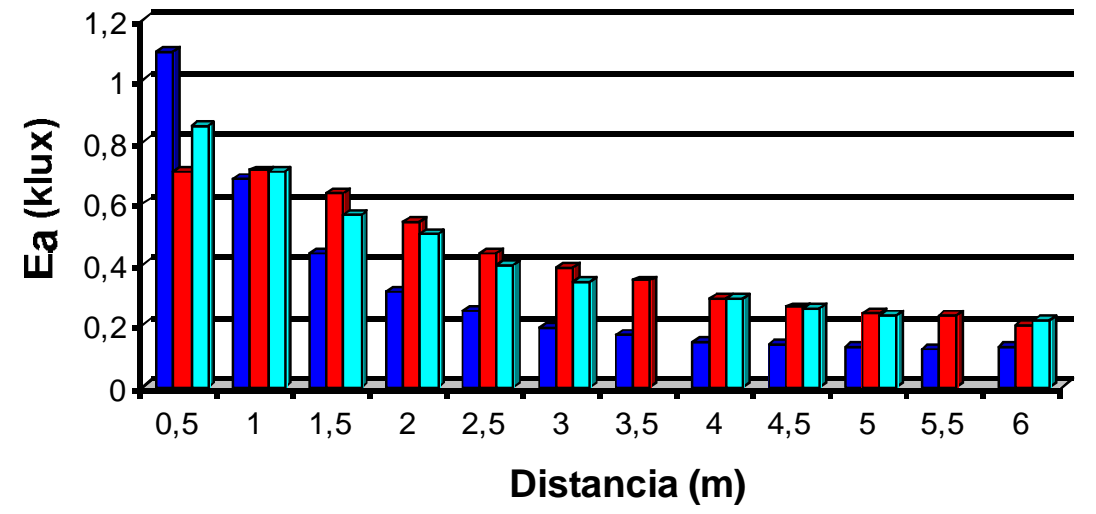

- SIMULACIÓN

口AULA

口ECUACIÓNZ2

(c) 13:00.

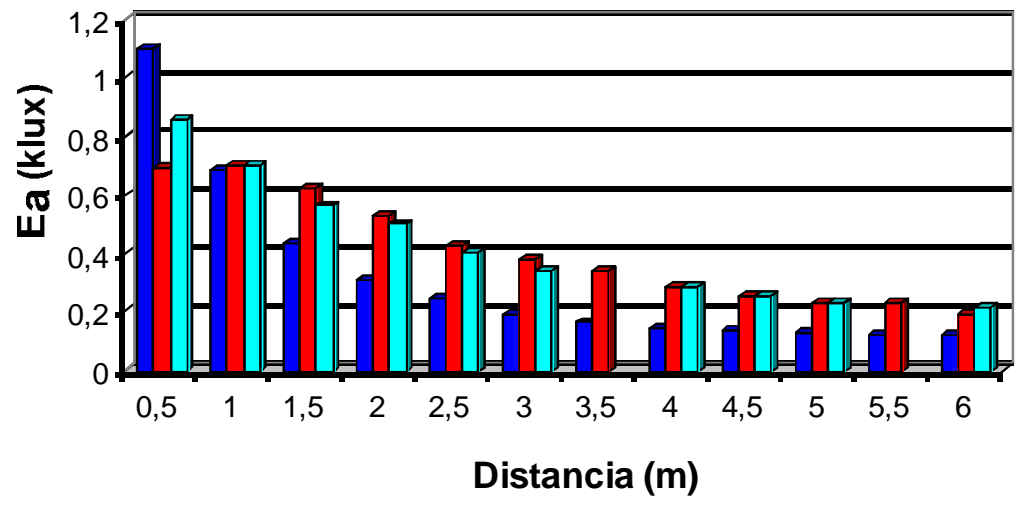

- SIMULACIÓN

口AULA

口ECUACIÓNZ2

(d) 14:00. 

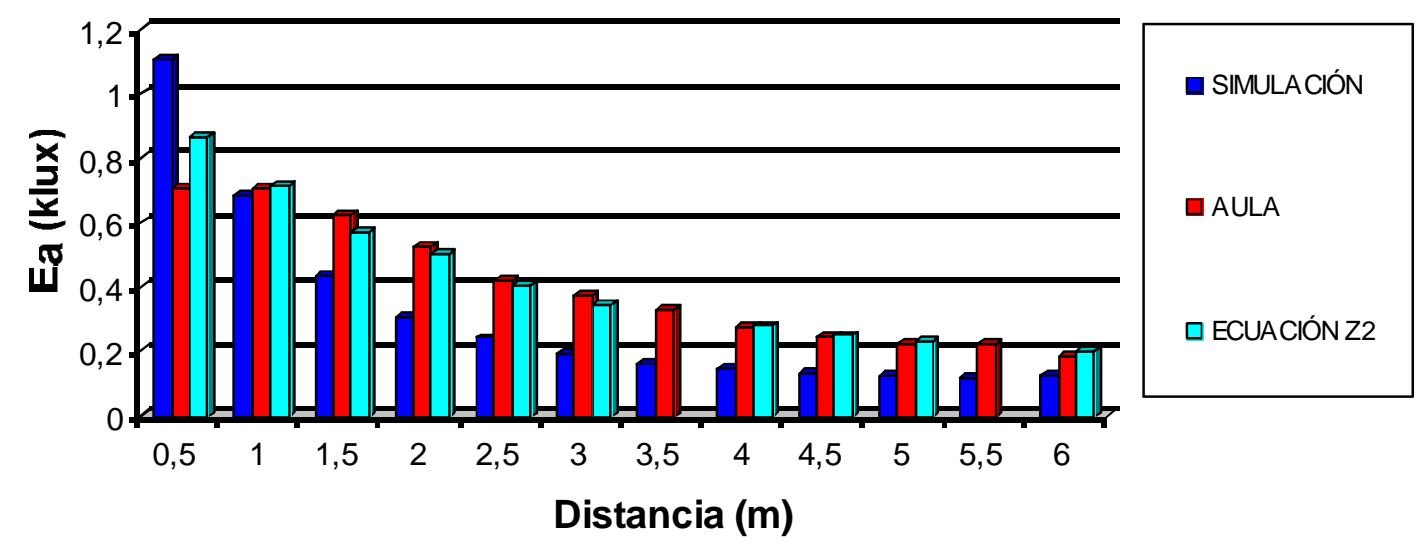

(e) 15:00.
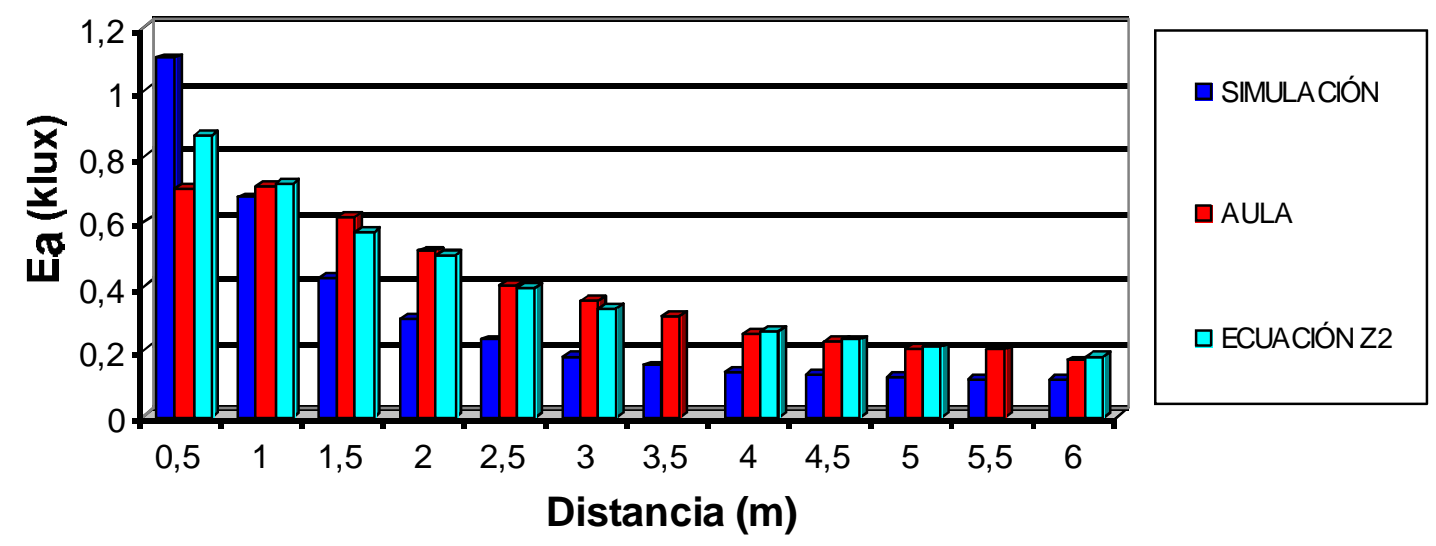

(f) $16: 00$.

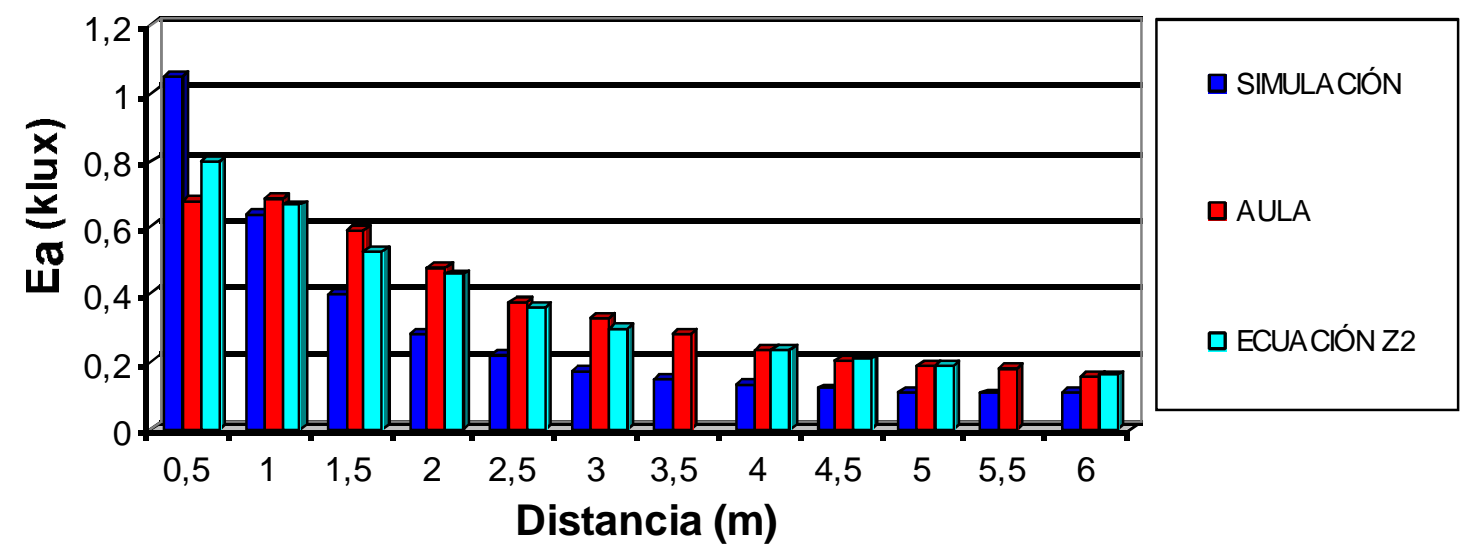

(g) 17:00.

Figura 6.36. Iluminancias del aula obtenidas midiendo directamente, por simulación y aplicando la ecuación Z2 a distintas distancias de la ventana el día 05/04/2015 a las horas, indicadas.

En los diagramas de barras de la figura 6.36 se observa también que las iluminancias medidas en el aula y las obtenidas por la ecuación Z2 son similares y mayores que las de la simulación, salvo a la distancia de $0.5 \mathrm{~m}$ de la ventana. 
Los resultados obtenidos del estudio comparativo de las iluminancias de la zona 2 para el día 04/04/2015 se muestran en los diagramas de barras de la figura 6.37 .

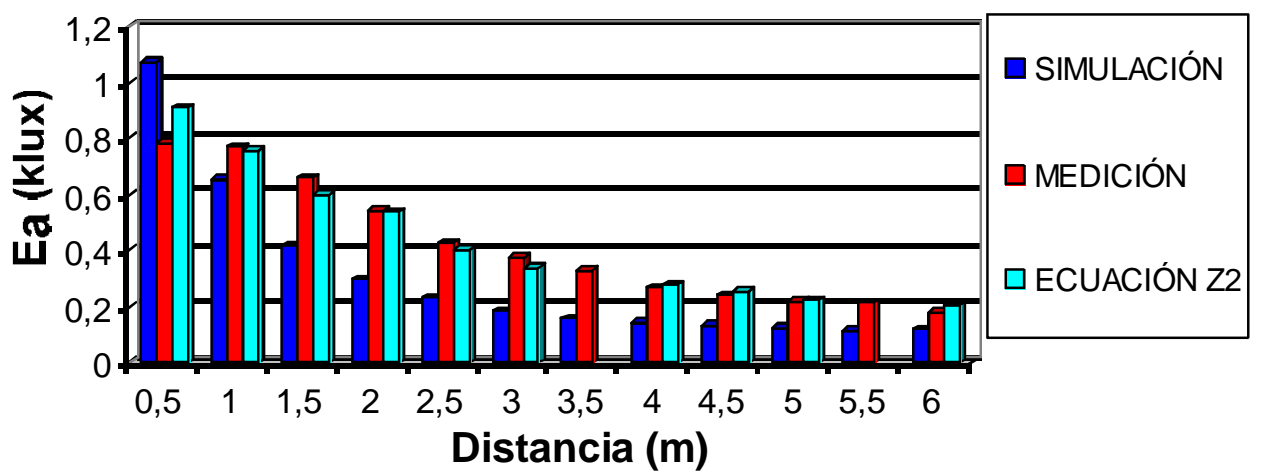

(a) 11:00.

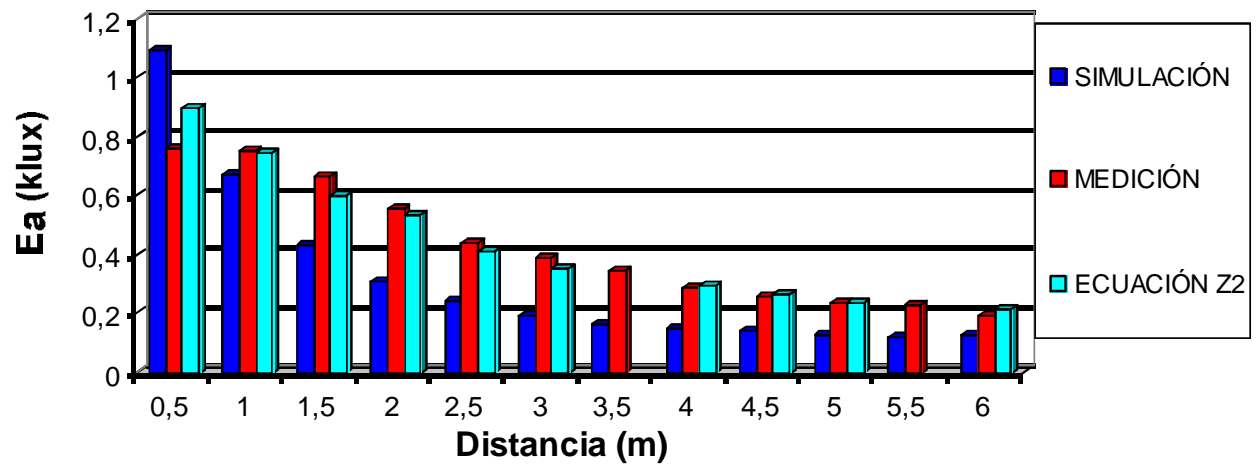

(b) $12: 00$.

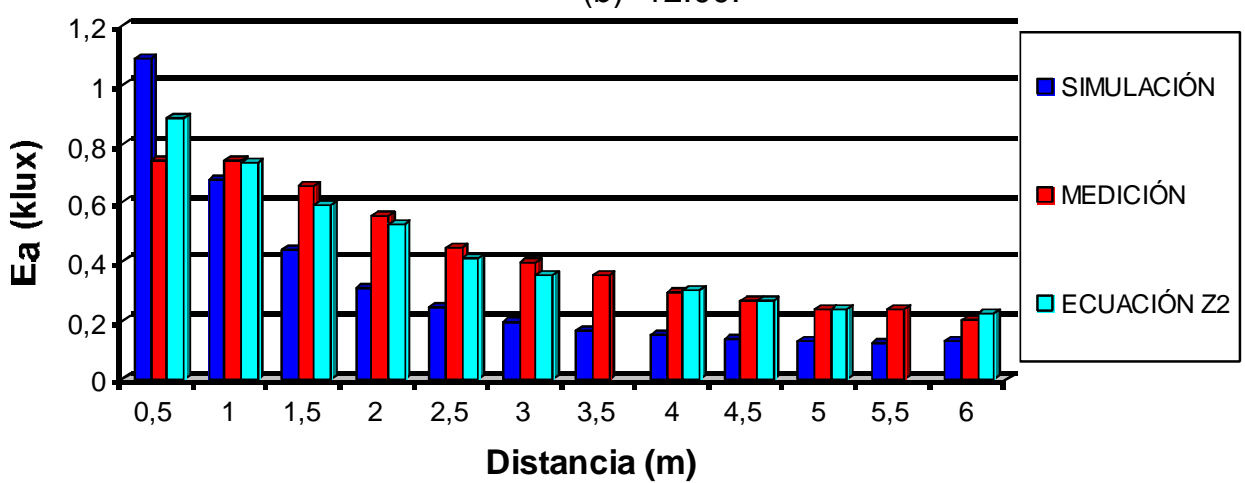

(c) 13:00.

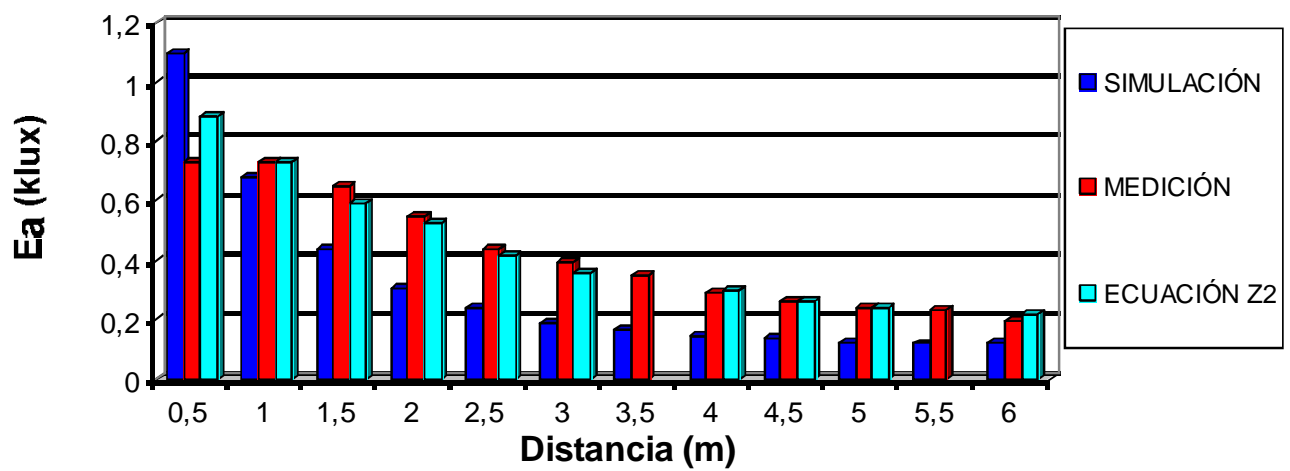

(d) $14: 00$. 


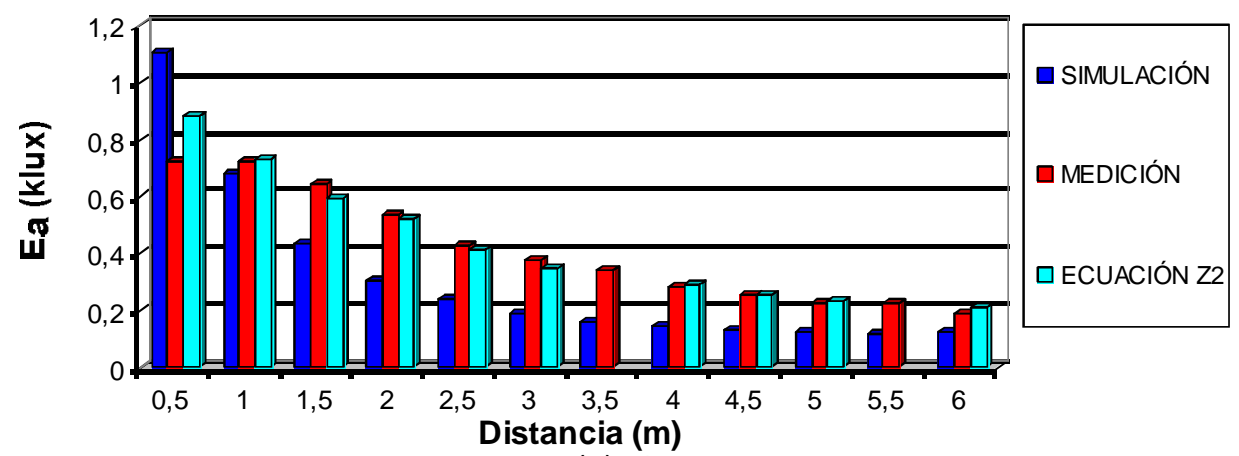

(e) $15: 00$.

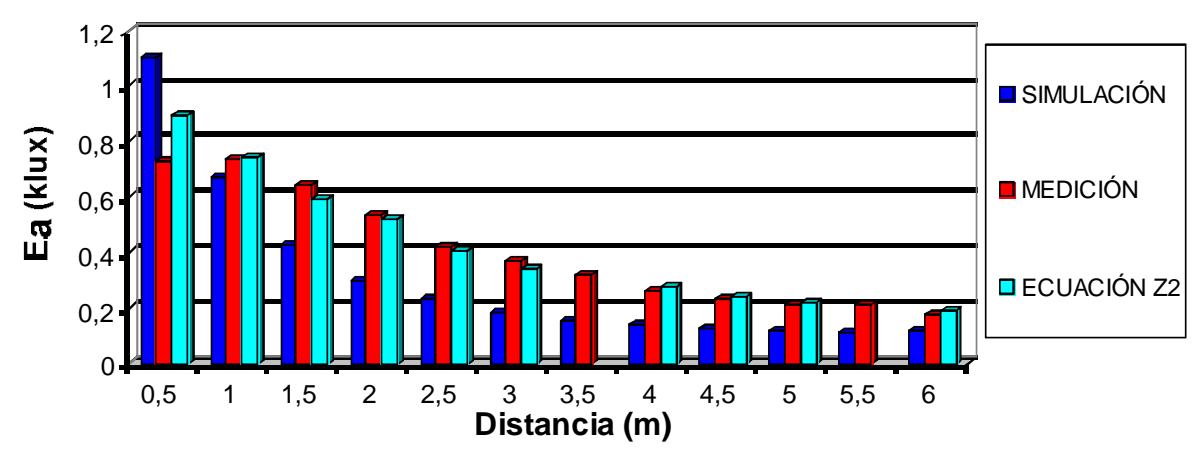

(f) $16: 00$.

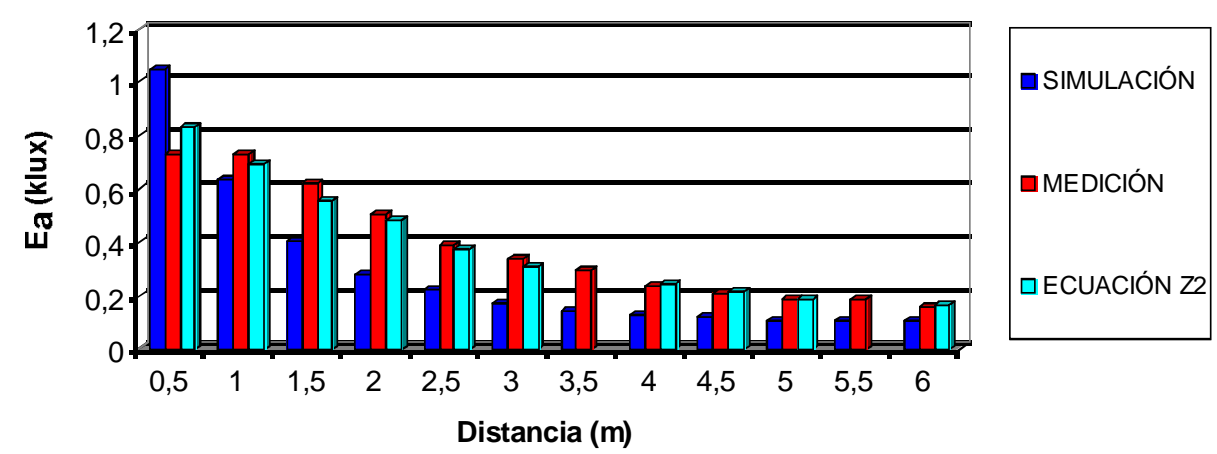

(g) 17:00.

Figura 6.37. Iluminancias del aula obtenidas midiendo directamente, por simulación y aplicando la ecuación Z2 a distintas distancias de la ventana el día 04/04/2015 a las horas, indicadas.

También en los diagramas de barras de la figura 6.37 se puede observar que las iluminancias medidas en el aula y las obtenidas por la ecuación Z2 son similares y mayores que las de la simulación, salvo a la distancia de $0.5 \mathrm{~m}$ de la ventana.

Finalmente, los resultados obtenidos en el estudio comparativo de las iluminancias de la zona 2 correspondiente al día 01/04/2015 se muestras a continuación. 

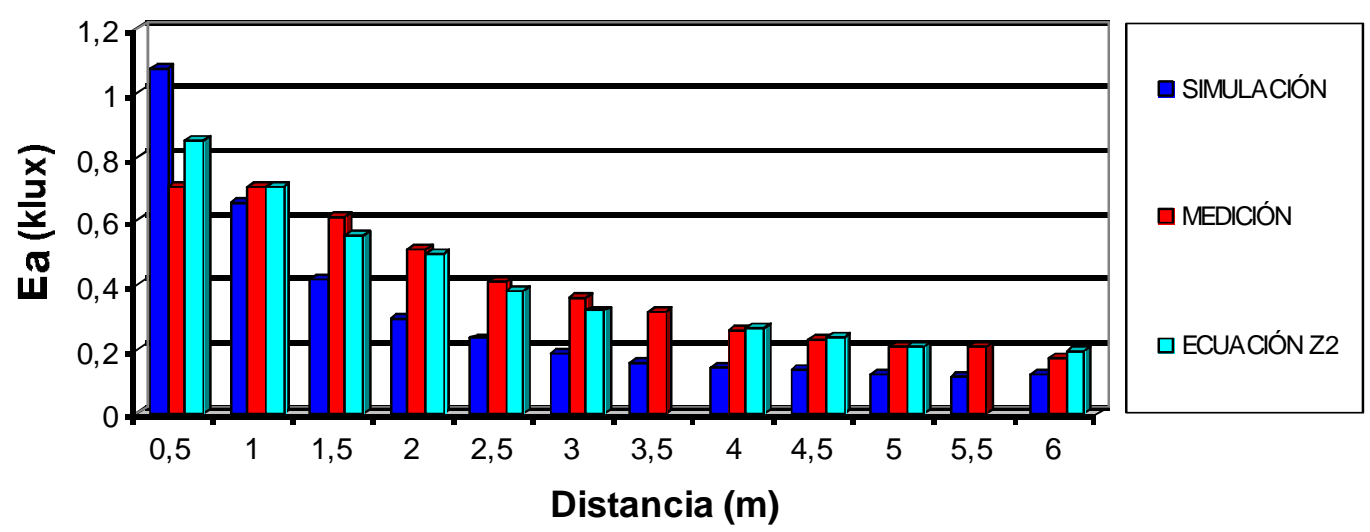

(a) 11:00.
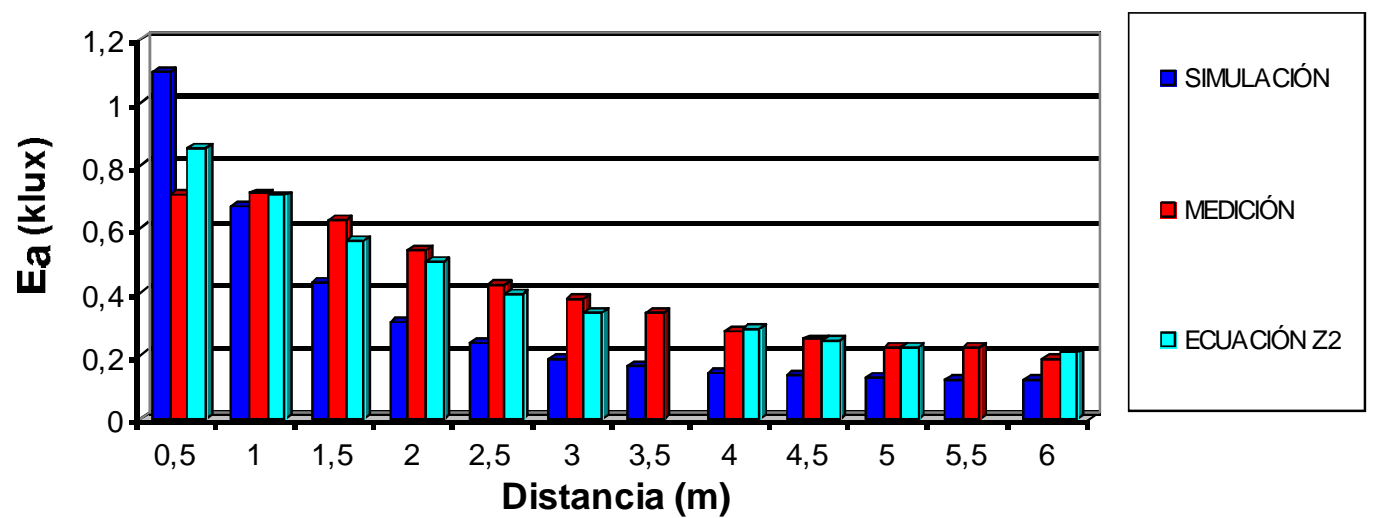

(b) $12: 00$

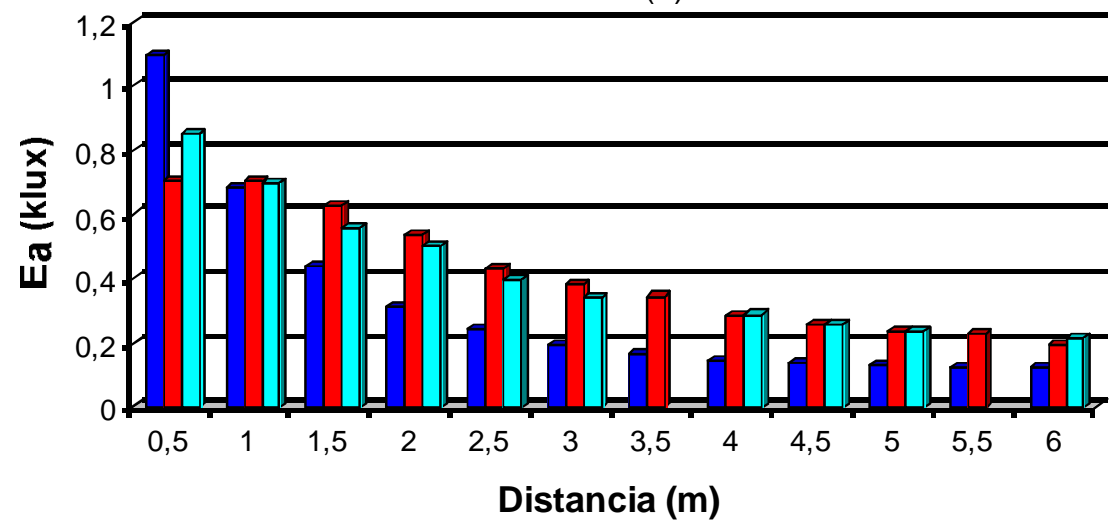

- SIMULACIÓN

MEDICIÓN

口ECUACIÓNZ2

(c) 13:00.

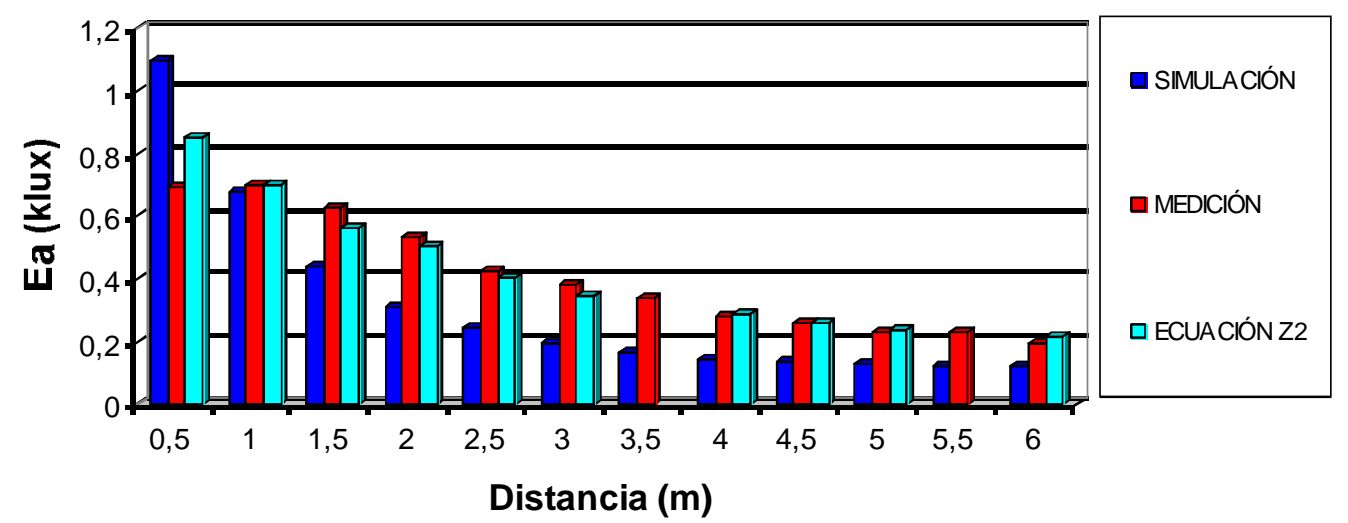

(d) $14: 00$. 


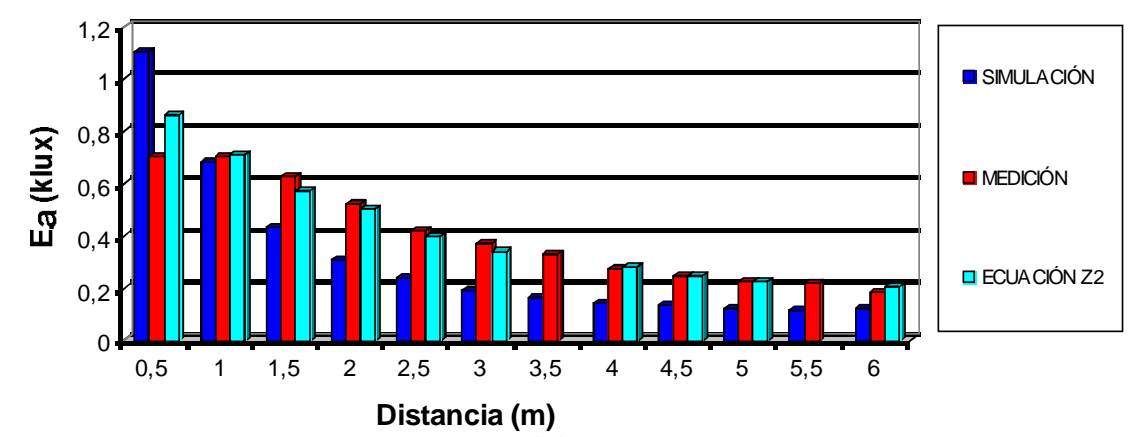

(e) 15:00.

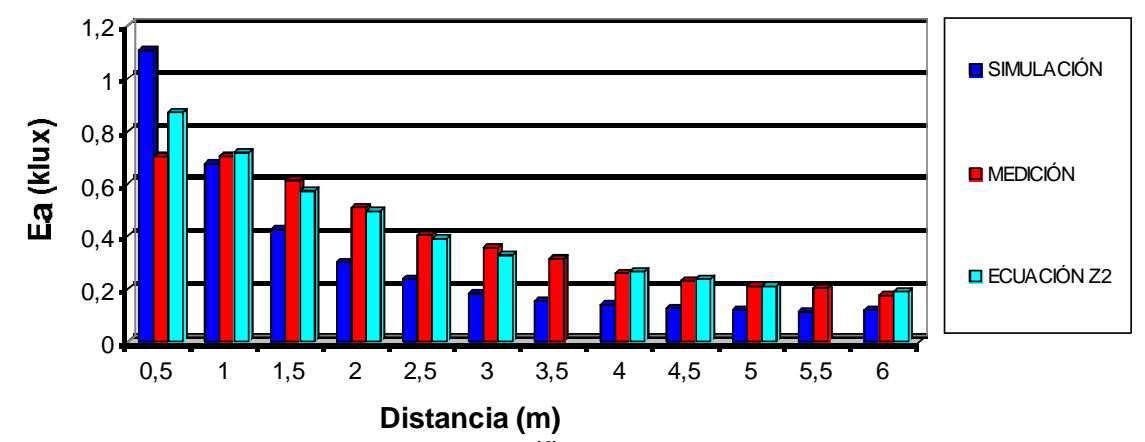

(f) $16: 00$.

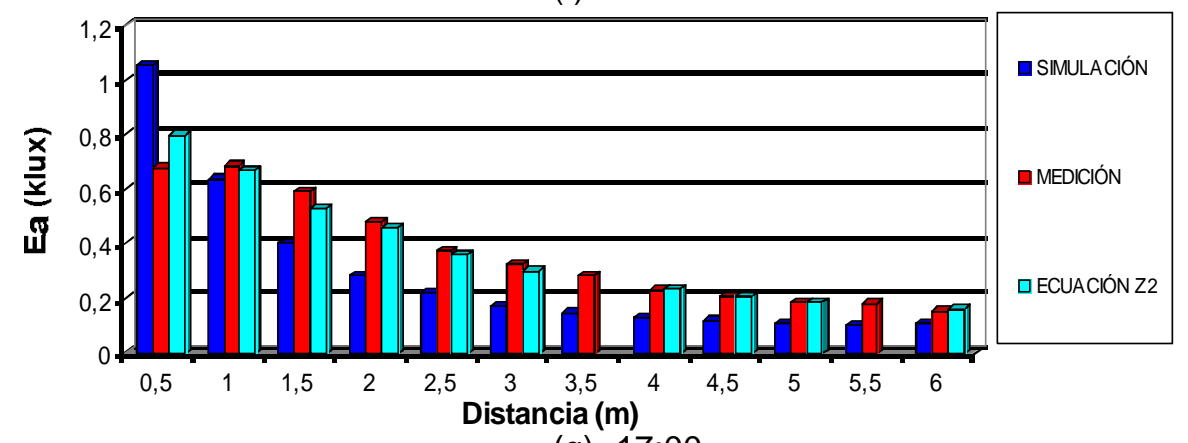

(g) $17: 00$

Figura 6.38. Iluminancias del aula obtenidas midiendo directamente, por simulación y aplicando la ecuación Z2 a distintas distancias de la ventana el día 01/04/2015 a las horas, indicadas.

De forma análoga a las figuras 6.36 y 6.37 , en la figura 6.38 , se ve cómo los valores de las iluminancias más bajos son los de la simulación, a excepción de la correspondiente a la distancia de $0.5 \mathrm{~m}$. Las iluminancias obtenidas por la ecuación Z2 y midiendo en el aula son similares a todas las distancias.

En el anexo II (apartado 10.2.2) se presentan las curvas ajustadas de las iluminancias del aula representadas en las figuras $6.36,6.37$ y 6.38 para las distintas horas consideradas, y las ecuaciones que describen dichas curvas con sus correspondientes coeficientes de ajuste, mayor que 0.9 en todos los casos. 


\subsubsection{Zona 3}

La zona que se va a analizar en este apartado, la zona 3, está situada a $3.17 \mathrm{~m}$ de la pared Este, centrado, con el machón central de la fachada del aula. La colocación y distribución de los sensores se muestra en las figuras 6.39 y 6.40 .

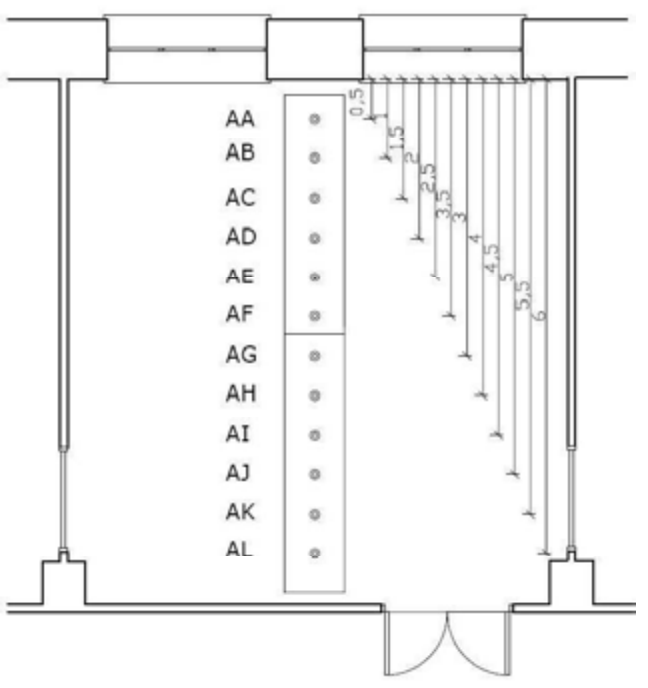

Figura 6.39. Esquema sensores zona 3.

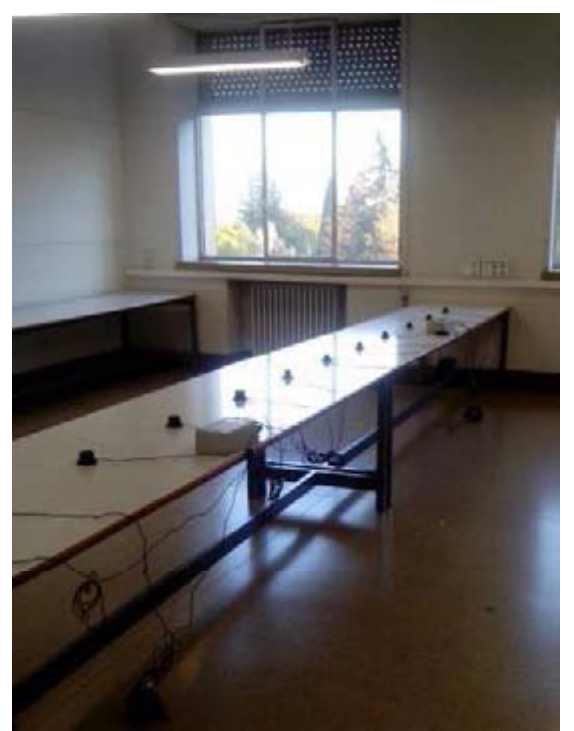

Figura 6.40. Colocación sensores zona 3.

El estudio de esta zona se ha realizado con las medidas de iluminación tomadas los días 05/12/2015 y 06/12/2015 con cielo totalmente despejado desde las 11:00 de la mañana hasta las 17:30 de la tarde (hora local), dado que en diciembre anochece mucho antes que en los casos anteriores.

Se han medido iluminancias en el exterior y a todas las distancias consideradas (0.5 m, 1 m, 1.5 m, 2 m, 2.5 m, 3 m, 3.5 m, 4 m, 4.5 m, 5 m, 5.5 m, 6 m), tanto en el aula objeto de estudio como en el modelo a escala 1/15 del mismo (a las distancias proporcionales). Sin embargo se han suprimido los datos correspondientes a la distancia de $3.5 \mathrm{~m}$, porque el sensor del modelo a esta distancia proporcional, tampoco funcionó de forma correcta.

\subsubsection{Evolución diaria de las iluminancias}

La evolución diaria de la iluminancia exterior $\left(\mathrm{E}_{\text {ext }}\right)$ de los días considerados en esta zona se muestra en la figura 6.41. 


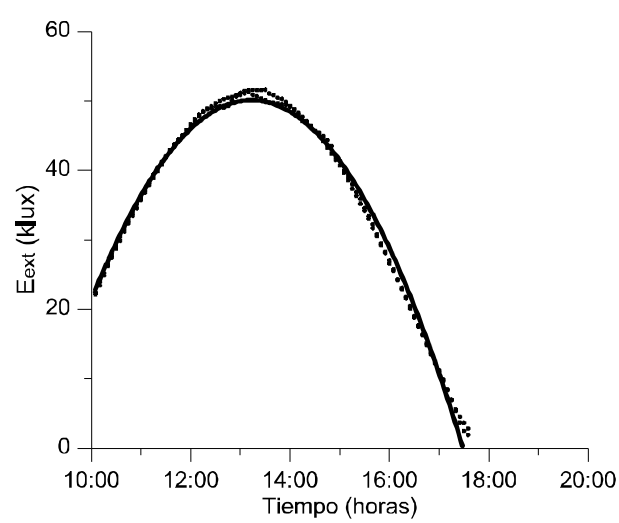

Figura 6.41. Evolución diaria de la iluminancia (Diciembre 2015).

Como se puede observar en la figura 6.41 las iluminancias exteriores son mucho menores que las de los casos anteriores dado que en este caso se ha medido en diciembre, cuando el sol está mucho más bajo. Esto mismo ocurre con las iluminancias del modelo $\left(E_{m}\right)$ y las del aula $\left(E_{a}\right)$ representadas en las figuras 6.42 y 6.43 , respectivamente. Cabe recordar que la fachada del aula de estudio tiene orientación norte. Por este motivo sólo se han considerado las iluminancias de aula y del modelo desde las 11.00 hasta las 17:30.

En el eje vertical aparecen representadas las iluminancias en klux y en el eje horizontal el tiempo expresado en horas. También se muestra la ecuación de segundo grado a la que se ajusta la nube de puntos, así como su coeficiente de ajuste $\left(r^{2}\right)$. En la columna de la izquierda en verde están representadas las iluminancias horizontales correspondientes al modelo y a la derecha en rojo las del aula.

\section{ZONA 3}

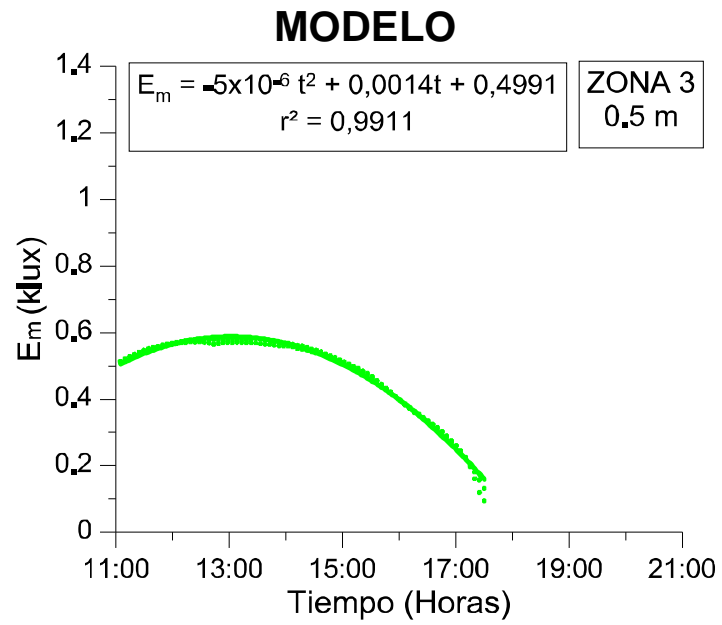

(a) $0.5 \mathrm{~m}$.

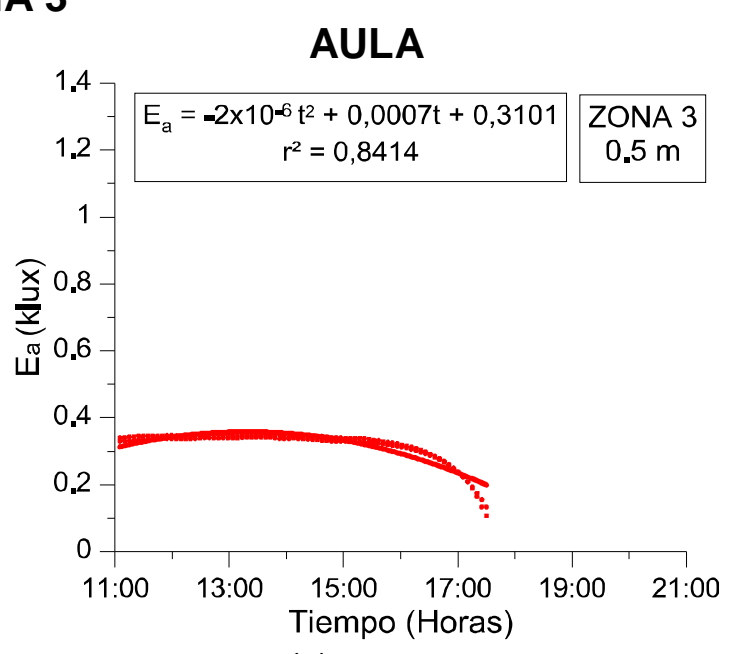

(a) $0.5 \mathrm{~m}$. 


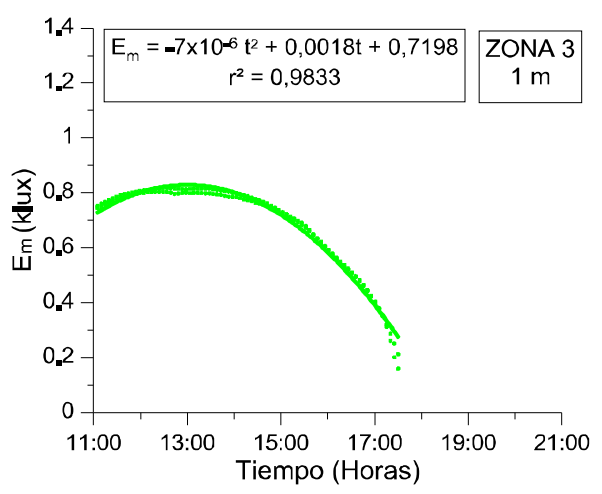

(b) $1 \mathrm{~m}$.

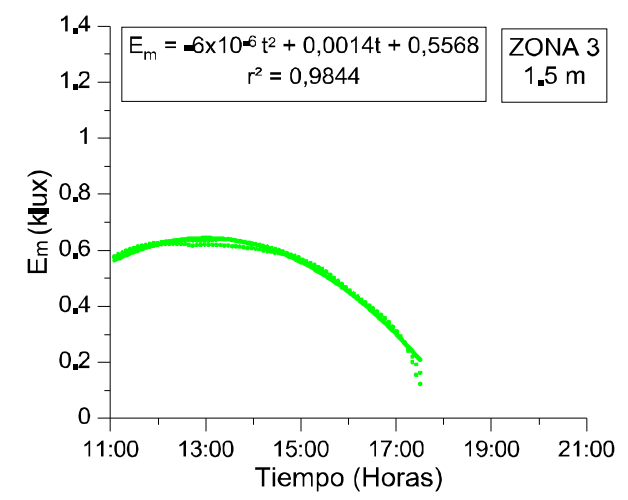

(c) $1.5 \mathrm{~m}$.

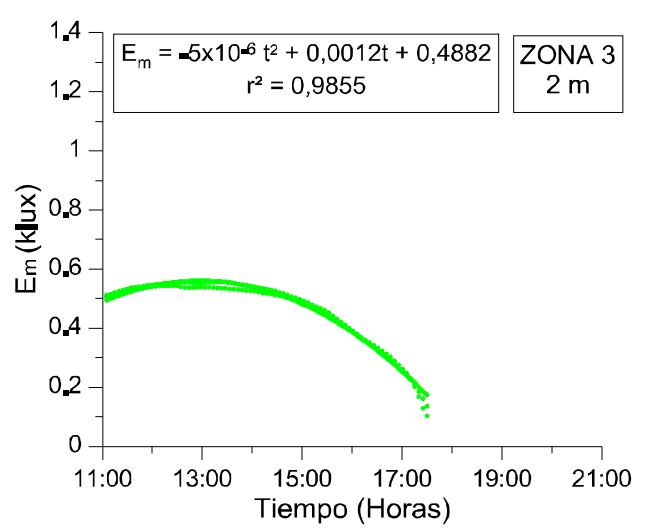

(d) $2 \mathrm{~m}$.

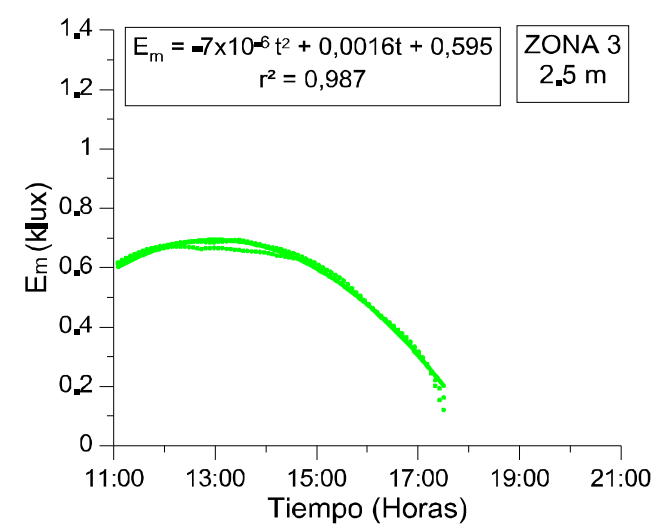

(e) $2.5 \mathrm{~m}$.

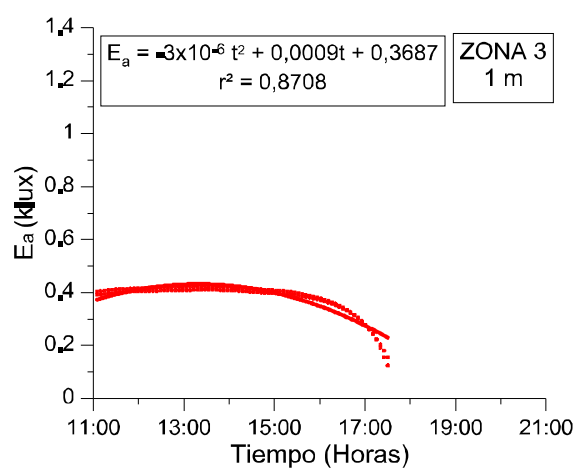

(b) $1 \mathrm{~m}$.

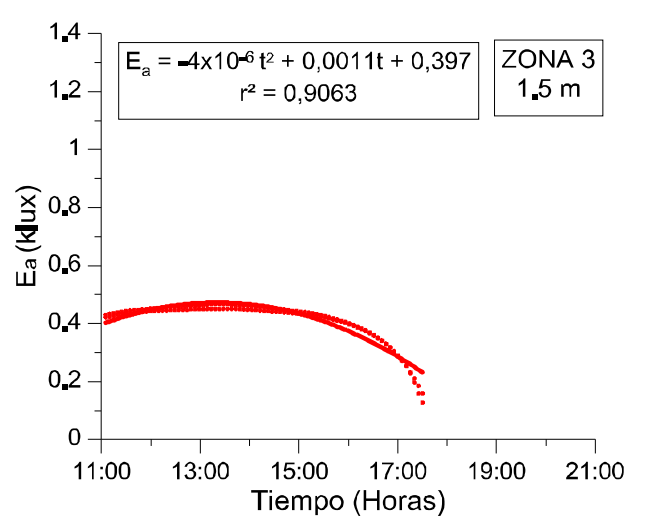

(c) $1.5 \mathrm{~m}$.

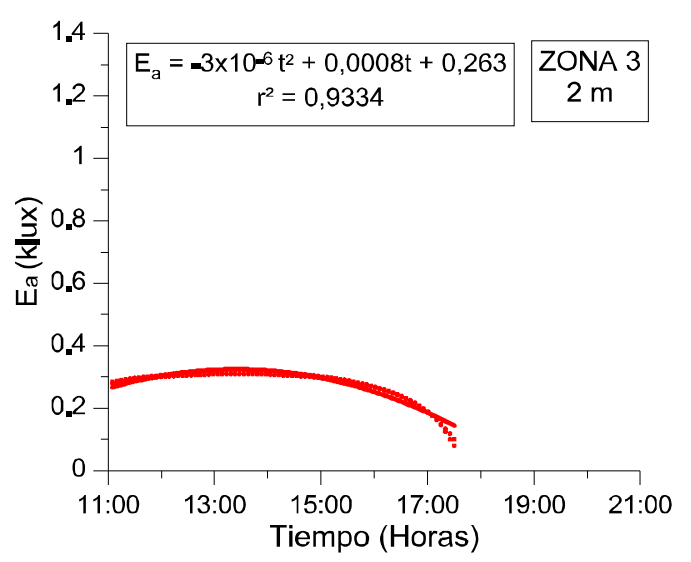

(d) $2 \mathrm{~m}$.

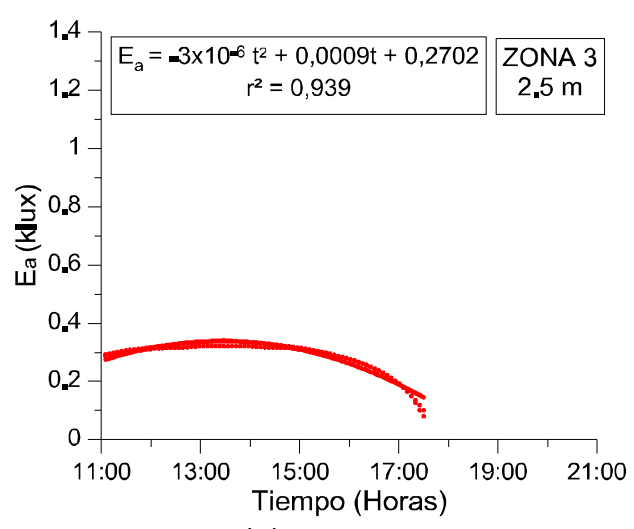

(e) $2.5 \mathrm{~m}$. 
Estudio experimental y modelización de la iluminación natural en la edificación mediante modelos a escala

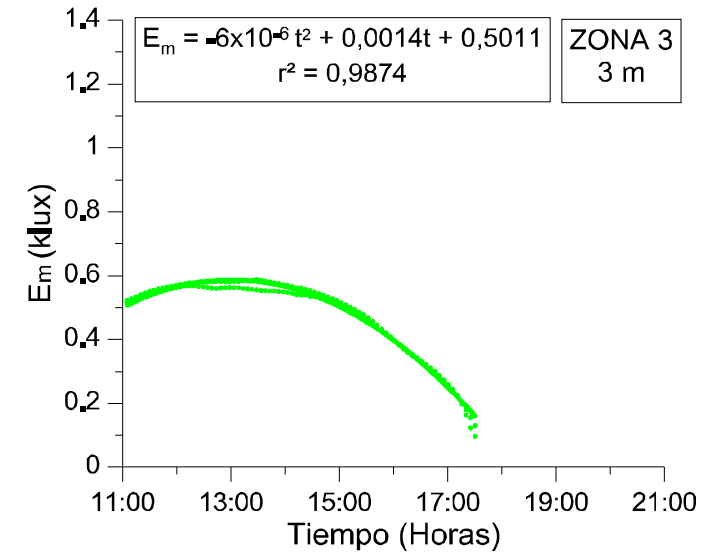

(f) $3 \mathrm{~m}$.

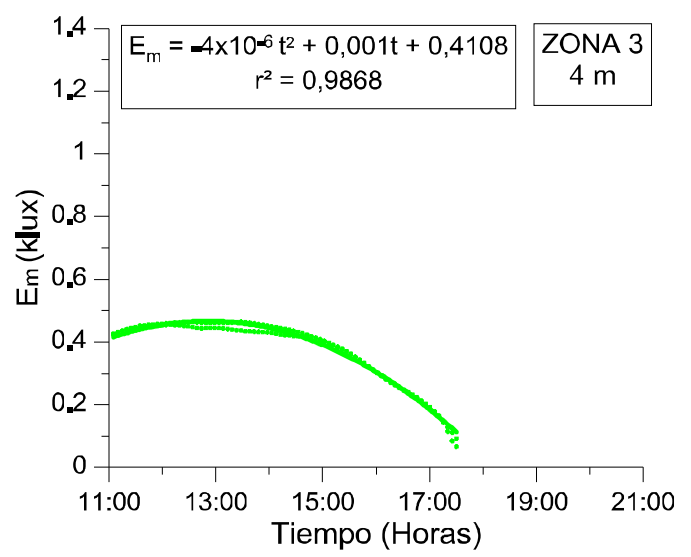

(g) $4 \mathrm{~m}$.

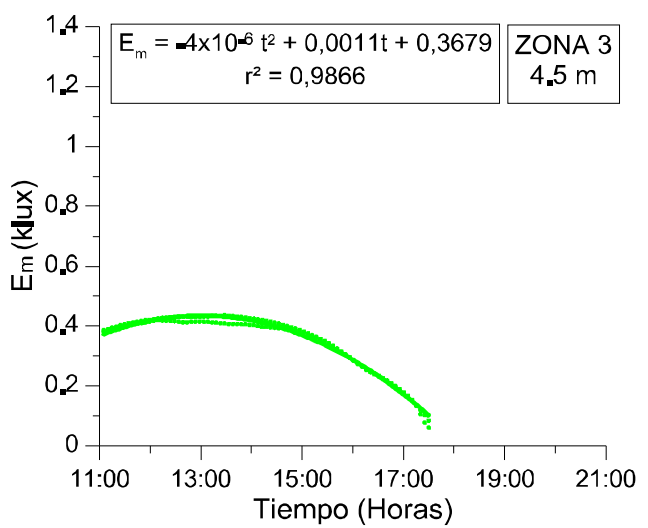

(h) $4.5 \mathrm{~m}$.

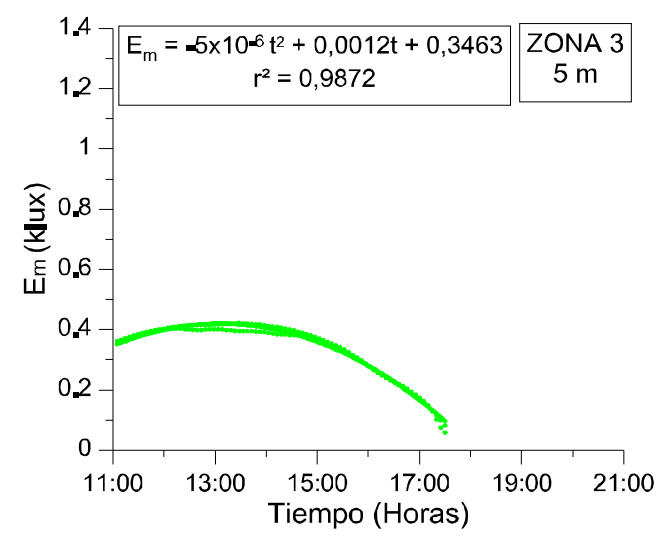

(i) $5 \mathrm{~m}$.

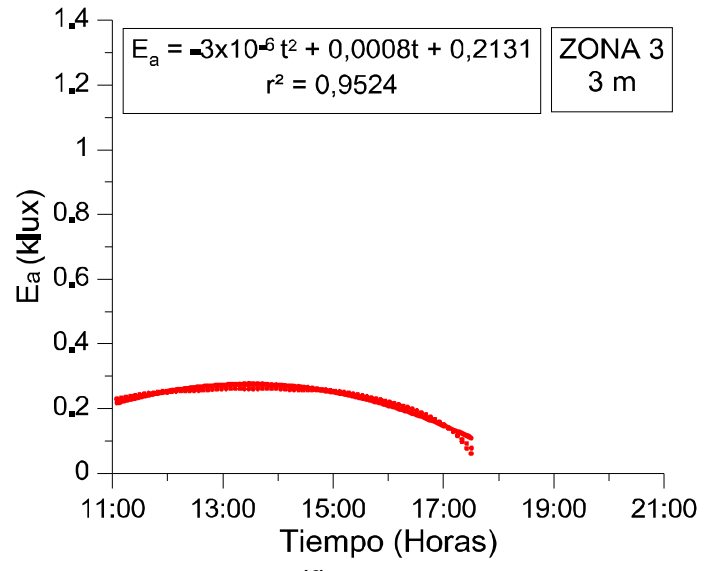

(f) $3 \mathrm{~m}$.

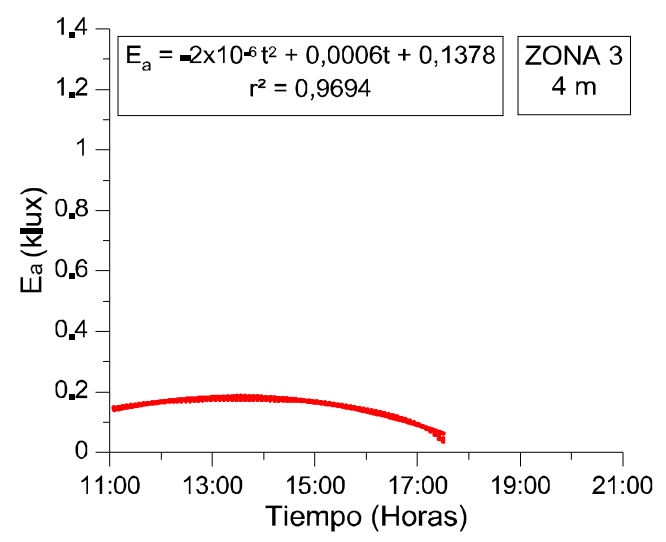

(g) $4 \mathrm{~m}$.

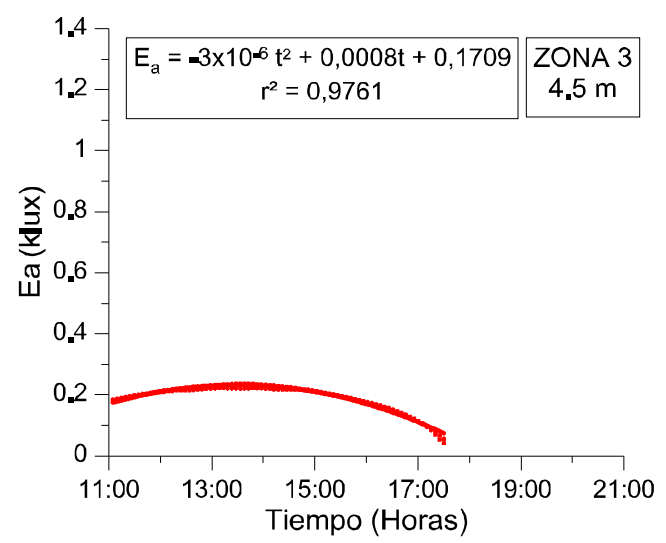

(h) $4.5 \mathrm{~m}$.

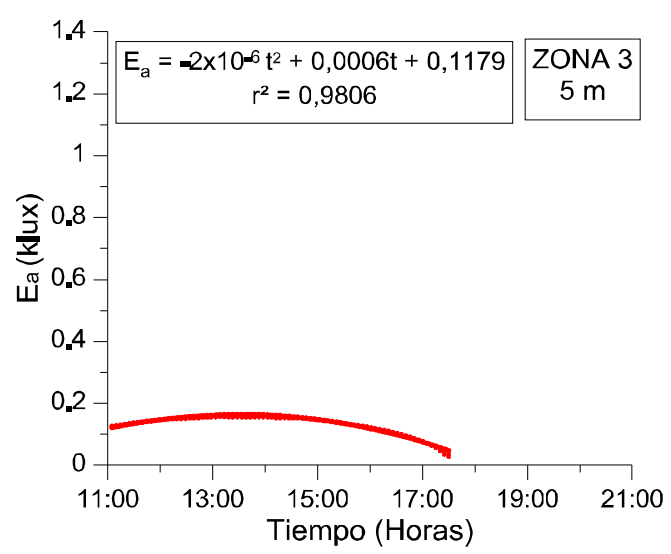

(i) $5 \mathrm{~m}$. 

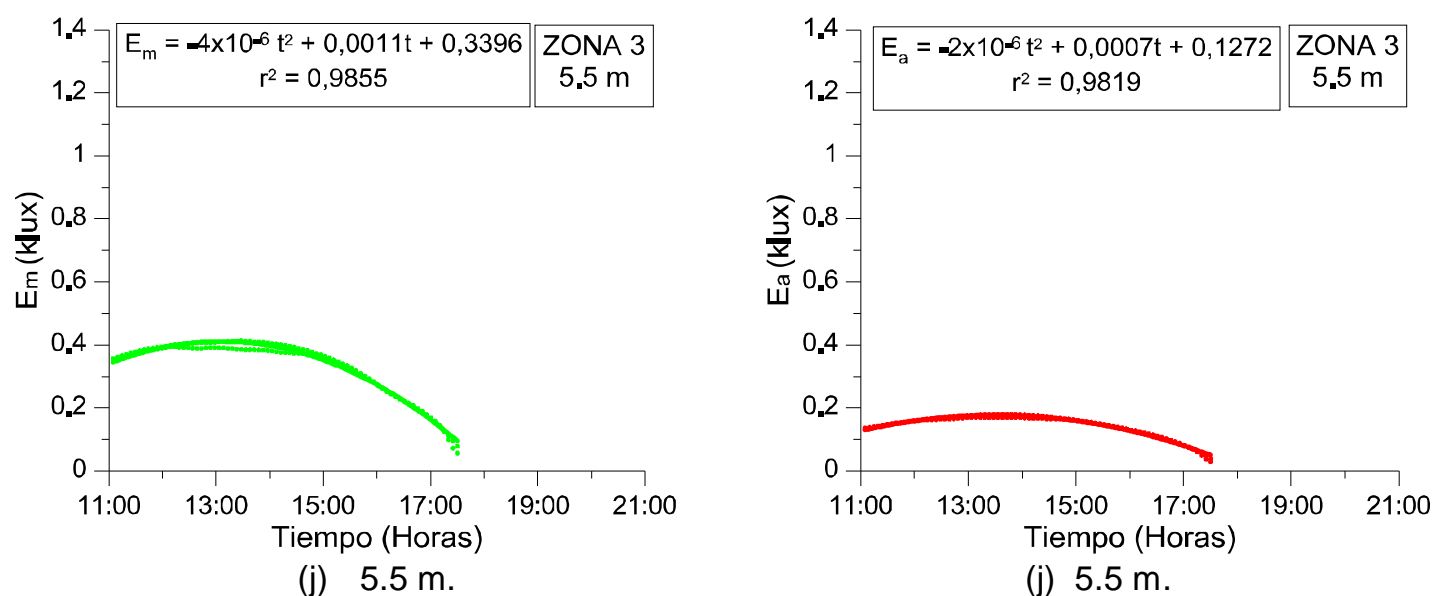

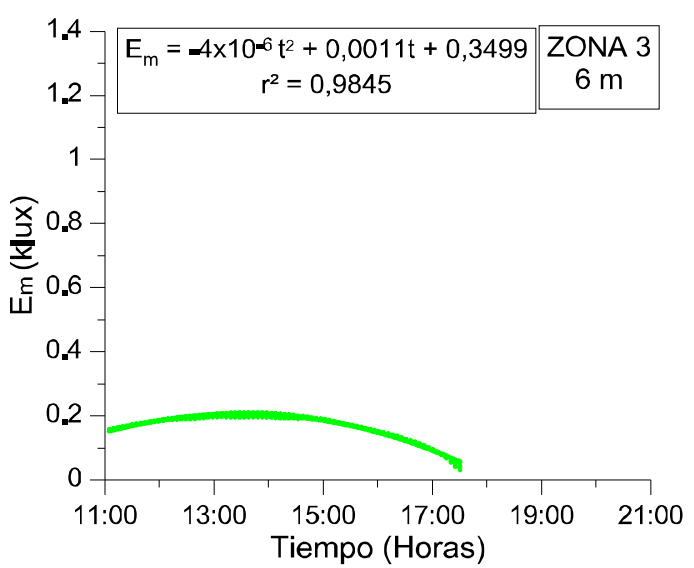

(k) $6 \mathrm{~m}$.

Figura 6.42. Evolución diaria de la iluminancia en el modelo a las distancias consideradas.

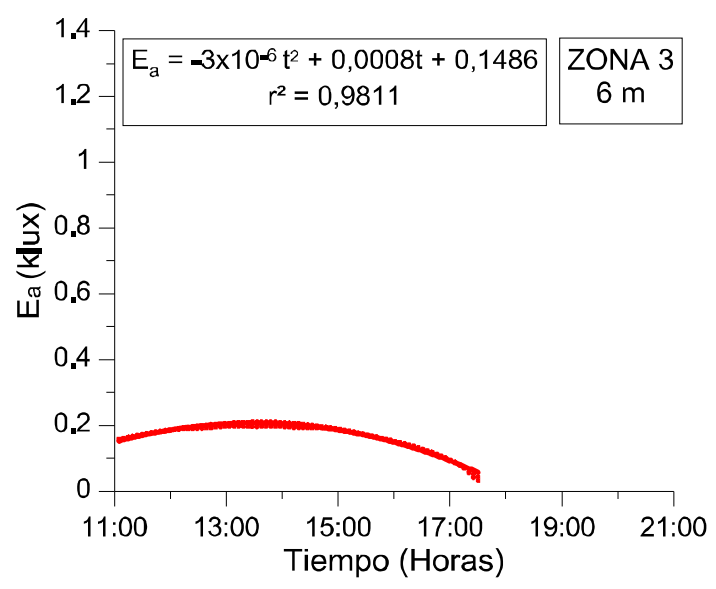

(k) $6 \mathrm{~m}$.

Figura 6.43. Evolución diaria de la iluminancia en el aula a las distancias consideradas

Como se puede apreciar en las gráficas, las iluminancias obtenidas en el modelo a lo largo del día son superiores a las obtenidas en el aula para todas las distancias consideradas.

A continuación, en las figuras 6.44 y 6.45 , se representan conjuntamente todas las curvas de ajuste, obtenidas anteriormente, tanto del modelo como del aula. En ellas se observa cómo a medida que aumenta la distancia a la fachada disminuye el valor de las iluminancias, salvo en el caso de los primeros sensores que al estar tan cerca de la fachada les llega menos luz de la ventana más próxima. 
MODELO

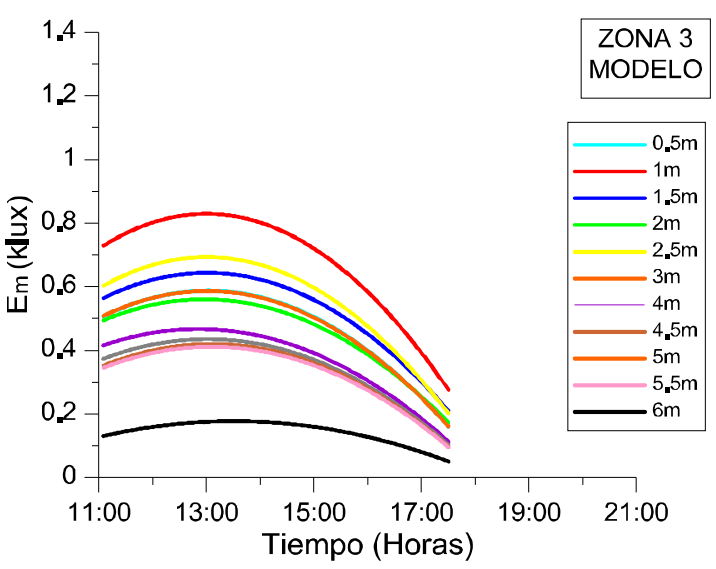

Figura 6.44. Curvas de ajuste de las iluminancias en el modelo a escala a las distancias consideradas.
AULA

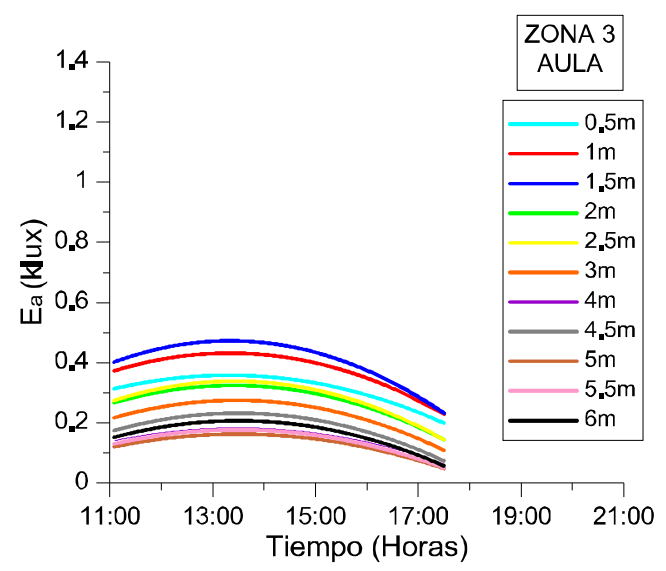

Figura 6.45. Curvas de ajuste de las iluminancias en el aula a las distancias consideradas

Las ecuaciones de las curvas ajustadas representadas anterior mente en las figuras 6.42 y 6.43 , así como el coeficiente de ajuste $\left(r^{2}\right)$, se han recogido en la tabla 6.10 .

\begin{tabular}{|c|c|c|c|c|}
\hline $\begin{array}{c}\text { ZONA 3 } \\
\begin{array}{c}\text { DISTANCIA } \\
(\mathbf{m})\end{array}\end{array}$ & ECUACIÓN & $r^{2}$ & ECUACIÓN & $r^{2}$ \\
\hline $\mathbf{0 . 5}$ & $E_{m}=-5 \times 10^{-6} t^{2}+0.0014 t+0.4991$ & 0.9911 & $E_{a}=-2 \times 10^{-6} t^{2}+0.0007 t+0.3101$ & 0.8414 \\
\hline $\mathbf{1}$ & $E_{m}=-7 \times 10^{-6} t^{2}+0.0018 t+0.7198$ & 0.9833 & $E_{a}=-3 \times 10^{-6} t^{2}+0.0009 t+0.3687$ & 0.8708 \\
\hline $\mathbf{1 . 5}$ & $E_{m}=-6 \times 10^{-6} t^{2}+0.0014 t+0.5568$ & 0.9844 & $E_{a}=-4 \times 10^{-6} t^{2}+0.0011 t+0.397$ & 0.9063 \\
\hline $\mathbf{2}$ & $E_{m}=-5 \times 10^{-6} t^{2}+0.0012 t+0.4882$ & 0.9855 & $E_{a}=-3 \times 10^{-6} t^{2}+0.0008 t+0.2630$ & 0.9334 \\
\hline $\mathbf{2 . 5}$ & $E_{m}=-7 \times 10^{-6} t^{2}+0.0016 t+0.5950$ & 0.987 & $E_{a}=-3 \times 10^{-6} t^{2}+0.0009 t+0.2702$ & 0.939 \\
\hline $\mathbf{3}$ & $E_{m}=-6 \times 10^{-6} t^{2}+0.0014 t+0.5011$ & 0.9874 & $E_{a}=-3 \times 10^{-6} t^{2}+0.0008 t+0.2131$ & 0.9524 \\
\hline $\mathbf{4}$ & $E_{m}=-4 \times 10^{-6} t^{2}+0.001 t+0.4108$ & 0.9868 & $E_{a}=-2 \times 10^{-6} t^{2}+0.0006 t+0.1378$ & 0.9694 \\
\hline $\mathbf{4 . 5}$ & $E_{m}=-4 \times 10^{-6} t^{2}+0.0011 t+0.3679$ & 0.9866 & $E_{a}=-3 \times 10^{-6} t^{2}+0.0008 t+0.1709$ & 0.9761 \\
\hline $\mathbf{5}$ & $E_{m}=-5 \times 10^{-6} t^{2}+0.0012 t+0.3463$ & 0.9872 & $E_{a}=-2 \times 10^{-6} t^{2}+0.0006 t+0.1179$ & 0.9806 \\
\hline $\mathbf{5 . 5}$ & $E_{m}=-4 \times 10^{-6} t^{2}+0.0011 t+0.3396$ & 0.9855 & $E_{a}=-2 \times 10^{-6} t^{2}+0.0007 t+0.1272$ & 0.9819 \\
\hline $\mathbf{6}$ & $E_{m}=-4 \times 10^{-6} t^{2}+0.0011 t+0.3499$ & 0.9845 & $E_{a}=-3 \times 10^{-6} t^{2}+0.0008 t+0.1486$ & 0.9811 \\
\hline
\end{tabular}

Tabla 6.10. Ecuaciones de ajuste de segundo grado de las iluminancias diarias a distintas distancias tanto del modelo como del aula.

En esta tabla se puede observar que prácticamente todos los coeficientes de ajuste son superiores 0,9 lo que indica un alto grado de fiabilidad. 


\subsubsection{Relación entre las iluminancias del aula y del modelo a escala en} función de las horas del día

Para saber si existe una relación lineal entre las iluminancias del aula y del modelo a escala, en este apartado se va a analizar el cociente de ambas con respecto al tiempo para las distintas distancias consideradas. Se han representado, en la figura 6.46, las gráficas del cociente obtenido entre las iluminancias del aula y del modelo, frente al tiempo (expresado en horas), según las distintas distancias a la fachada. La ecuación de la curva obtenida para cada distancia así como su correspondiente coeficiente de ajuste también se muestran en cada gráfica.

\section{ZONA 3}

MODELO

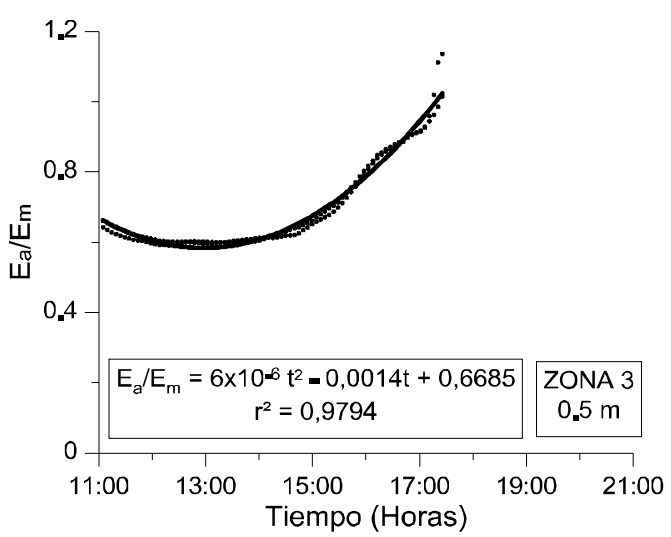

(a) $0.5 \mathrm{~m}$.

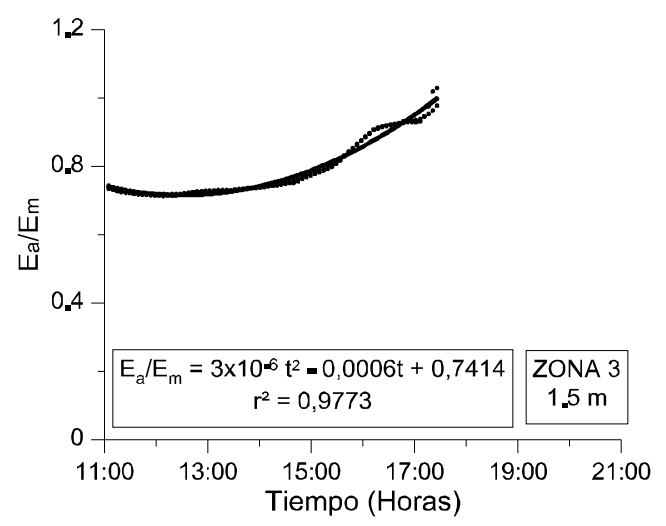

(c) $1.5 \mathrm{~m}$.

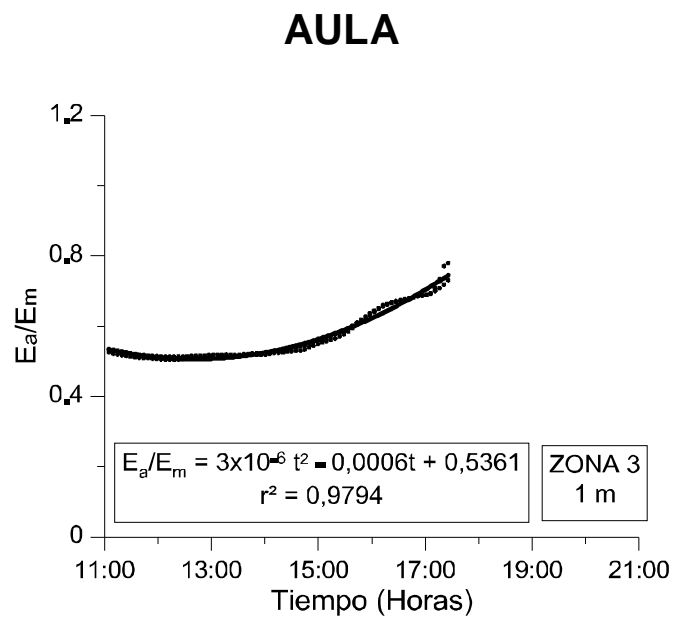

(b) $1 \mathrm{~m}$.

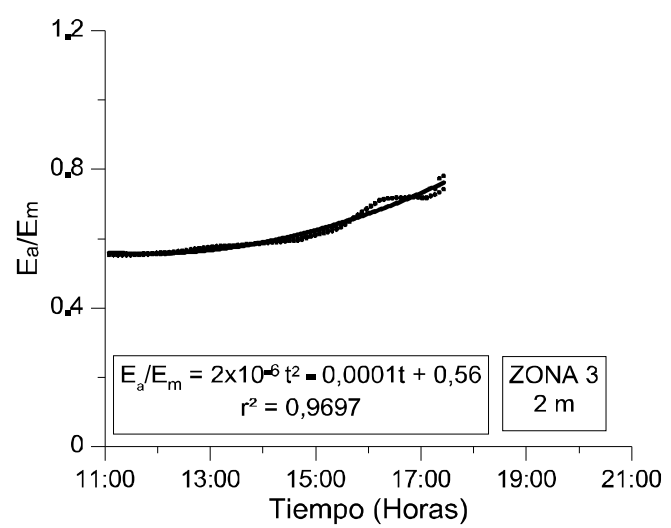

(d) $2 \mathrm{~m}$. 
Estudio experimental y modelización de la iluminación natural en la edificación mediante modelos a escala

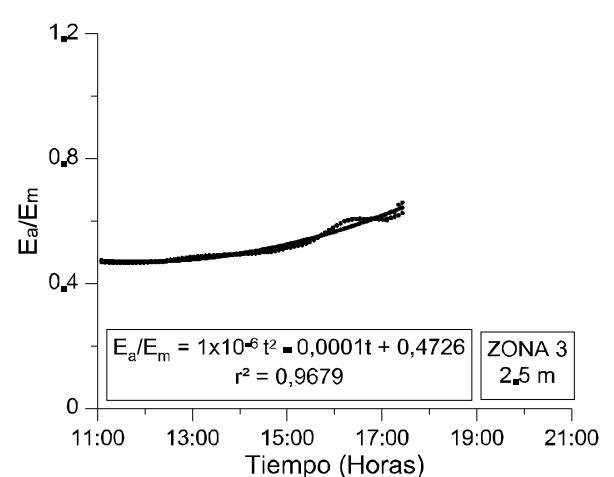

(e) $2.5 \mathrm{~m}$.

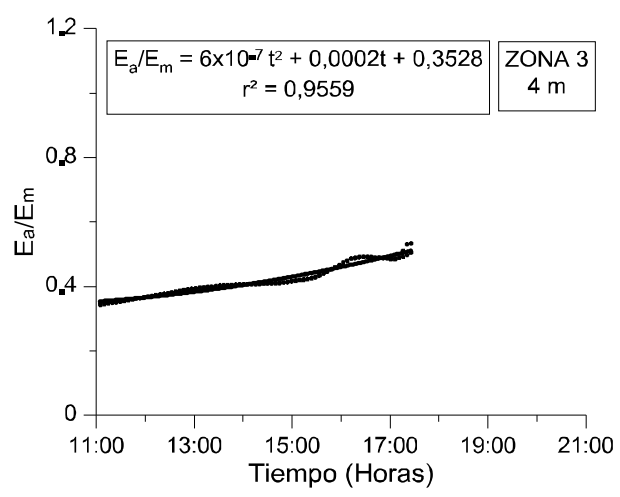

(g) $4 \mathrm{~m}$.

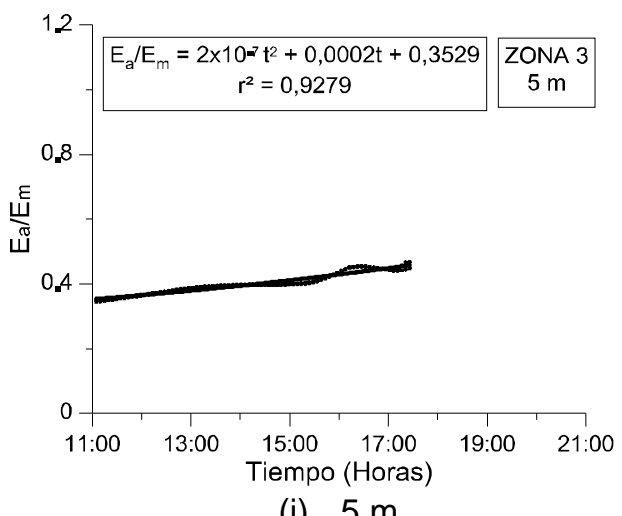

(i) $5 \mathrm{~m}$.

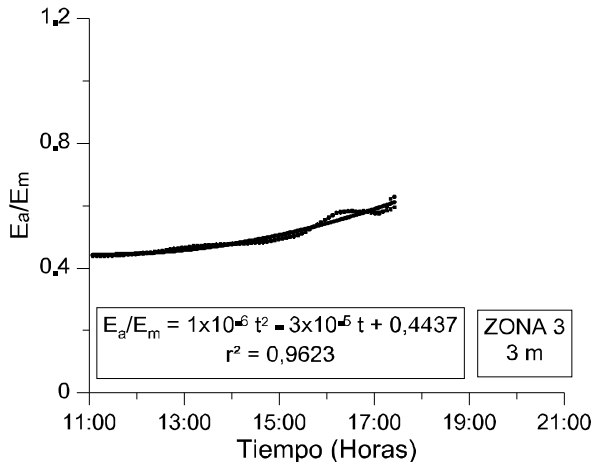

(f) $3 \mathrm{~m}$.

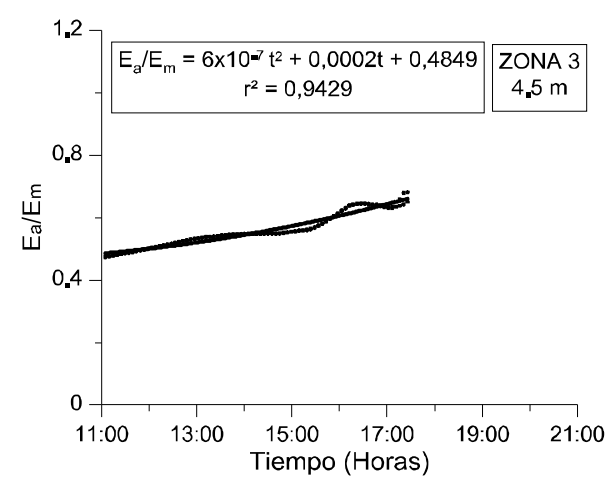

(h) $4.5 \mathrm{~m}$.

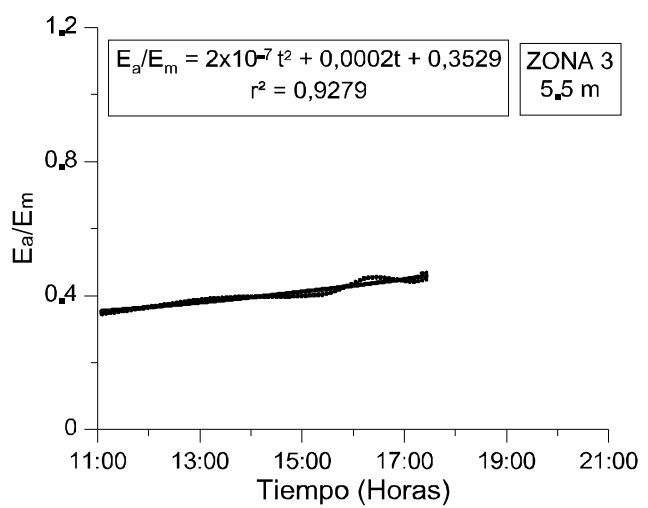

(j) $5.5 \mathrm{~m}$.

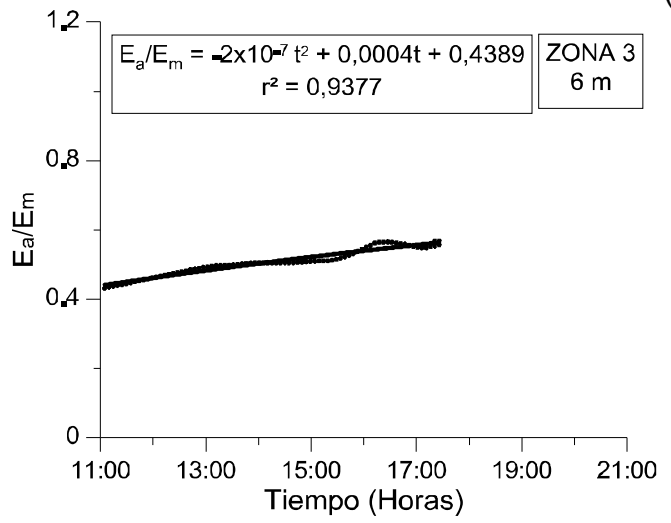

(k) $6 \mathrm{~m}$.

Figura 6.46. Cociente de las iluminancias del aula entre las del modelo frente al tiempo a las distancias indicadas. 
Se puede observar que no existe una relación lineal entre las iluminancias del aula y las del modelo. Para todas las distancias se sigue una ecuación de segundo grado.

A continuación se muestran de forma conjunta las curvas para todas las distancias, de los cocientes entre las iluminancias del aula y del modelo a escala frente al tiempo (figura 6.47). Se puede apreciar cómo según aumenta la distancia a la ventana la curvatura va disminuyendo.

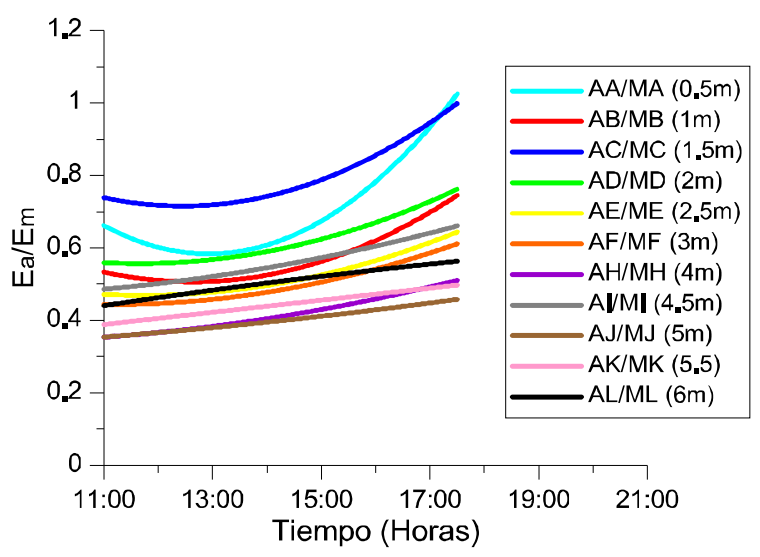

Figura 6.47. Curvas ajustadas de los cocientes las iluminancias aula/modelo frente al tiempo a las distintas distancias.

Las ecuaciones obtenidas de la representación del cociente de las iluminancias aula/modelo y el tiempo, así como su correspondiente coeficiente de ajuste, se muestran a continuación en la tabla 6.11. En dicha tabla se puede apreciar cómo todos los coeficientes de ajuste son superiores a 0.9 para todas las distancias consideradas, lo que indica una alta fiabilidad de los resultados.

\begin{tabular}{|c|c|c|}
\hline DISTANCIA (m) & ECUACIÓN & $\mathbf{r}^{2}$ \\
\hline $\mathbf{0 . 5}$ & $\mathrm{E}_{\mathrm{a}} / \mathrm{E}_{\mathrm{m}}=6 \times 10^{-6} \mathrm{t}^{2}-0.0014 \mathrm{t}+0.6685$ & 0.9767 \\
\hline $\mathbf{1}$ & $\mathrm{E}_{\mathrm{a}} / \mathrm{E}_{\mathrm{m}}=3 \times 10^{-6} \mathrm{t}^{2}-0.0006 \mathrm{t}+0.5361$ & 0.9794 \\
\hline $\mathbf{1 . 5}$ & $\mathrm{E}_{\mathrm{a}} / \mathrm{E}_{\mathrm{m}}=3 \times 10^{-6} \mathrm{t}^{2}-0.0006 \mathrm{t}+0.7414$ & 0.9773 \\
\hline $\mathbf{2}$ & $\mathrm{E}_{\mathrm{a}} / \mathrm{E}_{\mathrm{m}}=2 \times 10^{-6} \mathrm{t}^{2}-0.0001 \mathrm{t}+0.56$ & 0.9697 \\
\hline $\mathbf{2 . 5}$ & $\mathrm{E}_{\mathrm{a}} / \mathrm{E}_{\mathrm{m}}=1 \times 10^{-6} \mathrm{t}^{2}-0.0001 \mathrm{t}+0.4726$ & 0.9679 \\
\hline $\mathbf{3}$ & $\mathrm{E}_{\mathrm{a}} / \mathrm{E}_{\mathrm{m}}=1 \times 10^{-6} \mathrm{t}^{2}-0.00003 \mathrm{t}+0.4437$ & 0.9623 \\
\hline $\mathbf{4}$ & $\mathrm{E}_{\mathrm{a}} / \mathrm{E}_{\mathrm{m}}=6 \times 10^{-7} \mathrm{t}^{2}+0.0002 \mathrm{t}+0.3528$ & 0.9559 \\
\hline $\mathbf{4 . 5}$ & $\mathrm{E}_{\mathrm{a}} / \mathrm{E}_{\mathrm{m}}=6 \times 10^{-7} \mathrm{t}^{2}+0.0002 \mathrm{t}+0.4849$ & 0.9429 \\
\hline $\mathbf{5}$ & $\mathrm{E}_{\mathrm{a}} / \mathrm{E}_{\mathrm{m}}=2 \times 10^{-7} \mathrm{t}^{2}+0.0002 \mathrm{t}+0.3529$ & 0.9279 \\
\hline $\mathbf{5 , 5}$ & $\mathrm{E}_{\mathrm{a}} / \mathrm{E}_{\mathrm{m}}=-6 \times 10^{-9} \mathrm{t}^{2}+0.0003 \mathrm{t}+0,3873$ & 0.9267 \\
\hline $\mathbf{6}$ & $\mathrm{E}_{\mathrm{a}} / \mathrm{E}_{\mathrm{m}}=-2 \times 10^{-7} \mathrm{t}^{2}+0.0004 \mathrm{t}+0.4389$ & 0.9377 \\
\hline
\end{tabular}

Tabla 6.11. Ecuaciones, a las distintas distancias, de la relaciones entre el cociente de las iluminancias aula/ modelo y el tiempo. 


\subsubsection{Relación entre iluminancias del aula y del modelo en función de la iluminancia exterior}

Como en las zonas anteriores, se va a analizar la relación entre las iluminancias del aula $\left(E_{a}\right)$ y del modelo $\left(E_{m}\right)$ en función de la iluminancia exterior $\left(E_{\text {ext }}\right)$, expresada en klux, teniendo en cuenta los datos utilizados anteriormente, para las distintas distancias desde el interior a la fachada. En las gráficas de la figura 6.48 se ha representado en el eje vertical el cociente entre las iluminancias del aula y el modelo, y en el eje horizontal las iluminancias obtenidas con el sensor exterior.

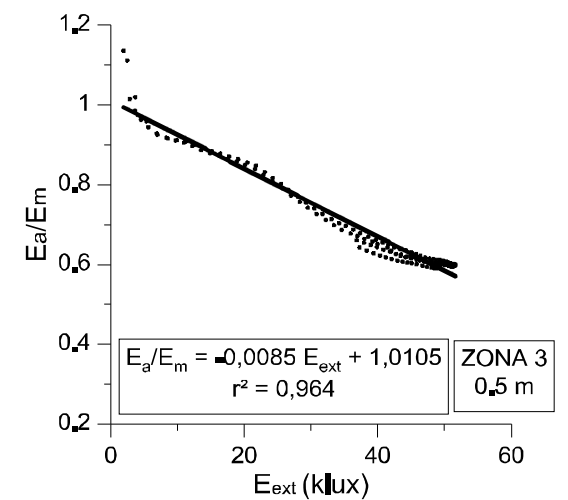

(a) $0.5 \mathrm{~m}$.

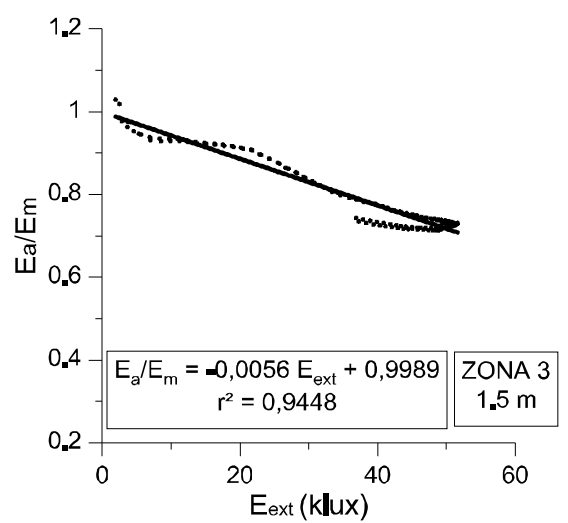

(c) $1.5 \mathrm{~m}$.

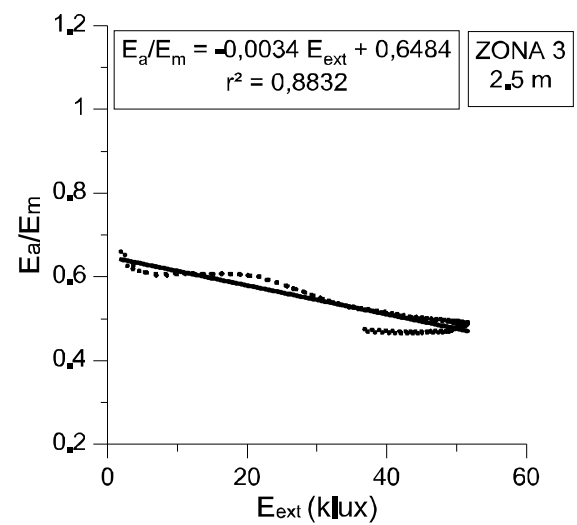

(e) $2.5 \mathrm{~m}$.

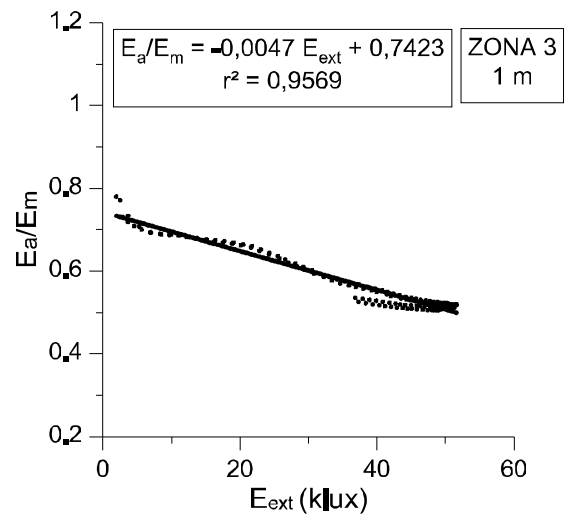

(b) $1 \mathrm{~m}$.

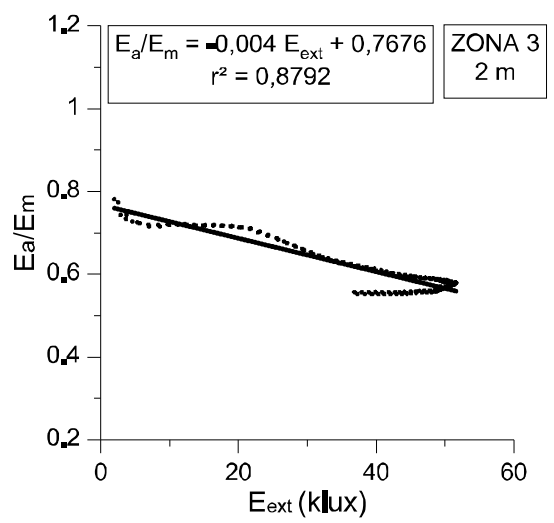

(d) $2 \mathrm{~m}$.

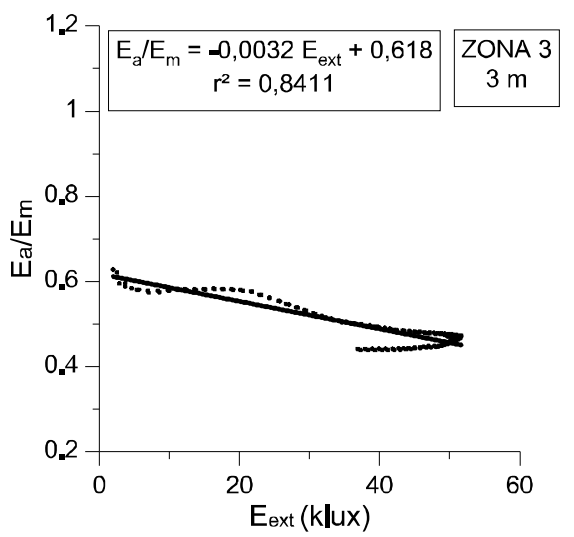

(f) $3 \mathrm{~m}$. 


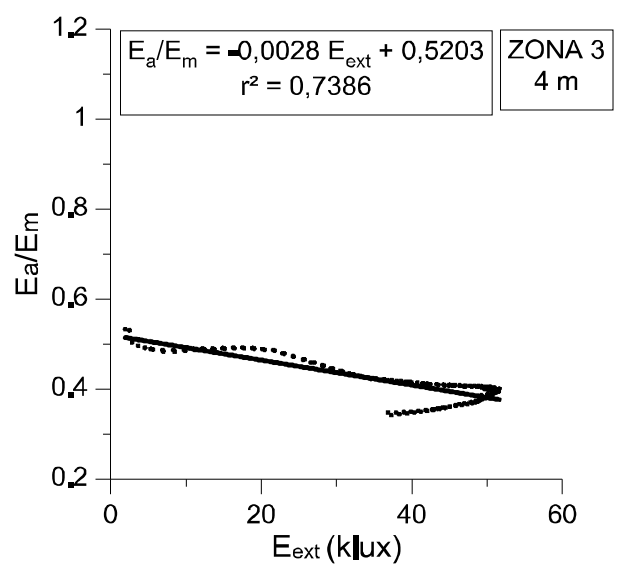

(g) $4 \mathrm{~m}$.

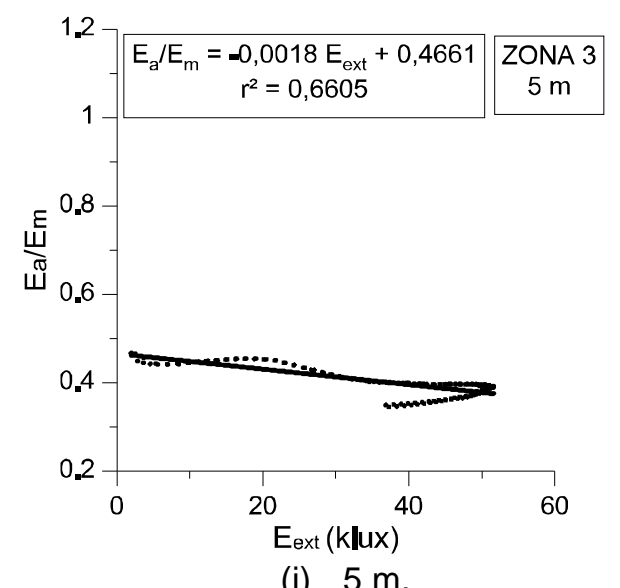

(i) $5 \mathrm{~m}$.

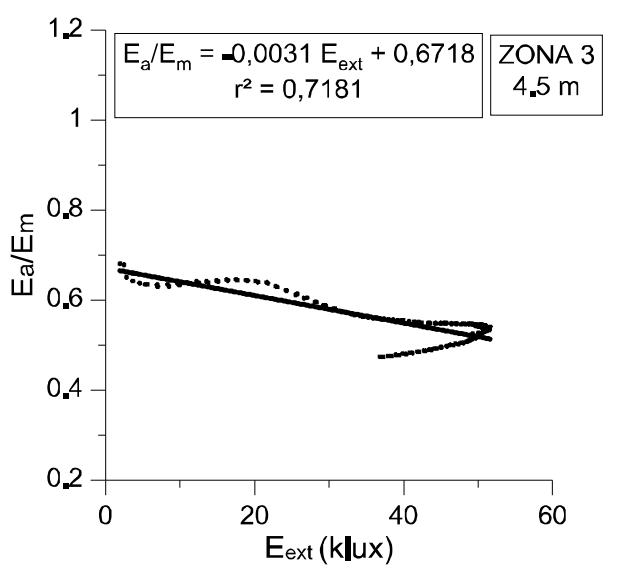

(h) $4.5 \mathrm{~m}$.

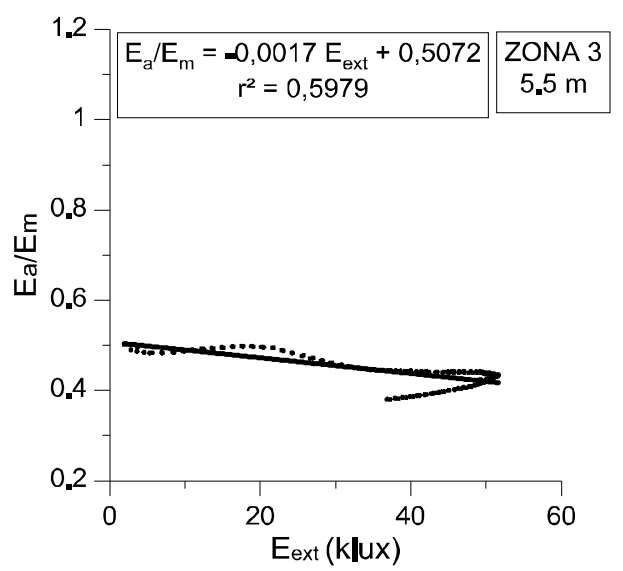

(j) $5.5 \mathrm{~m}$.

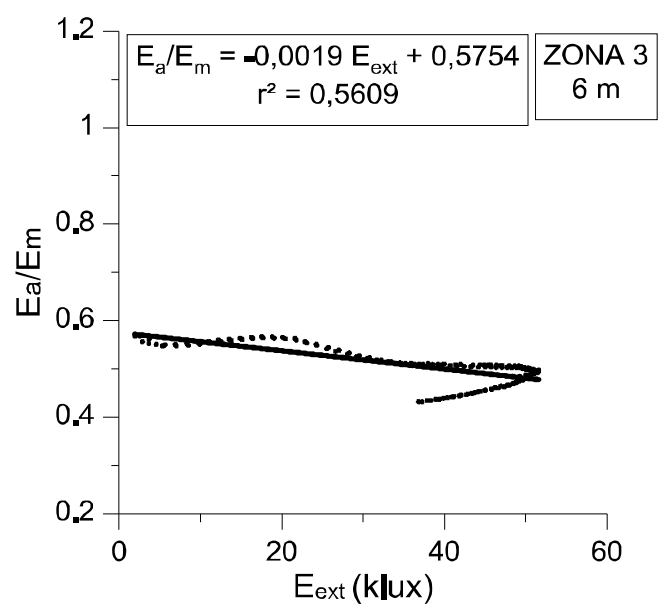

(k) $6 \mathrm{~m}$.

Figura 6.48. Cociente de las iluminancias del aula y del modelo frente a la iluminancia exterior a las distancias indicadas.

Se observa que la relación del cociente iluminancias aula/modelo frente a la iluminancia exterior, para cada distancia, es lineal. A medida que aumenta la distancia a la ventana el cociente iluminancia aula/modelo va disminuyendo. 
La representación conjunta de todas las rectas ajustadas anteriores, para las distancias consideradas, se muestra en la figura 6.49.

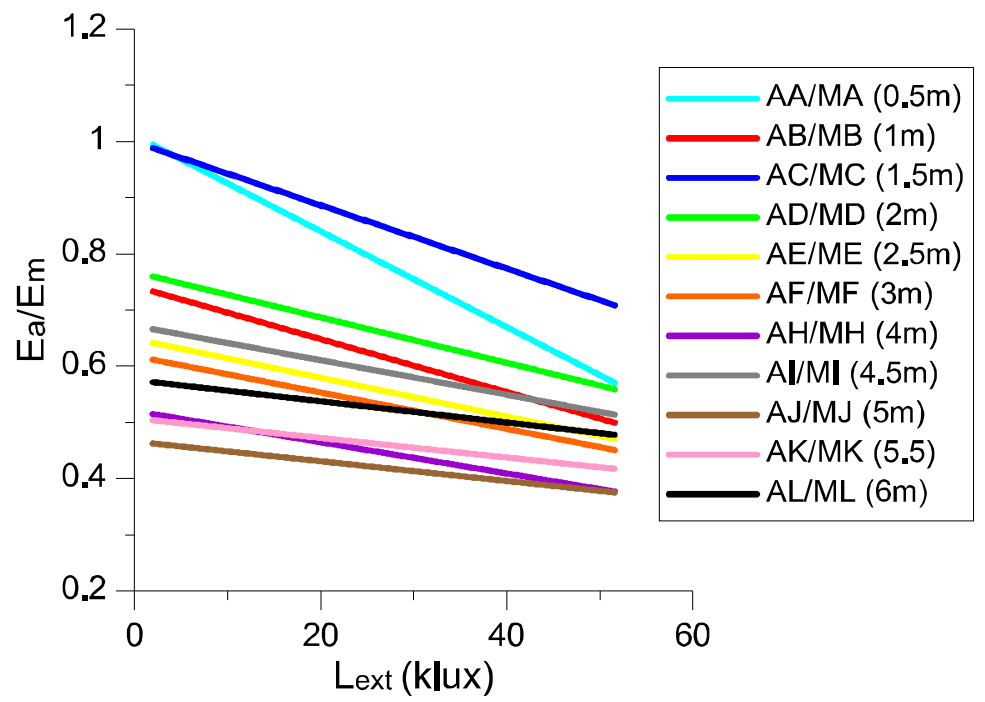

Figura 6.49. Rectas de ajuste a las distintas distancias de la relaciones entre el cociente de las iluminancias aula/ modelo frente a la iluminancia exterior.

De esta gráfica se deduce que al aumentar la distancia interior a la fachada, la pendiente de estas rectas va disminuyendo.

Las ecuaciones correspondientes a las rectas anteriores, así como su coeficiente de ajuste $\left(r^{2}\right)$ se recogen en la tabla 6.12. En ésta se puede apreciar cómo según aumenta la distancia a la ventana el coeficiente de ajuste va disminuyendo.

\begin{tabular}{|c|c|c|}
\hline DISTANCIA (m) & ECUACIÓN & $\mathbf{r}^{2}$ \\
\hline 0.5 & $E_{a} / E_{m}=-0,0085 E_{e x t}+1.0105$ & 0.964 \\
\hline$\overline{1}$ & $E_{a} / E_{m}=-0,0047 E_{e x t}+0.7423$ & 0.9569 \\
\hline 1.5 & $E_{a} / E_{m}=-0,0056 E_{e x t}+0.9989$ & 0.9448 \\
\hline 2 & $E_{a} / E_{m}=-0,004 E_{e x t}+0.7676$ & 0.8792 \\
\hline 2.5 & $E_{a} / E_{m}=-0,0034 E_{e x t}+0.6484$ & 0.8832 \\
\hline 3 & $E_{a} / E_{m}=-0,0032 E_{e x t}+0.618$ & 0.8411 \\
\hline 4 & $E_{a} / E_{m}=-0,0028 E_{e x t}+0.5203$ & 0.7386 \\
\hline 4.5 & $E_{a} / E_{m}=-0,0031 E_{e x t}+0.6718$ & 0.7181 \\
\hline 5 & $E_{a} / E_{m}=-0,0018 E_{e x t}+0,4661$ & 0.6605 \\
\hline 5.5 & $E_{a} / E_{m}=-0,0017 E_{e x t}+0,5072$ & 0.5979 \\
\hline 6 & $E_{a} / E_{m}=-0,0019 E_{e x t}+0.5754$ & 0.5609 \\
\hline
\end{tabular}

Tabla 6.12. Ecuaciones que relacionan las iluminancias del aula y el modelo con las del exterior a las distancias consideradas. 


\subsubsection{Modelo matemático para determinar la iluminancia en el aula}

Procediendo como en las zonas anteriores se han obtenido unas rectas que relacionan las iluminancias entre el aula $\left(E_{a}\right)$ y el modelo $\left(E_{m}\right)$ en función de la iluminancia exterior $\left(E_{\text {ext }}\right)$, para las distintas distancias desde la fachada a puntos del interior, de la forma:

$$
\frac{E_{a}}{E_{m}}=P \cdot E_{e x t}+O
$$

Siendo $P$ la Pendiente y $O$ la Ordenada en el origen de dichas rectas.

También en esta zona se busca una expresión que permita relacionar las variables anteriores con la distancia interior a la fachada. Para ello se van a analizar, por separado, las pendientes y las ordenadas en el origen de las ecuaciones de las rectas recogidas en la tabla 6.12 en función de la distancia. Estos datos se muestran en la tabla 6.13.

\begin{tabular}{|c|c|c|}
\hline Distancia $(\mathbf{m})$ & Pendiente & Ordenada \\
\hline 0,5 & $-0,0085$ & 1,0105 \\
\hline 1 & $-0,0047$ & 0,7423 \\
\hline 1,5 & $-0,0056$ & 0,9989 \\
\hline 2 & $-0,004$ & 0,7676 \\
\hline 2,5 & $-0,0034$ & 0,6484 \\
\hline 3 & $-0,0032$ & 0,618 \\
\hline 4 & $-0,0028$ & 0,5203 \\
\hline 4,5 & $-0,0031$ & 0,6718 \\
\hline 5 & $-0,0018$ & 0,4661 \\
\hline 5,5 & $-0,0017$ & 0,5072 \\
\hline 6 & $-0,0019$ & 0,5754 \\
\hline
\end{tabular}

Tabla 6.13. Pendientes y ordenadas en el origen a las distintas distancias de las rectas de ajuste procedentes del cociente de las iluminancias aula/ modelo frente a la iluminancia exterior.

La variación de la pendiente $(P)$ con la distancia $(d)$ se muestra en la figura 6.50. Es una variación lineal que se ajusta a la recta: $P=(0.924 d-6.685) \times 10^{-3}$ con $r^{2}=0.761$. La variación de la ordenada en el origen $(O)$ con la distancia $(d)$, que se muestra en la figura 6.51, también se ajusta a una recta, cuya ecuación es: $\mathrm{O}=-0.080 \mathrm{~d}+0.943$ con $r^{2}=0.674$. 


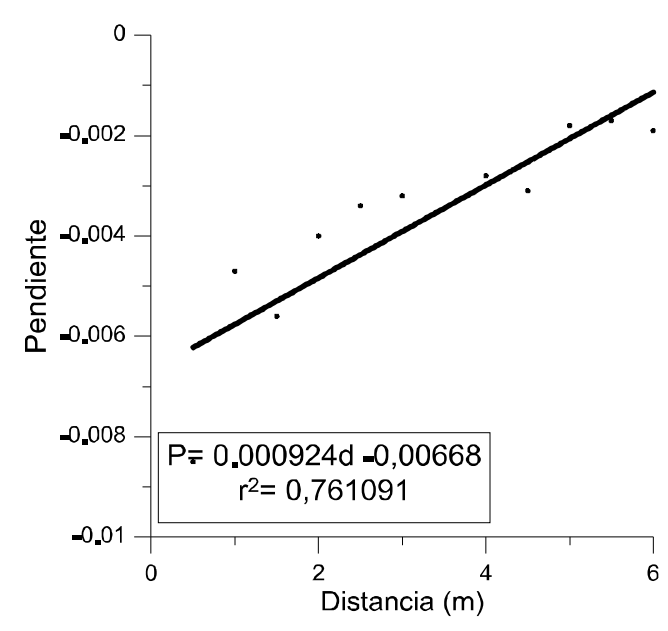

Figura 6.50. Pendiente en función de la distancia a la fachada.

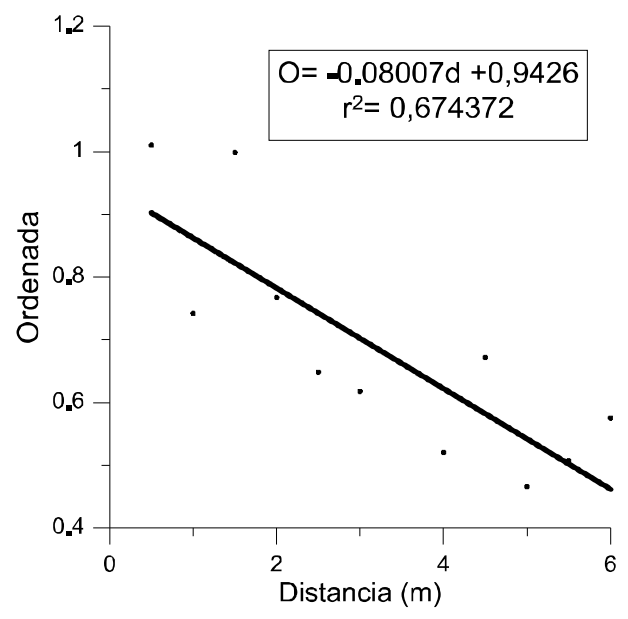

Figura 6.51. Ordenada en el origen en función de la distancia a la fachada.

En consecuencia el modelo matemático que permite determinar la iluminancia del aula a partir de la del modelo a escala, la exterior y la distancia es:

$$
E_{a}=\left[(0.925 d-6.685) \times 10^{-3} E_{e x t}-0.080 d+0.943\right] E_{m} \quad[Z 3]
$$

Siendo:

$E_{a}$ : lluminancia del aula (klux).

$E_{m}$ : lluminancia del modelo (klux).

$E_{\text {ext }}$ : lluminancia exterior (klux).

$d$ : Distancia a la fachada $(\mathrm{m})$

De esta manera se alcanza el objetivo de poder conocer las iluminancias de una estancia real (el aula) sin necesidad de medir en dicha estancia; bastaría conocer las iluminancias en el exterior y en el interior de la maqueta de la estancia estudiada así como las distancias a la que se hayan colocado los sensores.

\subsubsection{Análisis estadístico}

Para comprobar la fiabilidad del modelo matemático obtenido en esta zona se va a realizar un análisis estadístico de los valores obtenidos para las pendientes y las ordenadas en el origen a las distintas distancias. 
Se han analizado por separado la pendiente (eje vertical) frente a la distancia a la fachada (eje horizontal) y la ordenada en el origen (eje vertical) frente a la distancia a la fachada (eje horizontal).

En la gráfica en la que se han representado las pendientes de las rectas obtenidas $(P)$ en función de la distancia a la fachada (d) (figura 6.52) se observa que esta relación es lineal, que se ajusta a la recta con pendiente positiva: $P=(0.924 d-6.685) \times 10^{-3}$, como se había obtenido anteriormente. Se observa que la mayor parte de los datos se encuentran dentro de los límites de confianza al 95\% (verde) y todos los datos se encuentran entre los límites de predicción (rojo) por lo que no se aprecian valores atípicos.

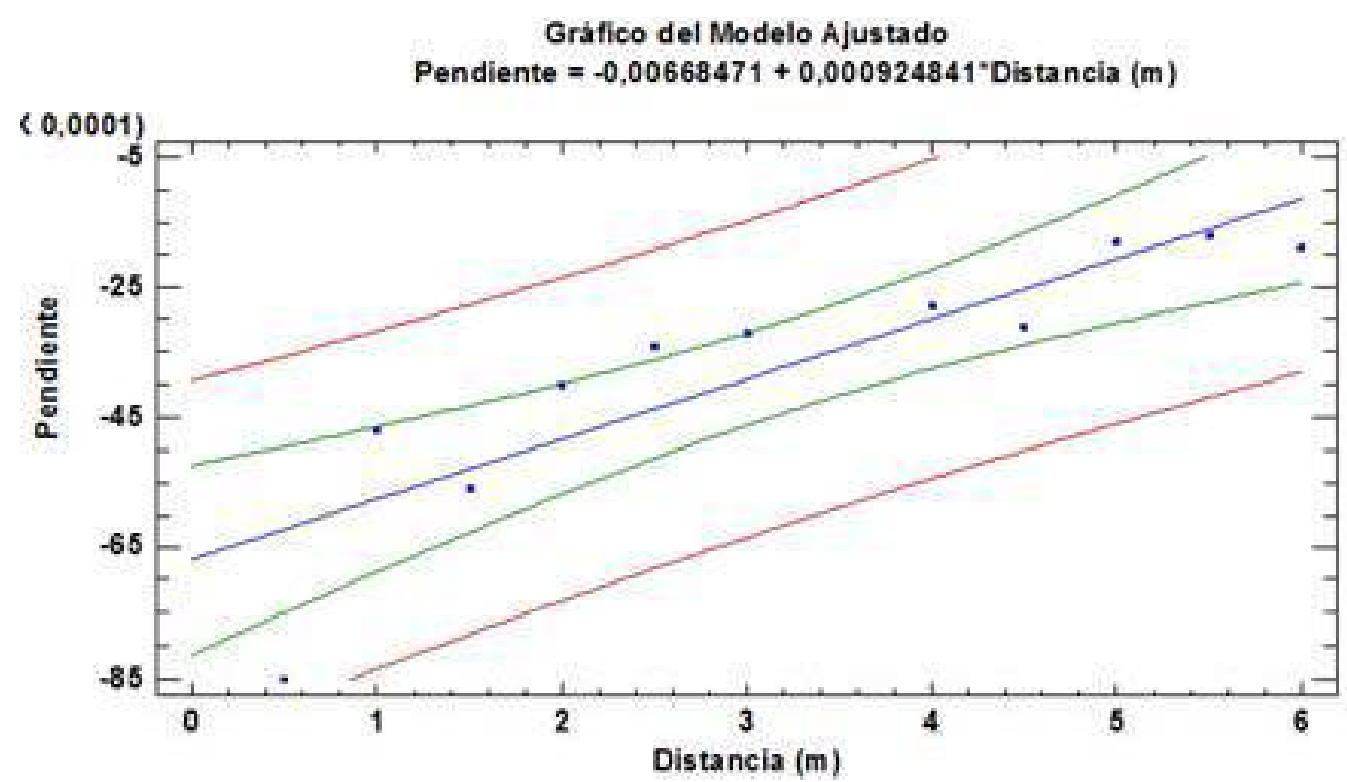

Figura 6.52. Gráfico del modelo ajustado de la pendiente en función de la distancia a la fachada.

En la gráfica de las ordenadas en el origen $(O)$ en función de la distancia a la fachada (d) (figura 6.53) también se obtiene que esta relación es lineal, que se ajusta a una recta con pendiente negativa: $\mathrm{O}=-0.080 \mathrm{~d}+0.943$. La mayor parte de los datos se encuentran dentro de los límites de confianza al 95\% (verde) y todos los datos se encuentran entre los límites de predicción (rojo), por lo que no se aprecian valores atípicos. 


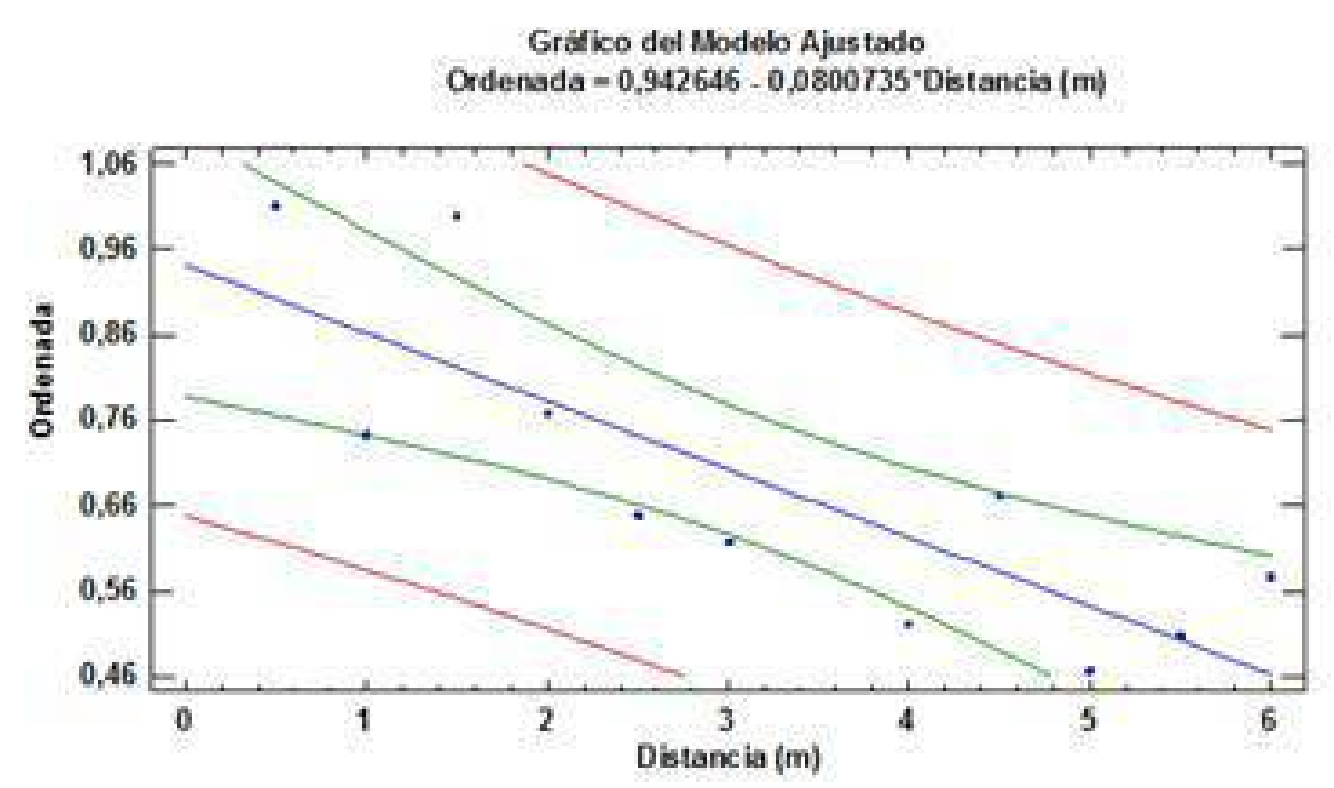

Figura 6.53. Gráfico del modelo ajustado de la ordenada en el origen, frente a la distancia a la fachada.

En ambos casos se obtiene un p-valor de $0.00<0.5$, lo que indica que hay una relación estadística significante entre las variables consideradas con un nivel de confianza de por lo menos el $95 \%$, es decir que la fiabilidad de los datos es $\geq 95 \%$. En el análisis de los residuos el p-valor es superior a 0.05 lo que indica que la probabilidad de que haya errores en este análisis es menor al $5 \%$.

\subsubsection{Simulación con software de iluminación y comprobación de resultados}

De forma análoga a como se ha realizado en las zonas 1 y 2 , se van a comparar las iluminancias del aula obtenidas midiendo directamente en el aula, mediante la ecuación Z3 y con un programa de simulación (Dialux 4.11), entre las 11:00 y las 16:00 horas. En este caso las gráficas se han acortado hasta las 16:00 horas porque el programa de simulación (Dialux 4.11) sólo da información en esta fecha hasta esta hora.

En la figura 6.54 se muestran gráficamente los resultados obtenidos en los tres casos para las distintas distancias consideradas en el estudio. Se han representado en rojo las iluminancias medidas directamente, en azul claro las obtenidas de la ecuación Z3 y en azul oscuro las del programa de simulación. 


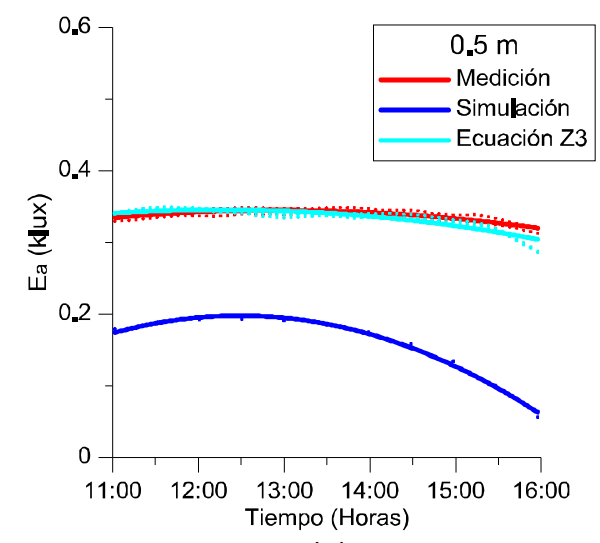

(a) $0.5 \mathrm{~m}$.

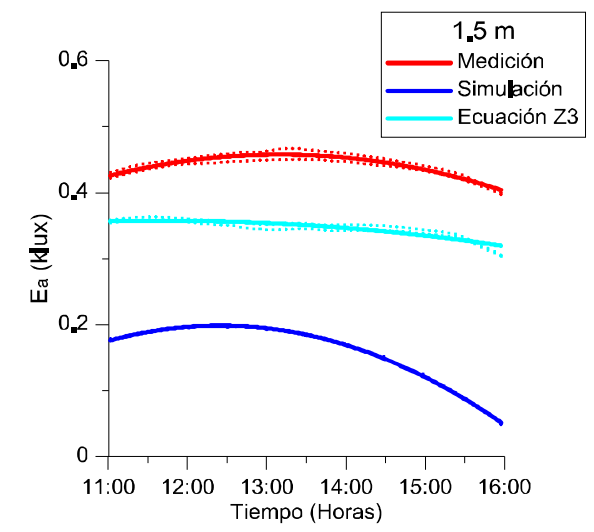

(c) $1.5 \mathrm{~m}$.

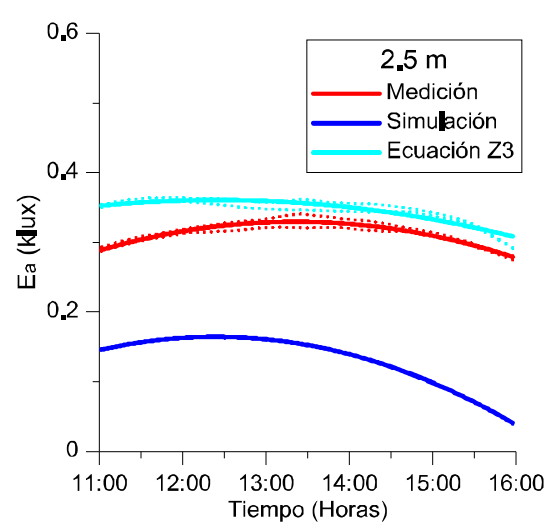

(e) $2.5 \mathrm{~m}$.

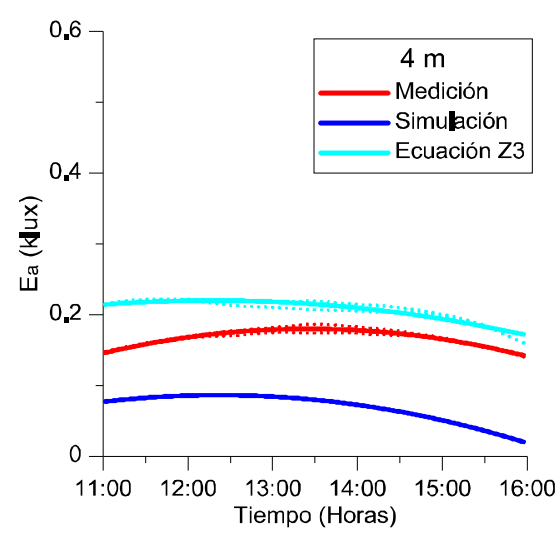

(g) $4 \mathrm{~m}$.

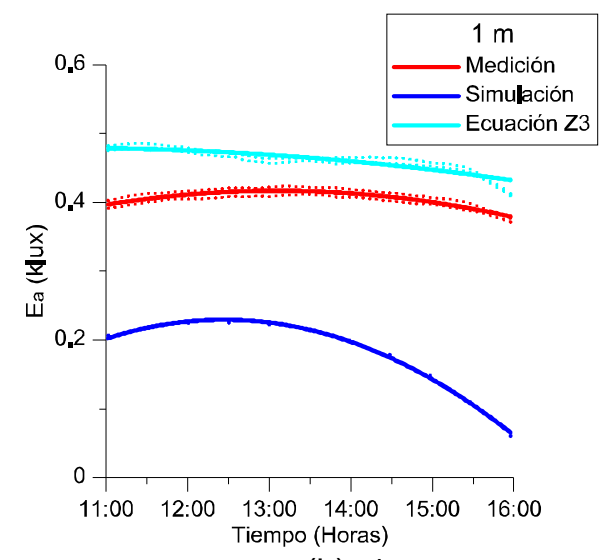

(b) $1 \mathrm{~m}$.

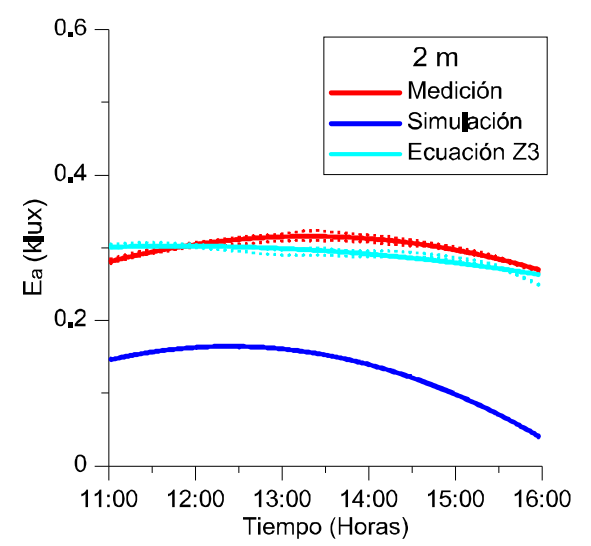

(d) $2 \mathrm{~m}$.

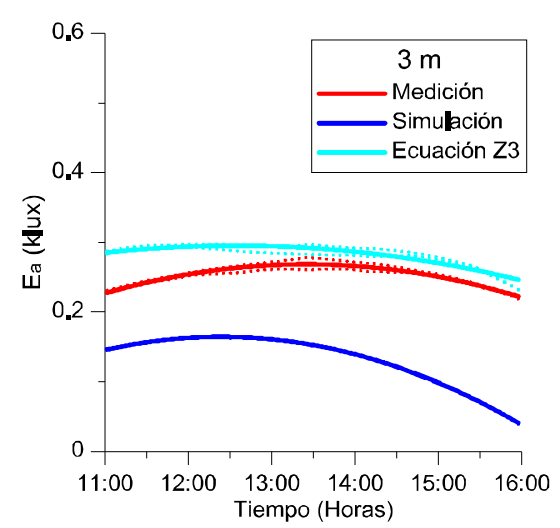

(f) $3 \mathrm{~m}$.

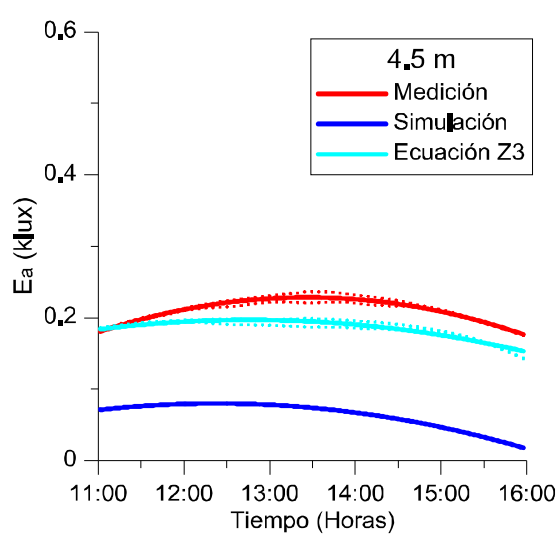

(h) $4.5 \mathrm{~m}$. 


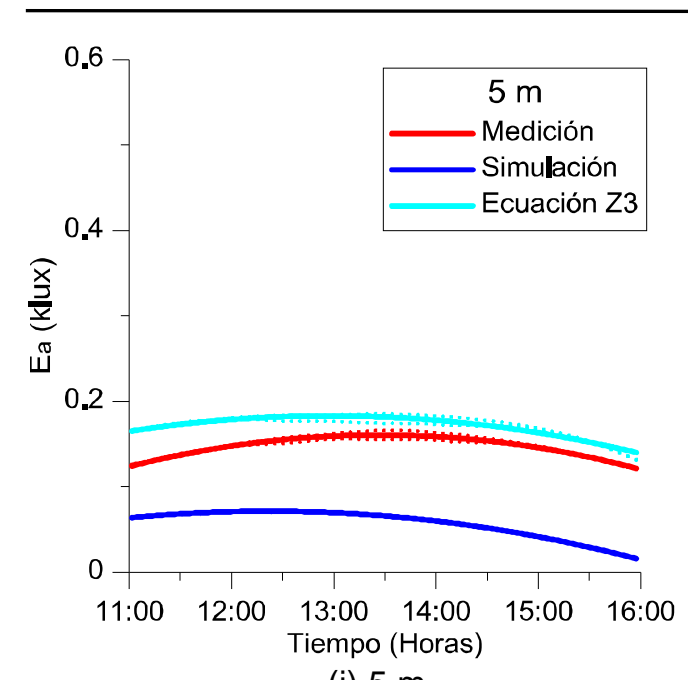

(i) $5 \mathrm{~m}$.

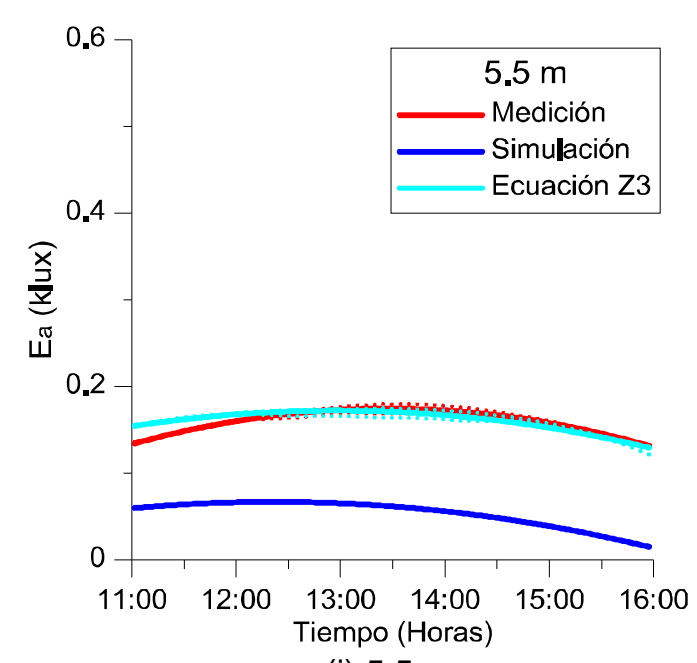

(j) $5.5 \mathrm{~m}$.

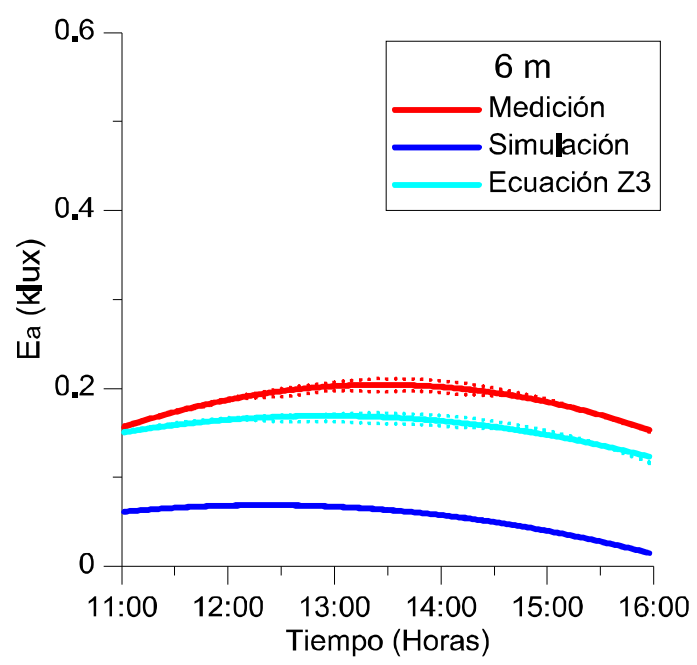

(k) $6 \mathrm{~m}$.

Figura 6.55. Iluminancias en el aula obtenidas de aplicar la ecuación Z3, por simulación y de forma experimental a las distancias de la fachada indicadas.

En la figura 6.55 se observa que para todas las distancias consideradas las iluminancias del aula obtenidas mediante la ecuación Z3 siguen un comportamiento similar al de los valores obtenidos por medición mientras que los de simulación son ligeramente inferiores.

Como complemento a este análisis comparativo, se van a representar a continuación los resultados obtenidos para todas las distancias simultáneamente a las siguientes horas: 11:00, 12:00, 13:00, 14:00, 15:00 y 16:00 (hora local), para los días 05/12/2015 (figura 6.56) y 06/12/2015 (figura 6.57). En este caso no se han ampliado las horas hasta las 17:00 como en el resto de zonas, debido a que el software de simulación sólo ofrece datos hasta las 16:00 en ésta época del año. 


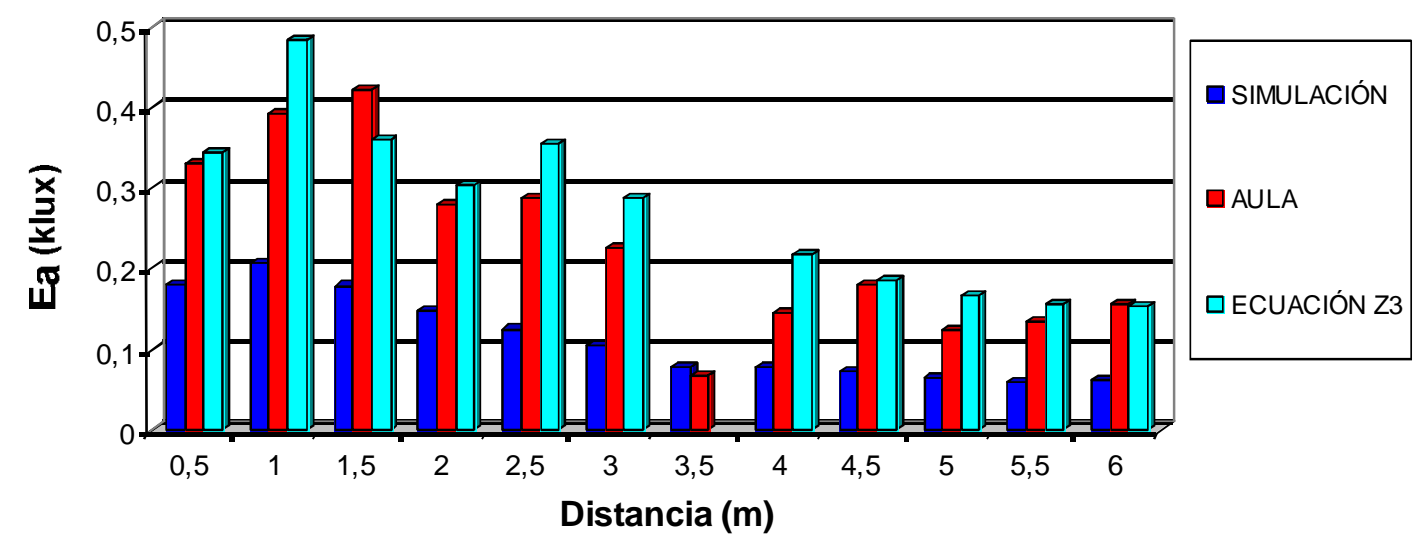

(a) 11:00.

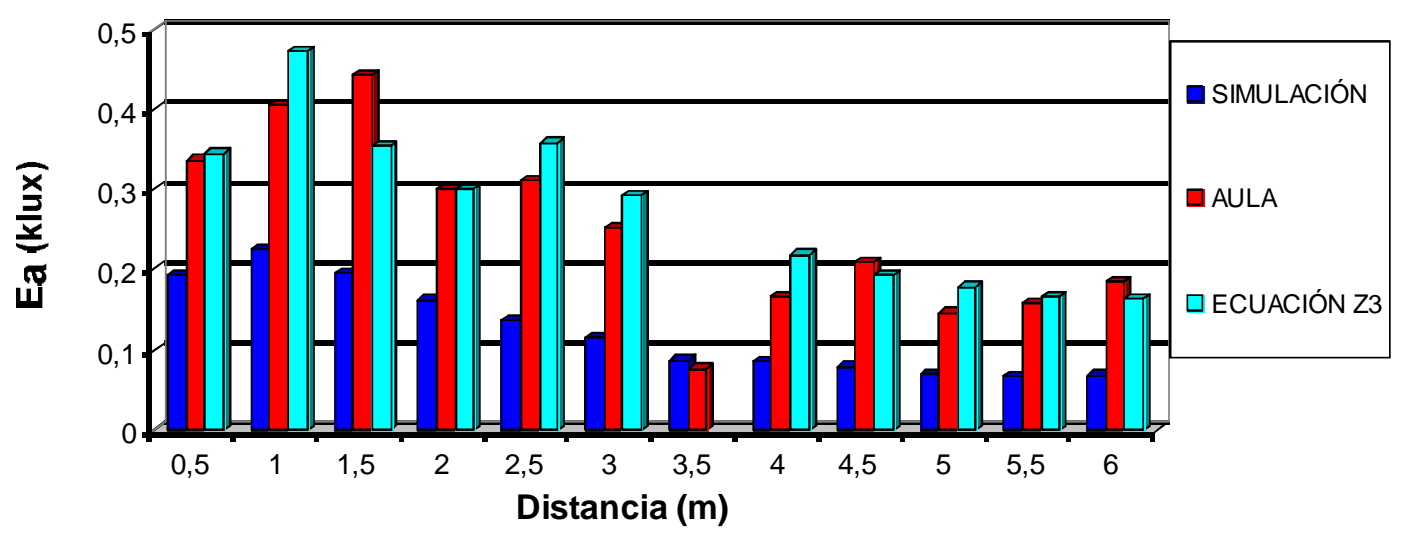

(b) 12:00.

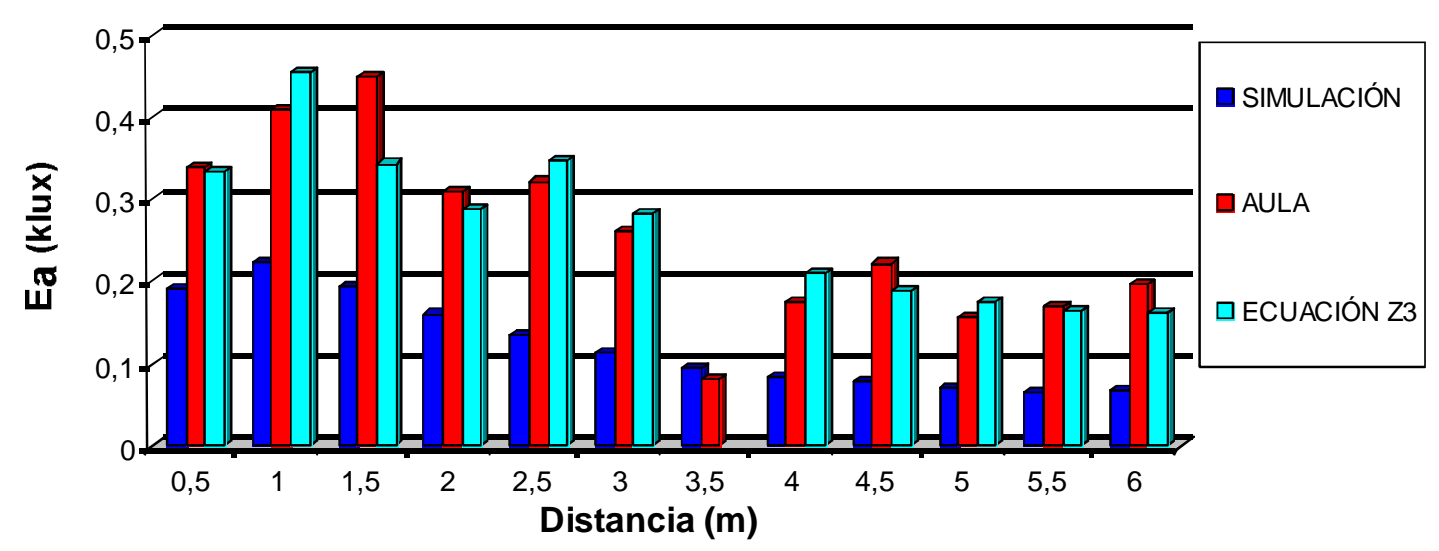

(c) $13: 00$. 


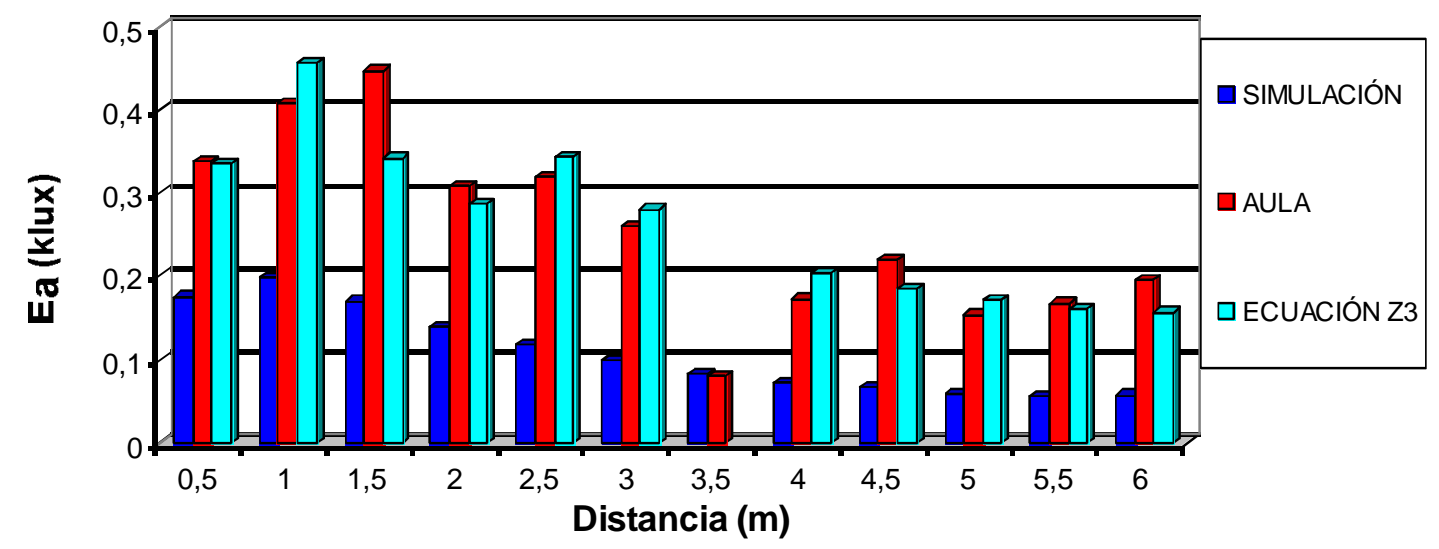

(d) $14: 00$.

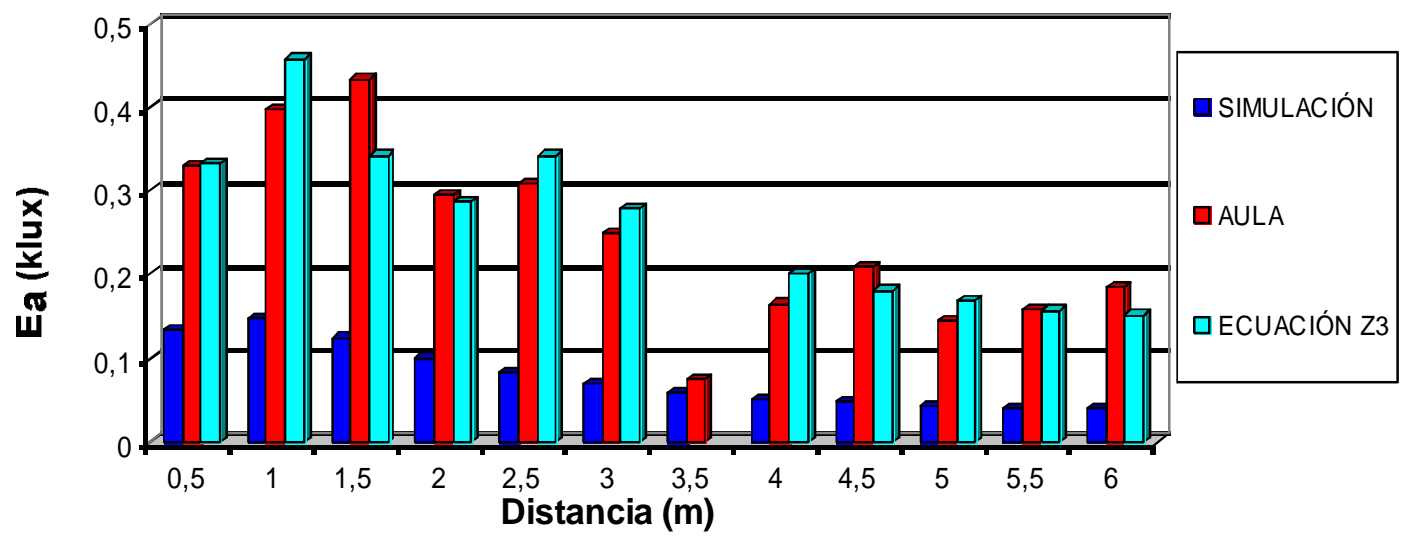

(e) 15:00.

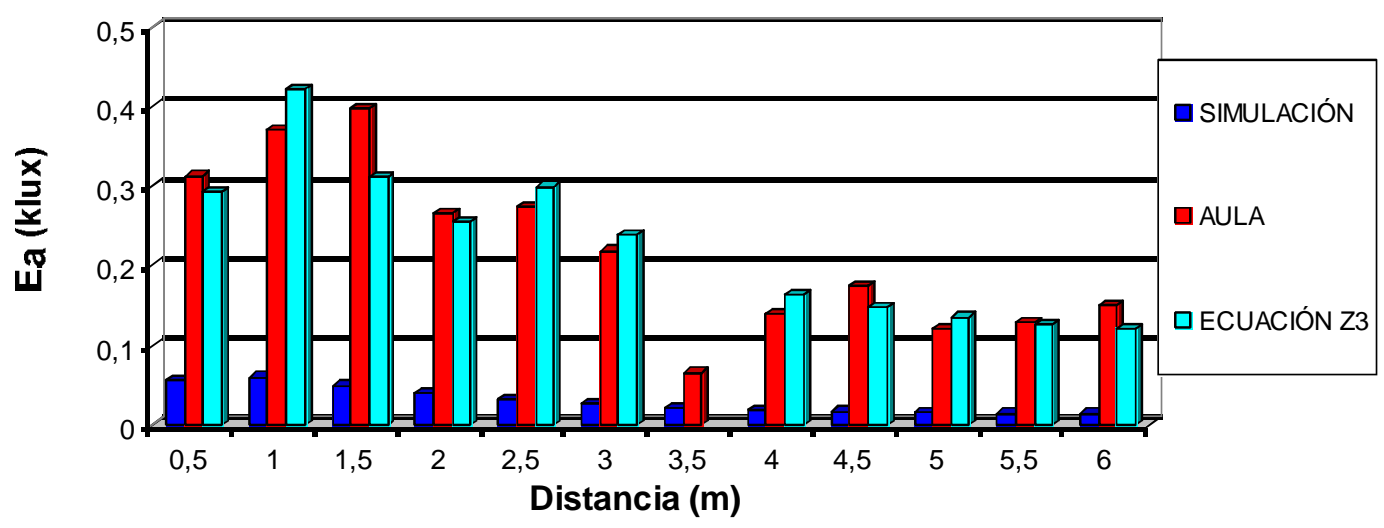

(f) $16: 00$.

Figura 6.56. Iluminancias del aula obtenidas midiendo en el aula, por simulación y aplicando la ecuación Z3 a distintas distancias de la fachada el día 05/12/2015 a las horas indicadas.

Los resultados obtenidos del estudio comparativo de las iluminancias del aula en la zona 3 para el día 06/12/2015 se muestra en los diagramas de barras de la figura 6.57 . 

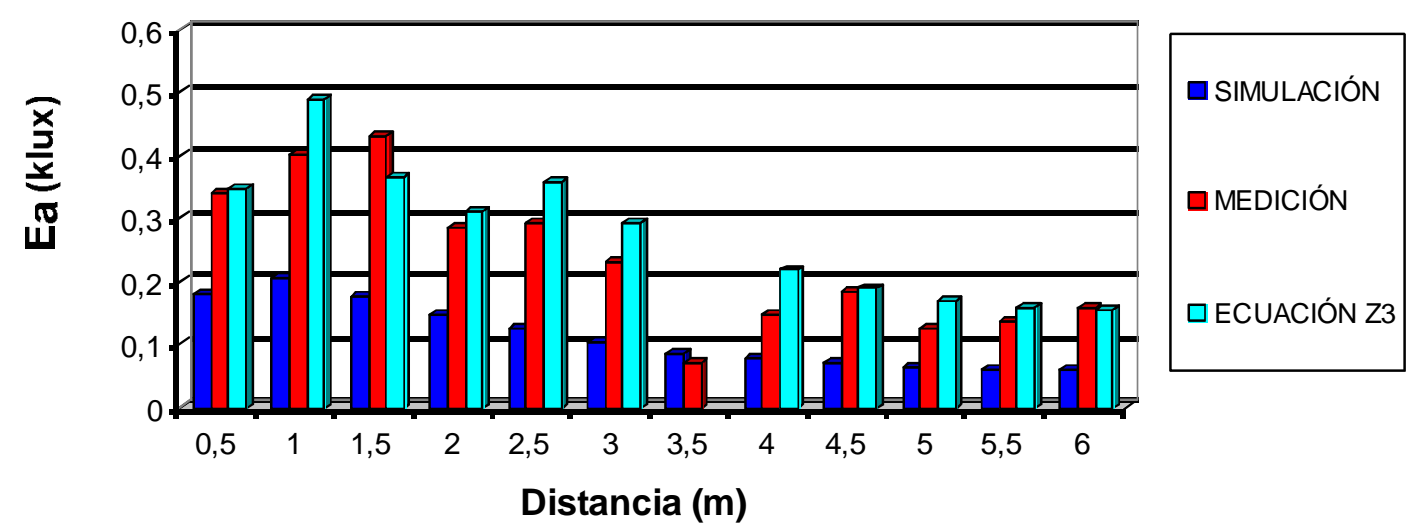

(a) 11:00.
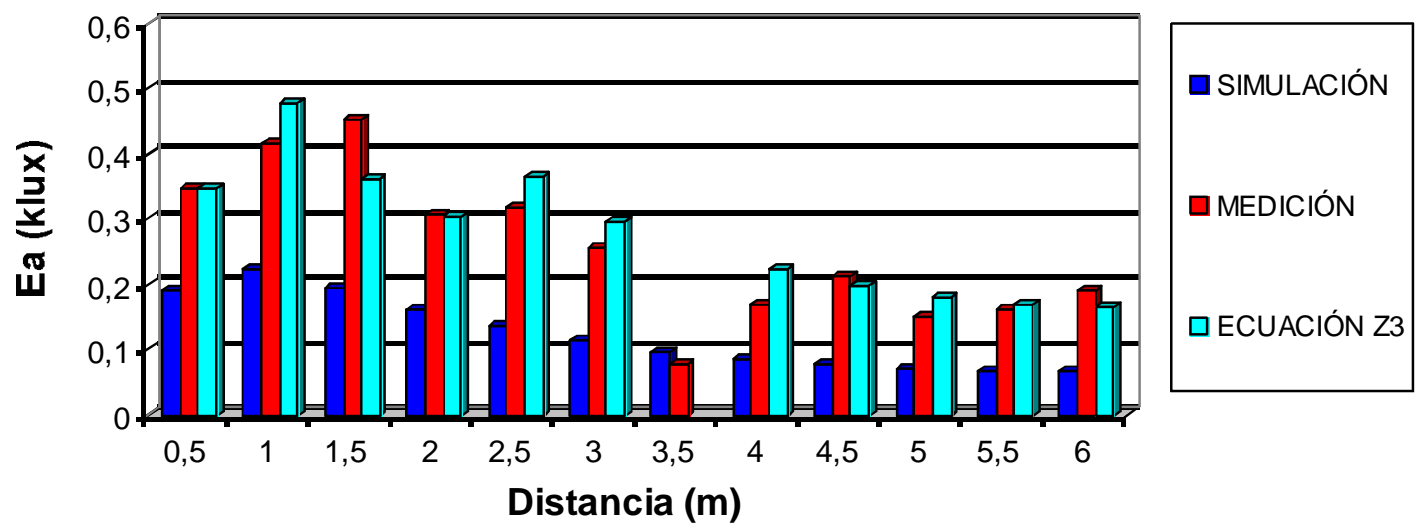

(b) 12:00.

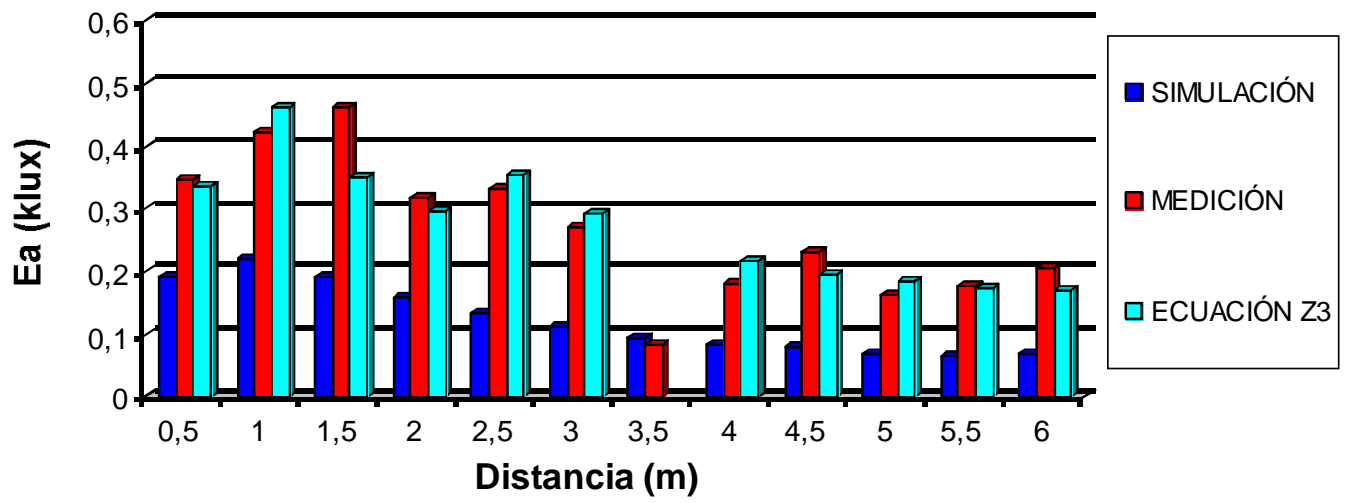

(c) 13:00.

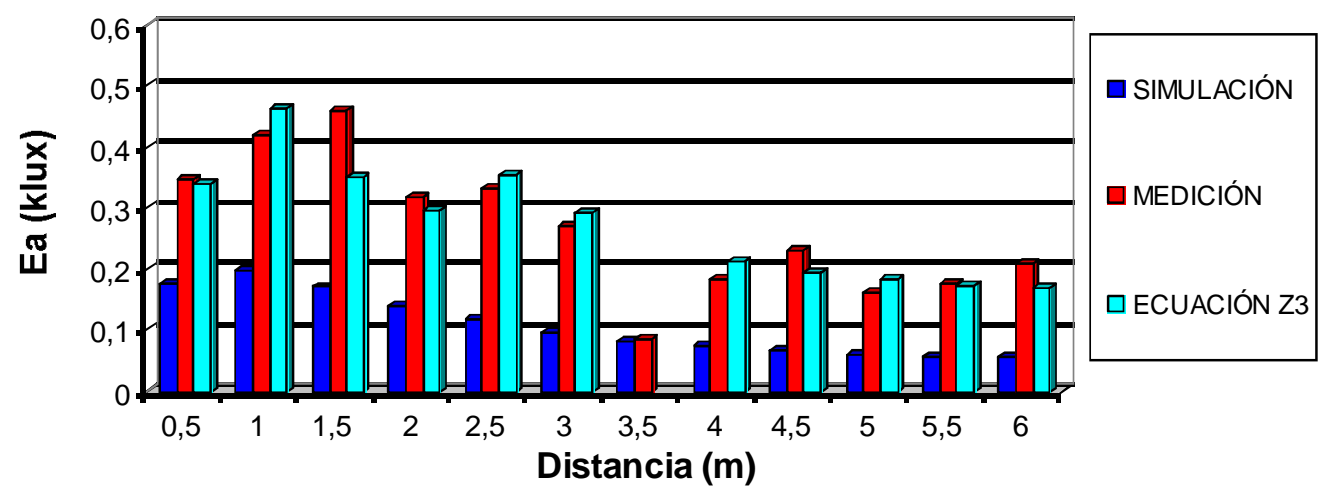

(d) $14: 00$. 


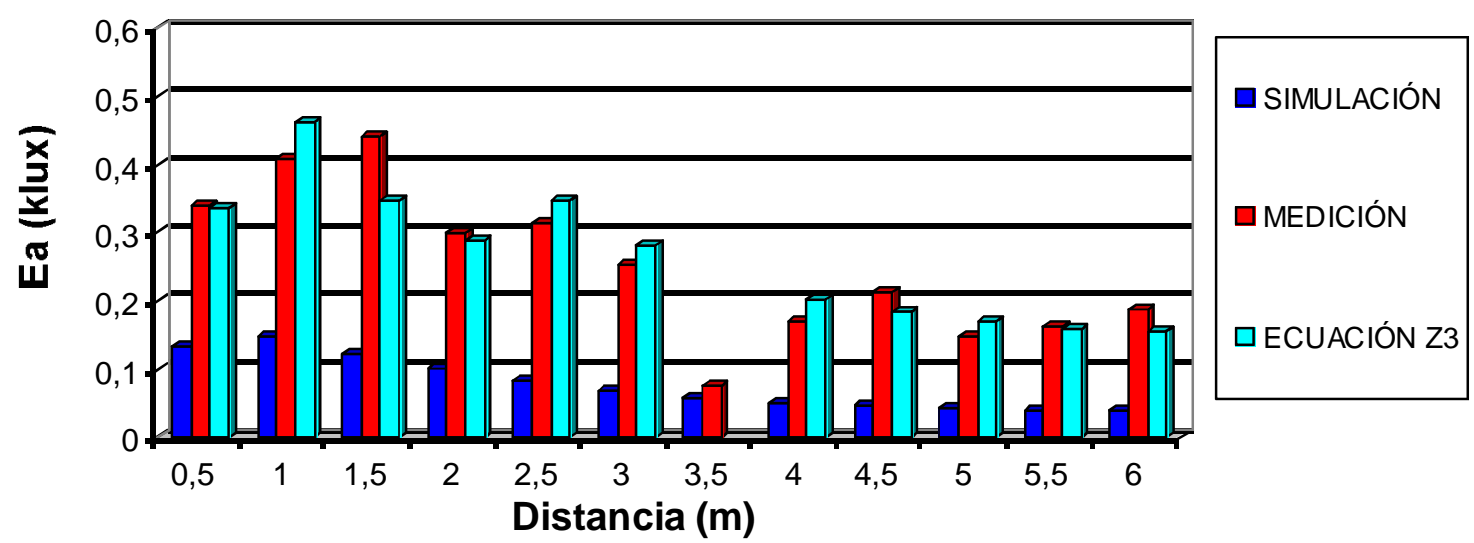

(e) 15:00.

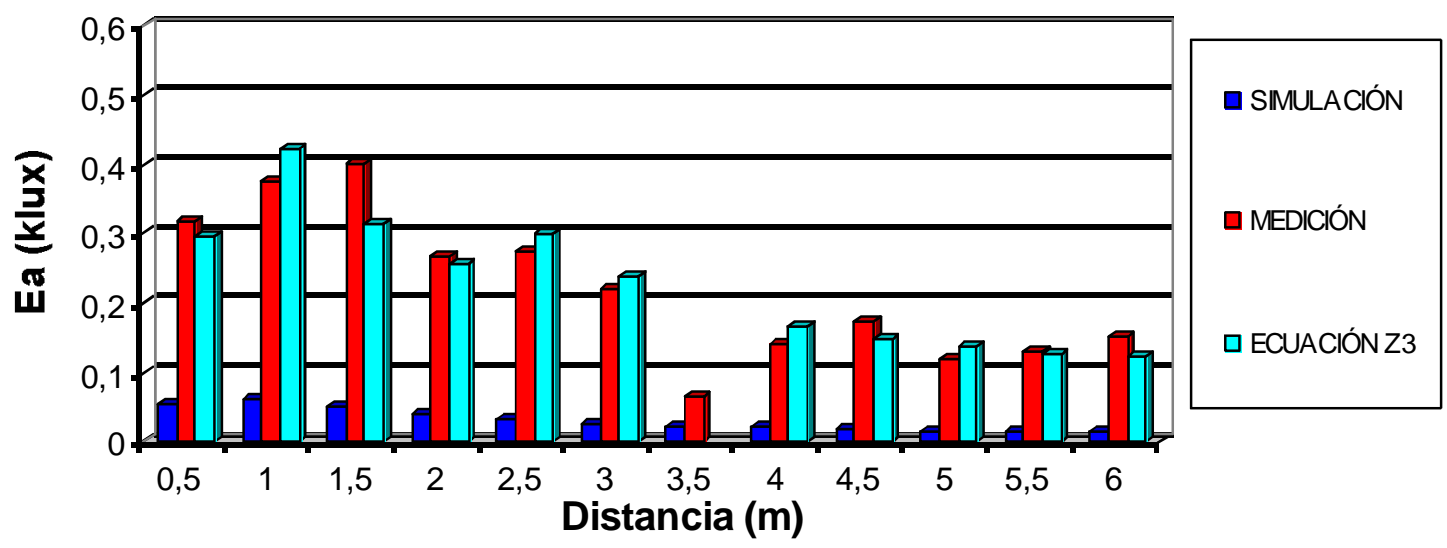

(f) $16: 00$.

Figura 6.57. Iluminancias del aula obtenidas midiendo en el aula, por simulación y aplicando la ecuación Z3 a distintas distancias de la fachada el día 06/12/2015 a las horas indicadas.

También en las figuras 6.56 y 6.57 , se aprecia cómo el comportamiento de las iluminancias obtenidas mediante la ecuación de la zona 3 se aproximan más a los valores obtenidos por medición en el aula que a los valores obtenidos por software de simulación.

En el anexo II (figuras 10.2.5 y 10.2.6) se presentan las curvas ajustadas de las iluminancias del aula representadas en las figuras 6.56 y 6.57 para las distintas horas consideradas, y las ecuaciones que describen dichas curvas con sus correspondientes coeficientes de ajuste, mayor que 0.9 en todos los casos. 


\subsubsection{Zona 4}

La última zona que se estudia individualmente en esta tesis es la zona 4, que está situada a $4.78 \mathrm{~m}$ de la pared Este, alineada a la mitad de la ventana situada más al Oeste del aula objeto de estudio. La colocación y distribución de los sensores en esta zona del aula se muestra en las figuras 6.58 y 6.59 .

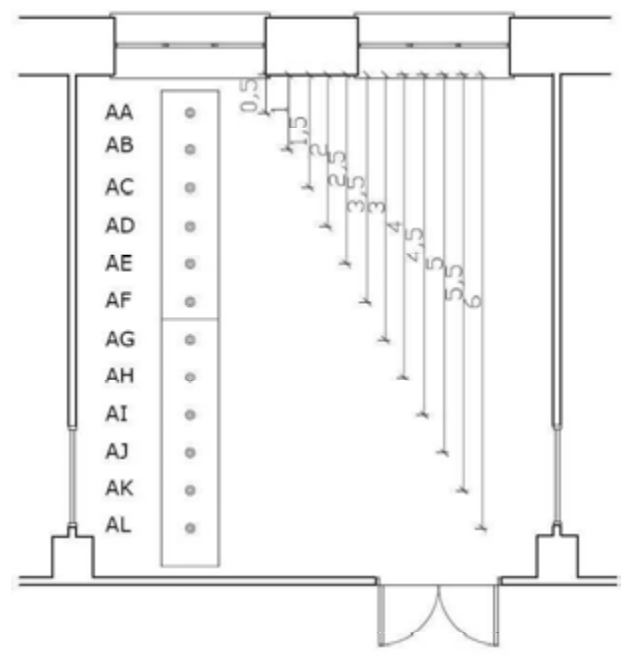

Figura 6.58. Esquema sensores zona 4.

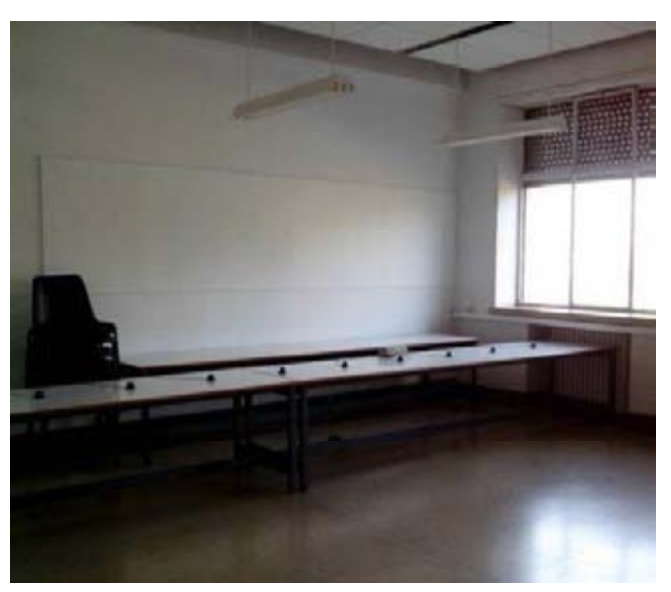

Figura 6.59. Colocación sensores zona 4.

Las medidas para el análisis de esta zona se han realizado el día 21/07/2015 con cielo totalmente despejado desde las 11:00 de la mañana hasta las 19:00 de la tarde (hora local).

Se han registrado datos en el exterior y a todas las distancias consideradas (0.5 m, 1 m, 1.5 m, 2 m, 2.5 m, 3 m, 3.5 m, 4 m, 4.5 m, 5 m, 5.5 m, 6 m), tanto en el aula como su modelo a escala 1/15 (a las distancias proporcionales). Sin embargo se han suprimido los datos correspondientes a la distancia de $5.5 \mathrm{~m}$, porque el sensor del modelo a escala a esta distancia proporcional tampoco funcionó de forma correcta.

\subsubsection{Evolución diaria de las iluminancias}

La evolución diaria de la iluminancia exterior $\left(E_{\text {ext }}\right)$ del día considerado para la zona 4 se muestra en la figura 6.60 . 


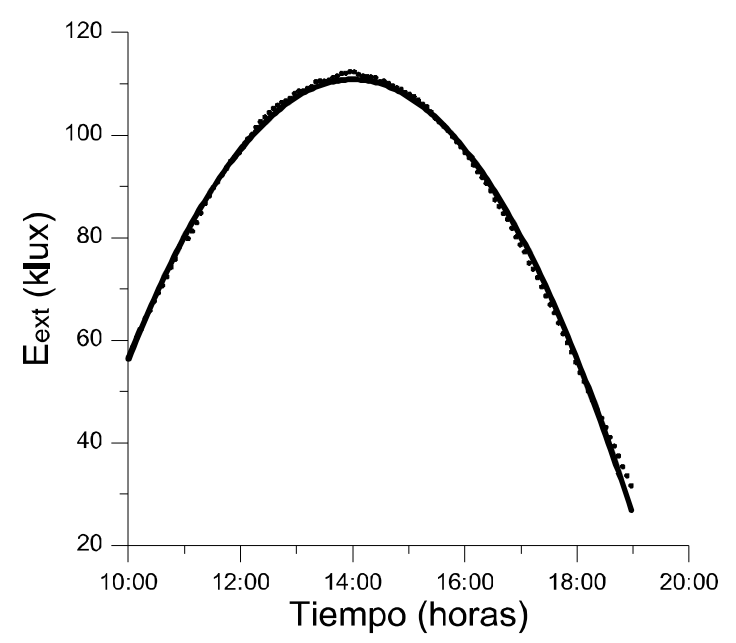

Figura 6.60. Evolución diaria de la iluminancia exterior (Julio 2015).

Con los datos de las iluminancias medidas en el aula y en el modelo a escala se han elaborado las gráficas que se muestran en las figuras 6.61 y 6.62. En el eje vertical aparecen representadas las iluminancias en klux y en el eje horizontal el tiempo expresado en horas.

También se muestra la ecuación de segundo grado a la que se ajusta la nube de puntos, así como su coeficiente de ajuste $\left(r^{2}\right)$. En la columna de la izquierda en verde están representados los datos correspondientes al modelo y a la derecha en rojo los del aula.

\section{ZONA 4}

MODELO

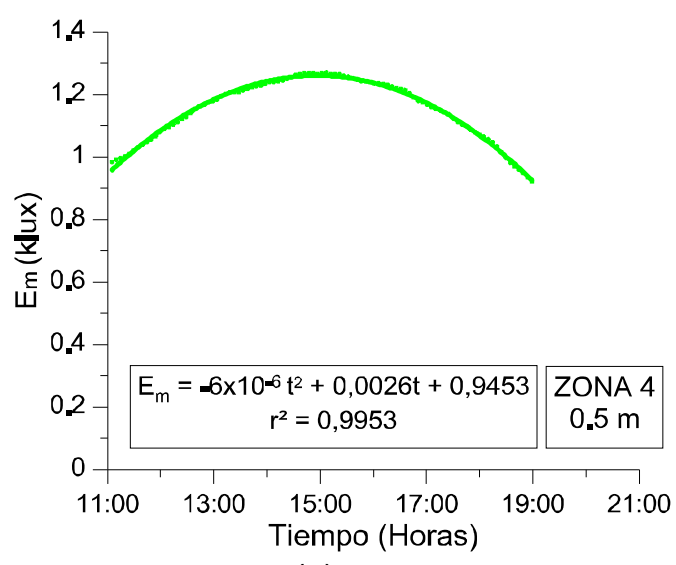

(a) $0.5 \mathrm{~m}$.

\section{AULA}

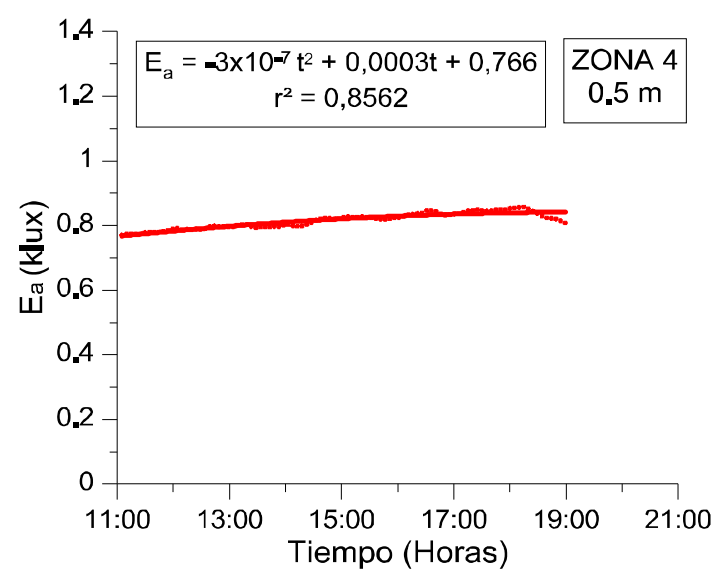

(a) $0.5 \mathrm{~m}$. 
Estudio experimental y modelización de la iluminación natural en la edificación mediante modelos a escala

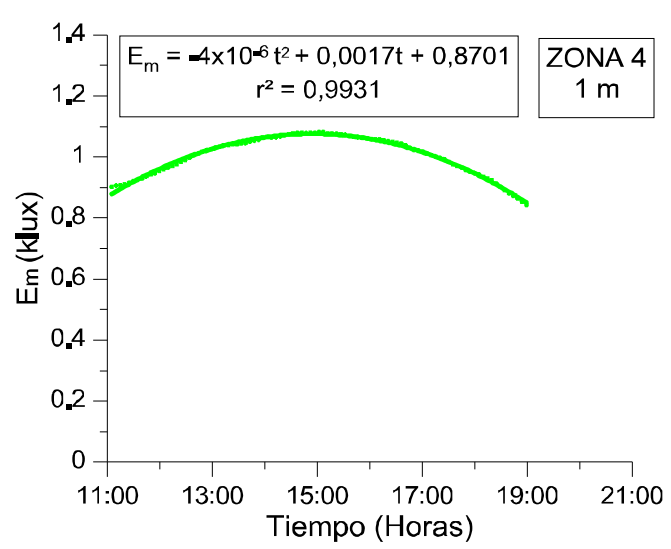

(b) $1 \mathrm{~m}$.

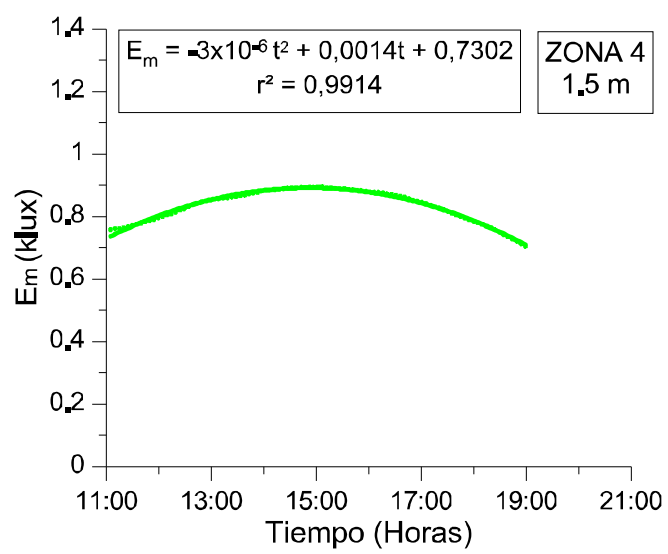

(c) $1.5 \mathrm{~m}$.

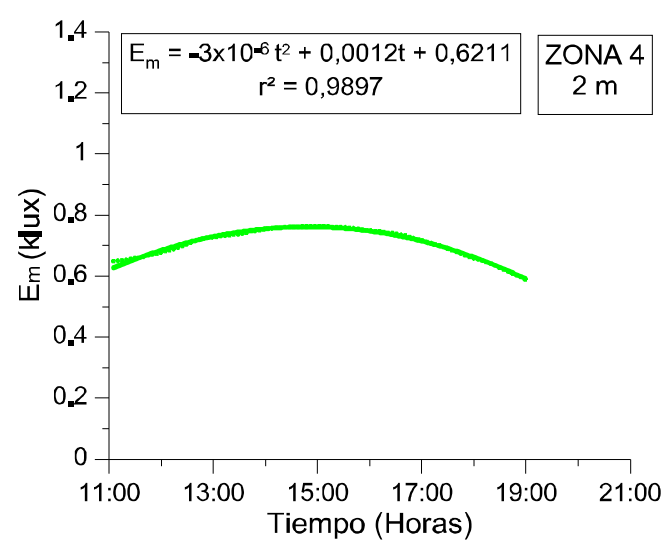

(d) $2 \mathrm{~m}$.

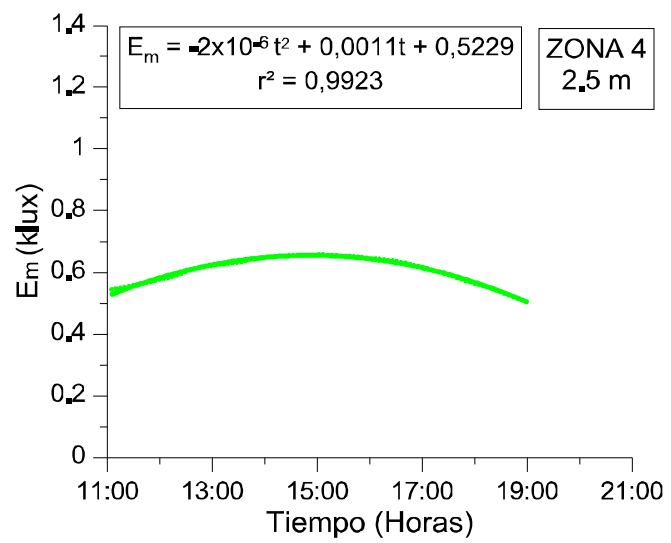

(e) $2.5 \mathrm{~m}$.

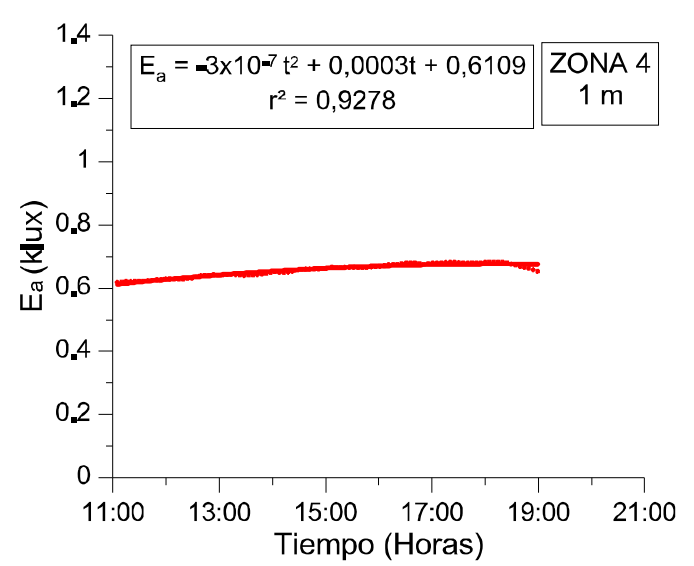

(b) $1 \mathrm{~m}$.

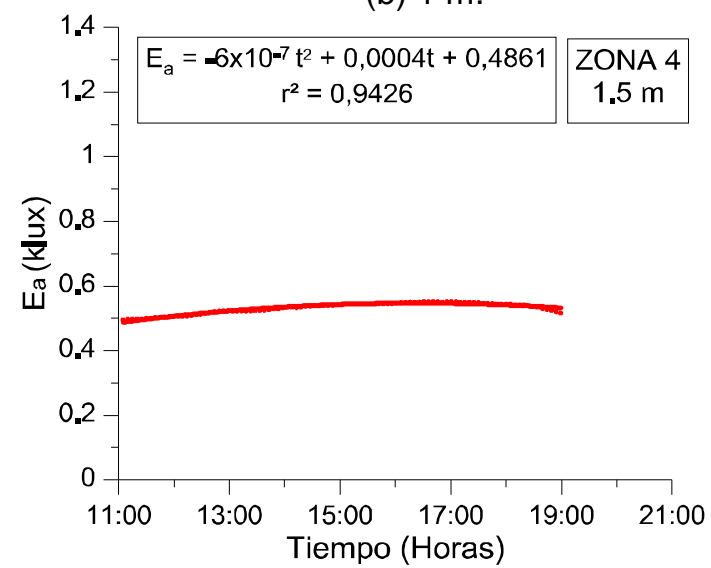

(c) $1.5 \mathrm{~m}$.

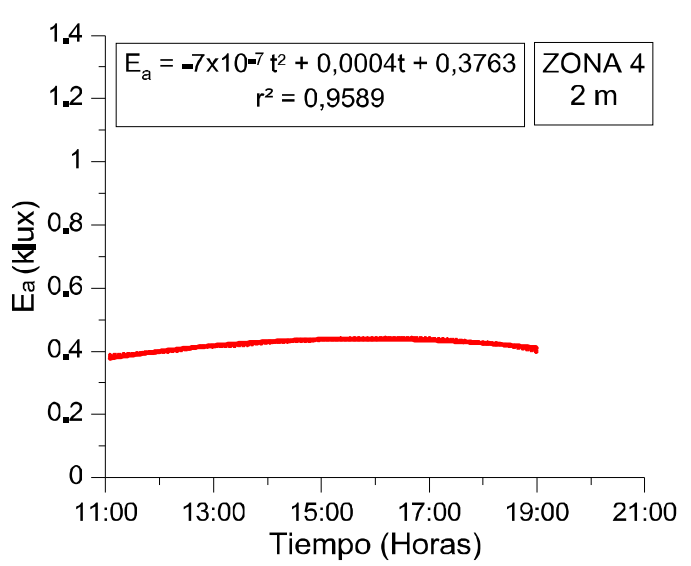

(d) $2 \mathrm{~m}$.

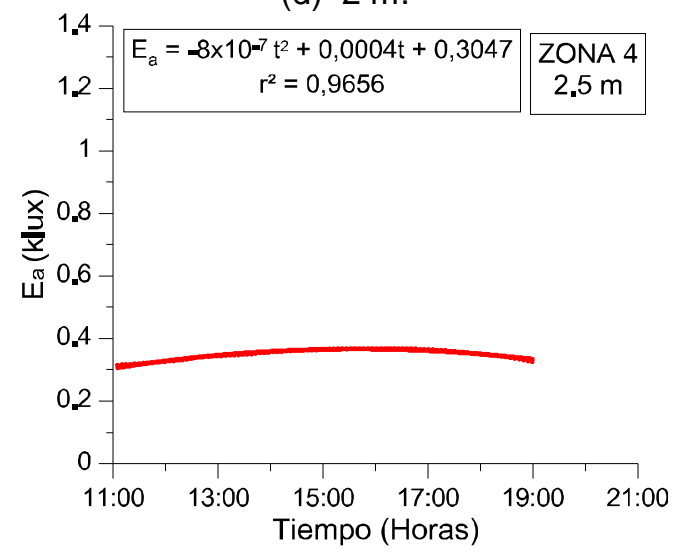

(e) $2.5 \mathrm{~m}$. 
Estudio experimental y modelización de la iluminación natural en la edificación mediante modelos a escala

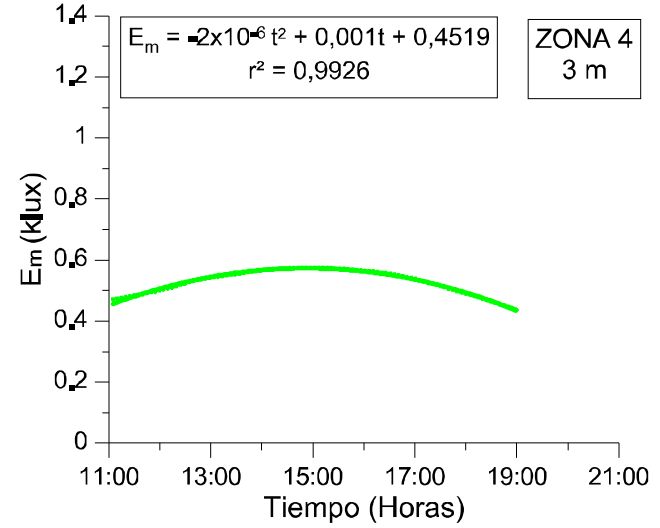

(f) $3 \mathrm{~m}$.

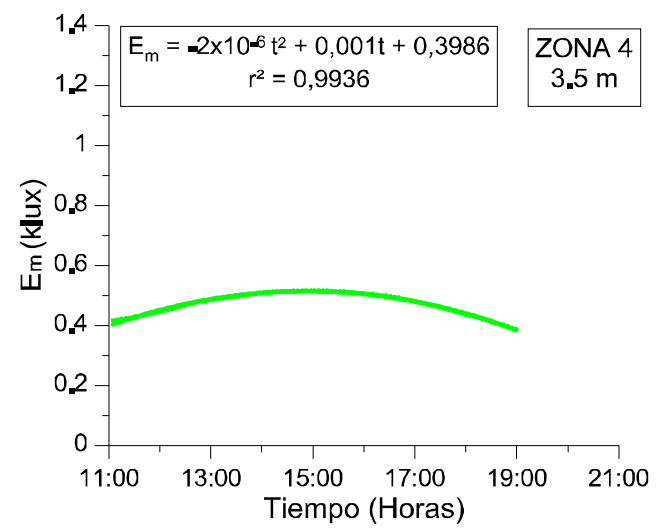

(g) $3.5 \mathrm{~m}$.

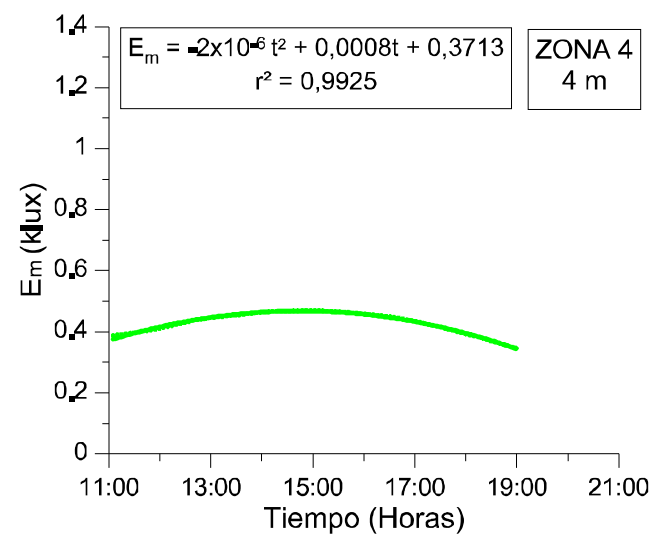

(h) $4 \mathrm{~m}$.

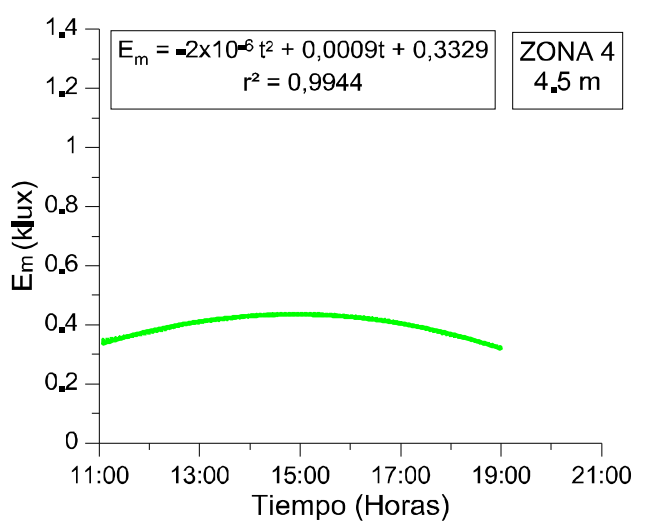

(i) $4.5 \mathrm{~m}$.

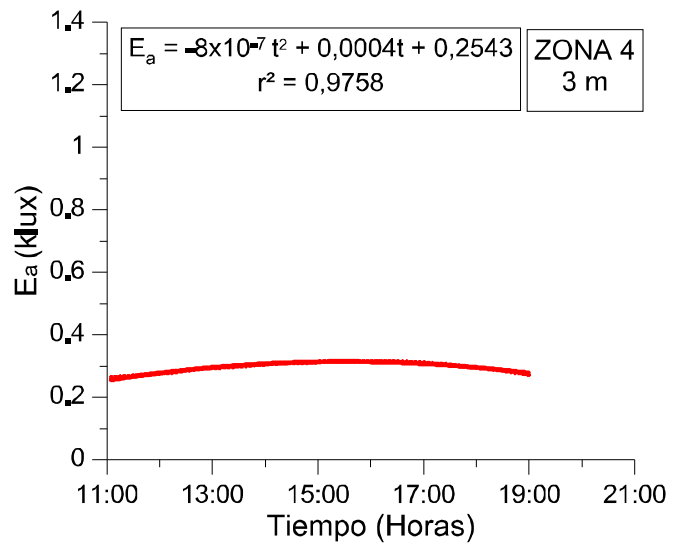

(f) $3 \mathrm{~m}$.

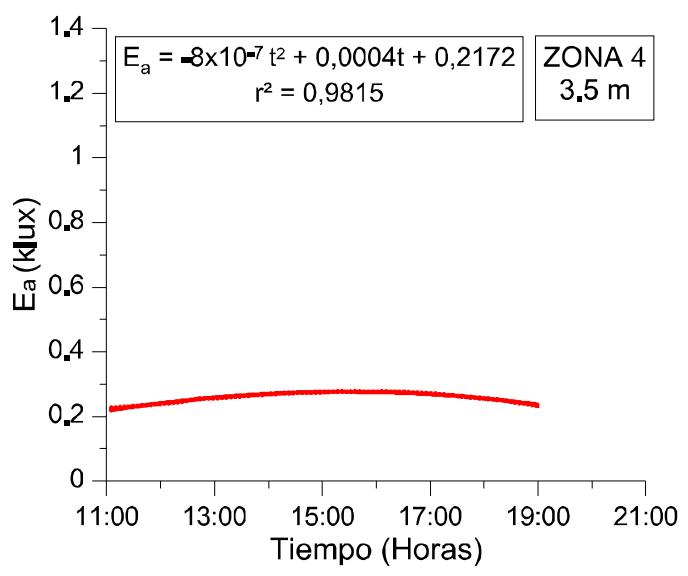

(g $3.5 \mathrm{~m}$.

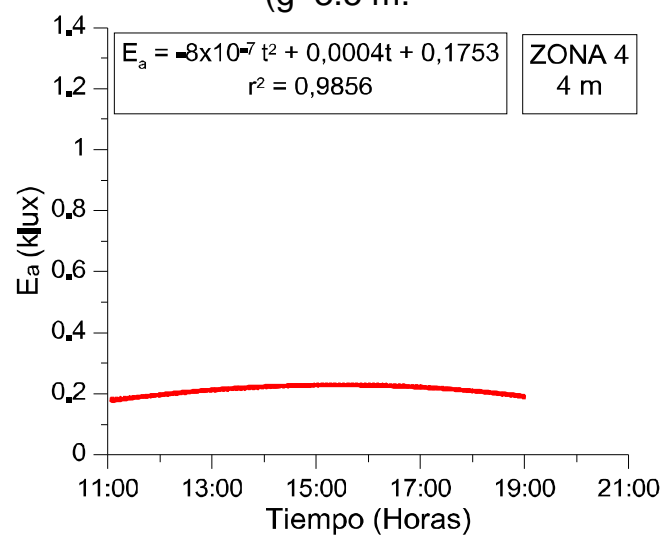

(h) $4 \mathrm{~m}$.

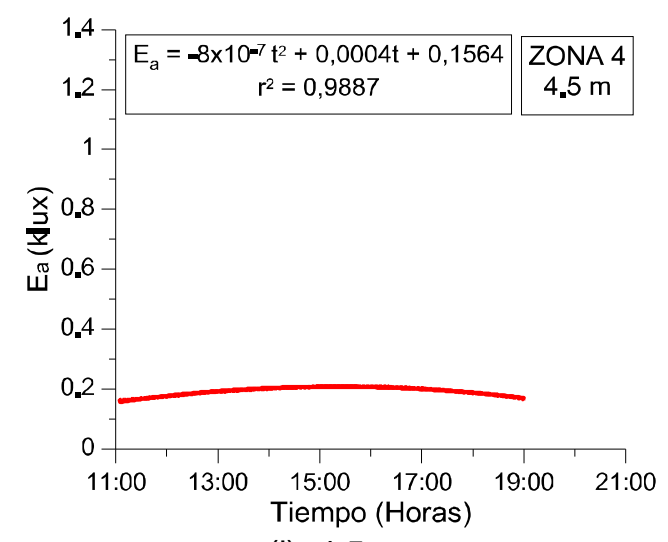

(i) $4.5 \mathrm{~m}$. 


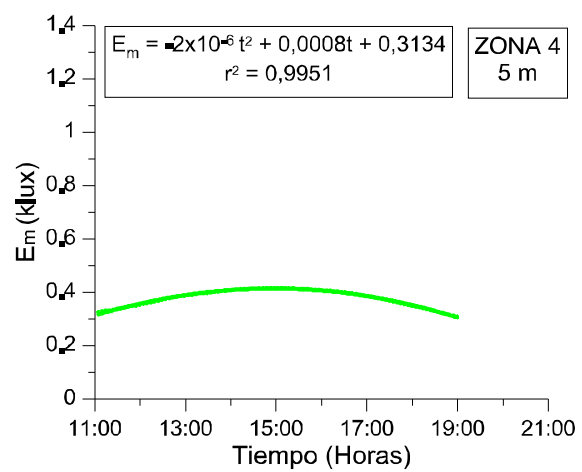

(j) $5 \mathrm{~m}$.

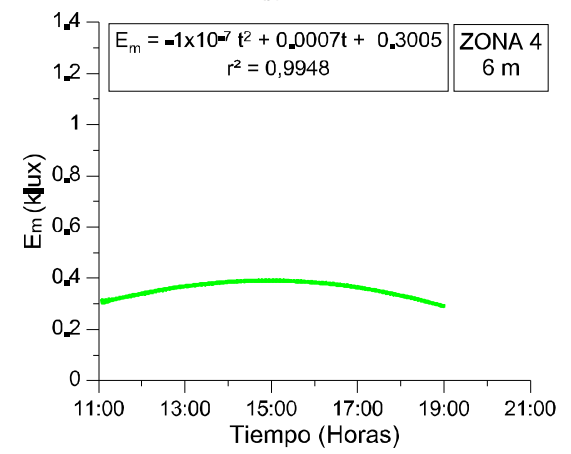

(k) $6 \mathrm{~m}$.

Figura 6.61. Evolución diaria de la iluminancia en el modelo a las distancias consideradas.

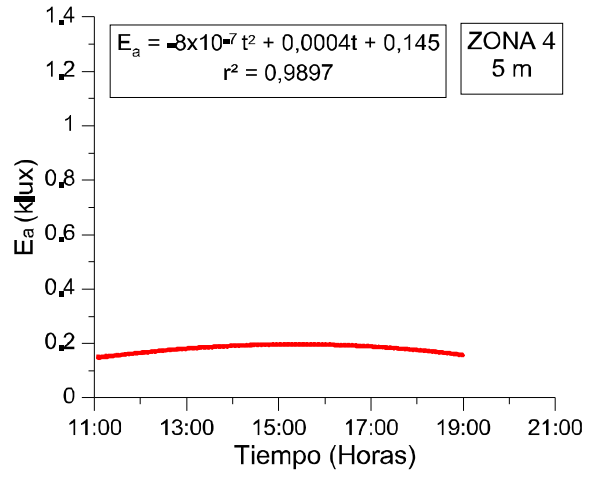

(j) $5 \mathrm{~m}$

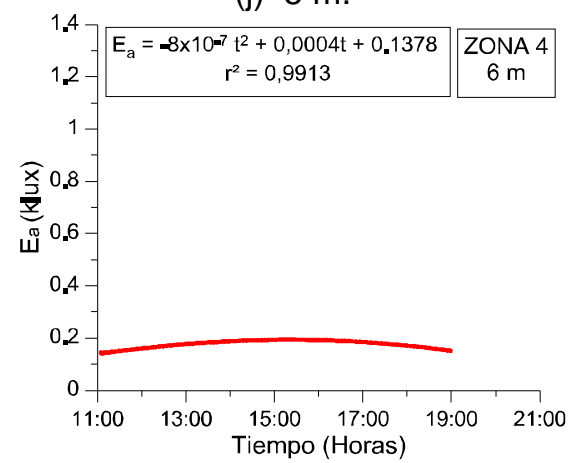

(k) $6 \mathrm{~m}$.

Figura 6.62. Evolución diaria de la iluminancia en el aula a las distancias consideradas.

Para ver cómo han variado las iluminancias del interior del aula y de la maqueta con la distancia, a continuación, se muestra una gráfica donde se recogen todas las curvas anteriores en el modelo (figura 6.63) y otra en el aula (figura 6.64).

En éstas se observa cómo a medida que aumenta la distancia a la fachada disminuye el valor de las iluminancias.

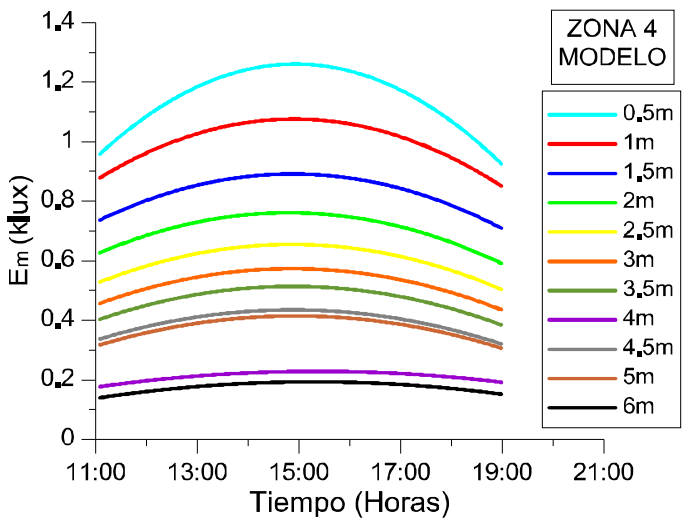

Figura 6.63. Curvas ajustadas de las iluminancias en el modelo a escala a las distancias consideradas.

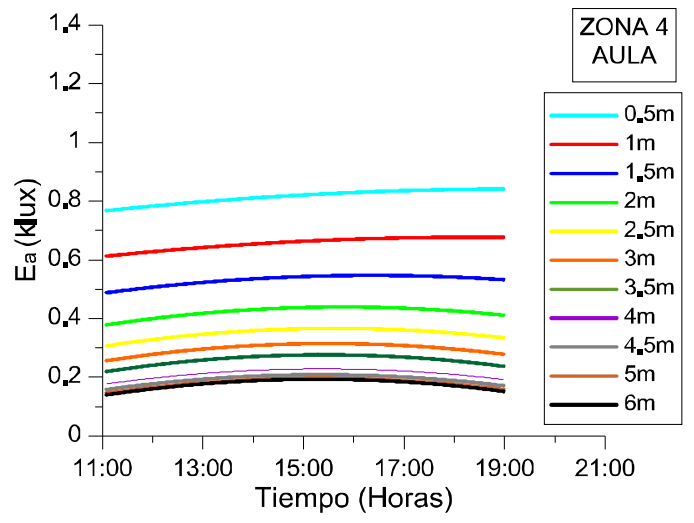

Figura 6.64. Curvas ajustadas de las iluminancias en el aula a las distancias consideradas. 
Las ecuaciones de las curvas representadas anteriormente en las figuras 6.63 y 6.64 , junto a sus correspondientes coeficientes de ajuste $\left(r^{2}\right)$ muestran en la tabla 6.14 .

\begin{tabular}{|c|c|c|c|c|}
\hline ZONA 4 & \multicolumn{2}{|l|}{ MODELO } & \multicolumn{2}{|l|}{ AULA } \\
\hline $\begin{array}{l}\text { DISTANCIA } \\
\text { (m) }\end{array}$ & ECUACIÓN & $r^{2}$ & ECUACIÓN & $r^{2}$ \\
\hline 0.5 & $E_{m}=-6 \times 10^{-6} t^{2}+0.0026 t+0.9453$ & 0.9953 & $E_{a}=-3 \times 10^{-7} t^{2}+0,0003 t+0,766$ & 0.8562 \\
\hline 1 & $E_{m}=-4 \times 10^{-6} t^{2}+0.0017 t+0.8701$ & 0.9931 & $E_{a}=-3 \times 10^{-7} t^{2}+0,0003 t+0,6109$ & 0.9278 \\
\hline 1.5 & $E_{m}=-3 \times 10^{-6} t^{2}+0.0014 t+0.7302$ & 0.9914 & $E_{a}=-6 \times 10^{-7} t^{2}+0,0004 t+0,4861$ & 0.9426 \\
\hline 2 & $E_{m}=-3 \times 10^{-6} t^{2}+0.0012 t+0.6211$ & 0.9897 & $E_{a}=-7 \times 10^{-7} t^{2}+0,0004 t+0,3763$ & 0.9589 \\
\hline 2.5 & $E_{m}=-2 \times 10^{-6} t^{2}+0.0011 t+0.5229$ & 0.9923 & $E_{a}=-8 \times 10^{-7} t^{2}+0,0004 t+0,3047$ & 0.9656 \\
\hline 3 & $E_{m}=-2 \times 10^{-6} t^{2}+0.001 t+0.4519$ & 0.9926 & $E_{a}=-8 \times 10-^{7} t^{2}+0,0004 t+0,2543$ & 0.9758 \\
\hline 3.5 & $E_{m}=-2 \times 10^{-6} t^{2}+0.001 t+0.3986$ & 0.9936 & $E_{a}=-8 \times 10^{-7} t^{2}+0,0004 t+0,2172$ & 0.9815 \\
\hline 4 & $E_{m}=-2 \times 10^{-6} t^{2}+0.0008 t+0.3713$ & 0.9925 & $E_{a}=-8 \times 10^{-7} t^{2}+0,0004 t+0,1753$ & 0.9856 \\
\hline 4.5 & $E_{m}=-2 \times 10^{-6} t^{2}+0.0009 t+0.3329$ & 0.9944 & $E_{a}=-8 \times 10^{-7} t^{2}+0,0004 t+0,1564$ & 0.9887 \\
\hline 5 & $E_{m}=-2 \times 10^{-6} t^{2}+0.0008 t+0.3134$ & 0.9951 & $E_{a}=-8 \times 10^{-7} t^{2}+0,0004 t+0,145$ & 0.9897 \\
\hline 6 & $E_{m}=-1 \times 10^{-7} t^{2}+0.0007 t+0.3005$ & 0.9948 & $E_{a}=-8 \times 10^{-7} t^{2}+0,0004 t+0.1378$ & 0.9913 \\
\hline
\end{tabular}

Tabla 6.14. Ecuaciones de ajuste de segundo grado de las iluminancias diarias a distintas distancias tanto del modelo como del aula.

En la tabla se muestra que todos los coeficientes de ajuste del modelo a escala, y prácticamente todos los del aula, son superiores a 0.95 lo que indica un alto grado de fiabilidad.

\subsubsection{Relación entre las iluminancias del aula y del modelo a escala en función de las horas del día}

Continuando el análisis como en zonas anteriores, se va a estudiar la relación que existe ente las iluminancias del aula y las de su modelo a escala a lo largo de todo el día, para las distintas distancias consideradas. Para ello se ha representado, en la figura 6.65 , el cociente obtenido entre las iluminancias del aula y del modelo durante el día, frente al tiempo (expresado en horas), según las distintas distancias a la ventana. La ecuación de la curva obtenida para cada distancia así como su coeficiente de ajuste correspondiente se muestran en cada gráfica. 
Estudio experimental y modelización de la iluminación natural en la edificación mediante modelos a escala

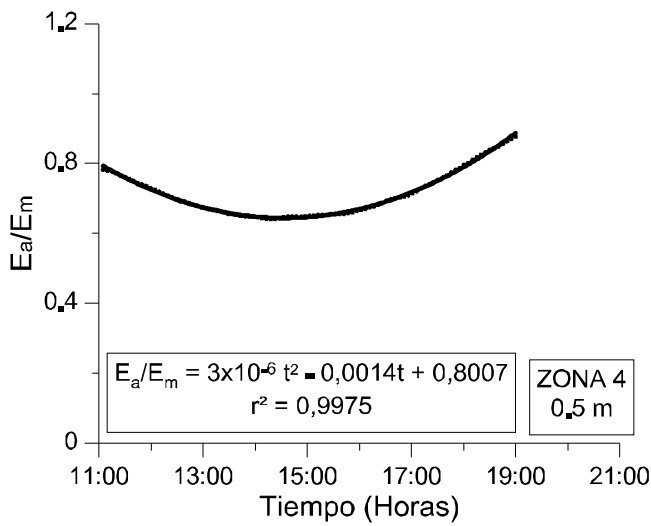

(a) $0.5 \mathrm{~m}$.

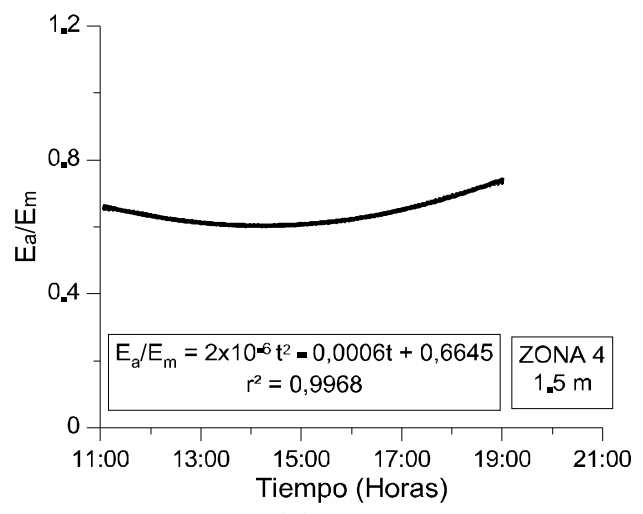

(c) $1.5 \mathrm{~m}$.

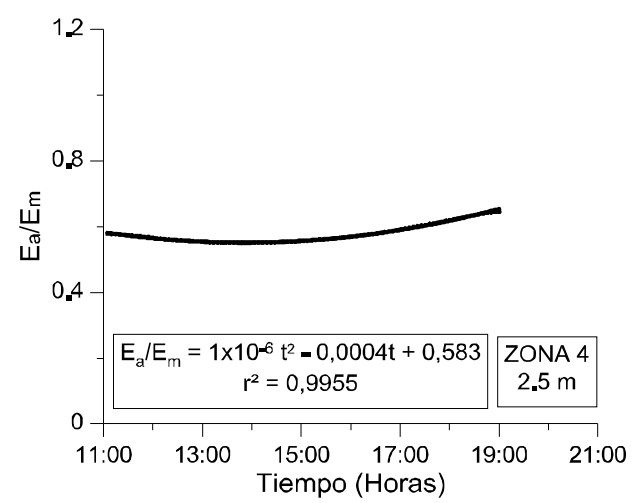

(e) $2.5 \mathrm{~m}$.

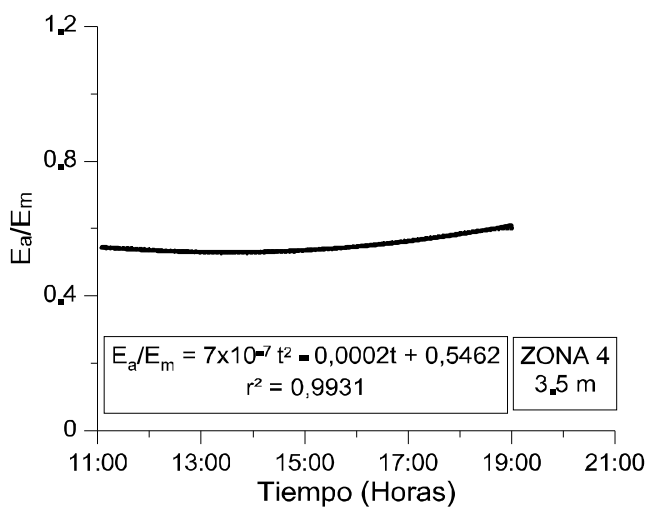

(g) $3.5 \mathrm{~m}$.

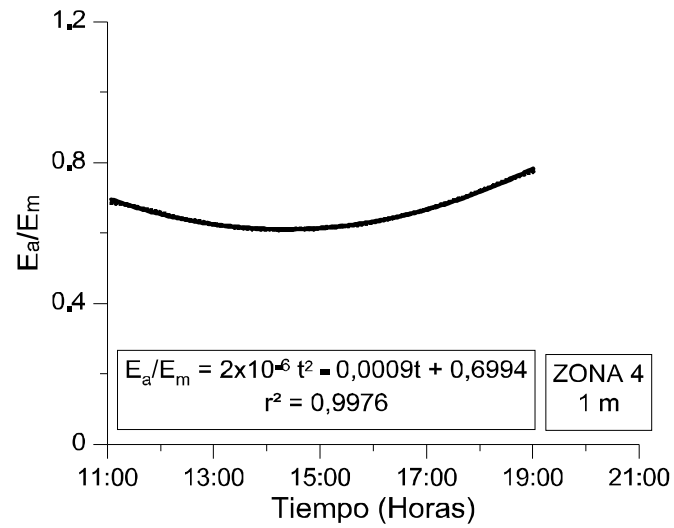

(b) $1 \mathrm{~m}$.

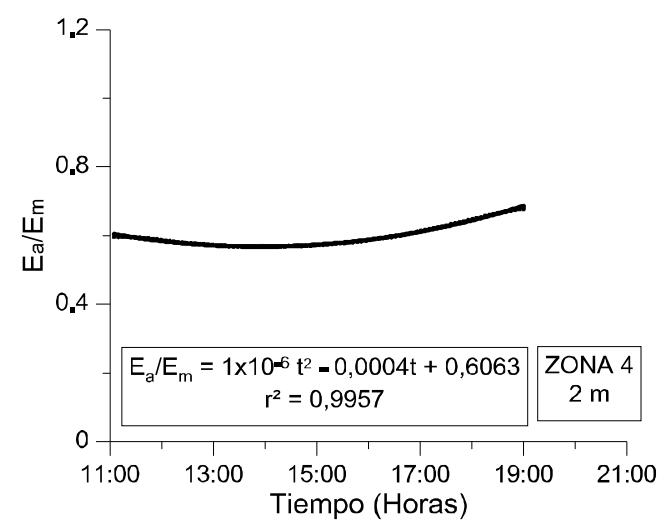

(d) $2 \mathrm{~m}$.

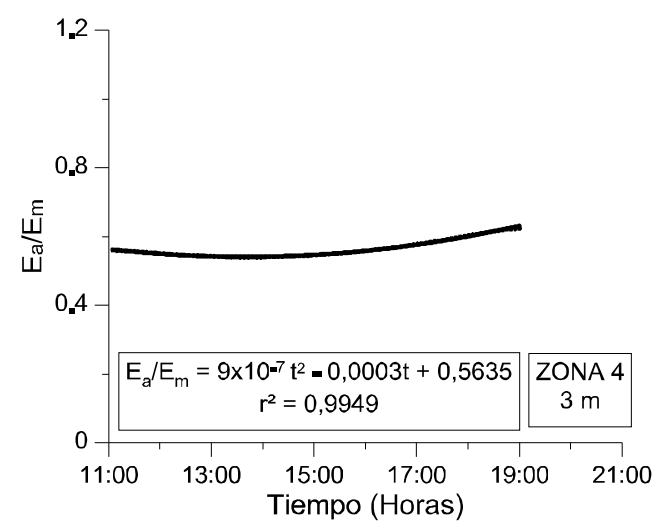

(f) $3 \mathrm{~m}$.

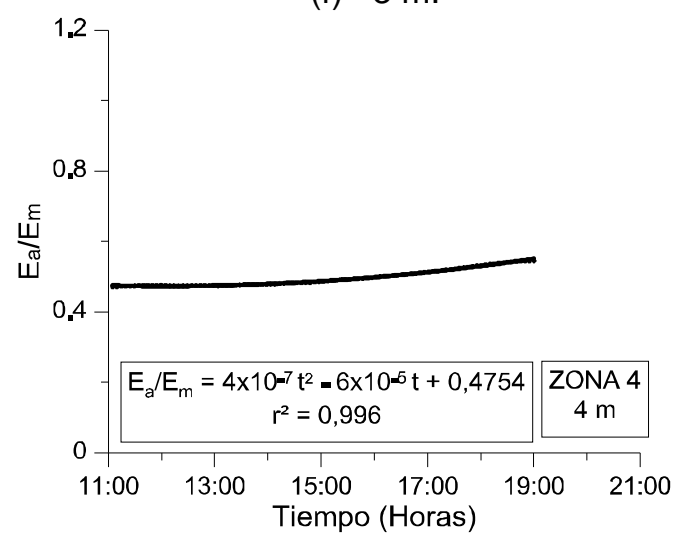

(h) $4 \mathrm{~m}$. 


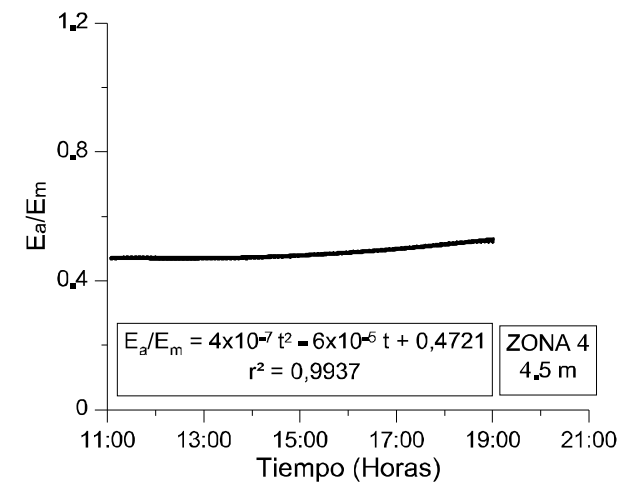

(i) $4.5 \mathrm{~m}$.

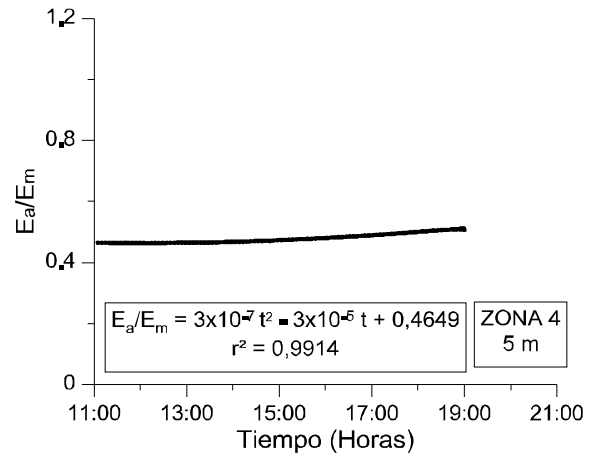

(j) $5 \mathrm{~m}$.

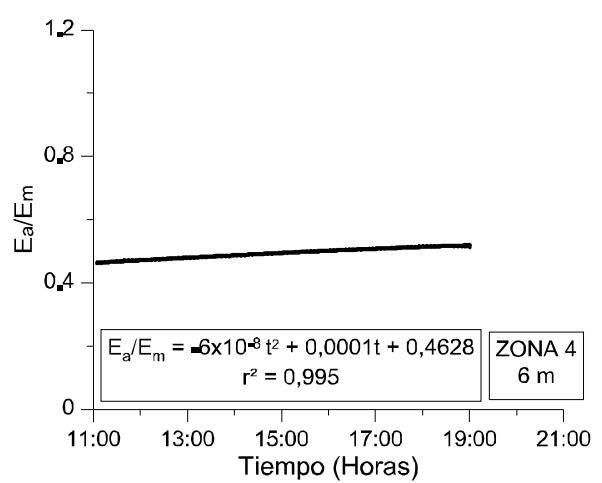

(k) $6 \mathrm{~m}$.

Figura 6.65. Cociente de las iluminancias del aula y del modelo frente al tiempo a las distancias indicadas.

Se puede observar en las gráficas que esta relación no es lineal sino que para todas las distancias, se ajusta a una ecuación de segundo grado.

A continuación se muestra de forma conjunta las curvas para las distintas distancias, de los cocientes de las iluminancias aula/ modelo frente al tiempo (figura 6.66), dónde se puede apreciar cómo según aumenta la distancia a la ventana la curvatura de estas ecuaciones de segundo grado van disminuyendo.

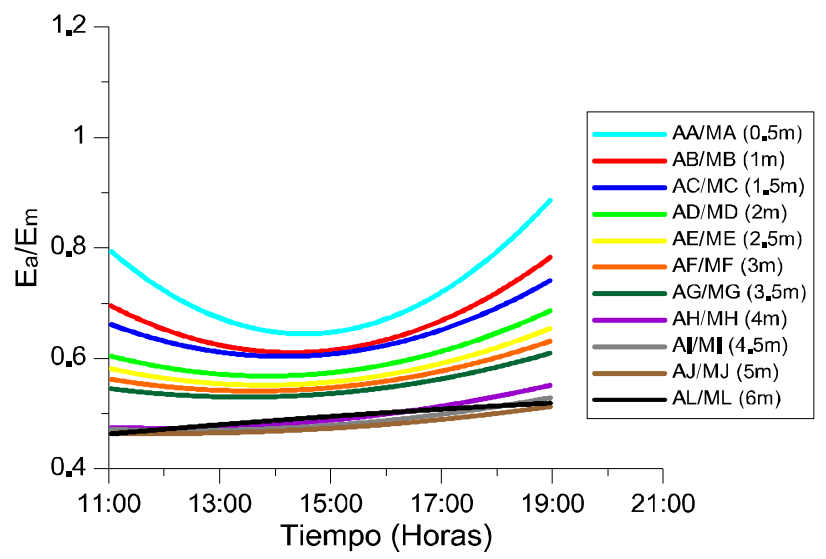

Figura 6.66. Curvas ajustadas, a las distintas distancias, de la relaciones entre el cociente de las iluminancias aula/ modelo frente al tiempo. 
Se observa cómo según aumenta la distancia a la ventana la curvatura de estas ecuaciones de segundo grado va disminuyendo, y el valor del cociente $E_{a} / E_{m}$ también.

En la tabla 6.15 se recogen las ecuaciones de las curvas representadas en la figura 6.66, así como su correspondiente coeficiente de ajuste. Se puede comprobar cómo todas las ecuaciones tienen un coeficiente de ajuste superior a 0.95 , lo que indica que tienen una alta fiabilidad.

\begin{tabular}{|c|c|c|}
\hline $\begin{array}{c}\text { DISTANCIA } \\
(\mathbf{m})\end{array}$ & ECUACIÓN & $\mathbf{r}^{\mathbf{2}}$ \\
\hline $\mathbf{0 . 5}$ & $\mathrm{E}_{\mathrm{a}} / \mathrm{E}_{\mathrm{m}}=3 \times 10^{-6} \mathrm{t}^{2}-0.0014 \mathrm{t}+0.8007$ & 0.9975 \\
\hline $\mathbf{1}$ & $\mathrm{E}_{\mathrm{a}} / \mathrm{E}_{\mathrm{m}}=2 \times 10^{-6} \mathrm{t}^{2}-0.0009 \mathrm{t}+0.6994$ & 0.9976 \\
\hline $\mathbf{1 . 5}$ & $\mathrm{E}_{\mathrm{a}} / \mathrm{E}_{\mathrm{m}}=2 \times 10^{-6} \mathrm{t}^{2}-0.0006 \mathrm{t}+0.6645$ & 0.9968 \\
\hline $\mathbf{2}$ & $\mathrm{E}_{\mathrm{a}} / \mathrm{E}_{\mathrm{m}}=1 \times 10^{-6} \mathrm{t}^{2}-0.0004 \mathrm{t}+0.6063$ & 0.9957 \\
\hline $\mathbf{2 . 5}$ & $\mathrm{E}_{\mathrm{a}} / \mathrm{E}_{\mathrm{m}}=2 \times 10^{-6} \mathrm{t}^{2}-0.0004 \mathrm{t}+0.583$ & 0.9955 \\
\hline $\mathbf{3}$ & $\mathrm{E}_{\mathrm{a}} / \mathrm{E}_{\mathrm{m}}=9 \times 10^{-7} \mathrm{t}^{2}-0.0003 \mathrm{t}+0.5635$ & 0.9949 \\
\hline $\mathbf{3 . 5}$ & $\mathrm{E}_{\mathrm{a}} / \mathrm{E}_{\mathrm{m}}=7 \times 10^{-7} \mathrm{t}^{2}-0.0002 \mathrm{t}+0,5462$ & 0.9931 \\
\hline $\mathbf{4}$ & $\mathrm{E}_{\mathrm{a}} / \mathrm{E}_{\mathrm{m}}=4 \times 10^{-7} \mathrm{t}^{2}-6 \times 10^{-5} \mathrm{t}+0.4754$ & 0.9960 \\
\hline $\mathbf{4 . 5}$ & $\mathrm{E}_{\mathrm{a}} / \mathrm{E}_{\mathrm{m}}=4 \times 10^{-7} \mathrm{t}^{2}-6 \times 10^{-5} \mathrm{t}+0.4721$ & 0.9937 \\
\hline $\mathbf{5}$ & $\mathrm{E}_{\mathrm{a}} / \mathrm{E}_{\mathrm{m}}=3 \times 10^{-7} \mathrm{t}^{2}-3 \times 10^{-5} \mathrm{t}+0.4649$ & 0.9914 \\
\hline $\mathbf{6}$ & $\mathrm{E}_{\mathrm{a}} / \mathrm{E}_{\mathrm{m}}=-6 \times 10^{-8} \mathrm{t}^{2}+0.0001 \mathrm{t}+0.4628$ & 0.9950 \\
\hline
\end{tabular}

Tabla 6.15. Ecuaciones de las relaciones entre el cociente de las iluminancias aula/ modelo y el tiempo a las distintas distancias.

\subsubsection{Relación entre iluminancias del aula y del modelo en función de la iluminancia exterior}

También en esta zona se va a analizar la relación entre las iluminancias del aula $\left(E_{a}\right)$ y del modelo $\left(E_{m}\right)$ en función de la iluminancia exterior $\left(E_{\text {ext }}\right)$, expresada en klux, teniendo en cuenta los datos utilizados anteriormente, para las distintas distancias desde el interior hasta la ventana. En las gráficas de la figura 6.67 se ha representado en el eje vertical el cociente entre las iluminancias del aula y el modelo, y en el eje horizontal las iluminancias obtenidas con el sensor exterior. 
Estudio experimental y modelización de la iluminación natural en la edificación mediante modelos a escala

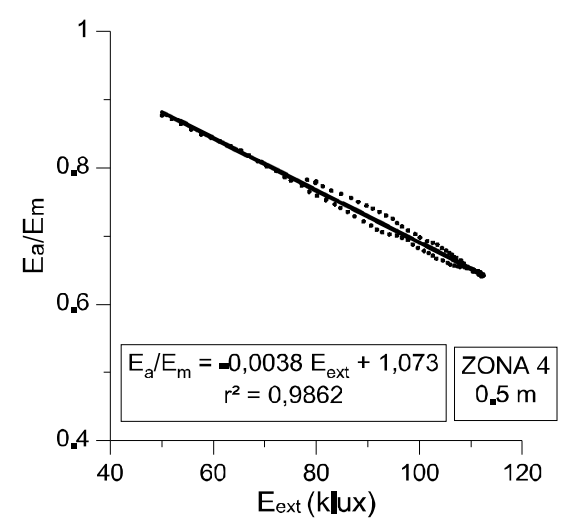

(a) $0.5 \mathrm{~m}$.

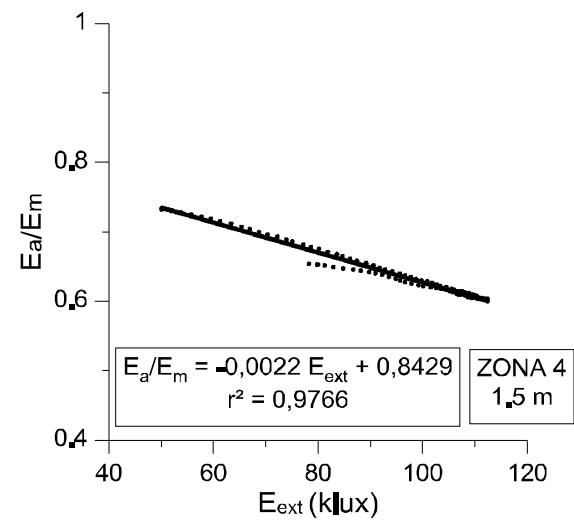

(c) $1.5 \mathrm{~m}$.

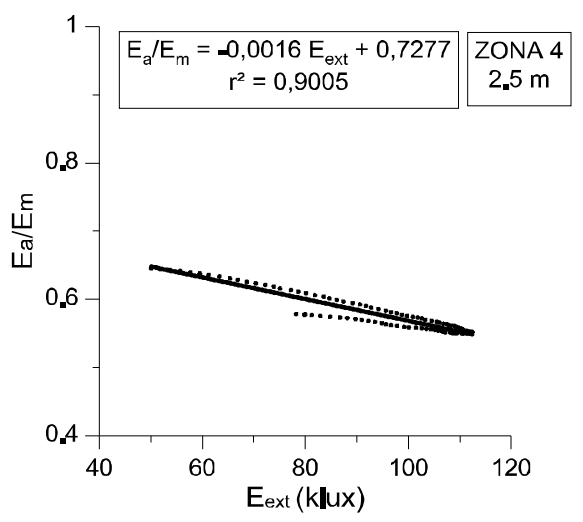

(e) $2.5 \mathrm{~m}$

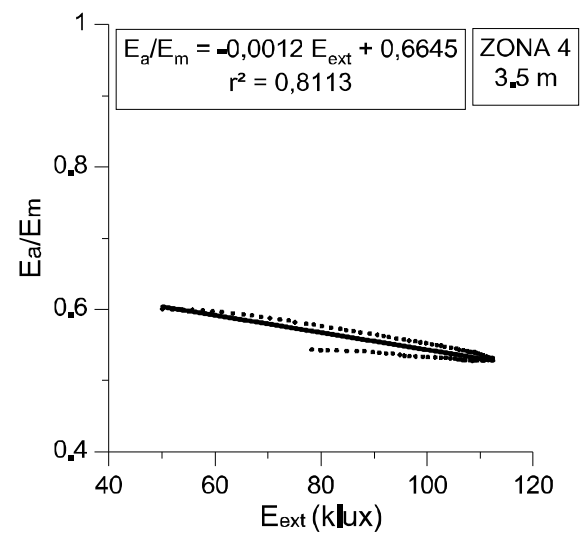

(g) $3.5 \mathrm{~m}$.

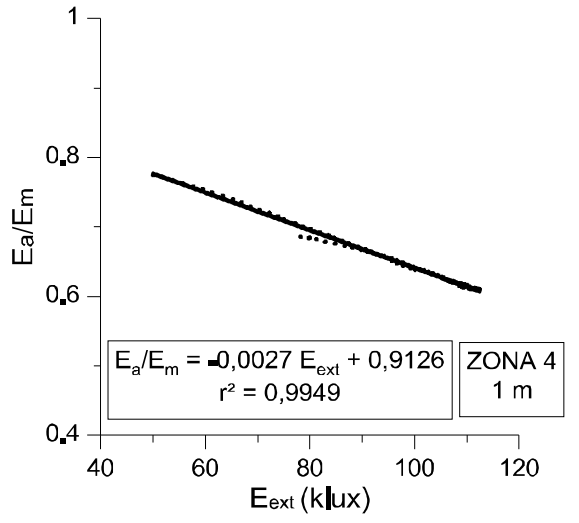

(b) $1 \mathrm{~m}$.

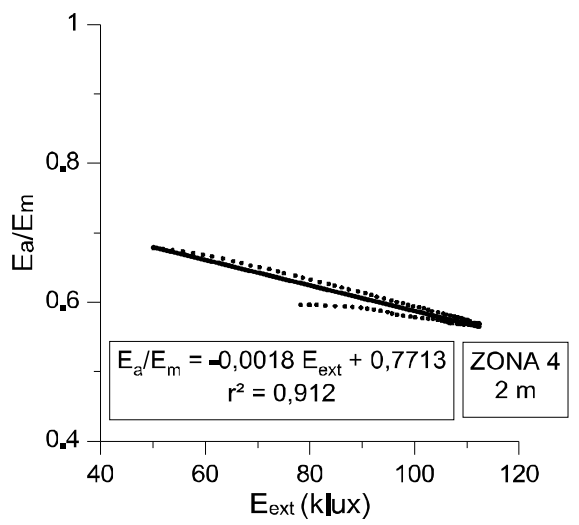

(d) $2 \mathrm{~m}$.

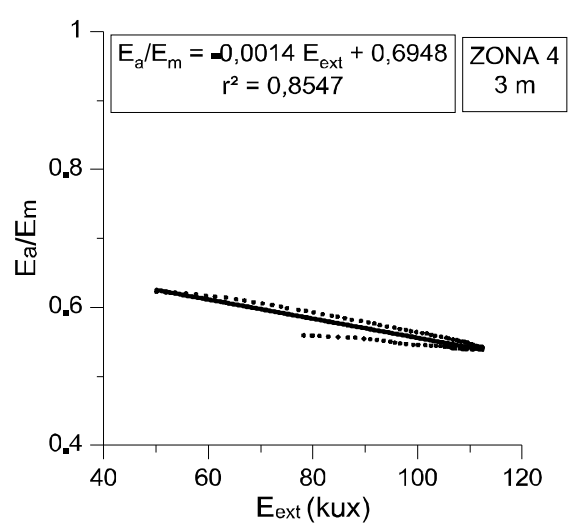

(f) $3 \mathrm{~m}$.

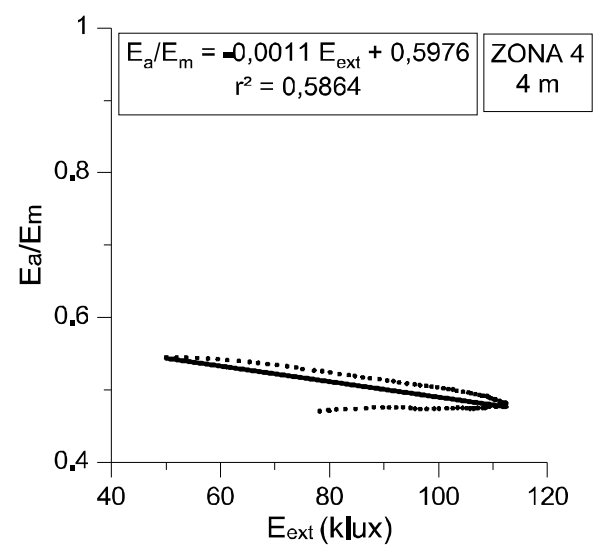

(g) $4 \mathrm{~m}$. 


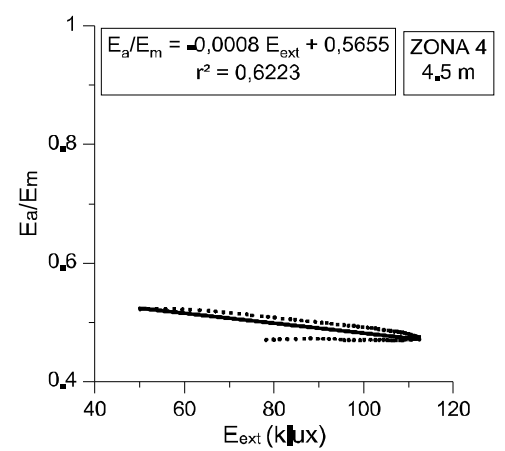

(h) $4.5 \mathrm{~m}$.

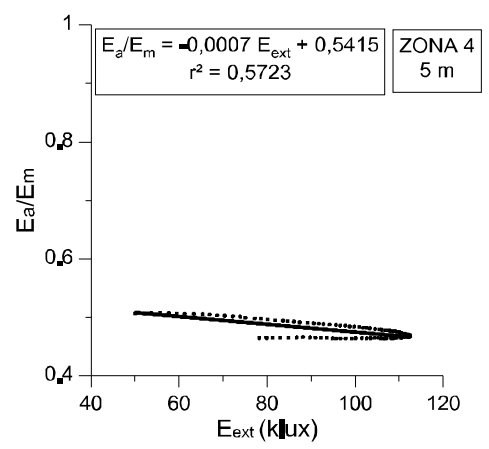

(i) $5 \mathrm{~m}$.

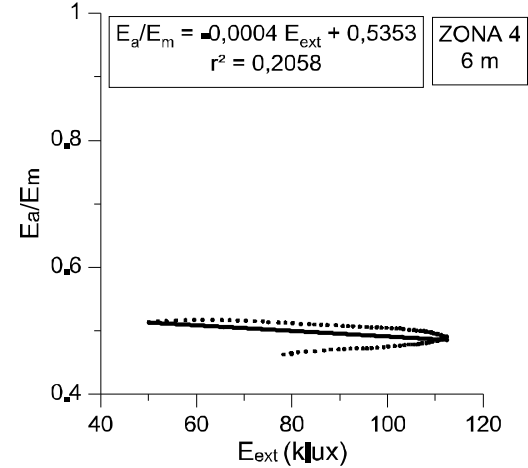

(j) $6 \mathrm{~m}$.

Figura 6.67. Cociente de las iluminancias del aula y del modelo frente a la iluminancia exterior a las distancias indicadas.

En las gráficas de la figura anterior se observa que, para cada distancia, la relación iluminancias aula/modelo frente a la iluminancia exterior es lineal. A medida que aumenta la distancia a la ventana el cociente iluminancia aula/modelo va disminuyendo. Si se representan las distintas rectas de ajuste, obtenidas anteriormente, de forma conjunta, se obtiene la gráfica de la figura 6.68 , de la que se deduce que al aumentar la distancia a la ventana va disminuyendo la pendiente de estas rectas

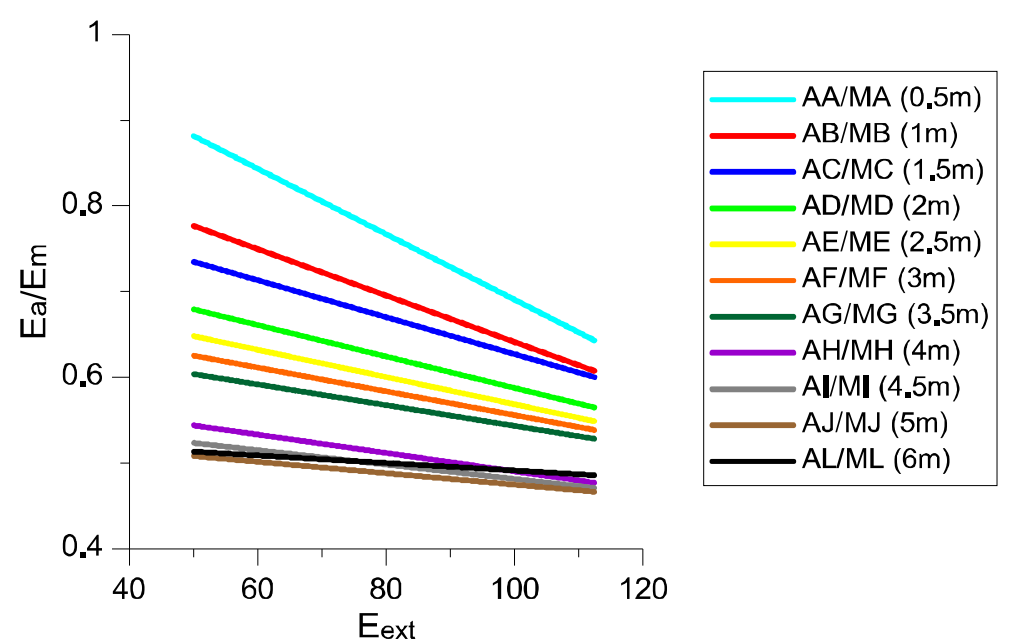

Figura 6.68. Rectas de ajuste de las relaciones entre el cociente de las iluminancias aula/modelo frente a la iluminancia exterior a las distintas distancias. 
Las ecuaciones correspondientes a las rectas anteriores, así como su coeficiente de ajuste $\left(r^{2}\right)$ se presentan en la tabla 6.16. Se puede apreciar como el coeficiente de ajuste va disminuyendo considerablemente según aumenta la distancia a la ventana, perdiendo fiabilidad a partir de $4 \mathrm{~m}$.

\begin{tabular}{|c|c|c|}
\hline DISTANCIA (m) & ECUACIÓN & $\mathbf{r}^{2}$ \\
\hline $\mathbf{0 . 5}$ & $\mathrm{E}_{\mathrm{a}} / \mathrm{E}_{\mathrm{m}}=-0,0038 \mathrm{E}_{\mathrm{ext}}+1.073$ & 0.9862 \\
\hline $\mathbf{1}$ & $\mathrm{E}_{\mathrm{a}} / \mathrm{E}_{\mathrm{m}}=-0,0027 \mathrm{E}_{\mathrm{ext}}+0.9126$ & 0.9949 \\
\hline $\mathbf{1 . 5}$ & $\mathrm{E}_{\mathrm{a}} / \mathrm{E}_{\mathrm{m}}=-0,0022 \mathrm{E}_{\mathrm{ext}}+0.8429$ & 0.9766 \\
\hline $\mathbf{2}$ & $\mathrm{E}_{\mathrm{a}} / \mathrm{E}_{\mathrm{m}}=-0,0018 \mathrm{E}_{\mathrm{ext}}+0.7713$ & 0.9120 \\
\hline $\mathbf{2 . 5}$ & $\mathrm{E}_{\mathrm{a}} / \mathrm{E}_{\mathrm{m}}=-0,0016 \mathrm{E}_{\mathrm{ext}}+0.7277$ & 0.9005 \\
\hline $\mathbf{3}$ & $\mathrm{E}_{\mathrm{a}} / \mathrm{E}_{\mathrm{m}}=-0,0014 \mathrm{E}_{\mathrm{ext}}+0.6948$ & 0.8547 \\
\hline $\mathbf{3 . 5}$ & $\mathrm{E}_{\mathrm{a}} / \mathrm{E}_{\mathrm{m}}=-0,0012 \mathrm{E}_{\mathrm{ext}}+0.6645$ & 0.8113 \\
\hline $\mathbf{4}$ & $\mathrm{E}_{\mathrm{a}} / \mathrm{E}_{\mathrm{m}}=-0,0011 \mathrm{E}_{\mathrm{ext}}+0.5976$ & 0.5864 \\
\hline $\mathbf{4 . 5}$ & $\mathrm{E}_{\mathrm{a}} / \mathrm{E}_{\mathrm{m}}=-0,0008 \mathrm{E}_{\mathrm{ext}}+0.5655$ & 0.6223 \\
\hline $\mathbf{5}$ & $\mathrm{E}_{\mathrm{a}} / \mathrm{E}_{\mathrm{m}}=-0,0007 \mathrm{E}_{\mathrm{ext}}+0.5415$ & 0.5723 \\
\hline $\mathbf{6}$ & $\mathrm{E}_{\mathrm{a}} / \mathrm{E}_{\mathrm{m}}=-0,0004 \mathrm{E}_{\mathrm{ext}}+0.5353$ & 0.2058 \\
\hline & & \\
\hline
\end{tabular}

Tabla 6.16. Ecuaciones que relacionan las iluminancias del aula y del modelo con la del exterior a las distancias consideradas

\subsubsection{Modelo matemático para determinar la iluminancia en el aula}

Hasta el momento, en esta zona 4, se han obtenido unas rectas cuyas ecuaciones relacionan las iluminancias entre el aula $\left(E_{a}\right)$ y el modelo $\left(E_{m}\right)$ en función de la iluminancia exterior $\left(\mathrm{E}_{\text {ext }}\right)$, para las distintas distancias desde puntas del interior hasta la ventana, de la forma:

$$
\frac{E_{a}}{E_{m}}=P \cdot E_{e x t}+O
$$

Siendo $P$ la Pendiente y $O$ la Ordenada en el origen de dichas rectas.

A partir de estas rectas se va a obtener una expresión que permita relacionar las variables anteriores también con la distancia interior a la ventana. Se van a analizar, por separado, las pendientes (figura 6.69) y las ordenadas en el origen (figura 6.70) de las ecuaciones de las rectas recogidas en la tabla 6.16 en función de la distancia. Estos datos se muestran en la tabla 6.17. 


\begin{tabular}{|c|c|c|}
\hline Distancia $(\mathbf{m})$ & Pendiente & Ordenada \\
\hline 0.5 & -0.0038 & 1.073 \\
\hline 1 & -0.0027 & 0.9126 \\
\hline 1.5 & -0.0022 & 0.8429 \\
\hline 2 & -0.0018 & 0.7713 \\
\hline 2.5 & -0.0016 & 0.7277 \\
\hline 3 & -0.0014 & 0.6948 \\
\hline 3.5 & -0.0012 & 0.6645 \\
\hline 4 & -0.0011 & 0.5976 \\
\hline 4.5 & -0.0008 & 0.5655 \\
\hline 5 & -0.0007 & 0.5415 \\
\hline 6 & -0.0004 & 0.5353 \\
\hline
\end{tabular}

Tabla.6.17. Pendientes y ordenadas en el origen a las distintas distancias de las rectas de ajuste procedentes del cociente de las iluminancias aula/modelo frente a la iluminancia exterior.

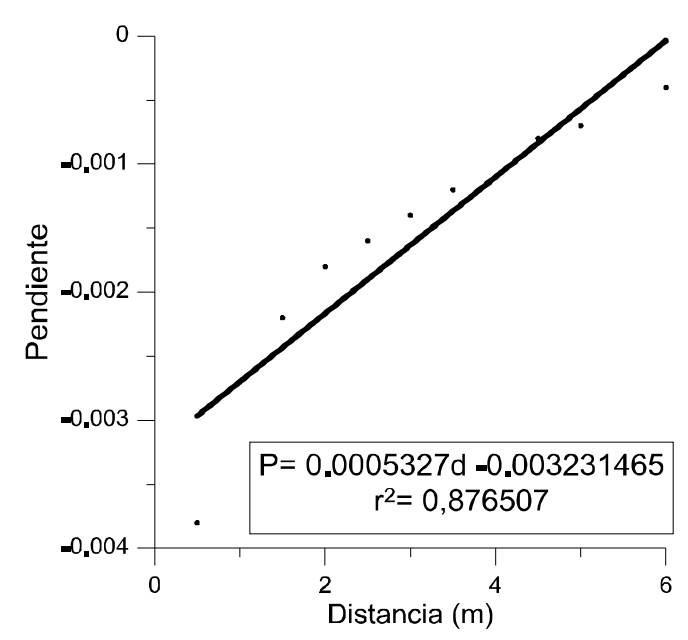

Figura 6.69. Pendiente en función de la distancia a la fachada.

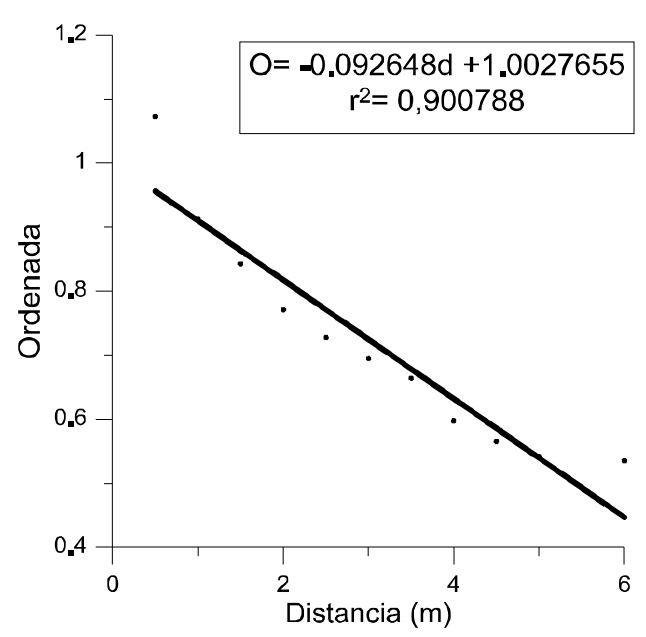

Figura 6.70. Ordenada en el origen en función de la distancia a la fachada.

En la figura 6.69 se han representado los valores de las pendientes $(P)$ para cada distancia $(d)$ a la ventana. Se obtiene una relación lineal definida por la recta: $P=(0.533 \mathrm{~d}-3.232) \times 10^{-3} ; r^{2}=0.975$.

Análogamente, en la figura 6.70 , se han representado las ordenadas en el origen $(O)$ frente a la distancia $(d)$ a la ventana, obteniéndose también una recta, definida por la ecuación: $O=-0.093 d+1.003 ; r^{2}=0.969$. 
En ambos casos el coeficiente de ajuste es superior a 0.95 lo que indica una alta fiabilidad del ajuste. De aquí se deduce que el modelo matemático que permite determinar la iluminancia del aula a partir de la del modelo a escala, la exterior y la distancia en la zona 4 es:

$$
E_{a}=\left[(0.533 d-3.232) \times 10^{-3} E_{e x t}-0.093 d+1.003\right] E_{m} \quad[Z 4]
$$

Siendo:

$$
\begin{aligned}
& E_{a} \text { : lluminancia del aula (klux) } \\
& E_{m} \text { : lluminancia del modelo (klux) } \\
& E_{\text {ext: }} \text { Iluminancia exterior (klux) } \\
& d \text { : Distancia a la fachada (m) }
\end{aligned}
$$

Esta ecuación permite calcular las iluminancias del aula a partir de la distancia del sensor a la ventana y las iluminancias medidas en la maqueta y en el exterior. De esta manera alcanzamos el objetivo de obtener las iluminancias de una estancia real (el aula) sin necesidad de medir en dicha estancia, sólo sería necesario conocer las iluminancias en el exterior y en el interior de la maqueta de la estancia estudiada así como la distancia a la que hayamos colocado los sensores.

\subsubsection{Análisis estadístico}

Como comprobación de la fiabilidad de la ecuación Z4 obtenida anteriormente, se va a realizar un análisis estadístico de los resultados. Por un lado se han analizado las pendientes frente a la distancia a la ventana y por otro lado las ordenadas en el origen también frente a la distancia a la ventana.

A partir de los datos de la tabla 6.17 , en la gráfica de la figura 6.71 se han representado las pendientes $(P)$ en función de la distancia a la ventana $(d)$. En ésta se puede observar que existe una relación lineal, que se ajusta a la recta con pendiente positiva: $P=(0.533 d-3.232) \times 10^{-3}$. La mayor parte de los datos se encuentran dentro de los límites de confianza al 95\% (verde) y todos los datos se encuentran entre los límites de predicción (rojo) por lo que no se aprecian valores atípicos. 


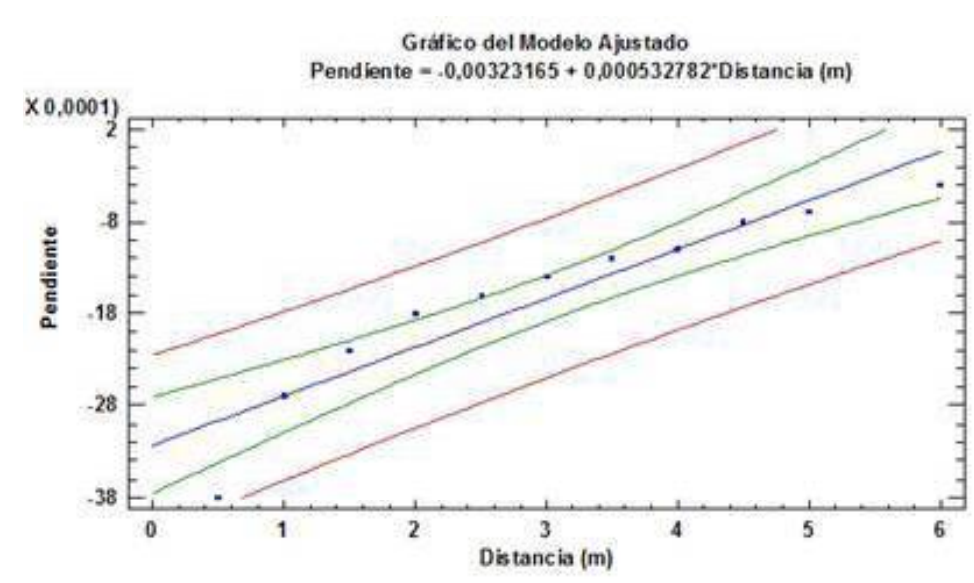

Figura 6.71. Gráfico del modelo ajustado de la pendiente frente a la distancia a la ventana.

Utilizando también los datos de la tabla 6.17 en la figura 6.72 , se han representado las ordenadas en el origen $(O)$ en función de la distancia a la ventana $(d)$. También existe una relación lineal entre ellas definida por la ecuación de primer grado con pendiente negativa: $O=-0.093 d+1.003$. La mayor parte de los datos se encuentran dentro de los límites de confianza al $95 \%$ (verde) y todos los datos se encuentran entre los límites de predicción (rojo), por lo que no se aprecian valores atípicos.

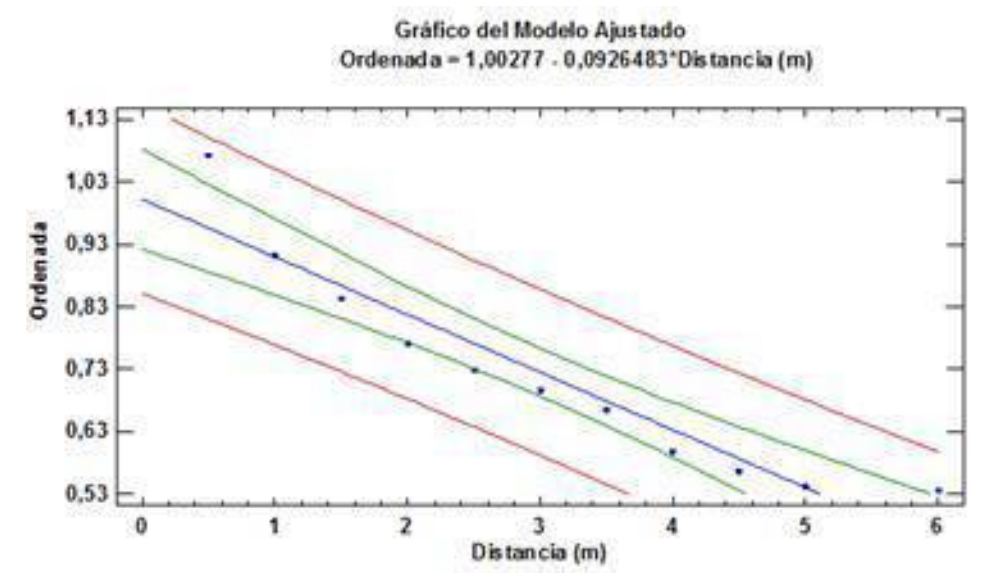

Figura 6.72. Gráfico del modelo ajustado de la ordenada en el origen frente a la distancia a la ventana

En ambos casos se obtiene un p-valor de $0.00<0.5$, lo que indica que hay una relación estadística significante entre las variables con un nivel de confianza de por lo menos el $95 \%$, es decir que la fiabilidad de los datos es $\geq 95 \%$. El p-valor del análisis de los residuos es superior a 0.05 lo que indica que la probabilidad de que existan errores en este análisis es menor al $5 \%$. 


\subsubsection{Simulación con software de iluminación y comprobación de resultados}

Siguiendo el procedimiento realizado en las zonas anteriores, en este apartado se van a comparar las iluminancias del aula obtenidas de medir directamente en el aula, de aplicar la ecuación Z4 y de aplicar el software de iluminación. En la figura 6.73 se muestran, para cada distancia a la ventana considerada, las iluminancias del aula obtenida por los tres métodos: en color rojo las medidas directamente en el aula, en azul claro las obtenidas de aplicar la ecuación Z4 y en azul oscuro las del programa de simulación.

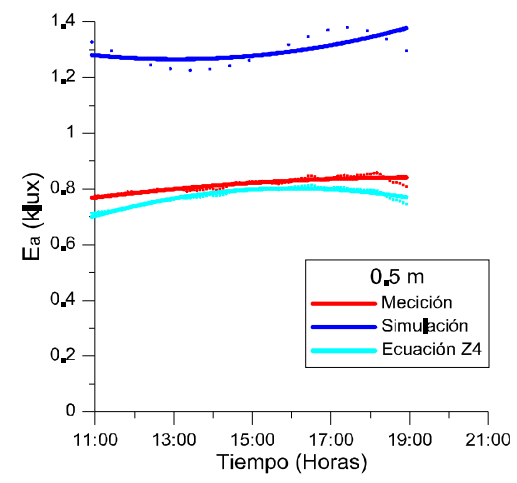

(a) $0.5 \mathrm{~m}$.

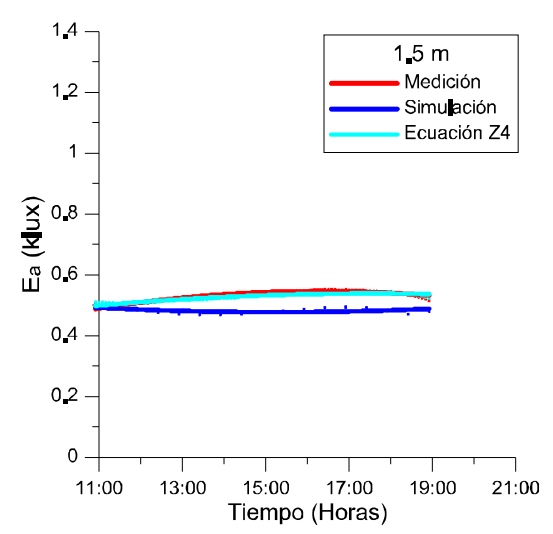

(c) $1.5 \mathrm{~m}$.

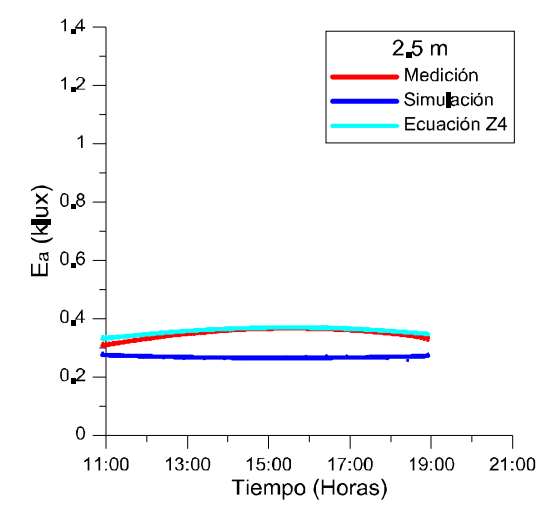

(e) $2.5 \mathrm{~m}$.

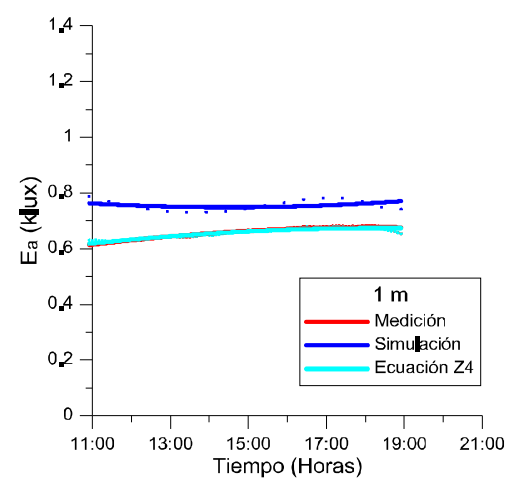

(b) $1 \mathrm{~m}$.

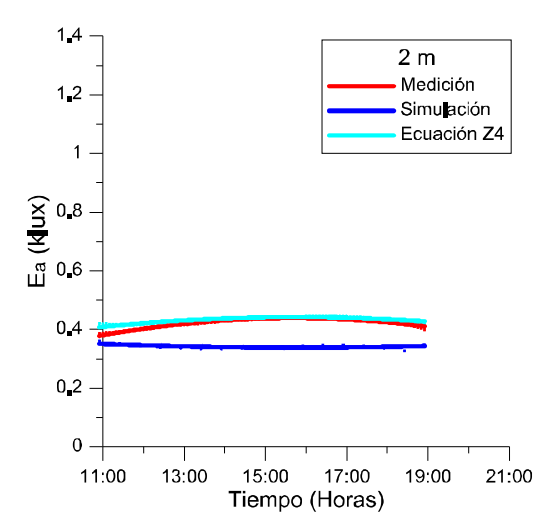

(d) $2 \mathrm{~m}$.

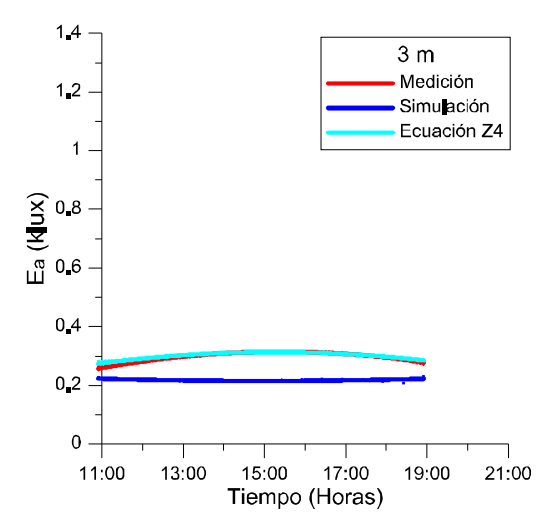

(f) $3 \mathrm{~m}$. 


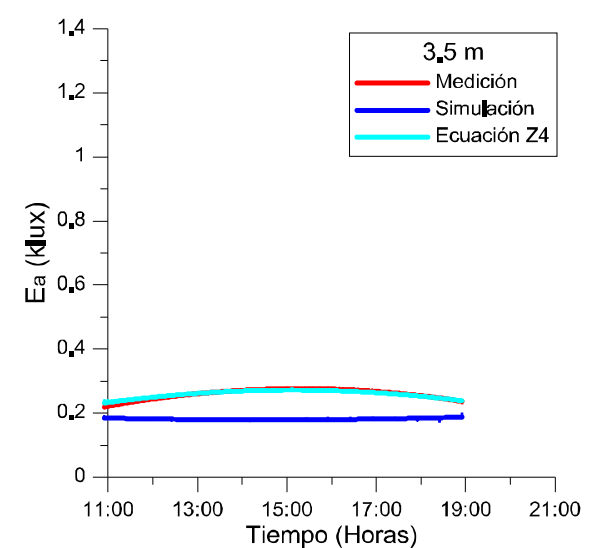

(g) $3.5 \mathrm{~m}$.

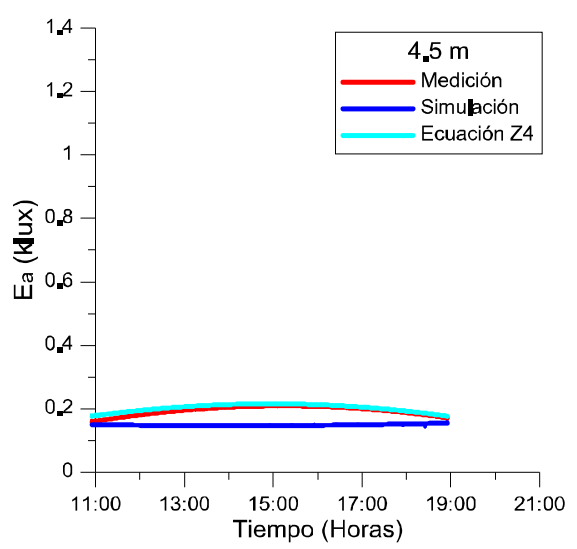

(i) $4.5 \mathrm{~m}$.

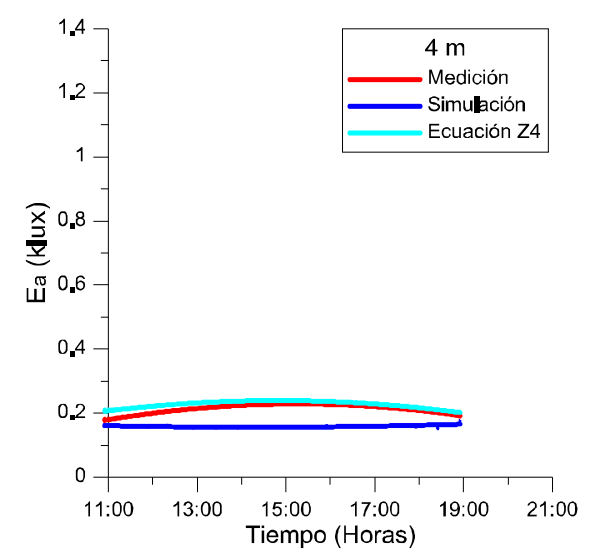

(h) $4 \mathrm{~m}$.

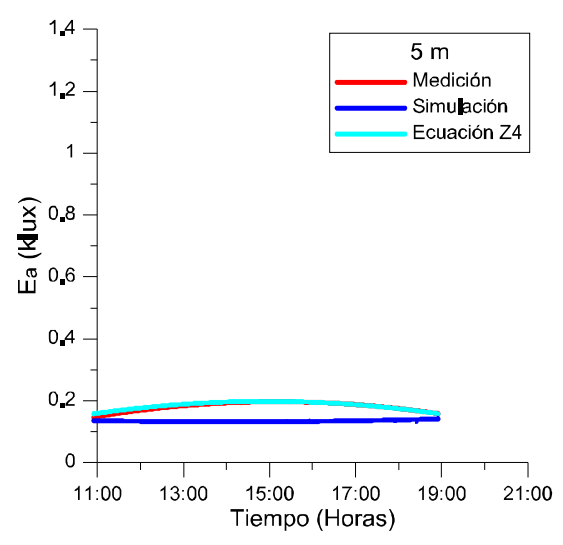

(j) $5 \mathrm{~m}$.

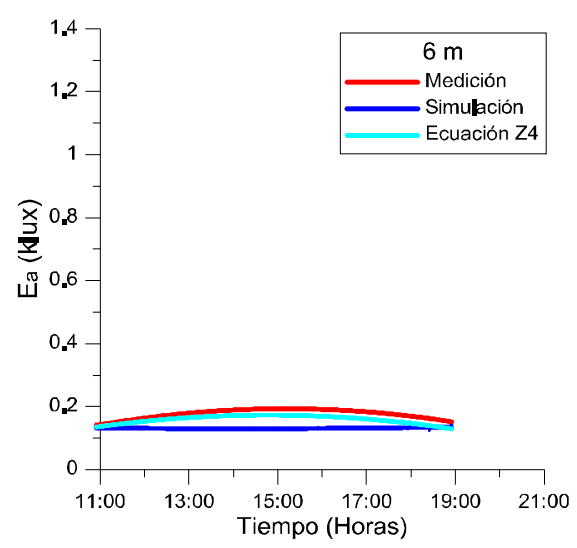

(k)

Figura 6.73. Iluminancias en el aula obtenidas de aplicar la ecuación Z4, por simulación y de forma experimental a las distancias de la ventana indicadas.

En las gráficas de la figura 6.73 se puede observar que a todas las distancias consideradas, salvo a 0.5 y $1 \mathrm{~m}$ de la ventana, los valores de iluminación más bajos se han obtenido en la simulación. Además, las iluminancias obtenidas aplicando la ecuación Z4 son más próximas a las del aula que las obtenidas por simulación. Esto indica que los valores obtenidos mediante esta ecuación son ligeramente más fiables que los obtenidos por simulación. 
Como complemento a esta comprobación de las iluminancias del aula, se van a analizar las iluminancias para todas las distancias simultáneamente, a distintas horas del día $(11: 00,12: 00,13: 00,14: 00,15: 00,16: 00$ y 17:00). Estos resultados se muestran en los diagramas de barras de la figura 6.74 .

En éstos no se han representado las iluminancias obtenidas de aplicar la ecuación Z4 a la distancia de $5.5 \mathrm{~m}$ porque, como se ha indicado anteriormente, el sensor del modelo a escala correspondiente a esta distancia no midió correctamente.

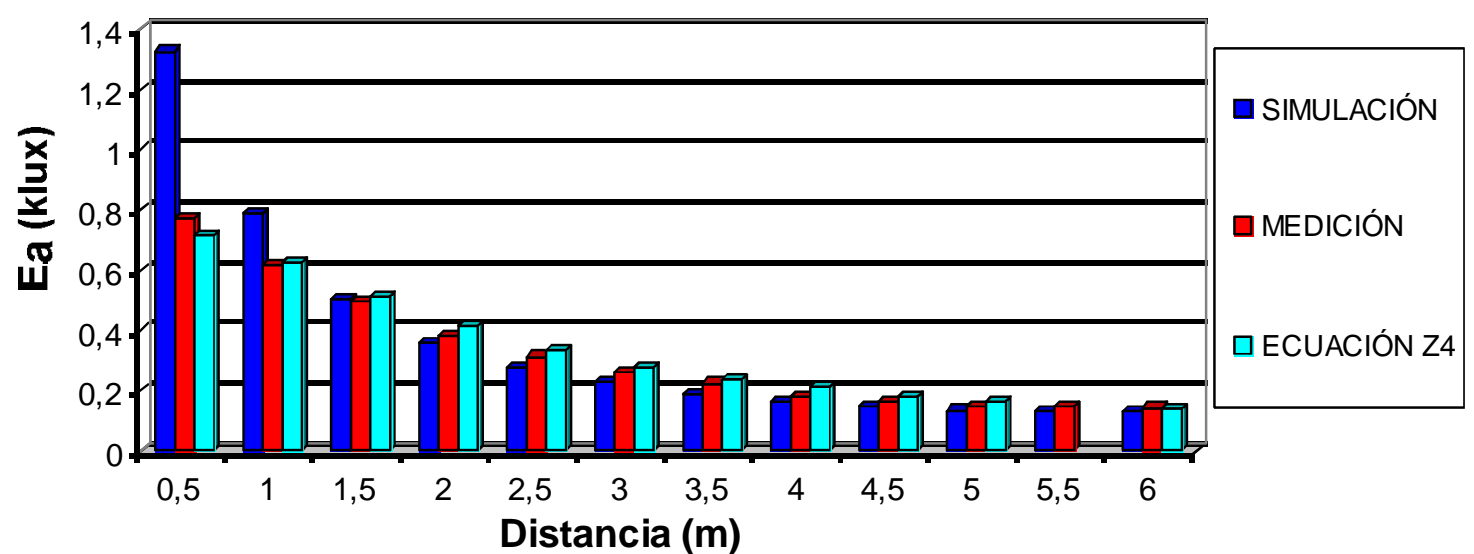

(a) $11: 00$.
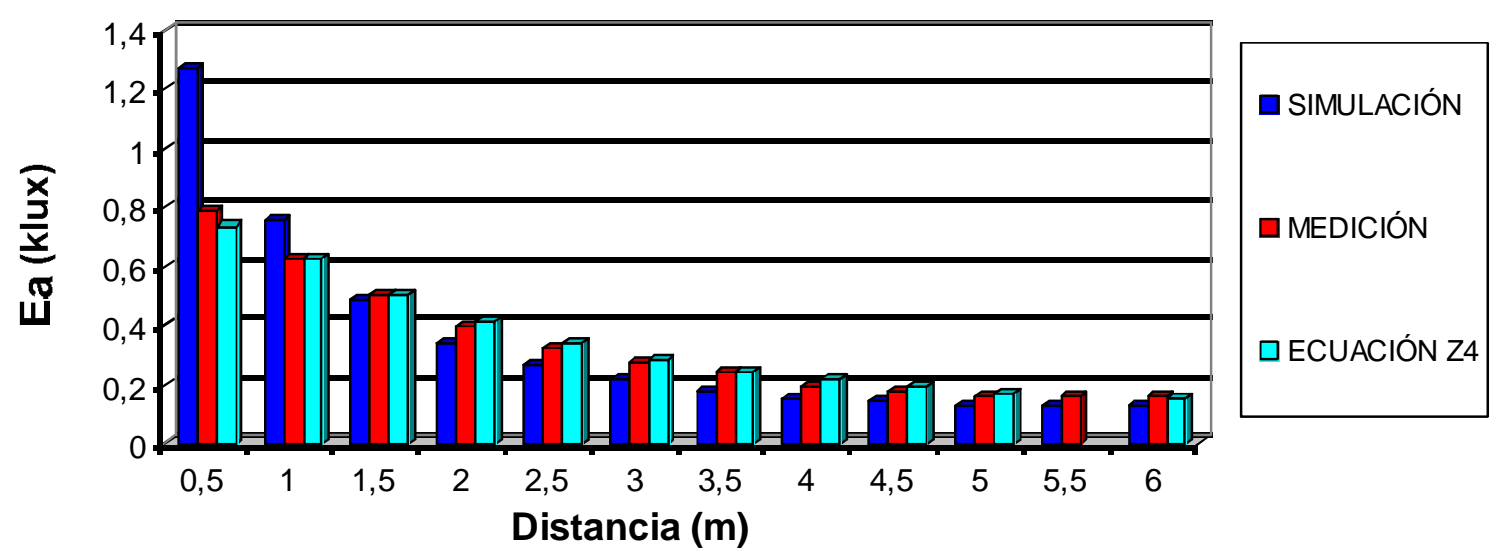

(b) $12: 00$. 


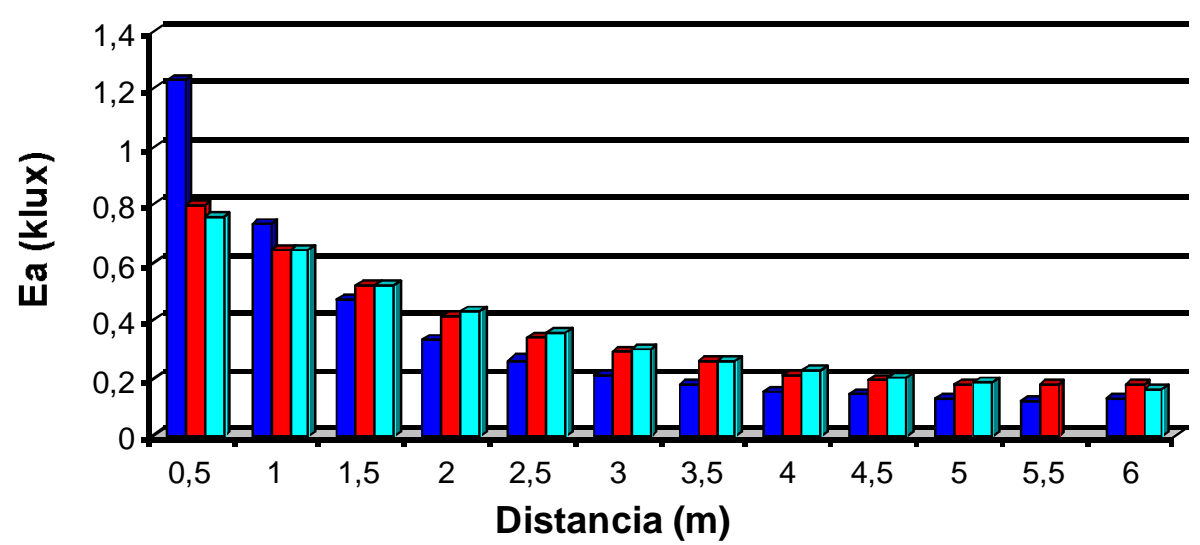

- SIMULACIÓN

口MEDICIÓN

口ECUACIÓN Z4

(c) $13: 00$

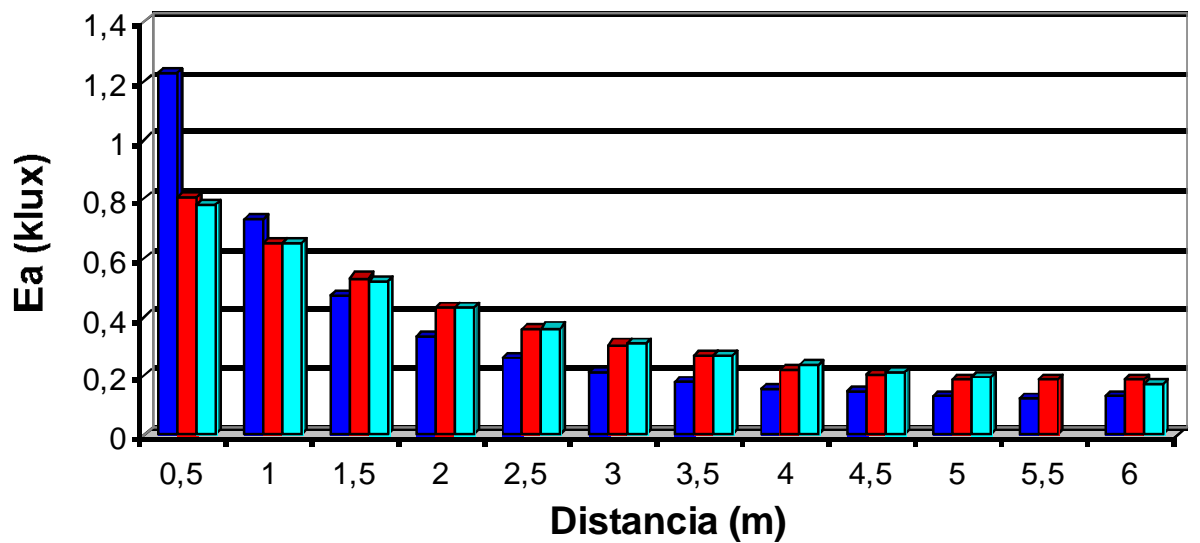

- SIMULACIÓN

口MEDICIÓN

口ECUACIÓN Z4

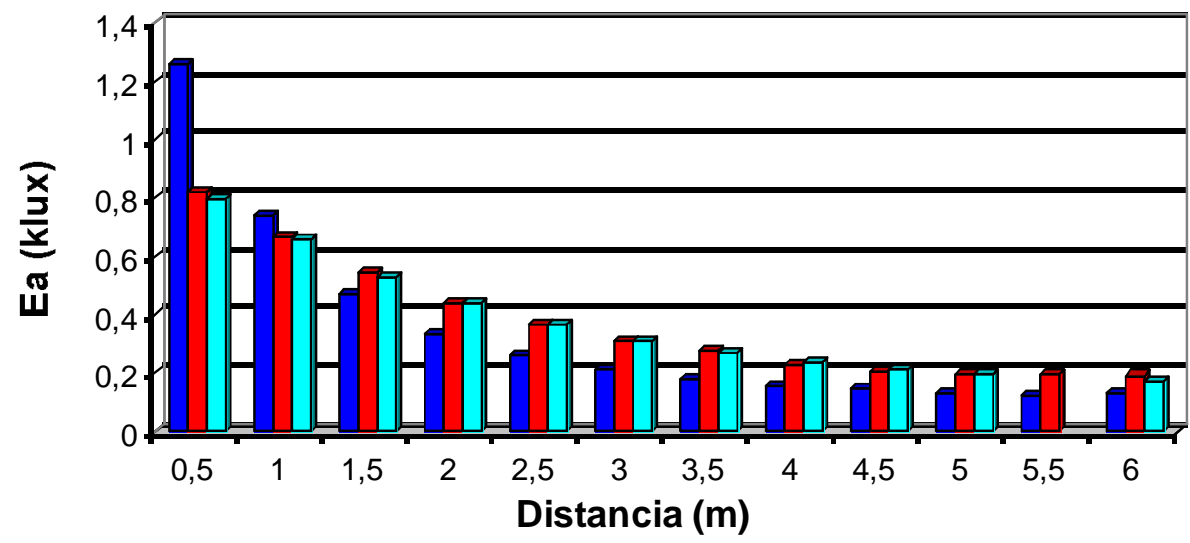

- SIMULACIÓN

口MEDICIÓN

口ECUACIÓN Z4

(e) 15:00. 

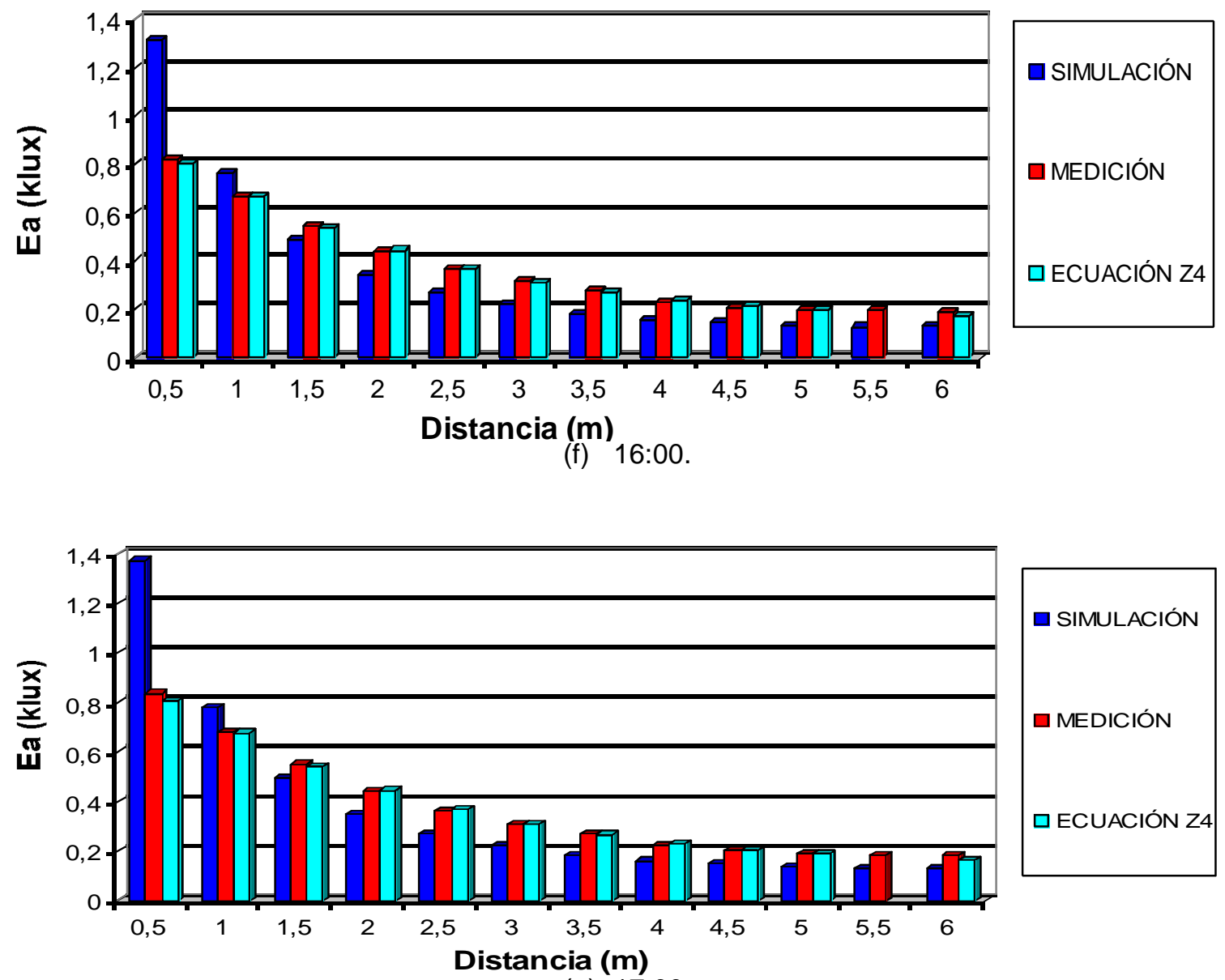

(g) 17:00.

Figura 6.74. lluminancias del aula obtenidas midiendo en el aula, por simulación y aplicando la ecuación Z4 a distintas distancias de la fachada el día 21/07/2015 a las horas indicadas.

Como en las anteriores zonas, también en la figura 6.74, se aprecia cómo el comportamiento de las iluminancias obtenidas mediante la ecuación de la zona 4 se aproximan más a los valores obtenidos por medición en el aula que a los valores obtenidos por software de simulación.

En el anexo II (figura 10.2.7) se presentan las curvas ajustadas de las iluminancias del aula representadas en la figura 6.74 para las distintas horas consideradas, y las ecuaciones que describen dichas curvas con sus correspondientes coeficientes de ajuste, mayor que 0.9 en todos los casos. 


\subsection{Resultados de las cuatro zonas conjuntamente}

En este apartado se presentan los resultados obtenidos al medir simultáneamente en las cuatro zonas en las que se ha dividido el aula 2S2 (zona 1, zona 2, zona 3 y zona 4) a tres distintas distancias (a $1 \mathrm{~m}, 2 \mathrm{~m}$ y $3 \mathrm{~m}$ ) de la fachada (figuras $6.75,6.76,6.77$ y 6.78 ). Se han registrado datos durante una semana, sin embargo, sólo se van a analizar aquellos días con cielo totalmente despejado. Estos días han sido el 01/05/2016 y el 02/05/2016.La colocación y distribución de los sensores en el aula y en el modelo a escala se muestran en las figuras 6.75 y 6.78

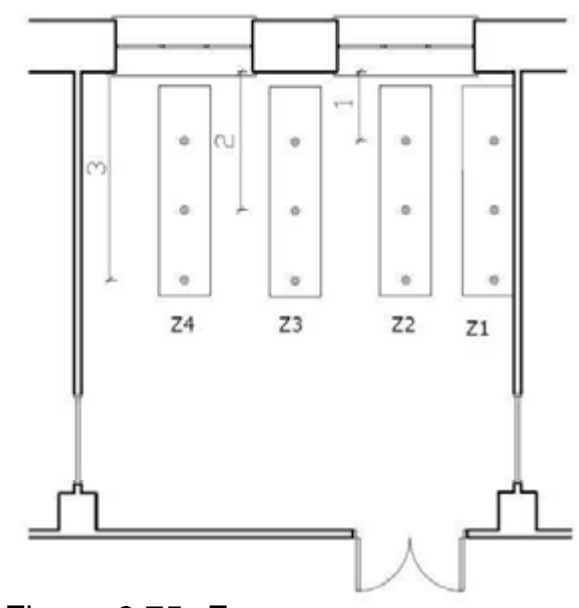

Figura 6.75. Esquema sensores en el aula en las cuatro zonas (cotas en $\mathrm{m}$ ).

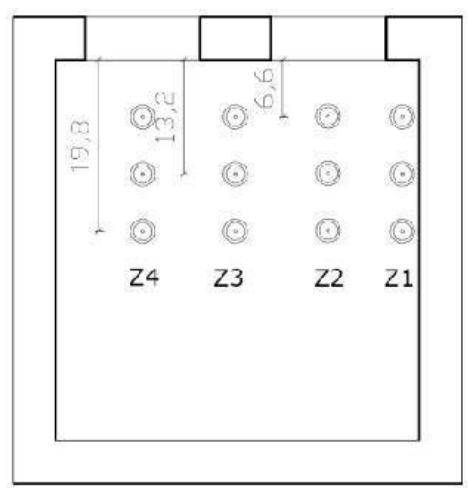

Figura 6.77. Esquema de los sensores en el modelo a escala en las cuatro zonas (cotas en $\mathrm{m}$ ).

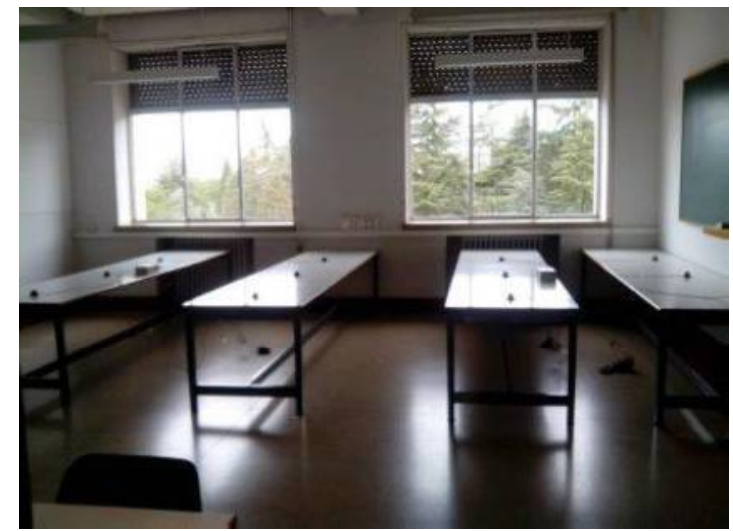

Figura 6.76. Colocación sensores en el aula en las cuatro zonas.

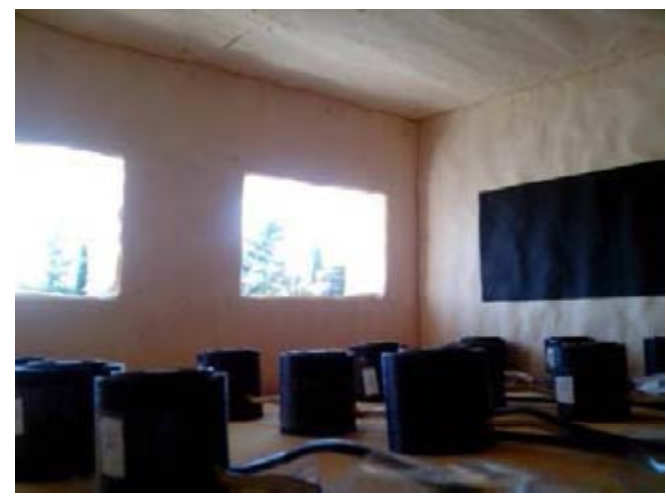

Figura 6.78. Colocación de los sensores en el modelo a escala en las cuatro zonas.

\subsubsection{Evolución diaria de las iluminancias}

La evolución diaria de la iluminancia exterior $\left(E_{\text {ext }}\right)$ los días considerados para este estudio se muestran en la figura 6.79 . 


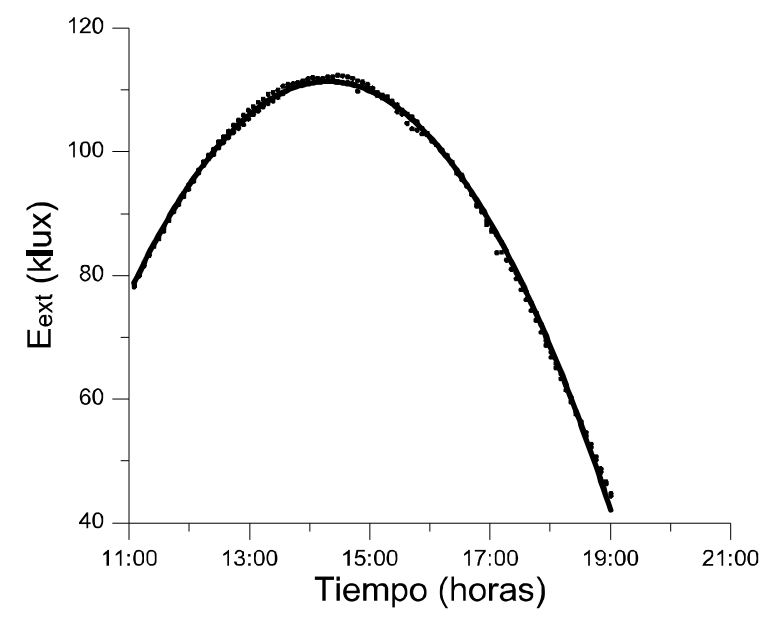

Figura 6.79. Evolución diaria de la iluminancia exterior (Mayo 2016).

Las iluminancias medidas en las 4 zonas del aula y del modelo a escala los días con cielo despejado considerados a las distancias de $1 \mathrm{~m}, 2 \mathrm{~m}$ y $3 \mathrm{~m}$ de la fachada se muestran en las gráficas de las figuras 6.80 y 6.81 . En el eje vertical se han representado las iluminancias en klux, y en el eje horizontal se ha representado el tiempo expresado en horas desde las 11:00 de la mañana hasta las 19:00 de la tarde (hora local).

\section{MODELO}

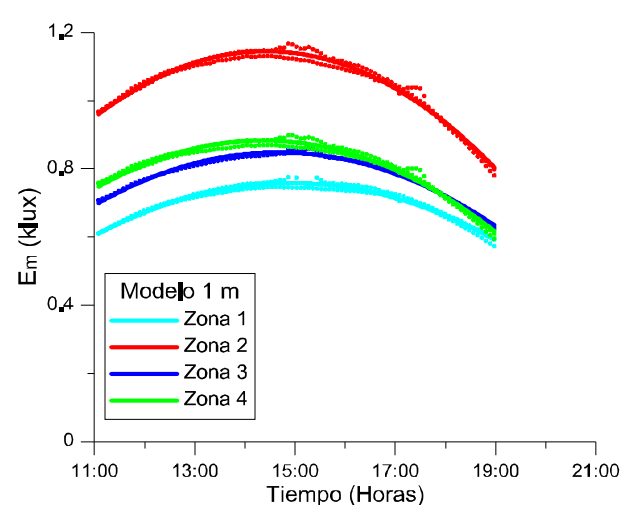

(a) $1 \mathrm{~m}$.

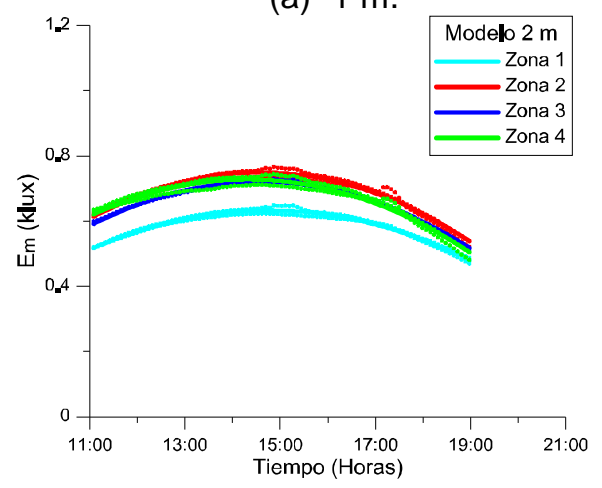

(b) $2 \mathrm{~m}$.

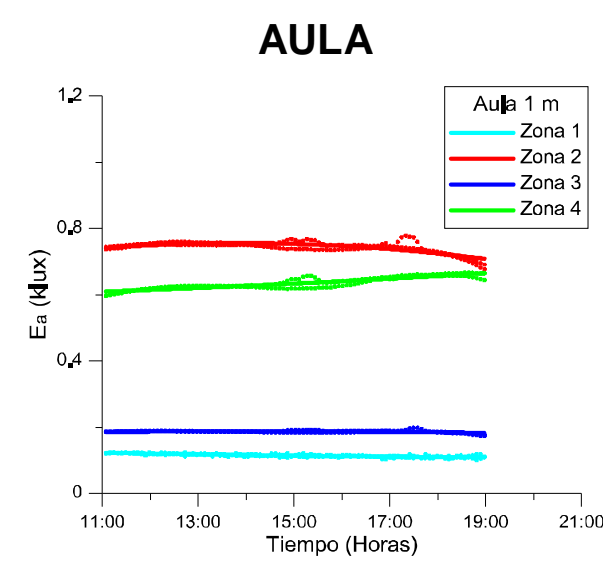

(a) $1 \mathrm{~m}$.

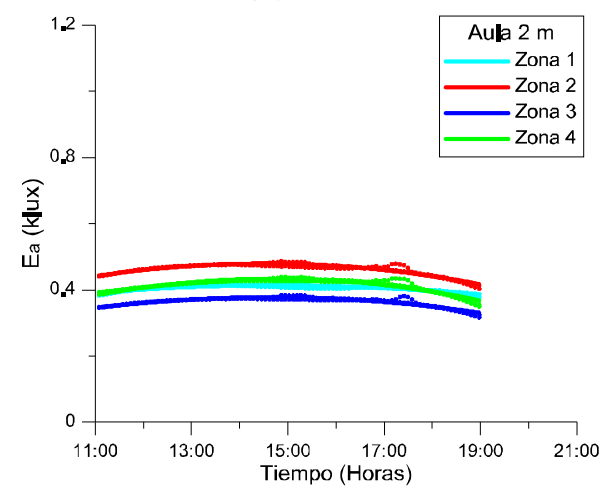

(b) $2 \mathrm{~m}$. 


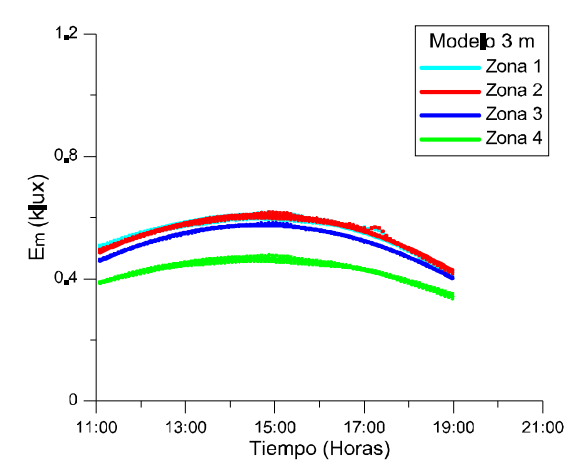

(c) $3 \mathrm{~m}$.

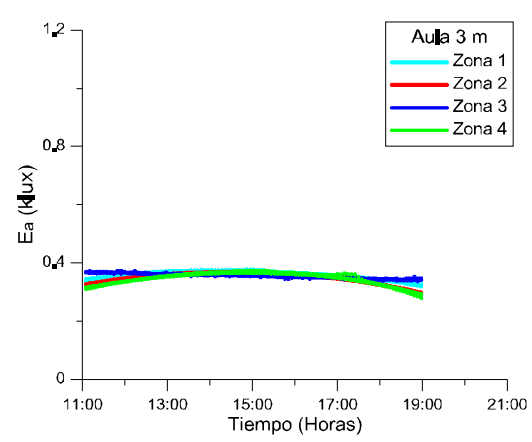

(c) $3 \mathrm{~m}$.

Figura 6.80. Evolución diaria de la iluminancia Figura 6.81. Evolución diaria de la iluminancia en el modelo a escala en todas las zonas a en el aula en todas las zonas a las distancias las distancias indicadas. indicadas.

En las figuras anteriores se puede observar que tanto en el aula como en el modelo a escala las iluminancias más altas se han dado en la zona 2 y a medida que aumenta la distancia a la ventana los valores de las iluminancias obtenidas en las cuatro zonas se aproximan entre sí.

\subsubsection{Relación entre iluminancias del aula y del modelo en función de la iluminancia exterior}

En este apartado se va a analizar la relación entre las iluminancias del aula $\left(E_{a}\right)$ $y$ del modelo a escala $\left(E_{m}\right)$ en función de la iluminancia exterior $\left(E_{\text {ext }}\right)$, expresada en klux, utilizando los datos obtenidos en las cuatro zonas simultáneamente.

En las gráficas de la figura 6.82 se ha representado en el eje vertical el cociente entre las iluminancias del aula y el modelo, y en el eje horizontal las iluminancias obtenidas con el sensor exterior.

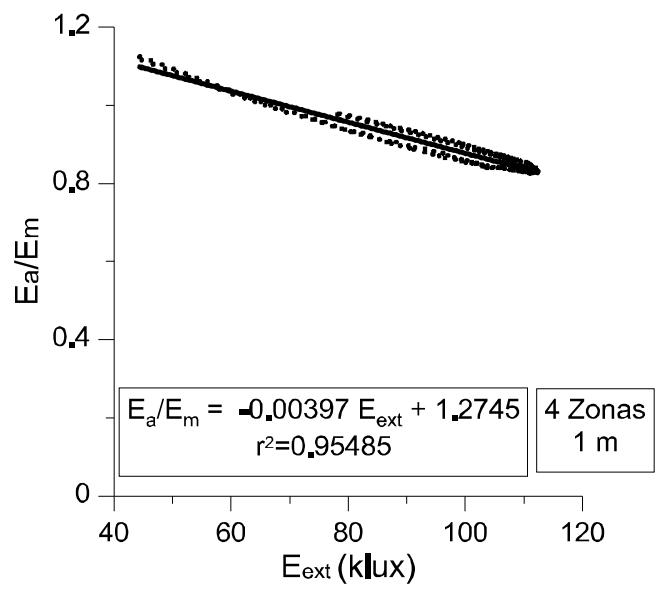

(a) $1 \mathrm{~m}$.

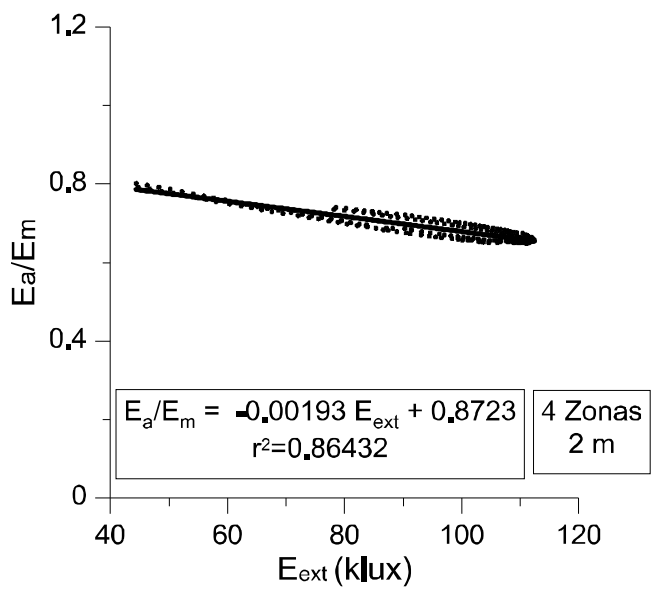

(b) $2 \mathrm{~m}$. 


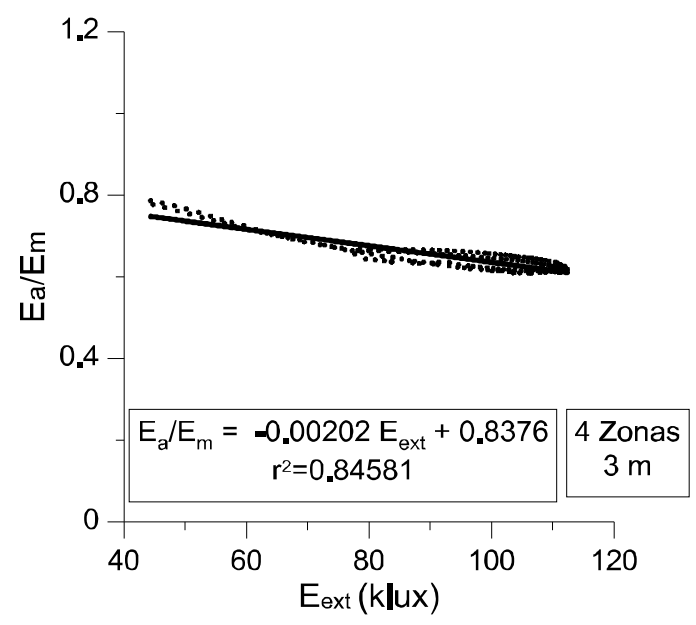

(c) $3 \mathrm{~m}$.

Figura 6.82. Cociente de las iluminancias del aula entre las del modelo frente a la iluminancia exterior a las distancias indicadas.

Como se puede observar la relación entre el cociente de las iluminancias aula/modelo frente a la iluminancia exterior es una relación lineal para las tres distancias a la ventana consideradas. A medida que aumenta esta distancia la relación iluminancia aula/modelo con la iluminancia exterior va disminuyendo.

Si se representan las distintas rectas, obtenidas anteriormente, de forma conjunta, se obtiene la gráfica de la figura 6.83 .

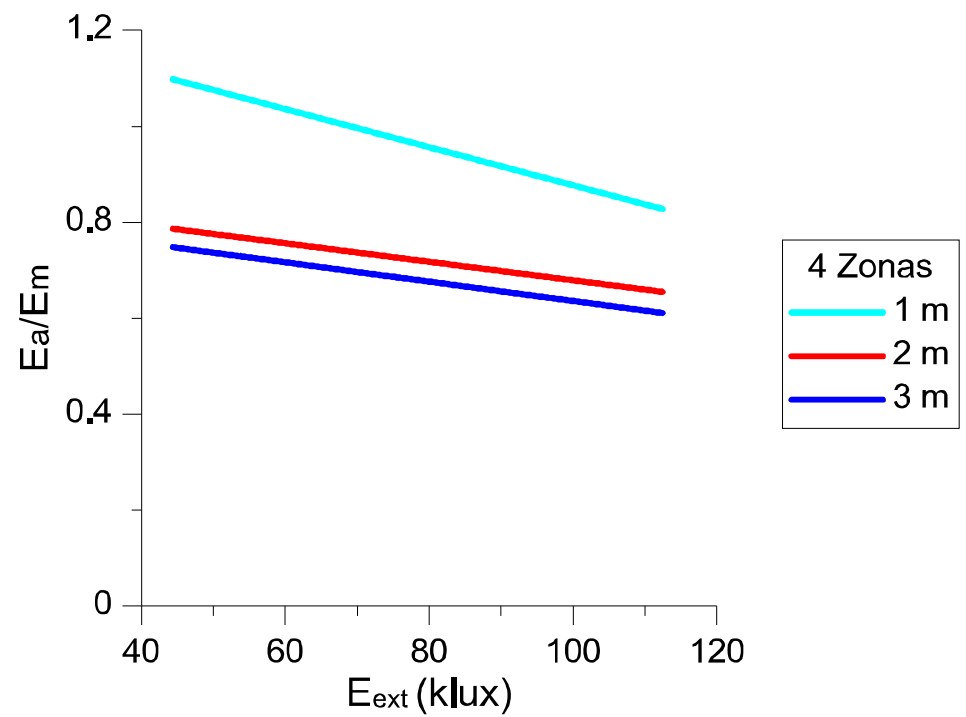

Figura 6.83. Rectas ajustadas a las distintas distancias de la relaciones entre el cociente de las iluminancias aula/modelo frente a la iluminancia exterior.

Se puede observar que según aumenta la distancia a la fachada, la pendiente de estas rectas va disminuyendo. 
Las ecuaciones correspondientes a las rectas anteriores, así como su coeficiente de ajuste $\left(r^{2}\right)$ se recogen en la tabla 6.18. En ésta se puede apreciar cómo según aumenta la distancia a la ventana el coeficiente de ajuste va disminuyendo.

\begin{tabular}{|c|c|c|}
\hline DISTANCIA (m) & ECUACIÓN & $\mathbf{r}^{\mathbf{2}}$ \\
\hline $\mathbf{1}$ & $\mathrm{E}_{\mathrm{a}} / \mathrm{E}_{\mathrm{m}}=-0,00397 \mathrm{E}_{\mathrm{ext}}+1,2745$ & 0,95485 \\
\hline $\mathbf{2}$ & $\mathrm{E}_{\mathrm{a}} / \mathrm{E}_{\mathrm{m}}=-0,00193 \mathrm{E}_{\mathrm{ext}}+0,8723$ & 0,86432 \\
\hline $\mathbf{3}$ & $\mathrm{E}_{\mathrm{a}} / \mathrm{E}_{\mathrm{m}}=-0,00202 \mathrm{E}_{\mathrm{ext}}+0,8376$ & 0,84581 \\
\hline
\end{tabular}

Tabla 6.18. Ecuaciones de ajuste que relacionan las iluminancias del aula y del modelo con la iluminancia exterior a las distancias consideradas.

\subsubsection{Modelo matemático para determinar la iluminancia en el aula}

En el apartado anterior se han obtenido unas ecuaciones que relacionan las iluminancias entre el aula $\left(E_{a}\right)$ y el modelo $\left(E_{m}\right)$ en función de la iluminancia exterior $\left(E_{\text {ext }}\right)$, para las distintas distancias desde la fachada a zonas del interior, de la forma:

$$
\frac{E_{a}}{E_{m}}=P . E_{e x t}+O
$$

Siendo $P$ la Pendiente y $O$ la Ordenada en el origen, de dichas ecuaciones recogidas en la tabla 6.18. A partir de estas ecuaciones se va a obtener una expresión que permita relacionar las variables anteriores con la distancia a la fachada. Para ello se va a analizar por separado, las pendientes (figura 6.84) y las ordenadas en el origen (figura 6.85) de las ecuaciones de las rectas recogidas en la tabla 6.18 en función de la distancia. Estos datos se muestran en la tabla 6.19.

\begin{tabular}{|c|c|c|}
\hline Distancia $(\mathbf{m})$ & Pendiente & Ordenada \\
\hline 1 & $-0,00397$ & 1,2745 \\
\hline 2 & $-0,00193$ & 0,8723 \\
\hline 3 & $-0,00202$ & 0,8376 \\
\hline
\end{tabular}

Tabla 6.19. Pendientes y ordenadas en el origen a las distintas distancias de las rectas de ajuste procedentes del cociente de las iluminancias aula/modelo frente a la iluminancia exterior. 
En este caso, al hacer el estudio de todas las zonas a la vez, sólo se tienen datos a tres distancias, lo que va a dar resultados menos fiables, aunque sirva para un análisis comparativo.

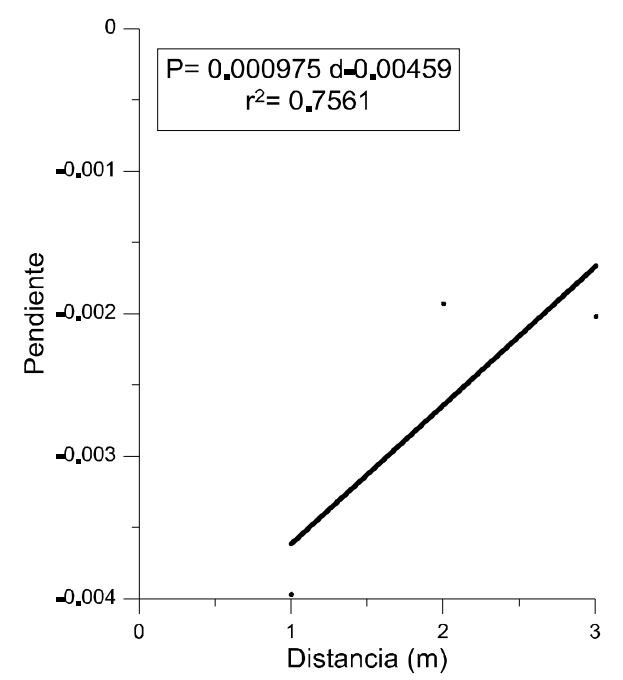

Figura 6.84. Pendiente en función de la distancia a la fachada.

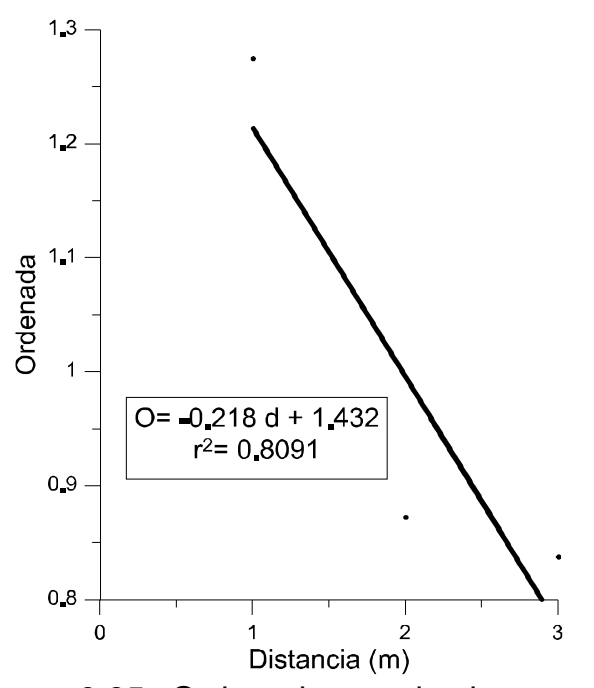

Figura 6.85. Ordenada en el origen en función de la distancia a la fachada.

La relación entre la pendiente $(P)$ y la distancia $(d)$ es lineal, y se expresa por la ecuación de la recta: $P=(0.975 d-4.590) \times 10^{-3}$ con $r^{2}=0.7561$. La relación entre la ordenada en el origen $(O)$ y la distancia a la ventana $(d)$ también es lineal, se define por la recta: $O=-0.218 d+1.432$ con $r^{2}=0.8091$. Como se ha indicado anteriormente, al sólo tener 3 datos los coeficientes de ajuste son menores.

Por lo tanto, el modelo matemático que permite determinar la iluminancia del aula a partir de la del modelo a escala, la exterior y la distancia, de forma única en todas las zonas es:

$$
E_{a}=\left[(0.975 d-4.590) \times 10^{-3} E_{e x t}-0.218 d+1.432\right] E_{m} \quad[G]
$$

Esta ecuación permite calcular las iluminancias del aula a partir de la distancia del sensor a la ventana y las iluminancias medidas en el modelo a escala y en el exterior. 


\subsubsection{Simulación con software de iluminación y comprobación de resultados}

En este apartado se van a comprobar las iluminancias del aula para cada zona obtenidas de cuatro formas distintas:

a) Midiendo directamente en el aula.

b) Calculado mediante la ecuación obtenida para cada zona de forma individual [Z1, Z2, Z3 ó Z4].

c) Calculado mediante la ecuación general obtenida de todas las zonas simultáneamente [G].

d) Utilizando el software de iluminación Dialux 4.11.

En la figura 6.86 se muestran gráficamente los resultados obtenidos para la zona 1, por las cuatro formas distintas, desde las 11:00 hasta las 19:00 horas (hora local).

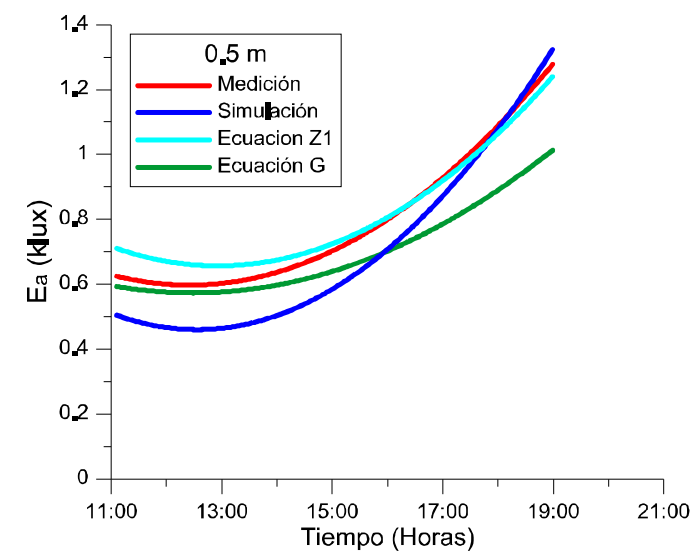

(a) $0.5 \mathrm{~m}$.

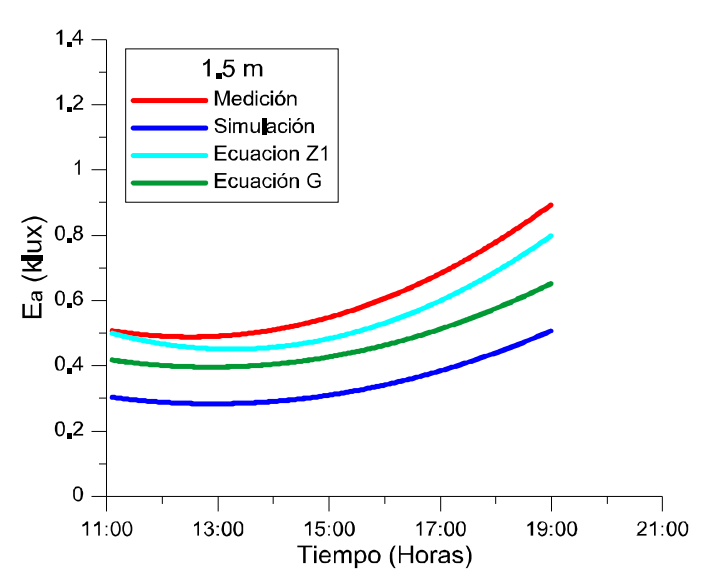

(c) $1.5 \mathrm{~m}$.

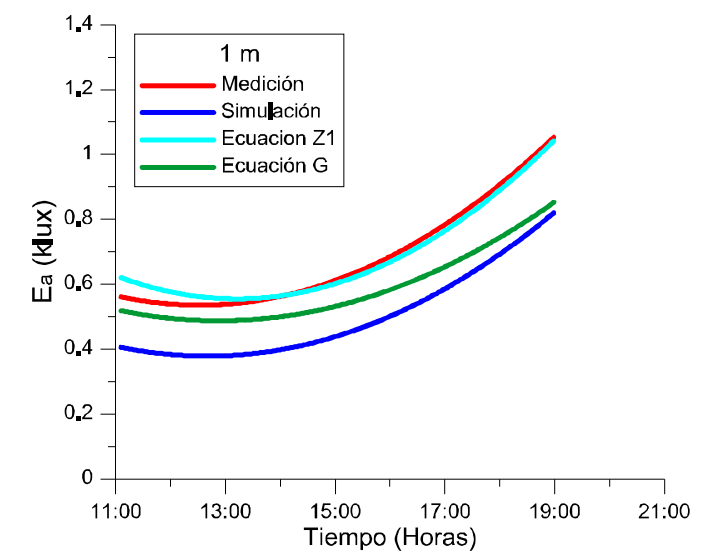

(b) $1 \mathrm{~m}$.

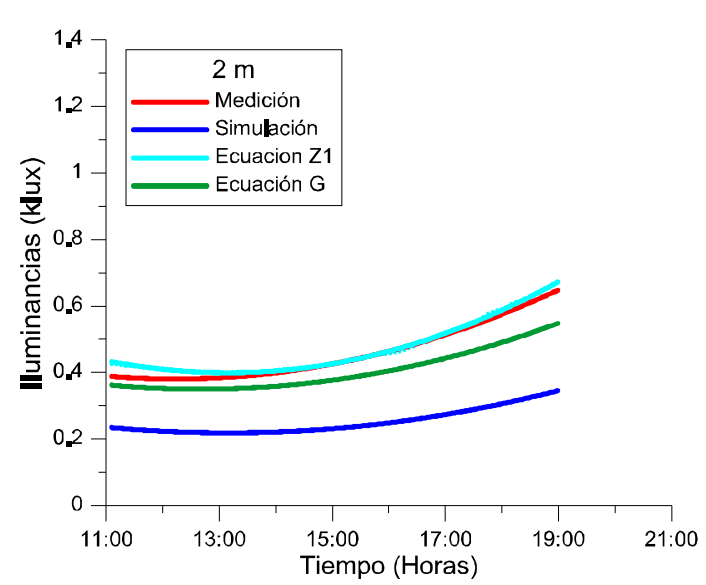

(d) $2 \mathrm{~m}$. 


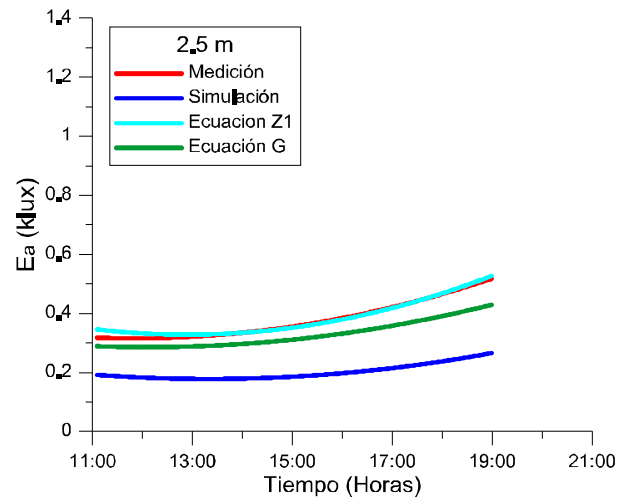

(e) $2.5 \mathrm{~m}$

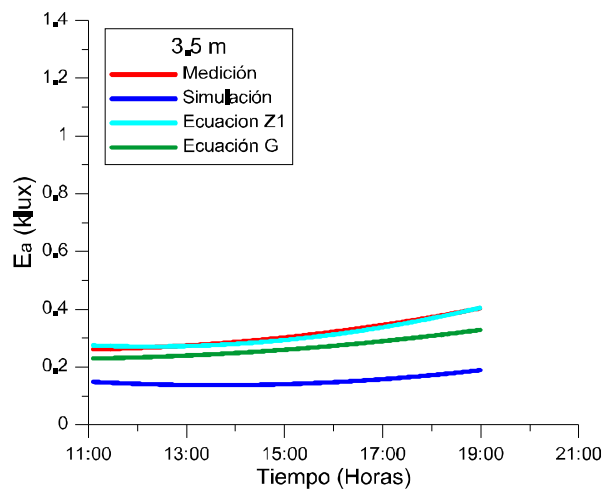

(g) $3.5 \mathrm{~m}$.

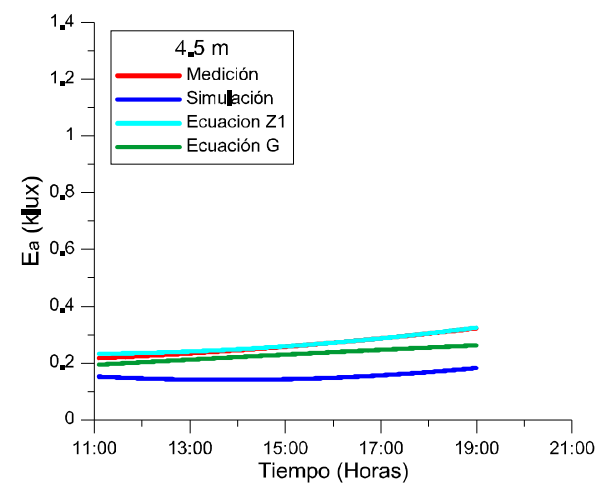

(j) $4.5 \mathrm{~m}$.

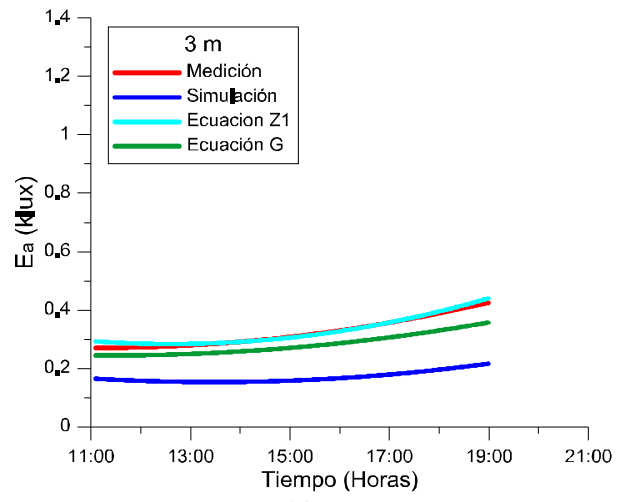

(f) $3 \mathrm{~m}$.

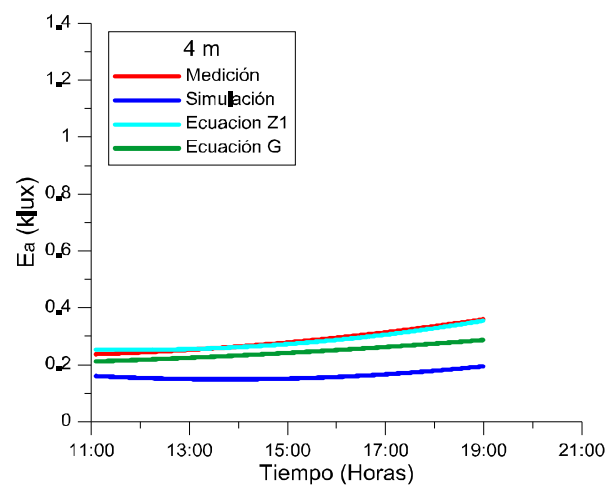

(h) $4 \mathrm{~m}$.

(i)

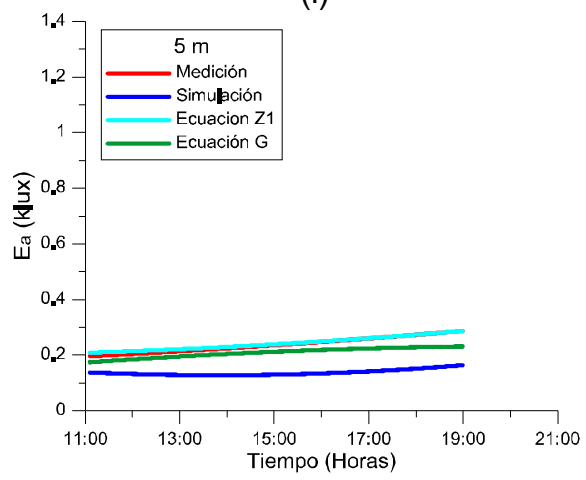

(k) $5 \mathrm{~m}$.

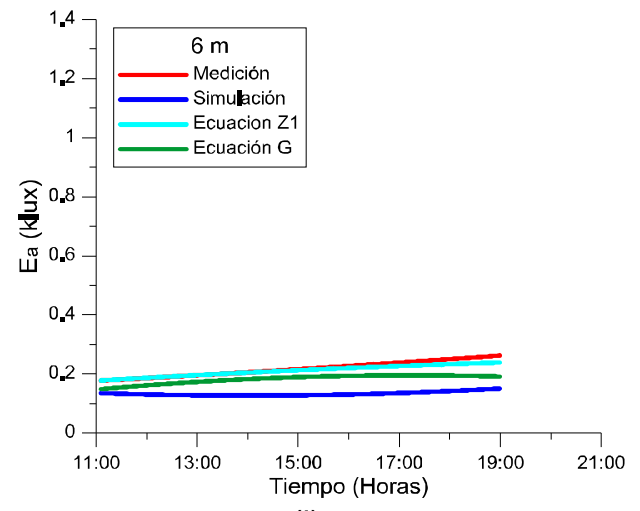

(I) $6 \mathrm{~m}$.

Figura 6.86. Iluminancias de la zona 1 del aula obtenidas midiendo en el aula, utilizando las ecuaciones Z1 y G, y por simulación, a las distancias indicadas. 
En la figura 6.86 se aprecia como las iluminancias obtenidas tras aplicar la ecuación Z1 son las que más se aproximan a las iluminancias medidas directamente en el aula. Las medidas obtenidas tras aplicar la ecuación $\mathrm{G}$ son ligeramente inferiores a las tomadas directamente en el aula, aunque los valores más bajos se han dado en las iluminancias obtenidas mediante simulación, a excepción de las iluminancias en el sensor situado a $0.5 \mathrm{~m}$ de la fachada a partir de las 16:00 horas. También se puede apreciar cómo según aumenta la distancia a la fachada la diferencia entre los valores de las iluminancias obtenidas por los 4 métodos se va acortando.

A continuación, en la figura 6.87 se muestran gráficamente los resultados de las iluminancias del aula obtenidos para la zona 2, de las cuatro formas distintas mencionadas, desde las 11:00 hasta las 19:00 horas.

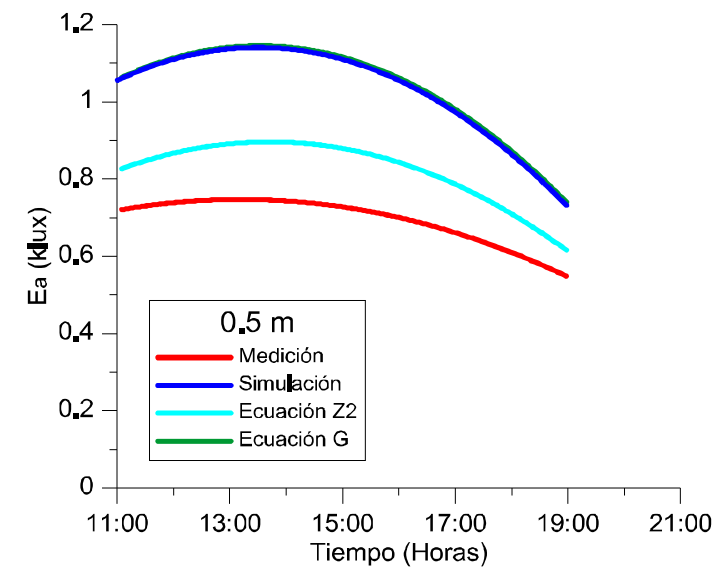

(a) $0.5 \mathrm{~m}$.

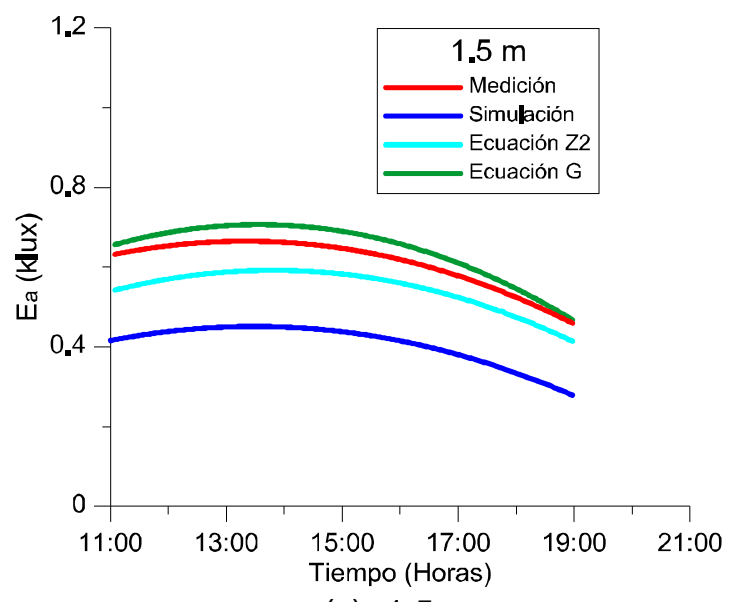

(c) $1.5 \mathrm{~m}$.

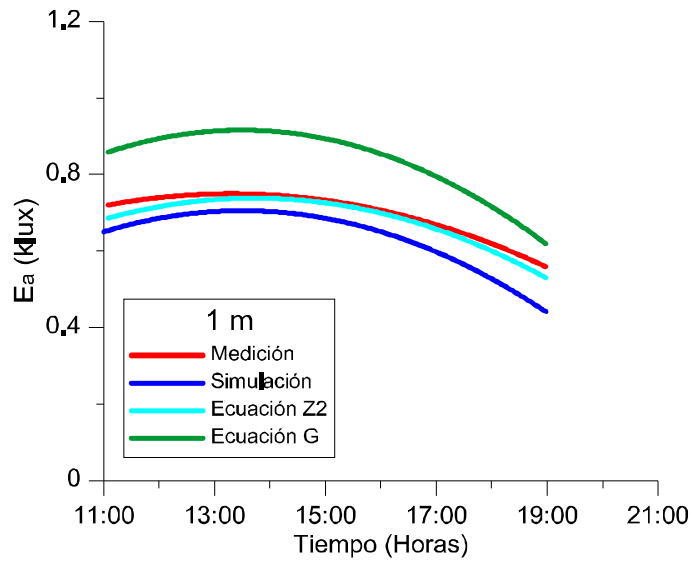

(b) $1 \mathrm{~m}$.

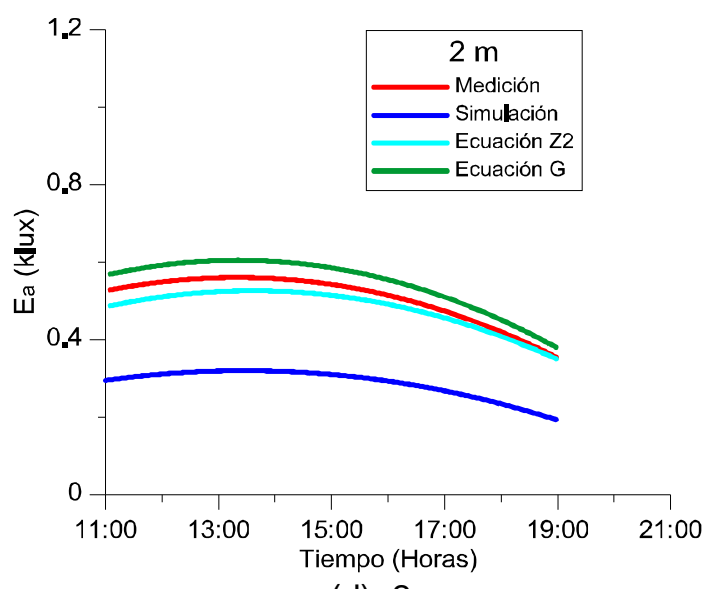

(d) $2 \mathrm{~m}$. 


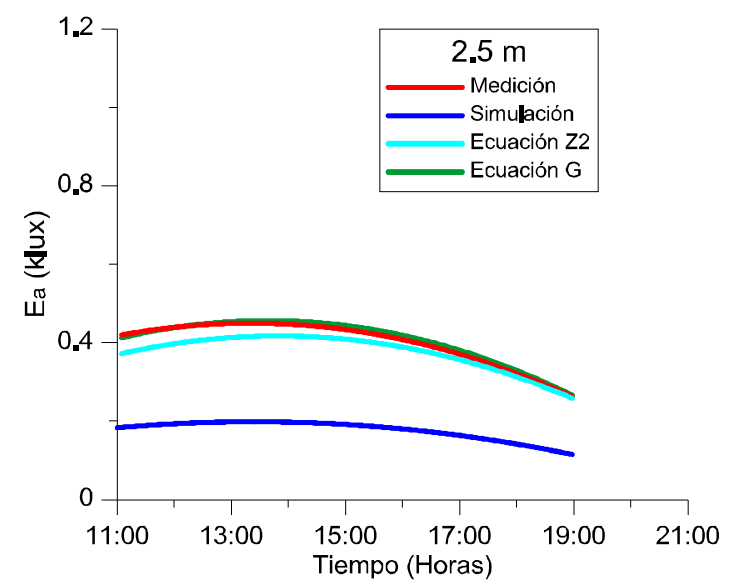

(e) $2.5 \mathrm{~m}$.

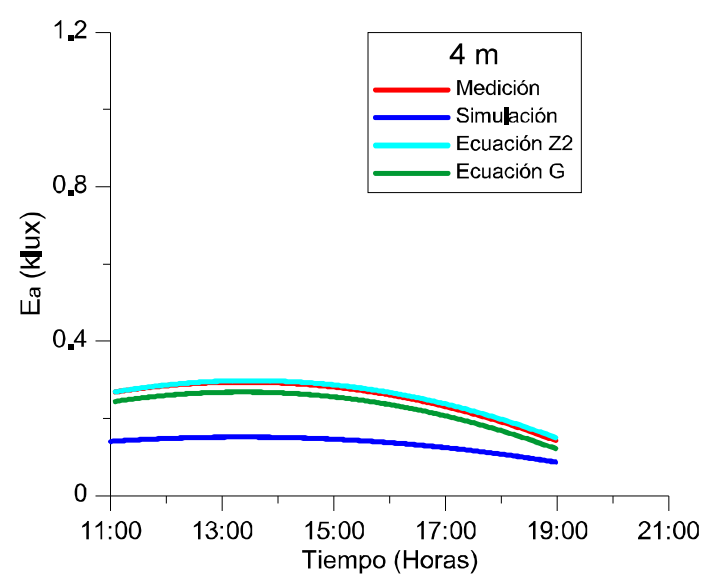

(g) $4 \mathrm{~m}$.

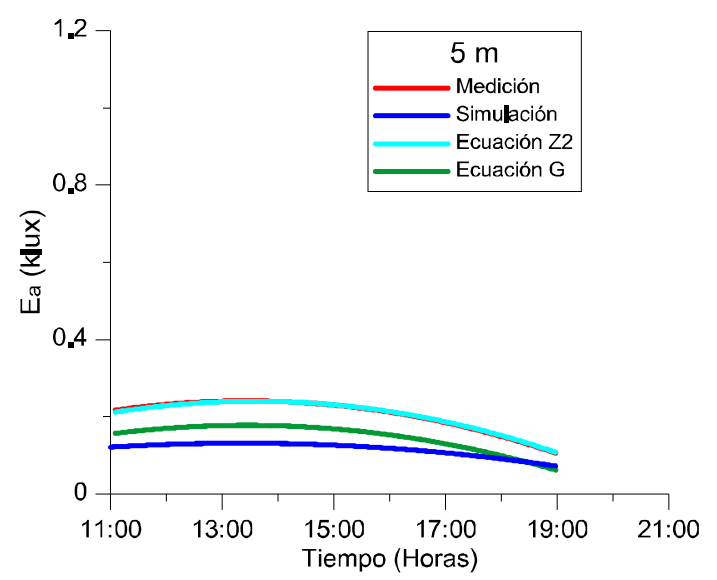

(j) $5 \mathrm{~m}$.

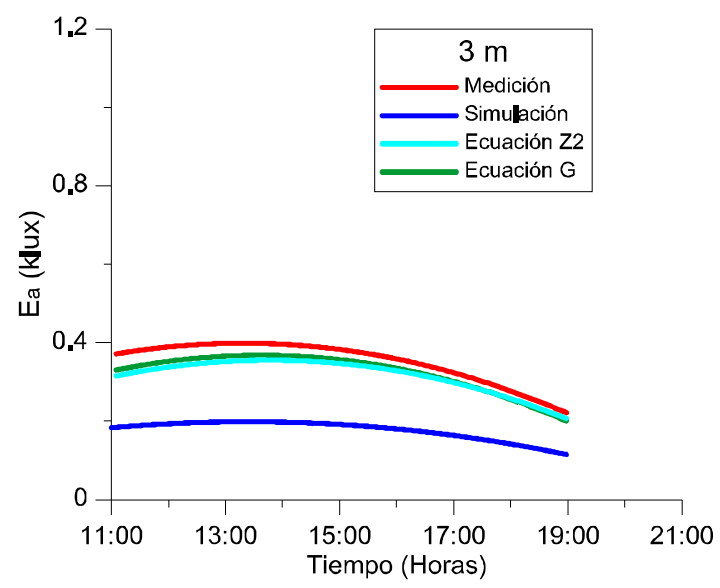

(f) $3 \mathrm{~m}$.

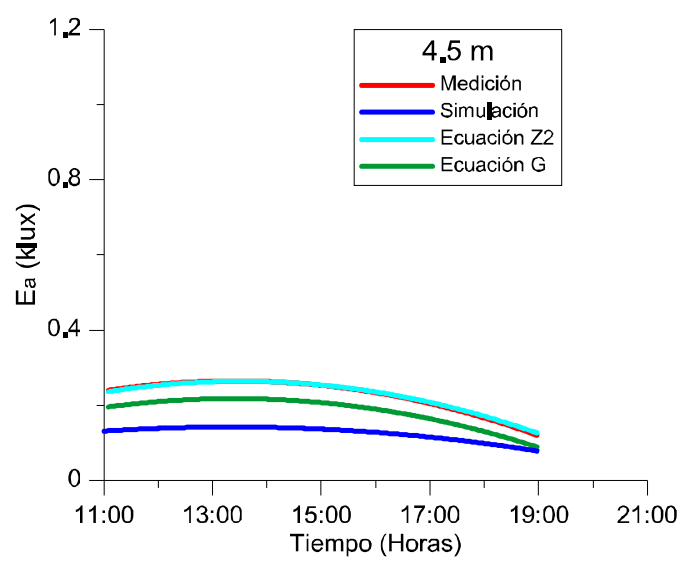

(h) $4.5 \mathrm{~m}$.

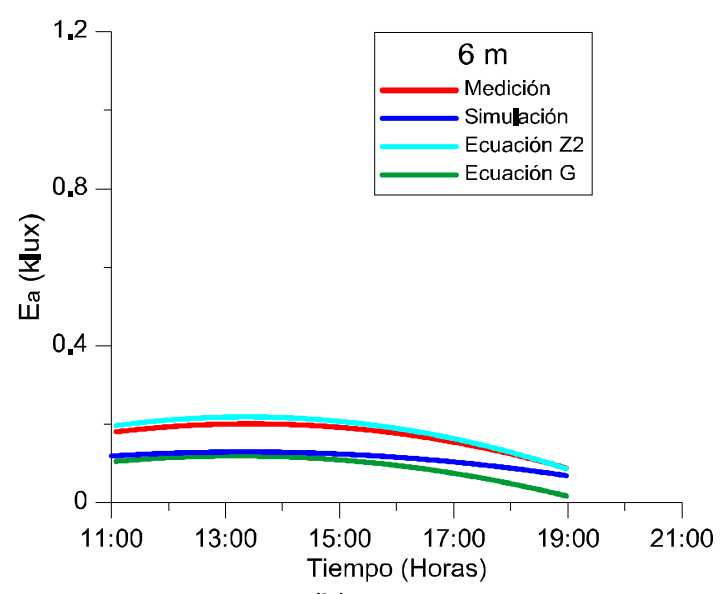

(k) $6 \mathrm{~m}$.

Figura 6.87. Iluminancias de la zona 2 del aula obtenidas midiendo en el aula, utilizando las ecuaciones Z2 y G, y por simulación, a las distancias indicadas.

Al igual que sucedía en la zona 1, podemos apreciar en la figura 6.87 como las iluminancias obtenidas tras aplicar la ecuación Z2 son las que más se aproximan a las iluminancias medidas directamente en el aula. 
Las medidas obtenidas tras aplicar la ecuación $\mathrm{G}$ son las más altas hasta llegar a la distancia de $2.5 \mathrm{~m}$ de la ventana donde empiezan a dar valores inferiores a los obtenidos midiendo directamente en el aula. Las iluminancias más bajas se han dado en las iluminancias obtenidas por simulación, a excepción del sensor situado a $0.5 \mathrm{~m}$ de la ventana, donde los valores de simulación y los obtenidos de aplicar la ecuación $\mathrm{G}$ son muy similares.

A continuación, en la figura 6.88 se muestran gráficamente los resultados de las iluminancias del aula obtenidos para la zona 3, de las cuatro formas distintas mencionadas, desde las 11:00 hasta las 17:30 horas, a excepción de los valores obtenidos mediante simulación, puesto q el software sólo aportaba información hasta las 16:00 horas.

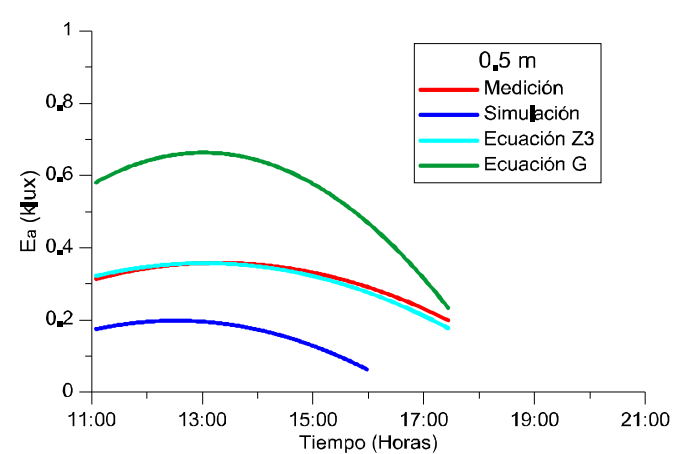

(a) $0.5 \mathrm{~m}$.

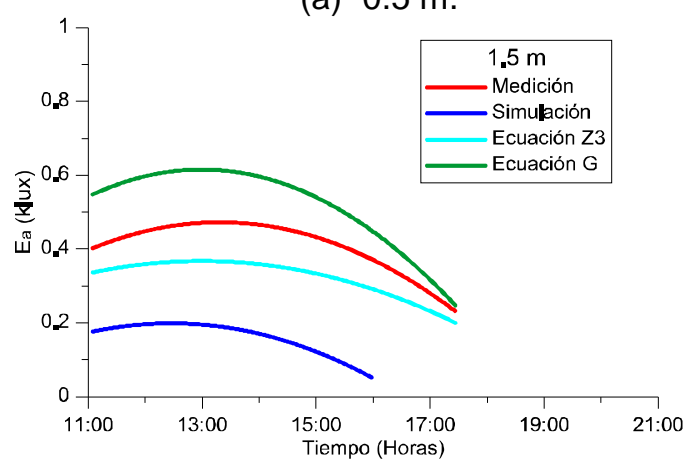

(c) $1.5 \mathrm{~m}$.

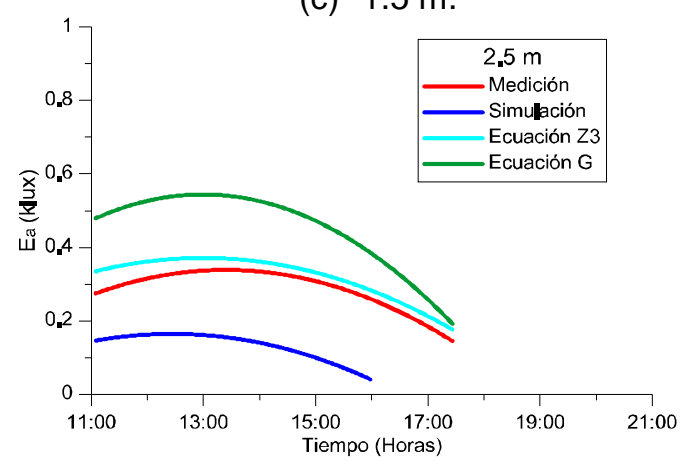

(e) $2.5 \mathrm{~m}$.

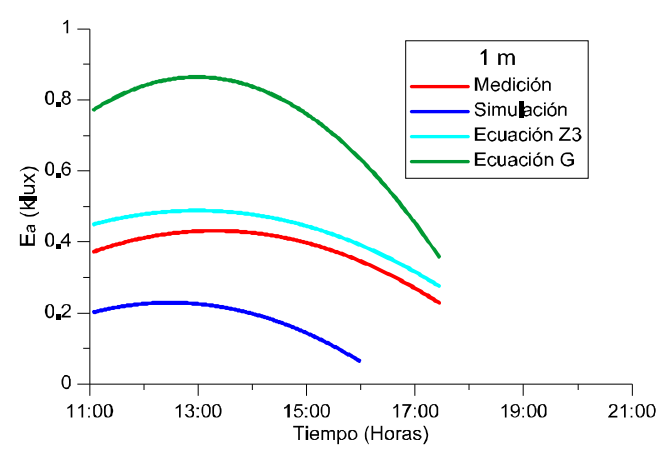

(b) $1 \mathrm{~m}$.

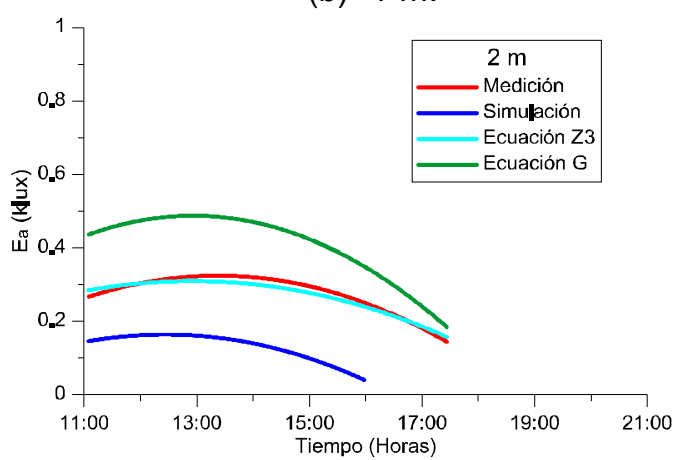

(d) $2 \mathrm{~m}$.

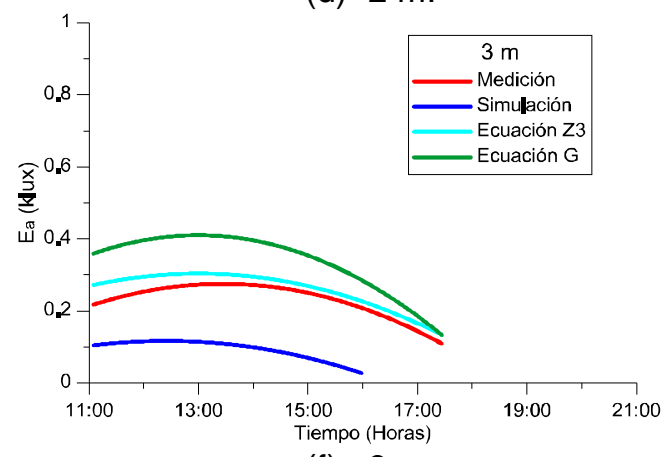

(f) $3 \mathrm{~m}$. 


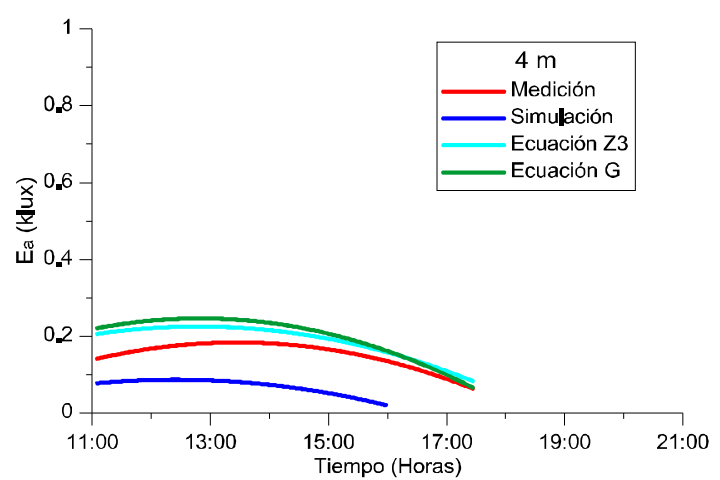

(g) $4 \mathrm{~m}$.

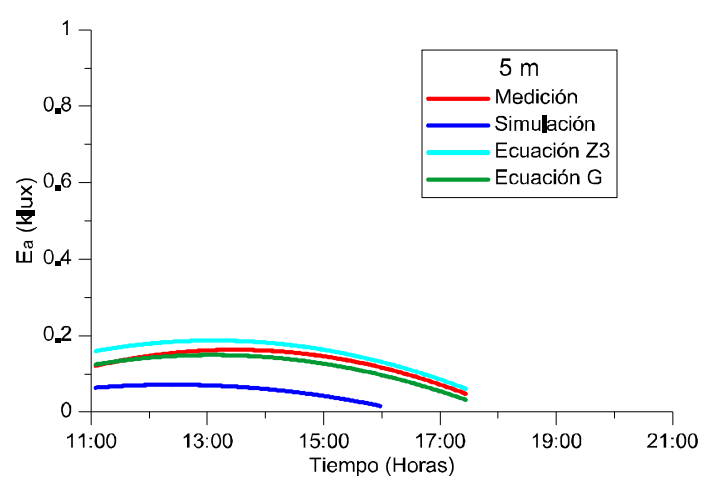

(i) $5 \mathrm{~m}$.

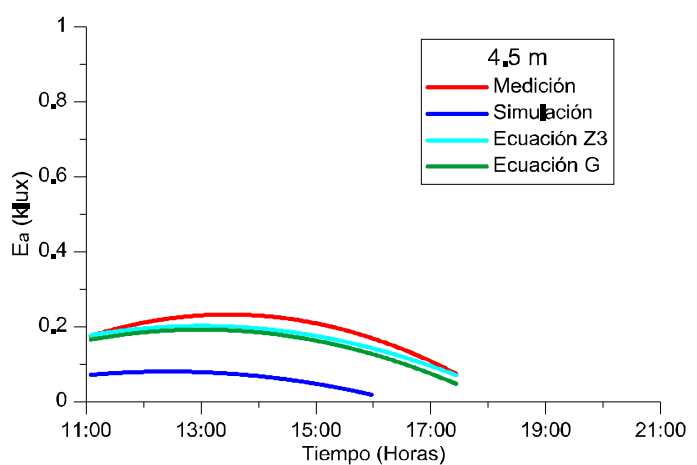

(h) $4.5 \mathrm{~m}$.

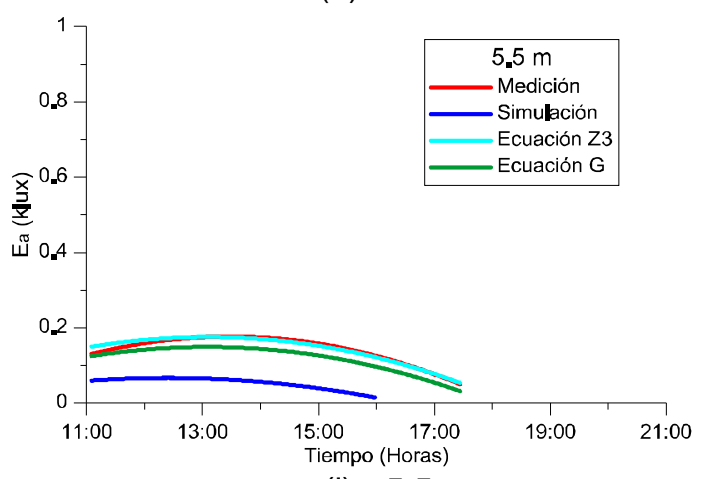

(j) $5.5 \mathrm{~m}$

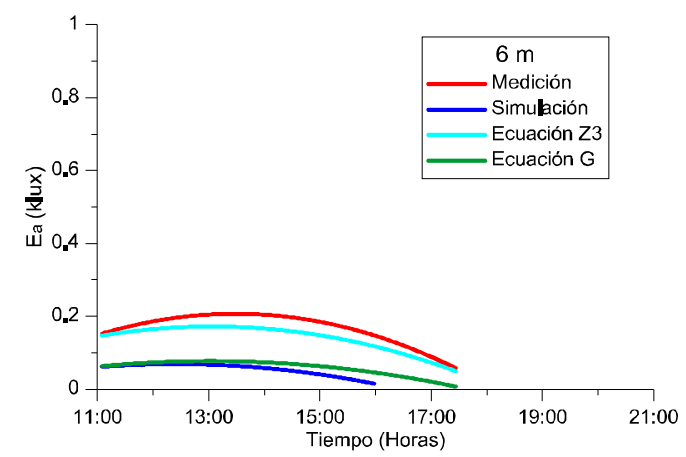

(k) $6 \mathrm{~m}$.

Figura 6.88. Iluminancias de la zona 3 del aula obtenidas midiendo en el aula, utilizando las ecuaciones Z3 y G, y por simulación, a las distancias indicadas.

En la figura 6.88 se aprecia como las iluminancias obtenidas tras aplicar la ecuación Z3 son las que más se aproximan a las iluminancias medidas directamente en el aula. Las iluminancias más bajas se han dado, al igual que en las zonas anteriores, en las obtenidas mediante simulación. Las iluminaciones más altas se han obtenido aplicando la ecuación $G$ hasta una distancia de $4.5 \mathrm{~m}$ de la fachada, a partir de esta distancia las iluminancias obtenidas tras aplicar la ecuación $G$ se aproximan a las obtenidas mediante la ecuación Z3 y midiendo directamente en el aula. A una distancia de $6 \mathrm{~m}$, las iluminancias obtenidas de aplicar la ecuación $\mathrm{G}$ se igualan a las obtenidas por simulación. 
Los resultados de las iluminancias del aula obtenidos para la zona 3, de las cuatro formas distintas mencionadas, desde las 11:00 hasta las 19:00 horas (hora local) se muestran en la figura 6.89.

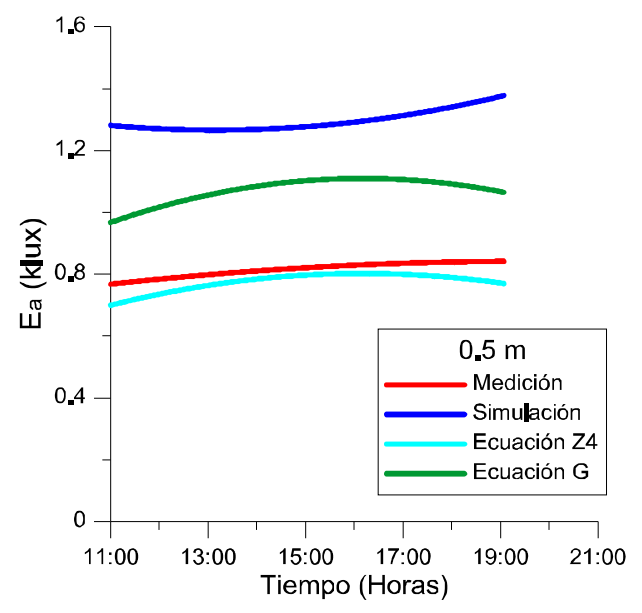

(a) $0.5 \mathrm{~m}$.

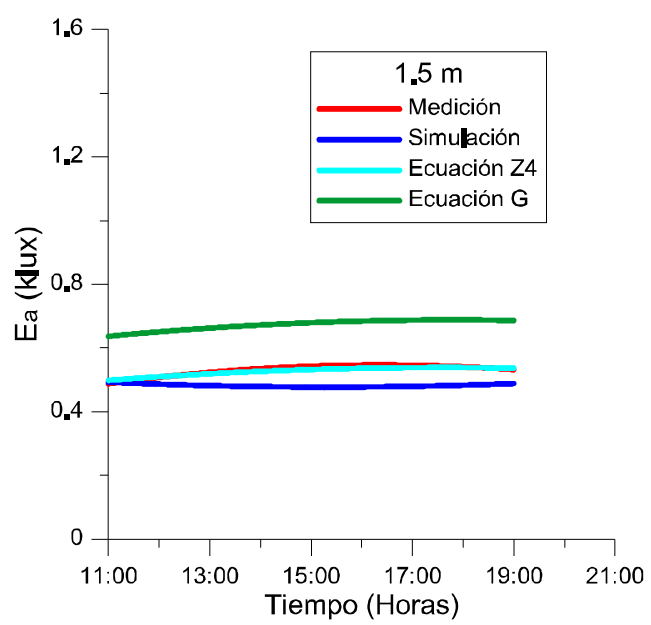

(c) $1.5 \mathrm{~m}$.

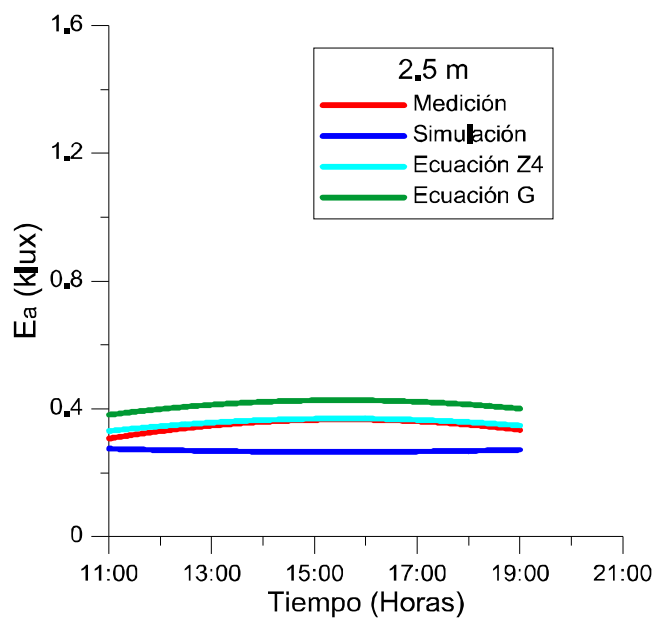

(e) $2.5 \mathrm{~m}$.

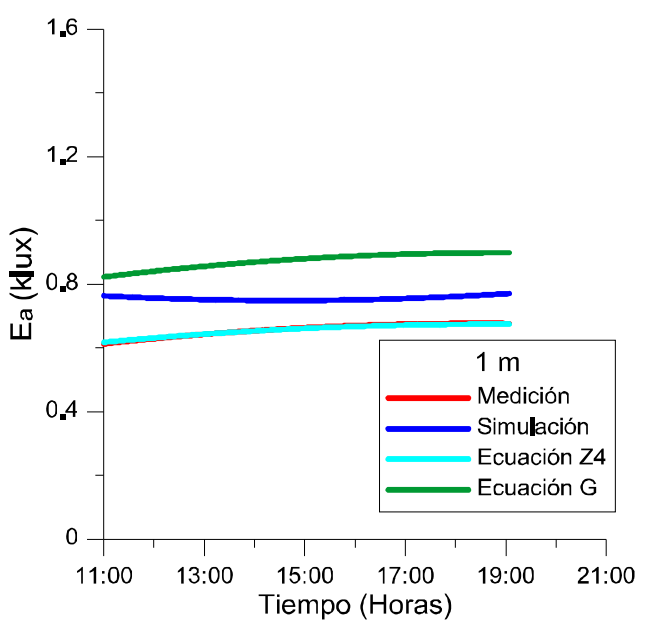

(b) $1 \mathrm{~m}$.

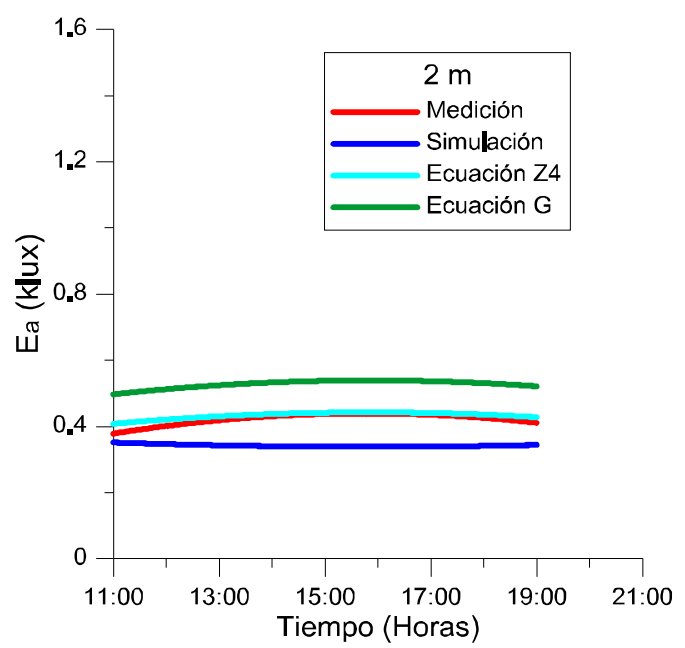

(d) $2 \mathrm{~m}$.

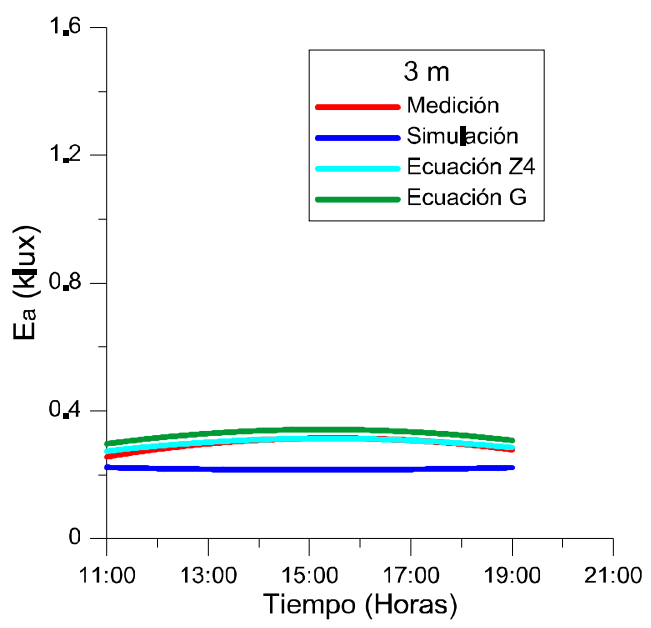

(f) $3 \mathrm{~m}$. 


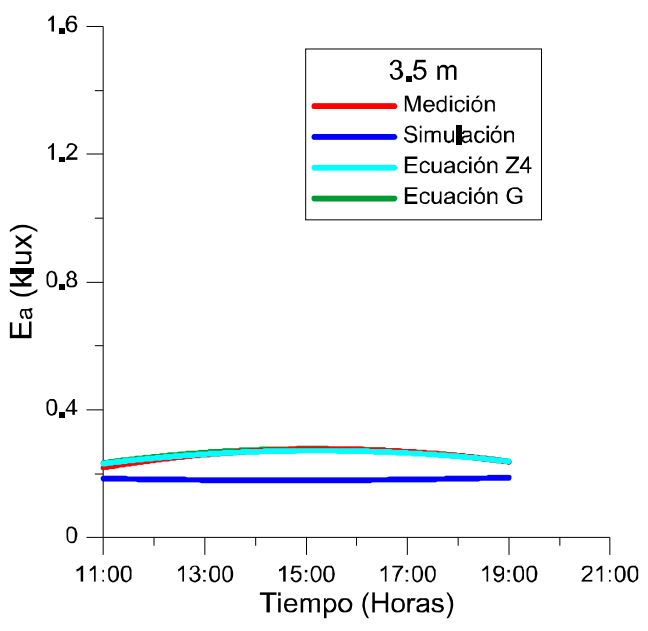

(g) $3.5 \mathrm{~m}$.

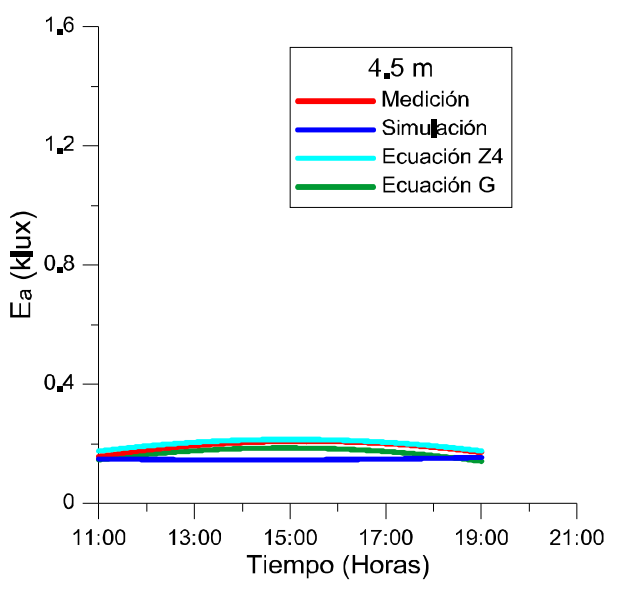

(i) $4.5 \mathrm{~m}$.

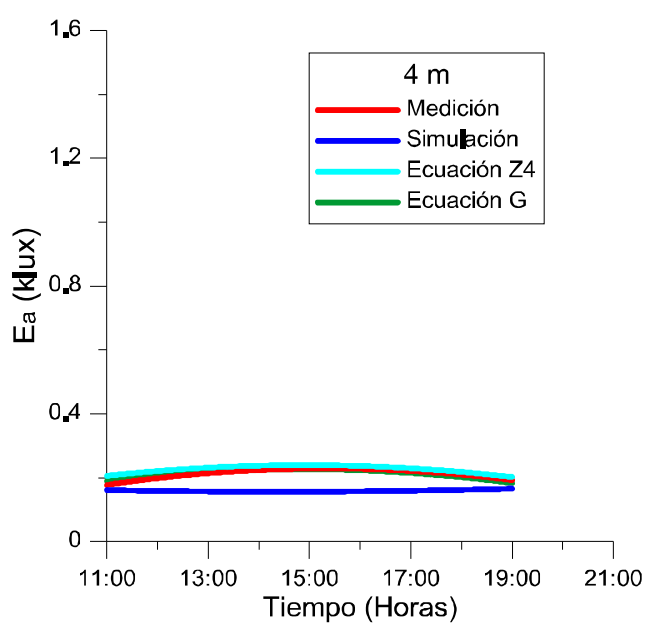

(h) $4 \mathrm{~m}$.

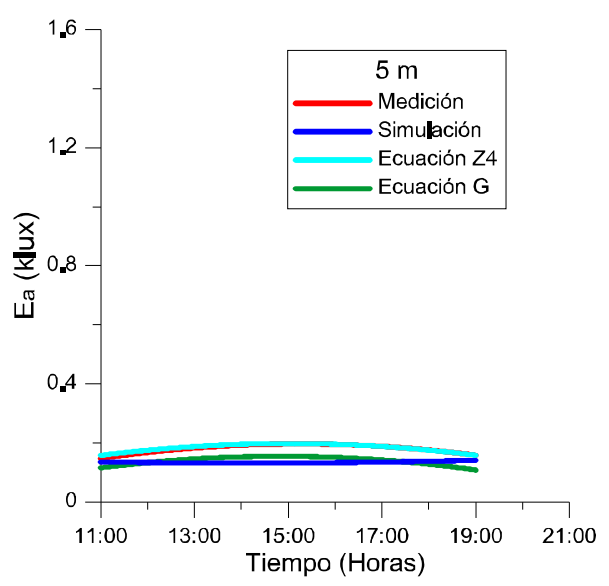

(j) $5 \mathrm{~m}$.

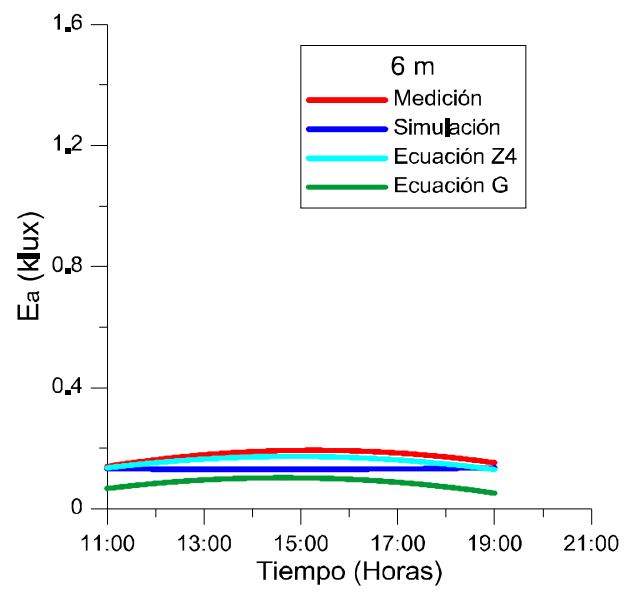

(k) $6 \mathrm{~m}$.

Figura 6.89. Iluminancias de la zona 4 del aula obtenidas midiendo en el aula, utilizando las ecuaciones Z4 y G, y por simulación, a las distancias indicadas. 
En la figura 6.89 se aprecia, tal y como sucede en las otras zonas estudiadas, que las iluminancias obtenidas tras aplicar la ecuación Z4 son las que más se aproximan a las iluminancias medidas directamente en el aula. Las iluminancias más bajas se han dado, mediante simulación, a excepción del sensor colocado a $0.5 \mathrm{~m}$ de la ventana (al igual que en la zona 2) y del sensor colocado a $6 \mathrm{~m}$ de ésta. Las iluminancias más altas se han obtenido aplicando la ecuación $\mathrm{G}$ hasta una distancia de $3 \mathrm{~m}$ de la ventana, a partir de la cual han ido disminuyendo hasta, a la distancia de $6 \mathrm{~m}$ de la ventana dar las iluminancias más bajas. 
7.CONCLUSIONES 



\section{CONCLUSIONES}

Tras un estudio detallado y un análisis personalizado de los resultados obtenidos, se va a concluir esta tesis doctoral con unas consideraciones finales acerca del trabajo de investigación realizado.

A continuación se exponen, a modo de conclusiones, las consecuencias más importantes de la presente tesis doctoral:

\section{Primera}

En las cuatro zonas consideradas la evolución diaria de las iluminancias tanto en el aula como en el modelo a escala responde a una ecuación de segundo grado, tomando siempre valores mayores en el modelo que en el aula. Esto último probablemente se deba a la ausencia de vidrios en las ventanas en el modelo y al factor de escala.

\section{Segunda}

A medida que aumenta la distancia a la fachada, no solo van disminuyendo el valor de las iluminancias, sino que el comportamiento de las iluminancias (tanto en el aula como en el modelo) tienden a igualarse. Es decir, que en el fondo del aula (a partir de los $3 \mathrm{~m}$ aproximadamente) no hay apenas distinción entre las cuatro zonas.

\section{Tercera}

La relación entre las iluminancias del aula y del modelo a escala con respecto al tiempo (hora diaria) no es lineal y va disminuyendo conforme aumenta la distancia a las ventanas.

\section{Cuarta}

La relación entre las iluminancias del aula y del modelo a escala en función de la iluminancia exterior, para cada distancia considerada, es lineal (con un coeficiente de ajuste mayor que 0.9 en la mayoría de los casos), y disminuye al aumentar la distancia a las ventanas. 


\section{Quinta}

Para cada zona considerada se ha obtenido una ecuación que permite determinar la iluminancia del aula a partir de la iluminancia del modelo a escala, de la iluminancia del exterior y de la distancia al paramento interior de la fachada.

\begin{tabular}{|c|c|}
\hline ZONA & ECUACIÓN \\
\hline $\mathbf{1}$ & $\mathrm{E}_{\mathrm{a}}=\left[(0.879 d-6.042) \times 10-3 \mathrm{E}_{\text {ext }}-0.145 d+1.462\right] \mathrm{E}_{\mathrm{m}}$ \\
\hline $\mathbf{2}$ & $\mathrm{E}_{\mathrm{a}}=\left[(0.700 \mathrm{~d}-4.060) \times 10^{-3} \mathrm{E}_{\mathrm{ext}}-0.119 d+1.152\right] \mathrm{E}_{\mathrm{m}}$ \\
\hline $\mathbf{3}$ & $\mathrm{E}_{\mathrm{a}}=\left[(0.925 d-6.685) \times 10^{-3} \mathrm{E}_{\mathrm{ext}}-0.080 \mathrm{~d}+0.943\right] \mathrm{E}_{\mathrm{m}}$ \\
\hline $\mathbf{4}$ & $\mathrm{E}_{\mathrm{a}}=\left[(0.533 \mathrm{~d}-3.232) \times 10^{-3} \mathrm{E}_{\mathrm{ext}}-0.093 d+1.003\right] \mathrm{E}_{\mathrm{m}}$ \\
\hline
\end{tabular}

\section{Sexta}

De los análisis estadísticos de los resultados obtenidos en cada zona se ha deducido que la fiabilidad de los resultados es del 95\%, y el p-valor del análisis de los residuos es superior a 0.05 lo que indica que la probabilidad de que aparezcan errores es menor del $5 \%$.

\section{Séptima}

Del análisis comparativo de las iluminancias en cada zona del aula al medir directamente en ella, aplicando la ecuación obtenida y mediante el software de simulación Dialux 4.11 se ha deducido que son más fiables las iluminancias calculadas con el modelo matemático obtenido que las suministradas por simulación.

\section{Octava}

Del estudio de la iluminación en todas las zonas simultáneamente se ha obtenido un modelo matemático único para todo el aula que, aunque no es tan preciso como los modelos obtenidos para cada zona por separado, también es más fiable que lo proporcionado por el software de simulación. 


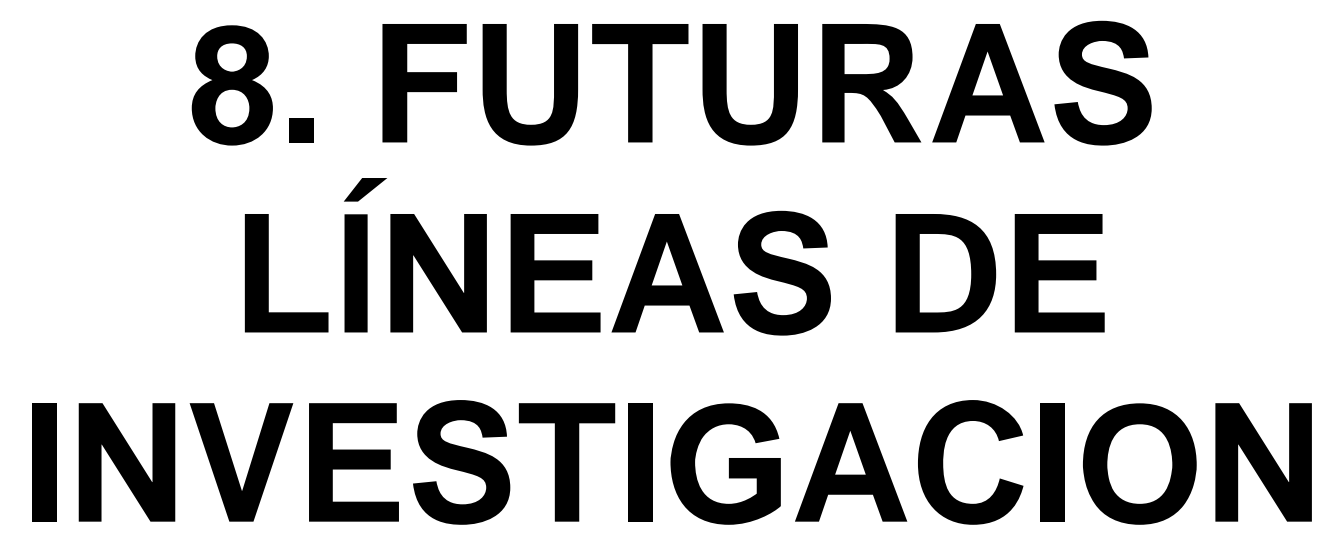





\section{FUTURAS LÍNEAS DE INVESTIGACIÓN}

En el presente trabajo de investigación se ha puesto de manifiesto la importancia del aprovechamiento de la luz natural en el campo de la edificación. Es necesario ampliar la investigación, en este tema, si se pretende incrementar el conocimiento del comportamiento de la luz natural en la edificación, y de este modo llevar a cabo las intervenciones necesarias para su máximo aprovechamiento en el interior de edificios. Por ello, futuros trabajos de investigación podrían continuar profundizando en los siguientes aspectos:

- Aplicar las correcciones necesarias a los parámetros del software de simulación y conseguir así simulaciones más aproximadas.

- Repetir el estudio para comprobar el comportamiento de las ecuaciones halladas para distintas orientaciones (Sur, Este y Oeste).

- Comprobar el comportamiento de las ecuaciones halladas y para distintos tipos de vidrios.

- Ampliar el estudio teniendo en cuenta días con cielo parcialmente cubierto y totalmente cubierto.

- Estudiar la posibilidad de simular vidrios en las ventanas del modelo a escala de tal manera que este no sufra sobrecalentamientos ni riesgos de incendio por el efecto lupa. 

9. BIBLIOGRAFÍA 



\section{BIBLIOGRAFÍA}

- Alexandridis, K., Dounis ,A. I. (2007). "Supervisor of Digital PI-like Fuzzy Logic Controllers for Indoor Lighting Control in Buildings", The Conference for the contribution of information Technology to Science, Economy, Society and Education, B.3.6. Greece 2007, p. 22-23.

- Bangali, J.A., Shaligram, A.D. (2012). "Energy Efficient Lighting Control System Design For Corridor illumination". International Journal of Scientific \& Engineering Research, Vol. 3, Issue 4, April-2012, ISSN 2229-5518.

-Baroncini, C., Boccia, O., Chella, F., Zazzini, P. (2010). "Experimental analysis on a 1:2 scale model of the double light pipe, an innovative technological device for daylight transmission". Solar Energy, Vol. 84, p. 296-307.

-Bellia, L., Pedace, A., Fragliasso, F. (Febrero2015). "The role of weather data files in climate-based daylight modeling". Sol Energy, Vol. 112, p. 169-182.

-Bellia, L., Pedace, A., Fragliasso, F. (Mayo 2015). "Dynamic daylight simulations: Impact of weather file's choice". Solar Energy, Vol.11, p. 224-235.

- Biana, Y., Mab, Y. (2017). "Analysis of daylight metrics of side-lit room in Canton, south China: Acomparison between daylight autonomy and daylight factor". Energy and Buildings, Vol. 138, p. 347-354.

-Boccia, O., Chella, F., Zazzini, P. (2014). "Natural light from a wall in buildings: Experimental analysis of the ventilated illuminating wall performances". Solar Energy, Vol. 108, p.178-188.

- Boccia, O., Zazzini, P. (2015). "Daylight in buildings equipped with traditional or innovative sources:A critical analysis on the use of the scale model approach". Energy and Buildings, Vol. 86, p. 376-393. 
- Boyce, P. (1998). "Why Daylight? In: Proceeding of Daylight". International conference on day lighting technologies for energy efficiency in buildings. Ottawa, Ontario, Canada, p. 359-366.

- Caicedo, D., Pandharipande, A., Willems, F.M.J. (2014). "Daylight-adaptive lighting control using light sensor calibration prior-information". Energy and Buildings, Vol. 48, p. 105-114.

- Çakir, A.; Çakir, G. (1990). "Licht und Gesundheit - Eine Untersuchung zum Stand der Beleuchtungstechnik in deutschen Büros - Gesundheits- und Befindlichkeitsstörungen bei der Büro- und Bildschirmarbeit "(authorized English translation Light and Health (1991 and 1998)). ERGONOMIC, Berlin. http://www.blueskywa.com.au/media/ciralight/downloads/Case\%20Studies/PDF /Daylight_for_Health_and_Efficiency.pdf. (Última visita 11/01/2017).

- Casablanca, G.A, Evans, J.M. (2003). "Estantes de luz: verificación de iluminación natural, asoleamiento y protección solar en maquetas de ensayo". Revista Avances en Energías Renovables y Medio Ambiente, Vol. 7, p. 25-30. ISSN 0329-5184.

- Cheng, C.L., Chen, C.L., Chou, C.P., Chan, C.Y. (2007). "A mini-scale modeling approach to natural daylight utilization in building design". Building and Environment, Vol. 42, p. 372-384.

- Cid, M. (2012). "Estudio comparativo de iluminancias globales sobre una superficie horizontal en distintas orientaciones y en distintas disposiciones de entradas de luz natural para la optimización de la misma en el interior". Trabajo fin de máster del Máster en Técnicas y Sistemas de Edificación de la EUATM.

- Claros, S.T., Soler, A. (2002). "Indoor daylight climate-influence of light shelf and model reflectance on light shelf performance in Madrid for hours with unit sunshine fraction". Building and Environment, Vol. 37, p. 587-598. 
- Cucumo, M.A., De la Rosa, A.,Ferraro, V., Kaliakatsos, D., Marinelli, V. (2008). "Predicting diffuse illuminance on vertical surfaces at Arcavacata di Rende (Italy) for various types of sky". Building and Environment, Vol. 43, Issue 10, p. 1678-1686.

- De Carli, M., De Giuli, V., Zecchin, R. (2008). "Review on visual comfort in office buildings and influence of daylight in productivity", Conference paper Indoor Air, 17-22 August 2008, Copenaghen, Denmark, Paper ID:112.

- De Carli, M., De Giuli, V. (2009). "Optimization of daylight in buildings to save energy and to improve visual comfort: analysis in different latitudes". Eleventh International IBPSA Conference, Glasgow, Scotland, July 27-30, 2009.

- De la Rosa, A., Ferraro, V., Kaliakatsos, D., Marinerlli, V. (2008)." Calculating diffuse illuminance on vertical surfaces in different sky conditions". Energy, Vol. 33, Issue 11, p. 1703-1710.

- De Rosa, A., Ferraro, V., Igawa, N., Kaliakatsos, V., Marinelli, V. (2009). "INLUX: A calculation code for daylight illuminance predictions inside buildings and its experimental validation". Building and Environment, Vol. 44, p.17691775.

- Du, J., Sharples, S. (2011). "Assessing and predicting average daylight factors of adjoining spaces in atrium buildings under overcast sky". Building and Environment, Vol. 46, p. 2142-2152.

- Edwards, L., Torcellini, P. (2002). "A literature review of the effects of natural light on building occupants". National Renewable Energy Laboratory, Golden (CO). http://www.nrel.gov/docs/fy02osti/30769.pdf. (Última visita 11/01/2017).

- European Union, Directive 2010/31/EU of the European Parliament and of the Council of 19 May 2010 on the Energy Performance of Buildings (recast), Off. J.Eur. Union L 153 (2010) 21, 18/06/2010, Strasbourg, France. 
- Fernandesa, L.L., Lee, E.S., Ward, G. (2013). "Lighting energy savings potential of split-pane electrochromic windows controlled for daylighting with visual comfort". Energy and Buildings, Vol. 61, p. 8-20.

- Fernández, E., Beckers, B., Besuievsky, G. (2016). "A fast daylighting method to optimize opening configurationsin building design". Energy and Buildings, Vol.125, p. 205-218.

- Fernández, M.A. (2011). "Estudio comparativo de iluminancias globales en superficies horizontales mediante distintas orientaciones de la fachada de un modelo a escala para optimizar la iluminación natural en el interior". Trabajo fin de máster del Máster en Técnicas y Sistemas de Edificación de la EUATM.

- Ferraro, V., Igawa, N., Marinelli, V. (2010). "INLUX-DBR e A calculation code to calculate indoor natural illuminance inside buildings under various sky conditions. Energy, Vol. 35, p. 3722-373.

- Gago, E.J., Muneer, T., Knez, M., Köster, H. (2015). "Natural light control sand guides in buildings. Energy saving for electrical lighting, reduction of cooling load". Renewable and Sustainable Energy Reviews, Vol. 4, p. 1-13.

- García, P. (2013)."Estudio comparativo de la iluminación natural en tres aulas de la ETSAM utilizando modelos a escala y software de simulación para su aplicación a la edificación". Trabajo fin de máster del Máster en Innovación Tecnológica de la Edificación de la EUATM

- Gonzalo, G., Ledesma, S., Nota,V., Márquez G. (2001). "Evaluación comparativa del comportamiento lumínico y térmico de diferentes soluciones de aventanamientos para escuelas y oficinas de la provincia de Tucumán". Revista Avances en Energías Renovables y Medio Ambiente Vol. 5, p.13-18. ISSN 0329-5184. 
- Hathaway, W.E., Hargreaves, J.A., Thompson, G.W., Novitsky, D. (1992). "A case of daylight robbery. Daylightingin Schools - Study into the Effects of Light on Children of Elementary School-Age". Alberta Dept. of Education, Edmonton, Canada.

- Hee, W.J.,.Alghoul, M.A,n, Bakhtyar, B., Elayeb, O., Shameri, M.A., Alrubaih, M.S., Sopian, K. (2015)."The role of window glazing on daylighting and energy saving in buildings". Renewable and Sustainable Energy Reviews, Vol. 42, p. 323-343.

- Hertog, W., Llenas, A., Carreras, J. (20015). "Optimizing indoor illumination quality and energy efficiency using a spectrally tunable lighting system to augment natural daylight". Optics Express, Vol. 23, Issue 24, p. A1564-A1574, doi:10.1364/OE.23.0A1564.

- Huang, Y., Niu, J., Chung, T. (2014). "Comprehensive analysis on thermal and daylighting performance of glazing and shading designs on office building envelope in cooling-dominant climates". Applied Energy, Vol. 134, p. 215-228.

- Ikuzwe, A., Sebitosi, A.B. (2015). "A novel design of a daylighting system for a classroom in rural South Africa". Solar Energy, Vol. 114, p. 349-355.

- Joseph, C., Danny, H. W. (1999). "An analysis of daylighting and solar heat for cooling dominated office buildings". Solar Energy Vol. 65, No. 4, p. 251-262, Pergamon PI I : S0038 - 092X(98)00136 - 4.

- Klein, D.C., Moore, R., Reppert, S.M. (1991). "Suprachiasmatic nucleus: the mind's clock". Oxford: Oxford UP, ISBN 0-19-506250-7P107-1023, p.107-123.

-Konstantzos, I., Tzempelikos , A., Chan, Y.C. (2015). "Experimental and simulation analysis of daylight glare probability in offices with dynamic window shades". Building and Environment, Vol. 87, p. 244-254. 
- Küller, R. (1987). "The effects of indoor lighting on well-being and the annual rhythm of hormones". CIE 21 stsession, Venice 1987, Vol. 1. No 601, p. 342-345.

- Küller R. (2002). "The influence of Light on Circarhythms in Humans". Journal of Physiological Anthropology and Applied Human Science, vol 2, Issue 21, p. 87-91.

- Küller, R.; Lindsten, C. (1992). "Health and behaviour of children in classrooms with and without windows". Journal of Environmental Psychology , Vol. 12, p. 305-317.

- Ledesma, S.L., Cisterna, M.S., Márquez Vega, G., Quiñones, G. , Nota, V.M., Gonzalo, G.E. (2005). "Evaluación del ahorro energético en iluminación artificial en aulas de edificios escolares en Tucumán. Avances en Energías Renovables y Medio Ambiente, Vol. 9, p. 19-24. ISSN 0329-5184.

-LI-1000 Datalogger Instruction Manual (1987), LI-COR (12/2008). "Instruction manual for the LI-1000 DataLogger". http://envsupport.licor.com/. (última visita 13/06/16)

- Li D.H.W., Cheung,G.H.W., Cheung, K.L., Lam, J.C. (2009). "Simple method for determining daylight illuminance in a heavily obstructed environment". Building Environment, Vol. 44, Issue 5, p. 1074-1080.

- Li D.H.W., Cheung,G.H.W., Cheung, K.L., Lam, J.C. (2010). "Determination of vertical daylight illuminance under non-overcast sky conditions". Building Environment, Vol. 45, Issue 2, p. 498-508.

- Li, D. H.W., Lau, C.C.S, Lam, L.C. (2004). "Overcast sky conditions and luminance distribution in Hong Kong". Building and Environment, Vol. 39, p.101108. 
- Li, D.H.W., Lam, J.C. (2001). "Evaluation of lighting performance in office buildings with daylighting controls". Energy and Buildings, Vol. 33, p. 793-803.

- Lim, Y.W, Heng, C.Y.S. (2016). "Dynamic internal light shelf for tropical daylighting in high-rise office buildings". Building and Environment, Vol.106, p. 155-166.

- Loisos G. (1999). "Daylinghting School, Daylighting Initiative". Design Tools and Information from The Pacific Gas and Electric Company. https://www.cashnet.org/resource-center/resourcefiles/127.pdf. (Última visita 10/01/2017).

- Malet-Damour, B., Boyer, H., Fakra, A.H., Bojic, M. (2014). "2013 ISES Solar World Congress Light Pipes Performance Prediction: inter model and experimental confrontation on vertical circular light-guides". Energy Procedia, Vol. 57, p. 1977-1986.

- Markou, M.T., Bartzokas, A., Kambezis, H.D. (2009). "Daylight climatology in Athens, Greece: types of diurnal variation of illuminance levels". International Journal of Climatology, Vol. 29, Issue 14, p. 2137-2145.

- Mohsenin, M., Hu, J. (2015). "Assessing daylight performance in atrium buildings by using Climate Based Daylight Modeling". Solar Energy, Vol.119, p. 553-560.

- Okogbue, E.C, Adedokun, J.A., Holmgren, B. (2008). "Hourly and daily global photometric illuminance and luminous efficacy at a tropical station, lle-lfe, Nigeria". International Journal of Sustainable Energy, Vol. 27, Issue 3, p. 105120.

- Olmo, F.J , Vida, J., Castro-Diez, Y., Alados-Arboledas, L. (1999). "Prediction of global irradiance on inclined surfaces from horizontal global irradiance". Energy, Vol. 24, Issue 8, August 1999, p. 689-704. 
- Ozdemir, A. (2010). "The effect of window views' openness and naturalness on the perception of rooms' spaciousness and brightness: A visual preference study "Scientific Research and Essays Vol. 5(16), p. 2275-2287.

- Pandharipande, A., Caicedo, D. (2011)."Daylight integrated illumination control of LED systems based on enhanced presence sensing". Energy and buildings, vol. 43 , p. $944-950$.

- Pattini A. y Kirschbaum C. (1998). Evaluación subjetiva de aulas iluminadas con luz natural. Revista Avances en Energías Renovables y Medio Ambiente, vol. 2, p. 03-63.

- Pattini, A. "Evaluación de la iluminación natural en edificio, modelos a escala". http://www.cricyt.edu.ar/asades/modulos/averma/trabajos/2000/2000-t005a004.pdf. (Última visita 10/01/2017).

-Pattini, A., CONICET, Argentina. http://www.edutecne.utn.edu.ar/eliiluminacion/cap11.pdf. (Última visita 11/01/2017).

- Pérez-Burgos, A., De Miguel, A., Bilbao, J. (2010). "Daylight illuminance on horizontal and vertical surfaces for clear skies. Case study of shaded surfaces". Solar Energy, Vol. 84, p. 137-143.

- Pérez-Burgos, A.,Bilbao, J.,de Miguel, A. (2007). "An evaluation on illuminance measurements at Valladolid (Spain)". Journal of Atmospheric and Solar-Terrestrial Physics, Vol. 69, issue 8, p. 939-946.

- Rincón, J. (2011). "Optimización de la iluminación natural en un aula de la ETSAM utilizando un modelo a escala y un modelo de simulación virtual". Trabajo fin de máster del Máster en Técnicas y Sistemas de Edificación de la EUATM. 
- Robledo, L.,Soler, A. (2000). "Estimation of global illuminance for clear skies at Madrid". Energy and Buildings, Vol 31, p. 25-28.

- Royce, P.R. (2003). "Human factors in lighting" (2nd ed.). London: Taylor and Francis, p 497-5004, ISBN 978-1-4398-7488-20.

- Ruiz, E., Soler, A., Robledo, L. (2002). "Statistical assessment of a model for global illuminance on inclined surfaces from horizontal global illuminance". Energy Conversion and Management, Vol. 43, p. 693-708.

- Ryckaert, R., Lootens, C., Geldof, J., Hanselaer, P. (2010). "Criteria for energy efficient lighting in buildings". Energy and Buildings, vol. 42, p 341-347.

- Shehabia , A., DeForesta, N., McNeila, A., Masanetb, E., Greenblatta, J., Lee, E.S., Helmsc, B.A., Milliron, D.J. (2013) "U.S. energy savings potential from dynamic daylighting controlglazings" Energy and Buildings, Vol. 66, p.415-423.

- Santiago-Tomás, C., Soler, A. (2002). "Indoor daylight climate-influence of light shelf and model reflectance on light shelf performance in Madrid for hours with unit sunshine fraction". Building and Environment, Vol. 37, p. 587- 598.

- Singh, M.C., Garg, S.N. (2010)."Illuminance estimation and daylighting energy savings for Indian regions". Renewable Energy, Vol. 35, p. 703-711.

- Soler, A., Gopinathan, K., Robledo,L., Ruiz, E. (2004). "Month-hour distributions of zenith luminance and diffuse illuminance in Madrid". Energy Conversion \& Management, Vol. 45, p. 1141-1152.

- Stanislav, D., Miroslav, K., Richard, K., Frantis"ek, K. (2010). "Illumination of interior spaces by bended hollow light guides: Application of the theoretical light propagation method". Solar Energy, vol. 84, p. 2112-2119. 
- Sudan, M., Tiwari, G.N. (). "Daylighting and energy performance of a building for composite climate: An experimental study". Alexandria Engineering Journal Vol. 55, p. 3091-3100.

- Surapong Chirarattananon, S., Chaiwiwatworakul, P., Pattanasethanon, S. (2002). "Daylight availability and models for global and diffuse horizontal illuminance and irradiance for Bangkok ". Renewable Energy, vol. 26, p 69-89.

- Tang, S., Kalavallya, V., Yew, K., Parkkinen, J. (2017). "Development of a prototype smart home intelligent lighting controlarchitecture using sensors onboard a mobile computing system". Energy and Buildings, Vol. 138, p.36-376.

- Thorington L. (1971). "Visual and biologic aspects of an artificial sunlight illuminant". Journal of the Illuminating Engineering Society, Vol.1 (10), p.33-41.

- Tregenza, P.R. (1983). "The Monte Carlo method in lighting calculations". Lightning Research and Technology, Vol 15, p. 163-170.

- Tsangrassoulis, A., Santamouris, M., Asimakopoulos, D. (1996). "Theoretical and experimental analysis of daylight performance for various shading systems". Energy and Buildings, Vol. 24, p. 223-230.

- Tzempelikosa, A., Chana,Y.C. (2016). "Estimating detailed optical properties of window shades from basic available data and modeling implications on daylighting and visual comfort". Energy and Buildings, Vol. 126, p. 396-407.

- Vallejo, J.J. (2002). Los secretos del antiguo Egipto: un recorrido inédito por el misterioso país de los faraones. Editorial Nowtilus, ISBN 9788497630078.

- Van de Meugheuvel, N., Pandharipande, A., Van den Hof, P.P.J.(2014). "Distributed lighting control with daylight and occupancy adaptation". Energy and Buildings, vol. 75, p. 321-329. 
- Wehr T.A. (1991). "The durations of human melatonin secretion and sleep respond to changes in daylength (photoperiod)". J Clin Endocrinol Metab, Vol. 73, p. 1276-1280.

-Xu, L., Pan, Y., Yao, Y., Cai, D., Huang, Z., Linder, N. (2017). "Lighting energy efficiency in offices under different control strategies". Energy and Buildings 138 (2017) 127-139.

- Yun, G.Y., Kong, H.J., Kim, H., Kim, J.T. (2012). "Effects of occupancy and lighting use patterns on lighting energy consumption". Energy and Buildings, Vol. 46, p. $152-158$.

- Zain-Ahmed, A., Sopian, K., Othman, M.Y.H., Sayigh, A.A.M., Surendran, P.N. (2002). "Daylighting as a passive solar design strategy in tropical buildings: a case study of Malaysia". Energy Conversion and Management, Vol. 43, p. 1725-1736.

- Zhou, X., Yan, D., Hong, T., Ren, X. (2015). "Data analysis and stochastic modelling of lighting energy use in large office buildings in China". Energy and Buildings, Vol. 86, p. 275-287.

\section{Normativa consultada:}

- CODIGO TÉCNICO DE LA EDIFICACIÓN. Ministerio de Vivienda, Gobierno de España. DB HE-3. 2013. Eficiencia energética de las instalaciones de iluminación.

- Norma europea UNE-EN 12464-1: 2012. Iluminación de los lugares de trabajo. Parte 1: lugares de trabajo interiores.

- Norma europea UNE-EN 12464-2: 2008. Iluminación de los lugares de trabajo. Parte 2: lugares de trabajo exteriores. 
- Norma europea UNE-EN 12665: 2012. Términos básicos y criterios para la especificación de los requisitos de alumbrado.

- Norma europea UNE-EN 12193: 2009. Iluminación. lluminación de instalaciones deportivas.

- Norma europea UNE-EN 15193: 2008. Eficiencia energética de los edificios. Requisitos energéticos para la iluminación.

- Norma europea UNE-EN 15193:2008/AC:2010. Eficiencia energética de los edificios. Requisitos energéticos para la iluminación.

- Norma europea UNE-EN 50172: 2005. Sistemas de alumbrado de seguridad.

- Norma europea UNE-EN 72153: 1985. Niveles de iluminación. Asignación a tareas visuales.

- Norma europea UNE-EN 72160: 1984. Niveles de iluminación. Definiciones.

- Norma europea UNE-EN 72161: 1985. Niveles de iluminación. Especificación.

- Norma europea UNE-EN 72162: 1985. Clasificación y designación.

- Norma europea UNE-EN 72163: 1984. Niveles de iluminación. Asignación a tareas visuales.

- Norma europea UNE-EN 72502: 1984. Sistemas de iluminación. Clasificación general.

- Norma europea UNE-EN ISO 28803: 2012. Ergonomía del ambiente físico. Aplicación de las normas internacionales a las personas con necesidades especiales.

- Real Decreto 486/1997, por el que se establecen las disposiciones mínimas de seguridad y salud en los lugares de trabajo. BOE no 97 de 23-04-1997.

- Real Decreto 1890/2008, por el que se aprueba el Reglamento de eficiencia energética en instalaciones de alumbrado exterior. BOE ํㅡ 279 de 19-11-2008. 
10. ANEXOS 



\section{ANEXOS}

10.1. ANEXO I

\section{LJ-210SA PHOTOMETRIC SENSOR}

LI-COR, Inc. Toll Free: 1-800-447-3576 (U.S. \& Canada) · Phone: 402-467-3576 · FAX: 402-467-2819 • E-mail: envsales@env.licor.com · Intemet: http:/umw.licor.com

\section{MeAsures IlLuminance AS RELATED TO THE CIE StANDARd OBSERVER CURVE}

The LI-210SA Photometric Sensor utilizes a filtered silicon photodiode to provide a spectral response that matches the CIE curve within $\pm 5 \%$ with most light sources. This photodiode and filter combination is placed within a fully cosinecorrected sensor head to provide the proper response to radiation at various angles of incidence.

Some of the applications for the LI-210SA Photometric Sensor include interior and industrial lighting, outdoor illuminance, passive solar energy, architecture and lighting models, illumination engineering, and biological sciences that require illuminance measurements. The LI-210SA is a research grade photometric sensor that is very reasonably priced.

\section{LI-210SA SPEGIFICATIONS}

Absolute Calibration: $\pm 5 \%$ traceable to NBS.

Sensitivity: Typically $30 \mu \mathrm{A}$ per $100 \mathrm{klux}$.

Linearity: Maximum deviation of $1 \%$ up to 100 klux.

Stability: $< \pm 2 \%$ change over a 1 year period.

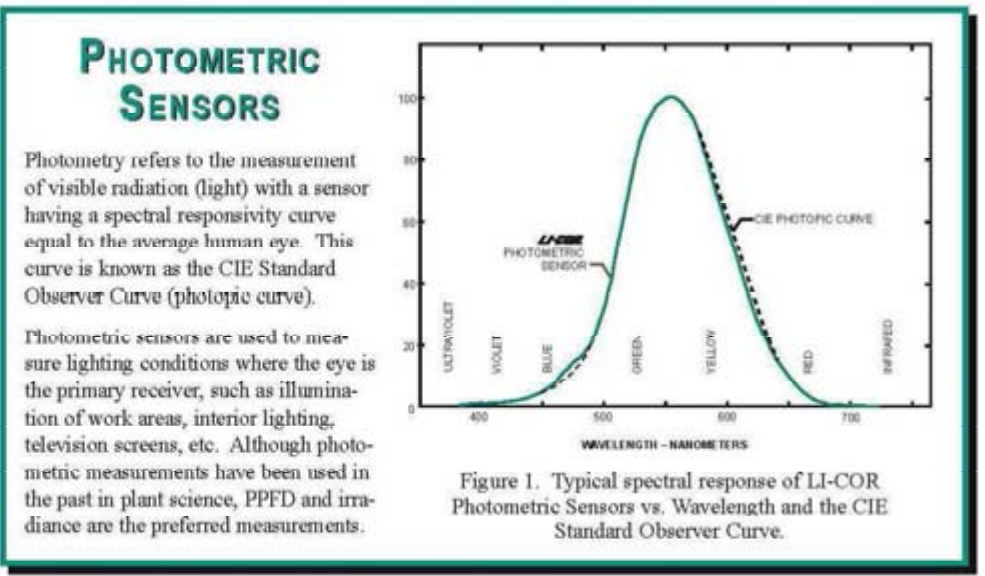

Response Time: $10 \mu \mathrm{S}$,

Temperature Dependence: $\pm 0.15 \%$ per ${ }^{\circ} \mathrm{C}$ maximum

Cosine Correction: Cosine corrected up to $80^{\circ}$ angle of ineidenos.

Azimuth: $< \pm 1 \%$ error over $360^{\circ}$ at $45^{\circ}$ elevation.

Tilt: No error induced from orientation.

Operating Temperature: -20 to $65^{\circ} \mathrm{C}$.

Relative Humidity: 0 to $100 \%$

Detector: High stability silicon photovoltaic detector (blue enhanced)

Sensor Housing: Weatherproof anodized aluminum case with acrylic diffuser and stainless steel hardware.

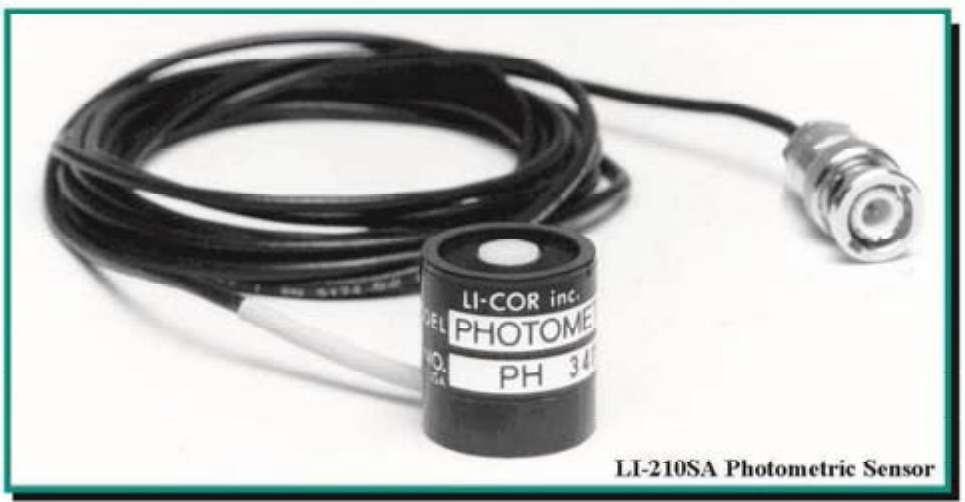

Size: 2.38 Dia. $\times 2.54 \mathrm{~cm} \mathrm{H}$ $\left(0.94^{\prime \prime} \times 1.0^{* \prime}\right)$

Weight: $28 \mathrm{~g}(1 \mathrm{oz})$.

Cable Length: $10 \mathrm{ft}$. standard.

\section{ORDERING INFORMATION}

The LI-210SA Photometric Sensor cable terminates with a $\mathrm{BNC}$ connector that connects directly to the LI-250 Light Meter or LI-1400 DataLogger. The 2290 Millivolt Adapter should be ordered if the LI-210SA will be used with a strip chart recorder or datalogger that measures millivolts. The 2290 uses a $604 \mathrm{Ohm}$ precision resistor to corvert the LI-210SA output from microamps to millivolts. The Photometric Sensor can also be ordered with bare leads (without the connector) and is designated LI-210SZ. The 2003S Mounting and Leveling Fixture is recommended for each sensor unless other provisions for mounting are made. Other accessories are described on the Accessory Sheet
LI-210SA Photometric Sensor
with $\mathrm{BNC}$ connector)
LI-210SZ Quantum Senso
(with bare leads)
2003S Mounting and Leveling Fixture 2222SB-50 Extension Cable (50 ft.) 2222SB-100 Extension Cable (100 ft.) 2290 Millivolt Adapter 


\subsection{ANEXO II}

A continuación se representan las curvas ajustadas del estudio comparativo de las iluminancias del aula a distintas horas y sus ecuaciones correspondientes para cada una de las zonas analizadas en la tesis.

\subsubsection{Zona 1}

Las curvas ajustadas del estudio comparativo de las iluminancias del aula obtenidas midiendo directamente en el aula, mediante la ecuación Z1 y mediante simulación representadas en la figura 6.19 se muestran a continuación:
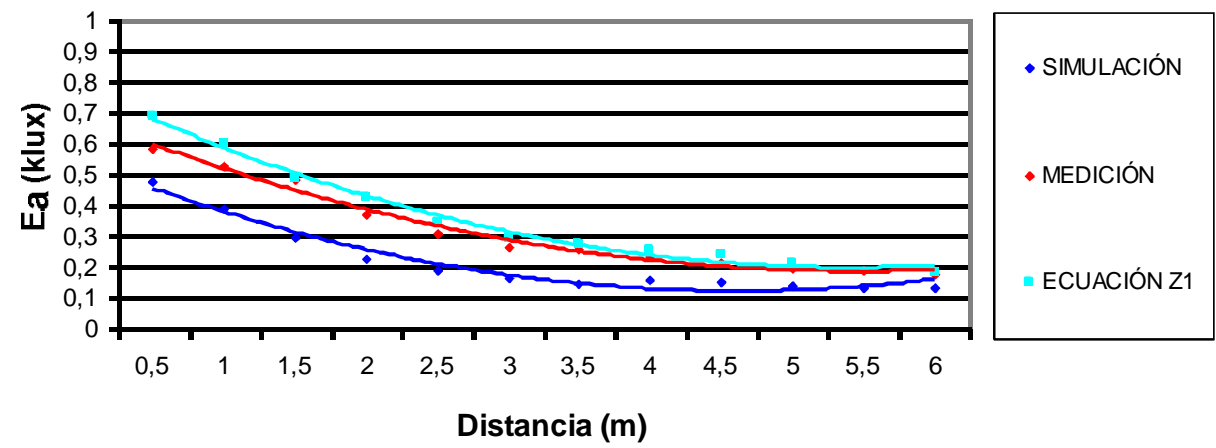

(a) 11:00.

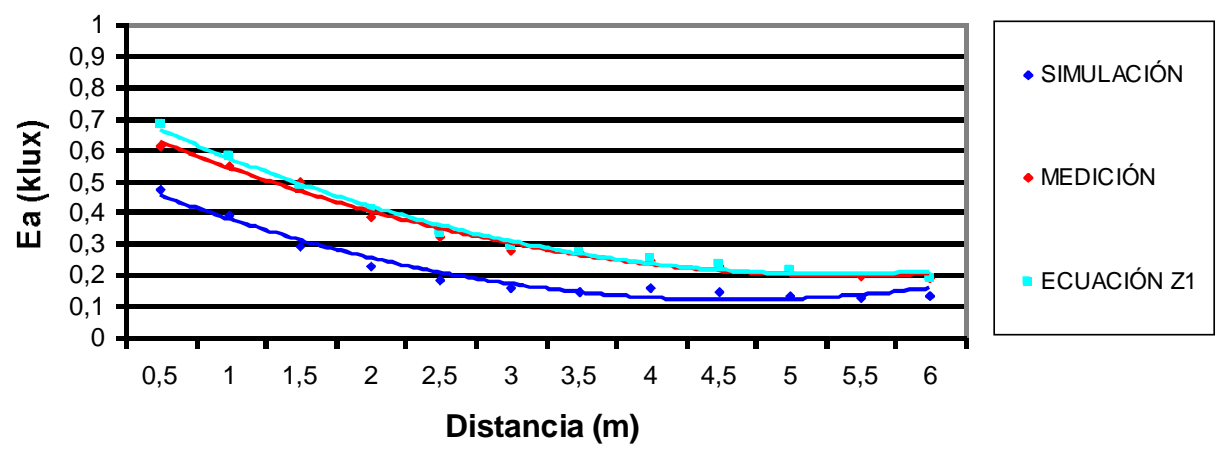

(b) $12: 00$

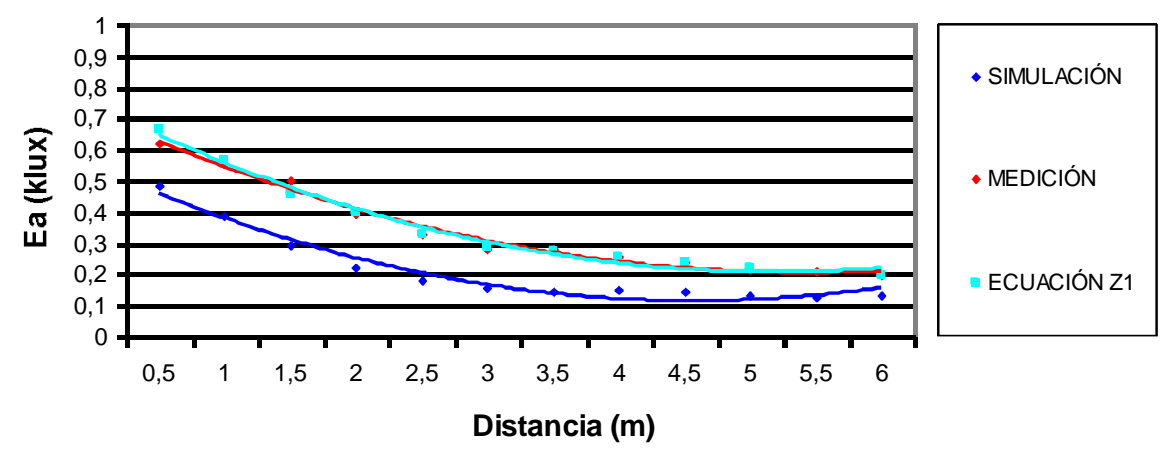

(c) $13: 00$ 

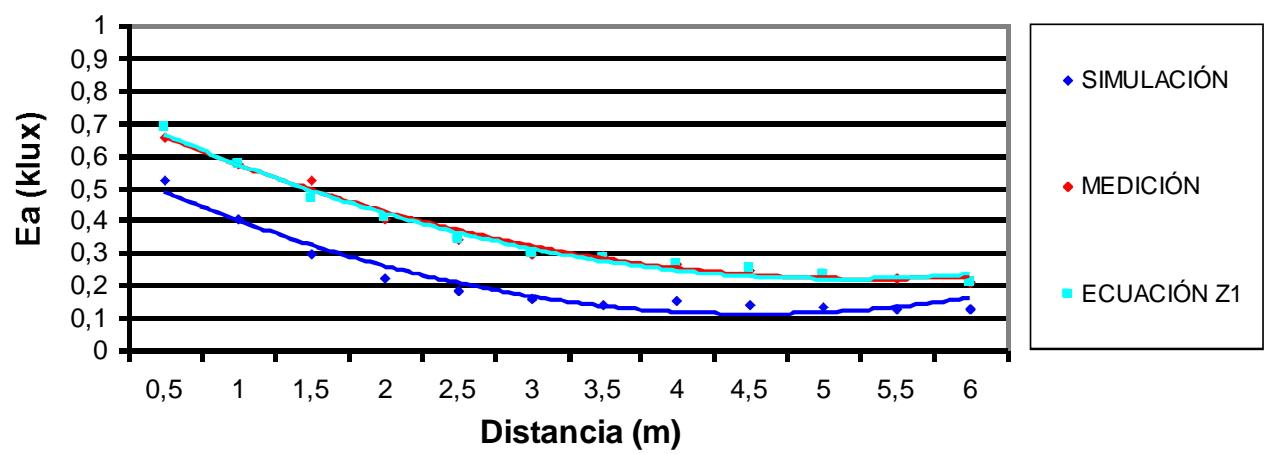

(d) 14:00.
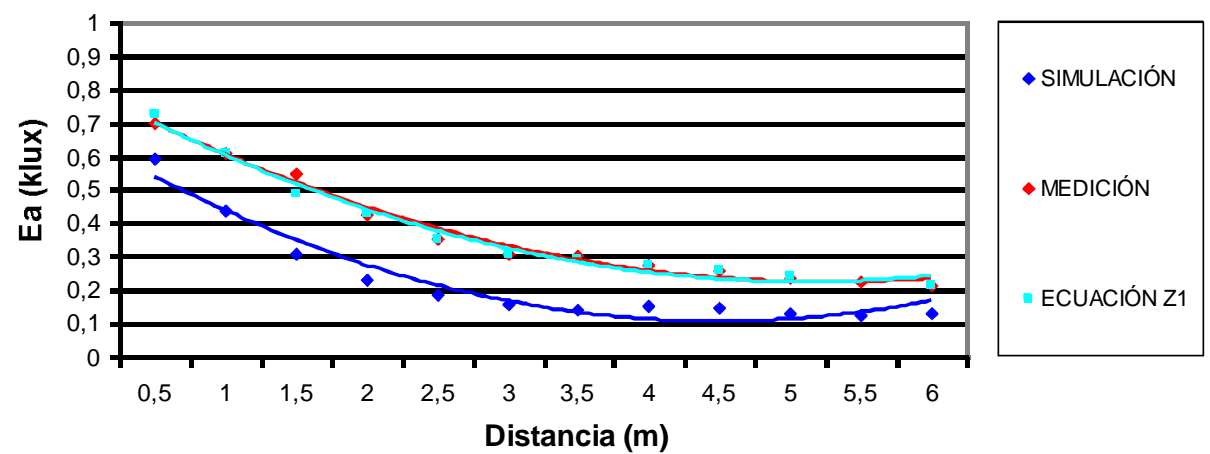

(e) 15:00
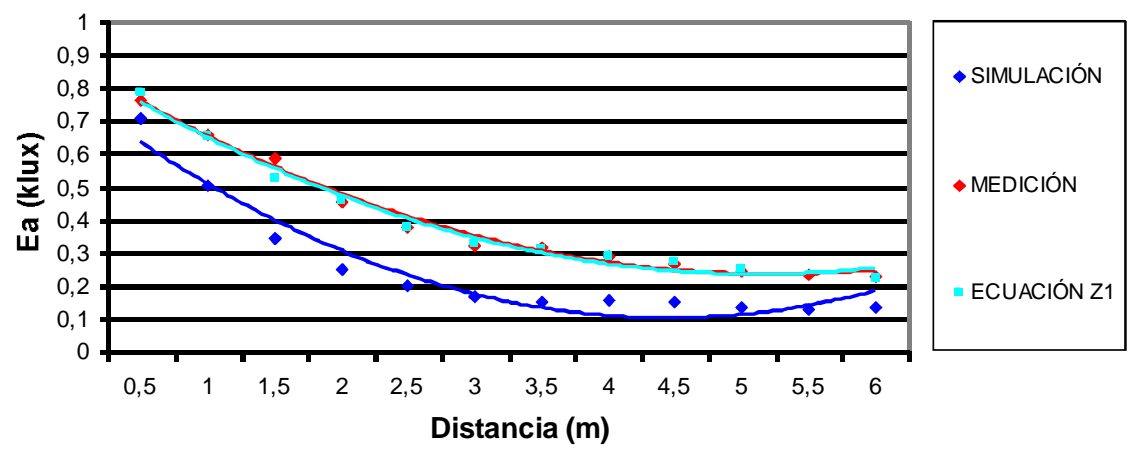

(f) 16:00.

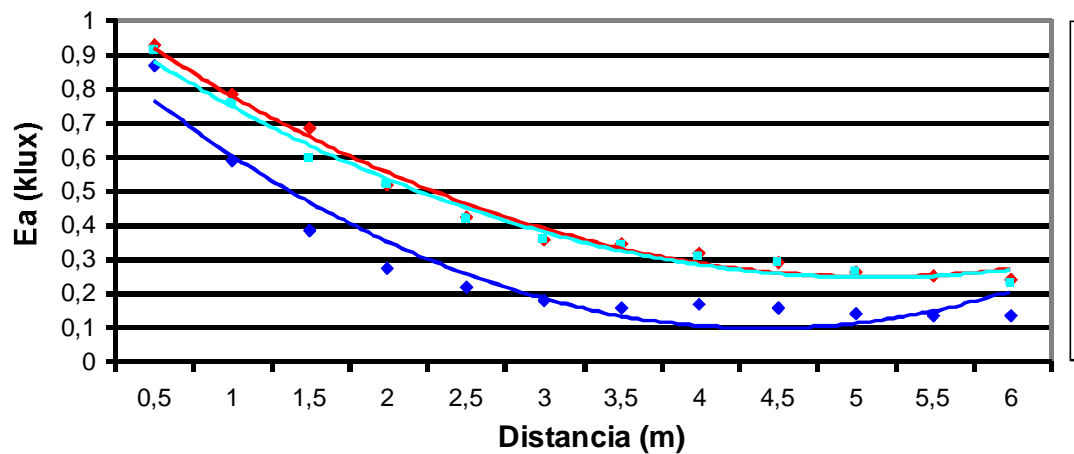

- SIMULACIÓN

- MEDICIÓN

ECUACIÓN Z1

(g) 17:00.

Figura 10.2.1. Curvas ajustadas de las iluminancias del aula recogidas en los diagramas de barras de la figura 6.19 a las distancias consideradas. 
Las ecuaciones correspondientes a las curvas son las siguientes:

\begin{tabular}{|c|c|c|}
\hline $11: 00$ & Ecuación & $r^{2}$ \\
\hline Aula & $E_{a}=0,0041 d^{2}-0,0909 d+0,6818$ & 0,9837 \\
\hline Simulación & $E_{a}=0,0049 d^{2}-0,0909 d+0,5387$ & 0,9658 \\
\hline Ecuación & $E_{a}=0,005 d^{2}-0,1079 d+0,78$ & 0,9890 \\
\hline
\end{tabular}

(a) 10:00.

\begin{tabular}{|c|c|c|}
\hline $12: 00$ & Ecuación & $r^{2}$ \\
\hline Aula & $E_{a}=0,0043 d^{2}-0,0945 d+0,71$ & 0,9842 \\
\hline Simulación & $E_{a}=0,005 d^{2}-0,0917 d+0,5389$ & 0,9651 \\
\hline Ecuación & $E_{a}=0,005 d^{2}-0,1065 d+0,7625$ & 0,9871 \\
\hline
\end{tabular}

(b) $11: 00$.

\begin{tabular}{|c|c|c|}
\hline $13: 00$ & Ecuación & $\mathbf{r}^{2}$ \\
\hline Aula & $\mathrm{E}_{\mathrm{a}}=0,0044 \mathrm{~d}^{2}-0,0946 \mathrm{~d}+0,7166$ & 0,9842 \\
\hline Simulación & $\mathrm{E}_{\mathrm{a}}=0,0052 \mathrm{~d}^{2}-0,0955 \mathrm{~d}+0,5491$ & 0,9628 \\
\hline Ecuación & $\mathrm{E}_{\mathrm{a}}=0,005 \mathrm{~d}^{2}-0,1039 \mathrm{~d}+0,7448$ & 0,9843 \\
\hline
\end{tabular}

(c) 12:00.

\begin{tabular}{|c|c|c|}
\hline $14: 00$ & Ecuación & $r^{2}$ \\
\hline Aula & $E_{a}=0,0047 d^{2}-0,1 d+0,7498$ & 0,9846 \\
\hline Simulación & $E_{a}=0,0058 d^{2}-0,105 d+0,5837$ & 0,9566 \\
\hline Ecuación & $E_{a}=0,0052 d^{2}-0,1065 d+0,7622$ & 0,9819 \\
\hline
\end{tabular}

(d) $13: 00$.

\begin{tabular}{|c|c|c|}
\hline $15: 00$ & Ecuación & $r^{2}$ \\
\hline Aula & $E_{a}=0,0052 d^{2}-0,1104 d+0,8044$ & 0,9846 \\
\hline Simulación & $E_{a}=0,0069 d^{2}-0,123 d+0,6552$ & 0,9479 \\
\hline Ecuación & $E_{a}=0,0056 d^{2}-0,1146 d+0,8086$ & 0,9817 \\
\hline
\end{tabular}

(e) 15:00.

\begin{tabular}{|c|c|c|}
\hline $16: 00$ & Ecuación & $r^{2}$ \\
\hline Aula & $E_{a}=0,0059 d^{2}-0,1226 d+0,874$ & 0,9851 \\
\hline Simulación & $E_{a}=0,0086 d^{2}-0,1528 d+0,7777$ & 0,9388 \\
\hline Ecuación & $E_{a}=0,0061 d^{2}-0,125 d+0,8731$ & 0,9828 \\
\hline
\end{tabular}

(f) $16: 00$.

\begin{tabular}{|c|c|c|}
\hline $\mathbf{1 7 : 0 0}$ & Ecuación & $\mathbf{r}^{2}$ \\
\hline Aula & $\mathrm{E}_{\mathrm{a}}=0,0078 \mathrm{~d}^{2}-0,1601 \mathrm{~d}+1,0667$ & 0,9856 \\
\hline Simulación & $\mathrm{E}_{\mathrm{a}}=0,011 \mathrm{~d}^{2}-0,1934 \mathrm{~d}+0,9462$ & 0,9280 \\
\hline Ecuación & $\mathrm{E}_{\mathrm{a}}=0,00741 \mathrm{~d}^{2}-0,1517 \mathrm{~d}+1,0205$ & 0,9836 \\
\hline
\end{tabular}

(g) 17:00.

Tabla 10.2.1. Ecuaciones de ajuste diagrama de barras de las iluminancias obtenidas en el aula, modelo a escala, simulación y aplicando la ecuación zona 1 a las distancias y horas consideradas el día 23/07/2014. 


\subsubsection{Zona 2}

Las curvas ajustadas del estudio comparativo de las iluminancias del aula obtenidas midiendo directamente en el aula, mediante la ecuación Z2 y mediante simulación representadas en la figura 6.36 , se muestran a continuación:
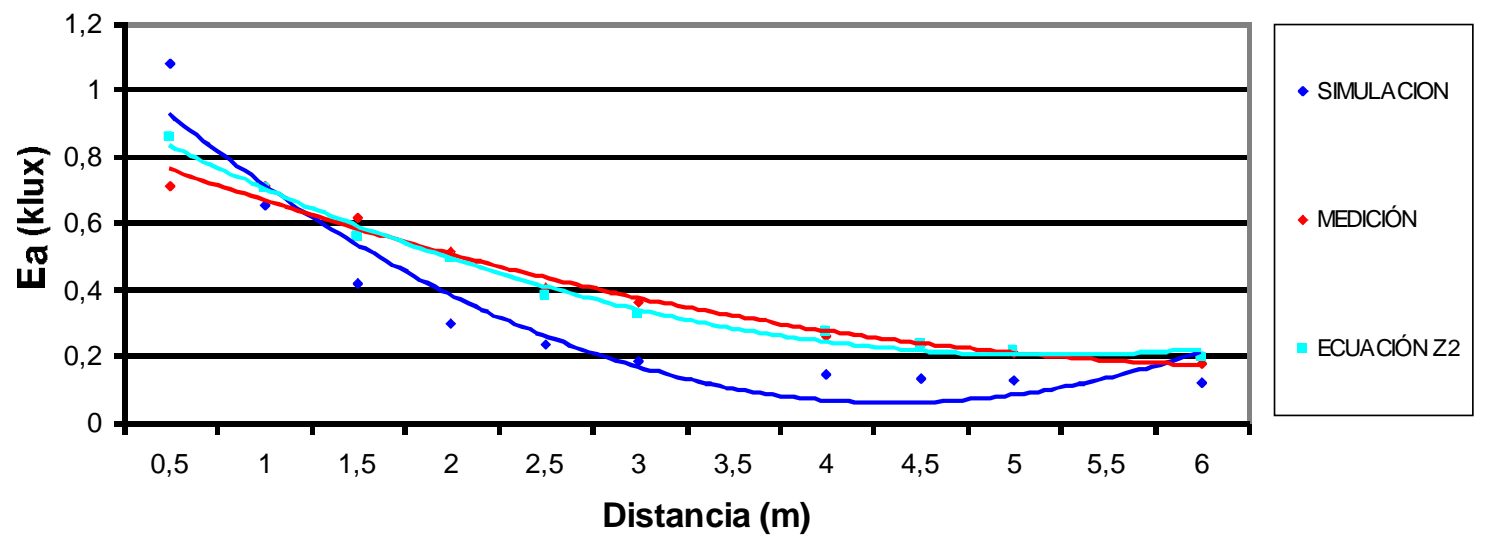

(a) 11:00.
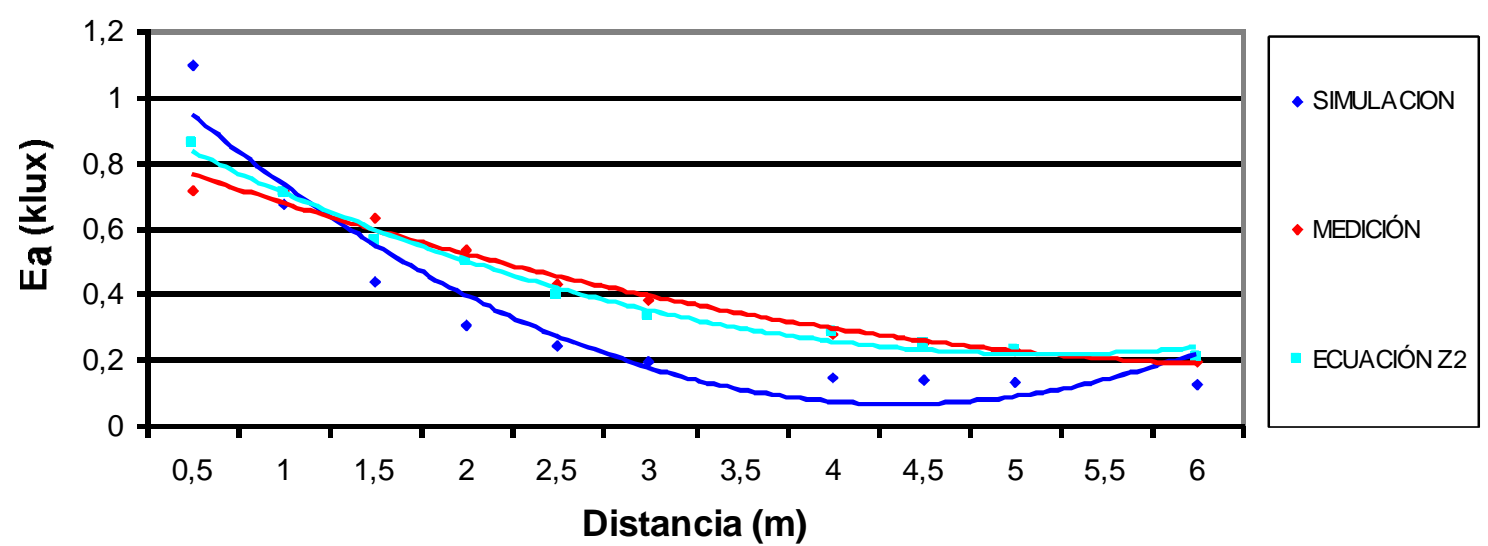

(b) $12: 00$

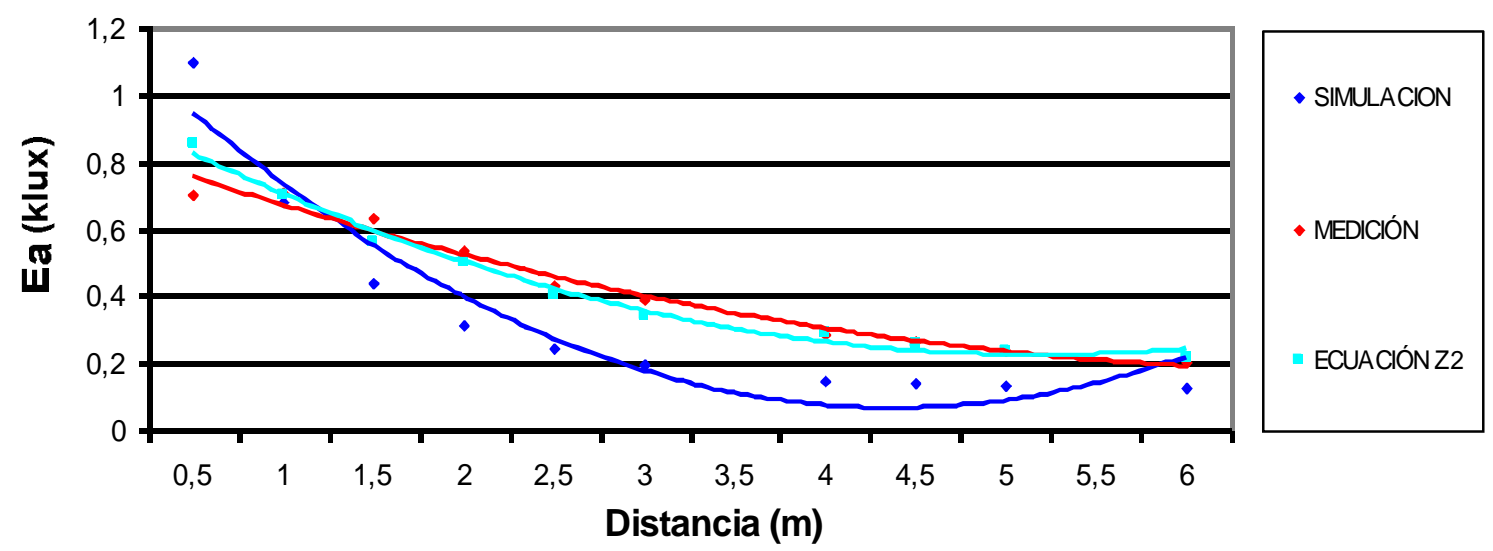

(c) 13:00 

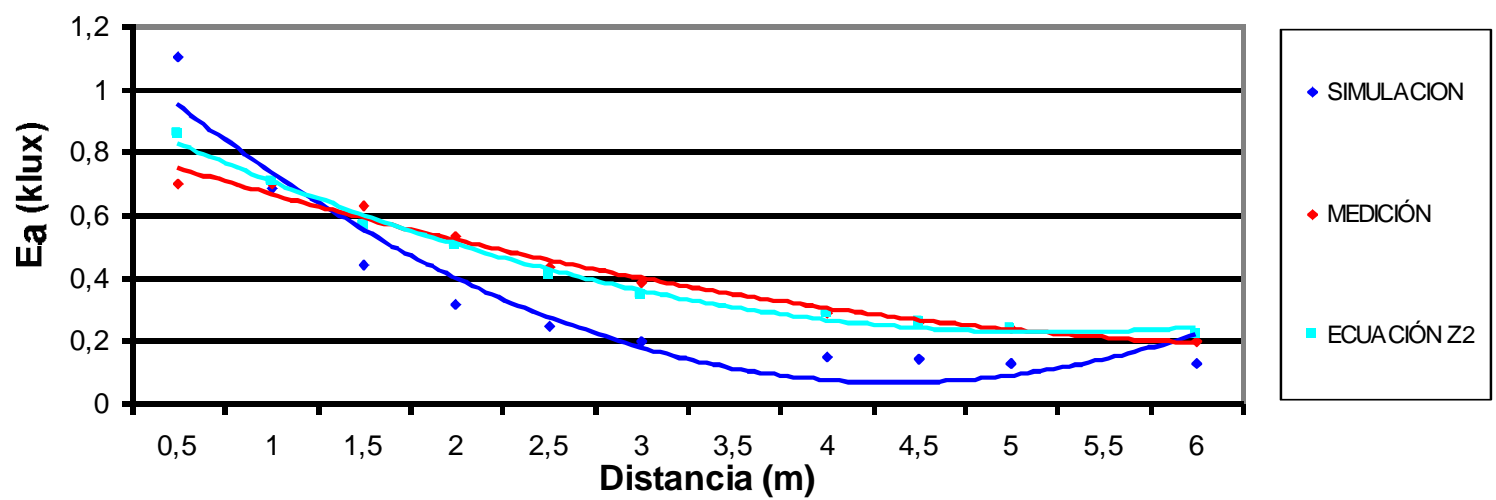

(d) 14:00.
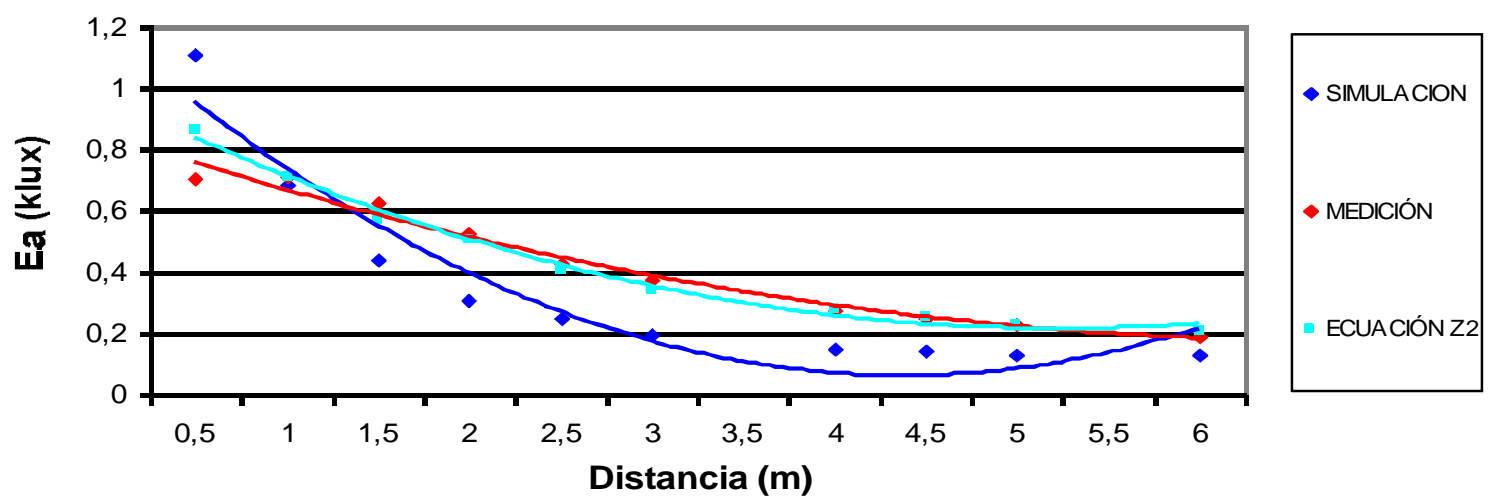

(e) 15:00.

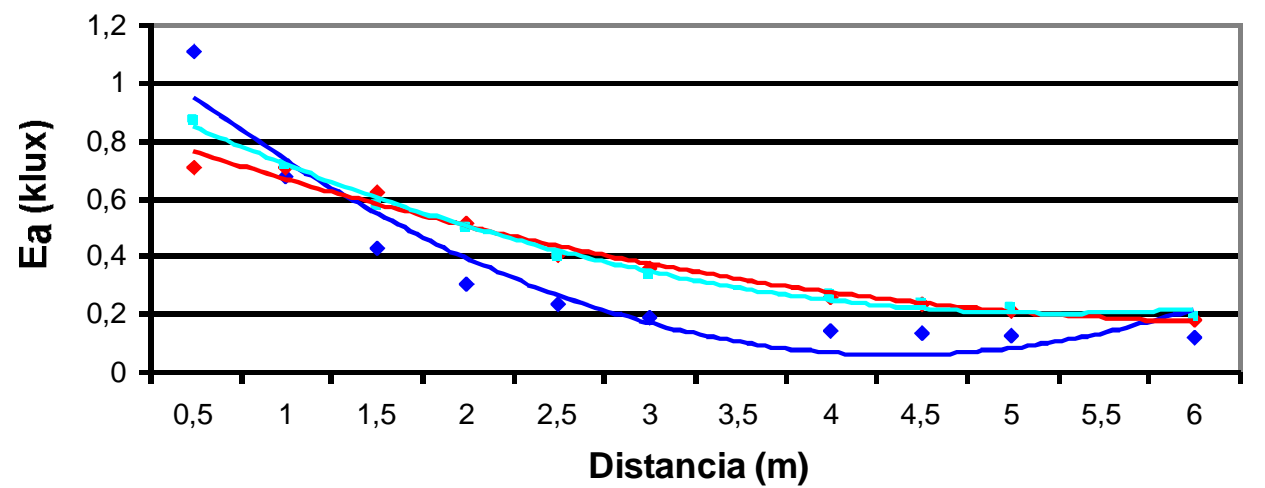

- SIMULACION

-MEDICIÓN

- ECUACIÓN Z2

(f) $16: 00$.
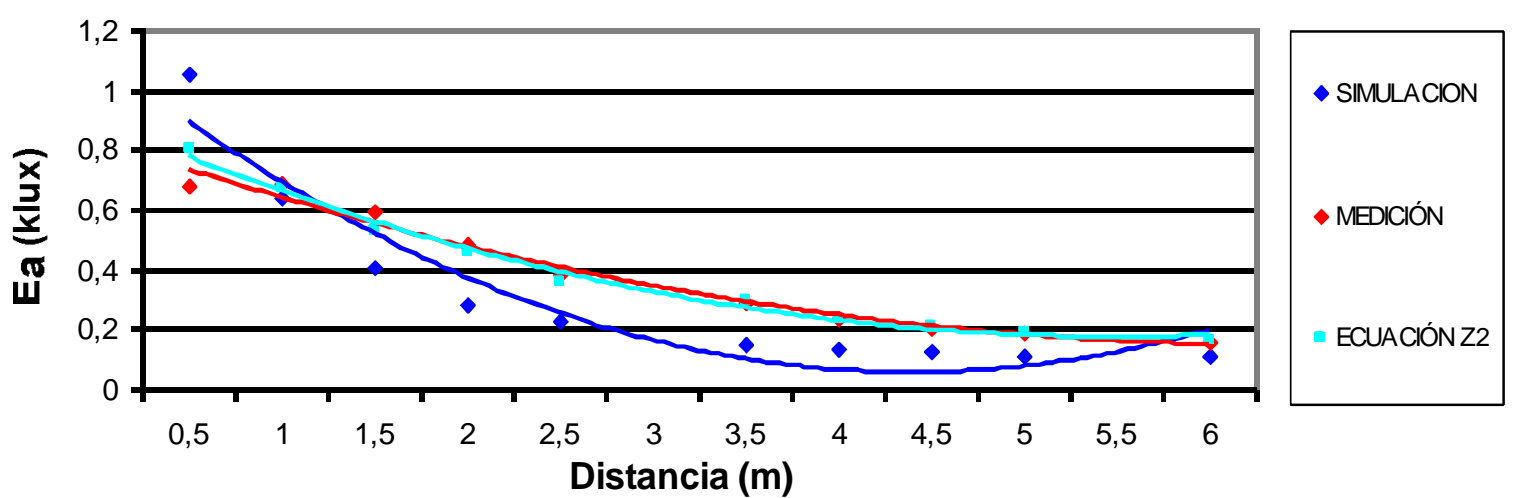

(g) 17:00.

Figura 10.2.2. Curvas ajustadas de las iluminancias del aula recogidas en los diagramas de barras de la figura 6.36 a las distancias consideradas. 
Las ecuaciones correspondientes a las curvas de la figura 10.2.2 son las siguientes:

\begin{tabular}{|c|c|c|}
\hline $11: 00$ & Ecuación & $\mathbf{r}^{2}$ \\
\hline Medición & $E_{a}=0,0041 d^{2}-0,1065 d+0,8625$ & 0,9835 \\
\hline Simulación & $E_{a}=0,0139 d^{2}-0,2452 d+1,1492$ & 0,9208 \\
\hline Ecuación & $E_{a}=0,0067 d^{2}-0,1435 d+0,9629$ & 0,988 \\
\hline
\end{tabular}

(a) 11:00.

\begin{tabular}{|c|c|c|}
\hline $12: 00$ & Ecuación & $r^{2}$ \\
\hline Medición & $E_{a}=0,0038 d^{2}-0,1007 d+0,8606$ & 0,9829 \\
\hline Simulación & $E_{a}=0,014 d^{2}-0,2487 d+1,1715$ & 0,9249 \\
\hline Ecuación & $y=0,0066 d^{2}-0,1402 d+0,9598$ & 0,9872 \\
\hline
\end{tabular}

(b) 12:00.

\begin{tabular}{|c|c|c|}
\hline $13: 00$ & Ecuación & $\mathbf{r}^{2}$ \\
\hline Medición & $\mathrm{E}_{\mathrm{a}}=0,0033 \mathrm{~d}^{2}-0,0941 \mathrm{~d}+0,8444$ & 0,9808 \\
\hline Simulación & $\mathrm{E}_{\mathrm{a}}=0,0146 \mathrm{~d}^{2}-0,2563 \mathrm{~d}+1,1873$ & 0,9244 \\
\hline Ecuación & $\mathrm{E}_{\mathrm{a}}=0,0068 \mathrm{~d}^{2}-0,1416 \mathrm{~d}+0,9574$ & 0,9892 \\
\hline
\end{tabular}

(c) 13:00.

\begin{tabular}{|c|c|c|}
\hline $14: 00$ & Ecuación & $r^{2}$ \\
\hline Medición & $E_{a}=0,0032 d^{2}-0,0929 d+0,8356$ & 0,9797 \\
\hline Simulación & $E_{a}=0,0147 d^{2}-0,2577 d+1,1913$ & 0,9248 \\
\hline Ecuación & $E_{a}=0,0068 d^{2}-0,1416 d+0,961$ & 0,9899 \\
\hline
\end{tabular}

(d) 14:00.

\begin{tabular}{|c|c|c|}
\hline $15: 00$ & Ecuación & $r^{2}$ \\
\hline Medición & $E_{a}=0,0036 d^{2}-0,0988 d+0,849$ & 0,9803 \\
\hline Simulación & $E_{a}=0,0149 d^{2}-0,2603 d+1,198$ & 0,9229 \\
\hline Ecuación & $E_{a}=0,0069 d^{2}-0,1456 d+0,9753$ & 0,9908 \\
\hline
\end{tabular}

(e) 15:00.

\begin{tabular}{|c|c|c|}
\hline $\mathbf{1 6 : 0 0}$ & Ecuación & $r^{2}$ \\
\hline Medición & $E_{a}=0,004 d^{2}-0,1054 d+0,8572$ & 0,9803 \\
\hline Simulación & $E_{a}=0,0149 d^{2}-0,2606 d+1,1922$ & 0,9206 \\
\hline Ecuación & $E_{a}=0,0071 d^{2}-0,1496 d+0,9847$ & 0,9918 \\
\hline
\end{tabular}

(f) $16: 00$.

\begin{tabular}{|c|c|c|}
\hline $17: 00$ & Ecuación & $r^{2}$ \\
\hline Medición & $E_{a}=0,0041 d^{2}-0,1067 d+0,837$ & 0,9803 \\
\hline Simulación & $E_{a}=0,0138 d^{2}-0,2436 d+1,1255$ & 0,917 \\
\hline Ecuación & $E_{a}=0,006 d^{2}-0,1324 d+0,9027$ & 0,9919 \\
\hline
\end{tabular}

(g) 17:00.

Tabla 10.2.2. Ecuaciones de ajuste diagrama de barras de las iluminancias obtenidas en el aula, modelo a escala, simulación y aplicando la ecuación zona 2 a las distancias y horas consideradas el día 05/04/2015. 
Las curvas ajustadas del estudio comparativo de las iluminancias del aula obtenidas midiendo directamente en el aula, mediante la ecuación Z2 y mediante simulación representadas en la figura 6.37 , se muestra a continuación:

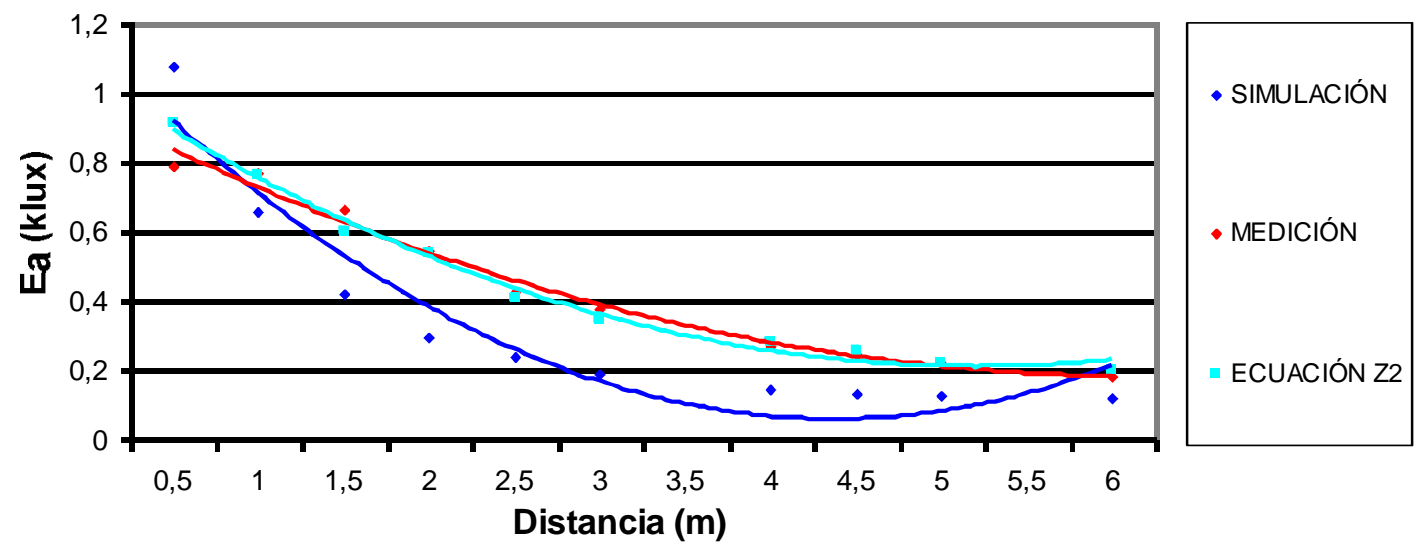

(a) 11:00.

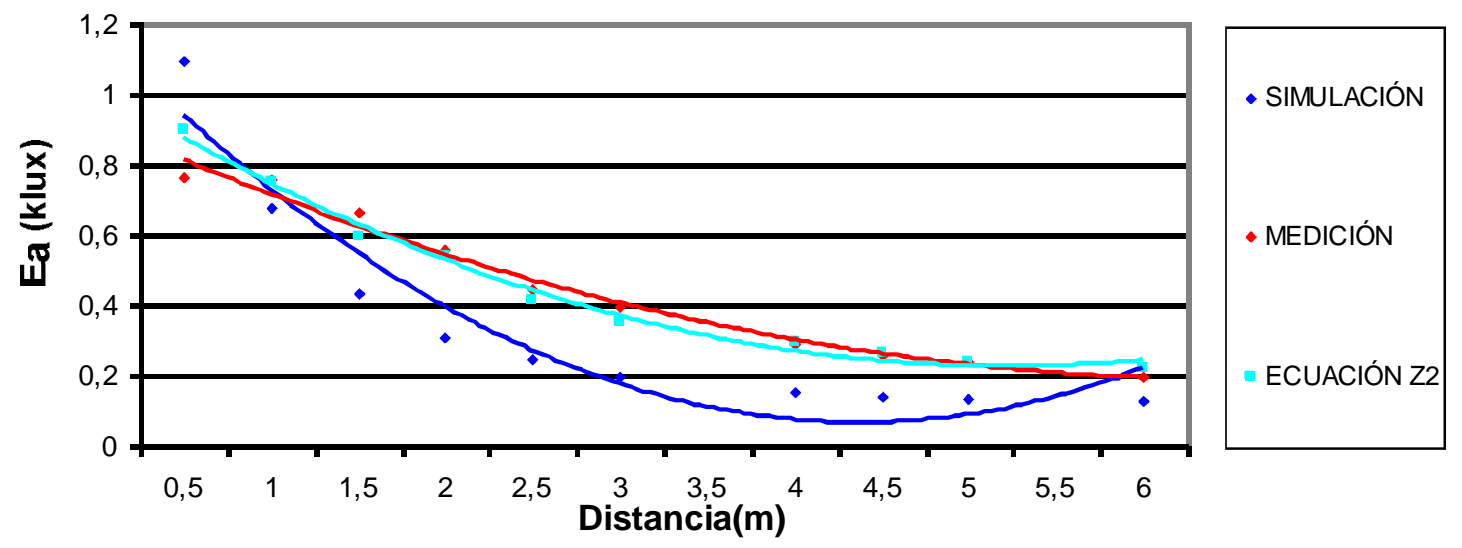

(b) $12: 00$
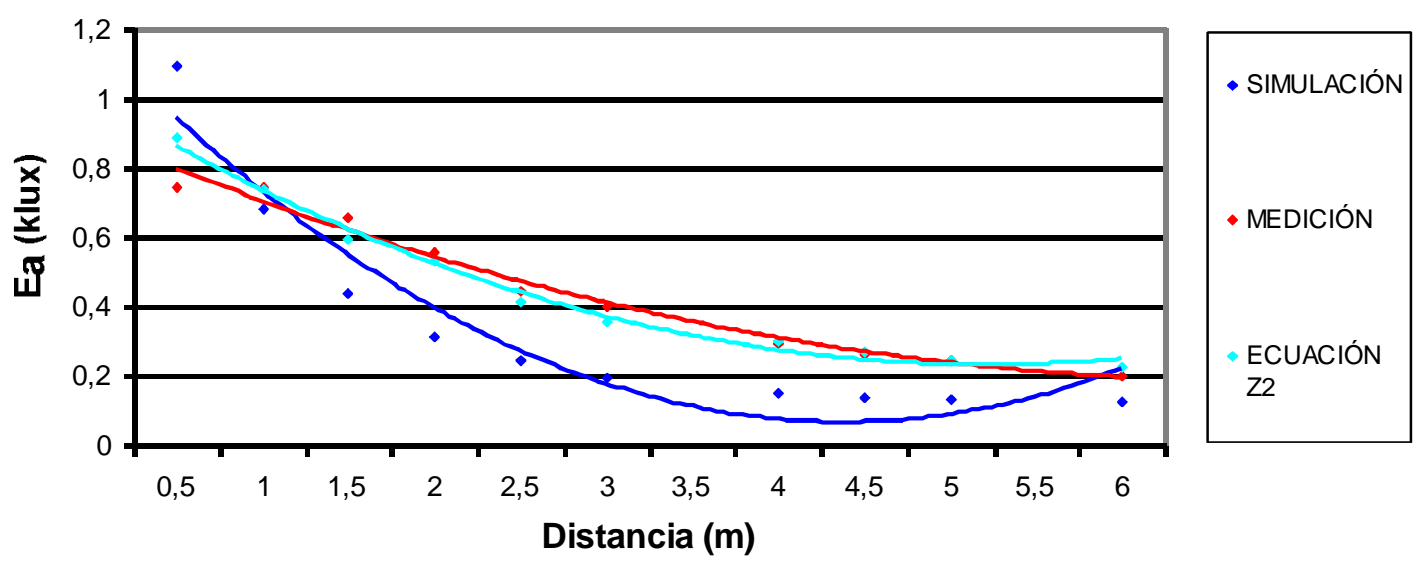

(c) $13: 00$ 
Estudio experimental y modelización de la iluminación natural en la edificación mediante modelos a escala

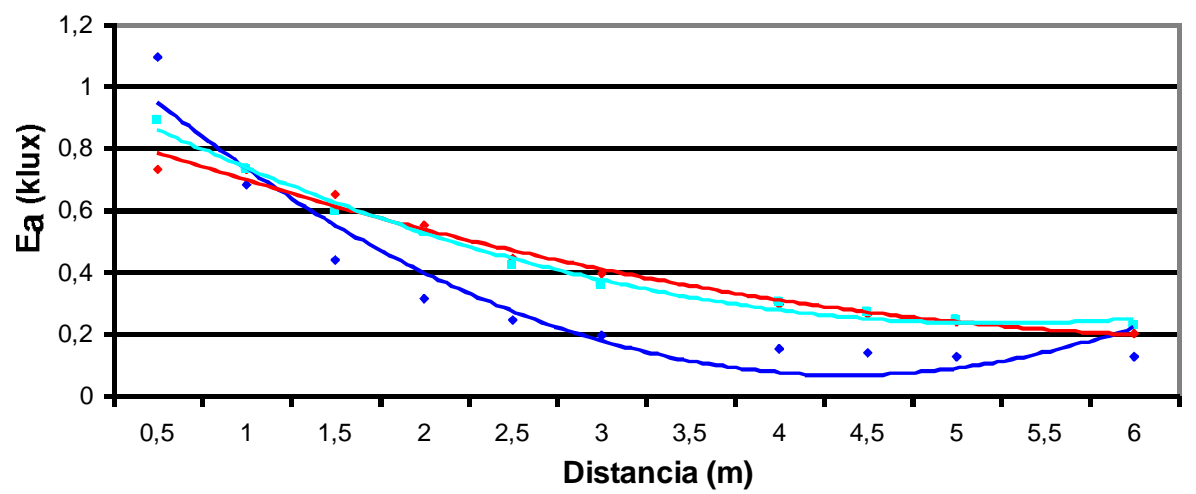

- SIMULACIÓN

- MEDICIÓN

ECUACIÓN Z2

(d) 14:00.
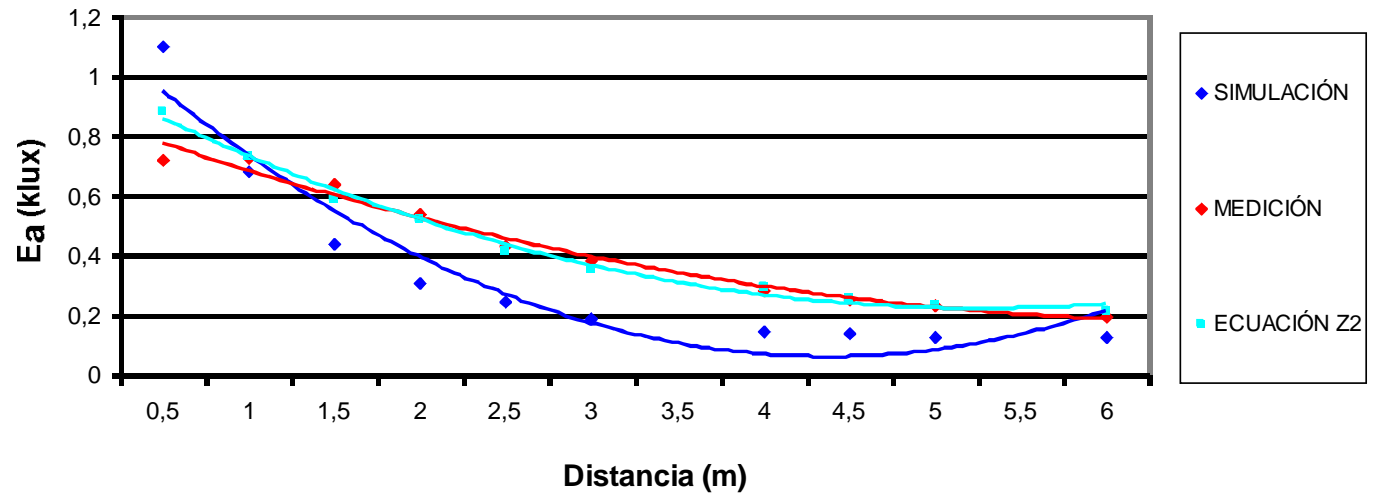

(e) 15:00.
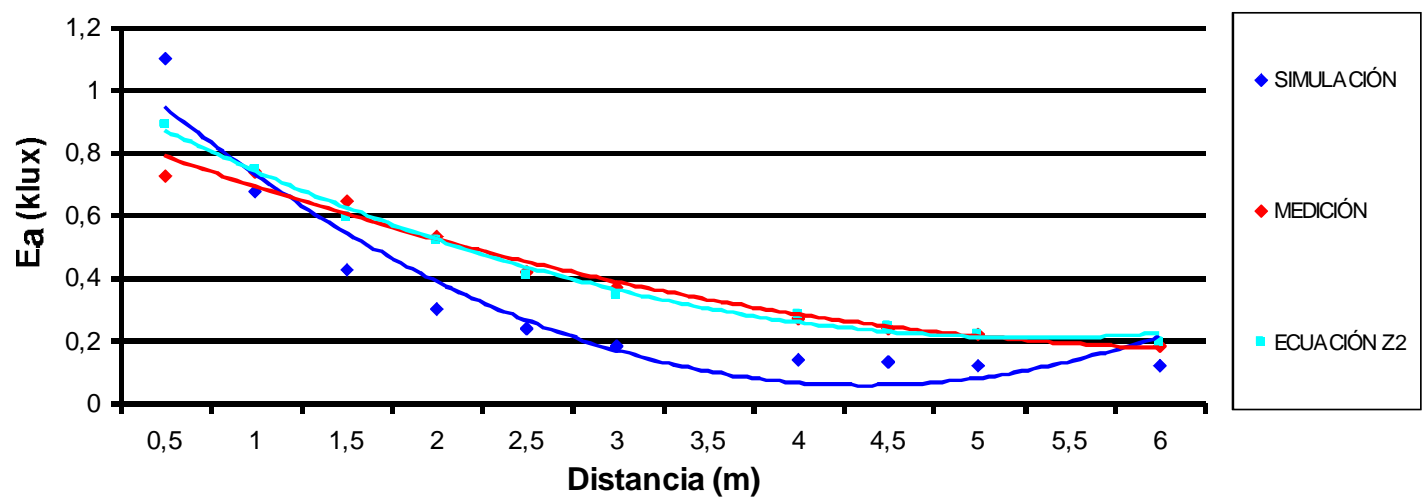

(f) $16: 00$.
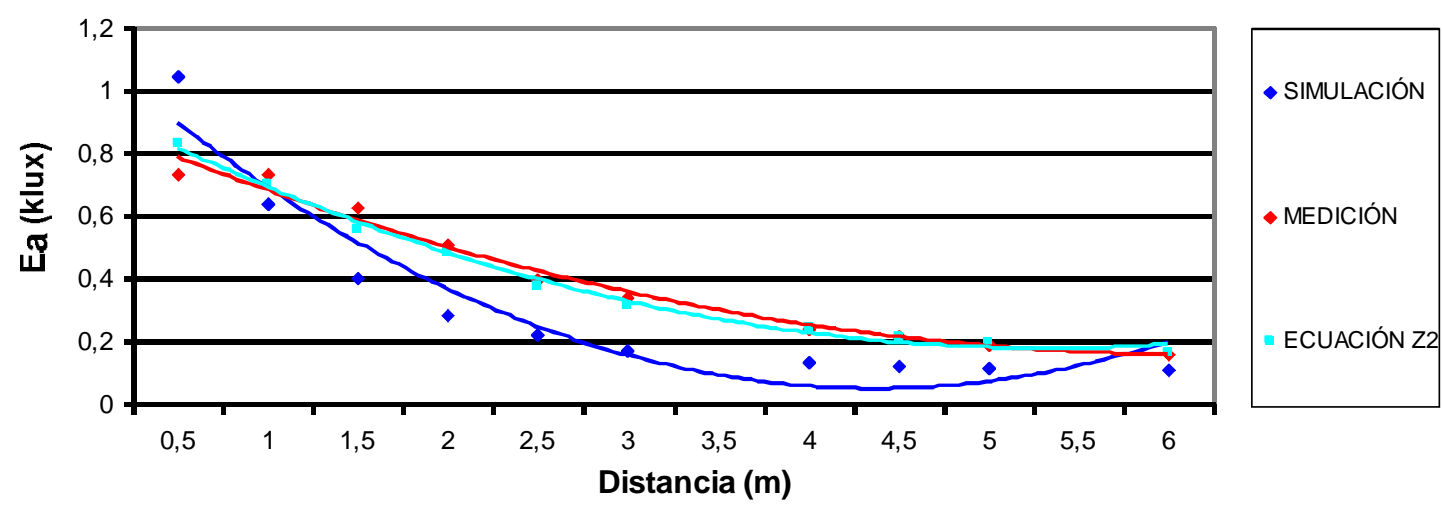

(g) 17:00.

Figura 10.2.3. Curvas ajustadas de las iluminancias del aula recogidas en los diagramas de barras de la figura 6.37 a las distancias consideradas. 
Las ecuaciones correspondientes a las curvas de la figura 10.2.3 son las siguientes:

\begin{tabular}{|c|c|c|}
\hline $11: 00$ & Ecuación & $\mathbf{r}^{2}$ \\
\hline Medición & $E_{a}=0,005 d^{2}-0,1246 d+0,9554$ & 0,9861 \\
\hline Simulación & $E_{a}=0,0144 d^{2}-0,2515 d+1,1566$ & 0,9184 \\
\hline Ecuación & $E_{a}=0,0077 d^{2}-0,1601 d+1,0439$ & 0,9901 \\
\hline
\end{tabular}

(a) 11:00.

\begin{tabular}{|c|c|c|}
\hline $\mathbf{1 2 : 0 0}$ & Ecuación & $\mathbf{r}^{2}$ \\
\hline Medición & $\mathrm{E}_{\mathrm{a}}=0,0042 \mathrm{~d}^{2}-0,1106 \mathrm{~d}+0,9179$ & 0,9837 \\
\hline Simulación & $\mathrm{E}_{\mathrm{a}}=0,0146 \mathrm{~d}^{2}-0,2554 \mathrm{~d}+1,1809$ & 0,9228 \\
\hline Ecuación & $\mathrm{E}_{\mathrm{a}}=0,0073 \mathrm{~d}^{2}-0,1528 \mathrm{~d}+1,0203$ & 0,99 \\
\hline
\end{tabular}

(b) 12:00.

\begin{tabular}{|c|c|c|}
\hline $13: 00$ & Ecuación & $r^{2}$ \\
\hline Medición & $E_{a}=0,0038 d^{2}-0,1037 d+0,8958$ & 0,9823 \\
\hline Simulación & $E_{a}=0,0146 d^{2}-0,2555 d+1,1844$ & 0,9249 \\
\hline Ecuación & $E_{a}=0,0071 d^{2}-0,1477 d+1,0013$ & 0,99 \\
\hline
\end{tabular}

(c) 13:00.

\begin{tabular}{|c|c|c|}
\hline $14: 00$ & Ecuación & $\mathbf{r}^{2}$ \\
\hline Medición & $E_{a}=0,0037 d^{2}-0,1012 d+0,8809$ & 0,9818 \\
\hline Simulación & $E_{a}=0,0147 d^{2}-0,2569 d+1,1884$ & 0,9251 \\
\hline Ecuación & $E_{a}=0,007 d^{2}-0,1468 d+0,9992$ & 0,9904 \\
\hline
\end{tabular}

(d) 14:00.

\begin{tabular}{|c|c|c|}
\hline $\mathbf{1 5 : 0 0}$ & Ecuación & $\mathbf{r}^{2}$ \\
\hline Medición & $\mathrm{E}_{\mathrm{a}}=0,0037 \mathrm{~d}^{2}-0,1022 \mathrm{~d}+0,8729$ & 0,9805 \\
\hline Simulación & $\mathrm{E}_{\mathrm{a}}=0,0148 \mathrm{~d}^{2}-0,2594 \mathrm{~d}+1,1947$ & 0,9237 \\
\hline Ecuación & $\mathrm{E}_{\mathrm{a}}=0,007 \mathrm{~d}^{2}-0,1479 \mathrm{~d}+0,9988$ & 0,9912 \\
\hline
\end{tabular}

(e)

\begin{tabular}{|c|c|c|}
\hline $16: 00$ & Ecuación & $\mathbf{r}^{2}$ \\
\hline Medición & $\mathrm{E}_{a}=0,0041 \mathrm{~d}^{2}-0,1091 \mathrm{~d}+0,8907$ & 0,9786 \\
\hline Simulación & $\mathrm{E}_{\mathrm{a}}=0,0148 \mathrm{~d}^{2}-0,2592 \mathrm{~d}+1,1866$ & 0,9213 \\
\hline Ecuación & $\mathrm{E}_{\mathrm{a}}=0,0072 \mathrm{~d}^{2}-0,1522 \mathrm{~d}+1,0139$ & 0,9926 \\
\hline
\end{tabular}

(f)

\begin{tabular}{|c|c|c|}
\hline $17: 00$ & Ecuación & $\mathbf{r}^{2}$ \\
\hline Medición & $\mathrm{E}_{\mathrm{a}}=0,0048 \mathrm{~d}^{2}-0,1195 \mathrm{~d}+0,8993$ & 0,9811 \\
\hline Simulación & $\mathrm{E}_{\mathrm{a}}=0,0141 \mathrm{~d}^{2}-0,2473 \mathrm{~d}+1,1276$ & 0,9197 \\
\hline Ecuación & $\mathrm{E}_{\mathrm{a}}=0,0068 \mathrm{~d}^{2}-0,1461 \mathrm{~d}+0,9548$ & 0,9936 \\
\hline
\end{tabular}

(g)

Tabla 10.2.3. Ecuaciones de ajuste diagrama de barras de las iluminancias obtenidas en el aula, modelo a escala, simulación y aplicando la ecuación zona 2 a las distancias y horas consideradas el día 04/04/2015. 
Las curvas ajustadas del estudio comparativo de las iluminancias del aula obtenidas midiendo directamente en el aula, mediante la ecuación Z2 y mediante simulación representadas en la figura 6.38 , se muestra a continuación:

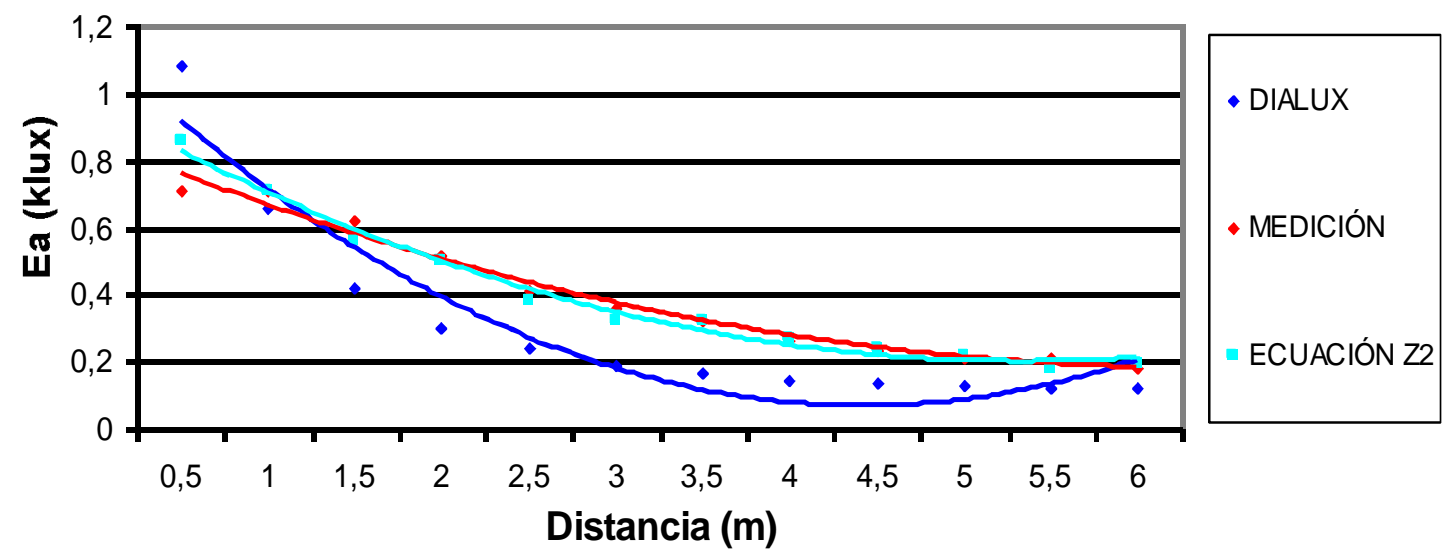

(a) 11:00.
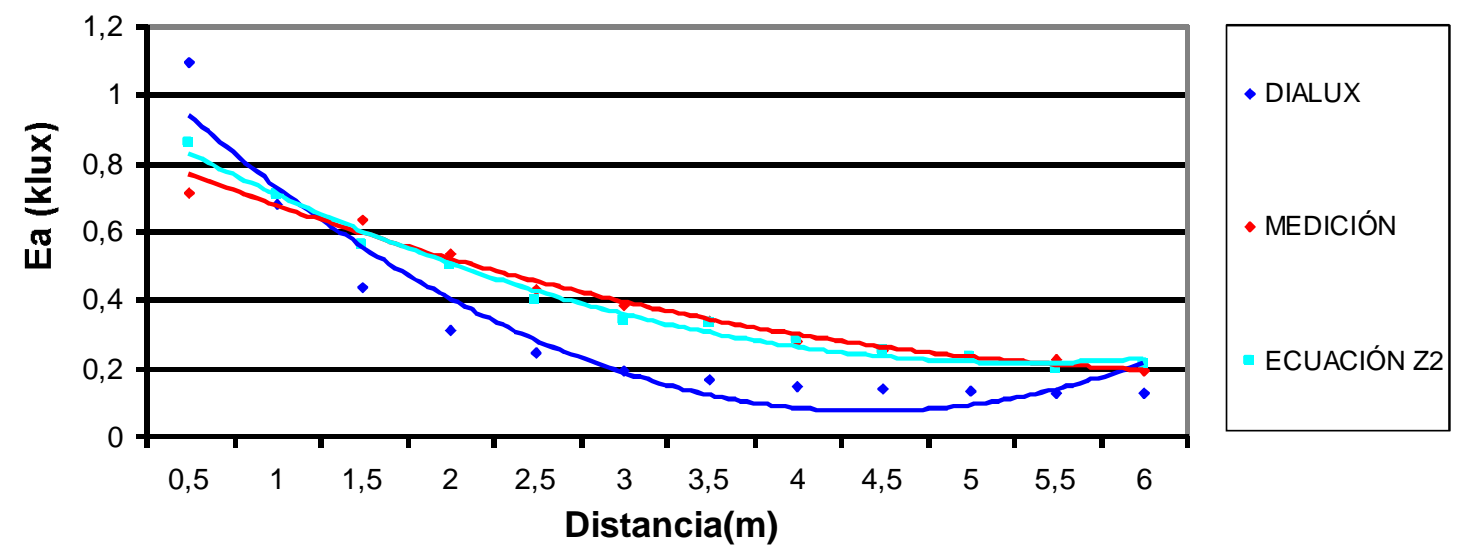

(b) $12: 00$

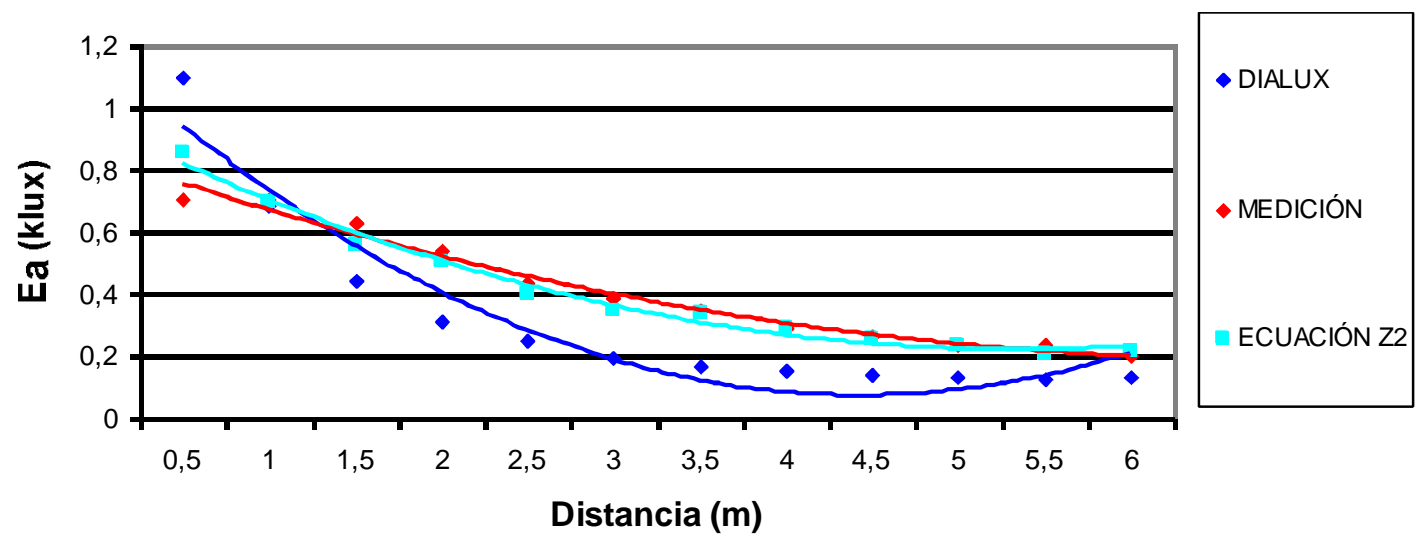

(c) $13: 00$ 

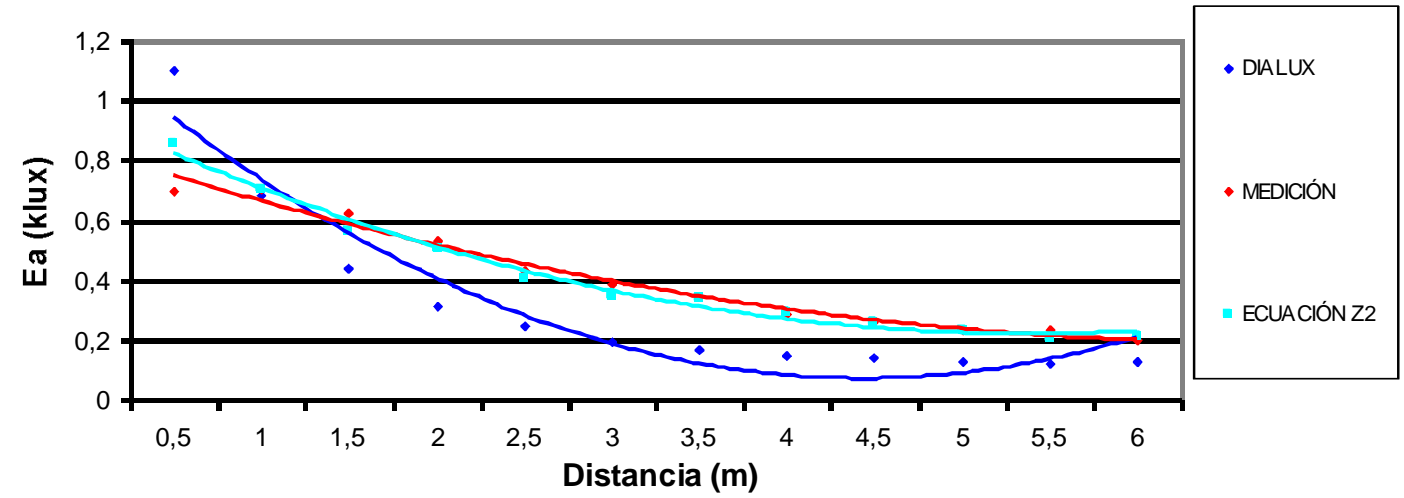

(d) $14: 00$

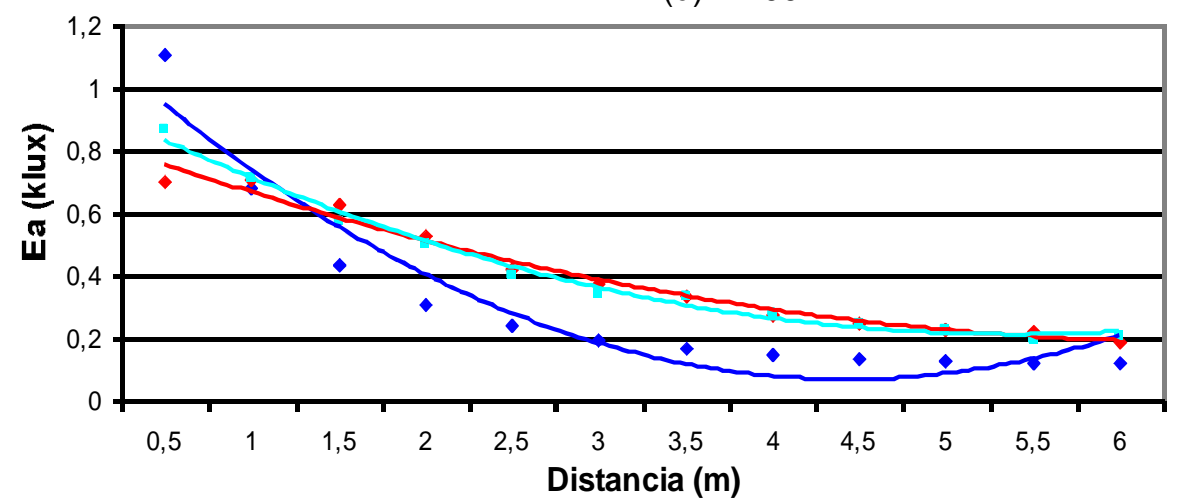

- DIALUX

(e) 15:00

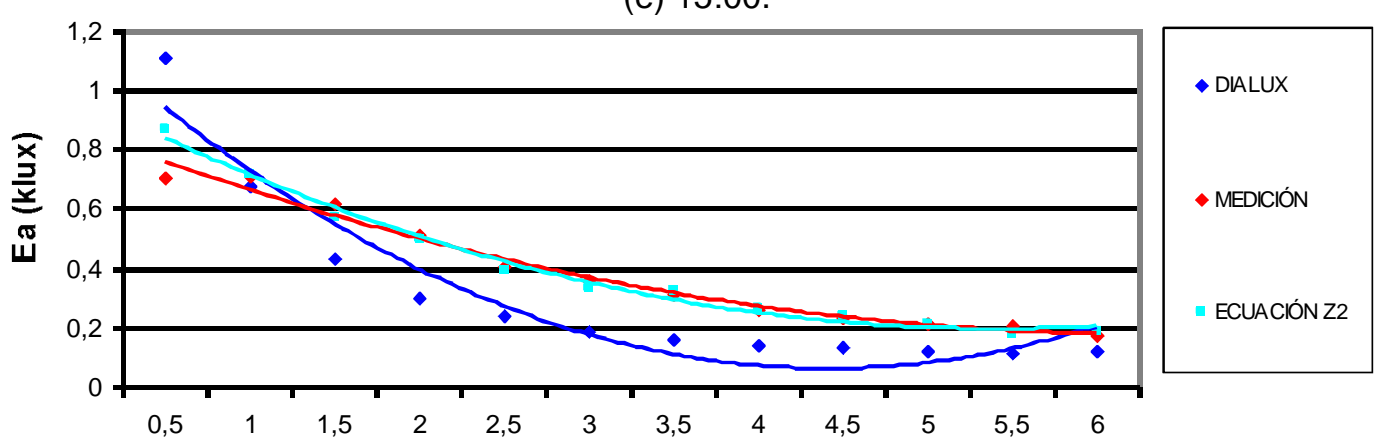

Distancia (m)

(f) $16: 00$.

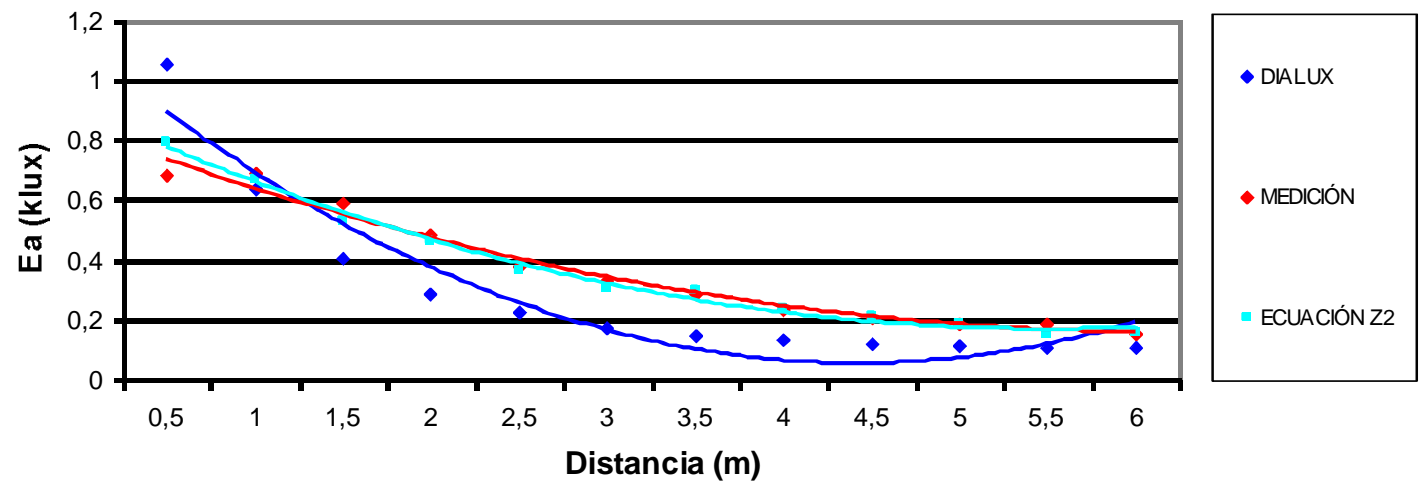

(g) 17:00.

Figura 10.2.4. Curvas ajustadas de las iluminancias del aula recogidas en los diagramas de barras de la figura 6.38 a las distancias consideradas. 
Las ecuaciones correspondientes a las curvas de la figura 10.2.4 son las siguientes:

\begin{tabular}{|c|c|c|}
\hline $11: 00$ & Ecuación & $\mathbf{r}^{2}$ \\
\hline Medición & $E_{a}=0,0041 d^{2}-0,1065 d+0,8625$ & 0,9835 \\
\hline Simulación & $E_{a}=0,0135 d^{2}-0,2397 d+1,1257$ & 0,9225 \\
\hline Ecuación & $E_{a}=0,0067 d^{2}-0,1435 d+0,9629$ & 0,988 \\
\hline
\end{tabular}

(a) $11: 00$.

\begin{tabular}{|c|c|c|}
\hline $12: 00$ & Ecuación & $\mathbf{r}^{2}$ \\
\hline Medición & $E_{a}=0,0038 d^{2}-0,1007 d+0,8606$ & 0,9829 \\
\hline Simulación & $E_{a}=0,0138 d^{2}-0,2449 d+1,1567$ & 0,9259 \\
\hline Ecuación & $E_{a}=0,0066 d^{2}-0,1402 d+0,9598$ & 0,9872 \\
\hline
\end{tabular}

(b) 12:00.

\begin{tabular}{|c|c|c|}
\hline $13: 00$ & Ecuación & $\mathbf{r}^{2}$ \\
\hline Medición & $\mathrm{E}_{\mathrm{a}}=0,0035 \mathrm{~d}^{2}-0,0955 \mathrm{~d}+0,8463$ & 0,9819 \\
\hline Simulación & $\mathrm{E}_{\mathrm{a}}=0,0138 \mathrm{~d}^{2}-0,2458 \mathrm{~d}+1,1632$ & 0,9285 \\
\hline Ecuación & $\mathrm{E}_{\mathrm{a}}=0,0064 \mathrm{~d}^{2}-0,1361 \mathrm{~d}+0,9481$ & 0,9871 \\
\hline
\end{tabular}

(c) 13:00.

\begin{tabular}{|c|c|c|}
\hline $14: 00$ & Ecuación & $\mathbf{r}^{2}$ \\
\hline Medición & $\mathrm{E}_{a}=0,0034 \mathrm{~d}^{2}-0,0943 \mathrm{~d}+0,8377$ & 0,981 \\
\hline Simulación & $\mathrm{E}_{\mathrm{a}}=0,0139 \mathrm{~d}^{2}-0,2471 \mathrm{~d}+1,1672$ & 0,9288 \\
\hline Ecuación & $\mathrm{E}_{\mathrm{a}}=0,0063 \mathrm{~d}^{2}-0,136 \mathrm{~d}+0,9516$ & 0,9877 \\
\hline
\end{tabular}

(d) 14:00.

\begin{tabular}{|c|c|c|}
\hline $15: 00$ & Ecuación & $r^{2}$ \\
\hline Medición & $E_{a}=0,0036 d^{2}-0,0988 d+0,849$ & 0,9803 \\
\hline Simulación & $E_{a}=0,0146 d^{2}-0,2567 d+1,1835$ & 0,9244 \\
\hline Ecuación & $E_{a}=0,0069 d^{2}-0,1456 d+0,9753$ & 0,9908 \\
\hline
\end{tabular}

(e) 15:00.

\begin{tabular}{|c|c|c|}
\hline $16: 00$ & Ecuación & $\mathbf{r}^{2}$ \\
\hline Medición & $\mathrm{E}_{\mathrm{a}}=0,004 \mathrm{~d}^{2}-0,1054 \mathrm{~d}+0,8572$ & 0,9803 \\
\hline Simulación & $\mathrm{E}_{\mathrm{a}}=0,0146 \mathrm{~d}^{2}-0,2552 \mathrm{~d}+1,1695$ & 0,9222 \\
\hline Ecuación & $\mathrm{E}_{\mathrm{a}}=0,0071 \mathrm{~d}^{2}-0,1496 \mathrm{~d}+0,9847$ & 0,9918 \\
\hline
\end{tabular}

(f) $16: 00$.

\begin{tabular}{|c|c|c|}
\hline $17: 00$ & Ecuación & $\mathbf{r}^{2}$ \\
\hline Medición & $\mathrm{E}_{\mathrm{a}}=0,0042 \mathrm{~d}^{2}-0,108 \mathrm{~d}+0,8387$ & 0,9794 \\
\hline Simulación & $\mathrm{E}_{\mathrm{a}}=0,0138 \mathrm{~d}^{2}-0,2413 \mathrm{~d}+1,1013$ & 0,9205 \\
\hline Ecuación & $\mathrm{E}_{\mathrm{a}}=0,0065 \mathrm{~d}^{2}-0,1392 \mathrm{~d}+0,9138$ & 0,9935 \\
\hline
\end{tabular}

(g) 17:00.

Tabla 10.2.4. Ecuaciones de ajuste diagrama de barras de las iluminancias obtenidas en el aula, modelo a escala, simulación y aplicando la ecuación zona 2 a las distancias y horas consideradas el día 01/04/2015. 


\subsubsection{Zona 3}

Las curvas ajustadas del estudio comparativo de las iluminancias del aula obtenidas midiendo directamente en el aula, mediante la ecuación Z3 y mediante simulación representadas en la figura 6.56 , se muestran a continuación:

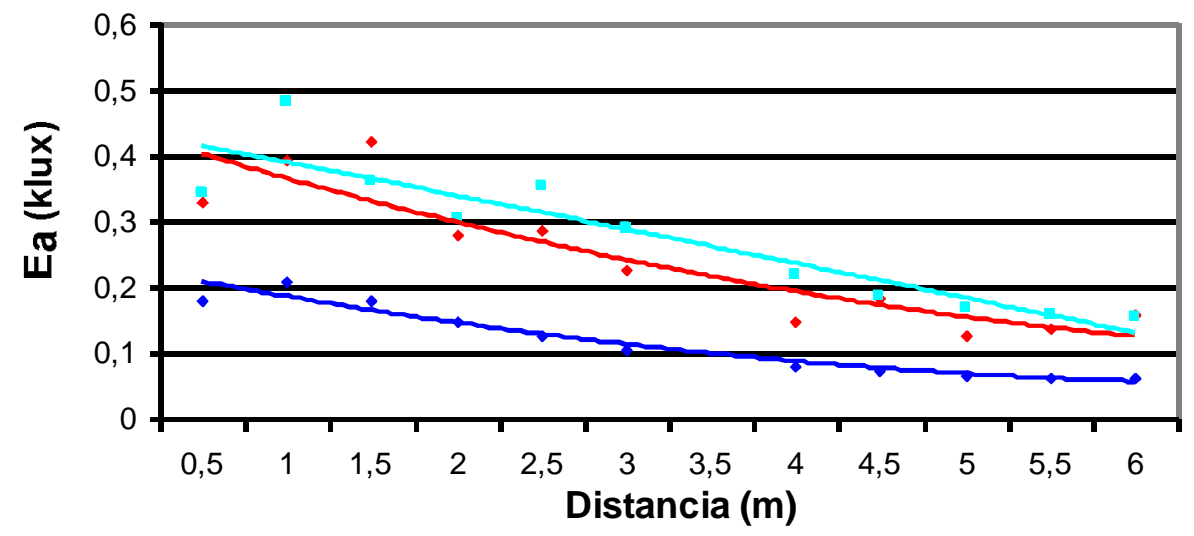

- SIMULACIÓN

- MEDICIÓN

ECUACIÓN Z3

(a) 11:00.

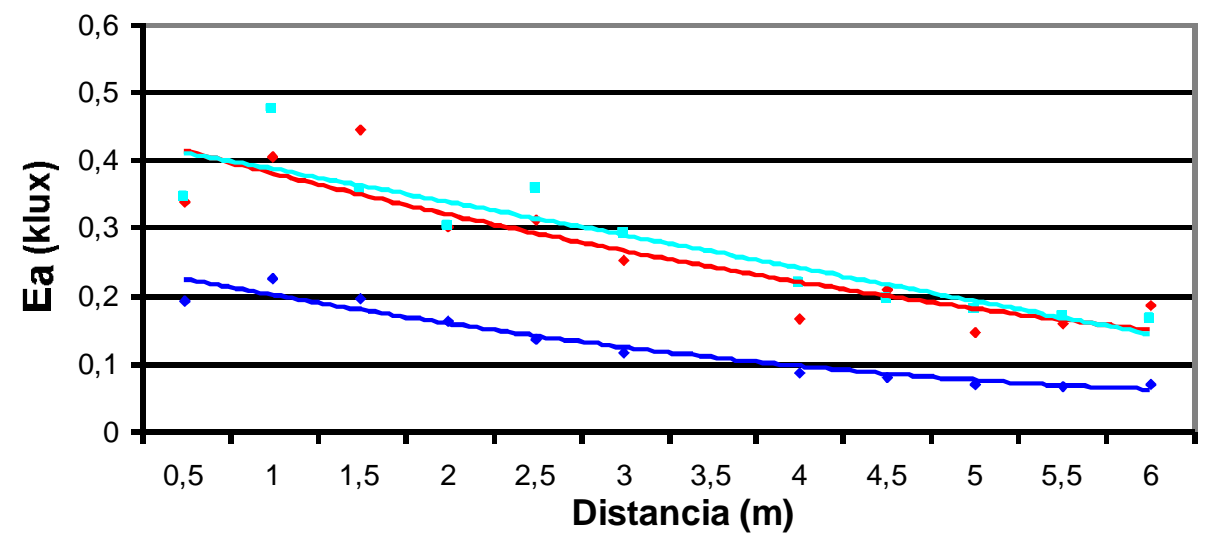

- SIMULACIÓN

MEDICIÓN

ECUACIÓN

Z3

(b) $12: 00$

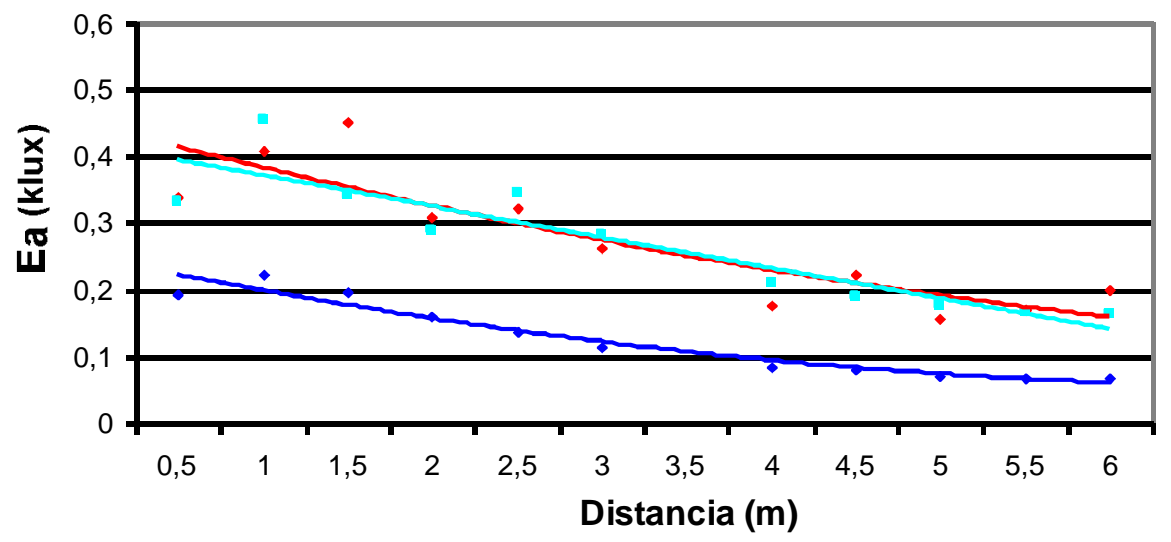

- SIMULACIÓN

- MEDICIÓN

- ECUACIÓN Z3

(c) $13: 00$ 

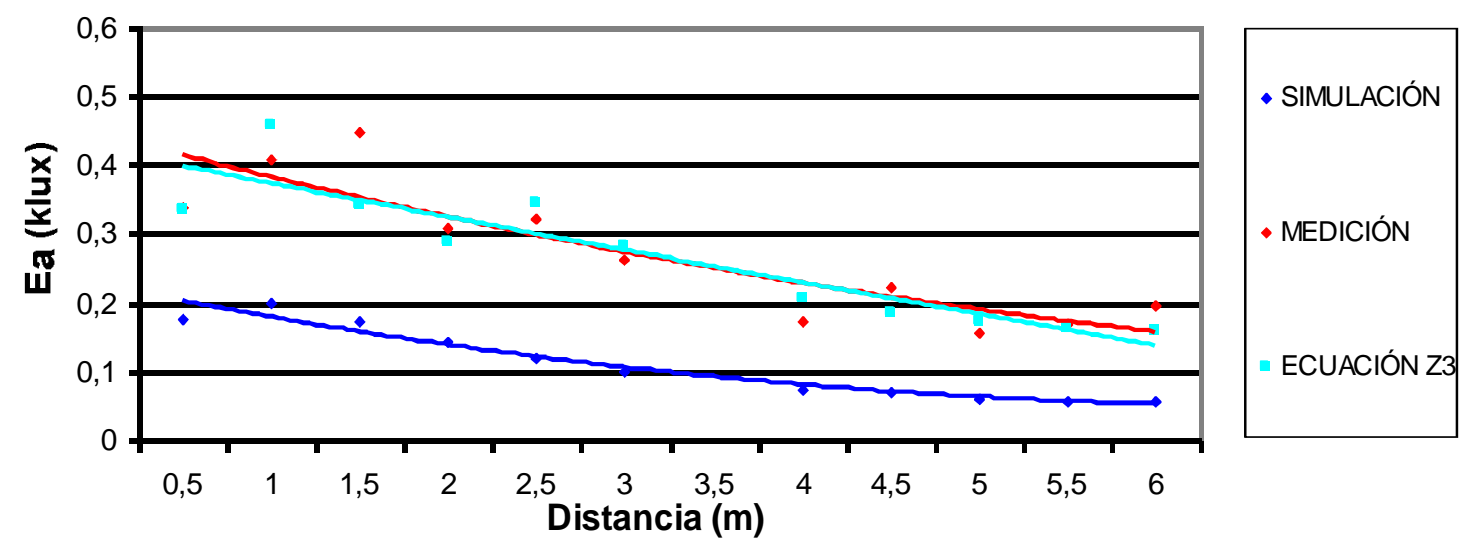

(d) 14:00.
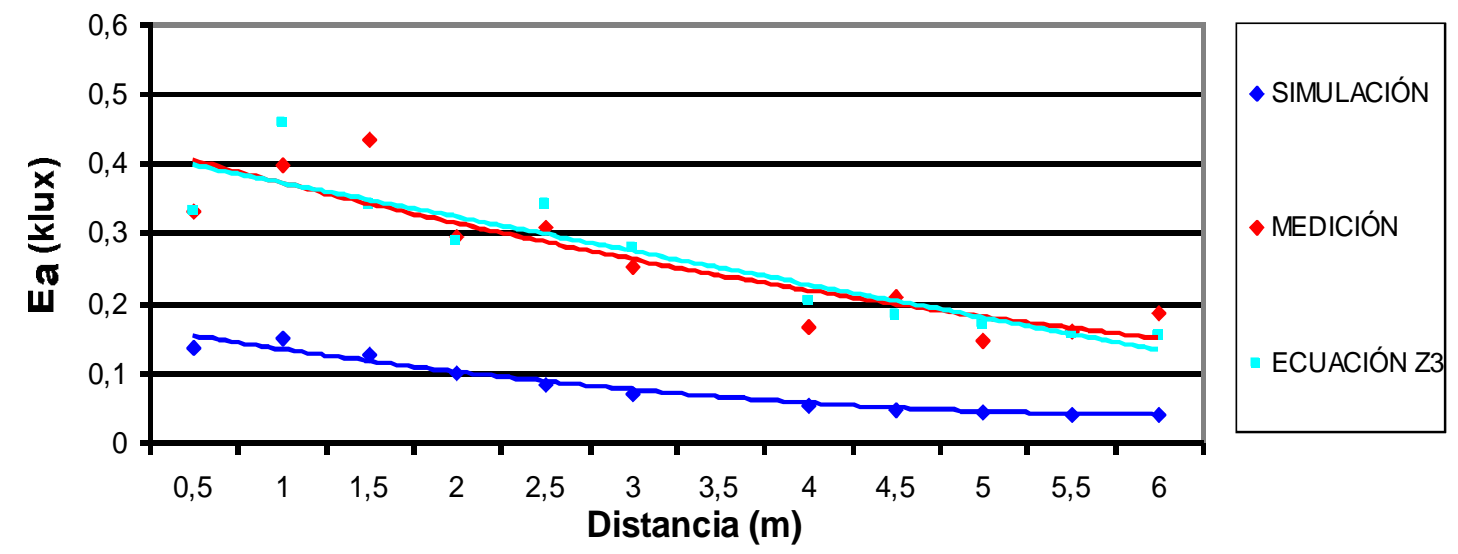

(e) 15:00.

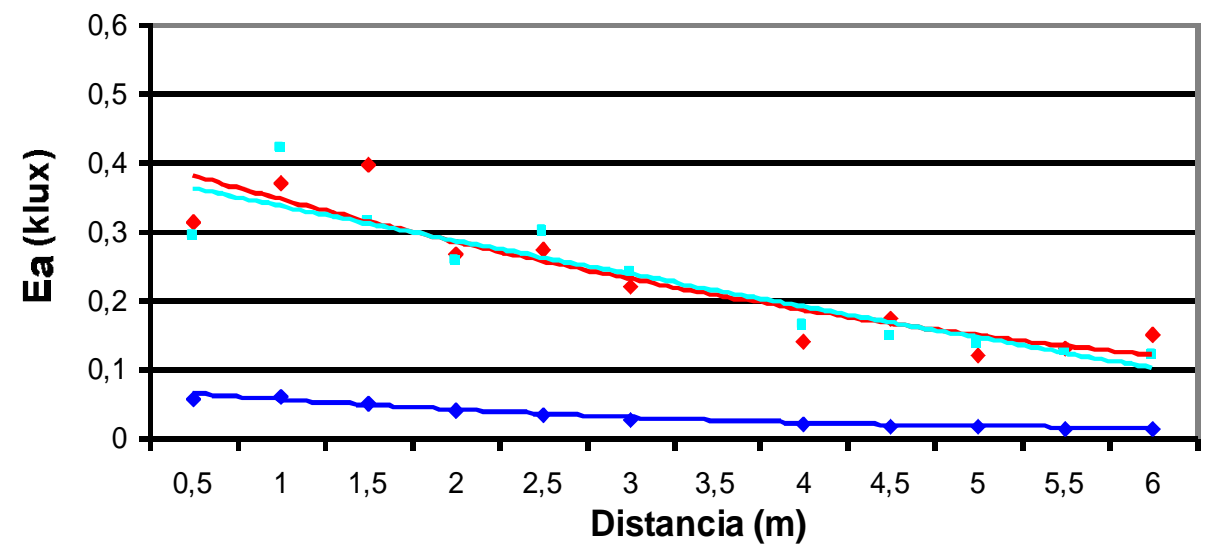

- SIMULACIÓN

- MEDICIÓN

ECUACIÓN Z3

(f) 16:00.

Figura 10.2.6. Curvas ajustadas de las iluminancias del aula recogidas en los diagramas de barras de la figura 6.56 a las distancias consideradas. 
Las ecuaciones correspondientes a las curvas de la figura 10.2.6 son las siguientes:

\begin{tabular}{|c|c|c|}
\hline $11: 00$ & Ecuación & $\mathbf{r}^{2}$ \\
\hline Medición & $E_{a}=0,0012 d^{2}-0,0404 d+0,4406$ & 0,8269 \\
\hline Simulación & $E_{a}=0,0009 d^{2}-0,0252 d+0,2312$ & 0,9439 \\
\hline Ecuación & $E_{a}=-0,0002 d^{2}-0,0175 d+0,2633$ & 0,8698 \\
\hline
\end{tabular}

(a) $11: 00$.

\begin{tabular}{|c|c|c|}
\hline $12: 00$ & Ecuación & $\mathbf{r}^{2}$ \\
\hline Medición & $E_{a}=0,0009 d^{2}-0,0358 d+0,4468$ & 0,7911 \\
\hline Simulación & $E_{a}=0,0009 d^{2}-0,0263 d+0,249$ & 0,9373 \\
\hline Ecuación & $E_{a}=-0,0003 d^{2}-0,0153 d+0,2411$ & 0,8545 \\
\hline
\end{tabular}

(b) $12: 00$.

\begin{tabular}{|c|c|c|}
\hline $13: 00$ & Ecuación & $r^{2}$ \\
\hline Medición & $E_{a}=0,0008 d^{2}-0,0337 d+0,4469$ & 0,7766 \\
\hline Simulación & $E_{a}=0,0009 d^{2}-0,0265 d+0,2476$ & 0,9394 \\
\hline Ecuación & $E_{a}=-0,0003 d^{2}-0,0143 d+0,2249$ & 0,847 \\
\hline
\end{tabular}

(c) $13: 00$.

\begin{tabular}{|c|c|c|}
\hline $14: 00$ & Ecuación & $r^{2}$ \\
\hline Medición & $E_{a}=0,0008 d^{2}-0,0336 d+0,4459$ & 0,7793 \\
\hline Simulación & $E_{a}=0,0009 d^{2}-0,0257 d+0,2258$ & 0,9491 \\
\hline Ecuación & $E_{a}=-0,0002 d^{2}-0,0156 d+0,2322$ & 0,8545 \\
\hline
\end{tabular}

(d) $14: 00$.

\begin{tabular}{|c|c|c|}
\hline $15: 00$ & Ecuación & $r^{2}$ \\
\hline Medición & $E_{a}=0,0008 d^{2}-0,0342 d+0,4367$ & 0,7897 \\
\hline Simulación & $E_{a}=0,0008 d^{2}-0,0214 d+0,1723$ & 0,9595 \\
\hline Ecuación & $E_{a}=-0,0002 x^{2}-0,0168 d+0,2479$ & 0,8648 \\
\hline
\end{tabular}

(e) $15: 00$.

\begin{tabular}{|c|c|c|}
\hline $16: 00$ & Ecuación & $r^{2}$ \\
\hline Medición & $E_{a}=0,001 d^{2}-0,0371 d+0,4154$ & 0,8277 \\
\hline Simulación & $E_{a}=0,0004 d^{2}-0,0096 d+0,0723$ & 0,9697 \\
\hline Ecuación & $E_{a}=-3 \times 10^{-5} d^{2}-0,0189 d+0,2467$ & 0,8747 \\
\hline
\end{tabular}

(f) $16: 00$.

Tabla 10.2.6. Ecuaciones de ajuste diagrama de barras de las iluminancias obtenidas en el aula, modelo a escala, simulación y aplicando la ecuación zona 3 a las distancias y horas consideradas el día 05/12/2015. 
Las curvas ajustadas del estudio comparativo de las iluminancias del aula obtenidas midiendo directamente en el aula, mediante la ecuación Z3 y mediante simulación representadas en la figura 6.56 y 6.57 se muestran a continuación:
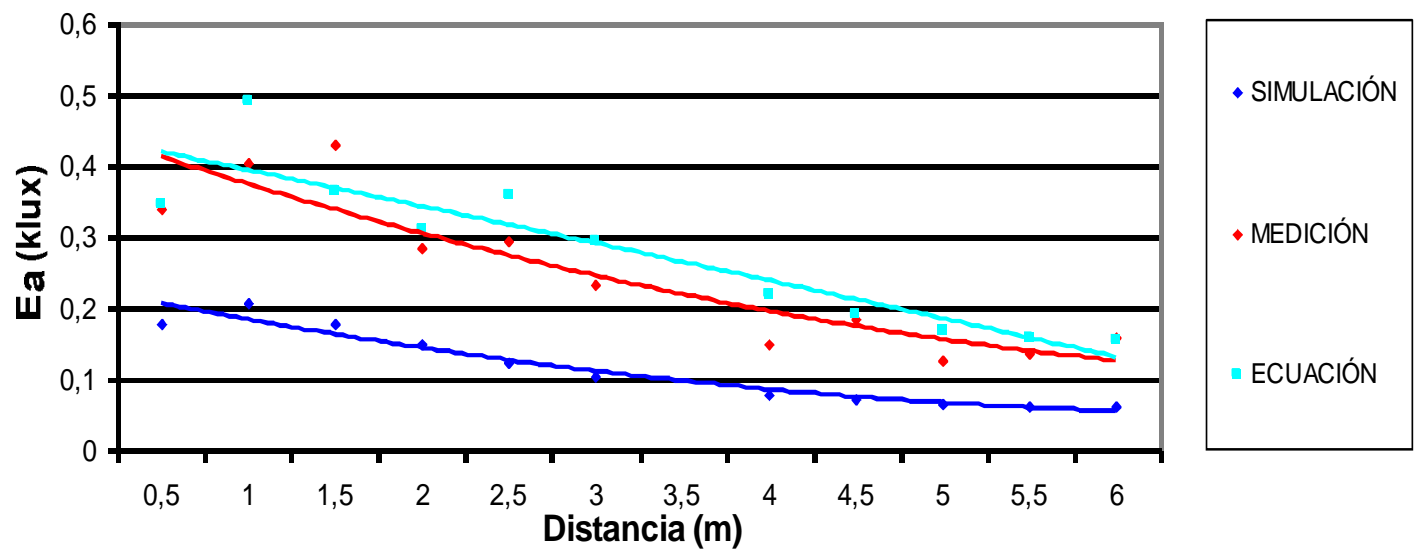

(a) $11: 00$
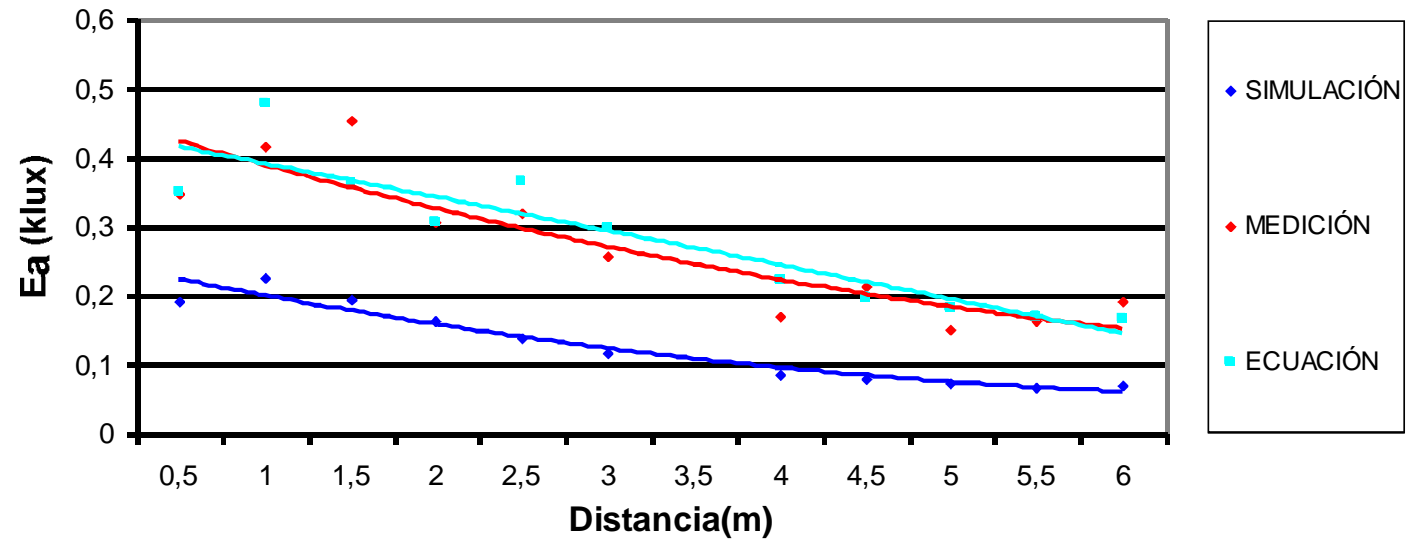

(b) $12: 00$
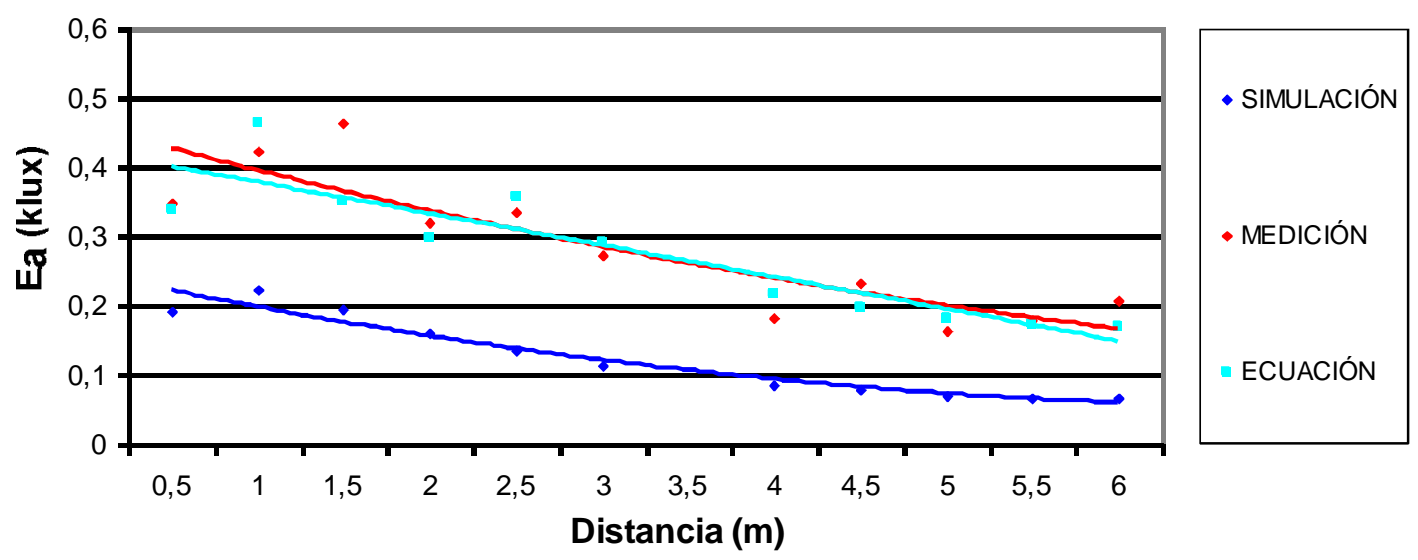

(c) $13: 00$ 

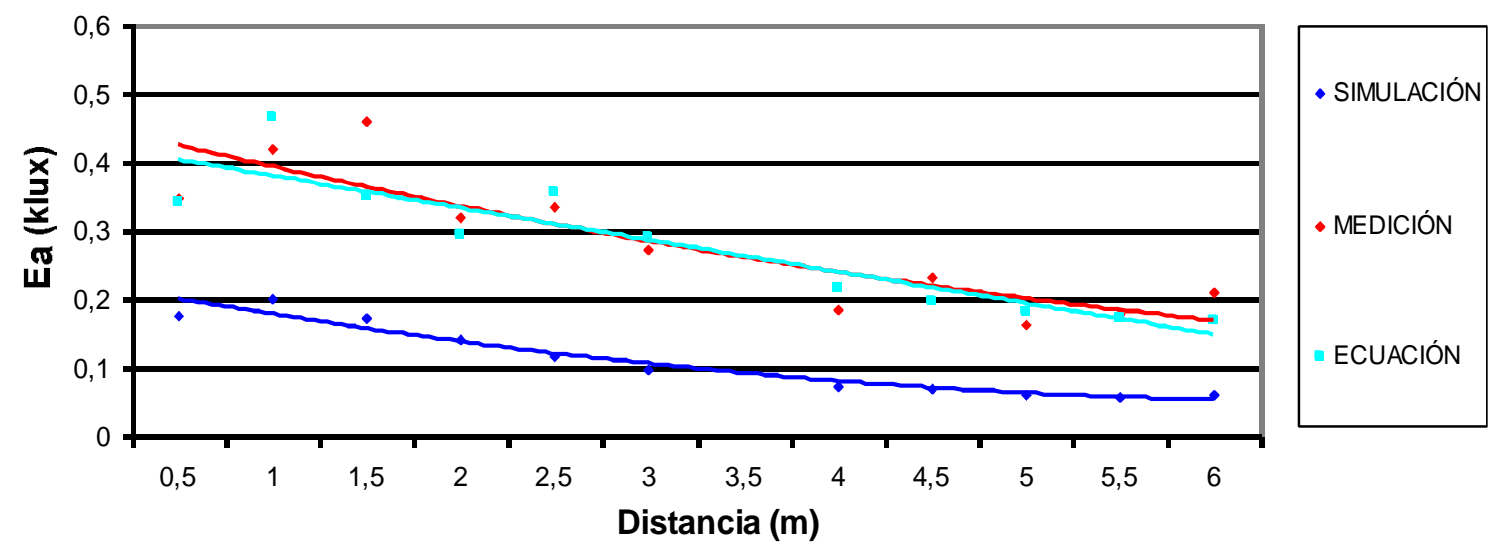

(d) 14:00.

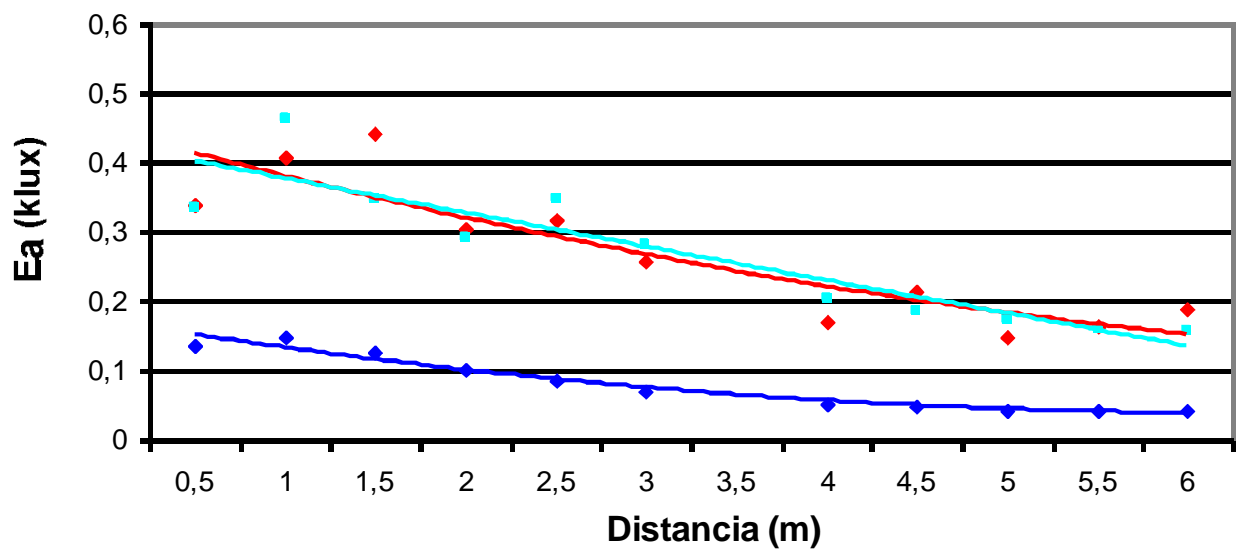

- SIMULACIÓN

- MEDICIÓN

ECUACIÓN

(e) 15:00.

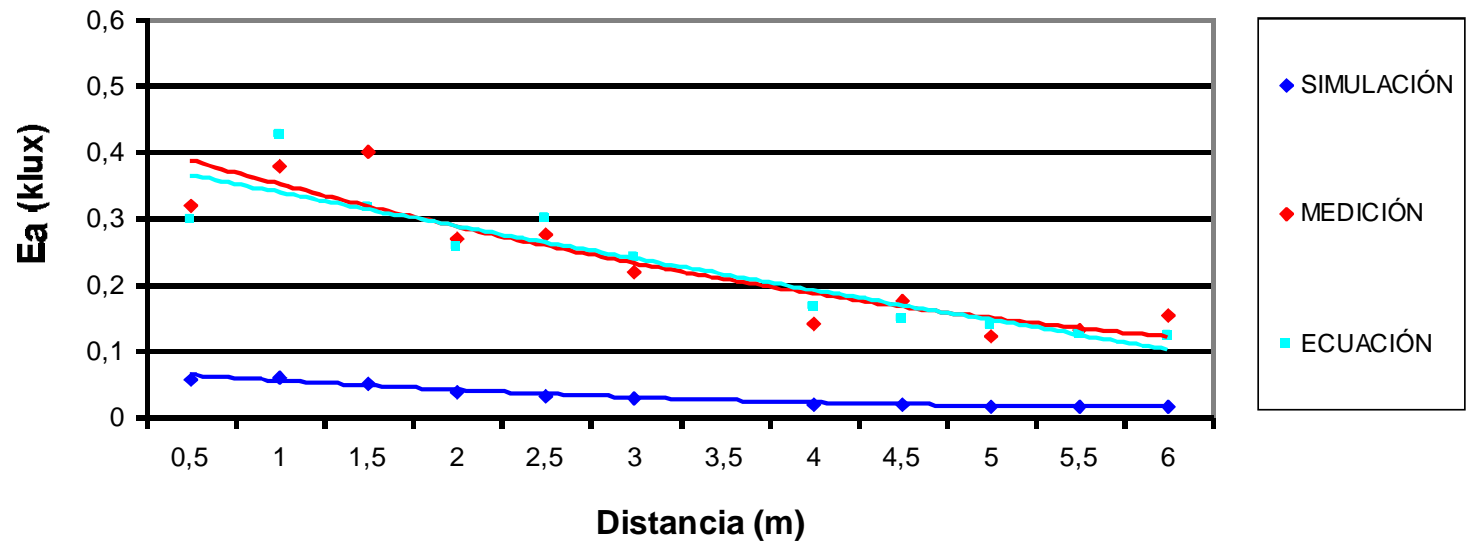

(f) 16:00.

Figura 10.2.5. Curvas ajustadas de las iluminancias del aula recogidas en los diagramas de barras de la figura 6.57 a las distancias consideradas. 
Las ecuaciones correspondientes a las curvas de la figura 10.2.5 son las siguientes:

\begin{tabular}{|c|c|c|}
\hline $11: 00$ & Ecuación & $\mathbf{r}^{2}$ \\
\hline Medición & $E_{a}=0,0012 d^{2}-0,0423 d+0,4536$ & 0,8324 \\
\hline Simulación & $E_{a}=0,0009 d^{2}-0,0252 d+0,2302$ & 0,9437 \\
\hline Ecuación & $E_{a}=-0,0003 d^{2}-0,0176 d+0,2668$ & 0,8683 \\
\hline
\end{tabular}

(a) $11: 00$.

\begin{tabular}{|c|c|c|}
\hline $12: 00$ & Ecuación & $\mathbf{r}^{2}$ \\
\hline Medición & $E_{a}=0,001 d^{2}-0,0376 d+0,4597$ & 0,797 \\
\hline Simulación & $E_{a}=0,0009 d^{2}-0,0261 d+0,2478$ & 0,9374 \\
\hline Ecuación & $E_{a}=-0,0003 d^{2}-0,0153 d+0,2449$ & 0,8542 \\
\hline
\end{tabular}

(b) 12:00.

\begin{tabular}{|c|c|c|}
\hline $13: 00$ & Ecuación & $\mathbf{r}^{2}$ \\
\hline Medición & $E_{a}=0,0008 d^{2}-0,0339 d+0,459$ & 0,7695 \\
\hline Simulación & $E_{a}=0,0009 d^{2}-0,0263 d+0,2463$ & 0,9389 \\
\hline Ecuación & $E_{a}=-0,0003 d^{2}-0,0135 d+0,2263$ & 0,8405 \\
\hline
\end{tabular}

(c) 13:00.

\begin{tabular}{|c|c|c|}
\hline $14: 00$ & Ecuación & $r^{2}$ \\
\hline Medición & $E_{a}=0,0008 d^{2}-0,0337 d+0,4568$ & 0,7693 \\
\hline Simulación & $E_{a}=0,0009 d^{2}-0,0256 d+0,2248$ & 0,948 \\
\hline Ecuación & $E_{a}=-0,0003 d^{2}-0,0147 d+0,2342$ & 0,846 \\
\hline
\end{tabular}

(d) 14:00.

\begin{tabular}{|c|c|c|}
\hline $15: 00$ & Ecuación & $r^{2}$ \\
\hline Medición & $E_{a}=0,0009 d^{2}-0,0358 d+0,4479$ & 0,7936 \\
\hline Simulación & $E_{a}=0,0008 d^{2}-0,0212 d+0,1711$ & 0,9594 \\
\hline Ecuación & $E_{a}=-0,0002 d^{2}-0,0169 d+0,251$ & 0,864 \\
\hline
\end{tabular}

(e) 15:00.

\begin{tabular}{|c|c|c|}
\hline $16: 00$ & Ecuación & $r^{2}$ \\
\hline Medición & $E_{a}=0,0011 d^{2}-0,0388 d+0,4232$ & 0,8347 \\
\hline Simulación & $E_{a}=0,0004 d^{2}-0,0094 d+0,0708$ & 0,9692 \\
\hline Ecuación & $E_{a}=-1 \times 10^{-5} d^{2}-0,0192 d+0,2489$ & 0,8745 \\
\hline
\end{tabular}

(f) $16: 00$.

Tabla 10.2.5. Ecuaciones de ajuste diagrama de barras de las iluminancias obtenidas en el aula, modelo a escala, simulación y aplicando la ecuación zona 3 a las distancias y horas consideradas el día 06/12/2015. 


\subsubsection{Zona 4}

Las curvas ajustadas del estudio comparativo de las iluminancias del aula obtenidas midiendo directamente en el aula, mediante la ecuación Z4 y mediante simulación representadas en la figura 6.74 se muestra a continuación:

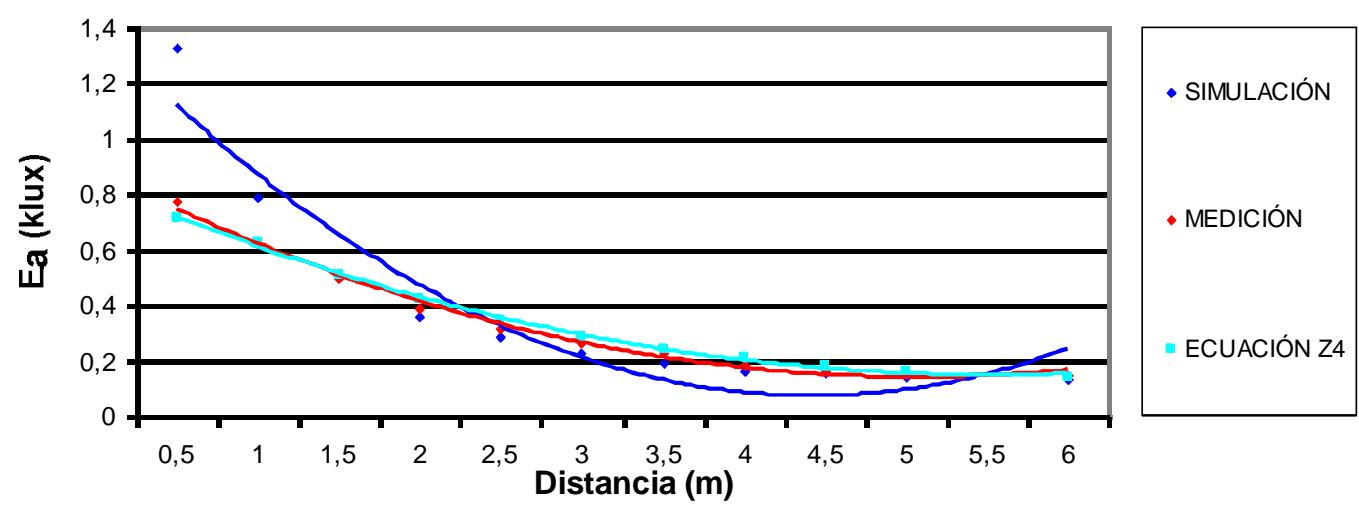

(a) 11:00.
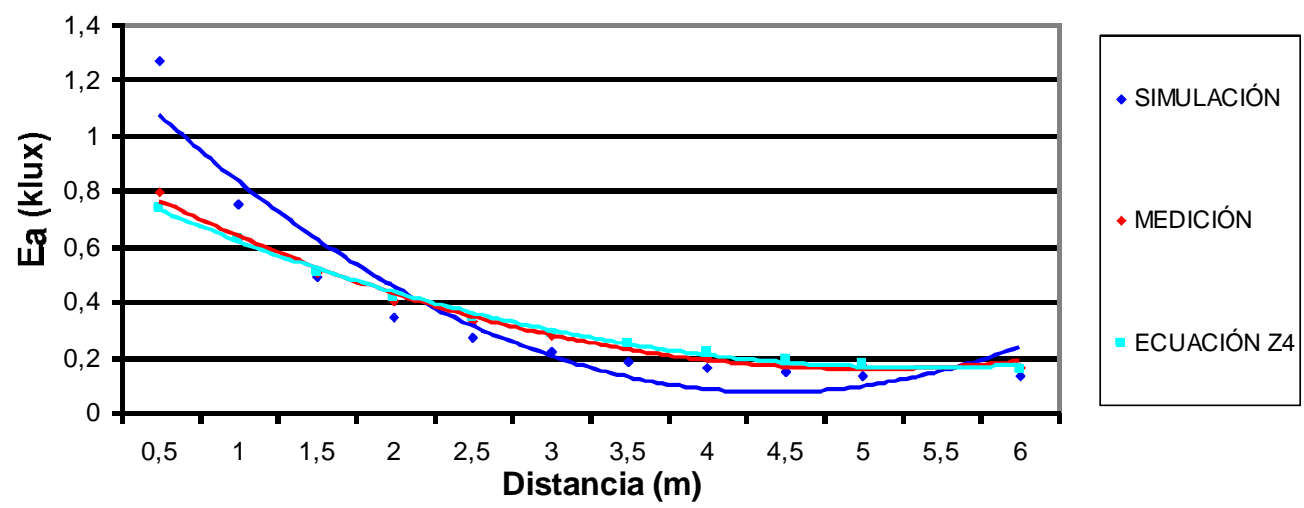

(b) $12: 00$
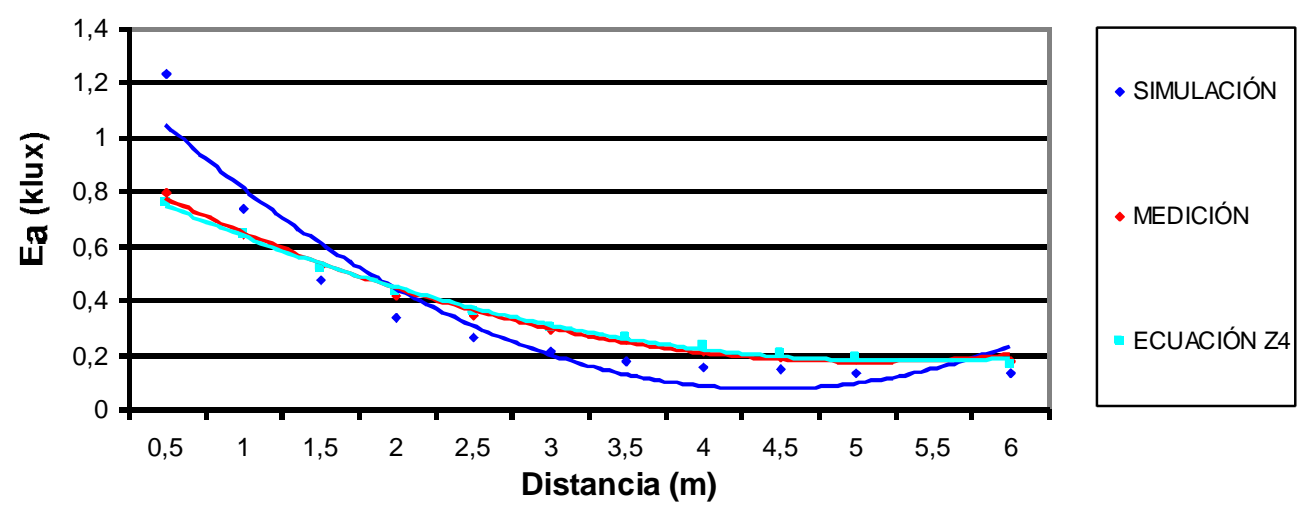

(c) $13: 00$ 

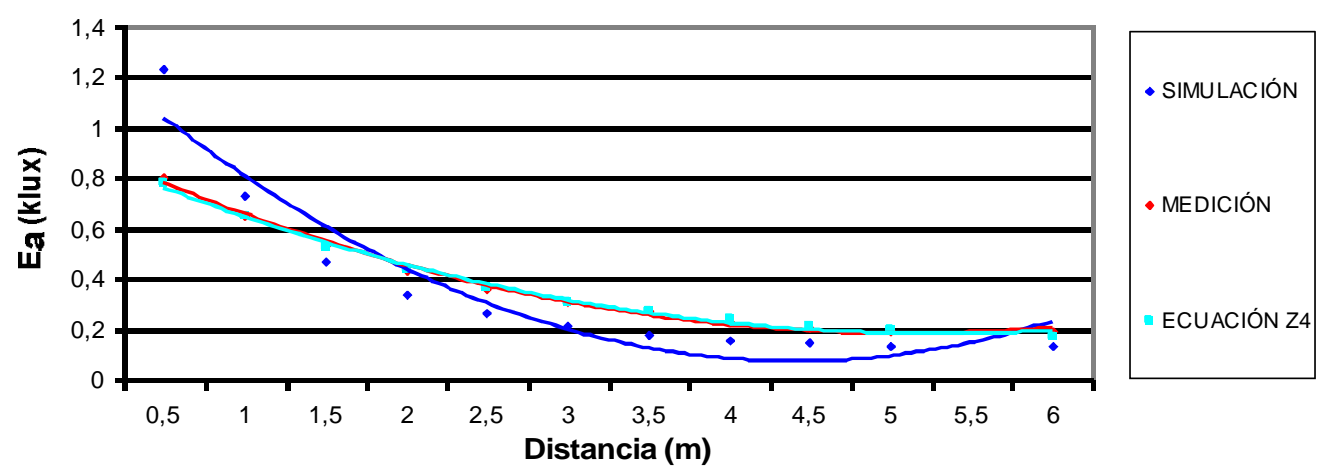

(d) 14:00.
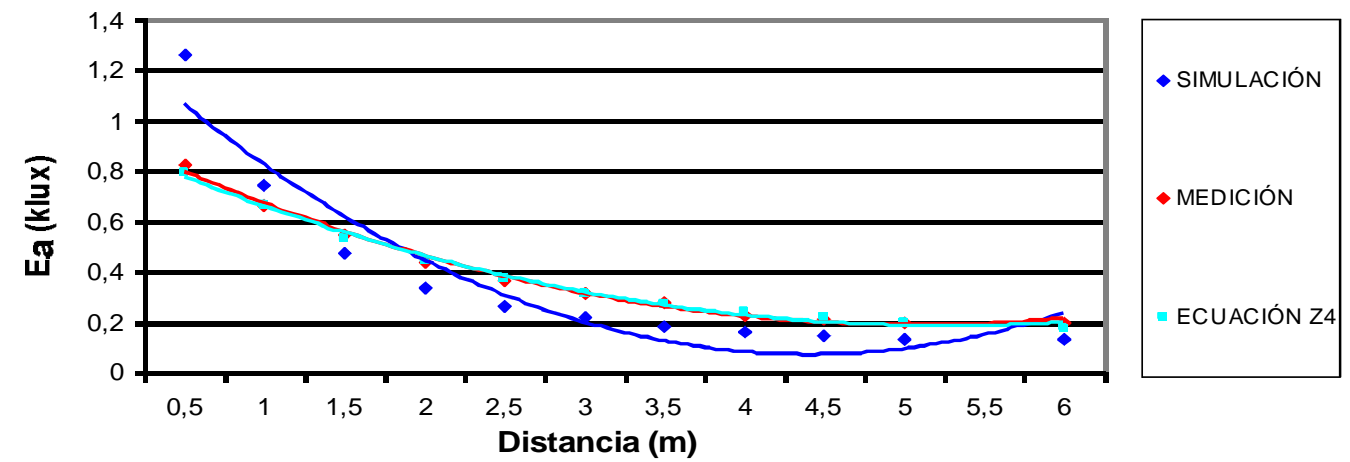

(e) 15:00
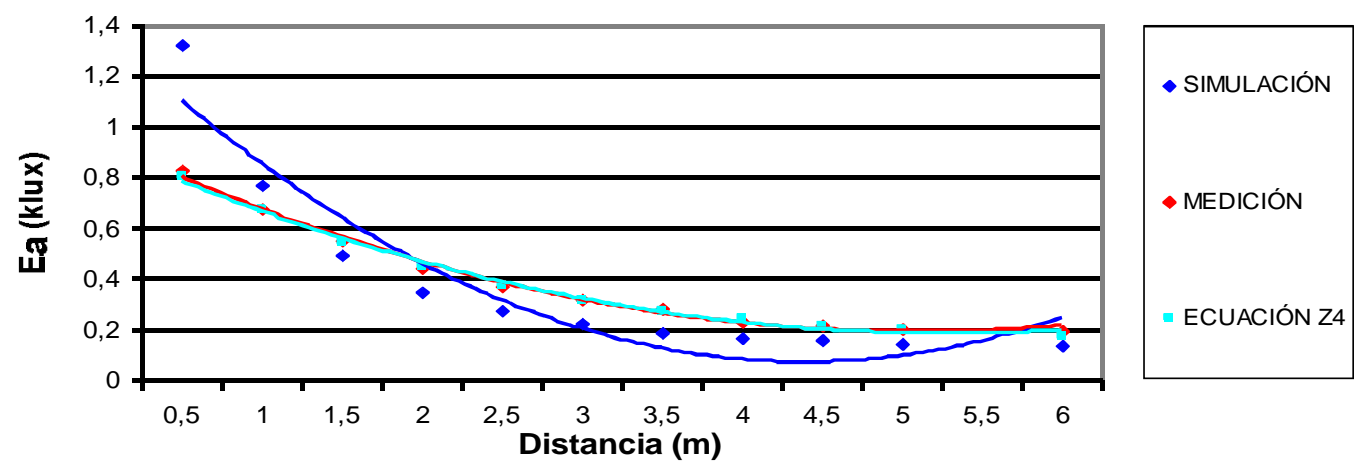

(f) $16: 00$.

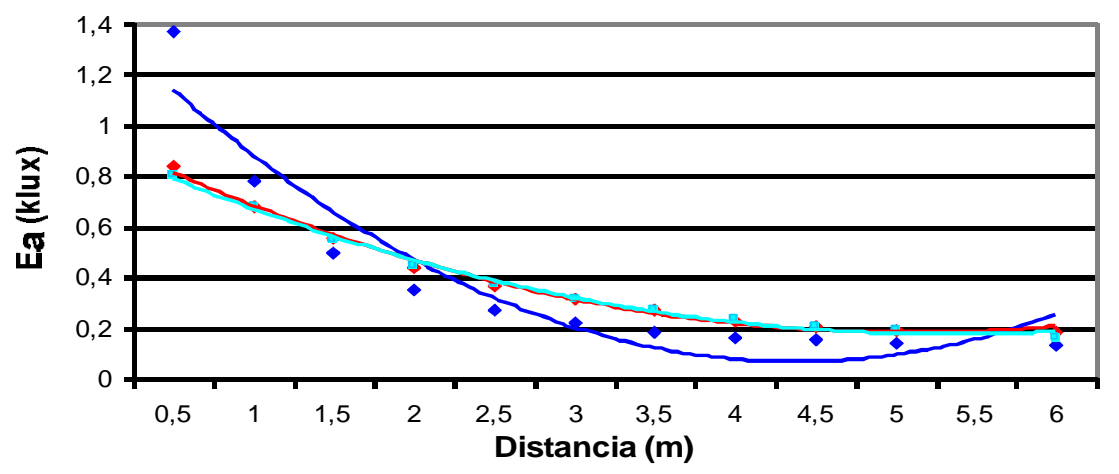

- SIMULACIÓN

- MEDICIÓN

ECUACIÓN Z4

(g) 17:00.

Figura 10.2.7. Curvas ajustadas de las iluminancias del aula recogidas en los diagramas de barras de la figura 6.74 a las distancias consideradas. 
Las ecuaciones correspondientes a las curvas de la figura 10.2.7 son las siguientes:

\begin{tabular}{|c|c|c|}
\hline $11: 00$ & Ecuación & $\mathbf{r}^{2}$ \\
\hline Medición & $E_{a}=0,0073 d^{2}-0,1474 d+0,8859$ & 0,9935 \\
\hline Simulación & $E_{a}=0,0171 d^{2}-0,3018 d+1,4055$ & 0,9153 \\
\hline Ecuación & $E_{a}=0,0056 d^{2}-0,1248 d+0,837$ & 0,9966 \\
\hline
\end{tabular}

(a) $11: 00$.

\begin{tabular}{|c|c|c|}
\hline $12: 00$ & Ecuación & $\mathbf{r}^{2}$ \\
\hline Medición & $\mathrm{E}_{\mathrm{a}}=0,0074 \mathrm{~d}^{2}-0,1489 \mathrm{~d}+0,9034$ & 0,9923 \\
\hline Simulación & $\mathrm{E}_{\mathrm{a}}=0,0163 \mathrm{~d}^{2}-0,2884 \mathrm{~d}+1,344$ & 0,9156 \\
\hline Ecuación & $\mathrm{E}_{\mathrm{a}}=0,006 \mathrm{~d}^{2}-0,1283 \mathrm{~d}+0,8496$ & 0,9957 \\
\hline
\end{tabular}

(b)12:00.

\begin{tabular}{|c|c|c|}
\hline $13: 00$ & Ecuación & $r^{2}$ \\
\hline Medición & $E_{a}=0,0072 d^{2}-0,1459 d+0,9096$ & 0,9937 \\
\hline Simulación & $E_{a}=0,0158 d^{2}-0,2796 d+1,3052$ & 0,9158 \\
\hline Ecuación & $E_{a}=0,0061 d^{2}-0,1308 d+0,8698$ & 0,9948 \\
\hline
\end{tabular}

(c) 13:00.

\begin{tabular}{|c|c|c|}
\hline $\mathbf{1 4 : 0 0}$ & Ecuación & r2 \\
\hline Medición & $E_{a}=0,0071 d^{2}-0,1444 d+0,9164$ & 0,9946 \\
\hline Simulación & $E_{a}=0,0158 d_{2}-0,2791 d+1,3019$ & 0,9147 \\
\hline Ecuación & $E_{a}=0,0063 d_{2}-0,1329 d+0,8857$ & 0,9941 \\
\hline
\end{tabular}

(d) $14: 00$.

\begin{tabular}{|c|c|c|}
\hline $15: 00$ & Ecuación & $\mathbf{r}^{2}$ \\
\hline Medición & $\mathrm{E}_{\mathrm{a}}=0,0073 \mathrm{~d}^{2}-0,1484 \mathrm{~d}+0,9388$ & 0,9937 \\
\hline Simulación & $\mathrm{E}_{\mathrm{a}}=0,0162 \mathrm{~d}^{2}-0,2865 \mathrm{~d}+1,3319$ & 0,9119 \\
\hline Ecuación & $\mathrm{E}_{\mathrm{a}}=0,0065 \mathrm{~d}^{2}-0,1372 \mathrm{~d}+0,9063$ & 0,9933 \\
\hline
\end{tabular}

(e) 15:00.

\begin{tabular}{|c|c|c|}
\hline $16: 00$ & Ecuación & $r^{2}$ \\
\hline Medición & $E_{a}=0,0073 d^{2}-0,1482 d+0,9405$ & 0,9943 \\
\hline Simulación & $E_{a}=0,0171 d^{2}-0,2999 d+1,3856$ & 0,9076 \\
\hline Ecuación & $E_{a}=0,0065 d^{2}-0,1387 d+0,9147$ & 0,9938 \\
\hline
\end{tabular}

(f) $16: 00$.

\begin{tabular}{|c|c|c|}
\hline $17: 00$ & Ecuación & $r^{2}$ \\
\hline Medición & $E_{a}=0,0075 d^{2}-0,1529 d+0,9568$ & 0,9945 \\
\hline Simulación & $E_{a}=0,0179 d^{2}-0,313 d+1,4347$ & 0,9025 \\
\hline Ecuación & $E_{a}=0,0066 d^{2}-0,1405 d+0,9221$ & 0,9949 \\
\hline
\end{tabular}

(g) $17: 00$.

Tabla 10.2.7. Ecuaciones de ajuste diagrama de barras de las iluminancias obtenidas en el aula, modelo a escala, simulación y aplicando la ecuación zona 4 a las distancias y horas consideradas el día 21/07/2015. 